Graduiertenkolleg Interdisziplinäre Umweltgeschichte

Patrick Masius, Ole Sparenberg und Jana Sprenger (Hg.)

\title{
Umweltgeschichte und Umweltzukunft
}

Zur gesellschaftlichen Relevanz einer jungen Disziplin

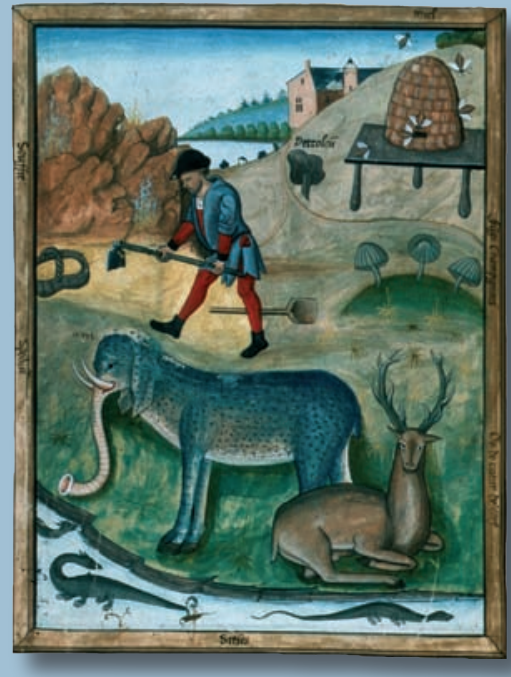



Patrick Masius, Ole Sparenberg und Jana Sprenger (Hg.) Umweltgeschichte und Umweltzukunft

This work is licensed under the Creative Commons License 2.0 "by-nd", allowing you to download, distribute and print the document in a few copies for private or educational use, given that the document stays unchanged and the creator is mentioned. You are not allowed to sell copies of the free version. 
erschienen im Universitätsverlag Göttingen 2009 
Patrick Masius, Ole Sparenberg und Jana Sprenger (Hg.)

\section{Umweltgeschichte und Umweltzukunft}

Zur gesellschaftlichen Relevanz einer jungen Disziplin

Graduiertenkolleg Interdisziplinäre Umweltgeschichte

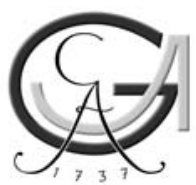

Universitätsverlag Göttingen 2009 


\section{Bibliographische Information der Deutschen Nationalbibliothek}

Die Deutsche Nationalbibliothek verzeichnet diese Publikation in der Deutschen Nationalbibliographie; detaillierte bibliographische Daten sind im Internet über $<$ http://dnb.ddb.de $>$ abrufbar.

\section{Anschrift der Herausgeber}

Graduiertenkolleg Interdisziplinäre Umweltgeschichte

Naturale Umwelt und gesellschaftliches Handeln in Mitteleuropa

Georg August Universität Göttingen

Bürgerstr. 50, 37073 Göttingen

http:/www.anthro.uni-goettingen.de/gk/

Gedruckt mit Unterstützung der Deutschen Forschungsgemeinschaft $\mathbf{D}=\mathbf{G}$

Dieses Buch ist auch als freie Onlineversion über die Homepage des Verlags sowie über den OPAC der Niedersächsischen Staats- und Universitätsbibliothek (http://www.sub.uni-goettingen.de) erreichbar und darf gelesen, heruntergeladen sowie als Privatkopie ausgedruckt werden. Es gelten die Lizenzbestimmungen der Onlineversion. Es ist nicht gestattet, Kopien oder gedruckte Fassungen der freien Onlineversion zu veräußern.

Satz und redaktionelle Bearbeitung: Patrick Masius, Ole Sparenberg, Jana Sprenger Umschlaggestaltung: Margo Bargheer, Jutta Pabst

Titelabbildung: Titelbild unter freundlich genehmigter Verwendung einer Abbildung aus MS 12322 Bibliothèque Nationale Paris, Section des Manuscriptes Occidentaux.

(C) 2009 Universitätsverlag Göttingen

http:/ / univerlag.uni-goettingen.de

ISBN: 978-3-940344-69-4 


\section{Inhaltsverzeichnis}

Vorwort

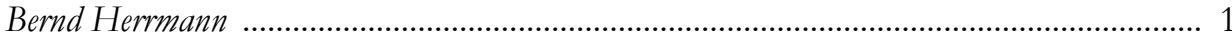

Einleitung: Verständnis und Institutionalisierung der

Umweltgeschichte

Lars Kreye, Markeus Schwarzer ......................................................................................... 3

\section{Spektrum der Umweltgeschichte}

Umweltgeschichte wozu?

Zur gesellschaftlichen Relevanz einer jungen Disziplin

Bernd Hermann

Bilder, die die Umwelt bewegten:

Naturwahrnehmung und Politik in der US-amerikanischen

Geschichte

Christof Mauch

Innenwelt der Umweltpolitik - Zu Geburt und Aufstieg eines

Politikbereichs

Edda Müller

Umweltgeschichte und Altlasten: zur anhaltenden Relevanz gefährdender Stoffe

Klaus Schlottau 87 


\section{Aus der Geschichte lernen}

Das Kyoto-Protokoll, oder: Was lässt sich aus der Geschichte umweltpolitischer Regulierung lernen?

Frank Uekötter 161

Naturkatastrophen: Was wurde aus ihnen gelernt?

Manfred Jakubowski-Tiessen

Invasive Arten - Freisetzungsexperimente in Vergangenheit und

Gegenwart

Josef H. Reichbolf

\section{Fast vergessene Debatten der Umweltgeschichte}

Was macht eigentlich das Waldsterben?

Roland Schäfer, Birgit Metzger 201

Was macht eigentlich das Geschwindigkeitslimit?

Kurt Möser 229

\section{Umweltgeschichte im Bildungssystem}

Wie vermittelt man Umweltgeschichte in der Schule?

Bodo von Borries

„Warum wir Umweltgeschichte studieren und erforschen“

Markus Schwarzer, Ole Sparenberg 259

Autoren 269 


\title{
Vorwort
}

\author{
Bernd Herrmann
}

Die vorliegende Veröffentlichung fasst Beiträge zum Workshop desselben Titels zusammen, der am 16. und 17. Juli 2008 in Göttingen stattfand. Organisiert wurde er durch das Göttinger Graduiertenkolleg „Interdisziplinäre Umweltgeschichte“. Der Ursprung dieses Workshops ging zurück auf einen früheren, der im März 2007 im Graduiertenkolleg zum Thema „Umweltgeschichte - Akademische Professionalisierung und Berufsfelder" durchführt wurde. Dort diskutierten Vertreter von Umwelteinrichtungen und Körperschaften der Politikberatung mit den Kollegiaten ${ }^{1}$ über deren Möglichkeiten, ihre Expertise, und damit sich selbst, zielgerichtet in die Berufswelt einzubringen. Der vormalige Präsident des Niedersächsischen Landesamtes für Ökologie, Meinfried Striegnitz, warf die provokante Frage auf, warum denn auf der Seite der Umwelt- und Politikberater das Bewusstsein für umwelthistorische Problemanalysen praktisch fehle. Die sich anschließende Diskussion spitzte die Frage sinngemäß auf die Formulierung zu „Welche Relevanz hat die Umweltgeschichte?" Die teilnehmenden Vertreter der Bundesstiftung Umwelt und der Volkswagenstiftung signalisierten spontan die Bereitschaft ihrer Einrichtungen, zu diesem Thema eine Anschluss-Tagung zu fördern. Sie ließen sich in der Zeit der auslaufenden Förderung der ersten Kollegiaten-Kohorte nicht mehr realisieren. Einige Kollegiaten waren zudem skeptisch, ob die Frage der gesellschaftlichen Relevanz für ihre eigene Forschungsarbeit Bedeutung habe, weil sich

\footnotetext{
${ }^{1}$ Hier, wie in allen folgenden Fällen dieses Aufsatzes/Buches, ist mit der männlichen stets auch die weibliche Form gemeint.
} 
Bernd Herrmann

Bewertungen und Verwertungszusammenhänge ihrer Forschungserträge allein aus dem fachlichen Umfeld ergäben, dem sie sich zugehörig fühlten.

Im nachfolgenden Sommer ist es dann auf der ESEH-Tagung in Amsterdam zur Planungsgruppe für diesen Workshop gekommen, mit Dorothee Brantz (Berlin), Christof Mauch (München), Joachim Radkau (Bielefeld), Frank Uekötter (München) und mir. Mein erster Dank gilt den beteiligten Kollegen für ihr Engagement. Die Deutsche Forschungsgemeinschaft hat das Göttinger Kolleg in die Lage versetzt, zu diesem Workshop einladen zu können. Mein zweiter Dank gilt folgerichtig der DFG. Workshops kommen bekanntermaßen nicht ohne Beiträge aus. Diejenigen Kollegen, die sich bereitwillig der Leitidee des Workshops zur Verfügung stellten, brachten das Wertvollste überhaupt mit, worüber Wissenschaftler verfügen: ihre Zeit und ihre Gedanken. Mein dritter Dank, das Zahlwort bezeichnet hier wie in den anderen Fällen eine Reihe und nicht die Rangordnung, gilt den Referenten. Und dann ist - viertens - unseren jetzigen Kollegiaten zu danken. Nicht nur, dass sie die Leitidee des Workshops teilten und sich inhaltlich beteiligten, sondern auch dafür, dass sie sich mit Engagement und Umsicht der technischen Realisierung des Workshops angenommen hatten und den Verlauf des Treffens als Moderatoren mitbestimmten.

Dass wir mit dem Workshop die historische wie die umwelthistorische Welt neu aufstellten, war weder beabsichtigt noch wäre es mit den Mitteln dieses Workshops erreichbar gewesen. Aber anregen wollten wir schon, in der Begeisterung für die wissenschaftliche Fragestellung gelegentlich innezuhalten, um sich oder anderen Rechenschaft über die Arbeit geben zu können. Und es sollte Einsicht, sollte Erkenntnis gewonnen werden, selbst, wenn der Dichterspott wie auf den Workshop gemünzt klingt:

«Ach? Gibt's die noch? Gibt's noch Erkenntnis? » Fragte mein Widersacher. So als fragte er: Trägt man noch Galoschen heute? «Erkenntnis! Die alte Schelle, mit der uns Kongressleiter in den Ohren bimmeln. $»^{2}$

Das Herausgeben der Beiträge ist üblicherweise den Organisatoren eines Workshops vorbehalten. In diesem Falle haben die Initiatoren, auf Bitte des Graduiertenkollegs, dankenswerter Weise die Herausgeberschaft an das von den Kollegiaten benannte Redaktionskomitee abgetreten. Das Einüben in und von Herausgebertätigkeiten entspricht einer in unserem Kolleg verfolgten Selbstprofessionalisierungspraxis. Ich danke den Kollegiaten Patrick Masius, Ole Sparenberg, und Jana Sprenger für die Übernahme dieser Aufgabe und danke den anderen Kollegiaten wie auch dem zweiten Sprecher des Kollegs, Herrn Kollegen JakubowskiTiessen, für ihre vielfältige Unterstützung.

2 Es handelt sich um Eingangssätze zur Kurzgeschichte „Staustufe“ von Botho Strauß, in der er ein Elementarereignis mit Konsequenzen für eine Traumlandschaft beschreibt. Beides fügt sich thematisch zu unserem Workshop, sowohl die Verwüstung von Landschaften als auch die spöttischselbstironisierende Frage nach der Erkenntnis. In: Botho Strauß, Mikado. Hanser, München Wien 2006, S. 171. 


\title{
Einleitung: Verständnis und Institutionalisierung der Umweltgeschichte
}

\author{
Lars Kreye und Markus Schwarzer
}

\section{Einleitung}

Klimawandel, Hungerkrise, Artensterben und Naturkatastrophen bestimmen vielerorts die Debatten um die Zukunft unserer globalen Welt. Hierbei erscheint es oft, als seien diese Phänomene völlig neuartige Entwicklungen, obwohl viele der gegenwärtig diskutierten Fragen historisch bedingt sind. Demzufolge erfordert die Bewältigung solcher Probleme nicht nur zukunftsorientiertes Denken, sondern auch ein historisches Verständnis der politischen, ökonomischen, sozialen und ökologischen Hintergründe unserer gegenwärtigen Situation. In diesem Sinn eröffnete die Konferenz „Umweltgeschichte und Umweltzukunft”, die im Juli 2008 in Göttingen stattfand und deren Beiträge in diesem Band veröffentlicht werden, ein fächerübergreifendes Forum, in dem die gesellschaftliche Relevanz der Umweltgeschichte diskutiert wurde.

Dabei lässt sich die in der Ankündigung der Tagung aufgeworfene Frage „Was können wir aus der (Umwelt)Geschichte lernen?“ - heute nicht in der Weise beantworten, dass diese ein auf die Gegenwart unmittelbar übertrag- und anwendbares Erfahrungswissen bereitstellen kann. ${ }^{1}$ Geschichte wiederholt sich nicht. Auch wenn bestimmte Auslöser von Naturkatastrophen in gewissen Zyklen wiederkehren, so ist doch der gesellschaftliche und historische Kontext immer ein

1 Vgl. zum Problem des Lernens aus der Geschichte: Herzog, B. 2002 Historia magistra vitae. In: Lexikon Geschichtswissenschaft. Hundert Grundbegriffe. Hrsg. von S. Jordan. Stuttgart. S. 145-147. 
Lars Kreye, Markus Schwarzer

anderer. Daher lässt sich der Topos vom Lernen aus der Geschichte für die Umweltgeschichte vielmehr dahingehend verstehen, dass sie Grundlagen für ein kritisches Korrektiv aktueller Debatten bereitstellt, indem sie die historische Bedingtheit von Umweltproblemen und die Geschichtlichkeit der Debatten selbst beleuchtet. Durch Thematisierung der historischen Dimension kann die Umweltgeschichte mit wissenschaftlichen Grundlagen zur Lösung gesellschaftlicher Fragen beitragen. Diese gesellschaftlich wichtige Möglichkeit darf jedoch weder dazu verleiten, Umweltgeschichte als anwendungsorientierte Disziplin zu betrachten noch eine Politisierung umweltgeschichtlicher Konzepte anzustreben. Um sich als junge wissenschaftliche Disziplin oder interdisziplinärer Forschungszusammenhang zu etablieren ist dagegen der Grundsatz, den Claus Leggewie in die Diskussion um eine kulturwissenschaftliche Perspektive der Klimadebatte einbrachte, auch für die Umweltgeschichte weitaus angemessener: „Die Praxis von Wissenschaft ist Wissenschaft".2

Die beiden einzigen einführenden Überblicksdarstellungen zur Umweltgeschichte in deutscher Sprache, erschienen 2007, geben auf die Frage, was unter Umweltgeschichte zu verstehen sei, unterschiedliche Antworten. So fassen Verena Winiwarter und Martin Knoll Umweltgeschichte weit als ein ,als historisches Fachgebiet“" mit interdisziplinärem Charakter. ${ }^{3}$ Während sie betonen, dass Umweltgeschichte mit anderen Fächern im Austausch steht, haben in Frank Uekötters enger Fassung von Umweltgeschichte interdisziplinäre Bezüge keine Bedeutung; er versteht Umweltgeschichte als eine ,historische Subdisziplin“. ${ }^{4}$ Eine solch enge Auffassung erscheint zwar für seinen Gegenstandsbereich der Umweltgeschichte im 19. und 20. Jahrhundert möglich, über deren Probleme und Tendenzen er auf einer breiten Basis an Forschungsliteratur einen pointierten Überblick gibt. Insbesondere aber für die umwelthistorische Erforschung der vorindustriellen Zeit kann, wie Winiwarter und Knoll betonen, die Integration naturwissenschaftlich gewonnener Daten eine wichtige Ergänzung zu historischen Methoden sein. In diesem Sinne lassen sich auch die beiden Säulen von „Rekonstruktion und Rezeption“ des Graduiertenkollegs „Interdisziplinäre Umweltgeschichte“ verorten ${ }^{5}$, indem sie einer weit gefassten Interpretation von Umweltgeschichte entsprechen: „Umweltgeschichte beschäftigt sich mit der Rekonstruktion von Umweltbedingungen in der

\footnotetext{
2 Vgl. Ahaus, B. 2008 Tagungsbericht Schnee von gestern? Zivilisationskritike und Überlebensperspektiven in Zeiten des Klimawandels. In: H-Soz-u-Kult, http://hsozkult.geschichte.hu-berlin.de/ tagungsberichte/ $\mathrm{id}=2311(30.10 .2008)$.

${ }^{3}$ Winiwarter, V., Knoll, M. 2007 Umweltgeschichte. Eine Einführung. Köln, S. 14. Bernd Herrmann spricht lockerer formuliert vom „Wissenszusammenhang Umweltgeschichte“, siehe seinen Beitrag in diesem Band.

${ }^{4}$ Uekötter, F. 2007 Umweltgeschichte im 19. und 20. Jahrhundert. In: Enzyklopädie Deutscher Geschichte. Hrsg. L. Gall. München, S. 2. Diese einseitige Engführung Uekötters bietet den unausgesprochenen Vorteil, das Gebiet als ein spezifisch geschichtswissenschaftliches überschaubar zu halten. Problematisch ist jedoch daran, dass die Beiträge von „Nachbardisziplinen“ zur umwelthistorischen Forschung, die im weiten Sinn als interdisziplinärer Forschungszusammenhang zu begreifen ist, völlig ausgeklammert bleiben.

${ }^{5}$ Vgl. http://www.anthro.uni-goettingen.de/gk/ (30.10.2008).
} 
Vergangenheit sowie mit der Rekonstruktion von deren Wahrnehmung und Interpretation durch die damals lebenden Menschen"..6 Diese Definition lässt sich als programmatischer Rahmen begreifen, mit dem sowohl natur- als auch geistes- und gesellschaftswissenschaftliche Zugangsweisen zu einer interdisziplinären Umweltgeschichte angesprochen werden. Dabei geht es insbesondere darum naturwissenschaftliche Daten, wie sie beispielsweise mit Methoden der Paläo- bzw. Archäobotanik und -zoologie, der Bodenkunde oder der Klimatologie gewonnenen werden können, einzubeziehen. Denn solche Zugangsweisen können eine Form von „Biooder Geoarchiv“" erschließen, das eine grundsätzlich andere Qualität hat als übliche schriftliche Quellen oder Bilder. Damit solche Daten jedoch als umwelthistorische Quellen verwendet werden können, müssen die Bedingungen reflektiert werden, unter denen solche gemeinhin als „objektiv“ geltenden Daten gewonnen werden, d. h. es muss eine spezifische Art von „Quellenkritik“ betrieben werden, in der die positivistische Annahme, solche Daten seien historische „Tatsachen“, relativiert wird. ${ }^{7}$ Letztlich entscheiden die Fragestellung und die Untersuchungsperspektive einer umwelthistorischen Arbeit, ob die Option, naturwissenschaftliche Methoden in eine Untersuchung einzubeziehen, sinnvoll ist. In diesem Verständnis sind naturwissenschaftlich gewonnene Daten ein Spezifikum und ein Potential der Umweltgeschichte, die damit als Erweiterung geistes- oder gesellschaftswissenschaftlicher Zugänge zur Geschichte aufgefasst werden kann. Diese Erweiterung besteht jedoch nicht nur darin, selber mit den genannten Methoden Daten zu erzeugen, sondern ein wichtiger Schritt liegt gerade darin, solchermaßen gewonnene Forschungsergebnisse zu rezipieren und ein grundlegendes Verständnis für die im Kontext der Fragestellung relevanten naturwissenschaftlich orientierten Umweltwissenschaften zu gewinnen. Ohne ein solches Grundverständnis wären umwelthistorische Analysen der zunehmend verwissenschaftlichten Konzepte und Institutionen der Umweltpolitik im 20. Jahrhundert und damit auch die gesellschaftlich relevante Stellungnahme zu aktuellen Umweltproblemen nur unzureichend möglich. Um das Geflecht umweltrelevanter Diskurse, Institutionen und Akteure auf beispielsweise den Ebenen Wissenschaft, Politik, Recht und Öffentlichkeit zu untersuchen, liegt grundsätzlich ein Schwerpunkt umwelthistorischer Forschung auf der Rekonstruktion von Wahrnehmungen und Deutungen sowie handlungsleitenden Konzepten und Praktiken.

Aufgrund ihres interdisziplinären Charakters kann es auch nicht wundern, dass sich die Umweltgeschichte als Spezialfach im deutschsprachigen Raum bisher nicht durchgesetzt hat. Sie weist insgesamt einen geringen, aber steigenden Grad der Institutionalisierung auf. Dabei distanzierte sie sich nach Uekötter zunehmend von der zunächst engen Verbindung zur Umweltbewegung und entwickelte sich in mehreren sukzessiven Phasen mit zum Teil lebhafter Kritik. So konnten Fehlurtei-

\footnotetext{
${ }^{6}$ Diese Definition von Umweltgeschichte geht auf Bernd Herrmann und Rolf Peter Sieferle zurück; siehe Winiwarter, Knoll, Umweltgeschichte, S. 14-15 sowie Herrmann in diesem Band.

${ }^{7}$ Winiwarter, Knoll, Umweltgeschichte, S. 24-27.
} 
Lars Kreye, Markus Schwarzer

le und Sackgassen produktiv verarbeitet werden, wodurch ein professioneller Fortschritt der Wissenschaft erzielt worden sei. Dabei zeichnet sich die Umweltgeschichte in Deutschland insbesondere durch ihre Offenheit gegenüber Außenseitern aus - eine separierende Schulbildung erfolgte bisher nicht. ${ }^{8}$

So richtig diese Einschätzung ist, vergisst sie doch die institutionelle Verankerung der Umweltgeschichte in verschiedenen wissenschaftlichen Disziplinen und Fachöffentlichkeiten, wie sie beispielsweise in der Historischen Geographie oder den Fachgeschichten der Biologie sowie der Agrar- und Forstwissenschaften vorhanden ist. ${ }^{9}$ Dieses Strukturmerkmal der Umweltgeschichte im deutschsprachigen Raum hat weitreichende Konsequenzen. So sind umwelthistorische Beiträge oft heterogen sowohl im Hinblick auf die Themen als auch hinsichtlich des konzeptuellen Rahmens. Dies spiegelt sich auch in einer nahezu unüberschaubaren Fülle lokaler und regionaler Zeitschriften wider, die umwelthistorisch relevante Beiträge enthalten. Eine deutschsprachige Zeitschrift für Umweltgeschichte gibt es hingegen nicht, wobei neben den beiden mehrfach zitierten Einführungen und einer Weltumweltgeschichte von Joachim Radkau ${ }^{10}$ einige deutschsprachige Sammelbände existieren, in denen grundlegende Fragen der Umweltgeschichte erörtert werden. ${ }^{11}$ Während die Literaturlandschaft im deutschsprachigen Bereich somit als eher zersplittert gelten kann, zeichnet sich der anglo-amerikanische Sprachraum vor allem durch die beiden zentralen Zeitschriften Environmental History (USA) und Environment \& History (GB/Europa) und einen wesentlich höheren Institutionalisierungsgrad in den USA aus. ${ }^{12}$

Obwohl die umweltgeschichtliche Etablierung in den USA Vorbildcharakter hat, wurde in der Abschlussdiskussion der Tagung konstatiert, dass diese für die zukünftige akademische Entwicklung des Fachs im deutschsprachigen Raum, kein direktes Vorbild sein könne. Im Gegensatz zu den USA, wo der hohe Grad akademischer Differenzierung eigene Lehrstühle erlaubt, wird im deutschsprachigen Bereich die Umweltgeschichte wohl immer im Zusammenhang mit anderen The-

\footnotetext{
8 Vgl. Uekötter, Umweltgeschichte, S. 88 f.

${ }^{9}$ Diese interdisziplinären Bezüge betonen zu recht Winiwarter und Knoll, Umweltgeschichte, S. 15.

${ }_{10}$ Radkau, J. 2000 Natur und Macht. Eine Weltgeschichte der Umwelt. München.

11 Hier währen u. a. zu nennen: Umweltgeschichte. Umweltverträgliches Wirtschaften in historischer Perspektive. Acht Beiträge. Hrsg. von W. Abelshauser. Göttingen 1994; Umweltgeschichte - Methoden, Themen, Potentiale. Hrsg. von G. Bayerl et al. Münster 1996; Umweltgeschichte. Themen und Perspektiven. Hrsg. von W. Siemann. München 2003.

12 Die „American Society for Environmental History (ASEH)“ gründete sich bereits im Jahr 1977, während die „European Society for Environmental History (ESEH)“ sich erst im Jahr 2001 konstituierte. Weitere umwelthistorische Gesellschaften gibt es auch für Asien / Südostasien und Australien, wobei an dieser Stelle auch darauf hingewiesen sein soll, dass unter der Schirmherrschaft des „International Consortium of Environmental History Organizations (ICEHO)" im Jahr 2009 der erste Weltkongress für Umweltgeschichte in Kopenhagen stattfinden wird. Während die Umweltgeschichte als junge Wissenschaft im Rahmen europäischer Nationalstaaten aufgrund ihres grenzüberschreitenden Gegenstandes institutionell nicht so stark verankert ist, scheint die internationale Integration des Faches auf Weltebene trotz mancher Sprachbarrieren im Fortschritt begriffen zu sein.
} 
menschwerpunkten an den Lehrstühlen verschiedener Disziplinen vertreten sein. ${ }^{13}$ Hierdurch bestehe einerseits die Gefahr einer Zerfaserung des Fachs, doch wird andererseits die Verknüpfung mit anderen Themenfeldern erleichtert, wie sich auch an den Beiträgen in diesem Band zeigt.

In seinem einleitenden Aufsatz zum Themenschwerpunkt Spektrum der Umweltgeschichte hebt Bernd Herrmann die Bedeutung eines inhaltlichen Grundkonsenses über den Gegenstandsbereich der Umweltgeschichte hervor. Einer Erörterung des Umweltbegriffs bei Jakob von Uexküll folgen als Anknüpfung einige Aspekte der Kulturtheorie Ernst Cassirers. Darin favorisiert Herrmann die auf ihn und Rolf Peter Sieferle zurückgehende o. g. Definition von Umweltgeschichte aus dem Lehrbuch von Winiwarter und Knoll, welche „Rekonstruktion und Rezeption“ als die beiden erkenntnisleitenden Säulen von Umweltgeschichte hervorhebt. Weiter erörtert er den Disziplincharakter der Umweltgeschichte unter wissenschaftssoziologischer und wissenschaftssystematischer Perspektive. Dabei sieht er die Gefahr einer Zersplitterung des Fachs, falls der interdisziplinäre Austausch zugunsten einer Verkapselung in einzelnen Wissenschaftsdisziplinen aufgegeben würde. Zum Abschluss stellt er die Frage nach der gesellschaftlichen Relevanz der Umweltgeschichte.

Die visuellen Repräsentationen von Umwelt stehen im Zentrum des Beitrags von Christof Mauch, in dem er das Verhältnis von landschaftlicher Ikone und Politik an Beispielen aus den USA des 19. und 20. Jahrhunderts in den Blick nimmt. Anhand einer Analyse von Gemälden, Fotographien, Filmen und Bildbänden zeigt er wie gerade Bilder einer unberührt erscheinenden Natur politisch instrumentalisiert wurden. Die Landschaftsgemälde insbesondere von Cole und der Hudson River School prägten sowohl den nordamerikanischen Naturschutzgedanken als auch die touristische Erschließung; sie beeinflussten nicht zuletzt auch die Gestaltung von Aussichtspunkten in Naturparks. Solche Repräsentationen avancierten im späten 19. Jahrhundert zu gesellschaftlichen Leitbildern. Dagegen standen in den 1930er-Jahren im Film die Bilder der katastrophalen Folgen des „Dust Bowl“ im Mittelpunkt, wodurch die maschinelle Eroberung der Natur kritisiert wurde. Seit den fünfziger Jahren spielten Bilder einer unberührten, aber zunehmend nun bedrohten Natur, die in Bildbänden vermittelt wurden, eine größere Rolle.

Edda Müller, die frühere Umweltministerin von Schleswig-Holstein, zeigt in ihrem Beitrag, dass die internationale Politik und die bundesstaatliche Verwaltung

13 Die einzige Professur explizit für Umweltgeschichte im deutschsprachigen Raum ist in Wien. Daneben gibt es mehrere Professuren in der Schweiz und Deutschland, die Umweltgeschichte neben anderen historischen Fächern vertreten. Des Weiteren sind jüngst zwei Juniorprofessuren für Umweltgeschichte in Bochum und Kiel eingerichtet worden. Bezeichnenderweise für den interdisziplinären Charakter der Disziplin befindet sich die Juniorprofessur in Bochum an der Fakultät für Geschichtswissenschaft, während die Juniorprofessur in Kiel an der MathematischNaturwissenschaftlichen Fakultät angesiedelt ist. Neben den Juniorprofessuren ist als neue Institution in Deutschland kürzlich das Kolleg Internationale Umweltgeschichte - Natur als kulturelle Herausforderung in München bewilligt worden. 
Lars Kreye, Markus Schwarzer

treibende Kräfte bei der Implementierung umweltrelevanter Regulierungen in Deutschland seit den 1970er-Jahren gewesen sind. Dabei stellte die Einführung nationaler Umweltprogramme in Deutschland während der frühen 1970er-Jahre eine neue Stufe der Umweltpolitik dar, wobei Müller den Erfolg von nationalen und internationalen Steuerungsmodelle in der Umweltpolitik betont. In diesem Zusammenhang favorisiert sie in der umweltpolitischen Entscheidungsfindung politische Eliten gegenüber der Umweltbewegung. Letzterer spricht sie im Rahmen des demokratischen Repräsentationsmodells die Möglichkeit ab, sich gegen wirtschaftliche Interessen durchzusetzen. Die Umweltbewegung könne allein das umweltpolitische Interesse in den Parteien wachhalten. Diese Einschätzung wurde während der Tagung von Frank Uekötter kritisiert, der historische Defizite bei der Implementierung übergeordneter Umweltprogramme ausmachte. Es entwickelte sich zwischen beiden Positionen eine Kontroverse, die zeigte, dass das Verhältnis und Zusammenspiel verschiedener politischen Ebenen bei umweltrelevanten Steuerungsfragen noch weiterer Forschung bedarf.

Den letzten Beitrag im Themenblock Spektrum der Umweltgeschichte liefert Klaus Schlottau. Er hatte während der Tagung auf den wichtigen Punkt eines berufspraktischen Feldes für Umwelthistoriker in der Altlastensanierung aufmerksam gemacht und war so freundlich, zu diesem Tagungsband einen längeren Beitrag beizusteuern. In seinem Aufsatz hebt er hervor, dass in der Vergangenheit oftmals Naturwissenschaftler, Mediziner und Ingenieure mit dem Nachweis, der Analytik und der Sanierung von Altlasten betraut worden seien. Doch habe sich durch das Aufstellen von Altlastenkatastern auch sukzessive ein neues Berufsfeld für Historiker mit interdisziplinären Interessen herauszubilden begonnen. Dieses hat mittlerweile durch die Gesetzgebung des Bundes und der Länder die konkrete Form des historischen Altlastensachverständigen angenommen, der durch seine Arbeit mit historischen Quellen, z. B. durch die Auswertung von Firmenarchiven einen Beitrag zur Beseitigung von Altlastenproblemen liefern kann.

Der Themenblock \$ us der Geschichte lernen versammelt klassisch umwelthistorische Themenfelder wie Luftverschmutzung, Naturkatastrophen aber auch das aktuell diskutierte Thema der invasiven Spezies. Am Beispiel von Luftverschmutzung thematisiert Frank Uekötter, wie umweltpolitische Reglementierungen historisch funktionieren. Sein Beitrag spannt das Feld zwischen staatlichen Regulierungsversuchen zur Emissionsminderung und deren Umsetzungsproblemen seit dem 19. Jahrhundert auf. Hier bestand das grundsätzliche Problem, die staatlichen Normen vor Ort umzusetzen, was zu Vollzugsdefiziten führte. Deshalb hätten seinerzeit schon zentrale ,top-down“-Modelle zur Steuerung umweltrelevanten Verhaltens, wie diese für Deutschland noch heute typisch seien, nicht zum gewünschten Erfolgt geführt. Deshalb schlägt Uekötter in Analogie zu diesem historischen Beispiel im Hinblick auf die „Umweltzukunft“" vor, in der Klimapolitik nicht allein auf globale Steuerungsinstrumente des Kyoto-Protokolls zu setzen, sondern diese durch nationale Traditionen berücksichtigende Instrumente zu er- 
gänzen. Im Ausblick geht er auch auf das historisch belegbare Potential der Umweltbewegung ein, durch innovative Lösungen politisch Akzente zu setzen.

Manfred Jakubowski-Tiessen stellt in seinem Beitrag heraus, dass bis in das 18 . Jahrhundert hinein schwere Naturkatastrophen als gottgewollte Schicksalsschläge hingenommen wurden, weshalb auch keine Prävention in heutigen Sinn gegen solche Ereignisse existierte. Erst als sich im Zuge der Aufklärung die Vorstellung durchsetzte, die Natur sei kausalgesetzlich erklärbar und technisch beherrschbar, konnten weitreichende Schutzmaßnahmen getroffen werden. Dies führte jedoch auch zu einer Illusion von Sicherheit, so dass mögliche Schäden unterschätzt wurden und die katastrophalen Auswirkungen von naturalen Extremereignissen bis heute groß sind. Deshalb könne historisch gelernt werden, dass neben einer naturwissenschaftlichen Risikoabschätzung auch die Betrachtung gesellschaftlicher Bewältigungsstrategien und der kollektiven Erinnerung potentieller Gefährdungen in einem Gebiet wichtig sei. Hierdurch könne die Anhäufung von Schadenspotentialen vermieden werden.

Dass die Invasion biologischer Arten zumeist eine nicht intendierte Folge menschlichen Handelns sei, stellt der Ökologe Josef Reichholf in seinem Beitrag heraus. Die hohen Stickstoffeinträge in Fließgewässer durch intensive landwirtschaftliche Nutzung fördern beispielsweise das üppige Wachstum eingewanderter Pflanzenarten. Aus naturschützerischer Sicht ist es Reichholf zufolge falsch und durchaus problematisch, fremde Arten, nur weil diese bisher nicht heimisch waren, grundsätzlich abzulehnen. Auch sei die Natur ständig in Bewegung, weshalb sich wissenschaftlich keine Vorgaben zu ihrer Normierung aus der Vergangenheit gewinnen ließen. Stattdessen sollte man die industriegesellschaftlichen Ursachen und die Auswirkungen der Invasionen untersuchen. Schließlich müsse sich der Naturschutz bei der politischen Bekämpfung der Ursachen bewusst sein, dass er selbst als Interessengruppe und nicht im Sinne eines allgemeinen Wohls handelt. Nur wenn er parteilich argumentiere, kann er seinem Akzeptanzdefizit entgegenwirken und politisch glaubwürdig agieren.

Im Themenblock zu den fast vergessenen Debatten der Umweltgeschichte werden die Diskussion um das Waldsterben und das Tempolimit neu beleuchtet. Birgit Metzger und Roland Schäfer geben in ihrem Beitrag einen Überblick über die Entwicklung der Waldsterbensdebatte seit den frühen 1980er Jahren. Dabei diskutieren sie neben realistischen und konstruktivistischen Ansätze zur Erklärung des Phänomens, inwieweit eine vergleichende Betrachtung des Waldsterbens zum Verständnis aktueller Umweltprobleme beitragen kann. Hier kommen sie zu dem Ergebnis, dass das Waldsterben heute weniger als Sachdebatte zu begreifen sei, sondern als Speerspitze eines themenübergreifenden Diskurses zur Implementierung von Umweltschutzmaßnahmen gelten müsse. Dabei ging es letztlich auch darum, in der Bevölkerung eine breite Akzeptanz für Maßnahmen des Umweltschutzes zu schaffen.

In seinem prägnanten Überblick über die Geschichte des Tempolimits in Deutschland vom Ende des 19. Jahrhunderts bis in die 1970er-Jahre stellt Kurt 
Möser heraus, dass es auch beim Tempolimit weniger um die Sache als solche ging, sondern um übergeordnete politische Leitbilder. Es könne davon gesprochen werden, dass sich in der Frage des Tempolimits der alte Gegensatz zwischen autoritärem und liberalem Staat offenbare. Dabei sei die Debatte immer ideologisch geführt und mit Fragen der Verkehrssicherheit, des Ressourcenschutzes oder anderen politischen Erwägungen verknüpft worden. Da die fortschrittlichen Kräfte hier auf eine Regulierung, die konservativen Kräfte hingegen auf eine Liberalisierung setzen, passe die Debatte um das Tempolimit paradoxerweise nicht in das übliche politische Unterscheidungsschema.

Im Themenblock Umweltgeschichte im Bildungssystem diskutiert zunächst Bodo von Borries die Frage, wie man Umweltgeschichte in der Schule vermittelt. In seiner deskriptiven Untersuchung führt er aus, dass die Umweltgeschichte in Lehrplänen nur unzureichend vertreten sei und in Schulbüchern mitunter ideologisch dargestellt werde. Schließlich betont von Borries die unzureichende Vermittlung des Fachs in der universitären Lehrerausbildung, die unzureichende Präsens der Umweltgeschichte in den Massenmedien sowie ihre kaum vorhandene Verankerung in zentralen geschichtswissenschaftlichen Institutionen. Die Umweltgeschichte müsse deshalb hier noch um ihre Kompetenz streiten.

Die Frage, warum wir Umweltgeschichte studieren und erforschen, behandeln im letzten Beitrag des Bandes Markus Schwarzer und Ole Sparenberg stellvertretend für die Doktorandinnen und Doktoranden des Graduiertenkollegs „Interdisziplinäre Umweltgeschichte“. Dabei betonen sie nicht nur die Bedeutung kultureller Aspekte und materieller Grundlagen für die Umweltgeschichte, sondern beleuchten exemplarisch das Verhältnis der Umweltgeschichte zu den geistes-, naturund ingenieurwissenschaftlichen Herkunftsdisziplinen der Stipendiaten. Darüber hinaus thematisieren sie die außerwissenschaftliche Relevanz von Umweltgeschichte in ihrem Ausblick.

Die behandelten Themen geben zwar einen gewissen Einblick in die Breite der umwelthistorischen Forschung. ${ }^{14}$ Deren Themenfelder werden jedoch keinesfalls abgedeckt. Während der Abschlussdiskussion der Tagung wurde betont, dass die umwelthistorische Forschung bisher häufig eine zu starke Konzentration auf materielle Aspekte vorgenommen hat. Es wurde darauf hingewiesen, dass emotionale und ästhetische Zugangsweisen in der Auseinandersetzung mit der Umwelt nicht unberücksichtigt bleiben dürften. ${ }^{15}$ Ein weiterer Gesichtspunkt, der künftig umweltgeschichtlich stärker beachtet werden sollte, ist die Demographie. Dabei gilt es

\footnotetext{
${ }^{14}$ Neben den hier veröffentlichen Beiträgen wurde die Tagung durch Vorträge von Joachim Radkau zur Öko-Ikonographie des SPIEGELS, von Gisela Mettele zur Gartenstadt und von Dorothee Brantz zu Viehseuchen bereichert.

15 Vgl. dazu die weiterführende Literatur im Kapitel „Gesellschaftliche Wahrnehmung von Umwelt“, Winiwarter, Knoll, Umweltgeschichte, S. 255-299. In ihrem Fazit dieses Abschnitts betonen sie, dass gesellschaftlich und kulturell geprägte Wahrnehmungen von Umwelt entscheidend sind für die Ausprägung von Weltbildern, in denen Natur als Projektionsfläche fungiert. Auch das Rechtssystem, das ein weiteres fruchtbares umweltgeschichtliches Forschungsfeld ist, lässt sich als kollektivierte und institutionalisierte Wahrnehmung begreifen.
} 
ihre Nähe zu rassistischen Ideologien und ihre Rolle im Dritten Reich sorgfältig zu reflektierten, bevor die dringend gebotenen Bevölkerungsfragen in die Umweltgeschichte einbezogen werden können. ${ }^{16}$

\section{Literatur}

Abelshauser, W. (Hrsg.) 1994 Umweltgeschichte. Umweltverträgliches Wirtschaften in historischer Perspektive. Acht Beiträge. Göttingen.

Ahaus, B. 2008 Tagunsbericht: Schnee von gestern? Zivilisationskritik und Überlebensperspektiven in Zeiten des Klimawandels. In: H-Soz-u-Kult. http://hsozkult.geschichte.hu-berlin.de/tagungsberichte/id=2311 (30.10.2008).

Bayerl, G., Fuchsloch, N. und Meyer, T. (Hrsg.) 1996 Umweltgeschichte Methoden, Themen, Potentiale. Münster.

Herzog, B. 2002 Historia magistra vitae. In: Lexikon Geschichtswissenschaft. Hundert Grundbegriffe. Hrsg. von S. Jordan. Stuttgart. S. 145-147.

Imhof, A. E. (Hrsg.) 1975 Demographie als Sozialgeschichte. Gießen und Umgebung vom 17. zum 19. Jahrhundert. Teil 1 und 2., Darmstadt (Als EQuelle 1995).

Radkau, J. 2000 Natur und Macht. Eine Weltgeschichte der Umwelt. München.

Siemann, W. (Hrsg.) 2003 Umweltgeschichte. Themen und Perspektiven. München.

Uekötter, F. 2007 Umweltgeschichte im 19. und 20. Jahrhundert. In: Enzyklopädie Deutscher Geschichte. Hrsg. L. Gall. München.

Winiwarter, V., Knoll, M. 2007 Umweltgeschichte. Eine Einführung. Köln.

\footnotetext{
${ }^{16}$ Eine Ausnahme, an die in diesem Kontext laut Herrmann angeknüpft werden könnte ist: Demographie als Sozialgeschichte. Gießen und Umgebung vom 17. zum 19. Jahrhundert. Teil 1 und 2. Hrsg. von A. E. Imhof, Darmstadt 1975 (Als E-Quelle 1995). Dem Bevölkerungsaspekt widmen auch Winiwarter und Knoll ein Kapitel ihrer Einführung.
} 



\title{
Umweltgeschichte wozu? Zur gesellschaftlichen Relevanz einer jungen Disziplin
}

\author{
Bernd Herrmann
}

\section{Umweltgeschichte}

Das Organisationskomitee hatte mir die Aufgabe des Eröffnungsreferates übertragen. Als wäre allein die Rede über den Gegenstand der „Umweltgeschichte“ nicht Aufgabe genug, sollte sie auch noch mit der Relevanzfrage verknüpft werden.

Die folgende Zweiteilung meines Beitrags folgt der inneren Logik des Auftrags. Zunächst wird es um Inhaltliches und Disziplinäres der Umweltgeschichte gehen müssen, anschließend wird die Relevanzfrage samt ihres Umfeldes erörtert.

Ia Umwelt

Dass die Umweltgeschichte als thematischer Rahmen älter ist als ihr Name, wird niemanden verwundern. Teile der Bibel, die als Parabel über die Neolithisierung gelesen werden können, der Mythos um die Gründung Athens, ${ }^{1}$ Guaman Poma de Ayalas ${ }^{2}$ Geschichte seines südamerikanischen Volkes bis zur Ankunft der Europä-

\footnotetext{
1 Vor allem der Zeugungsmythos um den sagenhaften Athenischen König Erichthonios (Laroux 1993).

2 Guaman Poma de Ayala (ca. 1550 - nach 1615), adeliger peruanischer Herkunft, zeitweilig in spanischen Diensten, beschrieb in „Nueva Crónica y Buen Gobierno“ eine Geschichte seines Volkes und seiner Kultur in vorspanischer Zeit. Eine umwelthistorische Aufarbeitung des Werks steht aus. Digital erreichbar unter: http://www.kb.dk/permalink/2006/poma/info/es/frontpage.htm
} 
er, die präzisen Beobachtungen, die uns Babur, ${ }^{3}$ der Gründer der indischen Mogulnherrschaft, hinterlassen hat, Voltaires Candide, Kants Anthropologie ${ }^{4}$ und Humboldts Kosmos sind nur einige namhafte Beispiele aus einer sehr langen Liste, die selbstverständlich bis in die vorschriftliche Tradition verlängert werden muss. Sie bezeugt, dass der reflektierende Geist dem Umgang des Menschen mit der "Natur" und ihren Auswirkungen auf den Menschen zu allen Zeiten eine erhebliche Bedeutung beimaß. Sicherlich ist hierin auch eine Anerkennung der Bedeutung materieller Faktoren für den Lauf der Geschichte zu sehen. Die Zuschreibung von Sinn und Bedeutung eben auch der naturalen Elemente verleihen der sinnlosen Unendlichkeit des Weltgeschehens ihre je spezifische kulturelle Qualität. ${ }^{5}$ Insofern muss die Auseinandersetzung mit „Natur“, mit „Umwelt“ für jede Gesellschaft, für jede Kultur zentral sein. Sie ist es selbst dann, wenn sie die Überwindung der Leiblichkeit sucht und eine vergeistigte Lebensführung in asketischer Reduktion aller Ansprüche gegen eine wie immer aussehende „Umwelt“ als Leitidee einführt.

Niemand kann der Umwelt entkommen, ${ }^{6}$ weil sie konstitutiver Teil seiner selbst ist. Seit ihrer spezifischen Entdeckung durch Johannes von Uexküll $(1864-1944)^{7}$ ist sie, gemeinsam mit der Genetik, das bestimmende Epistem der modernen Biologie geworden und hat darüber hinaus eigentlich alle Bereiche des gesellschaftlichen und kulturellen Lebens erreicht. Von Üxkuell hatte „Umgebung“ von „Umwelt“ geschieden und als entscheidende Differenz diejenige zwischen der bloßen Aufnahme von Objekten im Raum („Umgebung“) und dem „Weltbild“ eines Lebewesens benannt, das durch bestimmten Beziehungen des Lebewesens zu

\footnotetext{
${ }^{3}$ Das Baburnama, Das Tagebuch von Babur (1483 - 1530), enthält eine Fülle umwelthistorischer Details aus seinem Herrschaftsbereich (Stammer 1988). Aufgearbeitet sind bisher die zoologischen und botanischen Hinweise des Werks.

${ }^{4}$ Als einer der Gründerväter der Anthropologie hatte sich auch Kant mit den Auswirkungen der naturalen Determinanten als Ursache der Variabilität des äußeren Erscheinungsbildes des Menschen intensiv auseinander gesetzt. Seine Betonung u.a. der Klimafaktoren ist im Grundsatz und auf einer sehr allgemeinen Ebene richtig, die von ihm angeführten konkreten Kausalitäten sind jedoch naturwissenschaftlich nicht zutreffend. Im Prinzip führen ihn diese behaupteten Kausalitäten, wie andere zeitgenössische Autoren auch, zu Idealtypologien. Solche werden Ende des 19. Jh.s in den entstehenden ,geographischen Determinismus“ übernommen.

5 „, Kultur' ist ein vom Standpunkt des Menschen aus mit Sinn und Bedeutung bedachter endlicher Ausschnitt aus der sinnlosen Unendlichkeit des Weltgeschehens. Sie ist es für den Menschen auch dann, wenn er einer konkreten Kultur als Todfeind sich entgegenstellt und ,Rückkehr zur Natur' verlangt. Denn auch zu dieser Stellungnahme kann er nur gelangen, indem er die konkrete Kultur auf seine Wertideen bezieht und ,zu leicht' befindet.“ Max Weber (1988a, S. 180).

${ }^{6}$ Hier und im Folgenden wird ausschließlich auf den Umweltbegriff der Lebenswissenschaften bezogen. Ausschließlich aus diesem Bezug ergeben sich spezifische geschichtstheoretische Anschlüsse, weil die auch mögliche Lesart „Umgebung“ für „Umwelt“ sich für jede beliebige andere Geschichtsbetrachtung hergibt.

7 Umwelt und Innenwelt der Tiere 1909. Innerhalb dieses Aufsatzes beziehe ich mich auf die zweite, verbesserte Auflage von 1921.- In der Sekundärliteratur finden sich häufiger sinngemäße Hinweise, bei denen der Eindruck erweckt wird, sie griffen auf von Uexkülls „Umwelt und Innenwelt“ zurück. Tatsächlich lesen sich in seiner „Theoretischen Biologie“ [1928] einige Passagen als gereifte Erläuterungen zu „Umwelt und Innenwelt“, auf die dann stellvertretend zurückgegriffen wird. Auch stammt das berühmt gewordene Zeckenbeispiel nicht aus „Umwelt und Innenwelt“, sondern ist erst 1934 veröffentlicht worden (von Uexküll \& Kriszat 1934).
} 
seiner Außenwelt entsteht („Umwelt“, später auch synonym „Eigenwelt“). Umwelt ist bei von Uexküll als Relationsbegriff zu verstehen. ${ }^{8}$ Anders als „Natur“ ist „Umwelt" nicht er-lebbar, sondern nur lebbar. Sie lässt sich nicht verdinglichen.

Es handelt sich um einen Einflussbereich, der vom Individuum gestaltet wird, in den Dinge der Umgebung eintreten, der aber in jedem Falle ein ausschließlich individueller Bereich bleibt und sich zudem grundsätzlich der Erfahrbarkeit durch andere Lebewesen entzieht. Das je spezifische Lebewesen und der Raum „Umwelt“, in dem es sich bewegt, fallen zusammen. ${ }^{9}$

Anders als von Uexküll intendierte, operationalisierten die meisten Biowissenschaften „Umwelt“ hin zu einem Dingbegriff. Damit war Objektivierung und Verwendungsmöglichkeit für Gruppen von Lebewesen gewonnen. Die frühen Profiteure des Gedankens von Uexkülls haben außerdem seinen Umweltbegriff, der Anstoß gebend fruchtbar für die Entstehung der ökologischen Disziplinen und der Verhaltensforschung wurde, für „eng“ gehalten, ${ }^{10}$ und ihn ziemlich über seine Intentionen hinaus geändert. Die Biologen nehmen mit dem Umweltbegriff Bezug „auf dasjenige außerhalb des Subjekts, was dieses irgendwie angebt". ${ }^{11}$ Doch eigentlich han-

\footnotetext{
8 Das Wort „Umwelt“ ist ein Neologismus aus dem Jahre 1800, und beschreibt, vor dem späteren Gebrauch des Wortes „Milieu“, »die den Menschen umgebende Welt« (DWB). In diesem milieutheoretischen Sine scheint der Begriff auch heute von Historikern verstanden zu werden, selbst wenn sie sich auf von Uexküll beziehen, wie etwa Radkau (2002, S.14). Nur bei einem solchen Verständnis kann Umwelt ein ,anthropozentrischer Begriff“ sein. Der Anschluss an von Uexküll trifft an dieser Stelle jedoch nicht zu, da von Uexküll seine Umweltdefinition allgemein als artbezogen verstanden wissen wollte. Erst in einem spezielleren Verständnis kann „Umwelt“ aus menschlicher Perspektive zu einem anthropozentrischen Epistem werden. Wenn man jedoch so verfährt, kann es keine „Umwelt des Seeigels" usw. geben, weil in jedem Falle eine anthropozentrische Zuschreibung vorläge. Damit müsste ein allgemeiner Bezug auf von Uexküll entfallen und dürfte nur als spezieller Bezug zugelassen werden.

9 „Es gibt also reine subjektive Wirklichkeiten in den Umwelten. Aber auch die objektiven Wirklichkeiten der Umgebung treten nie als solche in den Umwelten auf. Sie werden stets in Merkmale oder Merkbilder verwandelt und mit einem Wirkton versehen, der sie erst zu wirklichen Gegenständen macht, obgleich vom Wirkton in den Reizen nichts vorhanden ist. Und schließlich lehrt uns der einfache Funktionskreis, dass sowohl die Merkmale wie Wirkmale Äußerungen des Subjekts sind und die Eigenschaften der Objekte, die der Funktionskreis einschließt, nur als ihre Träger angesprochen werden können. So kommen wir dann zum Schluss, dass ein jedes Subjekt in einer Welt lebt, in der es nur subjektive Wirklichkeiten gibt und die Umwelten selbst nur subjektive Wirklichkeiten darstellen. Wer die Existenz subjektiver Wirklichkeiten leugnet, hat die Grundlagen seiner eigenen Existenz nicht erkannt.“" (von Uexküll \& Kriszat 1934, S.93).

${ }^{10}$ So z.B. Thienemann (1958, S. 9). - Die unter den (deutschsprachigen) Biologen des 20. Jh.s gängige Verständnisformel für „Umwelt“ ist nach meiner Kenntnis von Friedrichs (1943), jenseits der ideologischen Einsprengsel (Friedrichs ist von 1940 -1945 Zoologe an der Reichsuniversität Posen), herausgearbeitet worden: Er definierte Umwelt „,ür den praktischen Gebrauch“ als „Komplex der direkten und der konkret greifbaren indirekten Beziehungen zur Außenwelt." Der Unterschied gegenüber der Definition von Uexkülls bestehe in der Aufnahme aller Beziehungen (Friedrichs 1943, S.157). Eine überdachte Begriffserläuterung durch Friedrichs (1950) kann als für die nachfolgenden Biologen leitend bezeichnet werden, ohne dass die Autoren sich jeweils explicit auf Friedrichs bezogen hätten. Friedrichs fasste die Leitlinien des biologischen Diskurses zusammen, wie er davor bestimmend war und es im Grunde bis heute ist. Von Uexkülls Einfluss auf die akademische Biologie blieb aus verschiedenen Gründen begrenzt, vor allem wohl, weil seine Ideen an vitalistische Vorstellungen anschließen, denen die moderne Biologie distanziert gegenüber steht.
}

${ }^{11}$ Friedrichs 1950, S.70. 
delt es sich nur darum, dass die Natur-Wissenschaft für ihre Aussagen über „Natur" bzw. die „Umwelt" messbare Größen, reproduzierbare analytische Kategorien und Zugangsmöglichkeit zum Forschungsgegenstand benötigt und das Subjektive aus der Naturwissenschaft entfernt. ${ }^{12}$ Eine Betrachtungsbeschränkung auf die Umwelt nur eines einzigen Lebewesens, eine subjektive Biologie, stünde zudem im Konflikt mit den Zielen der Naturwissenschaft, die Differenz zwischen Ereignis und Struktur, zwischen Zufall und Notwendigkeit aufzudecken, weshalb der Befund am Individuum stellvertretend als Regelverhalten der gesamten Art gilt. ${ }^{13}$ An diesem Missverständnis oder diesen Parallelpositionen hat sich bis heute wenig geändert. ${ }^{14}$ Nicht einmal, als Nagel (1974) in seinem berühmt gewordenen Aufsatz „What is it like to be a bat?" die Unmöglichkeit nachwies, sich in die Erlebniswelt der Fledermaus hinein zu versetzen. Während von Uexküll aber der Auffassung war, dass die Wissenschaft aus dem morphologisch-physiologischen Korrelat des Tiers hinreichend Aufschluss über dessen spezifische Erfahrungswelt gebe und das Verstehen des Korrelats gleichbedeutend mit dem Verständnis des Lebewesens wäre, ${ }^{15}$ entwickelt Nagel den Gedanken erkenntnistheoretisch weiter und kommt zu dem Schluss, dass die Kenntnis des Korrelats keine Erkenntnis über die Erlebniswelt des (anderen) Organismus liefert. ${ }^{16}$

\footnotetext{
${ }^{12}$ Sowohl das Subjektive des Beobachters oder Experimentators als auch das Individuelle des Untersuchungsobjekts.

13 Überraschender Weise ist von Uexküll selbst dieser Perspektive seiner Gedanken nicht weiter nachgegangen. Als Biologe hatte er die Umwelt der Art im Blick. Seine Entdeckung der Umwelt hätte logisch in die Zuschreibung je individueller Umwelten führen müssen. Solche individuellen Umwelttönungen akzeptiert er für Menschen, die von ihm aufgeführten Beispiele sehr subjektiven (individualistischen) Verhaltens bei Tieren klassifiziert er überraschend als aberrantes bis subpathologisches Verhalten.

14 Tatsächlich ist es heute gängige biologische Praxis, einen weitgehend von komplexen Bedeutungsinhalten befreiten Umweltbegriff zu verwenden. Zumeist beschreibt dieser, jenseits seines erkenntnistheoretischen Ursprungs, nur noch Forschungsfelder. Er ist damit auch in der Biologie zu einer Black-Box-Vokabel geworden, vergleichbar etwa der umgangssprachlichen Verwendung des Begriffs „Gesellschaft". Eine analytische, erklärende Qualität, wie sie sich von Uexküll vorstellte, wohnt ihm heute nicht inne. Spätestens seit die internationale (natur-)-wissenschaftliche Literatur anstelle von „Umwelt“ das Wort „environment" verwendet, sind die sub- und metatextlichen Bedeutungen ausgeblendet.

15 Mit der Kenntnis des artspezifischen sensorischen Apparates wäre dann auch die Umwelt der Lebewesen einer Art erschlossen. Deshalb kann von Uexküll sagen, dass es ,in der Welt der Mücke nur Mückendinge“ gebe, in der der Zecke nur Zeckendinge, für die Seeigel nur Seeigeldinge usw.

16 Der Anschluss Nagels an die bekannte „Ignoramus-ignorabimus“- Rede Emil Du Bois-Reymonds von 1872 ist unübersehbar, die Verbindung zu von Uexküll wird übersehen. Es handelt sich bei diesem Streit um erkenntnistheoretische Grundpositionen innerhalb der modernen Biologie, deren weitere Ausführung den Rahmen sprengen würde. Hier nur soviel: der Streit ist nicht entschieden und wird gegenwärtig zwischen Hirnforschern und Neurobiologen auf der einen, und Erkenntnisphilosophen auf der andern Seite geführt. Dabei geht es um die zentrale Frage, ob eine Willensfreiheit des Menschen existiere oder der menschliche Wille ein bloßes Produkt molekularer Konzentrationen und neurophysiologischer Kaskaden sei. Wenn Nagels Überlegung richtig ist, könnten konsequenterweise bestimmte Forschungsbereiche aufgegeben werden. Diese Perspektive täte sich dann auch für „Umwelt“-Forschung auf. Sie stellt sich deshalb nicht, weil die oberflächliche Rezeption der Gedanken von Uexkülls bzw. die Abgrenzung ihnen gegenüber allmählich in die heute gängige Begriffspraxis mündete (siehe Friedrichs 1950).
} 
Die inflationäre Verwendung des Begriffs „Umwelt“ und seine scheinbare Einfachheit haben in den letzten Jahrzehnten dazu geführt, dass kaum noch darüber nachgedacht wird, welches die Grundlagen des Begriffs waren, welches seine philosophischen Implikationen sind und welche Bedeutungen die Übernahme des Begriffs in außerbiologische Verwertungszusammenhänge hat. Dabei hatte doch spätestens Ernst Cassirer mit seinem „Versuch über den Menschen“ (1944) die Komplexität, aber auch die Produktivität, des Umweltbezugs demonstriert, in dem er die Aneignung der Welt durch Menschen als über eine Symbolwelt geleitet erkannte. Im direkten Anschluss an von Uexküll ist für Cassirer (beide hatten als Hamburger Kollegen Berührungen) der Mensch durch seine spezifischen Umweltbezüge als „animal symbolicum" definiert. Die geschichtstheoretisch nutzbare Konsequenz aus Cassirers Überlegung ist offensichtlich und zweifach:

Nichts auf der Welt ist dem Menschen einfach gegeben, alles muss von ihm symbolisch-kulturell dargestellt bzw. vermittelt werden und mit Sinnzuschreibung versehen werden. Damit wird selbstverständlich auch „Natur“ zu einem Kulturbegriff.

Fasst man weiterhin die alltagsweltlichen Bereiche oder die Institutionen zu je eigenen Symbolkomplexen zusammen, mündet dies in die bekannten Partikulargeschichten der Geschichtswissenschaften, wenn auch unter anderer Nomenklatur.

Es ist bemerkenswert, dass der umwelthistorischen Forschung das Wort „Umwelt“ im Wortgefüge „Umweltgeschichte“ bisher nicht Anlass zu einer theoriegerichteten Begriffsanalyse war. ${ }^{17}$ Nach meiner Einsicht scheint eine Umweltgeschichte mancher Autoren dem Umweltbegriff von Uexkülls unbewusst viel näher zu sein als dem umgangssprachlichen Verständnis von „Umwelt“, das dem der heutigen Bio-Disziplinen entspricht. Wer Umwelthandeln als subjektive Kategorie thematisiert, thematisiert zwar die Welt der „Menschendinge“. Nur redete er dann nicht mehr über eine objektivierbare Welt, nämlich diejenige Welt der Wissenschaft.

Eine objektivierbare Welt, also eine reproduzierbar vermittelbare Weltsicht, ist hingegen zum Teil sicherlich Folge subjektiven Umwelthandelns, sie ist aber ebenso Folge eines Prozessgeschehens außerhalb menschlicher Ursache oder Zuständigkeit. Daher ist die Aufnahme naturhistorischer Zustände in die Umweltgeschichte nach der Formel „Rezeption und Rekonstruktion“ des Göttinger Graduiertenkollegs zwangsläufig.

Neben den Vorbehalten, die von biologischer Seite gegenüber den Vorstellungen von Uexkülls geäußert wurden, steht zusätzlich jener ernste Einwand Adolf

\footnotetext{
${ }^{17}$ Ich danke Verena Winiwarter für den Hinweis auf ihren Aufsatz von 1994, der mir bei Abfassung der Vorversion dieses Beitrags nicht gewärtig war. Mein Urteil ist also entsprechend zu relativieren. Bei dem Aufsatz von Winiwarter handelt es sich um eine Begriffsanalyse, die weiter als für den von mir verfolgten Zweck reicht, da sich nach meiner Einsicht der Umweltbegriff der „Umweltgeschichte" historisch und inhaltlich einem ausschließlichen Bezug auf die lebenswissenschaftliche Verwendungspraxis des Umweltbegriffs verdankt. Parallelverwendungen des Begriffs, die Winiwarter mit untersucht hat, liegen daher außerhalb meines Bezugsrahmens.
} 
Portmanns, ${ }^{18}$ der von Uexküll ein Missverständnis seiner eigenen Theorie nachweist, wenn er beim Versuch der Übertragung seiner „Umwelt“-Idee einen entscheidenden Umstand vernachlässigt. Im Gegensatz zur arttypischen Umweltstruktur, die den Tieren zukomme, werden im Falle des Menschen diese arttypischen Differenzen gegenstandslos, weil ,alle diese verschiedenen Weltsichten ${ }^{19}$ an einer gemeinsamen Artwelt teilhaben, dass ,Verstehen' verschiedener derartiger Umwelten möglich ist - dass eine Aussprache über Gegensätze der Auffassung stattfinden kann."

Das nach meinem Verständnis Neue in der Geschichtsbetrachtung umwelthistorischer Ausrichtung besteht in dem von ihr praktizierten Perspektivenwechsel, in dem ich eine Erweiterung historischer Fragestellungen und Bewertungen durch das Weglassen oder Ergänzen bisheriger Bezüge sehe, wofür folgendes Beispiel steht. André Malraux wird die Bemerkung über das Niederländische Stillleben zugesprochen: „Dass man einen Fisch auf einen Teller legen kann, hat Holland nicht erfunden, dafür aber, dass dieser Fisch nicht mehr die Speise des Apostels zu sein braucht." In nämlicher Weise hat Svetlana Alpers (1998) argumentiert, dass in der entsakralisierten dinghaften Darstellung der Stilllebenmaler ,mit getreulicher Hand und ebrlichem Auge“ eine der mächtigen Wurzeln der neuzeitlichen Naturwissenschaft zu sehen ist. Das Neue entsteht durch das Weglassen alter Bezüge, durch ihre Neuordnung und ihre Erweiterung.

Kopfläuse, Wasserstände, die Kleine Eiszeit und dergleichen haben Umwelthistoriker nicht erfunden, wohl aber, dass ihre Beachtung den Blick auf den Prozess der Geschichte um entscheidende Determinanten weitet. „Natur“, „Umwelt“ und ihre Begleiter werden in der Umweltgeschichte in einer anderen, eigenständigen Weise als bisher in die Geschichtsbetrachtung eingebracht. Dem Naturalen wird Geschichtsmacht zugebilligt, die Ausrichtung der Rezeption und des Handelns auf sie werden anerkannt. ${ }^{20}$ Nur hat das ideengeschichtlich mit von Uexküll praktisch nichts zu tun. Die gedankliche Verbindung zwischen „Umwelt“ und „Geschichte“ musste eigenständig konstruiert werden. Dass diese Verbindung sich ggfl. in von Uexkülls Gedankenwelt einfügen lässt und deshalb letztlich auch fälschlich für eine von seiner Einsicht mitgetragenen Idee gehalten wird, ist eine andere Sache.

\footnotetext{
18 Adolf Portmann (1997 - 1982), bedeutender Zoologe und Verfasser biologisch-philosophischer Abhandlungen, im Vorwort zu von Uexküll \& Kriszat (1956, S.11).

19 Portmann, a.a.O. Hier meint Portmann die Differenzen zwischen verschiedenen Überzeugungssystemen und Kulturen.

${ }^{20}$ Beispielhaft angelegt bei Borst (1981)
} 
Die unlängst geäußerten Überlegungen von Haidle (2008) können beispielhaft für ein heute verbreitetes, verkürztes Verständnis und fast reflexhaftes Zitieren der angeblichen Gedanken von Uexkülls benannt werden, der sich dagegen wehren würde, „Umwelt als ein Ausschnitt aus der Umgebung“"21 zu verstehen. Heute, und so auch bei Haidle, wird philosophisch z.B. auf ein Urteil wie das von Sloterdijk

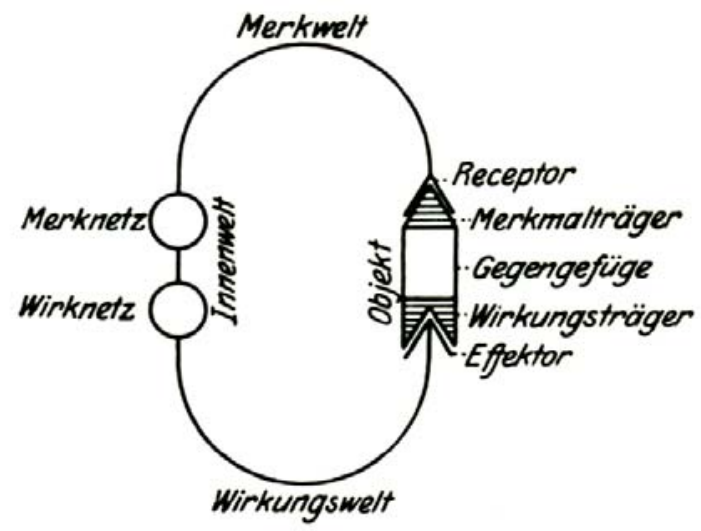

\begin{abstract}
Abb1. Schema des Funktionskreises (aus von Uexküll 1921:45). Die Wirkungswelt (Wirkwelt) bezeichnet alles, was ein Tier in seiner Umgebung aktiv zu bewirken vermag: Bau eines Stauwehres durch Biber, das Radnetz der Spinne, die Grabungstätigkeit des Maulwurfs, der Nestbau der Vögel. Die Merkwelt (engl. perceptual field) ist derjenige Ausschnitt der Außenwelt eines Tieres, der von seinen Sinnesorganen erfasst und seinem Zentralnervensystem zugeleitet wird. Nach von Uexküll existieren in der Umwelt des Tieres nur jene Dinge oder Zustände, die wahrgenommen werden. Diese Wahrnehmung und seine neuronale Verarbeitung sind individuenspezifisch bzw. artspezifisch. ${ }^{22}$ Das Diagramm stellt der Innenwelt des Tieres die verdinglichte Form der Wechselwirkung zwischen Objekt und Tier gegenüber. Zu weiteren Erläuterungen wird auf die Originalpublikation verwiesen.
\end{abstract}

(2001) vertraut. Dieser hatte ein passives bzw. reaktives tierliches „UmweltHaben“ einem aktiven bzw. kreativen menschlichen „In-der-Welt-Sein“ gegenüber gestellt. ${ }^{23}$ Die inhaltliche Nähe dieser Definition zur cartesisch-kantischen Unterscheidung zwischen behavioristischen Automaten (Tieren) und willensfreien Wesen (Menschen) ist unübersehbar, war aber überhaupt nicht von Uexkülls Thema,

\footnotetext{
${ }^{21}$ Haidle 2008, S.30.

22 „Es ist zweifellos richtig, wenn wir sagen, in der Welt der Mücke gibt es nur Mückendinge. Wie aber die Mückendinge gestaltet sind, das verlangt eine genaue Untersuchung. Der wichtigste effektorische Apparat einer Mücke, nämlich ihr Stachel, ist für unser Blut gebaut. Von unserem Blut aber erfahren die Rezeptoren der Mücke keine Einwirkung; dafür ist es der Duft unserer Hautdrüsen, der auf sie einwirkt. Die Hautdrüsen und das Blut des Menschen sind durch das anatomische Gegengefüge der menschlichen Haut miteinander verknüpft, das wohl innerhalb des Funktionskreises der Mücke liegt, aber gänzlich außerhalb jeder Merkmöglichkeit für den Mückenorganismus gelegen ist.“ (von Uexküll 1921, S. 217).

${ }^{23}$ Diese Position entspricht der Setzung von F.L.Ward (1903) aus soziologischer Perspektive, auf die in Fußnote 27 näher eingegangen wird.
} 
als er seine Theorie formulierte. ${ }^{24}$ Außerdem ergibt sich, unter Vernachlässigung der von Nagel geäußerten Bedenken, nicht nur Anschluss an die weiter unten kritisierte Position von Maurice Godelier. Die hier erwähnte Meinung Haidles führt schließlich auch zu der kritisch zu bewertenden Opposition „Mensch und Umwelt", die ich als ein folgenschweres Kategorienproblem begreife, der sich ein Teil des heutigen Umweltdilemmas überhaupt verdankt. ${ }^{25}$

Wer sich auf von Uexkülls Umweltbegriff (auch engl. „umwelt*) beruft, bezieht sich heute auf eine psychische Umwelt bzw. die „Eigenwelt“ eines Tieres, die aus Funktionskreisen besteht, welche sich aus der „Merkwelt“ und der „Wirkwelt“ des Tieres ergeben (Abb.1).

\section{IIb Umweltgeschichte}

Erprobte man nun den Begriff der „Umweltgeschichte“ vor diesem vielschichtigen Hintergrund, der hier nur sehr kursorisch skizziert ist, müsste man die zunächst individualistisch zu denkenden Umwelten einzelner Menschen zu einer gemeinsamen Geschichte verbinden und würde schließlich, über jeweils zusammenführende Ebenen von Klassen, Gesellschaften, Kulturen, dem naturalen Tableau usw., bei einem die gesamte Menschheit und die physischen Zustände einschließenden Umweltgeschichtsbegriff enden. Es ist offensichtlich, dass dies ein Synonymbegriff für eine „histoire totale“ wäre. Nur einmal, soweit ich das sehe, ist die Geschichtstheorie explizit 26 in die Nähe dieser Auffassung gekommen, und zwar durch Bündelung von Überlegungen zum Umweltgeschichtsbegriff unterschiedlicher Provenienz, also durch induktives Schließen (Lehmkuhl 2002). Freilich ist bei diesem erkenntnistheoretischen Umweg nicht an von Uexküll angeknüpft worden:

„Als Synthesewissenschaft analysiert sie [d.i. die Umweltgeschichte, $\mathrm{BH}$ ? $\mathrm{B}$. die Frage nach den Akteursqualitäten von Natur und Umwelt, und dies auf zwei Ebenen: zum einen,

\footnotetext{
${ }^{24}$ Die Übertragung auf menschliche Zusammenhänge erfolgt erst in „Streifzüge durch die Umwelten von Tieren und Menschen“ (1956 [1934], Kap. 13). - Nicht erst an dieser Stelle wird deutlich, dass die in Teilen der praktizierten Umweltgeschichte behauptete anthropologische Dimension bisher tatsächlich wenig von den Vorarbeiten der philosophischen Anthropologie profitiert hat.

25 Ansätze hierzu in Hermann 2008; sonst in Vorbereitung. Aus meiner Sicht sind „Mensch und Umwelt“ ebenso wenig äquivalente Kategorien wie „Körper und Geist“. Dieser „Descartsche Mythos" war durch Ryle [1969] dekonstruiert worden. Es scheint so, als würde der von Ryle identifizierte Fehler einer inkompatiblen ontologischen Kategorienbildung in den Umweltdiskursen mit dem Begriffspaar „Mensch und Umwelt“ wiederholt. Eine Position, die den Menschen neben der „Umwelt" oder ihr gegenüber sieht, spricht nicht von „Umwelt" sondern von „Umgebung“ und hat das Selbstverständnis von der menschlichen Sonderrolle auf der Erde noch nicht verlassen. „Umwelt“ (eigentlich „Umgebung“) und „Mensch“ sind dabei voneinander geschieden. Zahlreiche Missverständnisse resultieren aus der Tatsache, dass das Begriffspaar „Mensch und Umwelt“ häufiger nicht in einem ontologischen, sondern in einem analytischen Verständnis verwendet wird, ohne dass die Autoren dies besonders vermerken würden, allermeist scheint es ihnen sogar zu entgehen.

${ }^{26}$ Ich beziehe mich hier ausschließlich auf explizit geäußerte geschichtstheoretische Positionen. Ich übersehe nicht, dass in den großen Darstellungen zur Weltumweltgeschichte, etwa von Diamond, McNeill und Radkau, implizite theoretische Konzepte enthalten sind. Mir erscheint fraglich, ob die Autoren ihre Beiträge primär als dezidierte geschichtstheoretische Erweiterung oder Präzisierung aufgefasst wissen wollen. Deshalb werden sie hier in diesem Sinne auch nicht weiter diskutiert.
} 
indem Einflïsse von Natur und Umwelt auf menschliches Handeln und Verhalten in den Blick genommen werden; zum anderen, indem Ideen über Natur als ökologische Akteure konzeptualisiert werden. Umweltgeschichte beschäftigt sich mit Phänomenen, die sich nicht in ein nationalhistorisches Korsett zwängen lassen, sondern transnationale Geschichtsschreibung oder sogar einen „globalen" Ansatz im Sinne der neuen global history verlangen. Dabei werden Konzepte von Ökonomie, Kultur und Ökologie in neue analytische Zusammenhänge gestellt und heuristisch fruchtbar gemacht, und auch dies auf unterschiedliche Weise: durch eine ökologisch perspektivierte Kulturanalyse, oder durch die Analyse des Dialogs zwischen Ökologie und ÖKonomie oder [...] durch die Analyse der Interdependenz aller drei Faktoren, Ökologie, Ökonomie und Kultur." 27

Die Ablösung von „Umwelt“ vom Individuum und die ihr zugeschriebene eigenständige Akteursqualität, wie in diesem Beispiel, ist das am häufigsten anzutreffende gründliche Missverständnis des Umweltbegriffs von Uexkülls (s.o.).

Definitionen von „Umweltgeschichte“, die sich zudem nicht weiter mit Monita aufhalten, sind schnell zur Hand, wenn auch weniger zahlreich als man annehmen möchte. Das könnte zum einen daran liegen, dass in den Geschichtswissenschaften systematische Lehrbücher oder Review-Aufsätze nicht häufig sind. Gewiss liegt es aber auch daran, dass der Gegenstand der Umweltgeschichte vermeintlich ähnlich offensichtlich erscheint, wie z.B. derjenige der Botanik oder der Kirchengeschichte. Definitionen etwa der Botanik oder der Kirchengeschichte gehen für den Fachferneren kaum erkennbar über das intuitiv Selbstverständliche hinaus. In welche semantischen wie erkenntnistheoretischen Probleme man sich aber mit Definitionsversuchen hineinmanövrieren kann, sei an nur einem Beispiel skizziert:

Die Behauptung Maurice Godeliers, der Mensch habe deswegen Geschichte, weil er die Natur verändere, ist lediglich eine pointierte Spezialformulierung dafür, dass der Geschichtsbegriff an die aus menschlicher Ursache resultierenden Zustände in der Welt geknüpft ist. „... buman beings have a bistory because they transform nature. It is indeed this capacity which defines them as bumans. Of all forces which set them in movement and prompt them to invent new forms of society, the most profound is their ability to transform their relations with nature by transforming nature itself." (Godelier 1986, S. 2-3. Hervorhebungen im Original). ${ }^{28}$ Godelier schließt damit direkt an die bekannte Formulierung von Karl Marx an, nach dessen Einsicht es eine spezifisch menschliche Qualität ist, dass er der Natur selbst als Naturmacht gegenüber trete. ${ }^{29}$ Erprob-

\footnotetext{
${ }^{27}$ Lehmkuhl (2002, S.10). - Lehmkuhl wünscht sich in ihrem Abriss der umwelthistorischen Methoden (S.8) ein stärkeres Miteinander von natur- und geisteswissenschaftlichen Analyseinstrumenten, eine 2002 vielleicht immer noch nicht durchgängige, aber durchaus auch schon zu diesem Zeitpunkt verbreitete Praxis. Es kann hier aber vorweggenommen werden, dass die deutsche Umweltgeschichte viel stärkere Wahrnehmungsdefizite und -probleme gegenüber einschlägigen naturwissenschaftlichen Arbeiten hat als etwa die angelsächsische Umweltgeschichte.

28 Die Transformationsvorstellung findet sich neuerdings - unter dem Etikett „Metamorphose“ - in einer Geschichtsdefinition von Schatzki (2003) wieder. Sie bildet den Ausgang einer Überlegung, als deren Ergebnis Winiwarter \& Schmid (2008, S.161) vorschlagen, ,sozionaturale Schauplätze“ als Untersuchungsgegenstände der Umweltgeschichte einzuführen. „Die Metamorphose sozionaturaler Schauplätze, der Prozess ihres Wandels, ist Umweltgeschichte." (Hervorhebung im Original)

29 Marx [1968 S.192]. Ein ähnlich populäres Schlagwort lautet ,the environment transforms the animal, while man transforms the environment." Es handelt sich um eine Setzung von L.F.Ward
} 
te man Godeliers Setzung in einem evolutionsbiologischen Rahmen, müsste man den Kaninchen, den Bibern, den Radnetzspinnen und riffbauenden Korallen usw. auch Geschichtsmacht zuschreiben, wenn mit dem „Verhältnis zur Natur“ nicht zugleich ein reflektorisches Verbalten mit in Anspruch genommen wird. Zweifellos hat Godelier diesen Aspekt im Blick. Das Problem ist damit aber immer noch nicht befriedigend überwunden, weil die Reflexion von dem Ziel weggelenkt werden müsste, dass sich der Mensch setzt, hin auf das Ergebnis in der Natur. Nur wenn das sicbtbare Ergebnis in der Natur eine Folge seines von ihm selbst veränderten Verhältnisses zur Natur ist, fiele es unter Godeliers Geschichtsdefinition. Illustrierendes Beispiel: Die Existenz der Zuckerrübe ist das Ergebnis menschlicher Geschichte, weil ihr ein Züchtungsvorgang zugrunde liegt. In ihm ist das Verhältnis zur Natur verändert. Kritisches Beispiel: Hingegen wäre die Auswahl von Pflanzen als essbar bzw. nicht essbar zunächst kein geschichtsbildender Akt, weil ein Hamster genau so verfährt. Während wir vom Menschen aber wissen, dass diese Auswahl durchaus von seinem reflektierten Verhältnis zur Natur bestimmt sein kann, ${ }^{30}$ haben wir vom Hamster keine solche Kenntnis. Also verdankt sich Godeliers Einsicht einer emischen Betrachtung und keiner etischen. Sein Kriterium erfasst damit keine objektive Eigenschaft, wie seine Setzung suggeriert. Es müsste vielmehr im Einzelfall geprüft werden, ob einer anthropogenen Veränderung in der Natur tatsächlich auch eine Veränderung des menschlichen Verhältnisses zur Natur zugrunde liegt. Dies ist zumindest dann nicht der Fall, wenn das Ergebnis unbeabsichtigte Nebenfolge eines im Grundsatz gleich gebliebenen Verhältnisses zur Natur ist. Der Niedergang z.B. der indianischen Kulturen des amerikanischen Südwestens ist nicht Ergebnis eines veränderten Naturverhältnisses, sondern eines anhaltend gleichblei-

(1903). Spätestens seit Steward (1955) ist ein solcher Ansatz, seiner scheinbaren Richtigkeit zum Trotz, überholt. Das Zitat lautet im Zusammenhang: "It is this fact of permanent human achievement that makes the broad distinction between animal and human societies. Just as there is a radical difference between cosmic and organic evolution, $[\ldots]$ so there is a radical difference between organic and social evolution. The formula that expresses this distinction the most clearly is that the environment transforms the animal, while man transforms the environment. Now it is exactly this transformation of the environment that constitutes achievement. The animal achieves nothing. The organic world is passive. It is acted upon by the environment and adapted to it. And although it is true that in the structural modifications that constitute such adaptation the efforts and activities of the organism play a prominent part, still even this is only a reflex response to the pressure from without, and really constitutes a part of the environment. Man, on the contrary, as a psychically developed being, and in increasing degrees in proportion to his psychic development, is active and assumes the initiative, molding nature to his own use." (Ward, S. 16-17, Hervorhebung im Original).

Diese Textpassage wird von Park u. Burgess aufgegriffen (1921, S. 718), deren Veröffentlichung den Beginn der sozialwissenschaftlich vermittelten Humanökologie begründet. Von hier fand Wards Diktum seinen Weg auch in die umwelthistorischen Diskurse. Da Park u. Burgess nur den bei Ward kursiv gesetzten Ausdruck übernahmen, entgeht den Zitierern aus zweiter Hand vor allem die Vorstellung Wards von einer „passiven organischen Welt“. Seine Zeugenschaft ist von zweifelhaftem Wert, weil sie dem Tier-Mensch-Vergleich unterstellt, dass „molding nature to his own use“ ein besonderes Alleinstellungsmerkmal wäre, ein evolutionshistorisch teleologisches Element. Dies trifft zumindest auf einige Tierarten nicht zu und ist, wegen des verfolgten teleologischen Prinzips, ohnehin abzulehnen. Eine sozialwissenschaftliche Gegenposition vertreten z.B. Catton \& Dunlap (1980). $30 \mathrm{Zu}$ denken ist an Tabuisierungen; an komplexere Handlungsweise bei Coevolutionen (z.B. bei Glukose-6-Phosphat-Dehydrogenase-Mangel und gleichzeitigem Favabohnen-Konsum in MalariaGebieten), usw. 
benden, obwohl es nicht unabänderbar in seinem Beginn enthalten war. Absurder Weise wäre dieser Niedergang mit der Godelierschen Definition aus der Geschichte ausgeblendet. - Wenn dagegen eingewendet würde, dass es nicht auf die konkrete Handlung oder Handlungsfolge oder eine historische Tatsache ankäme, sondern vielmehr die potentielle Eigenschaft „des Menschen“ gemeint sei, dann endete die Godeliersche Pointe lediglich in der Tautologie, dass der Mensch Geschichte habe, weil er Mensch ist. Aber „Geschichte“ wäre dann keine singulär menschliche Angelegenheit.

Ungeachtet der Probleme, in die sie uns führt, ist Godeliers Setzung bei Umwelthistorikern ziemlich beliebt, vermutlich wegen ihrer scheinbaren Griffigkeit. Godelier geht fraglos vom Menschen als dem Homo-sapiens-Tier und von einer dinghaften Natur aus. Es bleibt aber offen, welche menschlichen Aktivitäten beweisgebend für ein verändertes Verhältnis zur Natur sind, zumal sich Godelier eigentlich nur für die sozialen Strukturen interessiert. Für die meisten menschlichen Aktivitäten mit Bezug auf diese Natur finden sich Parallelen in der Tierwelt, die irgendein animalischer Spezialist zum Vorteil seiner Spezies umgesetzt hat und die für diese konstitutiv wurden. Aber erst mit dem Ackerbau und mit den technischen Transformationsprozessen, die zur Schaffung von Materialien führen, die als solche in der Natur nicht vorkommen, wird ein geändertes Verhältnis des Menschen zur Natur sicher beweisbar. Würde damit die menschliche Geschichte erst vor 10.000 Jahren beginnen? ${ }^{31}$ Eine Bestattung aber, die ganz sicher mit der Existenz von Überzeugungssystemen zusammen hängt, ist denkbar ohne die Godeliersche Verhältnisänderung, obwohl gerade die Einführung von Bestattungen Ausdruck eines Geschichtsbewusstseins ist. Möglich, dass die paläolithische Kunst bereits Hinweis auf ein geändertes Naturverhältnis gibt. Sie ist datierbar, hingegen nicht die ihr vorgeordnete Denkleistung, die auch an anderer Stelle ein Problem aufwirft, wenn man z.B. Fernwaffen als Ausgang eines geänderten Naturverhältnisses annimmt. Steine und Grasbüschel werden zwar auch von Schimpansen (gegen Feinde) geschleudert, doch für Homo erectus wird die zweckvolle Herstellung von Speeren als Jagdwaffe nachweisbar. ${ }^{32}$ Die ältesten bekannten Speere sind mög-

\footnotetext{
31 Versuche, den Geschichtsbeginn früher anzusetzen, indem etwa über den Werkzeuggebrauch argumentiert wird, tragen nicht sehr weit, weil es hierfür viele Beispiele auch im Tierreich gibt. Hilfreicher wäre da schon der Feuergebrauch. Nur: wie will man Feuergebrauch als Beweis für ein verändertes Naturverhältnis verwenden, wenn er auch als opportunistische Indienststellung des Feuers verstanden werden kann, ähnlich der kulturell erworbenen Sitte bei Japan-Makaken, die Süßkartoffeln zu waschen oder im Winter in heißen Quellen zu baden. Wenn dieses Verhalten der Makaken nicht als opportunistische Nutzung eingeordnet würde: Hätten die Makaken damit im Godelierschen Verständnis für sich den Bereich einer Geschichte nach Art des Menschen erreicht? Schließlich: Die Beispiele paläolithischer Kunst sind unmittelbar auch nicht geeignet, ein neues Naturverhältnis zu beweisen.

32 Der nachgewiesene Gebrauch eines speziell angefertigten Gerätes als Fernwaffe für die Jagd setzt eine Reihe von Überlegungen bezüglich künftiger Erreichbarkeit von sonst nicht erreichbarer Jagdbeute voraus. Die Existenz eines solchen Speeres ist gleichzusetzen mit dem Nachweis einer bestimmten gedanklichen Leistung, die das Verhältnis des Jägers zu seiner Beute ändert, also das Verhältnis des Menschen zur Natur. Gegeben ist damit der Eintritt dieser speerschleudernden Menschen in eine Geschichte im Sinne Godeliers.
} 
licherweise 400.000 Jahre alt. ${ }^{33}$ Sie stammen, wie auch Zeugnisse paläolithischer Kunst, die z.B. Jagdtiere thematisieren, von einem Menschentypus, der Vorläufer des anatomisch modernen Menschen ist, aber doch von diesem morphologisch unterscheidbar ist und hinsichtlich seiner kognitiven Fähigkeiten für verschieden gehalten wird. Damit entstünde ein neues und weiteres Problem, nämlich das eines arten-übergreifenden Geschichtsbegriffs.

Einerseits, so lehrt es das Beispiel, ist Definitionsarbeit mühselig. Andererseits erhebt sich die Frage nach ihrem zielführenden Nutzen. Dass andere etablierte Wissenschaften ihre Gegenstandsbereiche nicht mit letzter Präzision der Grenzziehung bestimmen, könnte an deren Einsicht liegen, dass noch schließlich alle Erkenntnisgegenstände nur in ihrem Bedeutungszentrum sicher sind, zu den Randzonen hin die Unsicherheiten gehäuft auftreten und die Gegenstände sich schließlich in den Überlappungszonen zu anderen Kompetenzbereichen verflüchtigen. Es ist die aus der neuzeitlichen Physik gewonnene und übertragbare Einsicht, wonach zunehmende Aussagepräzision abnehmende Aussagerelevanz zur Folge hat. Es könnte sich daher erübrigen, die definitorischen Spielarten des Grundkonsenses aller Definitionen für „Umweltgeschichte“ mit spitzer hermeneutischer Feder oder dem Ockhamschen Rasiermesser zu autopsieren. Letztlich stimmen alle Definitionen in jener trivialen Schnittmenge überein, dass es um „das Verhältnis des Menschen zur Umwelt in der Geschichte“ gehe. Selbstverständlich liegt das Problem auch hier im Detail, nämlich in den Substantiva und ihren bestimmten Artikeln. Eine definitorische Entschärfung ist dadurch zu erreichen, dass die bestimmten Artikel ihrer grundsätzlichen ontologischen bzw. idealtypischen Zuweisungsqualität entkleidet werden und bei ihrer Verwendung lediglich eingeräumt wird, dass ein Präzisierungsbedarf bestehen kann. Ihm mag man aus pragmatischen Gründen nicht nachgehen wollen, weil ein allgemeiner Grundkonsens unterstellt werden kann. Definitorische Nachbesserung wird man erwarten dürfen, wenn einem Autor die pragmatische, unscharfe Definition nicht ausreicht. Es wäre dann die Aufgabe der scientific community, das von ihm gefundene Definitionsangebot kritisch zu prüfen und den Konsensbegriff ggfl. zu aktualisieren. Ein solches Selbstverständnis ist bisher von den wissenschaftlichen Gesellschaften, in denen sich Umwelthistoriker organisieren, nicht entwickelt worden.

Die genannten Substantiva fordern dann auch noch zu didaktisch-methodisch konzipierten geometrischen Figuren geradezu heraus. So sind in der Umweltgeschichte graphische Visualisierungsversuche verbreitet, die das grundsätzliche Mensch-Umwelt-Verhältnis mit Varianten des Bedeutungsinhalts zu Mensch (auch „Gesellschaft“ bzw. „Kultur“) bzw. zu Umwelt (auch „Natur“, auch i.S. von „Umgebung") im Hinblick auf eine geschichtstheoretische Funktionalität darstellen. ${ }^{34}$ Die analytische Qualität solcher Diagramme bleibt jedoch oft unklar. Vielmehr

\footnotetext{
${ }^{33}$ Strömungswissenschaftliche Bewertungen stufen die berühmt gewordenen Schöninger Speere als aerodynamische Hochleistungsgeräte ein. (Thieme 2007).

${ }^{34}$ Es genügt hier der Hinweis auf Winiwarter \& Knoll, Kap. 5.2., die einschlägige Graphiken (dort als „Modelle“ bezeichnet) zusammengestellt haben.
} 
scheint sich ihre Verwendung eher der Vorstellung zu verdanken, dass ein Diagramm als Ausweis gehobenen wissenschaftlichen Anspruchs gilt, als dass es einen Beitrag zur Erklärung eines konkreten Problems oder zur Systematisierung eines Forschungsfeldes leistet. Ich werde daher die diagrammatische Facette nicht weiter erörtern. ${ }^{35}$ Gleichfalls reizlos ist für mich die Demonstration einer fleißigen Belesenheit, die in die Präsentation einer Tabelle mit nuancenreichen Definitionsbemühungen mündete, wie sie aus der Literatur zutage gefördert werden könnten. Stattdessen genügen mir einige Beispiele:

Eine einflussreiche Schrift zur Frage „Was ist Umweltgeschichte?“ hat Radkau 1993 vorgelegt. Ihr folgten später Definitions-Varianten in Buchform. Donald Hughes definiert „Umweltgeschichte“ in einem Satz und hängt das erläuternde Buch dann an. ${ }^{36}$ Winiwarter u. Knoll (2007) definieren nicht explizit, sie machten ein Systematisierungsangebot, wie es eben die Aufgabe von Lehrbüchern ist. ${ }^{37}$ Lehmkuhls Aufsatz (2002) ist interessant, weil sie die Perspektive der histoire totale und das Zukunftspotential der Umweltgeschichte in den Blick nimmt. Der Aufsatz von Siemann \& Freytag (2003) erläutert die Bedeutung des Epistems Umwelt für die Geschichtswissenschaft und begründet seine gleichberechtigte Stellung neben den drei anderen Grundkategorien (Herrschaft, Wirtschaft, Kultur). ${ }^{38}$ Nach Auffassung der Autoren ist die Umweltgeschichte „letztlich Menschen- und Naturgeschichte in einem...“.39 Die Darstellung der „Umweltgeschichte im 19. und 20.Jhs. “ ${ }^{40}$ von Uekötter (2007) enthält im ersten Kapitel eine kurze, kritische Revision theoretischer Positionen und forschungspraktischer Themen, die in der deutschen Fachliteratur der Umweltgeschichte zuzurechnen sind. Definitorische Prob-

\footnotetext{
$35 \mathrm{Zu}$ den breiter rezipierten Naturdefinitionen von Holling \& Ludwig, die sehr einfache und damit einprägsame geometrische Darstellungen gefunden haben, vgl. Herrmann 2008. Sie sind aus den dort angegebenen Gründen m.E. für den umwelthistorischen Diskurs unbrauchbar.

36 "It is a kind of history that seeks understanding of human beings as they have lived, worked and thought in relationship to the rest of nature through the changes brought by time." (Hughes 2006, S. 1). Ungeachtet dieses Zitats konzentriere mich innerhalb des hier vorliegenden Aufsatzes auf den deutschsprachigen Raum, muss jedoch mindestens auf den etwas entlegen publizierten Reviewaufsatz von McNeill (2003) verweisen.

37 Anstelle eines Definitionskapitels versammeln Winiwarter \& Knoll Definitionsangebote aus der Literatur in der Einleitung (S.14-15). Sie folgen damit der Praxis vieler Lehrbücher unterschiedlichster Disziplinen, die ihren Gegenstand nicht eigens definieren, sondern vermutlich dem didaktischen Mittel folgen, mit dem Lehrbuchtext den Leser zu einer intuitiven Definition verhelfen zu wollen. Tatsächlich wird mit der Systematisierung des Stoffs in einem Lehrbuch implizit ein eigenes Definitionsangebot gemacht, was für praktische Belange zumeist ausreichend erscheint. - Eine ältere Definitionszusammenstellung für „Umweltgeschichte“ findet sich bei Fuchsloch.

38 Siemann \& Freytag haben eine nach meinem Urteil überzeugende Darstellung zur geschichtstheoretischen Bedeutung der „Umweltgeschichte“ verfasst. Umweltgeschichte wird von ihnen nicht eigens definiert, sondern pragmatisch ein Konsens von der „Erforschung der Wechselwirkungen zwischen Mensch und Natur" unterstellt (S.8).

39 A.a.O., S. 10.

40 Uekötter 2007. Der Kürze seiner Gesamtdarstellung fallen einige gewichtige Aspekte und manches differenzierter zu denkendes Urteil zum Opfer. Uekötter scheint dem Raisonnement einer Umweltgeschichte nicht-historiographischer Provenienz skeptisch gegenüber zu stehen, obwohl er in Kap.10 die „Vereinigung von menschlicher und natürlicher Geschichte als einen alten Historikertraum“ (S.90) bezeichnet.
} 
leme, die sich aus vergleichender Betrachtung von Naturaneignungen in der Organismenwelt ergeben, ${ }^{41}$ werden nicht allein von Uekötter abgelehnt, weil sie auf ein falsches Geschichtsverständnis gegründet wären. ${ }^{42}$ Greift man auf das Lehrbuch von Winiwarter \& Knoll zurück, dann gäbe es (wenigstens) zwei konsensfähige Definitionen:

„1) Umweltgeschichte befasst sich mit den Wechselbeziehungen zwischen Menschen und dem Rest der Natur in der Vergangenheit (nach Beinart \& Coates, 1995:1)

2) Umweltgeschichte beschäftigt sich mit der Rekonstruktion von Umweltbedingungen in der Vergangenheit sowie mit der Rekonstruktion von deren Wabrnehmung und Interpretation durch die damals lebenden Menschen (₹:B. Herrmann, 1996:21, Sieferle, 1997:13f)" 43

Der Vorzug der zweiten Definition besteht darin, dass sie sich über die kulturell und subjektiv kontextualisierten Mensch-Natur-Beziehungen hinaus auch die Rekonstruktion objektiver Naturzustände zur Aufgabe macht. Insofern ist die scheinbar allgemeiner gefasste Definition 1 tatsächlich die weniger umfassende, weil sie sich ausschließlich auf die anthropologische Dimension beschränkt. Wie immer der Gegenstandsbereich von „Umweltgeschichte“ be- oder entgrenzt wird, ihr wohnt nach meinem Urteil ein größerer Anteil wissenschaftsgeschichtlicher Selbstreflexion inne als anderen Geschichtsdisziplinen. ${ }^{44}$ Für die „Umweltgeschichte" wird er jedoch nur zum Teil betrieben und zumeist auch ohne als solcher begriffen zu sein. An die historischen Bedingungen, unter denen sich „Umweltgeschichte" als Wissenskorpus etablieren konnte, wird zwar gelegentlich erinnert. Aber das Abarbeiten der Elementarbegriffe des Wissenszusammenhangs „Umweltgeschichte" ist bislang in ihm selbst nur in geringem Umfang erfolgt, wie am Beispiel des „Natur"-Begriffs leicht erkennbar ist, der seit der Antike Dauerthema des philosophischen Diskurses ist, ohne von dort logisch in eine „Umweltgeschichte" geführt zu haben. Erschwert wird die wissenschaftshistorische Analyse allerdings durch methodischen Opportunismus (,anything goes", Feyerabend 1986) und methodische Vielseitigkeit, über die „Umweltgeschichte“ im Zuschnitt von „Rekonstruktion und Rezeption“ verfügt. Wenn die Definition 2 ein wissenschaftsförderliches Potential beinhaltet, dann dasjenige, das aus ihr die Einsicht abzuleiten ist, wonach „Umweltgeschichte“ mit der „Wahrnehmung und Interpretation" zugleich über die Bedingung der Freilegung dieser Wahrnehmung und Interpretation zu reflektieren hat. Eigentlich ist „Umweltgeschichte“ damit eine Wissenschaftsgeschichte des Gegenstandsbereichs „Umwelt". Als historische Epis-

\footnotetext{
${ }^{41}$ Das ist letztlich bei „Umwelt“-Wortzusammensetzungen nicht zu umgehen und müsste ein selbstverständlich erweitertes Verständnis von „Umweltgeschichte“ nach sich ziehen.

42 Eine solche Position führt nun ihrerseits u.a. zu Konflikten mit dem einflussreichen „new ecological paradigm“"von Catton \& Dunlap (1980) (siehe unten unter II).

43 Winiwarter \& Knoll 2007, S. 14-15.

${ }_{44}$ Die Rede ist nicht von einer Wissenschaftsgeschichte, die eine Nacherzählung einer Entwicklung ist. Unübersehbar hat ein Teil der „Umweltgeschichte“ so begonnen, z.B. als Verschmutzungsgeschichte. Vielmehr ist das hier gemeinte Verständnis von Wissenschaftsgeschichte eines, das der Ideengeschichte und Wissenschaftstheorie verpflichtet ist und von rein nacherzählenden Ansätzen abgegrenzt.
} 
temologie (i.S. von Rheinberger 2007) ist sie die Frage, wie und mit welchem Ziel die Facetten des Gegenstandsbereichs „Umwelt“ aus den Quellen zum Gegenstand des Erkennens gemacht werden. ${ }^{45}$

In der kursorischen Übersicht liest sich die Geschichte veröffentlichter umwelthistorischer Arbeiten eher als eine Geschichte der thematischen Selbstversicherung beteiligter Autoren als eine ausdrückliche Bemühung um die Inhaltsbestimmung und Wege der Erkenntnisgewinnung. Auch dies ist ein bekanntes Muster in der Wissenschaftsorganisation, wenn durch Gebrauch zirkulärer Techniken die Notwendigkeit von Begriffsbestimmungen und Reflexionen der Eingangsvoraussetzungen operational umgangen werden sollen, in dem simple Lösungen als zufrieden stellend gelten: „Umweltgeschichte ist das, was Umwelthistoriker erforschen." Findet man mit dieser Einstellung zu einer Gruppe von Gleichgesinnten (z.B. in der ESEH, in einem Graduiertenkolleg oder auf einem Workshop) und kann mit ihnen eine Zeitlang den produktiven Gedankenaustausch pflegen, dann ist offenbar ausreichende Selbstbestätigung gewährt. Durch sie hat sich seit dem Ende der 80er Jahre bis gegen heute in einem „Selbstfindungsprozess“ (Toyka-Seid 2003) die Einschätzung zumindest der unmittelbar Beteiligten verdichtet, die Umweltgeschichte sei allmählich zu einer Disziplin aufgewachsen. Wenn das so wäre, hätten wir sicheres Terrain erreicht.

\section{Ic Umweltgeschichte als Disziplin}

Ist „Umweltgeschichte“ eine wissenschaftliche Disziplin? Die Verwendung des Begriffs sagt nichts über die Etabliertheit des mit ihm umschriebenen Wissenszusammenhangs. Sie bezeichnet lediglich eine besondere Differenz zu den Inhalten anderer Wortzusammensetzungen, etwa in Umweltpolitik, Umwelttechnik, Umweltproblem bzw. in Kirchengeschichte, Wirtschaftsgeschichte, Herrschaftsgeschichte.

Folgt man einer wissenssoziologischen Bewertung, dann gilt der „Disziplinen“Status als Ritterschlag und Ausweis für dynamische Entwicklung und Leistungsfähigkeit eines Wissensgebiets. Wissenschaftliche Disziplinen wären grundsätzlich durch bestimmte inhaltliche Merkmale gekennzeichnet (Fragestellungen, Metho-

\footnotetext{
45 Rheinberger 2007, S. 11f: „Ich fasse unter dem Begriff der Epistemologie [...] die Reflexion auf die historischen Bedingungen, unter denen, und die Mittel, mit denen Dinge zu Objekten des Wissens gemacht werden, an denen der Prozess der wissenschaftlichen Erkenntnisgewinnung in Gang gesetzt sowie in Gang gehalten wird. An der Wende vom 19. zum 20. Jahrhundert [gibt es] einen Umschlag von der Erkenntnistheorie der klassischen philosophischen Tradition zur Epistemologie im genannten Sinne. Diese Verschiebung markiert zugleich eine Problemumkehr. Die Reflexion des Verhältnisses von Begriff und Objekt, die vom erkennenden Subjekt ihren Ausgang nahm, wird ersetzt durch die Reflexion des Verhältnisses von Objekt und Begriff, die am erkennenden Objekt ansetzt. Diese Problemverschiebung ist zugleich Kern der Epistemologie und Ausgangspunkt ihrer Historisierung. [...] Die Frage ist nicht mehr, wie das erkennende Subjekt seine Gegenstände unverstellt in den Blick bekommen kann, die Frage gilt jetzt vielmehr den Bedingungen, die geschaffen wurden oder geschaffen werden müssen, um Gegenstände unter jeweils zu bestimmenden Umständen zu Gegenständen empirischen Wissens zu machen.“ (Hervorhebung im Orig.).
} 
den, Problemlösungen) ${ }^{46}$ woraus ein Korpus akzeptierter Wissenselemente und akzeptierter Forschungsmethoden resultiert. Danach könnte Umweltgeschichte Disziplinen-Qualität besitzen.

Die außerdem erhobene Forderung nach einem homogenen Kommunikationszusammenhang ist für inter- und transdisziplinäre Wissensbereiche schon schwieriger zu erfüllen. Und damit deutet sich an, dass die Umweltgeschichte diesem soziologischen Kriterium nicht genügen könnte. ${ }^{47}$

Ganz sicher nicht erfüllt ist gegenwärtig schließlich das Kriterium von spezifischer Karriere und beruflicher Sozialisation in eigenen Institutionen.

Ergänzend ist diesem Kanon die Frage der Alimentierung beizufügen (die nicht zwingend mit eigenen Institutionen verbunden sein muss). Auf sie wird im Zusammenhang mit der Relevanzfrage noch ausführlicher einzugehen sein. Man wird nicht fehlgehen, wenn man der umwelthistorischen Forschung eher eine Unterförderung als eine Überalimentierung attestiert und daraus auf ihren zumindest unsicheren Platz in der Wissenschaftssystematik schließt. In der Bundesrepublik gibt es z.Zt. keine selbstständige akademische Institution, die Umweltgeschichte als alleinigen Namen führte. Es gibt keinen Lehrstuhl mit umwelthistorischer Denomination. Die ersten (Junior-)Professuren für Umweltgeschichte sind gerade besetzt, weitere sind ausgeschrieben. ${ }^{48}$ Forschungsverbünde mit umwelthistorischer Ausrichtung haben Schwierigkeiten, sich im Wettbewerb bis zur Bewilligung zu behaupten. Immerhin gibt es einzelne Studienangebote zur Umweltgeschichte (z.B. TU Berlin, TU Darmstadt, Univ. Göttingen). Erstaunlich ist, dass die Rahmenrichtlinien der gymnasialen Lehrerausbildung die Behandlung von Umweltgeschichte ausdrücklich einfordern (z.B. in Niedersachsen), universitär dem aber kaum Angebote gegenüber stehen (hierzu auch Beitrag von Borries in diesem Band).

Was sich inhaltlich zu einer disziplinären Qualität zusammenfügen ließe, wird auf der strukturellen Ebene nicht erreicht: Umweltgeschichte ist nach den gängigen Regeln des Wissenschaftsbetriebes zurzeit keine etablierte Disziplin. ${ }^{49}$ Gilt dies

\footnotetext{
46 Zusammengefasst nach Stichweh 1994.

${ }^{47}$ Unterstützung für meine Diagnose sehe ich u.a. auch in Kap.10 von Uekötter 2007.

48 Umweltgeschichte kann auch als historisierende Variante der Humanökologie verstanden werden. Für die humanökologische Umweltforschung insgesamt hatte der Wissenschaftsrat bereits vor vierzehn Jahre die Schaffung institutioneller Einrichtungen an den Universitäten - bislang folgenlos empfohlen (Wissenschaftsrat 1994, Bd.1, S. 104). Der Wissenschaftsrat hatte dabei die gesamte einschlägige Palette struktureller Maßnahmen vor Augen, die üblicherweise fachliche Gründungsinitiativen begleiten und vor allem darauf verwiesen, dass sich ohne institutionelle Strukturen der wissenschaftliche Nachwuchs nicht motivieren werden lasse, sich in diese Forschungsfelder hinein zu qualifizieren. Weiter heißt es (Bd.2, S.198): „Umweltgeschichte sollte hier [gemeint ist an der Biologischen Fakultät der Universität Göttingen, B.H.] zu einem weiteren Schwerpunkt entwickelt werden, der bis zu Aktivitäten im Rahmen von Global Change reichen kann." Eine Umsetzung erfolgte nicht.

49 Die Diagnose wird verstärkt durch den Umstand, dass im weitverbreiteten „Bücherverzeichnis zur deutschen Geschichte“ noch in der 16. Auflage weder im Register noch im Inhaltsverzeichnis „Umweltgeschichte“ vertreten ist (Baumgart 2006), ebenso fehlt „Umweltgeschichte“ in den Rezensionsrubriken von H-Soz-u-Kult bis auf den heutigen Tag (5.12.2008). Gegenüber diesem Ausdruck fach-
} 
schon für eine Betrachtung aus deutschsprachiger Perspektive mit Blick auf die deutschsprachige community, so sind die Wahrnehmungen dieser deutschen community durch die internationale Szene noch deprimierender. In ihrem Revisionsaufsatz über Inhalte und Ausrichtungen der Umweltgeschichte wird von Sörlin u. Warde (2007) auf den Text auch nicht eines deutschsprachigen Umwelthistorikers Bezug genommen. Dass hier wenigstens zum Teil die übliche angelsächsische Ignoranz ursächlich ist, wird man bei einiger Kenntnis des Metiers richtig vermuten. Es bleibt jedoch der Umstand der geringen internationalen Ausstrahlung, obwohl Deutsch traditionell eine lingua franca der Geschichtsdisziplinen zumindest war. Ein inhaltlicher Grund könnte allerdings auch sein, dass sich die deutsche umwelthistorische Gemeinde noch nicht als kohärente Gruppe gefestigt hat bzw. als solche nicht wahrnehmbar ist und dadurch ohne nennenswertes Gewicht wäre. ${ }^{50}$

Der wissenssoziologischen Disziplinenbetrachtung ist eine wissenschaftssystematische an die Seite zu stellen. ${ }^{51}$ Sie hat den Vorzug, dass sie sich nahezu ausschließlich auf die Qualitäten der wissenschaftlichen Vorgehensweise bezieht und den soziologischen Aspekt der Institutionalisierungsebene mit Folgen und Nebenfolgen ausblendet. Vier Antworten gäbe es nach Lorenz Krüger auf die Frage, wonach Disziplinen zu unterscheiden wären: ${ }^{52}$

1) es sind die Gegenstände, doch nicht diese allein, weil interessierende Aspekte, Fragen oder Probleme notwendige erläuternde Beifügungen wären.

2) es sind die Methoden, wobei allerdings begrenzte innerdisziplinäre Kompetenz den Einsatz bestimmter Methoden zunächst ausschließt.

3) ist es das Erkenntnisinteresse, etwa auf allgemeine Gesetzmäßigkeiten oder auf das Erkennen einmaliger Individualitäten hin; oder auf ein technisches Erkenntnisinteresse hin; oder auf ein praktisches, d.h. ein auf Verständigung im Handeln zielendes; oder auf ein emanzipatorisches, d.h. auf den Abbau der Herrschaft von Menschen über Menschen gerichtetes Interesse. ${ }^{53}$

4) schließlich sind es ihre Theorien. Auch ohne die Formulierung elaborierter theoretischer Konstrukte wären allein schon in der Absicht der Wissenschaften, die Fülle der Tatsachen, Regeln, Tatbestände und Entscheidungen zu ordnen, zu deuten oder zu erklären der theoretische Gehalt erkennbar,

lichen Mainstream-Verständnisses ist das verlegerisch dominierte Konzept der „Enzyklopädie deutscher Geschichte“ offener, das die Monographie von Uekötter (2007) aufgenommen hat.

${ }^{50}$ Dies ist u.a. ein Grund, warum m.E. von der deutschsprachigen Umweltgeschichte statt von einer Disziplin zumindest z.Zt. besser von einem Wissenszusammenhang zu sprechen wäre. Ausschlaggebend für diese begriffliche Bevorzugung ist allerdings anstelle des schwach entwickelten strukturellen Kriteriums eine Betonung des inter- bzw. transdisziplinären (und damit offenen) Kommunikationszusammenhangs.

51 Krüger 1987. Krüger stellt die Frage, was eine Disziplin sei, nicht direkt, sondern sucht nach den Merkmalen einer Disziplin, die sie von anderen abhebt.

52 Krüger 1987, $115 \mathrm{f}$.

53 Krüger bezieht hier die Zweiteilung auf Wilhelm Windelband, die Dreiteilung auf Jürgen Habermas. 
„,nämlich, dass die nächste durch das Leben herangetragene Einzelheit in vorgezeichneter Weise verarbeitbar wird.“

Folgte man diesen Überlegungen, ist der Wissenszusammenhang „Umweltgeschichte“ disziplinär organisierbar und von sich selbst und von anderen als Disziplin wahrnehmbar. Doch ist die Kohärenz auch in dieser Betrachtung nach wie vor gering, weil „die Begriffe Natur und Umwelt sehr vage sind, das methodische Vorgehen wenig geklärt und zudem unsicher ist, wer das Subjekt und welches die Bezugsgrößen dieser neuen Richtung sein sollen." 54 Auch acht Jahre nach diesem Verdikt sind „Natur“ und „Umwelt“ einer konsensualen innerdisziplinären Präzisierung nicht viel näher gekommen. Man mag darin das Symptom einer korporativen Kraftlosigkeit erkennen, die eine der Ursachen sein kann, warum sich keine selbstbewusste Gruppe formiert, um der Umweltgeschichte zu der entscheidenden Lobby zu verhelfen.

Letztlich könnte die Frage nach dem Disziplinencharakter als belanglos abgetan werden, wäre mit ihr andererseits nicht eine besondere, nämlich eine forschungspolitische Seite verbunden. Je nachdem, ob Umweltgeschichte als Disziplin oder als Subdisziplin begriffen wird, treten Vorbesitzer der Parzelle bzw. Besitzer benachbarter wissenschaftlicher Immobilien mit entsprechenden Ansprüchen auf. Wer von der Umweltgeschichte als von einer „historischen Subdisziplin“ spricht, ${ }^{55}$ und gleichzeitig die Definitionsmacht für den ihr zugrundeliegenden Geschichtsbegriff beansprucht, ${ }^{56}$ reklamiert Umweltgeschichte als Besitzstand bestimmter Fächer. Das blendet andere umwelthistorische Zugänge aus oder weist ihnen Ancilla-Funktionen zu. Die Diskussion ist älter ${ }^{57}$ und ich hatte sie für erledigt gehalten, weil die Wissenschaftspraxis und das Zusammenleben in der scientific community diese Differenzen überwunden zu haben schienen. Doch ein Blick in die Literaturlisten neuerer umwelthistorischer Arbeiten bzw. Sammelbände zeigt, dass Wahrnehmungsbeschränkungen immer noch bestehen. An manchen Stellen ist sogar ein erheblicher Entwicklungsrückstand bezüglich einer auf Inter- bzw. Transdisziplinarität ausgerichteten Umweltgeschichte mit entsprechender wissenschaftlicher Offenheit augenfällig.

Es ist immer zweischneidig, an professionelles Verhalten aller im Exempel agierenden Akteure zu appellieren. Gemeint ist sicher ein disziplinendienliches und gleichzeitig ein selbstdiszipliniertes Verhalten, das die Entwicklungsmöglichkeiten des Gebietes stärkt und fördert. Treffen wird man jedoch immer auch auf Machtund Interessenkonstellationen. Dennoch sei um der Sache willen für ein Miteinander geworben. Evolutionsbiologisch kann man zeigen, dass kooperatives Verhalten

\footnotetext{
${ }^{54}$ Brüggemeier 1996, S.9. Fraglos trifft die Diagnose Brüggemeiers zu, aber nicht nur aus Gründen inhaltlicher Komplexität allein, welche die Gegenstandsbereichen und ihre Beziehungen zueinander so diffus erscheinen lassen. Gründergeneration wie Protagonisten der (deutschsprachigen) Umweltgeschichte scheuen das Abarbeiten des Theoriedefizits.

55 z.B. Siemann u. Freitag 2003, S. 7; Uekötter 2007, S.2

56 z.B. Uekötter a.a.O.

${ }^{57}$ z.B. Herrmann 1996, S.28
} 
als Langfriststrategie für alle Beteiligten prämiert wird. Sollten allerdings die eben erwähnten Wahrnehmungsbeschränkungen Ergebnisse von stillschweigenden Kooperationsbünden sein, kann es einer beschränkten Umweltgeschichte nicht gelingen, sich gegen die Kompetenzen derjenigen anderen Disziplinen zu etablieren, die fruchtbar beizutragen hätten. Schlimmer noch, es würde die pointierte Unterscheidung zwischen den „Zwei Kulturen“ (C.P.Snow) der naturwissenschaftlichen und der geisteswissenschaftlichen Analyse weiter verfestigen. Damit wäre der interdisziplinäre Austausch, der für die Umweltgeschichte konstitutiv ist, durch eine neuerliche Diskriminierung zum Nachteil des Forschungsgegenstandes gefährdet, weil schon Erreichtes preisgegeben würde.

\section{Zur Relevanz der Umweltgeschichte}

\section{IIa Unabhängiges Forschen und}

Teilhabe an „aktuellen“ wissenschaftlichen Fragestellungen

Verbreitet ist eine naive Ansicht, nach der wissenschaftliche Themengebiete sich allein aus dem Interesse der einzelnen Forscher ergäben. Tatsächlich sind es überwiegend die Interessensverbünde aus Wissenschaft und übriger Gesellschaft, die wissenschaftliche Themen akademisch überhaupt zulassen, integrieren und zu „hoch-aktuellen“, zu ,innovativen“, zu „,anschlussfähigen“, zu „,zukunftsträchtigen" Themen machen, ihnen einen Aufmerksamkeitsstatus verleihen und als Attraktoren auf die Wissenschaftler zurückwirken. Damit wird eine sich selbst beschleunigende Spirale wissenschaftlicher Geschäftigkeit in Gang gesetzt, deren öffentliche Rezeption als Maßstab für die qualifizierteste, modernste und „hochaktuelle“ Forschung hergenommen wird. ${ }^{58}$ Solche wissenschaftlichen Konjunkturen, oder zumindest die Hoffnung auf diese, markierten auch Anfänge der deutschen wie der internationalen Umweltgeschichtsforschung.

Dennoch hält sich allgemein hartnäckig die Vorstellung einer allein aus dem persönlichen Interesse des Forschenden resultierenden Fokussierung auf sein Thema. So wurde auch Anlässlich der Übergabe seines Rektorats des Berliner Wissenschaftskollegs 2007 an Luca Giuliani von Dieter Grimm die Bedeutung dieser Institution mit folgenden Worten betont:

„...Die Forschung des Einzelnen ist wichtig, nicht nur sein Beitrag als Gruppenmitglied in Großprojekten. Die nicht von vornherein nutzenorientierte Forschung ist wichtig, nicht nur, weil sie manchmal ungeplanten Nutzen stiftet, sondern wegen des Selbstwertes von Erkenntnis. Die

\footnotetext{
${ }^{58}$ Es sei daran erinnert, dass nur extrem selten Nobelpreise verliehen werden, die zeitlich junge Arbeiten prämieren. Man kann darin einen Hinweis sehen, dass erst einmal die langfristige Tragfähigkeit „sensationeller, neuer Einsichten“ oder Befunde abgewartet wird, bevor man sich über deren wirklichen Wert sicher sein kann. Solche Beurteilungen zeigen sich unbeeindruckt vom wissenschaftlichen Tagesgeschäft, das sich an den Prinzipien des „Markgeschreis“ und der wissenschaftlichen wie der feuilletonistischen Seilschaftenpflege orientiert.
} 
Forschung, die nicht von Drittmitteln abhängig ist, ist wichtig, weil hier das Erkenntnisinteresse nicht extern, sondern intern bestimmt wird und weil man besser Distanz zu den beobachteten Systemen halten kann. Die Pflege der sogenannten kleinen Fächer ist wichtig, weil sie einen kulturellen Reichtum erhalten und Verständnishorizonte eröffnen, die unter Globalisierungsbedingungen nötiger denn je sind. "59 Einer solchen Bewertung wird man vermutlich vorbehaltlos zustimmen, denn Grimm trägt hier eben das bekannte und anerkannte Rechtfertigungsmantra für das individualistische, selbstbestimmte Forschen vor. Ein Institute for Advanced Studies anerkennt also die Bedeutung des Einzelnen, enthebt ihn der Drittmittelabhängigkeit und feiert den nur sich selbst verpflichteten Gelehrten. Im Kleinen, so deuten wir Grimms Beschreibung um, funktioniert ein Graduiertenkolleg letztlich wie ein Institute for Advanced Studies. Genauso sahen sich und es ja auch einige Kollegiaten des Göttinger Kollegs, wie im Vorwort geschildert. Mit dem Hinweis auf Grimm, auf die Idee der Institutes for Advanced Studies und dem Hinweis darauf, dass der Wissenschaftler allein entscheide, was relevant, was also im Wortsinne ,wichtig“ ist, könnte man die Sache als erledigt betrachten. Die „Relevanz" wird damit kategorial zur „Relevation“ hin verschoben, mit der die „Befreiung von einer Verbindlichkeit“ gemeint ist. Der Wissenschaftler sei frei von Verbindlichkeiten gegenüber wem auch immer.

Sollte Dieter Grimm tatsächlich entgangen sein, dass sein Kolleg mit der Fernhaltung der Fellows von den Niederungen praktischer Wissenschaftsorganisation und -alimentierung die damit verbundenen Probleme lediglich auf andere Ebenen verlagert hat, nämlich die der Administration seines Kollegs selbst? Käme man ohne Auswahl ans Wissenschaftskolleg? Gibt es keinen jährlichen Rechenschaftsbericht, mit dem die Geldgeber beruhigt werden? Verdanken sich die gemeinschaftlichen Forschungsprojekte des Kollegs wie auch der Fellows selbst nicht etwa auch jeweiligem gesellschaftlichem Interesse? ${ }^{60}$ Eine ähnliches Beispiel kann man in dem geldschweren britische Forschungsprogramm erkennen, dem der programmatischen Name „Blue Skies Research“ gegeben wurde, und das sich beispielsweise überraschend konventionell der Mitwirkung von Gutachtern versichert $^{61}$ - eine Rückversicherung, mit der das Entfernen der Projekte vom wissenschaftlichen Mainstream erschwert wird. Und warum veröffentlicht die Deutsche Forschungsgemeinschaft etwa mit den „Perspektiven der Forschung und ihrer Förderung" regelmäßig einen Fünfjahresplan, ${ }^{62}$ wenn von ihm nicht eine gewisse Selbstbindung an den dort skizzierten thematischen Rahmen ausgehen soll? Zweifel daran, dass die Bewegungsspielräume der Wissenschaft von gesellschaftlich gelenkten Vorstellungen und Geldmitteln abhängen, kann nur derjenige haben, der

\footnotetext{
59 Aus: Ansprache des Rektors des Wissenschaftskollegs zu Berlin, Dieter Grimm, aus Anlass der Rektoratsübergabe am 1.April 2007. Wissenschaftskolleg zu Berlin. Seiten 9- 10.

${ }^{60}$ Verf. glaubt, sich als ehem. Fellow-Sprecher (Class of 1995/96) diese Fragen erlauben zu dürfen.

${ }^{61}$ Der Geldgeber, das Natural Environment Research Council, NERC, weicht Erklärungen oder Diskussionen über diesen sonderbaren Umstand aus, wie Verf. aus eigener Gutachtertätigkeit weiß.

62 Zuletzt: Deutsche Forschungsgemeinschaft, Perspektiven der Forschung und ihrer Förderung 2007 - 2011. Wiley-VCH, Weinheim 2008
} 
noch nie dem Zwang zur Formulierung von Antragsprosa, der Suche nach den passenden Schüsselbegriffen ausgesetzt war und sich noch nie dem Wohlwollen einer Gutachtergruppe zu stellen hatte. So, wie die geldabhängige Wissenschaft organisiert ist, wohnt ihr der Zwang zur Anbiederung an die Strukturen und an die Philosophien der Drittmittelgeber inne. Allerdings tun beide Seiten so, als ginge es immer nur um die Sachen, nie um Interessen. Selbstverständlich ist der Wissenschaftler frei und darf denken, was immer er will. Aber sein Denken, für das er um Subventionierung nachsucht, wird begrenzt durch den Fluss des Geldes. Es liegt außerdem in der Logik eines solchen Systems, dass der gesellschaftliche bzw. institutionelle Einfluss auf die Forschung umso höher sein wird, umso höher diese Forschung subventioniert ist.

Anlässlich dieser Feststellung müsste man nach den Geldmengen suchen, die in umwelthistorische Projekte fließen, um auf diesem Wege eine erste Vorstellung von der gesellschaftlichen Relevanz der Umweltgeschichte zu erhalten. Dass die Mittel auf der institutionellen Seite bescheiden sind, wurde bereits festgestellt. Immerhin sind die Drittmittelgeber aufgeschlossener, als es zunächst erscheint. Doch konzentriert sich die Förderung dabei in einem allgemeineren Sinne auf geistesund sozialwissenschaftliche Behandlung von Umweltthemen und praktisch nicht auf umwelthistorische. ${ }^{63}$ Immerhin enthielt die „Nachwuchsförderung in der fächerübergreifenden Umweltforschung“ der VW-Stiftung (Förderzeitraum bis 2006) zwei archäologische Projekte.

Insgesamt gesehen liegt mit dem Göttinger Graduiertenkolleg 1024 daher eine bemerkenswerte Fördermaßnahme vor, aber abgesehen von singulären und eher persönlichen als strukturellen Erfolgen, binden umwelthistorische Projekte in der Bundesrepublik keine erheblichen Geldmengen. Nach diesem Maßstab ist Umweltgeschichte eine wenig relevante Wissenschaftsrichtung. Wegen fehlender Möglichkeiten der Nachwuchsförderung durch Stellenabsicherung wird sich absehbar daran nichts ändern (können). Die Situation am Stellenmarkt ist aber ein starkes Motiv für Graduierte, ihre weitere Qualifikation thematisch auszurichten. Die Perspektive einer persönlichen wirtschaftlichen Unabhängigkeit wird zu einem Kriterium der Qualifikations- und Zukunftsplanung und damit, neben dem Aspekt der reinen Wissenschaftlichkeit, zu einem offensichtlichen weiteren Relevanzkriterium. Für die Umweltgeschichte besteht auch hier ein strukturelles Defizit. „Forschen“, so stellt sich am Ende heraus, ist aus vielerlei Gründen nicht „unabhängig“, weil es sich auch an den Vorstellungen und Auswirkungen einer wie immer auch gearteten „Relevanz“ orientiert. Das gilt selbstverständlich auch für umwelthistorisches Forschen.

Der jeweils aktuelle Aufmerksamkeitsstatus lenkt Geldmengen zur gezielten Förderung wissenschaftlicher Arbeit um oder bewegt zusätzliche in die Forschung. Die Hoffnung auf eine solche Partizipation hat sich für die Umweltgeschichte

${ }^{63}$ Eine Skizze der einschlägigen Förderlandschaft der letzten Jahre und der größeren Einzelprojekte bei Knopf 2008, S.12-14. 
bisher nur im bescheidenen Maße erfüllt, obwohl der Zeitpunkt ihrer Anfänge aus dem Katastrophendiskurs und den umweltbezogenen Alarmismus-Debatten in einem technischen Sinn ,richtig“" lag. Obwohl die finanzielle und strukturelle Lage der Umweltgeschichte dies nahelegt und mit dem Hinweis auf die Relevation die Frage nach der Relevanz für möglicherweise erledigt erklärt wurde, ist sie selbstverständlich nicht erledigt, denn „Wichtigkeit“ bzw. „Erheblichkeit“, die Wortinhalte von „Relevanz“, sind keine objektiven Kategorien. Ist als ein Maßstab für „Relevanz" die in die Wissenschaft fließende Geldmenge ausgemacht, steht damit noch nicht fest, welche Relevanzerwartung der Geldgeber damit verbindet: wird Grundlagenforschung gefördert oder angewandte Forschung? Der gegenwärtige enorme Akquisitionserfolg beispielsweise biowissenschaftlicher Forschungsverbünde erklärt sich aus dem Besitz einer magischen Formel, die auf eine derartige Erwartung gründet. Was immer mit den Zauberworten „Stammzellen-Therapie“ oder „Besiegen des Krebses“ berührt wird, verwandelt sich augenblicklich in Geld. Heilsversprechen der Human- bzw. Biowissenschaften scheinen Garanten für die Steuerung der Geldströme. In der Gesundheitsforschung ist das deshalb einfach, weil die Erfindung der ultimativen Pille ein Wirtschaftsgut allerersten Ranges darstellt und „Gesundheit“ als allgemein schutzwürdiges und anzustrebendes materielles Gut und als mentaler Idealzustand akzeptiert ist. Könnte sich Umweltgeschichte - wenigstens in einem bescheidenen Umfange - an solchen strategischen Vorbildern orientieren und wenn, womit?

Tatsächlich sind Umweltthemen, jenseits eines marktgängigen Interesses an „öko“, durchaus prominent geförderte Forschungsfelder. Der Akquisitionserfolg der Klimaforschung verdankt sich gegenwärtig zweifellos der aufgeregt geführten Diskussion, die Anlass hat, die Welt dem Zustand des Hitzetodes mit allen ihren Vorboten nahe zu wähnen. Ein weiterer einschlägiger Geldgreifer sind die organismischen Biologen, denen es gelungen war, Mitte der 80er Jahre mit dem Schlagwort „Biodiversität“ einen Begriff zu etablieren, dessen Marketingwert auch den Begriffen der Molekulardisziplinen nicht sonderlich nachstand und neben dem Stichwort „Klimawandel“ heute ein bedeutendes Umweltthema besetzt. Drei Eigenschaften verbergen sich nach meiner Einsicht hinter dem Biodiversitätsbegriff: ${ }^{64}$ ein Tauf- und Missionsbefehl, ein Schlachtruf und ein Börsenbegriff. Der „Tauf- und Missionsbefehl“ bedient religiöse bzw. quasi-religiöse Bedürfnisse, auf die ich weiter unten noch eingehe. Der „Schlachtruf“ ist Instrument von NGOs und Körperschaften geworden und hat zu politischen Eckdaten geführt, die ihren Ausdruck u.a. im MEA ${ }^{65}$ finden. Sehr interessant ist die börsenaffine Seite des Begriffs. Sie beklagt den Rückgang der Artendiversität vor dem Hintergrund entgehender menschlicher Verwertungsmöglichkeit und damit entgehender wirtschaftlicher Nutzung, vor allem aus pharmakologischem Interesse. Umwelthistori-

\footnotetext{
64 Herrmann 2006, S. 187

65 Millenium Ecosystem Assessment. http://www.millenniumassessment.org - Wie man aus Biodiversitätsmodellen politische Kategorien macht, erzählen aufschlussreich Mann u. Plummer 1995.
} 
ker wissen, dass dieser Aspekt der Naturnutzung schon vor 250 Jahren den europäischen Blick in die Kolonien beflügelte: „Nutzung“ und „Verbesserung“ (der Natur) als Zentralbegriffe der Aufklärung. Allerdings bewegen sich heutige Ausbeutungsinteressen, die u.a. die Patentierung von Genombestandteilen anstreben, um dadurch wirtschaftliche Vormachtstellung zu erhalten, jenseits jeder Vorstellungsmöglichkeiten gutgläubiger Aufklärer. ${ }^{66}$

Fraglos sind „Biodiversität“ und sein mit der Rio-Konferenz etablierter Nachbarbegriff „Nachhaltigkeit“ sowie das Thema „Klimawandel“ gegenwärtige politikbestimmende Schlagwörter. ${ }^{67}$ Umweltthemen haben längst die Feuilletons erreicht, sind Gegenstand von unterhaltungsdienlichen Katastrophenszenarios. ${ }^{68}$ Die Werbung arbeitet mit umwelthistorischen Versatzstücken, wenn mit Chiffren des Paradiesverlustes die Lust auf einen Urlaub, ,im Südseeparadies“ geschürt oder ein beliebiger Konsumartikel als „Natur pur“ angepriesen wird. Alle sind Hinweis darauf, dass Umweltthemen breit rezipiert werden, mithin Relevanzcharakter haben. Ließe sich da der Umweltgeschichte nicht eine Teilhabe an Relevanzfragen zuweisen?

\section{IIb Aus der Geschichte lernen?}

Vielleicht sollte bei der Erwägung der vielbemühten Frage, „ob denn aus der Geschichte zu lernen sei“" und ihrer entsprechenden Abwandlung auf die Umweltgeschichte hin, zweckmäßig eine Unterscheidung zwischen geschichtstheoretischakademischem Purismus und praktischem Handeln gemacht werden. Die Theoretiker werden sich ad infinitum weiter streiten, weil sie den Kniff der ceterisparibus-Regel oder das Schließen aus normischen Gesetzen $^{69}$ nicht einsehen oder

\footnotetext{
${ }^{66}$ Beispielsweise ist mit dem geplanten Zugriff der Firma Monsanto, mit einer für diesen Agrarkonzern (bzw. Tochterunternehmen) patentierten Sequenz des Schweinegenoms die weltweite Schweineproduktion zu monopolisieren, bereits heute die bedrückendste Folge von Naturausbeutung durch Kommerzialisierung erreicht, weil davon möglicherweise selbst alte Landrassen nicht mehr ausgenommen wären. Die fatale Perspektive solcher Gen-Monopole liegt auf der Hand.

${ }^{67} \mathrm{Zu}$ den Themen, die u.a. auf dem Workshop nicht erörtert wurden, gehört auch die Frage: „Was macht eigentlich....das Artensterben?" Nach Jahren des hochrangigen politischen Arguments, scheint es im Moment etwas stiller um diese Frage geworden zu sein. Es lohnt sich, neben der Lektüre von Mann \& Plummer (1995) auch jene Prognose von Global 2000 (1980, besonders S. 86) heranzuziehen, in der ein Aussterben von 15-20\% aller auf der Erde lebenden Arten bis zum Jahre 2000 für möglich gehalten wurde. Dies scheint nicht eingetroffen zu sein. - Für den ersten AlarmismusBericht des Club of Rome „Grenzen des Wachstums“ (Meadows et al. 1973) spielten mögliche Biodiversitätsverluste noch keine Rolle.

68 z.B. die Filme „The Day after Tomorrow“ Regie: Roland Emmerich; „Deep Impact“ Regie: Mimi Leder; kriminalistische Bestseller: J.-C. Rufin, 100 Stunden, 2008

${ }^{69}$ Normische Gesetze gründen sich nicht auf eine monotone Logik, wie dies für streng monoton logische Gesetze, etwa die physikalischen, gilt. Vielmehr ermöglichen sie die Berücksichtung variabler Parameter mit variablen Ergebniskonstellationen. Sie erlauben das Schließen zur Erlangung von Hochwahrscheinlichkeitsaussagen nach dem Muster: „Das Licht geht normalerweise an, wenn der Lichtschalter betätigt wird.“ (Schurz 2006) - Ein solches alltagspraktisches Verständnis könnte ja auch dem Lernbedürfnis zugrunde gelegt werden, dass sich der Geschichte zuwendet. Das Schließen aus normischen Gesetzen ist die eigentliche Grundlage des größten Teils der Verabredungen, auf denen
} 
adaptieren wollen und Unvorhersehbarkeit menschlicher Entscheidungsfreiheit und Unbegrenztheit der menschlichen Ingeniosität reklamieren. ${ }^{70}$ Nicht nur durch die Fortschritte der Wissenschaftstheorie ist diese Position überholt, sie ist es auch durch die alltägliche Lebenswirklichkeit. Ausdrücklich hat z.B. die Arbeitsgruppe der Berlin Brandenburgischen Akademie „Zukunftsorientierte Nutzung ländlicher Räume“, 71 die „Visionen für abgelegne Räume entwickelt", an einen historischen Vorläufer angeschlossen. Vorbild ist ein Schlüsseltext der Aufklärung von Rudolph Zacharias Becker, ${ }^{72}$ dessen Anliegen „Wie man vergnügt leben, mit Ehren reich werden und sich und anderen helfen könne" ernsthaft zum Maßstab des Akademie-Projektes avancierte. ${ }^{73}$ Indes hatte bereits eine Klassikerin der Umweltgeschichte, Ester Boserup, ihre Analyse des Zusammenhangs zwischen Agrarwirtschaft und Bevölkerungswachstum ${ }^{74}$ vor dem Hintergrund ihrer profunden agrarbistorischen Kenntnisse entworfen. Historikern sei der Nutzen der Umweltgeschichte für die Geschichtsproduktion selbst wie auch für anschließende geschichtstheoretische Gedanken am Beispiel des Aufsatzes von Arno Borst über das Erdbeben von 1348 in Erinnerung gerufen. ${ }^{75} \mathrm{Er}$ ist ein außergewöhnliches historiographisches Zeugnis für die ebenso einfache wie wahre Tatsache, dass sich Vergangenheit durch Erinnerung in Erfahrung wandeln lässt und damit als Angebot für die Künftigen verfügbar wird. Hierauf gründet sich auch die Hoffnung, aus der Beschäftigung mit Umweltgeschichte lernen zu können. Denn sie befasst sich mit dem, was ich wiederholt als „Langzeitversuche unter natürlichen Bedingungen“ bezeichnet und dabei bewusst den Jargon der experimentellen Naturwissenschaften verwendet habe. Das eindrucksvollste Argument stammt vom Wegbereiter der deutschen Umweltgeschichte, Arno Borst (1990, S. 563), der die Bedeutung des Erdbebenereignisses von 1348 für heutige gesellschaftliche Aufgaben (in diesem Falle der Katastrophenforschung) auf einen allgemeingültigen Nenner brachte: „Nur wer die Geschichte nicht kennt, ist dazu verdammt, sie zu wiederholen.“

Die Anfänge der Umweltgeschichte lassen sich in einem damals aktuellen Katastrophendiskurs und in der Fortschrittsmüdigkeit der 60er Jahre ausmachen. Verschmutzung der Umwelt allenthalben, depravationstheoretische Erwägungen und die Hoffnung auf die Erklärungsmacht der aufstrebenden Ökologie bündelten

eine Gesellschaft gegründet ist, und nicht die akademische Abdeckung der letzten denktheoretisch möglichen Erwägung im Sinne monotoner Logik.

${ }^{70}$ Wenn es in der Geschichte keine Strukturen gibt, dann bleibt unklar, warum überhaupt die Beschäftigung mit ihr lohnend sein sollte, wenn alles nur kontingent und anekdotisch ist. Unter solchen Randbedingungen kann sich kein Verständnis historischer Abläufe ergeben, weil die historischen Ereignisse sich weder inhaltlich noch kausal systematisieren ließen. Schließlich untersagt die Existenz der Geschichtsdisziplinen selbst die Behauptung, dass es problematisch sei, aus der Geschichte zu lernen, bzw. dass aus ihr nichts gelernt werden könne.

$71 \mathrm{http}: / /$ landinnovation.bbaw.de

72 Becker 1788

${ }^{73}$ Das neue Hilfsbüchlein: Hüttl et al. 2008

${ }^{74}$ Boserup 1970. Siehe auch: Boserup 1988

${ }^{75}$ Borst 1981 
das Forschungsinteresse auch auf eine historische Perspektive. ${ }^{76}$ Niedergangsszenarien sollen nun für das europäische Selbstverständnis historisch geradezu konstitutiv sein. ${ }^{77}$ Solchen Erwartungshaltungen wäre eine Katastrophenfixiertheit dann auch in gewissem Maße selbstverständlich und damit erklärbar, warum die Anfänge der Umweltgeschichte übermäßig lange um einschlägige Themen kreisten.

Erkenntnis bzw. die ihr vorgeordneten Einsichten und Erfahrungen, hängen nun $\mathrm{ab}$ von den historischen Umständen ihrer Gewinnung. Man wird sich deshalb nicht wundern können, dass mit zunehmendem Verständnis für die Komplexität ökologischer Systeme sich die Einordnung umweltrelevanter Meinungen oder Beobachtungen mit den kulturellen Selbstverständnissen wandeln, somit auch der Blick darauf, was eigentlich eine Katastrophe sei oder in sie führe. So konnte noch zu Beginn des 19.Jh. die Idee von Charles Fourier (1772- 1837), durch spiegelgeführte Sonnenwärme die Polkappen abzuschmelzen, als bloße Phantasterei belächelt werden. ${ }^{78}$ Seine Vorstellung versetzte aber die Menschheit damals - anders als heute - keineswegs in „denkhemmende Angst“ (Riechelmann). Das 19. Jahrhundert hatte seinen Blick auf die Schornsteine des Gewerbefleißes gerichtet, sich an ihren Rauchfahnen erfreut und sie als Fortschrittssymbole gefeiert, konnte sich im selben Atemzug aber durchaus auch am Pathos einer Landschaften delektieren, die u.a. von den englischen Kontinentreisenden am Rhein oder sonst wo entdeckt worden waren und seitdem das Gemüt erheben. Im Namen des Fortschritts und der Hygiene hat Deutschland im 19. Jh. Flüsse begradigt, ${ }^{79}$ Feuchtbiotope trockengelegt $^{80}$ und begonnen, Abwässer auf Rieselfeldern zu klären. ${ }^{81}$ Es ist vermutlich das Jahrhundert mit den größten landschaftsverändernden Konsequenzen in Deutschland. ${ }^{82}$ Das Fortschrittsargument bringt nicht nur die Braunschweiger Müller vor dem Reichsgericht zum Verstummen, ${ }^{83}$ es wird im Grunde bis in die

76 „Es gehört zum Historismus unserer Gegenwartskultur, dass jedes neu auftauchende Problem alsbald auch in bistorischer Beleuchtung vorgeführt wird. Philosophen zumal sind, in gewissen Scbultraditionen, gewohnt, anstatt im Rabmen ibrer Möglichkeiten Antworten auf eine gestellte Frage zu geben, sich alsbald darüber zu verbreiten, in welchen evolutionären Variationen die einschlägige Frage früber auftrat und wie die einscblägigen Antworten früberer Philosophen lauteten. "Lübbe, S. 9

77 Das Geschichtsbild der europäischen Zivilisation ist nach der Einsicht Sieferles (2008) in hohem Maße von der Erfahrung von Zusammenbruch und Ruinerwartung geprägt.

${ }^{78}$ Cord Riechelmann, Frankfurter Allgemeine Zeitung Nr. 286 (2007), S.Z5; vgl. auch Benjamin, S. 699

${ }^{79}$ Es genügt hier der Hinweis auf die Rheinrektifikation durch Tulla (ab 1809) als eine der ersten Maßnahmen

${ }^{80}$ In Zusammenhang mit der erfolgreichen Malaria-Bekämpfung siehe Schuberg 1927

81 Anstoß gebend sind die Cholera-Epidemien, siehe z.B. Cordes 1861, 1868

82 Als Beispiel unter vielen: Der Landesplaner von Hazzi (1768 - 1845) hatte für Bayern entsprechende Ideen, die mindestens teilweise umgesetzt wurden (Beck 2003).

83 Zwei Braunschweiger Müller klagen 1881 bis 1883 erfolgreich gegen die Zuckerfabrik in Braunschweig-Rautheim, weil die Abwässer zur Eutrophierung ihrer Mühlenbäche führten und der überschießende Pflanzenwuchs die Mühlräder zum Stillstand brachte [(NLA-Staatsarchiv Wolfenbüttel) 37 A Neu Fb. 4 Nr. 30]. Die Geschichte ist Gegenstand des Romans „Pfisters Mühle“ von Wilhelm Raabe. Aber anders als bei Raabe, unterliegen die beiden Müller schließlich in der Revision 1884 vor dem Reichsgericht. Ihre Ansprüche werden dem Fortschrittsverständnis der wilhelminischen Gesellschaft untergeordnet. 
1960er Jahre das Zentralargument innerhalb der Gesellschaft bleiben. Fortschrittseuphorie brachte auch DDT in großen Mengen in die Nahrungskette ein. Vermutlich wäre aber die Rezeption der von Rachel Carson ${ }^{84}$ beschriebenen Umweltfolgen zumindest hierzulande erheblich gleichgültiger verlaufen, wenn sie nicht auf eine durch die Contergan-bedingten Missbildungen verstörte Öffentlichkeit gestoBen wäre. ${ }^{85}$ Der „Blaue Himmel über der Ruhr“"86 greift das Thema „Umwelt“ nicht mehr in der Tradition einer auf naturale Ausbeutung ausgerichteten Gesellschaft auf. Vielmehr geht er von der Setzung aus, wonach ein reduzierter Hemerobiegrad auch besser für die Menschen sei. Das Thema „Umwelt“ hatte damit die staatspolitische Ebene erreicht. Es war aus den Bürgerinitiativen und lokalen Interessenverbänden in die Großstädte (oder auch umgekehrt) geschwappt und hatte begonnen, einen allgemeinen Bewusstseinswandel einzufordern. Aber dieser skizzierte Wandel des Urteiles setzt ein jeweils früheres Urteil voraus und ist nur fassbar durch Kenntnis der historischen Kontexte.

Anders aber als um die anderen staatspolitischen Themen wie Herrschaft und Wirtschaft und Kultur, um die sich breite Betätigungsfelder der Geschichtsdisziplin herausgebildet haben, hatte sich beim Thema „Umwelt“ zunächst eine professionelle Abstinenz bezüglich einer ähnlichen Breite des Themas ergeben. Fortschrittskritik bildet, einschließlich einer Selbstkritik ihrer gelegentlich schlichten Argumentation, seitdem ein häufiges Element umwelthistorischen Raisonnements. ${ }^{87}$ Seine Pointe ist vorhersehbar und läuft immer auf einen Aufruf zum Verzicht hinaus, als Einsicht in die Endlichkeit der Welt und ihrer Ressourcen. Der geringe Erfolg der Vermittlung einer solchen Postulatenethik, der die Umsetzung in faktische Moral verwehrt bleiben wird, steht von vornherein fest, weil sie für den westlichen Kulturmenschen eine vierte negative existentielle Grunderfahrung zusätzlich zu jenen dreien bereit hält, die Siegmund Freud als narzisstische Kränkungen bestimmend für den Menschen der Moderne ausgemacht hatte. Sie betreffen sämtlich das Verhältnis von Menschen zur Natur:

o die erste Kränkung verdanke er Kopernikus, der nachwies, dass die Erde nicht der Mittelpunkt des Weltalls ist,

o die zweite Kränkung verdanke er Darwin, der nachwies, dass der Mensch nicht das Produkt einer kreationistischen Leistung ist,

o die dritte Kränkung verdanke er der Psychoanalyse, in deren Vermögen es liege, dem Bewußtsein erwachsener Menschen die unangenehme Einsicht vermitteln zu können, dass sein Anspruch auf Geborgenheit und Versorgung,

\footnotetext{
${ }^{84}$ Der Stumme Frühling, 1961, dtsch. 1962.

85 Thalidomid (Handelsname Contergan) wurde 1961 von Widukind Lenz als ursächlicher Wirkstoff für Missbildungen an Neugeborenen erkannt. Das Mittel wurde in der BRD unter Aufsehen erregenden Begleitumständen erst 1962 vom Markt genommen.

86 Willy Brandt in einer Rede am 28.4.1961; und wahlkampfpolitisches Ziel der SPD im Bundestagswahlkampf, dessen Themen vom Bau der Berliner Mauer 1961 überschattet werden.

${ }^{87}$ Eine beispielhafte und kritische Übersicht über oft gelesene Positionen bei Brüggemeier 1996,S. $7 \mathrm{f}$
} 
auf Zuneigung durch einen anderen Menschen, auf Halt in Einrichtungen und Institutionen, infantil und illusorisch ist.

Alle drei Kränkungen lassen sich in umwelthistorische Erwägungen einbeziehen. ${ }^{88}$ $\mathrm{Zu}$ den Konsequenzen der dritten Kränkungen gehört nun ein allmähliches Erschrecken darüber, dass Folgen und Nebenfolgen menschlichen Handelns den in „prästabilierter Harmonie“ begriffenen Zustand der Welt zu destabilisieren in der Lage ist. Damit wird eine vierte Kränkung unausweichlich: der „Selbstbedienungsladen Natur" wird sich künftig einem größeren Teil der Menschheit verweigern. Eine weltweite Konsumgesellschaft auf der Basis desjenigen Naturverbrauchs, wie er der euroamerikanischen Zivilisation zugrunde liegt, wird es nicht geben können. Die narzistische Kränkung derjenigen, die von der Partizipation ausgeschlossen werden, weil sie ein entsprechendes Niveau noch nicht erreicht hatten, und jener, denen der Abbau erreichter Konsumhöhe abverlangt wird, hat im Bedrohungspotential der nuklearen Selbstauslöschung ein Äquivalent. Nur ist die Bedrohung durch den Falle der zurück genommenen, der verweigerten oder der unmöglichen Teilhabe an der Ressourcenausbeutung ungleich realistischer als jene durch die Atombombe. Am schlimmen Ende verbinden sich beide womöglich sogar.

Auf die zweite und dritte narzisstischen Kränkung bietet sich, wie weiter oben mit dem Biodiversitätsbegriff angedeutet, eine Antwort. Sie ist enthalten in jenem durch die Konferenz von Rio de Janeiro 1992 formulierten Anspruch, dass der Prozeß der natürlichen Evolution von nun an in der Verantwortung der Menschen stehe. Mag man hierin die Annahme einer Verantwortung sehen, die aus dem menschlichen Handeln Konsequenzen für alle Lebewesen altruistisch Konsequenzen ziehen will, so ist diese Position ideengeschichtlich ein Rückschritt. Man braucht dabei gar nicht das Paradox der altruistischen Handlung zu bemühen, die dem Menschen immer nur zu einer egoistischen gelingen wird. Vielmehr ist an die in den Sozialdisziplinen früh erreichte Einsicht zu erinnern, dass die umweltverträglichste Position des Menschen diejenige seiner Einsicht in die Einbettung in den naturalen Gesamtzusammenhang ist (Catton und Dunlap 1980). Mit der RioAgenda ist faktisch vor diese Einsicht zurückgekehrt worden. Die Annahme der Herausgehobenheit des Menschen (hier durch selbstzugewiesene Verantwortung für den Prozess der Evolution) provoziert immer ein Gegenüber anstatt eines förderlichen Miteinanders. Insofern knüpft die Philosophie der Rio-Agenda letztlich an die alte Opposition „Mensch und übrige Natur“ an, statt das Miteinander zu

\footnotetext{
88 Diese Behauptung kann selbstverständlich nur für jene Menschen gelten, die von den kopernikanischen, darwinschen und freudschen Erklärungsmodellen betroffen sind, also den westlichen Kulturmenschen. Vier Jahrhunderte nach Kopernikus wird aber kaum noch jemand an dem von ihm entworfenen Weltbild verzweifeln. Hundertfünfzig Jahr nach Darwin waren dagegen zu einer allgemeinen Beruhigung nicht ganz ausreichend, wie u.a. der Blick auf den amerikanischen bible belt und dort tätige Kreationisten zeigt. In den fünfzig Jahren nach Freud selbst sind seine Hypothesen und Theorien noch nicht einmal allgemein rezipiert. In summa gilt auch den narzisstischen Kränkungen gegenüber, die Freud ausgemacht hatte, die Gnade der späten Geburt. Dagegen ist die vierte Kränkung aktuell und bestimmend, bis hinein in Entscheidungen von weltpolitischer Bedeutung.
} 
thematisieren, aus dem sich nachhaltige Verhaltensweisen und unverdächtige Rechtfertigungssätze für menschliches Umwelthandeln von selbst ergeben würden.

Hatte sich nach Darwin noch die Einsicht Bahn gebrochen, dass der Prozeß der Evolution selbstregulierend sei und in niemandes Verantwortung stände, ${ }^{89}$ wurde in Rio allgemein akzeptiert, dass Menschen Verantwortung für den Zustand der Naturräume der Welt trügen. Damit war endgültige bestätigt, dass der „biophile“ Lebensstil,90 d.h. die Sorge um und das Eintreten für die Biodiversität, auch quasi-religiöse Bedürfnisse bedient bzw. bedienen kann. Die Almosenspende wird in eine Spende an den WWF gewandelt. An die Stelle der Sorge um das Seelenheil tritt die Sorge um die Jahresdurchschnittstemperatur. Die Postulatenethik der Zehn Gebote wird durch eine Globalisierungsethik nach Art des Ökologischen Imperativs ersetzt. ${ }^{91}$ Nächstenliebe wird durch eine Fernstenliebe erweitert. Der aufgeklärten nach-darwinschen Einsicht, wonach ,in der Natur“ nichts so bleiben wird, wie es im Moment ist, entgegnen Menschen mit einem Beharren auf realitätsüberfordernden Konservativismus. Wenn schon die Blume welkt, Mutter und Vater sterben, dann soll wenigstens der Rest um uns herum so bleiben, wie er ist. Es ist die Furcht vor dem sicheren Ende des Vertrauten, das als Verlust erfahren und nur potentiell zur Chance wird. Aller Erfahrung nach besteht kaum je Grund, die Zukunft enthusiastisch zu erwarten. Nachdem die Sicherheit Gottes abhanden kam, die weltliche Sicherheit sich als unsicher herausstellte, müssen wir zur Kenntnis nehmen, dass auch „die Natur" keine Sicherheit bietet, weil sie keine Konstanz kennt. Im Moment ist diese Einsicht zugedeckt von den Problemen der Hyperhemerobie, die - paradoxer Weise - mit gesteigerter Zuwendung zur Natur überwunden werden soll. Sollten die dringendsten Probleme der Hyperhemerobie behoben worden sein, wird die Enttäuschung darüber erheblich ausfallen, dass eine Musealisierung der Natur durch ihr „Einfrieren“ auf dem status quo nicht gelingen kann. Wollte man einen aktiven „Rückbau“ naturaler Systeme betreiben, bereitet zudem deren Resilienzeigenschaft ein schwerwiegendes Problem. Denn resiliente Systeme haben durch ihre Nachlaufeigenschaften einen zum Zeitpunkt eines erkennbaren Systemumbruchs einen in seinem Umfang nicht bekannten Binnenwandel hinter sich. Wer resiliente Systeme zurückführen möchte, sollte zumindest wissen, bis vor welchen Zeitpunkt bzw. bis vor welche menschliche Verhaltensänderung. Wollte man beispielsweise den „Sauren Regen“ als Ursache des Waldsterbens beseitigen, müsste man den Autoverkehr abschaffen, die Kohlekraftwerke, die Hochschornsteine usw., bzw. die Technologien, die ursächlich für den Sauren Regen sind. Wenn es nicht möglich scheint, die Technologien in absehbaren Zeiträumen gänzlich zu ersetzen, bieten sich z.B. Biowissenschaften mit dem Konzept genetisch veränderter Organismen an. An Stelle einer Ursachenbeseitigung würde

\footnotetext{
${ }^{89}$ Herrmann 2006, S. 187

90 Wilson 1984

91 Jonas 1984 „Handle so, dass die Wirkungen deiner Handlung verträglich sind mit der Permanenz echten menschlichen Lebens auf Erden.“
} 
die Neutralisierung der Noxe durch entsprechend angepasste Individuen zu überwinden gesucht, was doch aber die Langfristfolgen des Sauren Regens für die Bodenqualität nicht berührte. Der Optimismus, mit dem molekulare Werkzeuge heute propagiert oder benutzt werden ist ungeheuer und mentalitätsgeschichtlich von Interesse. „Emanzipation“ war der zentrale Begriff der Aufklärung. Man kann sich von allem (Kulturellen) emanzipieren, nicht aber von seiner (der) Natur bzw. von den allgemeinen naturalen Voraussetzungen. In der Gentechnik ist der folgerichtige Versuch des Menschen angelegt, sich nun auch noch von der Natur zur emanzipieren. ${ }^{92}$ In diesem Zusammenhang wird allzu leicht übersehen, dass etwa eine gentechnisch hergestellte resistente Pflanze bei ihrer Schädlingsart sofort eine Coevolution in Gang setzt, die den erreichten Pflanzenschutz überwinden wird. Das Argument kann entsprechend auf andere Bereiche gentechnischer Bemühungen übertragen werden.

Jede ingenieurtechnische Beseitigung einer Kausalursache wird aber nur umsetzbar, wenn eine mentalitätengeschichtliche Begleitung die erforderliche Aufklärungsarbeit leistet, ohne die es kein zukunftsicherndes Umwelthandeln geben und sich die eingeforderte „neue Vorsorgekultur“ (Kofi Anan) nicht entwickeln kann. Manche ingenieurtechnische Lösung wäre entbehrlich, wenn die sozioökonomischen Bedingungen durch Bewusstseinswandel verändert würden. Auch in dieser Gewissheit steckt Umweltgeschichte.

\section{IIc Was aus der Geschichte lernen?}

Man wird es drehen und wenden können wie man will, eines Tages, wann immer sich selbst oder Dritten Rechenschaft zu geben sein wird, steht man unausweichlich vor der Frage, ob dem hedonistischen Adam im Wissenschaftler auch noch ein „politischer" Adam ${ }^{93}$ zur Seite stand? Der sollte ohne größeren Aufwand in der Lage sein, die Sinnhaftigkeit seiner Arbeit nicht nur allgemeinverständlich zu erläutern, sondern auch Perspektiven eines Nutzens aus dieser Arbeit aufzuzeigen. Eingefordert wird hier nicht einmal ein direkter Nutzen, sondern lediglich der perspektivische. Das entspräche in wissenschaftstheoretischer Formulierung einer „Anschlussfähigkeit“ für das konkrete Ergebnis, also der kleinstmöglichen Zweckbestimmung des Forschungsertrags. Allerdings sollten dabei jene Argumente keine Berücksichtigung finden, die sich lediglich des ablenkenden Hinweises bedienen: wenn eine Gesellschaft für Dieses oder Jenes Geld ausgebe, könne sie auch dieses oder jenes Projekt der Wissenschaft fördern.

Als entscheidende Voraussetzung für die Förderung wissenschaftlicher Fragestellungen gelten der Wettbewerb der Argumente und der Einfluss der Interessenvertreter. Im Falle der Umweltgeschichte müsste das Förderkonzept umgedreht

\footnotetext{
92 Herrmann 2003

93 Gemeint ist mit „politisch“ hier die Fortschreibung der aristotelischen Etikettierung des Menschen als „zoon politikon“", also eines gesellschaftlich verantwortlich handelnden Menschen. - Vgl. Seite 4; http://www.wbgu.de/wbgu_jg1996.pdf
} 
werden: Denn in einer Welt, die sich dem Wachstum des materiellen Wohlstandes aus naturalen Ressourcen verpflichtet hat, ist kaum zu erwarten, dass Argumente als mehrheits- bzw. wettbewerbsfähig gelten, die dem Individuum wie der Gesellschaft nahelegen, ein anderes Wertesystem zu entwickeln und die selbstzerstörerischen Eigenschaften des jetzigen Systems infrage zu stellen.

Damit wäre eine Relevanz der Umweltgeschichte benannt: Sie ist (erstens) zunächst einmal Anamnese. Sie betreibt die diachrone und diatopische Beobachtung und Analyse von naturalen, sozio-naturalen und sozio-ökonomisch-naturalen Systemen sowie an ihnen entwickelten Wertvorstellungen. Letztere beeinflussen die Entwicklung jener Wertesystemen, die nachfolgenden Generationen das (menschenwürdige) Leben in einer (intakten naturalen) Umwelt ermöglichen. Umweltgeschichte wird (zweitens) damit zu einem unveräußerlichen Bestandteil der ökologischen Grundbildung, die ihrerseits zu einem unveräußerlichen Bestandteil basaler Erziehung und Ausbildung werden muss.

Ein einfaches Beispiel von (drittens) der Verknüpfung historischer Umweltforschung mit zukunftsichernden Nachhaltigkeitsaspekten ist die Biodiversitätsforschung an Objekten naturkundlicher Sammlungen, die, z. T. über Jahrhunderte zusammengetragen, heute wertvollste diachrone Zeugnisse über genetische Vielfalt, über Arten, Lebensräume und Interaktionen sowie der Ökosysteme, ihrer Güter und Dienstleistungen geben (Leinfelder 2008). Aus ihnen sind Beiträge u.a. zur Beantwortung jener Probleme zu erwarten, die der WBGU-Jahresbericht bereits 1996 identifiziert: ${ }^{94}$ „Damit verbunden sind Forschungsfragen, deren Beantwortung in den kommenden Jahren weiter an Bedeutung zunehmen wird:

-Wie kommt es zu den Naturveränderungen, und wie sind sie mit der globalen Entwicklungsproblematik verknüpft?

- Wie kann man sie frühzeitig erkennen oder vorhersagen?

- Welche Risiken sind mit ibnen verbunden?

-Wie muss der Mensch bandeln, um negative Entwicklungen auf globaler Ebene zu verbindern, um drohenden Gefabren zu begegnen bəw. um die Folgen globaler Veränderungen zu minimieren?"

Solche Fragen sind ohne umwelthistorische Kenntnisse nicht beantwortbar, zumal in Wörtern wie ,negative Entwicklungen“ kultureller Erklärungsbedarf steckt. Allein mit der historischen Perspektive kann ein sicheres Verständnis für die prozessualen Abläufe bereitgestellt werden. Unschwer ist daher in dieser Agenda auch eine Handlungsanleitung für Umweltgeschichte erkennbar.

In der Raumplanung etwa ist seit jeher dieser Rückgriff auf und der Vergleich von historischen Szenarien erfolgt, um die Nutzungs- und Wertewandel angemessen zu berücksichtigen. ${ }^{95}$ Die Dimensionen der Bedeutung umwelthistorischer

\footnotetext{
94 http:/ /www.wbgu.de/wbgu_jg1996.pdf, Seite 4;

${ }^{95}$ Diese Aussage ist selbstverständlich nur von relativer Bedeutung und gilt vorderhand für mitteleuropäische Idealverhältnisse. Wie am Schadensausmaß des diesjährigen Erdbebens in China ersichtlich, hat die Raumplanung im betroffenen Gebiet historische Kenntnisse offenbar nicht hinreichend berücksichtigt.
} 
Datensätze werden besonders deutlich, wo sie zu prognostischen Szenarien herangezogen werden. Tatsächlich greifen Modellierer aktuell als drängend betrachteter Globalprobleme bereits auf umwelthistorische Daten zurück, wenn etwa die Situation des Weltklimas als von historischer agrarwirtschaftlicher Entwicklung abhängig betrachtet wird (Pongratz et al. 2008). Dabei wäre eine Einbindung des kompetenten umwelthistorischen Sachverstandes erforderlich, weil den Nutzern bloßer Literaturangaben der Stand des umwelthistorischen Diskurses entgehen kann. ${ }^{96}$

Ganz folgerichtig ist damit ein vierter Aufgabenbereich erreicht worden: will man Umweltgeschichte nicht nur um ihrer selbst willen betreiben, ${ }^{97}$ dann führt Umweltgeschichte zwangsläufig in eine Beratungsposition (siehe hierzu auch den Beitrag Schlottau in diesem Band). Beraten wird „die Gesellschaft“ über Abläufe und Folgen von Umwelthandeln, soweit es sich um wissenschaftlich zugängliche Gegenstandsbereiche oder Sachverhalte handelt. Dabei müsste nach Max Weber (1988b) allerdings die Trennung von „Wie?“ und „Ob?“ gewahrt bleiben.

Die Frage „Umweltgeschichte wozu?" ist gleichlautend vor einem Dutzend Jahren von Jost Hermand (1996) gestellt worden. Er hat ein vehementes Plädoyer dafür gehalten, der Umweltgeschichte eine weniger anthropozentrischen Ausrichtung zu geben und sie stärker auf Fragen der Zukunftssicherung auszurichten, eine „Mitweltgeschichte“ zu betreiben. Etwas nüchterner lässt sich feststellen, dass umwelthistorisch interessierte Wissenschaftler seit längerem den Beratungsbereich anvisieren, ihn z.T. sogar als konstitutiv für die Weiterentwicklung der Umweltgeschichte ansehen. ${ }^{98}$ Einige haben ihren Weg unmittelbarer gewählt und sind über die sozialwissenschaftlich geprägte Humanökologie direkt in die Politikberatung gelangt. ${ }^{99}$ Die aktuelle Hinwendung eines Teils der Umweltgeschichte zur Sozialgeschichte ist unübersehbar ${ }^{100}$ und nach meiner Einsicht diesem genannten Be-

\footnotetext{
96 Beispiel hierfür ist die Analyse von Pongratz et al. (2008), die in ihrer Darstellung die neueren Erkenntnisse über Vorkommen und Ausmaß fortgeschrittener Agrarkultur in Süd- und Nordamerika sowie Afrika nicht den Stand des heutigen Wissens abbilden, sondern sich auf Fortschreibungen älterer Arbeiten (im Anschluss an Whittlesley 1936) verlassen. Die sich ergebende Frage ist: Welche Aussagepräzision werden Modelle haben können, die sich auf unzulängliche Grundvoraussetzungen stützen? Ich danke William Woods, University of Kansas, dass er mich mit diesem Sachverhalt bekannt machte und für seine Zustimmung, darauf hinweisen zu dürfen, bevor er und Kollegen diesen Sachverhalt im Jahre 2009 auf dem 1st World Congress of Environmental History, Kopenhagen, genauer vorstellen werden.

97 Ich halte das zwar für einen auch akzeptablen Grund, sich mit Umweltgeschichte zu befassen, plädiere jedoch dafür, einige Mühe auch auf „Relevanzfragen“ zu verwenden.

98 Wie z.B. Sörlin \& Warde 2007

${ }_{99}$ Für das sozialwissenschaftliche Feld der Humanökologie seien stellvertretend die Publikationen Glaeser \& Teherani-Krönner (1992), Glaeser (1996) und Serbser (2003) genannt. - Als Beispiel für einschlägige Politikberatung kann das Wissenschaftszentrum Berlin gelten. - Die Zeitschrift GAIA ist Beispiel eines Periodikums, das mit wissenschaftlicher Legitimation auf umweltpolitische Wirkung zielt.

100 Z.B. die bevorstehende Tagung: „Terrains communs, Regards croisés. Intégrer le social et l'environmental en histoire." Centre de Recherches Historiques, UMR CNRS/EHSS, Paris 11-13 septembre 2008. - Ebenso ist die politische Motivation hinter dem Tagungsthema des ersten Weltkongresses für Umweltgeschichte in Kopenhagen unübersehbar: "Local Livelihoods and Global Changes: Understandig Human Interactions with the Environment“, August 2009.
} 
wusstseinswandel von der bloß wissenschaftlichen zur gesellschaftlich einflussreichen Tätigkeit hin verdankt. Für die „Disziplinierung“ der Umweltgeschichte ist dies eine zwiespältige Entwicklung. Sie kann an Einfluss gewinnen und gleichzeitig an disziplinärer Kontur verlieren, wenn das umwelthistorische Profil zugunsten des sozialgeschichtlichen bzw. des soziologischen Profils aufgegeben wird. Ob über Politik-Beratung eine wirkliche Verbesserung der fachlichen umwelthistorischen Position und eine allgemeinere Verbreitung ihrer Einsichten erreichbar sind, erscheint offen. Unstreitig erscheint mir allerdings, dass die Umweltgeschichte ihre Szenarien nicht fiktional entwickeln muss, sondern sich auf einen Erfahrungsschatz stützen kann, von dem man weiß, dass seine Inhalte reale Möglichkeiten sind. „Die Science-Profession folgt einem Verständnis von „guter Wissenschaft“, nach der im Zweifel Sicherheit ihrer Aussagen der Vorzug zu geben ist. Der Gesellschaft geht es demgegenüber unter „Sicherheit" um die reale Sicherheit von Infrastruktur und Vermögen." ${ }^{101}$ Die Umweltgeschichte ist hier in der komfortablen Situation, beide divergierenden Aspekte weitgehend verbinden zu können und die notwendige begleitende Moderation bereit zu stellen. Das Feld ist bereits ziemlich bestellt, doch sollte noch Platz für die spezifische Kompetenz der Umwelthistoriker sein.

\section{Literatur}

Alpers S (1998) Kunst als Beschreibung. Holländische Malerei des 17. Jahrhunderts. DuMont, Köln

Baumgart W (2006) Bücherverzeichnis zur deutschen Geschichte. Hilfsmittel, Handbücher, Quellen. 16. Auflage. dtv, München

Beck R (2003) Ebersberg oder das Ende der Wildnis. Eine Landschaftsgeschichte. $\mathrm{CH}$ Beck, München

Becker RZ (1788) Noth- und Hülfsbüchlein für Bauersleuthe oder lehrreiche

Freuden- und Trauergeschichte des Dorfs Mildheim. Gotha/Leipzig, Deutsche Zeitung/Göschen

Benjamin W [1974] Über den Begriff der Geschichte. In: W Benjamin, Gesammelte Schriften, Bd. 2, Teil 2. Hg. von R Tiedemann u. H Schweppenhäuser. Suhrkamp, Frankfurt. S. 698-699

Borst A (1981) Das Erdbeben von 1348. Historische Zeitschrift 233: 529-569

(hier zitiert nach der Fassung in: A Borst (1990) Barbaren, Ketzer und Artisten. Welten des Mittelalters. Serie Piper, München, Zürich. S. 528-563)

${ }^{101}$ Luhmann 2008, S. 30 
Boserup E (1970) The conditions of agricultural growth. Allen \& Unwin, London. (erste Auflage 1965)

Boserup E (1988) Environment, population, and technology in primitive societies. In: D Worster (Hg.) The ends of the earth. Cambridge University Press, Cambridge, New York. S. 23 - 38

Brüggemeier FJ (1996) Das unendliche Meer der Lüfte. Luftverschmutzung, Industrialisierung und Risikodebatten im 19. Jahrhundert. Klartext, Essen

Catton W, Dunlap R (1980) A new ecological paradigm for post-exuberant sociology. American Behavioral Scientist 24(1): 15-47.

Carson R (1987) [1962] Der Stumme Frühling. Beck'sche Reihe, München

Cassirer E (1996) Versuch über den Menschen [An Essay on Man, 1944]. Meiner, Hamburg

Cordes E (1861) Die Cholera in Lübeck: einige Worte an den Patriotismus und die Behörden, nebst einem Plan der Stadt, enthaltend die Todesfälle in den einzelnen Straßen. Aschenfeldt, Lübeck

Cordes E (1868) Die Cholera in Lübeck. Zeitschrift für Biologie 4(2)

Feyerabend P (1986) Wider den Methodenzwang. Suhrkamp, Frankfurt [hier zitiert nach der 4. Auflage 1993]

Friedrichs K (1943) Über den Begriff der „Umwelt“ in der Biologie. Acta Biotheoretica 7: 147-162

Friedrichs K (1950) Umwelt als Stufenbegriff und als Wirklichkeit. Studium Generale 3: 70-74

Fuchsloch N (1996) Einführung in „Methodenfragen der Umweltgeschichte“. In: G Bayerl, N Fuchsloch, T Meyer (Hg.) Umweltgeschichte - Methoden, Themen, Potentiale. (= Cottbuser Studien zur Geschichte von Technik, Arbeit und Umwelt 1). Waxmann, Münster u.a. S. 1-12

Glaeser B (1996) Huamnökologie: Der sozialwissenschaftliche Ansatz. Naturwissenschaften 83:145-152

Glaeser B, Teherani-Krönner P (Hg.) (1992) Humanökologie und Kulturökologie. Westdeutscher Verlag. Opladen

Global 2000 (1980) Der Bericht an den Präsidenten. Hg. der deutschen Übersetzung Reinhard Kaiser. Zweitausendeins, Frankfurt/M.

Godelier M [1984] L'idéel et le matériel. Pensée, économies, societés, Fayard, Paris. (hier zitiert nach der engl. Ausgabe: The mental and the material: thought, economy and society. Verso, London 1986) 
Haidle MN (2008) Verschiedene Welten: Umweltwahrnehmung und Umweltgestaltung im Laufe der menschlichen Evolution. In: T Knopf (Hg.) Umweltverhalten in Geschichte und Gegenwart. Vergleichende Ansätze. Attempo Verlag, Tübingen. S. $30-41$

Hermand J (1996) Umweltgeschichte wozu? In: G Bayerl, N Fuchsloch, T Meyer (Hg.) Umweltgeschichte - Methoden, Themen, Potentiale. (= Cottbuser Studien zur Geschichte von Technik, Arbeit und Umwelt 1). Waxmann, Münster u.a. S. 303-308

Herrmann B (1996) Umweltgeschichte als Integration von Natur- und Kulturwissenschaften. In: G Bayerl, N Fuchsloch, T Meyer (Hg.) Umweltgeschichte - Methoden, Themen, Potentiale. (= Cottbuser Studien zur Geschichte von Technik, Arbeit und Umwelt 1). Waxmann, Münster u.a. S. 21-30

Herrmann B (2003) Historische Humanökologie und Biodiversitätsforschung. In: SR Gradstein, R Willmann, G Zizka (Hg.) Biodiversitätsforschung. Die Entschlüsselung der Artenvielfalt in Raum und Zeit. S. 225 - 236. Schweizerbarth, Stuttgart (Kleine Senckenberg Reihe 45)

Herrmann B (2006) „Auf keinen Fall mehr als dreimal wöchentlich Krebse, Lachs oder Hasenbraten essen müssen!“- Einige vernachlässigte Probleme der „historischen Biodiversität““. In: H-P Baum, R Leng, J Schneider, (Hg.) Wirtschaft - Gesellschaft - Mentalitäten im Mittelalter. Festschrift zum 75. Geburtstag von Rolf Sprandel. (= Beiträge zur Wirtschafts- und Sozialgeschichte 107). Steiner, Stuttgart. S. 175 - 203

Herrmann B (2008) Empirische Zugänge zu historischen Biodiversitätsverdrängungen und Biodiversitätslenkungen: Die Beispiele Melioration und Schädlingsbekämpfung. In: T Knopf (Hg.) Umweltverhalten in Geschichte und Gegenwart. Vergleichende Ansätze. Attempo Verlag, Tübingen. S. 174192

Holling CS, Gunderson L, Ludwig D (2002) In Quest of a Theory of Adaptive Change. In: L Gunderson, CS Holling (Hg.) Panarchy. Understanding transformations in human and natural systems. Island Press, Washington. S. 322

Hüttl RF, Bens O, Plieninger T (Hg.) (2008) Zur Zukunft ländlicher Räume: Perspektiven der Landnutzung in peripheren Regionen Nordostdeutschlands. Akademie-Verlag, Berlin

Hughes JD (2006) What is Environmental History? Polity Press, Cambridge, Malden 
von Humboldt A [1847-62] Der Kosmos. Entwurf einer physischen Weltbeschreibung. Hg. v. O. Ette u. O. Lubbrich. Eichborn, Frankfurt/M. 2004

Jonas, H (1984) Das Prinzip Verantwortung. Versuch einer Ethik für die technologische Zivilisation. Suhrkamp, Frankfurt/M.

Knopf T (2008) Umweltverhalten als Forschungsaufgabe. In: T Knopf (Hg.) Umweltverhalten in Geschichte und Gegenwart. Vergleichende Ansätze. Attempo Verlag, Tübingen. S. 9-15

Krüger L (1987) Einheit der Welt - Vielheit der Wissenschaft. In: J Kocka (Hg.) Interdisziplinarität. Praxis - Herausforderungen - Ideologie. Suhrkamp, Frankfurt/M. S. 106-125

Laroux N (1993) The Children of Athena: Athenian ideas about citizenship and the division between the sexes. Princeton University Press, Princeton

Lehmkuhl U (2002) Histoire totale oder Bindestrich-Geschichte? In: Erfurter Beiträge zur Nordamerikanischen Geschichte 4, S. 2-10

http://www.uni-erfurt.de/nordamerika/erfurterbeit/umweltgeschichte.html\#a

Leinfelder R (2008) Der größte Schatz ist noch nicht gehoben. Leibniz - Journal der Leibniz-Gemeinschaft 1/2008: 3

Lübbe H (1986) Ökologische Probleme im kulturellen Wandel. In: H Lübbe u. E Ströker (Hg.) Ökologische Probleme im kulturellen Wandel. Finkh/Schöningh, Paderborn(= Ethik der Wissenschaften. Arbeiten aus einer Studiengruppe der Werner Reimers Stiftung V). S. 9-14

Lumann H-J (2008) Klimansensitivität, Leben und die Grenzen der ScienceKultur. Zum Vierten IPCC-Sachstandsbericht. Gaia 17(1): 25-30

Mann C, Plummer M (1995) Noah's choice. The future of endangered species. Alfred Knopf, New York

Marx K [1968] Die Produktion des absoluten Mehrwerts. 5. Kap. Arbeitsprozess und Verwertungsprozess. In: K Marx, F Engels, Werke, Band 23, "Das Kapital", Bd. I, Dritter Abschnitt, S. 192 - 213; Dietz Verlag, Berlin, DDR

McNeill J (2003) Observations on the nature and culture of environmental history. History and Theory, Theme Issue 42 (December 2003): 5-43

Meadows D, Meadows D, Zahn E, Milling P (1973) Die Grenzen des Wachstums. Bericht des Club of Rome zur Lage der Menschheit. Rowohlt, Reinbeck. [engl. u. dtsch. Erstveröffentlichung 1972]

Nagel T (1974) “What is it like to be a bat?” Philosophical Review 83: 435-450 
Park RE, Burgess EW (1921) Introduction to the science of sociology. The University of Chicago Press, Chicago [hier zitiert aus der zweiten Auflage 1924]

Pongratz J, Reick C, Raddatz T, Claussen M (2008) A global land cover reconstruction AD 800 to 1992 - Technical Description.

Max-Planck-Institut für Meteorologie, Berichte zur Erdsystemerforschung 51. http://www.mpimet.mpg.de/fileadmin/publikationen/Reports/WEB_Bze_51 .pdf

Radkau J (1993) Was ist Umweltgeschichte? In: C Simon (Hg.) Umweltgeschichte heute. Environmental History Newsletter. Special Issue No1, S. 86-97

Radkau J (2002) Mensch und Natur in der Geschichte. Klett, Leipzig (= Historisch Politische Weltkunde, Kursmaterialien Geschichte Sekundarstufe II/ Kollegstufe)

Rheinberger H-J (2007) Historische Epistemologie - zur Einführung. Junius, Hamburg

Ryle G [2002] Der Begriff des Geistes. Reclam, Stuttgart

Schaefer M (2003) Wörterbuch der Ökologie. Spektrum Akademischer Verlag, Heidelberg

Schatzki T (2003) Nature and technology in history. History and Theory, Theme Issue 42 (December 2003): 82-93

Schuberg A (1927) Das gegenwärtige und frühere Vorkommen der Malaria und der Anophelesmücke im Gebiet des Deutschen Reiches. Arbeiten aus dem Reichsgesundheitsamt 59: 1-424 (Springer, Berlin)

Schurz G (2006) Einführung in die Wissenschaftstheorie. Wiss. Buchgesellschaft, Darmstadt

Serbser W (Hg.) (2003) Humanökologie. Ursprünge - Trends - Zukünfte. LIT Verlag, Münster usw.

Sieferle RP (2008) Zusammenbruch von Zivilisationen. Eine konzeptuelle Analyse am Beispiel des Imperium Romanum. Gaia 17(2): 213-223

Siemann W, Freytag N (2003) Umwelt - geschichtswissenschaftliche

Grundkategorie. In: W Siemann, N Freytag (Hg.) Umweltgeschichte. Themen und Perspektiven. Beck, München. S. 7-20

Sloterdijk P (2001) Domestikation des Seins. Für eine Verdeutlichung der Lichtung. In: P Sloterdijk (Hg.) Nicht gerettet. Versuche nach Heidegger Suhrkamp, Frankfurt. S. 142-234 
Sörlin S, Warde P (2007) The problem of the problem of environmental history: a re-reading of the field. Environmental History 12: 107-130

Stammler, W. (Übers.) (1988) Die Erinnerungen des ersten Grossmoguls von Indien: d. Baburnama / Zahir-ad-Din Muhammad Babar. Manesse, Zürich

Steward JH (1955) Theory of cultural change. University of Illinois Press, Urbana

Stichweh R (Hg.) (1994) Wissenschaft, Universität, Professionen. Soziologische Analysen. Suhrkamp, Frankfurt/M.

Thieme H (Hg.) (2007) Die Schöninger Speere. Mensch und Jagd vor 400000 Jahren. Theiss, Stuttgart

Thienemann A (1958) Leben und Umwelt. Vom Gesamthaushalt der Natur. Deutsche Buchgemeinschaft, Berlin u. Darmstadt

Toyka-Seid M (2003) Mensch und Umwelt in der Geschichte. Neues aus dem produktiven Selbstfindungsprozess der Umweltgeschichte. Archiv für Sozialgeschichte 43: 423-447

Uekötter F (2007) Umweltgeschichte im 19. und 20 Jahrhundert. (Enzyklopädie Deutscher Geschichte Bd. 81). Oldenbourg, München

von Uexküll J (1921) Umwelt und Innenwelt der Tiere. Zweite Auflage, Springer, Berlin

von Uexküll J [1928] Theoretische Biologie. Mit einem Vorwort von Rudolf Bilz. Suhrkamp, Frankfurt/M. 1973

von Uexküll J, Kriszat G (1934) Streifzüge durch die Umwelten von Tieren und Menschen. Ein Bilderbuch unsichtbarer Welten. Springer, Berlin

von Uexküll J, Kriszat G [1934] Streifzüge durch die Umwelten von Tieren und Menschen. Ein Bilderbuch unsichtbarer Welten. Rowohlt, Hamburg 1956

Voltaire [1971] Candid oder Die Beste der Welten. Reclam, Stuttgart

Ward LF (1903) Pure Sociology. A treatise on the origin and spontaneous development of society. Macmillan; New York, London

Weber M (1988a) [1904] Die „Objektivität“" sozialwissenschaftlicher und sozialpolitischer Erkenntnis. In: M Weber, Gesammelte Aufsätze zur Wissenschaftslehre. Hg. von J Winckelmann. (UTB) Mohr, Tübingen, S. 146214

Weber M (1988b) [1922] Wissenschaft als Beruf. In: M Weber, Gesammelte Aufsätze zur Wissenschaftslehre, Hg. von J Winckelmann, (UTB) Mohr, Tübingen, S. 582-613

Wilson EO (1984) Biophilia. Harvard Univ. Press, Cambridge/Mass. 
Winiwarter V (1994) Umwelt-en. Begrifflichkeit und Problembewusstsein. In: G Jaritz, V Winiwarter (Hg.) Umweltbewältigung. Die historische Perspektive. Verlag f. Regionalgeschichte, Bielefeld. S. 130 - 159

Winiwarter V, Knoll M (2007) Umweltgeschichte. Eine Einführung. (UTB) Böhlau, Köln

Winiwarter V, Schmid M (2008) Umweltgeschichte als Untersuchung sozionaturaler Schauplätze?. Ein Versuch, Johann Colers »Oeconomia« umwelthistorisch zu interpretieren. In: T Knopf (Hg.) Umweltverhalten in Geschichte und Gegenwart. Vergleichende Ansätze. Attempo Verlag, Tübingen. S. 158-173

Wissenschaftsrat (1994) Stellungnahme zur Umweltforschung in Deutschland. 2 Bde. Wissenschaftsrat, Köln

Whittlesey D (1936) Major agriculture regions of the earth. Annals of Association of American Geographers 26(4): 199-240 


\title{
Bilder, die die Umwelt bewegten: Naturwahrnehmung und Politik in der US- amerikanischen Geschichte
}

\author{
Christof Mauch
}

\section{Politik und Ästhetik}

Die Amerikaner sind die Erfinder der Nationalparks. Früher als andere Regierungen hat die US-amerikanische damit begonnen, große Landstriche der privaten Nutzung zu entziehen. Hinter dieser Politik steckte weniger ein Nutzungskonzept als vielmehr eine ästhetische Vision, die auf die Bewahrung pittoresker, wildromantischer oder spektakulärer Landschaften zielte. Warum war für die Etablierung eines Parks wie Yosemite oder Yellowstone nicht das Leitbild einer Kulturlandschaft mit Bauernhöfen oder kleinen Städten ausschlaggebend, sondern das einer unberührten Landschaft? Warum wurden die Niagarafälle zum Naturpark erklärt und die anliegenden Sägemühlen und Brücken abgerissen? Woran liegt es, dass die Fotografien eines Ansel Adams, die eine menschenleere Landschaft darstellen, millionenfach in Form von Kalendern und Bildbänden verkauft und für den $\mathrm{Na}$ turschutz instrumentalisiert wurden? In den genannten Fällen, so argumentiert dieser Beitrag, gibt es eine enge Verbindung zwischen bildlichen Repräsentationen von Natur einerseits und Politik andererseits. Die Art und Weise, wie Landschaften seit dem frühen 19. Jahrhundert in Bildern präsentiert wurden, hat die Vorstellung von dem, was die zentralen Charakteristika der ,amerikanischen Natur“ sind, bis in die Gegenwart hinein geprägt. Entscheidungen US-amerikanischer Politiker und Planer, die sich entweder für Landschaftspflege und Naturschutz oder aber für die (industrielle oder gewerbliche) Nutzung und kulturelle Erschließung von Landschaften eingesetzt haben, sind aufs Engste mit den Bildern verknüpft, die 
sich die US-amerikanische Öffentlichkeit im Laufe der Geschichte von der Natur gemacht hat. Gemälde, Fotografien und Filme hatten, wie im Folgenden gezeigt wird, einen immensen Einfluss auf die Vorstellungswelt und auf die Gestaltung und Nutzung von Natur und Landschaft in den USA. Welche Visionen von Natur waren prägend? Welchen Einfluss hatten diese auf Politiker in Washington und auf Naturschützer im ganzen Land? Inwieweit hat die US-amerikanische Regierung selbst - etwa in Regierungsfilmen - Bilder erzeugt, die geschichtswirksam wurden? Und welche Rolle spielten Bilder im Kalkül des größten Umweltschutzverbandes der Welt, des Sierra Clubs? Dieser Aufsatz versteht sich als ein thought piece, welches weit verbreitete Naturbilder aus zwei Jahrhunderten USamerikanischer Geschichte exemplarisch in den Blick nimmt und deren politische Instrumentalisierung und Wirksamkeit reflektiert. ${ }^{1}$

\section{Die Erfindung der amerikanischen Landschaft}

Kein amerikanischer Künstler und keine Künstlergruppe haben das Bild von der amerikanischen Natur tiefer geprägt und nachhaltiger beeinflusst als Thomas Cole (1801-1848) und die so genannte Hudson River School. Auf den Staffeleien und Leinwänden von Cole, dessen Künstlerkollegen und Schülern findet sich eine Natur, in der Hügel und dichte Wälder, unberührte Bergseen und wilde Wasserfälle, knorrige Bäume und eine - abwechselnd Furcht und Ehrfurcht einflößende Wildnis regieren. „Nicht nur sinnlich“ oder ,selbstbezogen“ sei das Entzücken, das die derart dargestellte amerikanische Landschaft auf ihren Betrachter auslöse, erklärte der Künstler selbst in seinem Essay on American Scenery: ,in gazing on the pure creations of the Almighty, he feels a calm religious tone steal through his mind, and when turned to mingle with his fellow men, the chords which have been struck in that sweet communion cease not to vibrate". ${ }^{2}$ Die Landschaft war für Cole wie für viele seiner Schüler ein mit religiöser Bedeutung gefüllter Raum, ein Ort der Kontemplation und ein Spiegel göttlicher Präsenz. Die Natur, die er malte, zeichnete sich durch eine Ländlichkeit und Wildheit aus, in der der Mensch kaum Spuren hinterließ, in der er nicht als Bewohner, sondern allenfalls als Besucher auftauchte.

\footnotetext{
${ }^{1}$ Zum Zusammenhang von Naturwahrnehmung und Umweltgeschichte, vgl. Sieferle RP, Breuninger H, Hrsg. (1999) Natur-Bilder: Wahrnehmungen von Natur und Umwelt in der Geschichte. Campus, Frankfurt a.M., New York. Zur Rhetorik der Umweltbewegungen im postmodernen Kontext siehe DeLuca KM (1999) Image Politics. The New Rhetoric of Environmental Activism. The Guildford Press, New York and London.

${ }^{2}$ Cole T (1980) Essay on American Scenery. In: T. Cole, The Collected Essays and Prose Sketches. Ed. by M. Tymm, John Colet Press, St. Paul. S. 5
} 


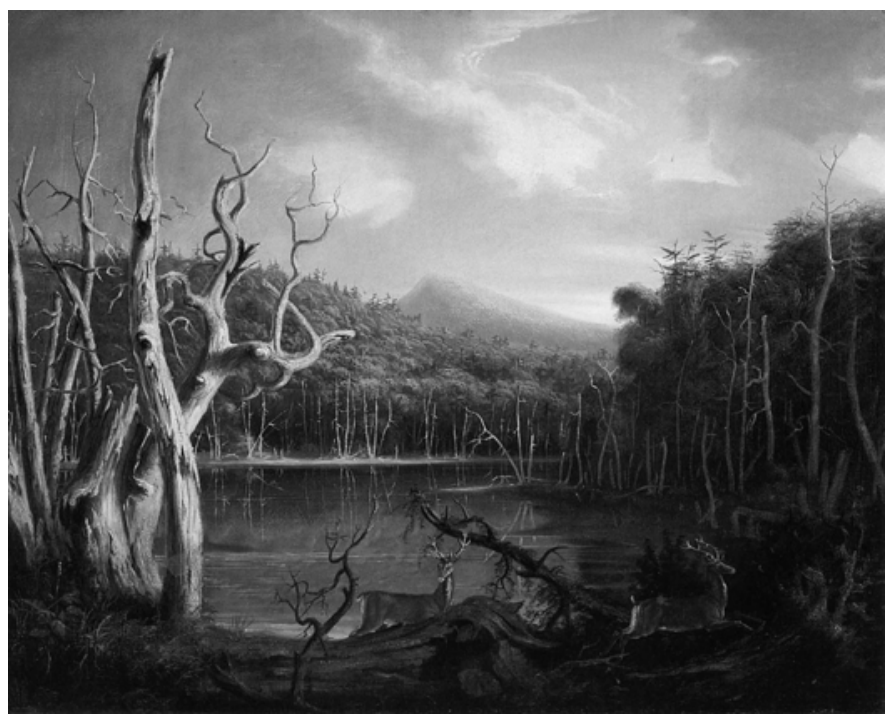

Abbildung 1: Thomas Cole „Lake with Dead Trees“

Für Cole, der aus Europa eingewandert war, machte die Wildnis der Landschaft das spezifisch Amerikanische aus. Der Dichter William Cullen Bryant erinnerte sich an den „Enthusiasmus“, den Coles frühere Werke bei ihm auslösten, es seien Bilder gewesen, ,which carried the eye over scenes of wild grandeur peculiar to our country, over our aerial mountain-tops with their mighty growth of forest never touched by the axe, along the banks of streams never deformed by culture, and into the depth of skies bright with the hues of our own climate". 3 Nach Cole waren es die Künstler Asher Durand, Jasper Cropsey und Frederic Church, die über Jahrzehnte hinweg eine Landschaft malten, die sich durch Blicke auf Felsen und tiefe Schluchten auszeichnete, durch eine raue, wilde Natur, wie sie in Europa längst Säge, Axt, Pflug, Kanal und Besiedelung zum Opfer gefallen war. Aber auch im Amerika Thomas Coles hatte die Vision der Unberührtheit, in der sich einzelne Menschen gleichsam heroisch an der gewaltigen Natur maßen, etwas Idealisiertes, Nostalgisches an sich. Während sich die junge Nation industrialisierte, malten die „Erfinder der amerikanischen Malerei“ Landschaften, die die dramatische Naturlandschaft als das eigentlich Erhabene feierten. ${ }^{4}$ Bis weit ins 19. Jahrhundert hinein war das Land auf beiden Seiten der Niagarafälle Privatbesitz; an manchen Stellen konnte man sich die Wasserfälle nur durch einen Zaun hindurch und gegen das Entrichten einer Gebühr ansehen.

\footnotetext{
${ }^{3}$ Bryant WC (1829) To Cole, the Painter, Going to Europe. In: Milford H (ed) Poems of William Cullen Bryant. London: Oxford University Press 1914. S. 14

${ }^{4}$ Vgl. Westheider O, Müller K (2007) Neue Welt. Die Erfindung der amerikanischen Malerei. Hirmer Verlag, München, Gaethgens TW ed. (2006) Bilder aus der Neuen Welt. Amerikanische Malerei des 18. und 19. Jahrhunderts. Prestel Verlag, München und Novak,B (1995) Nature and Culture. American Landscape and Painting, 1825-1875. Oxford University Press, New York.
} 


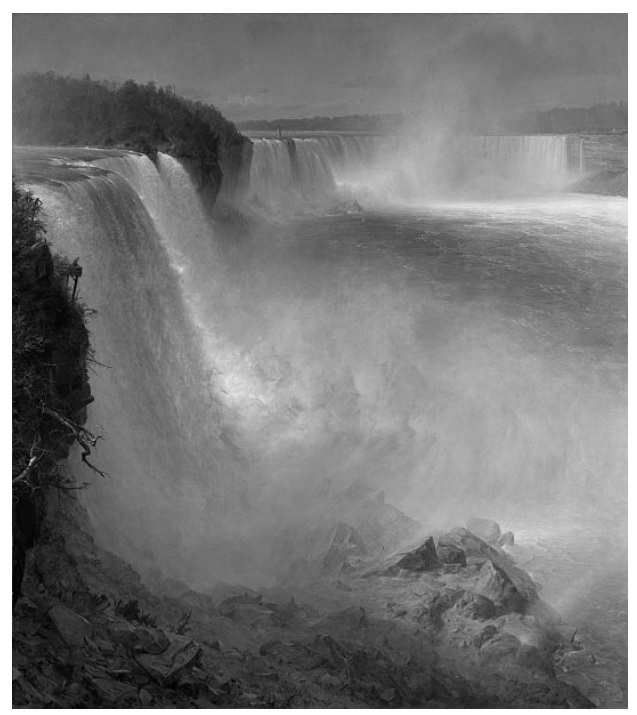

Abbildung 2: Frederick Church „Niagara Falls, from the American side"

Als Frederic Church, Thomas Coles berühmtester Schüler, im Jahr 1857 die Niagarafälle malte, gab es dort Sägemühlen, Kanäle und Brücken und ein hydraulisches Kraftwerk, das Getreidemühlen und Gerbereien mit Energie versorgte. Doch kaum ein Betrachter würde vermuten, dass die von Church dargestellten Niagarafälle industriell genutzt wurden. ${ }^{5}$ Die Ausgrenzung (nahezu) aller Spuren menschlichen Einflusses wurde zur Signatur der Hudson River School. Dass Church den Wunsch hegte, Naturspektakel wie Niagara sollten nicht nur auf der Leinwand unberührt bleiben oder die Illusion der Unberührtheit erwecken, wurde vollends deutlich, als sich der Maler Church mit dem Landschaftsarchitekten Frederick Law Olmsted in den 1860er Jahren an die Spitze des Free Niagara Movement stellte, das den Staat New York dazu veranlasste, das Land um die Niagarafälle von den Unternehmern zurückzukaufen und den dramatischen Anblick der Wasserfälle vor der drohenden Industrialisierung zu bewahren. ${ }^{6}$ So entstanden, gleichsam zur Bewahrung einer im eigentlichen Sinne „malerischen“ Naturvision, auf Drängen eines Künstlers und eines Architekten der Niagara Reservation State Park auf US-

\footnotetext{
${ }^{5}$ Allenfalls die ironische Anspielung auf den Salvator Rosa Baumstumpf im Vordergrund und ein Turm am Ufer im Hintergrund lassen erkennen, dass die Niagara-Natur nicht völlig unberührt war. ${ }^{6}$ Vgl. Dow CM (1914) The State Reservation at Niagara. A History. J.B. Lyon Co., Printers, Albany, New York; Gilbert GK et al. (1896) Niagara Falls and their history. New York, Cincinnati .Weitere relevante Unterlagen finden sich in den vom Secretary of the Niagara Falls Association zusammengestellten Papers of Charles Eliot Norton, 1880-1908. Darin finden sich in zwei Bänden Briefe an Norton von Frederick Law Olmsted and Henry Norman, Drucksachen und Pamphlete. Norton CE [1908] Papers of Charles Eliot Norton, 1880-1908, 2 Bde., Library of Congress, Washington, D.C. Archival Manuscript Material (Collection), MSS34926. Eine provokative neue Darstellung zur Geschichte von Niagara stammt von Strand G (2008) Inventing Niagara. Beauty, Power, and Lies. Simon \& Schuster, New York.
} 
amerikanischer Seite und der Queen Victoria Niagara Falls Park auf der Seite Kanadas. Das von den idealisierten Vorstellungen der Hudson River School evozierte Wunschbild einer intakten, unberührten, ursprünglichen Natur wurde zum Movens für die Realisierung des Naturschutzes in Amerika. Ob es ohne die romantische Vorstellung einer makellosen Natur, der der Mensch mit Ehrfurcht, Scheu und Schauer gegenübersteht, zur Kreation des Niagara-Naturparks gekommen wäre, darf bezweifelt werden. Dass die idealisierte Landschaft der Hudson River School, die den staunenden Blick des Betrachters nicht nur auf die Natur, sondern potenziell auch in eine metaphysische Sphäre lenkt, an Aktualität nichts eingebüßt hat, zeigen neuere Studien zur Kunstperzeption. Demnach bevorzugten Amerikaner auch im ausgehenden 20. Jahrhundert eine „Ersatz-Hudson-River-Landscape“ gegenüber allen anderen Formen und Typen der Landschaftsdarstellung. Ihnen gilt die Repräsentation einer ,wilden“, „unberührten“, alles Kulturelle ausschließenden Natur nicht nur als ideale Landschaft, sondern als perfekte Kunst. ${ }^{7}$

\section{Landschaftsmalerei in der politischen Metropole}

Dass der Maler Thomas Moran, der, 34-jährig, im Jahr 1871 mit einer Forschergruppe der US-Bundesregierung in den amerikanischen Westen aufbrach und die gigantischen Naturspektakel von Yellowstone malte, ausschlaggebend für die Entscheidung des US-Kongresses gewesen sei, Yellowstone zum ersten Nationalpark zu machen, ist in unzähligen Geschichtsbüchern nachzulesen. In Wirklichkeit ist die Geschichte komplizierter: Nicht die Bundesregierung, sondern die Northern Pacific Railroad hatte Moran eingeladen und dafür bezahlt, Aquarelle, Stiche, Radierungen und Ölgemälde zu produzieren, die später als Werbematerial für die Förderung des Tourismus im amerikanischen Westen eingesetzt werden konnten. ${ }^{8}$ Neben Moran spielte der Fotograf William Henry Jackson, der zusammen mit dem Maler unterwegs war, eine bedeutende Rolle. ${ }^{9}$ Jacksons Fotografien konnten, wie Zeitgenossen betonten, nicht „lügen“, die Landschaften nicht romantisch überhöhen, nichts hinzuerfinden. Sie trugen deshalb entscheidend dazu bei, den amerikanischen Westen in der Imagination der Amerikaner von einem mythischen

\footnotetext{
7 Woodward RB The Perfect Painting. In: NYT Magazine, February 20, 1994. S. 36-37

8 Vgl. Runte A (1990) Trains of Discovery. Western Railroads and the National Parks, Robert Rinehart, Niwot, Colorado sowie Davidson G et al. (2006) Frederic Church, Winslow Homer, and Thomas Moran. Tourism and the American Landscape. Bulfinch Press, New York u. Boston. S.3-73; Kinsey JL (2006) Thomas Moran's West. Chromolithography, High Art, and Popular Taste. University Press of Kansas, Lawrence, Kansas. S. 47-82, 159-182

${ }^{9}$ Eine wichtige Quelle ist die Autobiographie von Jackson WH (1994) Time Exposure. The Autobiography of William Henry Jackson. Profusely illustrated with photographs, paintings, and drawings by the author. The Patrice Press, Tucson, Arizona. Vgl. außerdem Moran T (1865) American Art and American Scenery. In: Philadelphia Photographer, II, March 1865. S.-33-35; zum Verhältnis von Fotografie und Kunst im 19. Jahrhundert in den USA vgl. Linquist-Cock E (1977) The Influence of Photography on American Landscape Painting, 1839-1880. Garland Publishing, Inc., New York and London. besonders S. 144-150
} 
in einen realen, aufsuchbaren Ort zu verwandeln. ${ }^{10}$ Dennoch lässt sich der politische Einfluss Morans kaum überschätzen. Zum einen lockte die Dramatik von Morans Bildern Zehntausende von Eisenbahntouristen zu den Naturschönheiten im Westen der USA. Weiterhin wurde die Perspektive, der Blickwinkel des Künstlers zum ausschlaggebenden Element bei der Gestaltung von Straßen und Aussichtspunkten. Dass die scenic overlooks Namen wie Moran's Point oder Artists Point tragen, ist ein Indiz dafür. Darüber hinaus wirkte Morans Schaffen aber auch konkret auf die Politik in Washington. ${ }^{11}$ Nachdem das gigantische Panoramagemälde Grand Canyon of the Yellowstone mit seinen warm glühenden Farben einige Wochen lang in New York gezeigt worden war, kam es 1872 nach Washington. ${ }^{12}$

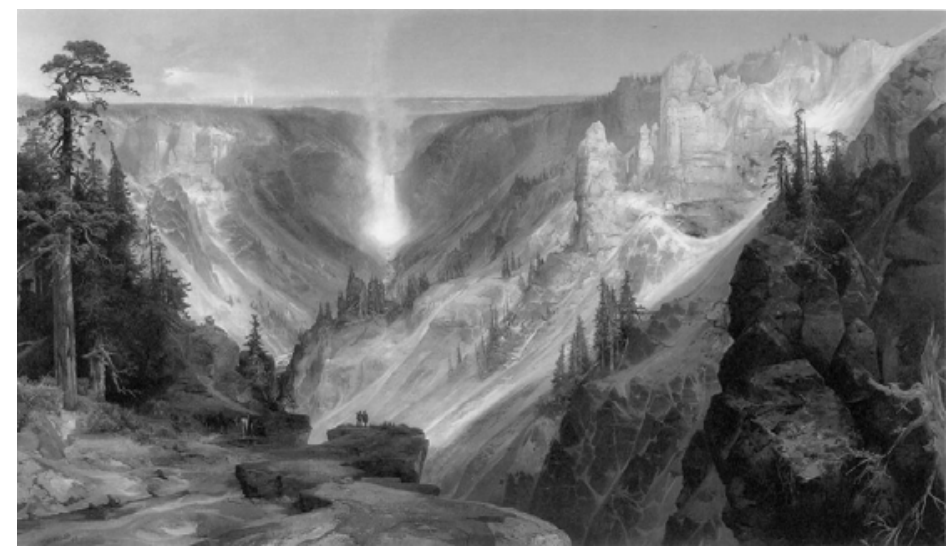

\section{Abbildung 3: Thomas Moran „Grand Canyon of the Yellowstone”}

Dort hing es zunächst in der Haupthalle des Smithsonian Castle. Von dort wechselte es, auf Betreiben Morans, in die „old Hall of Representatives“, konkret an die große Wand des Büros des House Speakers James G. Blaine. Dort, wo ein Abgeordneter nach dem anderen, Senatoren, Kongressabgeordnete und internationale Besucher das Bild zu sehen bekamen, wurde es zu einer großen Attraktion. Am 10. Juni 1872, drei Monate nach der Einrichtung des Yellowstone Nationalparks, votierte der Senat dafür, das Bild für 10.000 Dollar zu kaufen. Heute entspricht dies in etwa 180.000 Dollar. Kein anderes Bild in der US-amerikanischen Geschichte hatte bis dahin einen so hohen Preis erzielt. Grand Canyon of the Yellowstone war das erste Landschaftsgemälde, das Aufnahme in die Lobby des Senats fand; es hing zwischen Historienmalerei aus der Sammlung des Kongresses und machte allein schon durch seine Platzierung - deutlich, dass die gewählten US-

\footnotetext{
${ }^{10}$ Hales P (1988) William Henry Jackson and the Transformation of the American Landscape.Temple University Press, Philadelphia

${ }^{11}$ Wilkins T, Hinkley CL (1998) Thomas Moran. Artist of the Mountains. Foreword by Goetzmann W.H., 2nd edn. revised an enlarged. University of Oklahoma Press, Norman, Oklahoma.,S. 76-105 ${ }^{12}$ Morans Bild wurde zusammen mit Bildern prominenter Künstler aus der Düsseldorfer und Pariser Schule gezeigt. Der Erlös aus der Ausstellung kam den Opfern des Great Chicago Fire vom Oktober 1871 zugute.
} 
Volksvertreter die grandiose Landschaft des neuen Nationalparks als nationales Erbe par excellence ansahen und dass die überwältigende Dramatik der Landschaft als Inspiration für die Politik dienen konnte. ${ }^{13}$

\section{Bilder von der zerstörten Umwelt}

Eine neue Ästhetik und eine völlig neue Funktionalität im Verhältnis von Naturbildern und Politik machten sich in den 1930er Jahren breit. War es in den Gemälden von Cole, Church oder Moran und in den Fotografien eines William Henry Jackson die monumentale und spektakuläre amerikanische Landschaft, die gefeiert wurde, so erlebte in der darauf folgenden Zeit die desolate Landschaft eine regelrechte Konjunktur. Hatten die Bilder von Yellowstone, Yosemite, Monument Valley oder Niagara im späten 19. und frühen 20. Jahrhundert zum Besuch des amerikanischen Westens eingeladen, so wirkten die Fotografien der 1930er Jahre abweisend. Das hatte nicht zuletzt damit zu tun, dass die am weitesten verbreiteten Naturbilder die verlassene und zerstörte Landschaft der amerikanischen Prärie zum bevorzugten Sujet erhoben. In der Folge der Großen Depression und der großen Naturkatastrophen - insbesondere der Dust Bowl und der verheerenden Überflutungen des Mississippi ${ }^{14}$ - ließen die Tourismusströme in die Nationalparks nach, aber auch der Fortschrittsoptimismus, der sich im Feiern der nationalen Naturmonumente ausgedrückt hatte. Die Bilder einer von extremen Staubstürmen verheerten Landschaft, der Dust Bowl, wurden zum visuellen Ausdruck der nationalen Krise, ja zum Emblem der 1930er Jahre. Die amerikanische Prärie, die sich in der Folge des Ersten Weltkriegs - in Oklahoma, Texas, Colorado und New Mexico - zur globalen Weizenkammer entwickelt hatte, war nicht mehr wiederzuerkennen. Viele ihrer Bewohner hatten den Exodus in Richtung Kalifornien angetreten. Der Verlust von Heimat und Wohlstand spiegelte sich in zahlreichen Fotografien verlassener Landschaften wider, die sich bis heute in Lehrbüchern zur amerikanischen Geschichte finden. Etliche Dust Bowl Bilder sind zu Ikonen geworden: Bilder von versandeten Farmen und landwirtschaftlichem Gerät; und Bilder von den schicksalsgezeichneten Gesichtern der Farmer. Interessanterweise hatte die Bundesregierung selbst die Produktion dieser neuen Naturbilder veranlasst. ${ }^{15}$ Filmproduzenten wie Pare Lorentz und Robert Flaherty stellten sich in den Dienst von Franklin Delano Roosevelts New Deal. ${ }^{16}$ Wer sich heute

\footnotetext{
${ }^{13}$ Vgl. Wilkins T (1998), 76-105. Zur Geschichte der Nationalparks siehe Runte A (1997) National Parks. The American Experience, 3rd edn. University of Nebraska Press, Lincoln, Nebraska.

${ }^{14}$ Zur Dust Bowl siehe vor allem Worster D (2004) Dust Bowl. The Southern Plains in the 1930s. Oxford University Press, New York; zu Naturkatastrophen vgl Steinberg T (2000) Acts of God, The Unnatural History of Natural Disaster in America. Oxford University Press, New York.

15 Zur Ikonizität von Fotografien vgl. Hariman R, Lucaites JL (2007) No Caption Needed. Iconic Photographs, Public Culture, and Liberal Democracy. The University of Chicago Press, Chicago and London. S. 1-24

16 Die beste Darstellung zum Dokumentarfilm ist nach wie vor Barnouw E (1993) Documentary. A History of the Non-Fiction Film. Oxford University Press. Herausragend zum Genre der amerikani-
} 
wundert, wie ein ehemaliger US-Vizepräsident im frühen 21. Jahrhundert so offen und kritisch an die ,unbequeme Wahrheit" menschlicher Verantwortung für die Klimakatastrophe rührt ${ }^{17}$, tut gut daran, sich zu erinnern, dass die US-Regierung mit ihren Filmen in den 1930er Jahren nichts anderes getan hatte. In der Interpretation der New Deal-Filmemacher figurierte die Dust Bowl nicht als akzidentielles Phänomen; vielmehr galt der Mensch selbst als verantwortlich für die Zerstörung der Landschaft. In der Interpretation der Fotografen kam der Dust BowlKatastrophe apokalyptische Bedeutung zu. Die schrecklichen Staubstürme verwiesen auf die Endzeit (tatsächlich bedeuteten die Staubstürme für die meisten betroffenen Farmer das Ende ihrer landwirtschaftlichen Existenz); und sie repräsentierten zugleich die Zerstörung eines amerikanischen Paradieses. Das Land, das sich - so suggerierten es die Filme und Bilder - vor der Ankunft der Farmer in einem ausgewogenen Naturzustand befunden hatte, war wüst und leer geworden. Im Dokumentarfilm The Plow that Broke the Plains von 1936 sind die handelnden Personen, ganz anders als in traditionellen Hollywoodfilmen, austauschbar. ${ }^{18}$ Sie fungieren als Kulisse, nicht als Protagonisten. Stattdessen kommt dem Land, der Natur, der Umwelt emotionale und dramatische Akteursqualität zu. „We decided“, erklärte Lorentz „to use a very difficult and dangerous movie technique, seldom employed in this country, and in fact, successfully used only in Russian movie productions - that is we decided to use people as symbols and background for land and try to design a story that would make land itself emotional and dramatic". ${ }^{19}$ Für Lorentz und seine Zeitgenossen waren die schier endlosen Grasländer des Mittleren Westens, die zum Anbau von Weizen maschinell umgepflügt wurden, eine Art amerikanischer Garten Eden, den der Mensch aus seiner natürlichen Balance gebracht hatte. Eine Laissez-faire-Landwirtschaft, die sich allein an Aktienkursen für Weizen orientierte und sich mit eskalierender Geschwindigkeit ausbreitete, hatte nach dieser Interpretation die „Saat des Unheils“ gesät. ${ }^{20}$

schen Dokumentarfotografie der 1930er Jahre: Barnouw E (1993) Documentary Expression and Thirties America. Oxford University Press, New York; zum theoretischen und historischen Kontext, vgl. Trachtenberg A (1989) Reading American Photographs. Images as History. Matthew Brady to Walker Evans, Hill and Wang, New York und Sandweiss MA ed. (1991) Photography in NineteenthCentury America. Harry N. Abrams, Inc., New York.

${ }^{17}$ Gore A (2006) An Inconvenient Truth. The Planetary Emergency of Global Warming and what we can do about it. Bloomsbury, London.

${ }^{18}$ Lorentz P (1936) The Plow that Broke the Plains [Film]. Distributed by U.S. Resettlement Administration.

19 Pare Lorentz, „Confidential Report“, zitiert in: Dunaway F (2005) Natural Visions: The Power of Images in American Environmental Reform. Chicago University Press, Chicago and London. S. 45 ${ }^{20}$ Der Film bringt die Zusammenhänge mit schnellen Szenenwechseln und dramatischen Trommelwirbeln zum Ausdruck. 


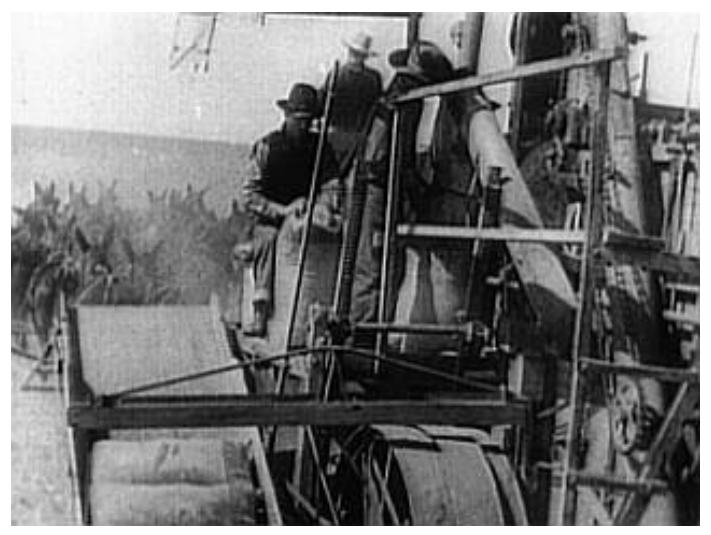

\section{Abbildung 4: Bild aus „The Plow that Broke the Plains“"}

Während die Bilder eines Church oder Moran in ihrer artistischen Rhetorik dafür plädierten, dass Landschaften wie die Niagarafälle oder Yellowstone aufgrund ihrer Erhabenheit vor menschlicher Intervention geschützt werden sollten, zeigten die Filme der New Deal-Produzenten, welche katastrophalen Folgen der mangelhafte Schutz weit weniger spektakulärer Landschaften haben konnte. Die maschinelle Eroberung der Natur, so lautete die Botschaft von Pare Lorentz, führte unweigerlich zur Rache der Natur. Das Nature-Fights-Back-Genre, das sich heute in großen Hollywoodproduktionen wie The Day after Tomorrow findet ${ }^{21}$, hat in den Regierungsdokumentationen der Roosevelt-Zeit seinen Ursprung.

Im März 1936 wurde Lorentz' Film The Plow im Weißen Haus gezeigt. „Sprudelnd vor Begeisterung“, hieß es im Time Magazin, habe der Präsident den Film aufgenommen. Sein Vorschlag, dass sich alle Kongressabgeordneten den Film ansehen sollten, ließ sich nicht realisieren, aber am Tag nach der offiziellen Premiere fanden sich in allen großen Zeitungen in den USA Berichte über The Plow - und nicht etwa im Unterhaltungsteil, sondern auf der ersten Seite. Und obwohl sich Hollywood ostentativ weigerte, einen von der US-Regierung veranlassten Film kommerziell zu vertreiben, lief The Plow in annähernd 3.000 Kinos und erreichte - zwischen 1936 und 1939 - nicht weniger als 10 Millionen Amerikanerinnen und Amerikaner. Dass der Dokumentarfilm von weiten Teilen der Bevölkerung im Mittleren Westen abgelehnt wurde, weil sie das Drama vom Ruin ihrer Landschaft als irreführende Propaganda der Ostküstenelite ansahen, und dass es einem Kongressabgeordneten aus South Dakota, dem späteren Kommunistenjäger Karl Mundt, gelang, den Film The Plow verbieten zu lassen, vermutlich nicht zuletzt, weil dieser die Rolle des Staates bei der Lösung von Umweltproblemen zu sehr ins Zentrum stellte, gehört zu den symptomatischen Folgen

${ }^{21}$ Emmerich R [director] (2004) The Day after Tomorrow [Film]. Distributed by Twentieth Century Fox. 
des Umweltengagements der US-Regierung. ${ }^{22}$ Was das Medium Film im Gegensatz zum Gemälde und zur Fotografie erreichte, war die Darstellung einer prozessualen Entwicklung: von der Evokation eines idealisierten ökologischen Ur- Naturzustands über die Destruktion bis zum mahnenden, quasi-apokalyptischen Ausblick: „Another decade of reckless use“, lautet der letzte Satz, den Lorentz den sonoren Erzähler sprechen lässt, ,and the grasslands will truly be the great American desert".

Hier wie dort - bei den von Washington instrumentalisierten Fotografien und Gemälden eines William Henry Jackson und eines Thomas Moran einerseits und im Falle der Dokumentarfotografien und -filme der 1930er Jahre andererseits - förderte die US-Bundesregierung die visuelle Verbreitung einer politischen Botschaft zum Verhältnis von Natur und Kultur. In beiden Fällen suggerierten die von Washington lancierten Bilder, dass ein Eingriff des Menschen in die Natur schadhaft sein könne und dass die Natur ,intakt" bleiben müsse. Allerdings stellen die drastischen Dokumentarbilder des New Deal kein Idealbild der amerikanischen Landschaft dar, sondern die Verkehrung des Ideals.

\section{$5 \quad$ Naturbilder und Umweltbewegung}

Interessanterweise war es nach dem Zweiten Weltkrieg nicht der Film, sondern der großformatige Bildband, das so genannte coffee table book, dem eine herausragende Bedeutung im Zusammenhang mit der sich breit formierenden Umweltbewegung zukommen sollte. Dafür war zuallererst David Brower, der Gründer der Stiftung Sierra Club, verantwortlich. Im Gegensatz zu den großformatigen Gemälden der Künstler des 19. Jahrhunderts, zu den Werbepostern der Eisenbahnen und zu den Filmen des New Deal, die allesamt in öffentlichen Räumen präsentiert wurden - in Museen und Ausstellungen, an öffentlichen Plätzen und in Filmtheatern -, transportierte das coffee table book Repräsentationen der Natur ins Wohnzimmer und erreichte den Einzelnen, nicht die Masse. ${ }^{23}$ Brower experimentierte auch mit anderen Medien, wie Dokumentarfilmen, aber dem Bild- und Essayband kam seiner Ansicht nach eine nachhaltende, mobilisierende Wirkung zu: „It has long been recognized that the book, for all that TV, radio, and periodicals may do, still has a status of its own in influencing thought. It lasts. It is kept and referred to. It is quoted. This is particularly true of the exhibit-format books". Die Filme von Lorentz und Flaherty wurden nach dem Zweiten Weltkrieg nicht mehr gezeigt, aber das coffee table book, das sich nicht zuletzt an führende Persönlichkeiten in der Industrie und Politik richtete, hatte nach Ansicht Browers das Potenzial, die nationale Politik zu beeinflussen. Inspiriert von der frühen, immens politischen Geschichte des Sierra Clubs - John Muir, der Gründer des Clubs, hatte sich um

\footnotetext{
22 Ironischerweise wurde ein Jahr nach Mundts Tod ein Nationalpark in South Dakota mit dem Namen The Karl Mundt National Wildlife Refuge versehen.

${ }^{23}$ Vgl. hierzu Dunaway F (2005) Natural Visions. The Power of Images in American Environmental Reform. The University of Chicago Press, Chicago and London. S.107-147
} 
die Wende zum 20. Jahrhundert mit Politikern und Lobbyisten zusammengetan, um seine Ziele zu erreichen - versuchte David Brower in den 1950er Jahren den Club aus der Existenz eines kalifornischen Wandervereins herauszukatapultieren und ihm nationale Sichtbarkeit und eine politische Stoßrichtung zu geben. Als das Washingtoner Bureau of Reclamation, das für die Wasserversorgung im Land zuständig war, Pläne für die Flutung des von Woodrow Wilson eingerichteten Dinosaur National Monument entwickelte, gab Brower beim Romanschriftsteller Wallace Stegner ein Buch in Auftrag, das die Einzigartigkeit und Schönheit des Monuments in Wort und Bild demonstrieren sollte. Stegner pries Dinosaur als ein ,sanctuary from a world paved with concrete, jet-propelled, smog-blanketed, sterilized, over-insured, aseptic; a world mass-produced with interchangeable parts, and with every natural beautiful thing endangered by the raw engineering power of the twentieth century". ${ }^{24}$ Für Stegner war die Anschauung der unzerstörten Natur ein Altar und Zufluchtsort in der zunehmend technischen, antlitzlosen Welt. Die Gesellschaft und Kultur der Moderne galten den Protagonisten des Sierra Clubs ähnlich wie der Pflug den New Dealern - als destruktiv. Ohne „Wilderness“, erklärte David Brower, ist „die Welt ein Käfig“.

Der Bildband This is Dinosaur wurde zur effektiven Waffe im Kampf gegen den Dammbau. Stegners Schlusssatz im Bildband, mit dem er sich direkt an den Leser richtete, lautete: ,This, you know now, is a country as grand and beautiful as any America can boast; and if the dams are built ... almost all of what you have seen ... will be wiped out" ${ }^{\text {“ }}{ }^{25}$ Browers Rechnung ging auf. Jedem Kongressabgeordneten wurde ein Exemplar des Bildbandes This is Dinosaur zugeschickt. Hunderttausende der Bildbände wurden verkauft; das Großprojekt des Bureau of Reclamation wurde nie gebaut; und der Sierra Club hatte weit über den kalifornischen Raum hinaus Prominenz und politische Bedeutung gewonnen. ${ }^{26}$

Die Amerikanisierung der Natur, das heißt die Identifizierung der Landschaft mit der Natur machte die Bildbände und Aktivitäten des Sierra Club zum Politikum par excellence. So kam es, dass die Sierra Club-Ausstellung This is the American Earth, die im Jahr 1955 entstand und in Text und Bild Amerikas Schönheit feierte, von der United States Information Agency (USIA) protegiert und international gezeigt wurde. ${ }^{27}$

\footnotetext{
${ }^{24}$ Stegner W (1955) The Marks of Human Passage, in: This is Dinosaur. Echo Park Country and Its Magic Rivers. ed. by Stegner W. Knopf, New York. S.15

25 Stegner (1955) o.P.

${ }^{26}$ Dunaway (2005) S.129

27 Adams A, Newhall N [1960] This is the American Earth, San Francisco, Sierra Club, San Francisco, California.
} 


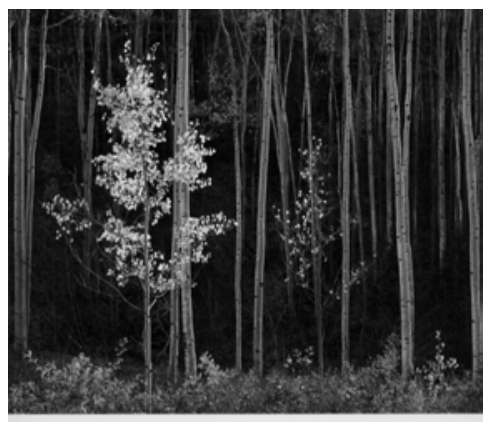

\section{THIS IS THE} AMERICAN EARTH

ANSEL ADAMS E NANCY NEWHALL

A Sierra Club Book

\author{
Abbildung 5: Der 1960 \\ erschienene Bildband zur \\ Sierra Club Ausstellung \\ „This is the American \\ Earth"
}

Die Schönheit der ,amerikanischen Natur“, wie sie in Fotos unter anderem von Ansel Adams zum Tragen kam, wurde zur Propagandawaffe in den internationalen Rivalitäten des Kalten Krieges. Ansel Adams polemisierte gegen die Sowjets, von denen die Wildnis nur so lange geschützt wird, wie sie keinen anderweitigen Nutzen findet. „It might be well to impress on our public that the Russian concept of a National Park is ... an area ... set aside for relaxation and recreation until the timber is ready to cut!“'28 Die Realisierung des Naturschutzgedankens, die Etablierung von Wildnisgebieten durch den Wilderness Act im Jahr 1964 und die religiöse Dimension der vom Menschen „unberührten“ Natur wurde zum Beweisgrund für die Überlegenheit der freien Welt gegenüber der des sowjetischen Rivalen.

Die Bilder des Fotografen Ansel Adams (1902-1984), der zu einem der prominentesten Naturschützer Amerikas avancieren sollte, stellten eine Natur dar, die an Cole, Church und Moran erinnerte: Neben dem Panoramablick bzw. der Weitwinkeloptik des Fotografen wurde die Unberührtheit der Landschaft und ihr spiritueller Charakter zum Hauptmerkmal von Adams’ Fotografien.

28 Ansel Adams zit. in Dunaway (2005) S.132 


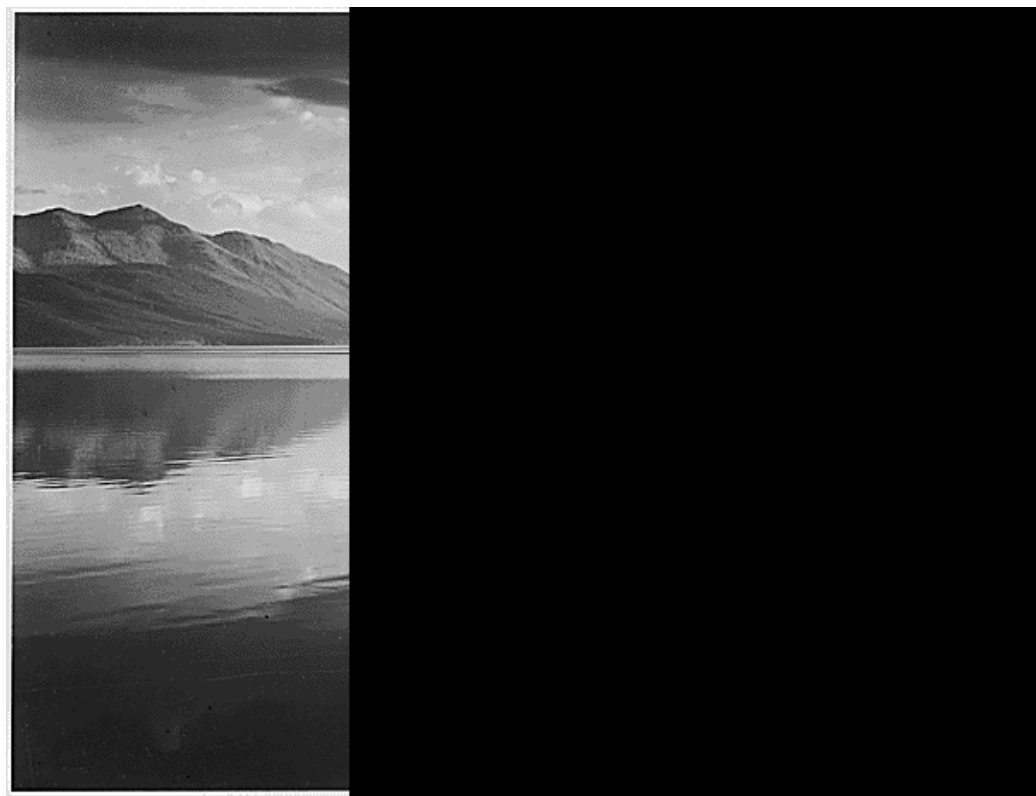

\section{Abbildung 6: Ansel Adams „Evening McDonald Lake, Glacier} National Park“

\section{Unberührte Natur}

Es gehört zu den Besonderheiten der amerikanischen Kunst- und Fotografiegeschichte, dass die idealisierte Natur - von Thomas Cole über Ansel Adams bis zu Clyde Butcher ${ }^{29}$ - immer dann als genuin amerikanische gilt, wenn sie unberührt ist.

Anders als in den europäischen Ländern waren es in den USA nicht die Kulturlandschaft, nicht die Bauernhöfe von Vermont, die Ranches von Kansas, nicht die grünen Vororte der US-amerikanischen Städte, nicht die Dämme des Westens oder die Holzbrücken im Nordosten, die die künstlerische Präsentation der amerikanischen Natur dominierten; es war die Wildnis.

Die wilde Natur wurde damit zur Gegenkultur schlechthin; ihr kam, wie in keinem anderen Land, subversive, politische Funktion zu. Die Welt war und ist für die Amerikaner, in der Bildtradition und in der politischen Realität, in zwei Teile eingeteilt, die paradiesische wilde Natur und die höllische Kultur: „Hell we are building here on earth“"30, hieß es in einem der großen Sammelbände des Sierra Club, der den Bau von Vororten mit der Vertreibung aus dem Paradies gleichsetzte. Kein Wunder, dass es die USA waren, die - mit dem Wilderness Act von 1964 als erstes Land der Erde Wildnisgebiete einrichteten. 3\% der Fläche der USA wur-

\footnotetext{
${ }^{29}$ Butcher C (1994) Portfolio I : Florida landscapes. Foreword by G.A. Long ; Beyond the frame by

C. Ellinghausen, $2^{\text {nd }}$ edn. Shade Tree Press, Fort Myers, Florida.

30 Adams A, Newhall N (1960) S. 36
} 
den von jeder Bebauung ausgenommen. Diese Flächen waren jeweils definiert als „an area where the earth and its community of life are untrammeled by man, where man himself is a visitor who does not remain". ${ }^{31}$

Wer verstehen will, wieso es den Amerikanern früher und konsequenter gelang, Naturparks und Wildnisgebiete einzurichten, findet eine gewichtige, wenn auch nicht die einzige, Erklärung in der Dominanz einer ästhetischen Bildtradition, die Natur und Kultur radikal und willkürlich voneinander trennte und das Natürliche mit quasi-religiöser Bedeutung ausstattete. Das Spektakuläre, Monumentale, Erhabene war in der Imagination immer separiert von Kultur und Technik. Natur galt als potenziell heilig, Kultur als profan. Dem entspricht, dass es in der amerikanischen Umweltgeschichte unzählige Untersuchungen zur Geschichte der Naturparks und zur Geschichte der Wildnis gibt, während in Frankreich, Deutschland oder Großbritannien Darstellungen zur urbanen Sphäre und zur Umweltverschmutzung dominieren. Der Umwelthistoriker William Cronon hat dafür plädiert, den Blick von der Wildnis abzuwenden und die Natur in urbanen und suburbanen Räumen ins Zentrum von Natur- und Umweltdebatten zu stellen. Nur so könne die Dichotomie im Denken überwunden werden, die die Unberührtheit der Natur glorifiziere, die kulturelle Dimension dagegen ignoriere. Eine Analyse von Gemälden, Fotografien, Filmen und Bildbänden aus der amerikanischen Geschichte hilft nicht nur zu verstehen, wie tief die Trennung von Natur und Kultur in Bildern verankert ist. Sie zeigt auch, dass die Bilder politische Funktion besitzen: dass sie subtilen Einfluss üben, indem sie neuartige Sehweisen vermitteln und dass sie von Politikern und Umweltverbänden instrumentalisiert wurden, um kulturelle und politische Ziele zu realisieren.

\section{Literatur}

\section{Primärquellen}

Adams, A and Newhall, N (1960): This is the American Earth, San Francisco, Sierra Club. San Francisco, California

Bryant, W C (1914): Poems of William Cullen Bryant. Ed. H. Milford. Oxford University Press, London and Oxford

Butcher, C (1994): Portfolio I : Florida landscapes. Foreword by G.A. Long ; Beyond the frame by C. Ellinghausen, $2^{\text {nd }}$ edn. Shade Tree Press, Fort Myers, Florida

\footnotetext{
${ }^{31}$ Wilderness Act. Public Law 88-577 (16 U.S. C. 1131-1136). 88th Congress, Second Session, September 3, 1964. http:/ / www.wilderness.net/index.cfm?fuse=NWPS\&sec=legisAct. Zum größeren Zusammenhang siehe Callicott JB and Nelson M.P. eds. (1998), The Great New Wilderness Debate. An Expansive Collection of Writings Defining Wilderness from John Muir to Gary Snyder. University of Georgia Press, Athens, Georgia.
} 
Cole. T (1980): Essay on American Scenery. In: T. Cole, The Collected Essays and Prose Sketches. Ed. by M. Tymm, John Colet Press, St. Paul

Emmerich, R. [director] (2004): The Day after Tomorrow [Film]. Distributed by Twentieth Century Fox

Jackson, W H (1994): Time Exposure. The Autobiography of William Henry Jackson. Profusely illustrated with photographs, paintings, and drawings by the author. The Patrice Press, Tucson, Arizona

Lorentz, P (1936): The Plow that Broke the Plains [Film]. Distributed by U.S. Resettlement Administration

Moran, T (1865): American Art and American Scenery, in: Philadelphia Photographer, II, March 1865. S. 33-35

Norton, CE [1908] Papers of Charles Eliot Norton, 1880-1908, 2 Bde., Library of Congress, Washington, D.C. Archival Manuscript Material (Collection), MSS34926

Stegner, W ed. (1955): This is Dinosaur. Echo Park Country and Its Magic Rivers. Knopf, New York

Wilderness Act. Public Law 88-577 (16 U.S. C. 1131-1136). 88th Congress, Second Session, September 3, 1964. http://www.wilderness.net/index.cfm?fuse $=$ NWPS\&sec=legisAct. zuletzt besucht am 26. September 2008

2. Sekundärliteratur

Barnouw, E (1993) Documentary. A History of the Non-Fiction Film, Oxford University Press

Callicott, J B and Nelson M P, eds. (1998): The Great New Wilderness Debate. An Expansive Collection of Writings Defining Wilderness from John Muir to Gary Snyder. University of Georgia Press, Athens, Georgia

Cronon, C., ed. (1995): Uncommon Ground. Toward Reinventing, W.W. Norton \& Co, New York

Davidson, G S et al. (2006): Frederic Church, Winslow Homer, and Thomas Moran. Tourism and the American Landscape. Bulfinch Press, New York u. Boston

DeLuca, K M (1999): Image Politics. The New Rhetoric of Environmental Activism. The Guildford Press, New York and London

Dunaway, F (2005): Natural Visions. The Power of Images in American Environmental Reform. The University of Chicago Press, Chicago and London 
Gaethgens TW ed. (2006) Bilder aus der Neuen Welt. Amerikanische Malerei des 18. und 19. Jahrhunderts, Prestel Verlag, München

Gore, A (2006): An Inconvenient Truth. The Planetary Emergency of Global Warming and what we can do about it. Bloomsbury, London

Hariman, R \& Lucaites, J L (2007) No Caption Needed. Iconic Photographs, Public Culture, and Liberal Democracy. The University of Chicago Press, Chicago and London

Kinsey, J L (2006): Thomas Moran's West. Chromolithography, High Art, and Popular Taste. University Press of Kansas, Lawrence, Kansas

Linquist-Cock, E (1977): The Influence of Photography on American Landscape Painting, 1839-1880. Garland Publishing, Inc., New York and London

Novak, B (1995): Nature and Culture. American Landscape and Painting, 18251875. Oxford University Press, New York

Runte, A (1990): Trains of Discovery. Western railroads and the National Parks. revised edn. Robert Rinehart, Niwot, Colorado.

Runte, A (1997) National Parks. The American Experience, 3rd edn. University of Nebraska Press, Lincoln, Nebraska

Sandweiss, M A, ed. (1991): Photography in Nineteenth-Century America. Harry N. Abrams, Inc., New York

Sieferle, R P \& Breuninger H, Hrsg. (1999): Natur-Bilder: Wahrnehmungen von Natur und Umwelt in der Geschichte. Campus, Frankfurt a.M. and New York

Steinberg, T (2000): Acts of God, The Unnatural History of Natural Disaster in America. Oxford University Press, New York

Trachtenberg, A (1989): Reading American Photographs. Images as History. Matthew Brady to Walker Evans. Hill and Wang, New York

Westheider, O \& Müller, K (2007): Neue Welt. Die Erfindung der amerikanischen Malerei. Hirmer Verlag, München

Wilkins, T \& Hinkley, C L (1998): Thomas Moran. Artist of the Mountains. Foreword by Goetzmann W.H., 2nd edn. revised and enlarged. University of Oklahoma Press, Norman, Oklahoma

Woodward, R B (1994): The Perfect Painting. In: New York Time Magazine, February 20, 1994, S. 36-37

Worster, D (2004): Dust Bowl. The Southern Plains in the 1930s. Oxford University Press, New York 


\section{Abbildungen}

Adams, A (1942): Evening McDonald Lake, Glacier National Park. National Archives, Washington, D.C.

Adams, A \& Newhall N (1960): This is the American Earth [Sierra Club exhibit format series, 1]. Sierra Club, San Francisco, California

Church, F (1867): Niagara Falls, from the American side. National Galleries of Scotland, Edinburgh, Scotland

Cole, T (1825): Lake with Dead Trees. Allen Memorial Art Museum, Oberlin College, Oberlin, Ohio

Lorentz, P [Director] (1936): The Plow that Broke the Plains. Library of Congress, Washington, D.C.

Moran, T (1872): Grand Canyon of the Yellowstone. Department of the Interior Museum, Washington, D.C. 



\title{
Innenwelt der Umweltpolitik - Zu Geburt und Aufstieg eines Politikbereichs
}

\author{
Edda Müller
}

\section{Einleitung}

Mein Thema sind Geburt und Aufstieg der Umweltpolitik in der Zeit von 1969 bis 1982. Dabei geht es zum einen um den politisch-administrativen Prozess des Aufbaus eines neuen Politikbereichs - ich nenne es die „Innenwelt der Umweltpolitik“ $^{\text {“1 }}$ Es geht zum anderen um die „Außenwelt der Umweltpolitik“, d.h. die externen Kräfte und Faktoren, die das Entstehen und die Ausformung der Umweltpolitik beeinflusst haben.

Einem Auditorium von Historikern werde ich die Frage zu beantworten haben, wie ich auf das Geburtsjahr 1969 gekommen bin und was einen 'Politikbereich' ausmacht. Politisches Handeln gab es in den verschiedensten Teilbereichen des Umweltschutzes bereits vor 1969. Die Umweltgeschichte lehrt uns, dass der Umgang mit dem Umweltschutz nicht selten auch ,politisch“ in dem Sinne war, dass der Interessenkampf und die Suche nach einem Konsens die Bewältigung von Umweltproblemen begleiteten. Wie verblüffend die Parallelen zu den heutigen politischen Kontroversen sein können, zeigt die folgende Geschichte, die ich meinem Vortrag voranstellen möchte:

\footnotetext{
1 Vgl. Edda Müller: Innenwelt der Umweltpolitik. Sozial-liberale Umweltpolitik. (Ohn)Macht durch Organisation, Opladen 1986. Der nachfolgende Beitrag fußt in weiten Teilen auf dieser Untersuchung.
} 
Im Juli 1890 - zwei Jahre vor der großen Cholera-Epidemie, die im Spätsommer 1892 über 10000 Bewohnern Hamburgs den Tod brachte - fiel in der Hamburger Bürgerschaft die Entscheidung zum Bau einer Filtrationsanlage für die Trinkwasserversorgung der Hansestadt. Es war zu spät, um die Epidemie zu verhindern. Zwischen dem ersten Vorschlag zum Bau einer solchen Anlage - er war nach der Cholera-Epidemie von 1873 gemacht worden - waren 17 Jahre vergangen. Die der Entscheidung vorangegangenen Auseinandersetzungen sind ein Lehrstück für die Widerstände gegen Umweltmaßnahmen, die aktueller nicht sein könnten. Richard J. Evans beschreibt sie in seinem Buch „Tod in Hamburg“2. Ich gebe sie hier sinngemäß wieder.

Im Streit um die Finanzierung der verbesserten Wasserversorgung forderte der Hamburger Grundeigentümerverein, dass die vom Senat geplante Wassersteuer nicht von den Vermietern sondern den Mietern gezahlt werden solle. Dazu sollten die Wasserwerke mit jedem einzelnen Verbraucher einen gesonderten Liefervertrag schließen. Der Senat verwies auf den Verwaltungsaufwand: Es seien bereits 14000 Einzelverträge abgeschlossen worden; gäbe man der Forderung der Hausbesitzer nach, wäre ein Heer von Beamten von Nöten. Die höheren Verwaltungskosten müsse man dann natürlich in Form höherer Steuern auf die Hausbesitzer abwälzen. Streit gab es auch über die Pläne des Senats, in jedem Haus - nicht aber in jeder Wohnung - einen genormten Wassermesser von den Behörden einbauen zu lassen. Der Grundeigentümerverein lehnte dies kategorisch ab. Er befürchtete Manipulationen des Staates zu seinem Nachteil und wollte stattdessen das Recht durchsetzen, eigene Wasseruhren zu installieren ${ }^{3}$.

Das Beispiel Hamburg zeigt nicht nur Parallelen zum Gewicht von Wirtschafts- und Kapitalinteressen in der Abwägung mit gemeinwohlorientierten Umweltschutzmaßnahmen, die nach 1969 die umweltpolitischen Debatten bestimmten. Es zeigt auch, dass zentrale Teilaufgaben der Umweltpolitik seit langem zu den öffentlichen, insbesondere kommunalen Aufgaben gehören und keine Erfindung des 20. Jahrhunderts sind. Das gilt für die Hygiene inklusive der Abfallbeseitigung und der Wasserreinhaltung. Auch die Luftreinhaltung und der Lärmschutz wurden im Zuge der Industrialisierung zu öffentlichen Aufgaben und waren Gegenstand von Gesetzgebung. Bemerkenswert ist das Wirken einer erster „Bürgerbewegung“ zum Naturschutz, die als Reaktion auf die Industrialisierung zum Ausgang des 19. Jahrhundert und zu Beginn des 20. Jahrhunderts insbesondere im Bildungsbürgertum entstand. ${ }^{4}$

Dennoch gibt es die Umweltpolitik als eigenes Politikfeld in Deutschland erst seit Ende der 1960er/Anfang der 1970er Jahre. Was die neue Dimension ausmacht, werde ich im folgenden beschreiben. Dabei werde ich zunächst die „In-

\footnotetext{
2 Richard J. Evans: Tod in Hamburg. Stadt, Gesellschaft und Politik in den Cholera-Jahren 18301910, Hamburg 1991

3 vgl. Evans: a. a. O., S. $207 \mathrm{ff}$

${ }^{4}$ vgl. zur Geschichte der Umweltpolitik insbesondere Klaus-Georg Wey: Umweltpolitik in Deutschland. Kurze Geschichte des Umweltschutzes in Deutschland seit 1900, Opladen, 1982
} 
nenwelt der Umweltpolitik“, d.h. den internen politisch-administrativen Prozess des Aufbaus und der Ausformung des Politikbereichs behandeln. Mit den externen Einflussfaktoren - der Rolle der Wissenschaft und der Bürgerbewegung sowie dem Einfluss der internationalen Politik befasst sich der dritte Teil des Vortrags. Am Schluss werde ich Bilanz ziehen.

\section{Innenwelt der Umweltpolitik - ein neuer Politikbereich wird aufgebaut}

Spätestens jetzt muss ich die Frage beantwortet, was eigentlich das Neue an der Umweltpolitik war. Im Prinzip wurden die alten Aufgaben: Luftreinhaltung, Wasserreinhaltung, Abfallbeseitigung, Umwelthygiene, Naturschutz und Landschaftspflege weitergeführt. Neu war das „Denkgebäude“ und die Erkenntnis, dass die verschiedenen Problembereiche als Teil einer Gesamtproblematik verstanden werden müssten, „die durch die sozio-ökonomische Zielvorstellung, die Steigerung des Wohlstands verursacht wurde“" . Neu war die übergreifende Programmatik sowie die Schaffung institutioneller Strukturen, die den Anspruch erhoben, Umweltpolitik sektorübergreifend und querschnittsorientiert zu betreiben. Die Preisfrage ist, wie kam es zu diesem neuen Anspruchsniveau und der Bündelung der bisherigen öffentlichen Aufgaben in einem integrativen Politikbereich, der ab 1970 als Umweltpolitik bezeichnet wurde? ${ }^{6}$

Die Entstehungsgeschichte der Umweltpolitik ist politikwissenschaftlich bemerkenswert, weil sie so gar nicht mit gängigen Annahmen zum politischen Agenda-setting übereinstimmt. Der neue Politikbereich war 1969 nicht das „Kind einer akuten Krise“. Der Umweltschutz hatte im Bundestagswahlkampf 1969 keine Rolle gespielt. In keiner Partei gab es auch nur Ansätze eines umweltpolitischen Programms. Von einem politischen Wettbewerb und der Umsetzung eines umweltpolitischen Versprechens konnte beim Start der neuen Bundesregierung daher keine Rede $\operatorname{sein}^{7}$.

Der neue Politikbereich Umweltschutz entstand vielmehr ,in einem weitgehend von externen Faktoren unbeeinflussten autonomen politisch-administrativen Prozeß. An dessen Anfang stand der Regierungswille, die Verbesserung der Umweltbedingungen in den Katalog innerer Reformen aufzunehmen. Die programmatische und institutionelle Ausgestaltung wurde den Experten in der Ministerialverwaltung und im Parlament ${ }^{8}$ überlassen. "9 Diese agierten allerdings nicht im „luft-

\footnotetext{
5 Klaus-Georg Wey, a.a.O., S. 153

6 Die Begriffe Umweltpolitik und Umweltschutz wurden im Januar 1970 durch die Übersetzung des englischen Begriffs 'environmental protection' in das deutsche politische Vokabular eingeführt als man im Bundesministerium des Innern einen Namen für die aus dem Bundesministerium für das Gesundheitswesen übernommene Abteilung suchte; vgl. Edda Müller, a.a.O., S. 55

7 vgl. zum folgenden Edda Müller, a.a.O., S. 45-143

8 Bereits 1952 hatten sich Parlamentarier der Landtage und des Bundestages aus allen Parteien in der Interparlamentarischen Arbeitsgemeinschaft für naturgemäße Wirtschaft (IPA) zusammengeschlos-
} 
leeren Raum“. Wie ich später erläutern werde, spielte die internationale Politik eine wichtige Rolle. Auch begünstigte die allgemeine sozio-ökonomische Stimmung und öffentliche Meinung den Start der Umweltpolitik.

„Die Arbeit der Experten wurde von einem breiten politischen und gesellschaftlichen Konsens getragen. Die hohe Konsensfähigkeit des Umweltschutzes erleichterte die Implementation der neuen Aufgabe gegenüber Ressortegoismen und Widerständen im administrativen System, bewirkte jedoch auch ihre unzureichende machtpolitische Untermauerung. Parteien, Medien und die gesellschaftlichen Gruppen griffen das neue Thema bereitwillig auf und akzeptierten dankbar die Unterstützung der administrativen Experten bei der Formulierung von Programmen, der Informationsvermittlung und Bereitstellung finanzieller Mittel für die Verbandsarbeit" 10 (hierzu später mehr). Als das allgemeine Klima für den Umweltschutz jedoch rauer wurde, fehlten die „starken Bataillone“, um den Umweltschutz auf der „Siegerstraße“ zu halten.

Ich unterscheide zwischen 1969 und 1982 drei zeitliche Phasen der Umweltpolitik:

- die Phase offensiver weitgehend ,autonomer“ Umweltpolitik mit einem starken Gewicht der Verwaltung in den Jahren 1969 bis 1974 ,

- die Phase einer defensiven Umweltpolitik in den Jahren 1974 bis 1978, in der ökonomische Kräfte den politischen Prozess dominierten und zum Teil blockierten,

- eine „Erholungsphase“ in den Jahren 1978 bis 1982; sie wurde durch sozio-kulturelle Veränderungen und Verschiebungen im politischen Kräftefeld bewirkt ${ }^{11}$.

Im folgenden werde ich die wesentlichen Merkmale dieser Phasen skizzieren.

\subsection{Erste Phase: Die Ministerialverwaltung legt das Fundament und zimmert das Haus}

Am Anfang der Umweltpolitik stand die Umorganisation der Bundesregierung. Durch Organisationserlass von Bundeskanzler Willy Brandt wurde Bundesinnenminister Hans-Dietrich Genscher die Abteilung „Wasserwirtschaft, Reinhaltung der Luft, Lärmbekämpfung“" aus dem Bundesgesundheitsministerium übertragen.

sen. Sie haben 1950er und 1960er Jahren wichtige Umweltgesetze angestoßen. Ihre Wirkung beruhte auf ihrer Überparteilichkeit und der Betonung der fachlichen Expertenarbeit. Verbunden war damit zugleich der geringe politische Einfluss auf die Programmatik ihrer Parteien. Vgl. Edda Müller, a.a.O, S. 54

${ }^{9}$ Edda Müller, a.a.O., S. 95

10 ebenda

11 vgl. Edda Müller, a.a.O., S. 45 
Die ursprünglich ebenfalls beabsichtigte Verlagerung der Zuständigkeit für den Naturschutz und die Landschaftspflege aus dem Bundeslandwirtschaftsministerium ins BMI scheiterte am Widerstand des FDP-Landwirtschaftsministers. ${ }^{12}$ Sie ließ sich erst 1986 nach der Tschernobyl-Katastrophe bei der Schaffung des Bundesministeriums für Umwelt, Naturschutz und Reaktorsicherheit (BMU) verwirklichen. Die Ressortaufteilung des Naturschutzes und des Umweltschutzes hat im übrigen die Verwirklichung des integrativen Anspruchs der Umweltpolitik erheblich behindert zumal dies auch mit der Existenz zweier nachgeordneter Behörden verbunden war. ${ }^{13}$

Den Elan der Umweltschützer im BMI hat das nicht gebremst. Die Beamten des BMI legten mit Unterstützung ihrer Kollegen aus dem Bundeskanzleramt ab 1970 in rascher Folge die Bausteine des neuen Politikbereichs vor und schufen sich zielstrebig ihre „Hilfstruppen“:

- Am 17. September 1970 wurde das Sofortprogramm vom Bundeskabinett verabschiedet.

- Es folgte im September 1971 das eigentliche Umweltprogramm.

- Im Dezember 1971 wurde der unabhängige Sachverständigenrat für Umweltfragen - SRU - errichtet.

- Ebenfalls 1971 schuf sich der BMI mit der Arbeitsgemeinschaft für Umweltfragen - AGU einen „Runden Tisch“ mit Beteiligten aus Verwaltung, Politik, Gesellschaft, Wirtschaft und der Wissenschaft als Diskussionsforum und Gremium zum Meinungs- und Erfahrungsaustausch.

- 1974 nahm das Umweltbundesamt in Berlin seine Arbeit auf. Die Schaffung dieser ,integrativen“ Behörde und die Verhinderung der ursprünglichen Absicht, drei getrennte Fachbehörden für Immissionsschutz, für Abfallwirtschaft und für Umweltschutz einzurichten, ist aus meiner Sicht eine der wichtigsten Weichenstellungen für die Umweltpolitik gewesen. ${ }^{14}$

Parallel hierzu wurde innerhalb der Bundesregierung und für die Zusammenarbeit mit den Bundesländern eine Reihe politischer Abstimmungsgremien geschaffen: Anfang 1970 der Kabinettausschuss für Umweltfragen (sog. Umwelt-Kabinett); im selben Jahr der Ständige Abteilungsleiterausschuss-Bund (STALA-Bund) für die Vorbereitung von Entscheidungen des Umweltkabinetts sowie zur Abstimmung ressortübergreifender Umweltfragen; die Umweltministerkonferenz von Bund und

\footnotetext{
12 vgl. Edda Müller, a.a.O., S. 56 f.

${ }^{13}$ Für die wissenschaftliche Zuarbeit zum BML in Sachen Naturschutz und Landschaftspflege war die Bundesanstalt für Naturschutz und Landschaftsökologie zuständig, für die wissenschaftliche Unterstützung der Umweltaufgaben des BMI das Umweltbundesamt.

${ }^{14}$ vgl. Edda Müller: 25 Jahre Umweltbundesamt - Spuren in der Umweltpolitik, Erster Teil in: Jahrbuch Ökologie 1999, S. 207-224, S. 210 f.
} 
Ländern tagte erstmals im Oktober 1972. Ihre Beschlüsse wurden und werden seit 1972 vom STALA - Ständiger Bund/Länder- Abteilungsleiter- Ausschuss vorbereitet.

Mit dem Sofortprogramm konnte der BMI das Überraschungsmoment nutzen - die Gegner hatten ihre Schützengräben noch nicht bezogen. Am deutlichsten war dies bei der raschen Verabschiedung der ersten Stufe des Benzin-Blei Gesetzes (im August 1971). Gewollt war aber vor allem, den Umweltschutz rasch in die öffentliche Diskussion zu bringen und eine breite gesellschaftliche Unterstützung zu mobilisieren.

Das Umweltprogramm war das eigentlich intellektuell Neue an der Umweltpolitik. Es setzte erstmals die verschiedenen Teilaufgaben - wie Luftreinhaltung, Wasserreinhaltung usw. - in einen größeren Zusammenhang. Formuliert wurden Leitlinien der Umweltpolitik, die später in allen von Parteien, Verbänden und anderen gesellschaftlichen Gruppen vorgelegten Umweltprogrammen wieder auftauchten. Richtschnur der Umweltpolitik sollten das Vorsorge-, Verursacherprinzip- und das Kooperationsprinzip sein. Die Programmschreiber verstanden die Umweltpolitik als „Querschnittspolitik“ und formulierten ihre Ansprüche an andere Politikbereiche.

Die Gesetzgebungsarbeit bis 1974 war dann mit Ausnahme der Verfassungsänderungen, mit denen der Bund die Gesetzgebungskompetenz für den Immissionsschutz und die Abfallwirtschaft sowie die Rahmengesetzgebungskompetenz für die Wasserwirtschaft und den Naturschutz erhielt, eher konventionell - weil überwiegend sektoral. Bis Mitte 1974 wurden sechs Gesetze, für die der BMI selbst federführend zuständig war über die gesetzgeberischen Hürden gebracht ${ }^{15}$. Daneben wurden zahlreiche Rechtsverordnungen und Verwaltungsvorschriften erlassen. ${ }^{16}$ Hier ist nicht der Ort, die einzelnen Maßnahmen detailliert zu beschreiben. Eine Erwähnung verdient jedoch das Bundes-Immissionsschutzgesetz von 1974. Es band die Genehmigung von Anlagen an eine doppelte Voraussetzung: zum einen an die Einhaltung bestimmter Immissionsgrenzwerte in Abhängigkeit von der lokalen und regionalen Belastungssituation und zum anderen an die Einhaltung des Standes der Technik hinsichtlich der Emissionen. Trotz aller Schwierigkeiten und Unzulänglichkeiten in der Umsetzung - ich erinnere an die „Hohe Schornsteinpolitik“ - hat die „Emissionspolitik nach dem Stand der Technik“ eine Dynamik entfaltet, ohne die der inzwischen relativ hohe anlagenbezogene Stand der Luftreinhaltung und der Umweltschutztechnik in Deutschland wahrscheinlich nicht möglich gewesen wäre. Ich werde hierauf zurückkommen.

\footnotetext{
15 Das im BML ab 1970 ebenfalls vorbereitete Bundesnaturschutzgesetz konnte erst 1976 verabschiedet werden.

16 Ein vollständiger Überblick über die Aktivitäten der Bundesregierung im Umweltschutz bis 1974 findet sich in BMI-Umwelt 29/74, S. 1 ff.
} 
2.2 Zweite Phase: Die Bäume wachsen nicht in den Himmel - Konflikte mit der Wirtschaft

Die Ölkrise vom Winter 1973/74 und der darauf folgende Wirtschaftseinbruch leiteten den Klimawechsel in der Umweltpolitik ein. Er hielt bis 1978 an und zwang die Umweltpolitik zu einem „Defensiv- und Stellungsspiel“. Den Auftakt bot die vom Bundeskanzleramt initiierte Tagung auf Schloß Gymnich vom Juni 1975. Die dort gemeinsam von Industrie und Gewerkschaften formulierten Vorwürfe beherrschten die umweltpolitische Auseinandersetzung der kommenden Jahre. Sie lauteten ${ }^{17}$ :

Der Umweltschutz sei für einen enormen Investitionsstau verantwortlich. Durch langwierige Genehmigungsverfahren und hinsichtlich ihres Ausgangs unvorhersehbare Gerichtsverfahren würden Investitionen in einer Größenordnung von $50 \mathrm{Mrd}$ DM behindert.

Der Umweltschutz gefährde durch zu strenge Auflagen zur Luftreinhaltung die Energieversorgung.

Der Umweltschutz bedrohe und vernichte Arbeitsplätze aufgrund der vorgenannten Probleme und der allgemeinen Überforderung der wirtschaftlichen Leistungsfähigkeit.

Die innerhalb der Bundesregierung eingerichteten Koordinationsgremien entwickelten sich nun zunehmend zu Aufpassern der Umweltschützer im BMI. Den administrativen Blockaden der „Verursacherressorts“ - allen voran das Bundeswirtschaftsministerium - hatten die BMI-Beamten wenig entgegenzusetzen. Die Aufmerksamkeit des FDP-Bundesinnenministers war durch die Terrorismusgefahr absorbiert. Überdies gab es weder in der FDP, in der sich das Kräfteverhältnis zugunsten des Wirtschaftsflügel verschoben hatte, noch in den anderen Parteien von Einzelpersönlichkeiten abgesehen - eine Umweltlobby. Auch die inzwischen sich formierende Umweltbewegung war wenig hilfreich. Sie war weitgehend mit den Protesten gegen die Kernenergie beschäftigt. Da wegen der Terrorismusgefahr durch die RAF bei den Parteien Sicherheit, Ruhe und Ordnung hoch im Kurs standen, bewirkte sie bei den Politikern eher Berührungsängste gegenüber dem Umweltschutz.

„Der Ministerialverwaltung blieb daher nichts anderes übrig, als den erreichten Stand der Umweltschutzgesetzgebung und Umweltschutzprogrammatik gegen Abschwächungstendenzen zu verteidigen, im übrigen aber Konflikten - wenn möglich - aus dem Weg zu gehen. Es kann daher zumindest als „Verteidigungserfolg“ der Umweltpolitik gewertet werden, dass in den schwierigen Jahren zwischen 1975 und 1978 kein Umweltgesetz zurückgenommen oder abgeschwächt wurde“18

\footnotetext{
17 zum folgenden vgl. Edda Müller, a,a,O., S. 98

18 Edda Müller, a.a.O., S. 113 f.
} 
Als hilfreich erwiesen sich in dieser Zeit die ,institutionellen Hilfstruppen“, allen voran das Umweltbundesamt ${ }^{19}$. Es managte ein umfangreiches Umweltforschungsprogramm und bereitete ein Altanlagensanierungsprogramm vor. Ab Mitte der 1980er Jahre wurde es zu einem generellen Investitionsprogramm zur Förderung umweltgerechter Techniken und Produkte erweitert. ${ }^{20}$ Beide sorgten dafür, dass mit öffentlichen Finanzmitteln der „Stand der Technik“ vorangetrieben und auf der Grundlage des Bundes-Immissionsschutzgesetzes zum Teil auch rechtlich verbindlich vorgeschrieben werden konnte.

\subsection{Dritte Phase: Macht kommt ins Spiel - der Umweltschutz politisiert sich}

Die politischen Rahmenbedingungen - nicht die ökonomischen - änderten sich ab 1978 grundlegend. Im Juni 1978 kandidierten neue „Grüne Parteien“ für die Landtage in Niedersachsen und Hamburg. Sie blieben zwar unter der $5 \%$ Grenze, sorgten aber dafür, dass die FDP an der $5 \%$ Hürde scheiterte und nicht mehr in die Landtage einzog. Die Auswirkungen auf die innerparteilichen Willensbildungsprozesse - nicht nur in der FDP - waren enorm. Zwar glaubten die etablierten Parteien noch nicht, dass ihnen mit den „Grünen Parteien“ auf Dauer ein ernstzunehmender Konkurrent entstanden sei, sie registrierten aber ein neues Wählerpotential, das für den Wahlausgang entscheidend sein konnte. In allen Parteien wurde daher der bisher kaum beachtete „Umweltflügel“ gestärkt, und es setzte eine rege Programmtätigkeit ein ${ }^{21}$ :

Im Dezember 1979 legte die CDU ihr neues „Umweltpolitisches Programm“ vor. Die CSU folgte im April 1980 mit einem Positionspapier „Umweltpolitik in den 80er Jahren“. 1981 verabschiedete die FDP auf ihrem Kölner Parteitag das „Ökologische Aktionsprogramm“. Im Mai 1981 stellte die SPD in ihrem Papier „Ökologische Orientierungen“ die Umweltvorsorge in den Mittelpunkt und formulierte Anforderungen an umweltbelastende Politikbereiche wie z.B. die Verkehrspolitik und die Wirtschaftspolitik. In ihrem auf dem Berliner Parteitag 1979 verabschiedeten „ökologisch-ökonomischen Gesamtkonzept" war sie bereits für eine „Versöhnung von Ökologie und Ökonomie“ eingetreten. Sie wollte damit in erster Linie die Gewerkschaften besänftigen.

Die neue „Grüne Partei“ konnten sie damit nicht aufhalten. Im Januar 1980 wurde die Bundespartei der Grünen gegründet. Sie erreichte bei der Bundestagswahl 1980 zwar nur 1,5 \% der Stimmen. Zwischen 1979 und 1982 zogen „Die Grünen“ jedoch in sechs Landtage ein. 1983 betraten sie erstmals auch die parlamentarische Bühne des Deutschen Bundestages.

19 vgl., Edda Müller: 25 Jahre Umweltbundesamt - Spuren in der Umweltpolitik, Erster Teil, in: Jahrbuch Ökologie 1999, S. 207-224, Zweiter Teil, in: Jahrbuch Ökologie 2000, S. 199-220

${ }^{20}$ vgl. Edda Müller: 25 Jahre Umweltbundesamt - Spuren in der Umweltpolitik. Erster Teil, in: Jahrbuch Ökologie 1999, S. 218

21 Zum folgenden vgl. Edda Müller, a.a.O., S. 115 ff. 
Es ist bemerkenswert, dass der neue politische Handlungsspielraum von den ehemals so aktiven „beamteten Umweltschützern“ im BMI kaum genutzt werden konnte. Die interministeriellen Arbeiten an einem ökologischen Aktionsprogramm verliefen äußerst zäh. Hinderlich war vor allem der Zuständigkeitskonflikt zwischen dem BMI und dem für Naturschutz und Landschaftspflege zuständigen BML. Ebenso kamen die Arbeiten an einem Bodenschutzprogramm nicht voran ${ }^{22}$. Eine der Gründe war, dass die Politisierung des Umweltschutzes bei der Ressourcenausstattung folgenlos blieb. Weder wurde die Umweltabteilung des BMI personell verstärkt noch wurden aktive Instrumente wie die Verbändeförderung oder die Öffentlichkeitsarbeit finanziell verstärkt. Mein Fazit an dieser Stelle: „In der dritten Phase blies die Politik zwar zum Angriff, signalisierte und praktizierte auch Konfliktbereitschaft, sie sorgte jedoch nicht dafür, dass die ,administrative Truppe“ auch von ihren Fesseln befreit wurde, die aus Verhandlungsroutinen, Zuständigkeitsegoismen und Kapazitätsengpässen bestanden“. ${ }^{23}$

\section{Außenwelt der Umweltpolitik - was waren die treibenden Kräfte und wer die Helfer der Umweltpolitik?}

Ich kann weder die Wissenschaft noch die in Verbänden und Bürgerinitiativen organisierte „Umweltbewegung“ zu den originären treibenden Kräften und Helfern der Umweltpolitik bei ihren Anfängen zählen. Generell spielten bei der Entstehung des neuen Politikbereichs populärwissenschaftliche Veröffentlichungen an erster Stelle das 1962 veröffentlichte Buch „Silent Spring“ von Rachel Carsons, das die Umweltpolitik in den USA anschob - eine wichtigere Rolle als die Wissenschaft an sich. Dies gilt gleichermaßen für das 1972 vom Club of Rome veröffentlichte Buch zu den „Grenzen des Wachstums“, das später von der „seriösen“ Wissenschaft in Grund und Boden kritisiert wurde.

Der Druck der „Basis“ in Gestalt von Bürgerinitiativen, Naturschutz- und Umweltverbänden war beim Aufbau der Umweltpolitik in Deutschland ab 1969 nicht existent. In den schwierigen Zeiten, in denen die Umweltpolitik mit erheblichen Widerständen aus Wirtschafts- und Gewerkschaftskreisen konfrontiert war, waren diese „Verbündeten“ keine Helfer. Das änderte sich erst als sich mit Hilfe der Medien und der nun mit öffentlicher Hilfe politisch agierenden Umweltverbände in der Bevölkerung und der Wählerschaft ein politisch relevantes Umweltbewusstsein und Umweltengagement aufgebaut hatte. Hilfreich im politischen Prozess war vor allem die internationale Politik. Im Folgenden will ich diese Behauptungen begründen.

\footnotetext{
$22 \mathrm{vgl} \mathrm{Edda} \mathrm{Müller:} \mathrm{Ministerialverwaltung} \mathrm{im} \mathrm{Prozess} \mathrm{der} \mathrm{Normgenese} \mathrm{am} \mathrm{Beispiel} \mathrm{des} \mathrm{Bodenschut-}$ zes, in: Edmund Brandt/Ulrich Smeddinck/Ralf Tils (Hrsg.) Gesetzesproduktion im administrativen Binnenbereich, Baden-Baden, 2001, S. 17-29

${ }^{23}$ Edda Müller: a.a.O., S. 143
} 


\subsection{Zur Rolle der Wissenschaft}

Die Situation und Rolle der Wissenschaft beim Start der Umweltpolitik ist weitgehend identisch mit der Entwicklung des sektoralen Umweltschutzes. Wey hat das sehr schön beschrieben. ${ }^{24}$ Biologen, Forstwissenschaftler, Wasserwissenschaftler, Wissenschaftler, die sich mit den Umweltschäden durch Rauch und Abgasen beschäftigten, haben bei politischen Entscheidungen zum Umgang mit diesen sektoralen Umweltproblemen bereits im 19. Jahrhundert eine wichtige Rolle gespielt. Zum Teil haben sie durch Streitigkeiten innerhalb der jeweiligen Disziplinen und durch interdisziplinäre Kontroversen der Politik eher Steine statt Brot gegeben. Die Bewertung der politischen Rolle der Biologen durch Wey mag daher auch für die anderen Disziplinen gelten. Im Zusammenhang mit der Rolle von Ernst Haeckel zur Begründung des Wissenschaftszweiges der Ökologie stellte er fest: „Umweltpolitisch wurde der Beitrag der Biologen jedoch kaum wirksam. Vielfach waren sich die deutschen Professoren wohl zu schade für die Niederungen des alltäglichen praktischen Ringens um eine naturnahe Umwelt für die Menschen. “25

Beim Start der Umweltpolitik konnten die administrativen Umweltschützer daher zwar auf ein erhebliches wissenschaftliches Potential zurückgreifen. Es fand sich vor allem in öffentlichen Forschungsanstalten und Ämtern ${ }^{26}$. Es bedurfte aber für den weiteren Aufstieg der Umweltpolitik eines mit öffentlichen Mitteln gut ausgestatteten Umweltforschungsprogramms, um das Engagement der Wissenschaft auf Themen zu lenken, die für die Umsetzung politischer Umweltziele wichtig waren. Hierbei hat das Umweltbundesamt eine zentrale Rolle in dem Sinne gespielt, dass es die Wissenschaft ertüchtigte, als Helfer der Umweltpolitik eine positive und hilfreiche Rolle zu spielen.

Bis weit in die 80er Jahre hinein erhielt die Umweltpolitik daher vor allem Rückendeckung durch die Disziplinen der „Problemursachenforschung“ - allen voran die Naturwissenschaften und die Analytik. Hilfestellungen bei der Problemlösung leistete insbesondere die Ingenieurwissenschaft. Die Beiträge zur Entwicklung umweltpolitischer Instrumente waren anfangs auf die Rechtswissenschaft und Finanzwissenschaft beschränkt. Seit Mitte der 1980er Jahre kamen die Wirtschaftswissenschaften hinzu, nachdem das Umweltbundesamt in seinem Umweltforschungsplan (UFOPLAN) hier einen Förderungsschwerpunkt gelegt hatte. Richtig ist aber auch, dass es zu jedem Gutachten auch ein Gegengutachten geben konnte, dessen Auftraggeber die Wissenschaft dann je nach ihrem Interesse für oder gegen politische Umweltmaßnahmen instrumentalisierten.

Mindestens so wichtig wie die öffentliche Forschungsförderung war aus meiner Sicht daher der Aufbau umweltwissenschaftlicher Studiengänge an den Universitäten und Hochschulen. In Ergänzung der sektorspezifischen umweltrelevanten

\footnotetext{
${ }^{24}$ vgl Klaus-Georg Wey, a.a.O., S. 27 ff.

25 Klaus-Georg Wey, a.a.O., S. 28

${ }^{26}$ vgl. Hans-Peter Vierhaus: Umweltbewußtsein von oben. Zum Verfassungsgebot demokratischer Willensbildung, Berlin 1994, S. 66 ff
} 
Ausbildung wurden hier einer neuer Generation von Studierenden Kenntnisse und ein Verständnis für die Gesamtproblematik des Umweltschutzes sowie für Methoden einer integrativen Umweltpolitik vermittelt. Die Nachfrage nach solchen Studiengängen war etwa ab Mitte der 1970er Jahre größer als das Angebot. Sie war Ausdruck des wachsenden Umweltbewusstseins in der Bevölkerung und führte allmählich auch zu neuen Studiengängen sowie zur Veränderung vorhandener Lehrpläne.

\subsection{Die Rolle der „Zivilgesellschaft“ und der Umweltbewegung}

Mit Ausnahme der „sozialen Frage“ hat wohl kaum ein Thema die gesellschaftlichen Strukturen und Bewusstseinshaltungen stärker verändert als der Umweltschutz. Diese Veränderungen standen jedoch nicht am Anfang der Umweltpolitik, sie verliefen vielmehr parallel zur Entwicklung der staatlichen Umweltpolitik. Vierhaus spricht sogar von einem „Umweltbewusstsein von oben“27 der „Lenkung der öffentlichen Meinung durch die Bundesregierung“"28 und resümiert: „Die Jahreswende 1969/70 beschreibt ... den Zeitpunkt, zu dem sich - auf Druck der Bundesregierung - Umweltschutz vom Expertenwissen zum Gegenstand der öffentlichen Meinung zu wandeln beginnt." 29

In seinem Umweltgutachten 1978 hat der Sachverständigenrat für Umweltfragen die Entstehung des Umweltbewusstseins, die Rolle der Bürgerinitiativen sowie von Presse, Rundfunk und Fernsehen beschrieben. ${ }^{30}$ Die Ergebnisse sind eindeutig:

Während im September 1970 der Begriff Umweltschutz erst $41 \%$ bekannt war, konnten sich nach der Vorlage des Umweltprogramms im November 1971 bereits $92 \%$ der Befragten unter Umweltschutz etwas annähernd Richtiges vorstellen. Alle Meinungsumfragen ergaben zwischen 1971 und 1974 eine hohe Einschätzung der politischen Bedeutung und Wichtigkeit des Umweltschutzes ${ }^{31}$.

In die gleiche Zeitspanne fiel das rasche Anwachsen der Bürgerinitiativbewegung. Der wichtigste Multiplikator waren Presse, Rundfunk und Fernsehen. Die Bundesregierung hatte die Weckung des Umweltbewusstseins in allen Teilen der Bevölkerung und die Unterstützung von Bürgerinitiativen zu einem der zentralen Themen ihres Umweltprogramms gemacht. ${ }^{32}$ Im Errichtungsgesetz des Umweltbundesamtes wurde der Auftrag zur Aufklärung der Öffentlichkeit in Umweltfragen explizit verankert.

\footnotetext{
27 Vierhaus, a.a.O., Buchtitel

${ }^{28}$ Hans-Peter Vierhaus, a.a.O., S. 139

${ }^{29}$ Hans-Peter Vierhaus, a.a.O., S. 160

${ }^{30}$ vgl. SRU. Umweltgutachten 1978, hrsg. vom BMI als BT-Drs. 8/1938

31 vgl. Edda Müller, a.a.O., S. 87

32 Umweltprogramm der Bundesregierung von 1971, in: BMI (Hrsg) Umweltschutz. Das Umweltprogramm der Bundesregierung mit einer Einführung von Hans-Dietrich Genscher, Stuttgart, 1972, S. 42
} 
Es wurde ein Haushaltstitel zur Verbändeförderung eingerichtet, mit dem Aktionen und Veranstaltungen der Umweltverbände finanziell unterstützt wurden. Vor allem aber wurden Anlässe geschaffen, über die die Medien berichten konnten. Der SRU stellte dazu in seinem Umweltgutachten 1978 fest:

„Es fällt auf, dass viele Themen von Verbänden und Behörden gezielt in die öffentliche Diskussion gebracht worden sind. Hinter fast jedem Pressebericht ist der Anstoß und das Informationsmaterial eines solchen Interessenten spürbar; man schuf Anlässe für die Berichterstattung, indem man Tagungen veranstaltete oder Aufrufe vorlegte. Der Rückblick zeigt hier eine besonders preiswerte Methode der Aufklärung der Öffentlichkeit". ${ }^{33}$

Für den politisch-administrativen Prozess bei der Vorbereitung von Gesetzen und Programmen war diese breite öffentliche Unterstützung hilfreich solange die professionellen Umweltschützer und Umweltpolitiker selbst in der Offensive waren. Als nach Gymnich die Umweltpolitik unter Druck und in die Defensive geriet, kam es zum „Zauberlehrlingseffekt" - man wurde die Geister, die man gerufen hatte, nicht mehr los. Das hohe Umweltbewusstsein äußerte sich nun in einer wachsenden Unzufriedenheit mit der Umweltpolitik und den Leistungen der „etablierten Parteien“ und bildete das Sympathiepotential, dem die „grünen Parteien“ ab 1978 ihren Aufstieg zu verdanken haben.

Sehr bald schon hatten die „beamteten Umweltschützer“ im BMI gemerkt, dass öffentliche Unterstützung allein nicht ausreichte, um im Interessenstreit erfolgreich zu sein. Gesellschaftliche Interessen werden in erster Linie durch Verbände gegenüber dem politisch-administrativen Raum artikuliert. Dies vollzieht sich in einem institutionalisierten Rahmen: in durch Geschäftsordnungen der Bundesregierung und des Bundestages geregelten Verbändeanhörungen bei Gesetzgebungsverfahren und durch gesetzliche Beteiligungsverfahren wie sie z.B. im $\ 29$ Bundesnaturschutzgesetz und $\ 51$ des Bundes-Immissionsschutzgesetzes festgelegt sind. Gebraucht wurden daher Umwelt- und Naturschutzverbände, die diese Aufgaben professionell wahrnahmen.

In den Anfängen der Umweltpolitik gab es bereits Naturschutz- und Umweltverbände, die zum Teil auf eine lange Tradition zurückblickten, wie z.B. den Deutschen Naturschutzring (DNR), den Deutschen Heimatbund (DHB), den Deutschen Bund für Vogelschutz (DBV), ab 1975 auch den Bund für Umwelt und Naturschutz Deutschland (BUND). Ihr umweltpolitisches Engagement wurde jedoch in den ersten Jahren der Umweltpolitik kaum erkennbar. Ellwein u.a. stellten in einer - vom Umweltbundesamt in den 80er Jahre in Auftrag gegebenen empirischen Untersuchung der Umweltverbände ${ }^{34}$ fest, dass diese in der Mehrzahl eher einen „unpolitischen Umweltschutz“ betrieben. Sie konzentrierten sich auf lokale Aktionen etwa die Säuberung eines Waldstücks oder den Schutz eines bedrohten

\footnotetext{
33 SRU, a.a.O., Tz. 1400

34 vgl. Thomas Ellwein/Martin Leonhard/ Peter M. Schmidt: Umweltschutzverbände in der Bundesrepublik Deutschland, MS-Forschungsbericht 10101 031/02 im Auftrag des Umweltbundesamtes, Berlin 1985
} 
Biotops. Nur beim BUND und beim DBV waren Ansätze für ein politisch wirksames Agieren erkennbar. ${ }^{35}$

Diese Lücke wollte 1972 der BBU - der Bundesverband der Bürgerinitiativen Umweltschutz- schließen. Der BMI stellte für die Gründungsversammlung in Frankfurt-Mörfelden hierfür die Reisekosten bereit ${ }^{36}$. Die Bürgerinitiativbewegung wollte sich damit zwar einerseits auf die institutionellen Erfordernisse einer wirksamen Einflussnahme auf die Politikvorbereitung auf Bundesebene einstellen. Sie wollte andererseits jedoch den Anspruch nicht aufgeben, die Umweltpolitik durch direkte spontane Aktionen voranzutreiben. Die Doppelstrategie gelang nur unvollkommen. Sie hat vermutlich den späteren Bedeutungsverlust des BBU mit herbeigeführt. „Ständig in der Furcht, seine Integrationskraft gegenüber der Bürgerinitiativbewegung durch zu große Nähe zur Macht zu verlieren, war (der BBU) für die Umweltschützer in der Ministerialverwaltung ein wenig verlässlicher und kalkulierbarer Verhandlungspartner, ..."37

Die Zivilgesellschaft und die Umweltbewegung haben - so mein Zwischenfazit - die Umweltpolitik gestärkt und vorangebracht. Dies war jedoch erst möglich als sie in Gestalt der „Grünen Partei“ sowie der erstarkten Umweltflügel in den anderen Parteien einen ,institutionellen Zugang“ zum politisch-administrativen Prozess gefunden hatten. Heute gehören der BUND, der NABU (als Nachfolgeorganisation des DBV) sowie der DNR zum festen Bestandteil des Kräfteparallelogramms in der umweltpolitischen Auseinandersetzung.

\subsection{Die internationale Politik als treibende Kraft}

Die internationale Politik war die treibende Kraft bei der Entstehung der deutschen Umweltpolitik. Die entscheidenden Impulse kamen aus den USA. „Der Umweltschutz wurde in die Bundesrepublik gleichsam importiert". ${ }^{38}$ Die Programmschreiber im BMI hatten die US-amerikanische Umweltpolitik und Umweltprogrammatik studiert und in wesentlichen Teilen „abgekupfert“"

Die Politisierung des Umweltthemas in den USA in den 1960er Jahren hatte zudem eine Reihe multilateraler Umweltinitiativen und Vereinbarungen ausgelöst. Außerdem gingen vom Europarat wichtige Anstöße aus. Ob sie durch die Entwicklung in den USA beeinflusst wurden oder auf eigenen Initiativen beruhten, weiß ich nicht. Vielleicht war ja der ,Zeitgeist" reif für das Thema ohne dass einem bestimmten Land oder einzelnen Personen und Institutionen das Urheberrecht zugesprochen werden kann. Umwelthistoriker hätten hier noch ein interessantes Betätigungsfeld. Ich will die wichtigsten Aktivitäten hier nennen:

\footnotetext{
35 vgl. Thomas Ellwein u.a, a.a.O., S. 390

${ }^{36}$ vgl. Edda Müller, a.a.O., S. 88

${ }^{37}$ vgl. Edda Müller, a.a.O., S. 119

38 vgl. Edda Müller, a.a.O., S. 53
} 
Die UNESCO veranstaltete 1968 in Paris den internationalen Kongress „Mensch und Biosphäre“. Er initiierte das aus meiner Sicht interessanteste und am meisten unterschätzte internationale „Programm zur Nachhaltigkeit“ lange bevor die Brundtland-Kommission mit dem Konzept der "nachhaltigen Entwicklung“" Furore machte. 1968 verkündete der Europarat eine Wassercharta und eine Charta zur Luftreinhaltung und erklärte das Jahr 1970 zum Europäischen Naturschutzjahr.

1969 fand in Neu Delhi der Kongress der Internationalen Union zum Schutz der Natur und der natürlichen Ressourcen statt, auf dessen Legitimation sich z.B. die deutsche Nationalparkpolitik beruft. 1971 wurde das internationale Übereinkommen zum Schutz von Wasser- und Watvögeln (Ramsar-Konvention) verabschiedet, dem natürlich ein längerer Verhandlungsprozess voran gegangen war. Es folgte 1973 das Washingtoner Artenschutzabkommen zum internationalen Handel gefährdeter Arten freilebender Tiere und Pflanzen, dem mit Sicherheit ebenfalls ein längerer Verhandlungsprozess vorausging.

Auffällig ist, dass ein großer Teil der internationalen Aktivitäten den Naturschutz betreffen. Aus meiner Sicht spricht dies für das gute internationale Netzwerk, mit dem die Naturschutzpolitik ihre Durchsetzungsschwäche und mangelnde Unterstützung in den nationalen politischen Prozessen zu kompensieren versuchte.

Das wichtigste Ereignis für die „moderne Umweltpolitik“ ist die UNKonferenz über die Umwelt des Menschen, die im Juni 1972 in Stockholm stattfand. Sie geht auf eine UN-Resolution vom 3. 12. 1968 zurück. Der Vorbereitungsprozess für diese Konferenz hat den Umweltschutz nicht nur auf der politischadministrativen Bühne enorm vorangebracht. Er hat insbesondere auch dafür gesorgt, dass in den Wirtschaftsverbänden - BDI und DIHT - und bei den deutschen Gewerkschaften spezielle Arbeitskreise und Verantwortliche eingerichtet und benannt wurden, die nunmehr die internen Willensbildungsprozesse organisierten und als Ansprechpartner für die Umweltpolitik zur Verfügung standen.

Im Konkreten war die Stockholm-Konferenz für Deutschland und die deutsche Umweltpolitik „kein Zuckerschlecken“. Schweden und die nordischen Länder warfen u.a. Deutschland vor, für die Versauerung ihrer Seen durch den weiträumigen Transport insbesondere von SO2 aus der Verbrennung und Verstromung fossiler Energien verantwortlich zu sein. Die offizielle deutsche Politik wies diesen Vorwurf zurück. Man fürchtete Schadenersatzforderungen an den deutschen Fiskus. Die Umweltpolitik der 1970er Jahre war dennoch - und vielleicht gerade deshalb - von der Luftreinhaltepolitik geprägt. So war die Begrenzung der SO2- und NOX-Emissionen aus Großfeuerungsanlagen ein jahrelanges Streitthemen zwischen Umweltschutz und Energiewirtschaft. Es wurde erst nach dem Scheitern der sozial-liberalen Koalition im Jahre 1983 durch die Verabschiedung der Großfeuerungsanlagenverordnung zugunsten des Umweltschutzes entschieden. 


\section{Bilanz: Wo steht die Umweltpolitik heute?}

Meine Untersuchungen zu Geburt und Aufstieg der Umweltpolitik enden mit der Beendigung der sozial-liberalen Bundesregierung 1982. Zum Schluss meines Vortrags will ich deshalb den Bogen spannen vom Anspruchsniveau der Umweltprogrammatik 1970/71 hin zur Situation der Umweltpolitik heute. Ich beurteile sie wie folgt:

1. Die Umweltpolitik kämpft noch immer um die Durchsetzung des Vorsorge- und Verursacherprinzips. Die Klimapolitik ist im Prinzip ein „Glücksfall“ für die Umweltpolitik, weil sie ihr die Legitimation verschafft, auf die „Verursacherbereiche“ klassischer Umweltbelastungen einzuwirken und für grundsätzliche Strukturveränderungen unserer Produktions- und Konsummuster zu sorgen.

2. Das allgemeine Umweltbewusstsein ist weniger stabil, opfer- und handlungsbereit als Meinungsumfragen suggerieren. Das Paradigma der Nachhaltigkeit - wonach ökologische, soziale und ökonomische Ziele gleichermaßen befriedigt werden können, bietet hier Chancen.

3. Der internationalen Politik kommt nach wie vor eine wichtige `push- und pull-Funktion' zu. Zunehmend gilt dies auch für die Rolle der Europäischen Union.

Zu 1. Bei der Beurteilung der deutschen Umweltpolitik dominieren in der öffentlichen Meinung die Kritiker. Sie werfen der Umweltpolitik vor, dass sie bisher zu wenig ganzheitlich vorgegangen sei und sich auf die Reparatur vorhandener Schäden anstelle der Vermeidung und Vorsorge konzentriert habe.

Ich halte diese Kritik für wenig praxisgerecht und unpolitisch. Programmatische allgemeine Ziele sind als Richtungsvorgabe wichtig. Sie müssen aber letztendlich in Handlungen überführt werden. Ohne eine Arbeitsteilung sind diese nicht denkbar und machbar. Unter dem Dach der generellen auf eine ganzheitliche Betrachtung ausgerichteten „Philosophie der Umweltpolitik“ war deshalb eine Abarbeitung der Umweltprobleme nach den jeweiligen Sektoren unvermeidlich. Der sektorale Reparaturbetrieb der deutschen Umweltpolitik war nötig und insbesondere bei der Begrenzung der Emissionen aus industriellen Anlagen erfolgreich. Er hat die klassische Luftverschmutzung reduziert, die anlagenbezogene Lärmbelästigung vermindert, die Gewässerqualität deutlich verbessert und hinsichtlich der Abfallentsorgung für eine Revolution gesorgt. Abfälle sind seitdem Sekundärrohstoffe. Die Abfallwirtschaft ist ein maßgeblicher Teil einer Ressourcenschonungspolitik geworden.

Die Umweltpolitik war wenig erfolgreich, wenn es darum ging für eine frühzeitige Integration und Berücksichtigung von Umweltbelangen in den verschiedenen Verursacherbereiche - insbesondere der Produktpolitik und Strukturpolitik zu sorgen. Besonders augenscheinlich ist dies bei der Verkehrs- und Autopolitik, der 
Agrarpolitik, der Siedlungspolitik und dem Tourismus. Umweltpolitische Querschnittsinstrumente wie die Umweltverträglichkeitsprüfung (UVP) waren hilfreich. Sie haben aber außer einem enormen Untersuchungs- und Bürokratieaufwand nicht den Durchbruch geschafft. Die von den Wirtschaftswissenschaften als Allheilmittel propagierten ökonomischen Instrumente, mit denen die externer Umweltkosten in die Preise integriert werden sollen, stoßen aus ökonomischen und sozialen Gründen auf erhebliche politische Widerstände. Sofern sie nicht zumindest innerhalb der Europäischen Union abgestimmt eingeführt werden können, stellen sie für die betroffene deutsche Wirtschaft erhebliche Wettbewerbsnachteile und eine Gefährdung heimischer Arbeitsplätzen dar. Sozialpolitisch sowie aus Gründen allgemeiner politischer Akzeptanz sind ihrer effizienten Nutzung ebenfalls Grenzen gesetzt, weil sich keine demokratisch legitimierte Politik aufgrund noch so rationaler Maxime leisten kann, den sozialen Sprengstoff politischer Entscheidungen zu ignorieren.

Die Klimapolitik ist für die Umweltpolitik - so zynisch dies klingen mag - ein Glücksfall. Der globale Klimawandel ist weitgehend energiebedingt. Es gilt in erster Linie die CO2 - Emissionen aus der Verbrennung fossiler Energieträger zu vermindern. Hierfür gibt es - wenn man von der Erhöhung der Speicherkapazität etwa durch Aufforstung absieht - nur zwei Wege: Das Wegsparen von CO2 durch die Steigerung der Energieeffizienz sowie die Veränderung des Energiemixes durch den Einsatz nicht-fossiler Energien. Beide Strategien sind wegen der Endlichkeit fossiler Energieträger sowie der Einsparung von Energiekosten auch aus ökonomischen Gründen notwendig und sinnvoll. Die Umsetzung erfordert zum einen technologische Innovationen etwa in der Produkt- und Materialpolitik, sie erfordert zum anderen eine grundlegende Veränderung der Strukturen unserer Energieversorgung sowie aller auf Energie angewiesener Bereiche: Produktion, Konsum, Bauen, Wohnen und Verkehr. Für all diese Bereiche ist die Umweltpolitik nicht zuständig. Niemand macht ihr jedoch die Verantwortung für die Klimapolitik und die CO2-Verminderung streitig. Dies bedeutet in der Logik der Mechanismen des politisch-administrativen Prozesses: Die Zuständigkeitsfrage ist geklärt und damit zugleich bei wem die Initiativfunktion und die Verfahrensherrschaft für den Klimaschutz angesiedelt ist. Die Umweltpolitik hat den Hebel in die Hand bekommen, um mit Hilfe von Minderungszielen und nicht zuletzt der internationalen und EU-Politik für eine Integration von Umweltanforderungen in die jeweiligen Lebens- und Politikbereiche zu sorgen.

Zu 2. Das allgemeine Umweltbewusstsein ist gegenüber den Anfängen der Umweltpolitik sicherlich auf einem höheren Niveau angekommen ${ }^{39}$. Es aus meiner Sicht aber immer noch „ein scheues Reh“, d.h. es ist leicht zu verschrecken, wenn Umweltschutz mit Einschränkungen im Alltag und im Geldbeutel der Menschen

\footnotetext{
39 vgl. BMU, Hrsg.: Umweltbewusstsein in Deutschland 2004. Ergebnisse einer repräsentativen Bevölkerungsumfrage. Förderkennzeichen 20317132/01, Bonn 2004
} 
verbunden ist. Das gilt vor allem dann, wenn breite Bevölkerungsschichten mit Arbeitslosigkeit und sinkenden Nettoeinkommen konfrontiert sind.

Die Umweltverbände sind heute präsent. Ob sie damit bessere Helfer der Umweltpolitik gegen massive wirtschaftliche Widerstände bei konkreten politischen Entscheidungen geworden sind, wage ich zu bezweifeln. Mit Sicherheit trägt ihr Protestpotential jedoch politisch Früchte, indem es das „Umweltgewissen“ in den Parteien wach hält. Die Umweltverbände und ihre Mitglieder leisten zudem vor Ort und zusammen mit anderen gesellschaftlichen Gruppen überaus wertvolle Beiträge, in denen Umwelt- und Naturschutz sehr konkret gelebt und beispielhaft demonstriert wird. Das gilt für Lokale Agenda-Gruppen sowie Aktivitäten aus kirchlichen Kreisen ebenso wie das Wirken vieler sonstiger Vereine und Netzwerke. ${ }^{40}$

Aus meiner Sicht wird es aber nach wie vor auf die „Funktionseliten“ ankommen, um die Umweltpolitik weiter voranzubringen. Sie ist gegenüber den 1970er Jahren vielfältiger geworden. Vorkämpfer für den Umweltschutz finden sich heute nicht mehr nur im politisch-administrativen Raum sondern auch in Unternehmen, in der Kunst- und Kulturszene, unter Publizisten und nicht zuletzt in der Wissenschaft. Entscheidend für ihre Wirksamkeit ist das konkrete Tun, nicht mehr allein die Vermittlung moralischer Appelle und „Weltuntergangsszenarien“. Es ist hier, wo m.E. das Paradigma der Nachhaltigkeit überzeugend mit Leben gefüllt werden kann. Da ist z.B. der Unternehmer, der auf eine ökologische Produktion ebenso achtet wie auf soziale, familiengerechte Arbeitsbedingungen und eine angemessene Bezahlung seiner Mitarbeiter und Mitarbeiterinnen. Oder da ist der Prominente aus dem Showbusiness, der etwa durch seinen Konsumstil, die Wahl seines Autos und seines Stromlieferanten persönliche Umweltverantwortung praktiziert und auf diese Weise hilft, eine neue Form gesellschaftlicher Reputation „salonfähig“ zu machen.

Zu 3. Von herausragender Bedeutung für die deutsche Umweltpolitik sind nach wie vor die internationale Politik und zunehmend auch die EU-Politik. Diese Aussage ist ein Allgemeinplatz, weil wir allenthalben von der „Globalisierung aller Lebensbereiche“ und den Zwängen der EU-Politik zu hören gewohnt sind. Zumeist wird damit aber auf die Abhängigkeiten und Einschränkungen der innenpolitischen Handlungsfähigkeit hingewiesen nicht aber auf ihre Anstoßfunktion und Unterstützung für nationale politische Aufgaben.

Natürlich sind gerade in der internationalen Politik - ich erinnere nur an die Klimapolitik - Fortschritte mühsam, zäh und langwierig. Für die nationalen umweltpolitischen Akteure können solche Verhandlungen mit ihren Terminsetzungen jedoch immer wieder genutzt werden, um ein Thema auf die „politische Agenda“ zu bringen, Öffentlichkeit herzustellen und nationale Widersacher unter Druck zu setzen und zu Zugeständnissen zu bewegen. Hilfreich ist dabei, dass seit vielen

\footnotetext{
${ }^{40}$ vgl. u.a. Rat für Nachhaltige Entwicklung, www.mission-sustainibility.org sowie www.verbrauchertag.de/Projektdatenbank-Nachhaltigkeit.7.0 html
} 
Jahren solche Verhandlungen nicht mehr im Stil der „Geheimdiplomatie“ ablaufen, sondern ein großer Kreis von Beobachtern aus Wirtschaft, den NichtRegierungsorganisationen, der Wissenschaft die Verhandlungen aktiv begleitet. Sie reisen zu solchen Konferenzen ebenso wie die Regierungsvertreter nicht unvorbereitet an. Vielmehr geht den Verhandlungen in allen beteiligten Kreisen ein intensiver nationaler und internationaler Vorbereitungs- und Abstimmungsprozess voraus. Dabei können z.B. Vorreiter aus den Kreisen der Wirtschaft Unterstützung für ihre Position mobilisieren und Vertreter aus Kreisen der NichtRegierungsorganisationen können sich weltweit vernetzen, neue Allianzen schmieden und medienwirksame Aktionen und Resolutionen vorbereiten. Bei der Umweltkonferenz Umwelt und Entwicklung in Johannesburg im Jahr 2002 waren es z.B. Frauenorganisationen aus Entwicklungsländern, die den Verhandlungsprozess positiv beeinflussten.

Von anderer Art ist die unterstützende Rolle der EU-Politik. Im Gegensatz zu den Verhandlungen im Rahmen der Vereinten Nationen gibt es hier in Gestalt der EU-Kommission einen eindeutigen Akteur, der mit seinem Initiativrecht Vorschläge auf die politische Agenda setzen kann. Mit dem Europäischen Parlament gibt es eine politische Kraft, die von nationalen Interessen ausreichend unabhängig ist und in Gestalt der Ministerräte gibt es Entscheidungsorgane, die inzwischen in vielen Feldern Mehrheitsbeschlüsse herbeiführen können. In diesem mehrstufigen Verfahren kann es auch immer wieder bei wichtigen Umweltfragen zu Blockaden, zu Verzögerungen und zu „faulen Kompromissen“ kommen. Das jüngste Beispiel ist die Verschiebung der verbindlichen Einführung der CO2- Werte für Kraftfahrzeuge, die in erster Linie auf den deutschen Widerstand zurückgeht. In der Bilanz überwiegen aber m.E. die positiven Einflüsse. Beispiele sind die Liberalisierung des Strom- und Gasmarktes, die Diskussion zur eigentumsrechtlichen Entflechtung von Stromproduktion und -Verteilung sowie die jüngsten Beschlüsse der EU zur Klimapolitik. Eine besonders förderliche Rolle hat die EU auch im Naturschutz gespielt. So haben die Flora-Fauna-Habitat-Richtlinie und das Programm Natura 2000 die Naturschutzpolitik in Deutschland vorangebracht.

Politik im Allgemeinen und Umweltpolitik im Besonderen ist ein mühsames Geschäft. Max Webers Bild vom „Bohren dicker Bretter mit Augenmaß und Leidenschaft" kennt vermutlich jeder, der sich mit Politik beschäftigt. Was ich hier beschrieben habe, war das Drum und Dran des Bohrens. Am Anfang der Umweltpolitik wurde das Brett definiert. Es wurde festgelegt, wer bohrt und wo die Helfer waren. Die Astlöcher, die den Bohrenden immer wieder Widerstand leisten, waren und sind weniger kalkulierbar. Von ihnen wird auch künftig die Wirksamkeit und Geschwindigkeit umweltpolitischen Handelns abhängen. 


\title{
Umweltgeschichte und Altlasten: zur anhaltenden Relevanz gefährdender Stoffe
}

\author{
Klaus Schlottau
}

Den Historikern, auch den Umwelthistorikern, sind Altlasten nur in wenigen Fällen geläufig. Ein systematischer Zugriff und eine Einordnung in den Gegenstandbereich der Umweltgeschichte fehlen bis auf ganz wenige Ausnahmen. Ein Blick aber in z.B. die öffentlich zugängliche Bibliothek für Umwelt in Dresden ergibt in der Auswertung der Systematik zu den Begriffen Altlasten und Umweltgeschichte ein Verhältnis der Veröffentlichungen von 127 : 87. Die Veröffentlichungen zu Altlasten sind mithin eindeutig in der Überzahl und eine Durchsicht der Autoren zeigt, dass darunter keine bekannten Umwelthistoriker, ganz überwiegend aber Ingenieurwissenschaftler und Naturwissenschaftler, vertreten sind. Die Geschichte der Altlasten wurde bislang von Historikern nicht als Umweltgeschichte oder als Gegenstand der allgemeinen Geschichte wahrgenommen.

Wahrnehmung ist ein zentrales Kriterium des Gegenstandbereiches der Geschichtswissenschaft. Es stellt sich daher die Frage, warum die Historiker den Altlasten keine Bedeutung zumessen, warum sie nicht wahrgenommen werden? Eine Antwort wäre, dass die stoffliche Beschaffenheit der Altlasten eine Wahrnehmung durch historische Subjekte und eine daraus resultierende Tradierung nicht ermöglichte.

Im Jahr 1998 trat das Bundesbodenschutzgesetz in Kraft. Mit diesem Gesetz ist intendiert, dem Boden den notwendigen Schutz gegenüber gegenwärtigen und künftigen Ansprüchen zu gewähren. Er wird als das „Archiv der Menschheit“ beschrieben. Er ist aber auch das Archiv der vergangenen Bodenbelastungen: Ab- 
fälle sind darin vergraben worden und industrielle Altstandorte gefährden nach wie vor in großem Maße die Nutzung des Bodens.

Aus diesem Grunde sind die Ordnungsbehörden befugt, Sachverständige für die Untersuchung und Bewertung der Altstandorte zu berufen. Die erste Aufgabe der Sachverständigen besteht gemäß den Ausführungsbestimmungen zum \ 31 des Bundesbodenschutzgesetzes in der „Durchführung und Beurteilung von standortbezogenen Erhebungen (historische Recherche)“. Da der Gesetzgeber davon ausgeht, dass kein Sachverständiger alle sechs Aufgabengebiete ausreichend beherrschen kann, gibt er vor, dass möglichst jedes der Teilgebiete von einem speziell vorgebildeten Sachverständigen bearbeitet wird. Gefordert ist daher der „,sachverständige Historiker“ als erster Gutachter für ein Grundstück, das unter einem Altlastenverdacht steht. Altlasten gehen von Stoffen aus, die die gesetzlichen Schutzgüter, also Menschen, Tiere, Wasser, Boden, Bodenorganismen und Luft, gefährden. Sie befinden sich in der Regel auf oder in Altablagerungen oder Altstandorten - Altablagerungen können landläufig als Deponien bezeichnet werden, während Altstandorte als Grundstücke, auf denen mit gefährdenden Stoffen umgegangen wurde, zu bezeichnen sind.

Altlasten sind daher komplexe Sachverhalte, die in der Vergangenheit entstanden sind, in der Gegenwart noch nicht bewältigt wurden und zudem drohen, in der Zukunft weiteres Unheil anzurichten: es handelt sich mithin im Einzelfall um einen Sachverhalt, der in der Retrospektive auf die Entstehungsgeschichte ein genuin historisches Arbeitsfeld umfasst, wegen seiner fortdauernden Aktualität und der Prolongierung in die Zukunft aber auch ein Gegenstandsbereich der handlungsorientierten Wissenschaften ist. Wegen der stofflichen Eigenschaften der Altlasten, ihrer Beharrung in den Umweltmedien und der daraus resultierenden Gefährdung beschäftigen sich insbesondere Naturwissenschaftler, Mediziner und Ingenieure mit dem Nachweis, der Analytik und der Sanierung von Altlasten.

Die gesellschaftliche Relevanz der Altlasten ist unbestritten. In einer jahrzehntelangen systematischen Suche haben die zuständigen Behörden der Bundesländer- auch mit Hilfe von Historikern - Altlastenkataster angelegt, die, je nach Bundesland, dessen Größe und dessen Industriegeschichte, von wenigen tausenden bis zu vielen zehntausenden altlastverdächtige Standorte umfassen. Als Beispiel sei hier die Schweiz genannt. Für die Auffindung, Untersuchung und eventuelle Sanierung der 50.000 verdächtigen Standorte wird, gemäß einer Kostenschätzung aus dem Jahre 2003, ein Betrag von 50 Milliarden Franken erforderlich sein. ${ }^{1}$ Die Kosten sind jedoch nicht der einzige bedeutsame Faktor: Bei jeder planerischen Neugestaltung treten höhere Kosten und ein deutlicher Zeitverzug in der Umsetzung ein, weil sich Altlasten auf wenige Bereiche konzentrieren. Altlasten verteilen sich in der Schweiz nicht gleichmäßig über die Fläche. Sie befinden sich in den Tälern, besonders aber in den Städten, also an standorttheoretischen Optimallagen, die

1 Vgl:: Beat Jordi: Teure Aufräumaktion zum Schutz der Gesundheit. In: Umwelt 3/2003, Altlasten, S. 6-9. hier S. 8 . 
auch jetzt und in Zukunft und in vermutlich allen Ländern von Bedeutung sind. In der Bundesrepublik Deutschland, die im Vergleich zur Schweiz eine weit größere Industriedichte, eine viel stärkere Urbanisierung und sehr viel mehr militärische Altlasten aufweist, gehen Behörden von einer Gesamtzahl von nur ca. 300.000 verdächtiger Altstandorte aus. Die einfache Übertragung Schweizer Verhältnisse, ein Altstandort je 70 Einwohner, würde ergeben, dass in der Bundesrepublik mehr als eine Million Altstandorte vorhanden wären und Sanierungskosten in Höhe von mehr als 650 Milliarden Euro entstünden; wenn nicht Historiker bereits jetzt sowohl die Zahl der Verdachtsflächen reduzieren und die Kosten beträchtlich senken würden. Der Grund für die Abweichung von der durch eine Hochrechnung zu erwartenden Gesamtzahl verdächtiger Flächen liegt darin, dass bereits bei der systematischen Suche nach den Altlasten in hohem Maße historische Arbeit geleistet wurde, um zu selektieren, was als Altlastverdacht zu bezeichnen ist.

Im Folgenden wird daher zunächst darzustellen sein, welche Hilfsmittel, Quellen und Methoden von einem Altlastensachverständigen eingesetzt werden. Exempliziert wird die Arbeit sodann an zwei Beispielen, die in mehrfacher Hinsicht von Bedeutung sind: mit dem Beispiel Milzbrand wird eine Altlast diskutiert, die im herkömmlichen Sinne keine stofflich vergleichbaren Eigenschaften besitzt, aber dennoch durch Sporen über Jahrhunderte gefährlich bleibt; mit dem folgenden Beispiel der chemischen Reinigungen wird eine Problematik dargestellt, die sich nicht der Produktion, sondern der Dienstleistung, einem in der Umweltgeschichte kaum wahrgenommenen Problembereich, zuordnen lässt. Beide Beispiele wiederum entziehen sich der Wahrnehmung durch den Menschen, so dass zu fragen ist, ob es sich überhaupt um historische Gegenstände handeln kann. Dies wird am Ende der Ausführungen zu diskutieren sein.

\section{$1 \quad$ Historiker als Altlastensachverständige}

In modernen Gesellschaften werden nicht mehr nutzbare Abfall- und Reststoffe aus der gewerblichen Produktion und den Dienstleistungsgewerben freigesetzt. Dies ist ein Problemfeld, mit dem sich die Disziplin der Umweltgeschichte seit den letzten dreißig Jahren an aussagekräftigen Beispielen beschäftigt. Gegenstände der Forschung sind häufig singuläre Katastrophen, spektakuläre Langfristschäden, Umweltgeschichte von Städten Regionen, Flüssen oder z.B. intertemporale oder interregionale Vergleiche für die Umweltmedien Wasser, Boden und Luft. Zugleich aber begann sich im letzten Jahrzehnt des 20. Jahrhunderts, seit der Veröffentlichung eines Gutachtens des Sachverständigenrates für Umweltfragen, ein neues Berufsbild für Historiker mit interdisziplinären Interessen herauszubilden, das durch die Gesetzgebung des Bundes und der Länder konkrete Formen annahm. ${ }^{2}$

\footnotetext{
2 Der Rat von Sachverständigen für Umweltfragen: Altlasten. Sondergutachten, Dezember 1989, Stuttgart 1990, S. 74-84.
} 
Die Ziele des historischen Altlastensachverständigen bewegen sich zwischen der Intention, auf einer Untersuchungsfläche den ,hot spot', den Ort, von dem die Gefährdung mit einem oder mehreren gefährdenden Stoffen ausgeht, zu finden und der allgemeineren Aufgabe, zu ermitteln, welche Branchen der Wirtschaft zu welchem Zeitpunkt als gefährdend anzusehen sind. Letzteres ist eine Forschungstätigkeit mit übergreifender Aufgabe, die als umweltgeschichtliche Strukturgeschichte betrachtet werden kann und jedem Historiker sicherlich verständlich ist. Ersteres hingegen erfordert, neben der grundsätzlichen Kenntnis der Branchentätigkeit, einen mikrohistorischen Zugriff auf eine Fläche, die sowohl die Geschichte der ansässigen Unternehmen als auch die der Produkte und deren Zwischen-, Halb- und Abprodukte räumlich zeitlich erfassen und derartig zuzuordnen beabsichtigt, dass z. B. bei einer alten vergessenen Gasanstalt genau der Teerkeller unter der Gasregeneration bei Probebohrungen getroffen wird, von dem aus der benachbarte Kinderspielplatz kontinuierlich mit polyaromatischen Kohlenwasserstoffen (PAK), Zyankali und Aromaten vergiftet wird. Der Altlastenhistoriker hat mithin die konkrete Aufgabe, den Ingenieuren und Naturwissenschaftlern, die nach ihm tätig werden, genau vorzugeben, welche Teilflächen eines gewerblich genutzten Areals mit bestimmten Schadstoffen verunreinigt sind, damit die Probebohrungen und die Probenahme den richtigen Ort mit bestimmten Schadstoffen und daraus resultierenden Gefährdungen der Umweltmedien betreffen. Es handelt sich mithin um eine gezielte Suche, die kosten- und zeitintensive Rasterbeprobungen und damit auch die Perforation wasserundurchlässiger Bodenschichten vermeidet.

Der historische Sachverständige hat weiterhin die Aufgabe, Altlasten zu erfassen und alle historischen Daten für die Erstbewertung und Gefährdungsabschätzung einer Untersuchungsfläche zu sammeln und zu bewerten. Betrachtet man die Stoffmengenbilanz eines Unternehmens, so werden Roh- oder Hilfsstoffe eingesetzt, um Zwischen- oder Fertigprodukte zu erzeugen. Stoffe, die nicht verarbeitet wurden oder sich im Rahmen eines chemischen Prozesses als unveräußerliches Abfallprodukt bilden sowie flüssige oder gasförmige Abfälle bilden eine Schadstoffmenge, die, bei entsprechender Abbildung der Stoffflüsse innerhalb des Unternehmens, prinzipiell als umweltschädigende Substanzen im Gelände verortet werden können.

Ungeachtet der Diskussion unter den Historikern, ob die Analyse von Stoffflüssen Bedeutung für die Umweltgeschichte besitzt, hatten Ingenieure und Naturwissenschaftler, die mit der Untersuchung von ehemaligen Betriebsflächen beauftragt wurden, die These von Ulrich Troitzsch bereits seit Jahren als Arbeitshypothese benutzt und vielfach verifiziert. ${ }^{3}$ Behörden und Forschungsinstitute

\footnotetext{
${ }^{3}$ Die These, dass die Kenntnis der eingesetzten und umgesetzten Stoffe auch Aufschluß über die Umweltgeschichte eines Betriebes oder einer Branche vermittelt, wurde von Ulrich Troitzsch aufgestellt. Vgl. Ders.: Umweltprobleme im Spätmittelalter und der Frühen Neuzeit aus technikgeschichtlicher Sicht. In: Bernd Herrmann (Hg.): Umwelt in der Geschichte. Beiträge zur Umweltgeschichte, Göttingen 1989, S. 95-97. Die Gegenthese wurde von Ralf Henneking vertreten. Vgl.: Ralf Henneking: Chemische Industrie und Umwelt. Konflikte um Umweltbelastungen durch die chemische
} 
haben nach flächendeckenden regionalen Untersuchungen Kataloge mit branchentypischen Kontaminationen entwickelt, deren Menge vom Produktionsverfahren und von der Betriebsgröße abhängig ist. ${ }^{4}$ Die Praxis der historischen Altlasten- und Umweltuntersuchung ist folglich über den Stand der historischen Umweltforschung hinausgeschritten und es bleibt zu fragen, wie die Arbeit der Altlastenhistoriker sich gegenwärtig gestaltet und ob die Ergebnisse von den historischen Disziplinen integriert werden können?

Ohne die gesetzlichen Vorschriften und die Entwicklung bis zum gegenwärtigen Stand im Detail zu referieren, lässt sich der Beginn bis auf die Einführung des Wasserhaushaltsgesetzes zurückführen, mit dem 1972 die Notwendigkeit der Erfassung von zugelassenen und ,wilden“ Müllablagerungsplätzen eintrat, weil diese ein öffentliches Schutzgut, nämlich Grund- und damit auch Trinkwasser, gefährdeten. Im Laufe der katastermäßigen Erfassung der Müllkippen zeichnete sich ab, dass von den Standorten der gewerblichen Produktion, von Handwerks- und Industriebetrieben, die mit gefährdenden Stoffen umgingen, eine Gefährdung ausging, die jener der alten und neuen Abfalldeponien nicht nachstand. Das Ordnungs- und das Baurecht boten die einzig verfügbare Rechtsgrundlage, derartige Altstandorte auf ihre schädlichen Auswirkungen zu untersuchen und gegebenenfalls eine Sanierung oder Sicherung von dem Verursacher oder Eigentümer zu fordern. Ein wesentlicher Nebeneffekt besteht darin, dass Baugenehmigungsverfahren und damit neue Nutzungen der Fläche, das „Flächenrecycling“, nur unter Aufwendung erheblicher Kosten und nach erheblichem Zeitverzug durchgeführt werden können. Gewöhnlich wurden die notwendigen Untersuchungen von Ingenieurunternehmen und Laborbetriebe durchgeführt, die in einem geometrischen Raster Suchbohrungen in den verdächtigen Untergrund niederbrachten, Boden und Wasser auf Schadstoffe jeder Art untersuchten und dann aufgrund der Analysenwerte Vorschläge für die Flächensanierung erarbeiteten. Dieses Verfahren war und ist kostenintensiv, dauert lange und bietet keine hinreichende Sicherheit, Schadstoffnester im Untergrund zu erfassen. Darüber hinaus wurden durch die Bohrungen nur zu häufig die vorhandenen Schadstoffe mobilisiert, indem sie durch die Perforationen der Bohrlöcher in das nächste Grundwasserstockwerk abflossen und damit zu einer unmittelbaren Gefährdung wurden.

Weil mit der traditionellen Methodik der Altlastenuntersuchung größere Kosten, Zeitverzug und eine zusätzliche Gefährdung verbunden ist, entwickelte sich allmählich die Methode einer destruktionsfreien Voruntersuchung des Untersuchungsareals. Sie besteht einerseits aus einer multitemporalen Karten- und Luft-

Industrie am Beispiel der schwerchemischen, Farben- und Düngemittelindustrie der Rheinprovinz (ca. 1800-1914). (Zeitschrift für Unternehmensgeschichte, Beiheft 86), Stuttgart 1994, S. 26-27.

${ }^{4}$ Vgl. z.B.: Branchenkatalog zur historischen Erhebung von Altstandorten, herausgegeben von der Landesanstalt für Umweltschutz Baden-Württemberg, 2. Auflage, Karlsruhe 1993. In jüngster Zeit bietet besonders der Altlastenleitfaden Schleswig-Holstein eine Fülle von Anregungen. Vgl.: Altlasten-Leitfaden Schleswig-Holstein, herausgegeben vom Landesamt für Natur und Umwelt des Landes Schleswig-Holstein, Kiel-Flintbek 2003. 
bildanalyse, die von der Geographie, die als Disziplin ohnehin bereits an den Altlastuntersuchungen beteiligt war, methodisch herausgebildet wurde. ${ }^{5}$ Parallel hierzu wurde von den beteiligten Ingenieur- und Naturwissenschaften in Behörden und Gutachterbüros eine historische Standortuntersuchung initiiert, um mehr über die Entwicklung und Nutzung des Altstandortes zu erfahren. Die destruktionslose historische Voruntersuchung hat die Aufgabe, die Grundlage für die anschließende gezielte Probenahme und für die analytische Untersuchung von Boden und Wasser auf bestimmte Schadstoffe oder Schadstoffgruppen zu liefern. Sie führt mithin zu einer schonenden, beschleunigten und, wie sich nach der Durchführung von vielen derartigen Untersuchungen am Beispiel der Rüstungsaltlasten gezeigt hat, auch deutlich kostengünstigeren Sanierung. ${ }^{6}$

Nachteilig war, dass die mit der Untersuchung beauftragten Ingenieurbüros in der Regel keine historisch ausgebildeten Mitarbeiter hatten, so dass auf allen Ebenen des öffentlichen Archivwesens Verständigungs- und Interpretationsprobleme auftraten, Quellenkritik nicht betrieben wurde und zu häufig Leseschwierigkeiten der Untersuchenden gerade die wichtigsten Passagen eines Behördenvorgangs, die handschriftlichen „Aktennotizen“ und die handschriftlichen Originalbetriebskonzessionen, übersehen ließen. Die Folge war, dass, vor allem im Zuge größerer Regionaluntersuchungen, z.B. der Suche nach Rüstungsaltlasten in der ehemaligen DDR, immer mehr Mitarbeiter mit einer historischen Ausbildung und Archivkenntnissen hinzugezogen werden mussten. Nach und nach begannen Historiker die Ingenieure und Naturwissenschaftler während der ersten Untersuchungsphase einer Altlast, der Verdachtsflächenerhebung und der Erstgefährdungsabschätzung, zu unterstützen. ${ }^{7}$

Am 7. Februar 1994 wurde der Referentenentwurf für das Bundesbodenschutzgesetz erstmals vorgestellt. ${ }^{8}$ Demgemäß wird die Untersuchung von Altlasten Sachverständigen übertragen, deren Rechte und Pflichten nach $\ 31$ des Entwurfs und den näheren Bestimmungen der Behörden geregelt werden. Obgleich der Entwurf damals noch keine Gesetzeskraft erlangt hatte, hat die Konferenz der Umweltminister die „Ländergemeinschaft Abfall“ (LAGA) und die „Ländergemeinschaft Boden“ (LABO), bereits damit beauftragt, in einer „Arbeitsgruppe Qualitätssicherung" die näheren Bestimmungen zur Formulierung des untergesetz-

\footnotetext{
5 Vgl.: Jürgen Dodt: Die Verwendung von Karten und Luftbildern bei der Ermittlung von Altlasten. Ein Leitfaden für die praktische Arbeit, Düsseldorf 1987; Hans-Walter Borries: Altlastenerfassung und Erstbewertung durch multitemporale Karten- und Luftbildauswertung, Würzburg 1992.

${ }^{6}$ Vgl.: Manfred Tiedemann: Archivalien als Mittel zur historisch-genetischen Erkundung von Rüstungsaltlasten. In: Altlasten-Spektrum, Jg. 1994, Heft 1, S. 5-8; Klaus-Jürgen Berief und Wolfgang Koch: Die Folgenutzungsplanung beginnt mit der Historischen Recherche. In: TerraTech, Jg. 1996, Heft 1, S. 23-27.

${ }^{7}$ In den Niederlanden z.B. ermittelt die Forschungsgruppe „de geschiedeniswinkel“ an der Universität Groningen aus den Archiven ein bis 1800 zurückreichendes Altlastenkataster für das ganze Land.

8 Referenten-Entwurf Bundes-Bodenschutzgesetz in der mit den Ressorts abgestimmten Fassung vom 7. Februar 1994. In: Verhandlungen des sechzigsten Deutschen Juristentages, Münster 1994, Band 1, Teil B, München 1994, S. B5-B87.
} 
lichen Regelungswerkes über die Sachverständigen zu treffen. Demgemäß wurden in Übereinstimmung mit den Bestimmungen des Landes Nordrhein-Westfalen im Landesabfallgesetz, \31a, Abs. 3, sechs Teilgutachter für die Altlastenuntersuchung gefunden, die 1998 auch in den $\int 18$. des Bundesbodenschutzgesetzes übernommen wurden.

Unter der Voraussetzung, dass es den Sachverständigen für Altlasten aufgrund vieler beteiligter Wissenschaftsdisziplinen nicht geben kann, wurde eine Aufteilung der Aufgaben in Teilgutachter für folgende Arbeitsbereiche vorgenommen:

- Durchführung und Beurteilung von standortbezogenen Erhebungen (historische Recherche),

- Untersuchung und Beurteilung von Gewässergefährdungen und Gewässerschäden (Grundwasser, Oberflächengewässer),

- Untersuchung und Beurteilung von Gesundheitsrisiken,

- Untersuchung und Bewertung von Kulturböden und Pflanzen,

- Beurteilung von Probenahme, Analytik und chemischem Stoffverhalten,

- Bewertung der Eignung und Kostenwirksamkeit von Sanierungsmaßnahmen.

Jeder Gutachter hat neben der persönlichen Zuverlässigkeit sowohl eine allgemeine als auch eine besondere Sachkunde nachzuweisen. Zu den persönlichen Voraussetzungen zählen Zuverlässigkeit und Integrität, Objektivität, Verschwiegenheit, die Bereitschaft zur ständigen fachlichen Weiterbildung sowie die erforderliche gerätetechnische Ausstattung. Die allgemeinen fachlichen Voraussetzungen sind in der Regel durch ein einschlägiges Studium und mehrjährige praktische Tätigkeit in der Untersuchung und Beurteilung von Altlastverdachtsflächen und Altlasten sowie durch die Kenntnis der einschlägigen Gesetze, Verordnungen und der ständigen Rechtsprechung erfüllt. Durch mehrjährige Berufserfahrung in der Untersuchung von Altlastverdachtsflächen, durch Erfahrungen im Projektmanagement und in der Projektplanung sind zudem die besonderen fachlichen Voraussetzungen für eine eigenverantwortliche Untersuchung und gutachterliche Stellungnahme in der Regel gegeben.

Für den Teilbereich der historischen Untersuchung von Altlastverdachtsflächen und Altlasten mittels der historischen Recherche des Standortes sind von Seiten der Gutachterausschüsse der Handelskammern, die mit dem Verfahren der Bestellung von öffentlich vereidigten Sachverständigen betraut sind, Anforderungsprofile erstellt worden. Grundvoraussetzung ist ein abgeschlossenes Studium in natur- oder ingenieurwissenschaftlichen Fächern oder vergleichbare Kenntnisse. Zusätzlich müssen Kenntnisse oder Erfahrungen in der historischen Methodik, im Archivwesen und in der Paläographie vorhanden sein. Vor der Zulassung zum Anerkennungsverfahren muss der Bewerber eine fünfjährige Berufstätigkeit in der Altlastenerkundung nachweisen. Für die Auswertung von Karten und Luftbildern sind der Nachweis von Stereoskop, Panthograph sowie entprechender Computer- 
programme, z.B. AutoCad und ArcView, sowie die üblichen Bürosoftwareprogramme erforderlich. Bezüglich der 'gerätetechnischen Ausstattung' hat sich auch für den Historiker ein erheblicher Bedarf ergeben. Reichten vor Jahren noch die üblichen Schreib-, Tabellenkalkulations- und Datenbankprogramme, so hat sich durch die Einführung von geographisch orientierten Altlastinformationssystemen auf allen Behördenebenen ein breites Spektrum notwendiger Investitionen ergeben, das noch dadurch gesteigert wird, dass viele dieser Informationssysteme untereinander nicht kompatibel sind, dass vielfach noch Eigenlösungen mit Inselcharakter vorhanden sind, dass die großen Systeme fast nur unter einem UnixBetriebssystem auf Workstations betrieben werden können und nur selten Schnittstellen zu PC-Programmen bieten. Die Folge ist, dass der Sachverständige neben den marktführenden Schreib- und Datenbankprogrammen immer auch eine Reihe von weniger gebräuchlichen Datenbankprogrammen und Tabellenkalkulationen besitzen und zudem in der Lage sein muss, Datenbanken selber zu entwerfen oder derart zu modifizieren, dass die Ergebnisse der Recherche von des Auftraggebers EDV-System akzeptiert wird, bzw. in die vorgegebene Maske eingefügt werden kann.

Die Altlast-, Wasser- oder Bodeninformationssysteme, in die die Daten zu den Untersuchungsflächen eingefügt werden sollen, sind in der Regel mit 'Geographischen Informationssystemen' (GIS) gekoppelt, so dass die Sachbearbeiter der Ämter im Idealfall nur mit dem Cursor auf einen Punkt ihrer digitalisierten Karte zeigen müssen, um alle bereits gesammelten Daten einer Verdachtsfläche aus den Datenbanken des Systems eingespielt zu bekommen, bzw., um aus der Datenbank heraus die Lage der Fläche angezeigt zu erhalten. Die Schnittstelle zwischen der Datenbank und der digitalen Karte wird durch die Gauß-Krüger-Koordinaten bestimmt. Die Folge ist, dass der Historiker mindestens Grundkenntnisse der Geographie besitzen sollte und in der Lage sein muss, seine Rechercheergebnisse zeichnerisch in Form mehrerer signifikanter Zeitschnitte in das vorgegebene Raster einzufügen, bzw. die Zeichnungen mit einer Georeferenzierung versehen muss. Gerätetechnisch bedeutet dies, dass eine überdurchschnittlich ausgestattete Rechenanlage mit einem modernen CAD-System (Computer aided Design), mit einem Digitalisiertablett, einem Scanner, mit einem Großformatdrucker oder Plotter sowie mit mehreren GIS-Programmen und Zusatzprogrammen für die Konvertierung unterschiedlich formatierter vektorisierter und georeferenzierter Kartengrundlagen vorhanden sein sollten.

Die allgemeinen fachlichen Anforderungen sind in der Regel nicht durch ein Studium der Geschichtswissenschaften abgedeckt. Es sollte mindestens ein Studium der neueren Geschichte oder der Wirtschafts- und Sozialgeschichte abgeschlossen worden sein. Zusätzlich sollten Grundkenntnisse der Unternehmensgeschichte sowie vertiefte Kenntnisse der Geschichte mechanischer und chemischer Verfahrenstechniken erworben worden sein. Unerlässlich sind vertiefte Grundkenntnisse historischer Methoden und Techniken wissenschaftlichen Arbeitens, Quellen- und Methodenkritik, Kenntnisse historischer Hilfswissenschaften sowie 
die Fähigkeit, handschriftliche Quellen der Neuzeit fließend zu lesen und fehlerfrei zu übertragen. Viele Historiker, die sich in den vergangenen Jahrzehnten der neuen historischen Disziplin „Umweltgeschichte“ zugewandt haben, haben große Erfahrungen in einer Reihe dieser allgemein-fachlichen Anforderungen, selten jedoch sind sie mit Behördenschriftgut vertraut, das ihnen im Rahmen einer allgemeinhistorischen Darstellung nicht zur Verfügung gestellt wird. Häufig reicht es zwar aus, sich dem Fachwissen des Sachbearbeiters in großen Archiven anzuvertrauen, jedoch sollte der Gutachter in der Lage sein, dem Sachbearbeiter im Archiv die richtigen Hinweise zu geben, in welchen Teilbeständen des Behördenschriftgutes eine Genehmigung, eine Konzession, Anliegereinsprüche, die Auflagen der diversen beteiligten Ämter etc., zu finden sein werden. Gleiches gilt für die Recherche in den Archiven der Firmen, der Kammern, der Verbände und in dem Behördenschriftgut, das den öffentlichen Archiven noch nicht übergeben wurde, weil es sich um noch laufende Vorgänge handelt. Der historische Gutachter erhält während seiner Untersuchung, die in der Regel von den Behörden oder von den untersuchungspflichtigen Eigentümern oder Nutzern in Auftrag gegeben wird, nahezu uneingeschränkte Akteneinsicht und hat damit einen weit größeren Quellenfundus als der Historiker, der sich mit einem Betrieb aus sozialhistorischen, unternehmensgeschichtlichen, umwelt- oder lokalhistorischen Absichten auseinandersetzt. All diese Akten müssen, unter strenger Wahrung datenschutzrechtlicher Belange, aufgenommen, ausgewertet und bewertet werden - häufig ist es angesichts der Überfülle an Dokumenten und widersprüchlicher Aussagen ein organisatorisches Problem, mit einer systematischen Quellenkritik ein individuell angepasstes Bewertungsmuster $\mathrm{zu}$ schaffen und damit auch zu erkennen, welche Quellengattung vorzuziehen ist, welche eventuell auch noch zu suchen ist.

Handelt es sich bei den zuvor genannten allgemeinen fachlichen Anforderungen noch um solche, die mit einer 'normalen' Laufbahn des Historikers vereinbar sind, so sollte der historische Sachverständige doch einige Kenntnisse von anderen Disziplinen besitzen, um mit den Gutachtern anderer Disziplinen zu kommunizieren, um seine Untersuchungsergebnisse dem Untersuchungszweck entsprechend schriftlich und mündlich formulieren zu können, und, nicht zuletzt, um im Falle einer unmittelbar notwendigen Gefahrenabwehr die Tatsachen korrekt zu interpretieren und zu vermitteln, ohne auf das Fachgebiet anderer Gutachter überzugreifen. Daher sollten folgende Kenntnisse vorhanden sein:

Grundkenntnisse der Geographie, der Geologie und der Hydrologie, die soweit gefestigt sind, dass ein Verständnis entsprechender fachwissenschaftlicher Ausführungen für das eigene Untersuchungsfeld sowie eine Kommunikation mit Wissenschaftlern dieser Disziplinen gewährleistet ist;

Grundkenntnisse der mechanischen Technologie, die notwendig sind, um aus dem Nachweis bestimmter Maschinentypen oder auf der Basis des zeitgenössischen Standes der Technik die Produktion einer zeitgenössischen branchentypischen Fabrik oder die Stückzahl pro Zeiteinheit mit hinreichender Sicherheit einschätzen zu können; 
Vertiefte Grundkenntnisse der chemischen Technologie. Diese sind notwendig, um einerseits den zeitgenössischen Stand der Technik verstehen und bewerten zu können, andererseits aber dringend erforderlich, um eine detaillierte Liste der auf dem Untersuchungsgelände während des Nutzungszeitraumes als Roh- oder Hilfsstoff benutzten, als Zwischen-, Abfall- oder Endprodukt hergestellten Stoffe und Waren für die nachfolgenden chemischen Untersuchungen zu erstellen. Die Kenntnisse sollten soweit reichen, dass die historische Bezeichnung chemischer Stoffe oder Verfahren in die Systematik der Genfer Nomenklatur übersetzt wird;

Historische und aktuelle Kenntnisse des Wasserrechts, des Bergrechts, der Gewerbeordnung, des Abfallrechts, des Verwaltungsrechts, des Baurechts, der Polizeiordnung, der Honorarordnung, der Qualitätssicherung etc.;

Grundkenntnisse der Betriebswirtschaft und der Materialwirtschaft, die notwendig sind, um Bilanzen, Geschäftsberichte, Warenausstoß, Personalstand und andere Kenndaten für den Materialfluß in einem Gewerbe- oder Industriebetrieb erfassen und interpretieren zu können.

In der Regel wird die Berufstätigkeit in Teilbereichen der historischen Standortuntersuchung für Behörden, Ingenieurbüros oder Gutachtergemeinschaften beginnen, bevor nach und nach durch ständige Weiterbildung und durch die Praxis ein gesicherter Fundus an Kenntnissen und Fähigkeiten vorliegt, der dann auch die besonderen fachlichen Kenntnisse umfasst. Es handelt sich hierbei einerseits um Organisationswissen, das im Kern die Tätigkeit einer Projektleitung umfasst, andererseits aber um das Erfahrungs- und Fachwissen, das aus der Untersuchung einer Vielzahl von Altlasten aus diversen Branchen entsteht. Das Organisationswissen eines Gutachters beginnt mit scheinbar trivialen Dingen wie der Zeit- und Kostenkalkulation in Form eines Angebotes. Für die Erstellung eines zutreffenden Kosten- und Leistungskatalogs unter Vorgabe einer Frist ist es notwendig, die vorhandenen Mitarbeiter und deren Qualifikationen gezielt einzusetzen und deren Tätigkeit genau zu überwachen, bzw. bestimmte Leistungen an andere Gutachter oder Unternehmen abzugeben. Unabhängig von der Leistungsfähigkeit des eigenen Büros muss ein Katalog notwendiger Leistungen für den Auftrag entworfen werden: Es müssen alle Archive und Behörden erfasst werden, die Vorgänge und Daten verwahren, deren Kenntnis für die Untersuchung notwendig ist. Es ist zu ermitteln, wie viele Vorgänge oder Aktenmeter voraussichtlich zu bearbeiten sind. Je nach Struktur der Daten müssen durchschnittliche Zeitvorgaben und Nebenkosten kalkuliert werden, so dass im Angebot jede Position mit der Angabe eines bestimmten Zeitbedarfs und der voraussichtlichen Schätzung der Kosten erscheint da der größte Teil einer historischen Recherche sich auf die Arbeit in Archiven und Bibliotheken erstreckt, ist es notwendig, zu wissen, welche Informationen dort zu erwarten sind, wie das Archiv gegliedert ist, wann es geöffnet hat, ob Kopier- oder Reproduktionsmöglichkeiten vorhanden sind, welche Bereitstellungsfristen zu beachten sind, wie lange es dauert, bis Reproduktionen geliefert werden und, nicht zuletzt, in welcher Höhe Gebühren erhoben werden. In der Regel werden mindestens zehn Archive und Behörden auf der Ebene der Gebietskörperschaften, auf 
Landes- und auf Bundesebene sowie zusätzlich noch Firmenarchive, Kammerund Verbandsarchive zu berücksichtigen sein. Der Gutachter hat die Aufgabe, die Relevanz der in den jeweiligen Archiven vermuteten Daten einzuschätzen, um dem Auftraggeber Untersuchungsprioritäten vorzuschlagen. Neben den Archiven und dem Behördenschriftgut ist auch die Sekundärliteratur zu berücksichtigen: Regional- und Lokalgeschichte, Unternehmensgeschichte, Branchengeschichte, Verfahrensgeschichte, die Auswertung von Wirtschaftshandbüchern, Patentschriftgut, die Auswertung der Handelsregistereinträge und die Zeitzeugenbefragung sind hinsichtlich des Zeitbedarfs wie auch der erforderlichen Qualifikation der Mitarbeiter und der damit verbundenen Kosten zu berücksichtigen.

Für die Dokumentation der Topografie und der Hydrogeologie ist es notwendig, sich geologische Kartenwerke zu beschaffen, die Daten von Bohrungen und Untersuchungen bei den geologischen und hydrologischen Landesämtern zu besorgen, die Grundwasserstockwerke sowie die Grundwasserfließrichtung und die Geschwindigkeit zu erfassen, meteorologische Daten zu sammeln, bei den Wasserwirtschaftsämtern die Entnahme- und Einleitungsgenehmigungen zu erfragen sowie die wasserwirtschaftliche Situation im näheren Umfeld zu erkunden. Für die sogenannte „multitemporale Karten- und Luftbildauswertung“, die manchmal die einzige Möglichkeit zur Verifizierung der in Schriftstücken tradierten Absichtserklärungen von Firmen ist, müssen bei den Kataster- und Vermessungsverwaltungen alle Kartenchronologen des Untersuchungsgebietes beschafft werden. Um auch die Karten und Skizzen aus den Bauarchiven verifizieren zu können, ist es zudem notwendig, alle verfügbaren Senkrecht-, Geneigt- und Ansichtsfotografien zu beschaffen, damit der jeweilige zeitgenössische Bau- und Nutzungszustand des Untersuchungsgebietes belegt werden kann. Diese werden teils in den Archiven, Planungsämtern, Kataster- und Vermessungsämtern, Landesbildstellen, Denkmalschutzämtern, teils aber auch als deponierte Archive von Luftbildfirmen bei einigen großen Landesarchiven, z.B. Hauptstaatsarchiv Düsseldorf, geführt. Ein sehr wichtiger Bestand, die alliierten Luftaufklärungsfotos, stehen über den Kampfmittelräumdienst der Länder lizenzgemäß auch den Recherchen für Altlasten offen. Die Beschaffung und Umzeichnung der Karten, Luftbildkarten und anderer Senkrechtaufnahmen ist in der Regel zeit- und kostenintensiv, so dass qualifizierte Mitarbeiter in jedem Fall eine Vorauswahl der anzuschaffenden Karten und Aufnahmen hinsichtlich Abbildungsstand, Qualität und Aussage vornehmen müssen, damit die Bearbeitung der Unterlagen innerhalb einer akzeptablen Frist möglich ist.

Auch für die Bewertung aller gesammelter Daten und Unterlagen ist ein Zeitbedarf zu kalkulieren, weil daraus einerseits die gutachterliche Stellungnahme entsteht und andererseits die Vorarbeiten für die Dokumentationen abgeleitet werden. In der Regel wird der historische Gutachter wenigstens folgende Dokumentationen erstellen, um seine Stellungnahme zu belegen:

Eine schriftliche Dokumentation der baulichen Entwicklung des Untersuchungsgebietes in chronologischer Folge der Bau-, Abbruch- und Nutzungsgenehmigungen für das gesamte Untersuchungsgebiet. Dabei ist idealerweise für 
jedes Bauwerk, jede Grube, Halde oder Freifläche eine Kennziffer zu vergeben und die Nutzungsgeschichte sowie typische Baudaten, Einbauten, Anzahl der Beschäftigten oder Leistungskennziffern von installierten Maschinen anzugeben.

Ein Teil der schriftlichen Dokumentation betrifft Fragen zur Firmengeschichte: Was hat die Firma in welcher Menge und in welchen Zeiträumen produziert, welche Verfahren, Patente, wurden wann eingeführt, welche Maschinen und wie viel Personal, Transportmittel etc. standen wann zur Verfügung, welche Mengen Roh- und Hilfsstoffe wurden wann für welche Verfahren genutzt, wie hoch war die Stoffausbeute insgesamt, bzw. zwischen den einzelnen Verfahrensstufen? Grundlagen dieser Dokumentation sind in der Regel die Betriebskonzessionen und deren Änderungen, die vor dem Hintergrund der zeitgenössischen Technik mit den Daten der Bauchronologie und den zu ermittelnden Wirtschaftsdaten des Betriebes verglichen werden. Im Idealfall gelingt es, den Materialfluß auf dem Betriebsgelände in Form einer Stoffmengenbilanz zu rekonstruieren, Umschlags- und Abfüllbereiche zu lokalisieren und die eigentlichen Produktionsgebäude nach Art und Nutzung zu identifizieren.

Schriftliche und zeichnerische Erstellung mehrerer signifikanter Zeitschnitte für die Lokalisierung von Gebäuden, Nutzungen und Umschlags- oder Ablagerungsflächen. Die Anzahl der Zeitschnitte wird sich danach richten, ob es sich nur um den kontinuierlichen Ausbau eines Betriebes mit einer bestimmten Produktion gehandelt hat, oder ob nach und nach Teilflächen mit anderen Vornutzungen hinzuerworben, bzw. durch Grundstücksteilungen wieder abgetrennt wurden, woraus jeweils neue Teiluntersuchungsflächen entstehen.

Dokumentation der ausgewerteten Kartenchronologen sowie der historischen Senkrecht- und Geneigtfotografien. Ferner eine Bilddokumentation der aktuellen Situation aus der die Art und die Ausdehnung der Bodenversiegelung, die Zugänglichkeit für die Öffentlichkeit sowie besondere Problembereiche der Fläche zu entnehmen sind.

Dokumentation aller benutzten Quellen, Behördenvorgänge, Karten und Fotografien, unabhängig davon, ob sie für die gutachterliche Aussage genutzt wurden. Eingehende Bewertung der benutzten Quellen sowie Begründung für die Verwendung bestimmter Quellengruppen.

Die gutachterliche Stellungnahme wird in der Regel weit weniger umfangreich als die Dokumentationen sein. Sie enthält in einem ersten Teilbericht die beauftragte Fragestellung für das Gutachten und dessen Beantwortung unter Berücksichtigung der möglichen Gefährdungspfade und sensibler Nutzungen im Umkreis sowie eine Empfehlung für die weitere Bearbeitung durch andere Fachgutachter. Die Begründung für die Stellungnahme folgt in einem ausführlichen Textteil, der deskriptiv die Geomorphologie, Topografie und Hydrologie der Untersuchungsfläche, die Art der historischen Nutzung und die wirtschaftliche und verfahrenstechnische Entwicklung der nutzenden Firmen darstellt. Unter Rückgriff auf die Baugeschichte und die Nutzung der Freiflächen und Umschlaganlagen wird sodann eine Darstellung der vermutlich kontaminierten Teilflächen folgen, in der für jede 
Teilfläche die belastenden Stoffe nach Art, Zeitraum der Exposition und vermutlicher Menge der Abfälle oder Handhabungsverluste aufgelistet wird. Zur Veranschaulichung dient hierbei eine maßstäbliche Zeichnung der Untersuchungsfläche, in der durch verschiedene Signaturen die belastenden Stoffe, Abfallhalden, Gruben, Entwässerungen, Kläranlagen, singuläre Schadens- oder Störfälle und die Gefährdungspfade eingezeichnet sind. Obgleich keineswegs davon ausgegangen werden kann, dass die Abfallmenge in einer simplen Gleichung der Gefährdung von Schutzgütern entspricht, kann der nachfolgende Fachgutachter, der durch Beprobung und Analyse das konkrete Ausmaß einer Gefährdung festzustellen hat, die Aussagen des historischen Gutachtens unmittelbar für die Bohr- und Analyseplanung verwenden: denn die Art der Stoffe, das Stoffverhalten, das Produktionsverfahren, der Stoffumsatz und andere Kenndaten sind hinsichtlich des Ursprungs unmittelbar auf dem Untersuchungsgelände verortet und ermöglichen bei gegebener Hydrologie, Bodenart, Bodenschichtung und Niederschlagsmenge eine Prognose über die vergangene und gegenwärtige Belastung von Wasser und Boden.

Zusammenfassend lassen sich drei Arbeitsstufen für historische Altlastgutachten und deren Ziele wie folgt darstellen:

1. Verdachtsflächenermittlung

- Standortgetreue Ermittlung aller altlastenrelevanten Nutzungen seit der Industrialisierung.

- Erstellung von Mittelpunkts- oder Umgebungskoordinaten für die größte Ausdehnung.

- Ermittlung der aktuellen Nutzer, Eigentümer, Flächengröße und Flächenbezeichnung.

- Hilfestellung bei der Bewertung der Standorte im Rahmen einer Prioritätenbildung.

- Hilfestellung für die Bau- und Stadtplanung.

2. Historischen Standortuntersuchung:

- Erarbeitung einer vollständigen Chronologie aller Nutzungen, Nutzer und Eigentümer.

- Erarbeitung einer vollständigen Chronologie aller baulichen Veränderungen.

- Flächengetreue Zuordnung aller differenzierbaren Nutzungen.

- Übertragung der Bau- und Nutzungschronik in Zeichnungen für signifikante Zeitschnitte.

- Flächengetreue Zuordnung aller relevanten Rohrleitungssysteme.

- Flächengetreue Zuordnung aller singulären Schadensfälle.

- Flächengetreue Zuordnung aller historische eingesetzten Verfahren.

- Erstellung einer Liste aller ein- und freigesetzten Stoffe und deren Menge.

- Flächengetreue Zuordnung aller ein- und freigesetzten Stoffe. 
- Dokumentation mit Quellennachweis für alle Sachaussagen.

\section{Historischen Gefährdungsabschätzung:}

- Erarbeitung einer vollständigen Chronologie aller Nutzungen, Nutzer und Eigentümer.

- Erarbeitung einer vollständigen Chronologie aller baulichen Veränderungen.

- Flächengetreue Zuordnung aller differenzierbaren Nutzungen.

- Übertragung der Bau- und Nutzungschronik in Zeichnungen für signifikante Zeitschnitte.

- Flächengetreue Zuordnung aller relevanten Rohrleitungssysteme.

- Flächengetreue Zuordnung aller singulären Schadensfälle.

- Flächengetreue Zuordnung aller historische eingesetzten Verfahren.

- Erstellung einer Liste aller ein- und freigesetzten Stoffe und deren Menge.

- Flächengetreue Zuordnung aller ein- und freigesetzten Stoffe.

- Dokumentation mit Quellennachweis für alle Sachaussagen.

- Dokumentation bisheriger Analysen, Schichtprofile, Oberflächenbeschreibung.

- Ermittlung der Grundwasserfließrichtung, Höhen, Richtung und Geschwindigkeit.

- Ermittlung der Niederschlagsmengen und der überwiegenden Windrichtungen.

- Ermittlung der Feuchtgebiete, Fließ- und Oberflächengewässer.

- Ermittlung der nächstgelegenen gefährdeten Nutzungen.

- Positionierung eines hydrologischen Dreiecks sowie der Bohrungen und Analysen.

- Vorschläge zum weiteren Vorgehen, Arbeitsschutz etc.

Das beschriebene Standardverfahren für die historische Gutachtertätigkeit steht in seiner abschließenden Sachaussage über die vergangene und gegenwärtige Belastung von Wasser und Boden in einem krassen Gegensatz zu der oben zitierten Ansicht, dass es unmöglich sei, mit historischen Methoden zu ermitteln, „welche Mengen an Abgasen, Abwässern und sonstigen Abfällen ein Unternehmen verursacht hat" ${ }^{\text {" }}{ }^{9}$ Diese Ansicht wird aber verständlich, wenn berücksichtigt wird, dass der historische Sachverständige für seine Arbeit eine umfassende Vollmacht zur Akteneinsicht benötigt, um ein annähernd zutreffendes Bild der jeweiligen Situati-

\footnotetext{
${ }_{9}$ Ralf Henneking: Chemische Industrie und Umwelt. Konflikte um Umweltbelastungen durch die chemische Industrie am Beispiel der schwerchemischen, Farben- und Düngemittelindustrie der Rheinprovinz (ca. 1800-1914). (Zeitschrift für Unternehmensgeschichte, Beiheft 86), Stuttgart 1994, S. 27.
} 
on zu erhalten. Ohne diese Vollmacht bleiben die Firmenarchive, die Archive der Bauaufsicht, der Bauplanungsämter, der Wasserbehörde, der Bergbehörde, der Umweltbehörde, Gesundheitsbehörde etc. verschlossen. Wenn der Sachverständige allein auf den Quellenbestand angewiesen wäre, der nach einer Aktenkassation, die in der Regel mindestens 95 Prozent der Vorgänge vernichtet, noch in öffentlichen Archiven nach entsprechender Wartezeit vorhanden ist, wäre eine Sachaussage und damit die Sachverständigentätigkeit in der Tat unmöglich. Der historische Sachverständige genießt daher ein Privileg gegenüber seinen wissenschaftlich tätigen Kollegen, die in der Regel niemals aus einer annähernd vergleichbaren Datenfülle werden schöpfen können. Erkauft wird es damit, dass die Gutachten nicht veröffentlicht werden können und damit auch für die Weiterentwicklung der historischen Umweltwissenschaft verloren sind.

\subsection{Methodik und Quellen der Altlastenbearbeitung}

Ein erstes Kriterium, das eine Altlastverdachtsfläche zu erfüllen hat, ist die Nutzung durch eine Branche, die, nach heutigen Kenntnissen, mit gefährdenden Stoffen umging oder solche herstellte. Für die Selektion der Branchen als erster Stufe der Bearbeitung eines flächendeckenden Katasters ist in hohem Maße die Kenntnis ehemaliger und aktueller mechanischer und chemischer Verfahrenstechnik erforderlich. Da die Ausbildung von Ingenieuren und Naturwissenschaftlern stets am neuesten Produktionsverfahren, dem Stand der Technik oder der Forschung orientiert ist, wurde und wird von Technik- oder Naturwissenschaftshistorikern kontinuierlich an der Verbesserung von Branchenlisten gearbeitet. Quellen für die Bearbeitung von Branchen sind insbesondere die Monographien zur Untersuchung einzelner branchenzugehöriger Betriebe und, nicht minder wichtig, die Synopse der jeweiligen Auflagen von Standardwerken zu den Branchen sowie den Handbüchern und Enzyklopädien der technischen Chemie, Physik, Technikgeschichte etc. Zur Kontrolle der Einschätzung dienen die Untersuchungsergebnisse bereits analytisch erkundeter Standorte.

Nur wenige der auf diese Weise vorselektierten Branchen haben dauerhaft den oder die gleichen gefährdenden Rohstoffe, Zusatz-, Zwischen- und Halbfabrikate genutzt, so dass, wie im unten stehenden Beispiel der chemischen Reinigung zu bemerken ist, Abstufungen im Grad der Gefährdung einzuführen sind. Welche Stoffe grundsätzlich und dauerhaft gefährden, welche Stoffe durch chemische oder mikrobielle Vorgänge unter günstigen Voraussetzungen in vorhersehbaren Zeitspannen abgebaut werden, wird von den beteiligten Naturwissenschaften vorgegeben, der Historiker hingegen untersucht, ab wann diese Stoffe grundsätzlich verfügbar waren, von welchem Zeitpunkt an sie unter technischen und ökonomischen Gesichtspunkten eingesetzt wurden und wann sie, im, Hinblick auf die vorgenannten Kriterien und gesetzliche Vorgaben, von anderen, substituierenden Stoffen, abgelöst wurden. Parallel hierzu wird die eingesetzte Maschinentechnik historisch untersucht, um deren Einfluss auf die Stoffe und die Stoffverluste an die Umwelt 
einschätzen zu können. Ein dritter historischer Untersuchungsbereich dieser Phase der Erarbeitung von Vorgaben erstreckt sich auf die übliche Betriebsgröße und die Arbeitskräftestruktur sowie die Sachkenntnis der Arbeitskräfte: ungelernte und angelernte Arbeitskräfte haben öfter Bedienfehler und größere Handhabungsverluste als von Fachkräften oder Inhabern geführte Betriebe.

Sobald eine „Branchenliste“ mit Gefährdungseinstufung für abgrenzbare Zeiträume vorliegt, kann eine systematische Suche nach den Flächen, auf denen diese Branchen anzutreffen waren, einsetzen. In der Vergangenheit wurden von Nichthistorikern gerne Telefonbücher und Branchenverzeichnisse genutzt, um erste Kataster der Altstandortverdachtsflächen zu erstellen. Dass diese Verzeichnisse einer Quellenkritik nicht standhielten erwies sich sehr bald: zwar waren bis zum Ende der 1980er Jahre fast alle Haushalte mit Telefonen ausgestattet, aber niemand musste, schon aus Kostengründen, seinen Betrieb, seine Zweigstellen oder Lagerplätze etc. näher bezeichnen. Auch die Branchenbücher nehmen nur jene Anbieter in das Verzeichnis auf, die gezahlt haben. Warum aber sollte ein Betrieb, der auf Laufkundschaft im Nahbereich vertraut, inserieren, warum sollte die Tankstelle an der Ausfallstraße inserieren? Nach einer fundamentalen Quellenkritik an den beiden einfachen Verzeichnissen breitete sich bald die Nutzung des Straßenverzeichnisses in amtlichen Adressbüchern aus: für jede Straße und jede Hausnummer, selbst für die Hinterhofbebauung, sind die Bewohner oder Nutzer mit Namen, Beruf, Stand und Betrieb verzeichnet.

Wie sich bereits an diesem kurzen Abriss einer historischen Darstellung der ersten Versuche, den Gegenstand der historischen Altlastenforschung durch Quellen zu ermitteln zeigt, besteht ein wesentliches Problem darin, geeignete Quellen für die Rekonstruktion der historischen Nutzungen zu finden und diese auch kritisch zu bewerten. Da es sich hierbei um ein Aufgabenfeld handelt, das die Umweltgeschichte grundsätzlich betrifft, sei im Folgenden aus Platzgründen eine tabellarische Kurzdarstellung und Bewertung der Quellen für verschieden Ziele der Altlastenforschung vorgestellt. 
Tabelle 1: Historische Standortuntersuchung - Quellenbeurteilung, Behördenakten.

\begin{tabular}{|c|c|c|c|}
\hline Amtliche Quelle & Flächige Untersuchung & Einzeluntersuchung & $\begin{array}{l}\text { Erfassungsgrad für } \\
\text { Ersterfassung }\end{array}$ \\
\hline Bundesarchive & Ungeeignet. & $\begin{array}{l}\text { In jedem Fall zu } \\
\text { benutzen bei Bun- } \\
\text { desliegenschaften, } \\
\text { Rüstungsaltlasten, } \\
\text { alten Wasser-, Bo- } \\
\text { den- und Luftanaly- } \\
\text { sen. }\end{array}$ & $\begin{array}{l}\text { Nur für bestimm- } \\
\text { te Branchen ge- } \\
\text { eignet, z.B. Rüs- } \\
\text { tungsstandorte, } \\
\text { Tankstellen, Hüt- } \\
\text { ten etc. }\end{array}$ \\
\hline $\begin{array}{l}\text { Landes-, Stadt- } \\
\text { oder Kreisar- } \\
\text { chive }\end{array}$ & $\begin{array}{l}\text { Gering geeignet, } \\
\text { weil nach Prove- } \\
\text { nienzen geordnet. }\end{array}$ & $\begin{array}{l}\text { Für jede Einzelunter- } \\
\text { suchung unerlässlich, } \\
\text { weil Altakten aus } \\
\text { jeder der folgenden } \\
\text { Behörden sowie } \\
\text { Firmen- und Privat- } \\
\text { archive verwahrt } \\
\text { werden. }\end{array}$ & Sehr hoch. \\
\hline $\begin{array}{l}\text { Bauaufsichts- } \\
\text { amt }\end{array}$ & $\begin{array}{l}\text { Mit Einschränkun- } \\
\text { gen geeignet, sofern } \\
\text { z.B. nur Sondernut- } \\
\text { zungen gesucht } \\
\text { werden. }\end{array}$ & $\begin{array}{l}\text { Unbedingt erforder- } \\
\text { lich. }\end{array}$ & $\begin{array}{l}\text { Außerordentlich } \\
\text { hoch im Rahmen } \\
\text { bisheriger Akten- } \\
\text { kassation. }\end{array}$ \\
\hline $\begin{array}{l}\text { Gewerbeauf- } \\
\text { sichtsamt }\end{array}$ & $\begin{array}{l}\text { Mit Einschränkun- } \\
\text { gen geeignet, sofern } \\
\text { klare Kriterien für } \\
\text { die Auswahl vor- } \\
\text { handen sind. }\end{array}$ & $\begin{array}{l}\text { Unbedingt erforder- } \\
\text { lich. }\end{array}$ & $\begin{array}{l}\text { Vollständige Er- } \\
\text { fassung im Rah- } \\
\text { men der bisheri- } \\
\text { gen Aktenkassati- } \\
\text { on. }\end{array}$ \\
\hline Gewerbeamt & $\begin{array}{l}\text { Im Rahmen der } \\
\text { Aktenkassation } \\
\text { bedingt geeignet. }\end{array}$ & $\begin{array}{l}\text { Notwendig wegen } \\
\text { Firmierung und Fir- } \\
\text { menzweck. }\end{array}$ & $\begin{array}{l}\text { Lückig, kurze } \\
\text { Archivierungszeit- } \\
\text { räume, daher nur } \\
\text { bedingt nutzbar. }\end{array}$ \\
\hline $\begin{array}{l}\text { Amtsgericht, } \\
\text { Handelsre- } \\
\text { gister }\end{array}$ & Schlecht geeignet. & $\begin{array}{l}\text { Firmierung, Hand- } \\
\text { lungs- und Nut- } \\
\text { zungsstörer nach- } \\
\text { weisbar. }\end{array}$ & $\begin{array}{l}\text { Bei genügendem } \\
\text { Arbeitseinsatz } \\
\text { hoch. }\end{array}$ \\
\hline
\end{tabular}




\begin{tabular}{|c|c|c|c|}
\hline Amtliche Quelle & Flächige Untersuchung & Einzeluntersuchung & $\begin{array}{l}\text { Erfassungsgrad für } \\
\text { Ersterfassung }\end{array}$ \\
\hline $\begin{array}{l}\text { Katasteramt, } \\
\text { Grundbuch- } \\
\text { amt }\end{array}$ & $\begin{array}{l}\text { Notwendiger Be- } \\
\text { standteil der Arbeit, } \\
\text { um historische und } \\
\text { aktuelle Fläche, } \\
\text { Flächenbezeich- } \\
\text { nung und Eigentü- } \\
\text { mer zu ermitteln. }\end{array}$ & $\begin{array}{l}\text { Aus gleichen Grün- } \\
\text { den unabdingbar. }\end{array}$ & Hier irrelevant. \\
\hline $\begin{array}{l}\text { Stadtentwäs- } \\
\text { serung, Müllab- } \\
\text { fuhr }\end{array}$ & $\begin{array}{l}\text { Im Archiv häufig } \\
\text { alle Entwässerungs- } \\
\text { akten und Sonder- } \\
\text { nutzungen bzgl. } \\
\text { Wasserschädigung. }\end{array}$ & $\begin{array}{l}\text { Weist häufig die } \\
\text { Abfallstoffe und } \\
\text { damit die Problem- } \\
\text { bereiche einer Fir- } \\
\text { ma aus. }\end{array}$ & $\begin{array}{l}\text { Für die Zeit ab } \\
1972 \text { nahezu voll- } \\
\text { ständig. }\end{array}$ \\
\hline $\begin{array}{l}\text { Wasserwirt- } \\
\text { schaftsamt }\end{array}$ & $\begin{array}{l}\text { Weist alle noch } \\
\text { existierenden Ge- } \\
\text { nehmigungen zur } \\
\text { Entnahme von } \\
\text { Wasser und zur } \\
\text { Abwassereinleitung } \\
\text { nach. }\end{array}$ & Unabdingbar. & $\begin{array}{l}\text { Für Betriebe mit } \\
\text { wasserrechtlichen } \\
\text { Genehmigungen } \\
\text { nahe } 100 \% .\end{array}$ \\
\hline Bergamt & Ungeeignet. & $\begin{array}{l}\text { Bei Verdacht auf } \\
\text { bergrechtliche Tä- } \\
\text { tigkeiten (Altablage- } \\
\text { rungen) unabding- } \\
\text { bar. }\end{array}$ & Irrelevant. \\
\hline $\begin{array}{l}\text { Geologisches } \\
\text { Landesamt }\end{array}$ & Ungeeignet. & $\begin{array}{l}\text { Für jede Gefähr- } \\
\text { dungsabschätzung } \\
\text { notwendig. }\end{array}$ & Irrelevant. \\
\hline Umweltamt & $\begin{array}{l}\text { Nur für die jüngste } \\
\text { Vergangenheit. }\end{array}$ & $\begin{array}{l}\text { Bedingt geeignet, } \\
\text { gelegentlich gute } \\
\text { Detailkenntnisse } \\
\text { und Analysener- } \\
\text { gebnisse. }\end{array}$ & $\begin{array}{l}\text { Gering bis irrele- } \\
\text { vant. }\end{array}$ \\
\hline $\begin{array}{l}\text { Gesundheits- } \\
\text { amt }\end{array}$ & Ungeeignet. & $\begin{array}{l}\text { Sollte auf besondere } \\
\text { Vorkommnisse hin } \\
\text { aufgenommen wer- } \\
\text { den. }\end{array}$ & Ungeeignet. \\
\hline $\begin{array}{l}\text { Handwerks- } \\
\text { kammer }\end{array}$ & $\begin{array}{l}\text { Geeignet für die } \\
\text { letzten Jahrzehnte. }\end{array}$ & $\begin{array}{l}\text { Notwendiger Be- } \\
\text { standteil, weil hier } \\
\text { bestimmte Tätigkei- } \\
\text { ten genehmigt wer- } \\
\text { den. }\end{array}$ & $\begin{array}{l}\text { Für den Bereich } \\
\text { Handwerk und für } \\
\text { die jüngste Ver- } \\
\text { gangenheit bis zu } \\
95 \% \text {. }\end{array}$ \\
\hline
\end{tabular}




\begin{tabular}{|c|c|c|c|}
\hline Amtliche Quelle & Flächige Untersuchung & Einzeluntersuchung & $\begin{array}{l}\text { Erfassungsgrad für } \\
\text { Ersterfassung }\end{array}$ \\
\hline $\begin{array}{l}\text { Handels- } \\
\text { kammer }\end{array}$ & $\begin{array}{l}\text { Geeignet für die } \\
\text { letzten Jahrzehnte. }\end{array}$ & $\begin{array}{l}\text { Notwendige Re- } \\
\text { cherche, da das } \\
\text { Archiv noch weit } \\
\text { mehr als eine Stan- } \\
\text { dardauskunft bieten } \\
\text { kann. }\end{array}$ & $\begin{array}{l}\text { Notwendig und für } \\
\text { die jüngste Ver- } \\
\text { gangenheit mit } \\
\text { einer Treffsicher- } \\
\text { heit von nahezu } 80 \\
\text { - } 90 \% \text {. }\end{array}$ \\
\hline Feuerwehr & Ungeeignet. & $\begin{array}{l}\text { Für die Kontrolle } \\
\text { von Schadensfällen, } \\
\text { aber auch für Ge- } \\
\text { nehmigungen den } \\
\text { Akten des Gewer- } \\
\text { beaufsichtsamtes } \\
\text { vergleichbar. }\end{array}$ & Ungeeignet. \\
\hline $\begin{array}{l}\text { Meteorologi- } \\
\text { sches Lan- } \\
\text { desamt } \\
\end{array}$ & Ungeeignet. & $\begin{array}{l}\text { Für Gefährdungs- } \\
\text { abschätzungen not- } \\
\text { wendig. }\end{array}$ & Ungeeignet. \\
\hline $\begin{array}{l}\text { Liegenschafts,- } \\
\text { Wirtschaftsamt } \\
\text { und andere }\end{array}$ & $\begin{array}{l}\text { Ungeeignet, sofern } \\
\text { es sich nicht um } \\
\text { Sonderbehörden, } \\
\text { z.B. Hafenämter } \\
\text { etc. handelt. }\end{array}$ & $\begin{array}{l}\text { Bedingt geeignet, } \\
\text { sofern andere Quel- } \\
\text { len durch Aktenver- } \\
\text { luste versagen. }\end{array}$ & Gering. \\
\hline
\end{tabular}


Tabelle 2: Historische Standortuntersuchung - Quellenbeurteilung, Gedruckte Quellen.

\begin{tabular}{|c|c|c|c|c|}
\hline $\begin{array}{l}\text { Gedruckete } \\
\text { Quelle }\end{array}$ & $\begin{array}{l}\text { Flächige Un- } \\
\text { tersuchung }\end{array}$ & $\begin{array}{l}\text { Einzeluntersu- } \\
\text { chung }\end{array}$ & Vorteil & Nachteil \\
\hline $\begin{array}{l}\text { Branchen- } \\
\text { verzeichnisse } \\
\text { von Städten } \\
\text { oder Kreisen }\end{array}$ & $\begin{array}{l}\text { Geeignet, } \\
\text { sofern Stra- } \\
\text { Benver- } \\
\text { zeichnisse } \\
\text { vorhanden. }\end{array}$ & $\begin{array}{l}\text { Geeignet und } \\
\text { für einen } \\
\text { schnellen Über- } \\
\text { blick zur Nut- } \\
\text { zerchronologie } \\
\text { empfohlen. }\end{array}$ & $\begin{array}{l}\text { Große zeitliche } \\
\text { Reichweite; } \\
\text { Schnell durch- } \\
\text { führbar, auch } \\
\text { mit unqualifi- } \\
\text { ziertem Perso- } \\
\text { nal. }\end{array}$ & $\begin{array}{l}\text { Schlechte Tref- } \\
\text { ferquote infolge } \\
\text { vieler Kontor- } \\
\text { adressen und } \\
\text { fehlender Inse- } \\
\text { rate. }\end{array}$ \\
\hline $\begin{array}{l}\text { Einwohner- } \\
\text { verzeichnisse } \\
\text { von Städten } \\
\text { und Kreisen }\end{array}$ & $\begin{array}{l}\text { Gut geeig- } \\
\text { net, sofern } \\
\text { ein Sy- } \\
\text { nonymver- } \\
\text { zeichnis der } \\
\text { Straßen } \\
\text { vorhanden } \\
\text { ist. }\end{array}$ & $\begin{array}{l}\text { Unbedingt für } \\
\text { eine vollständi- } \\
\text { ge Nutzerchro- } \\
\text { nologie zu be- } \\
\text { arbeiten. }\end{array}$ & $\begin{array}{l}\text { Hohe Treffsi- } \\
\text { cherheit auch } \\
\text { für kleine, ein- } \\
\text { gemietete Be- } \\
\text { triebe. }\end{array}$ & $\begin{array}{l}\text { Hohe Kosten } \\
\text { und nur mit } \\
\text { geschultem } \\
\text { Personal durch- } \\
\text { führbar. }\end{array}$ \\
\hline $\begin{array}{l}\text { Fachbran- } \\
\text { chenbücher }\end{array}$ & $\begin{array}{l}\text { Notwendig } \\
\text { für ländliche } \\
\text { Gebiete } \\
\text { ohne eigene } \\
\text { Einwohner- } \\
\text { und Bran- } \\
\text { chenver- } \\
\text { zeichnisse. }\end{array}$ & $\begin{array}{l}\text { Notwendig für } \\
\text { alle Gebiete } \\
\text { ohne lokale } \\
\text { Verzeichnis- } \\
\text { druckwerke. }\end{array}$ & $\begin{array}{l}\text { Bedingter Nut- } \\
\text { zen unter den } \\
\text { genannten Ein- } \\
\text { schränkungen. }\end{array}$ & $\begin{array}{l}\text { Geringe Treff- } \\
\text { sicherheit, häu- } \\
\text { figer falsche } \\
\text { Adressangaben } \\
\text { oder Firmierun- } \\
\text { gen. }\end{array}$ \\
\hline $\begin{array}{l}\text { Wirtschafts- } \\
\text { auskunfts- } \\
\text { fachbücher } \\
\text { („Hop- } \\
\text { penstedt“" } \\
\text { oder „Kos- } \\
\text { mos" }^{\text {") }} \text {. }\end{array}$ & $\begin{array}{l}\text { Bedingt } \\
\text { geeignet, } \\
\text { nachgewie- } \\
\text { sen werden } \\
\text { nur Mittel- } \\
\text { und Groß- } \\
\text { betriebe. }\end{array}$ & $\begin{array}{l}\text { Für die Firmen- } \\
\text { struktur, Be- } \\
\text { schäftigte, An- } \\
\text { teilseigner und } \\
\text { Kapitalvertei- } \\
\text { lung unabding- } \\
\text { bar. }\end{array}$ & $\begin{array}{l}\text { Schneller in- } \\
\text { formativer } \\
\text { Überblick über } \\
\text { Geschichte, } \\
\text { Produkte, Pa- } \\
\text { tente und Ver- } \\
\text { hältnisse der } \\
\text { Firma. }\end{array}$ & $\begin{array}{l}\text { Kaum vorhan- } \\
\text { den. }\end{array}$ \\
\hline $\begin{array}{l}\text { Patent- und } \\
\text { Warenzei- } \\
\text { chenliteratur }\end{array}$ & Ungeeignet. & $\begin{array}{l}\text { Sofern eine } \\
\text { Rekonstruktion } \\
\text { der Verfahrens- } \\
\text { schritte gefor- } \\
\text { dert ist. }\end{array}$ & $\begin{array}{l}\text { Leichtere und } \\
\text { vollständigere } \\
\text { Ausführung der } \\
\text { Stoffliste. }\end{array}$ & $\begin{array}{l}\text { Nur in Mün- } \\
\text { chen und Ham- } \\
\text { burg gut zu- } \\
\text { gänglich. }\end{array}$ \\
\hline
\end{tabular}




\begin{tabular}{|c|c|c|c|c|}
\hline $\begin{array}{l}\text { Gedruckte } \\
\text { Quelle }\end{array}$ & $\begin{array}{l}\text { Flächige Un- } \\
\text { tersuchung }\end{array}$ & $\begin{array}{l}\text { Einzeluntersu- } \\
\text { chung }\end{array}$ & Vorteil & Nachteil \\
\hline $\begin{array}{l}\text { Ortschroni- } \\
\text { ken und } \\
\text { lokalhistori- } \\
\text { sche Litera- } \\
\text { tur }\end{array}$ & $\begin{array}{l}\text { Nur für eine } \\
\text { erste Orien- } \\
\text { tierung ge- } \\
\text { eignet. }\end{array}$ & $\begin{array}{l}\text { Manchmal mit } \\
\text { wertvollen Hin- } \\
\text { weisen verse- } \\
\text { hen. }\end{array}$ & $\begin{array}{l}\text { Schneller Über- } \\
\text { blick, gelegent- } \\
\text { lich Hinweise. }\end{array}$ & $\begin{array}{l}\text { Große Menge } \\
\text { irrelevanter, oft } \\
\text { nicht belegter } \\
\text { Angaben. }\end{array}$ \\
\hline $\begin{array}{l}\text { Firmenhisto- } \\
\text { rische Litera- } \\
\text { tur }\end{array}$ & Ungeeignet. & $\begin{array}{l}\text { Für Einzelun- } \\
\text { tersuchungen } \\
\text { unabdingbar. }\end{array}$ & $\begin{array}{l}\text { Häufig mit } \\
\text { Bilanzen, Um- } \\
\text { satzziffern und } \\
\text { Bildern ausges- } \\
\text { tattet }\end{array}$ & $\begin{array}{l}\text { Quellenkritisch } \\
\text { kaum zu über- } \\
\text { prüfen. Liegt } \\
\text { meist in ver- } \\
\text { streuten Ex- } \\
\text { emplaren vor. }\end{array}$ \\
\hline $\begin{array}{l}\text { Technikhis- } \\
\text { torische } \\
\text { Literatur }\end{array}$ & Ungeeignet. & $\begin{array}{l}\text { Manchmal mit } \\
\text { detaillierten } \\
\text { Hinweisen und } \\
\text { weiterführender } \\
\text { Literatur verse- } \\
\text { hen. }\end{array}$ & $\begin{array}{l}\text { Schneller Über- } \\
\text { blick über die } \\
\text { Entwicklung } \\
\text { einer Branche. }\end{array}$ & $\begin{array}{l}\text { Schlecht er- } \\
\text { reichbar, lang- } \\
\text { wieriges Suchen } \\
\text { im Text erfor- } \\
\text { derlich. }\end{array}$ \\
\hline $\begin{array}{l}\text { Verfahrens- } \\
\text { historische } \\
\text { Literatur }\end{array}$ & Ungeeignet. & $\begin{array}{l}\text { Für die Erstel- } \\
\text { lung einer Stoff- } \\
\text { liste und für das } \\
\text { Verständnis } \\
\text { zum histori- } \\
\text { schen Betriebs- } \\
\text { ablauf ein un- } \\
\text { bedingtes Muss. }\end{array}$ & $\begin{array}{l}\text { Geeignete Lite- } \\
\text { ratur, z. B. alte } \\
\text { Ausgaben von } \\
\text { 'Ullmann', bie- } \\
\text { tet einen } \\
\text { schnellen Über- } \\
\text { blick, einen } \\
\text { historischen } \\
\text { Abriss und } \\
\text { weiterführende } \\
\text { Literatur. }\end{array}$ & $\begin{array}{l}\text { In alten Ausga- } \\
\text { ben nur schwer } \\
\text { zugänglich. }\end{array}$ \\
\hline
\end{tabular}


Tabelle 3: Historische Standortuntersuchung - Quellenbeurteilung - Multitemporale Karten- und Bildauswertung, Zeitzeugenbefragung

\begin{tabular}{|c|c|c|c|c|}
\hline Quelle & $\begin{array}{l}\text { Flächige Un- } \\
\text { tersuchung }\end{array}$ & $\begin{array}{l}\text { Einzeluntersu- } \\
\text { chung }\end{array}$ & Vorteil & Nachteil \\
\hline $\begin{array}{l}\text { Kampfmit- } \\
\text { telräum- } \\
\text { dienst }\end{array}$ & $\begin{array}{l}\text { Nur bedingt } \\
\text { geeignet. }\end{array}$ & $\begin{array}{l}\text { Große Zahl } \\
\text { unveröffentlich- } \\
\text { ter Aufklärungs- } \\
\text { aufnahmen, die } \\
\text { gemäß Lizenz- } \\
\text { vertrag auch für } \\
\text { die Umweltun- } \\
\text { tersuchung } \\
\text { nutzbar sind. }\end{array}$ & $\begin{array}{l}\text { Historische } \\
\text { Gebäudesituati- } \\
\text { on und Flä- } \\
\text { chennutzung ist } \\
\text { gut sichtbar. }\end{array}$ & $\begin{array}{l}\text { Differierende } \\
\text { Maßstäbe, } \\
\text { schwer zugäng- } \\
\text { lich. }\end{array}$ \\
\hline \begin{tabular}{|l} 
Alliierte \\
Luftbildar- \\
chive
\end{tabular} & $\begin{array}{l}\text { Nur bedingt } \\
\text { geeignet. }\end{array}$ & $\begin{array}{l}\text { Verfügt über } \\
\text { alle alliierten } \\
\text { Aufklärungsfo- } \\
\text { tos. }\end{array}$ & \begin{tabular}{|l} 
Gute Rekon- \\
struktion von \\
Gebäudezerstö- \\
rungen und \\
Nutzungen \\
möglich.
\end{tabular} & $\begin{array}{l}\text { In Montgome- } \\
\text { ry/Alabama } \\
\text { und in der } \\
\text { University of } \\
\text { Keele schwer } \\
\text { zugänglich: } \\
\text { Kosten- und } \\
\text { Zeitintensiv. }\end{array}$ \\
\hline $\begin{array}{l}\text { Hansa- } \\
\text { Bildarchiv, } \\
\text { Deutsche } \\
\text { Luftbild und } \\
\text { andere }\end{array}$ & $\begin{array}{l}\text { Manchmal } \\
\text { geeignet. }\end{array}$ & $\begin{array}{l}\text { Für jede Einzel- } \\
\text { untersuchung } \\
\text { notwendig, falls } \\
\text { die Fotos nicht } \\
\text { anderweitig } \\
\text { beschafft wer- } \\
\text { den. }\end{array}$ & $\begin{array}{l}\text { Gute Mischung } \\
\text { von Senkrecht- } \\
\text { und Geneigtau- } \\
\text { ahmen in vielen } \\
\text { Zeitschnitten. }\end{array}$ & $\begin{array}{l}\text { Wenn nicht in } \\
\text { Katasterämtern } \\
\text { oder lokalen } \\
\text { Archiven vor- } \\
\text { handen, zu- } \\
\text { meist kostenin- } \\
\text { tensiv aus Düs- } \\
\text { seldorf zu be- } \\
\text { schaffen. }\end{array}$ \\
\hline $\begin{array}{l}\text { Denkmal- } \\
\text { schutzamt }\end{array}$ & Ungeeignet & $\begin{array}{l}\text { Bei älteren } \\
\text { Standorten und } \\
\text { sonst fehlendem } \\
\text { Bildmaterial } \\
\text { eine notwendige } \\
\text { Adresse. }\end{array}$ & $\begin{array}{l}\text { Kaum vorhan- } \\
\text { den. }\end{array}$ & $\begin{array}{l}\text { Künstlerisch } \\
\text { wertvolle Bil- } \\
\text { der, kostenin- } \\
\text { tensiv. }\end{array}$ \\
\hline $\begin{array}{l}\text { Landesbild- } \\
\text { stelle }\end{array}$ & Ungeeignet. & $\begin{array}{l}\text { Bei sonst feh- } \\
\text { lendem Bildma- } \\
\text { terial eine not- } \\
\text { wendige Re- } \\
\text { cherche. }\end{array}$ & $\begin{array}{l}\text { Umfangreiche } \\
\text { Bilddokumenta- } \\
\text { tion, gut geglie- } \\
\text { dert. }\end{array}$ & $\begin{array}{l}\text { Vielfach Auf- } \\
\text { nahmen „wert- } \\
\text { voller“ Bauten. }\end{array}$ \\
\hline
\end{tabular}




\begin{tabular}{|c|c|c|c|c|}
\hline Quelle & $\begin{array}{l}\text { Flächige Un- } \\
\text { tersuchung }\end{array}$ & $\begin{array}{l}\text { Einzeluntersu- } \\
\text { chung }\end{array}$ & Vorteil & Nachteil \\
\hline $\begin{array}{l}\text { Landes- } \\
\text { /Stadtarchiv }\end{array}$ & Ungeeignet. & $\begin{array}{l}\text { Notwendige } \\
\text { Recherche nach } \\
\text { Bildmaterial und } \\
\text { alten Flurkarten } \\
\text { sogenannte } \\
\text { „Inselkarten“ } \\
\text { oder „Mutati- } \\
\text { onskarten“. }\end{array}$ & $\begin{array}{l}\text { Sammlung von } \\
\text { Profi- und Ama- } \\
\text { teuraufnahmen, } \\
\text { daher häufig } \\
\text { auch Industrie- } \\
\text { gebäude vor- } \\
\text { handen. }\end{array}$ & $\begin{array}{l}\text { Kaum vorhan- } \\
\text { den, zeit- und } \\
\text { kostenintensiv. }\end{array}$ \\
\hline $\begin{array}{l}\text { Firmenan- } \\
\text { sichten auf } \\
\text { Briefköpfen }\end{array}$ & Ungeeignet. & $\begin{array}{l}\text { Manchmal für } \\
\text { die Einzelunter- } \\
\text { suchung gut } \\
\text { geeignet. }\end{array}$ & $\begin{array}{l}\text { Bestandteil des } \\
\text { Firmenschrift- } \\
\text { wechsels, daher } \\
\text { leicht beschaff- } \\
\text { bar. }\end{array}$ & $\begin{array}{l}\text { Nur nach ein- } \\
\text { gehender Quel- } \\
\text { lenkritik zu } \\
\text { nutzen. }\end{array}$ \\
\hline $\begin{array}{l}\text { Ansichtskar- } \\
\text { ten, Ama- } \\
\text { teuraufnah- } \\
\text { men }\end{array}$ & Ungeeignet. & $\begin{array}{l}\text { Als Quellenkri- } \\
\text { tik sehr gut zu } \\
\text { verwenden, } \\
\text { bringt häufig } \\
\text { Hinweise auf } \\
\text { Sondernutzun- } \\
\text { gen von Flä- } \\
\text { chen. }\end{array}$ & $\begin{array}{l}\text { Zum Teil in den } \\
\text { Bauakten bereits } \\
\text { enthalten (hier } \\
\text { besonders in } \\
\text { den Generalak- } \\
\text { ten). }\end{array}$ & $\begin{array}{l}\text { Sofern unver- } \\
\text { öffentlicht } \\
\text { oder in An- } \\
\text { tragsakten } \\
\text { enthalten, nur } \\
\text { schwer zu } \\
\text { beschaffen und } \\
\text { zu datieren. }\end{array}$ \\
\hline Bauarchiv & $\begin{array}{l}\text { Ungeeignet, } \\
\text { es sei denn, } \\
\text { es wird ein } \\
\text { Viertel oder } \\
\text { ein Straßen- } \\
\text { zug bearbei- } \\
\text { tet. }\end{array}$ & $\begin{array}{l}\text { Einzeluntersu- } \\
\text { chungen müs- } \\
\text { sen jede aussa- } \\
\text { gefähige bemaß- } \\
\text { te Karte oder } \\
\text { Zeichnung mit } \\
\text { der Gebäude- } \\
\text { und Nutzungssi- } \\
\text { tuation der Un- } \\
\text { tersuchungsflä- } \\
\text { che erfassen. }\end{array}$ & $\begin{array}{l}\text { Die chronologi- } \\
\text { sche Erfassung } \\
\text { der Bauvorgän- } \\
\text { ge und der Kar- } \\
\text { ten veranschau- } \\
\text { licht die Abläufe } \\
\text { in der Firma. }\end{array}$ & $\begin{array}{l}\text { Zeit- und Kos- } \\
\text { tenintensiv. }\end{array}$ \\
\hline $\begin{array}{l}\text { Topographi- } \\
\text { sche Kar- } \\
\text { tenwerke: } \\
(1: 5000 / 250 \\
00), \text { Luft- } \\
\text { meßflüge } \\
(1: 2500) \text { und } \\
\text { Luftbildat- } \\
\text { lanten }\end{array}$ & $\begin{array}{l}\text { Gut geeignet } \\
\text { für Zeiträu- } \\
\text { me ab ca. } \\
\text { 1880, die } \\
\text { DGK erst } \\
\text { ab ca. } 1925\end{array}$ & $\begin{array}{l}\text { Unabdingbar } \\
\text { auch in der } \\
\text { Einzelflächen- } \\
\text { nalyse, Gefähr- } \\
\text { dungsabschät- } \\
\text { zung sowie für } \\
\text { Abtragungen } \\
\text { und Aufhöhun- } \\
\text { gen. }\end{array}$ & $\begin{array}{l}\text { Schneller Über- } \\
\text { blick über Lage, } \\
\text { bauliche Aus- } \\
\text { dehnung in } \\
\text { entsprechenden } \\
\text { Zeitschnitten. }\end{array}$ & $\begin{array}{l}\text { Häufige Ver- } \\
\text { änderung der } \\
\text { Signaturen, } \\
\text { quellenkritisch } \\
\text { zu betrachten } \\
\text { sind besonders } \\
\text { die Jahrgänge } \\
1934 \text { bis } 1945 .\end{array}$ \\
\hline
\end{tabular}




\begin{tabular}{|c|c|c|c|c|}
\hline Quelle & $\begin{array}{l}\text { Flächige Un- } \\
\text { tersuchung }\end{array}$ & $\begin{array}{l}\text { Einzeluntersu- } \\
\text { chung }\end{array}$ & Vorteil & Nachteil \\
\hline $\begin{array}{l}\text { Hydrogeolo- } \\
\text { gische Kar- } \\
\text { tenwerke }\end{array}$ & $\begin{array}{l}\text { Nicht geeig- } \\
\text { net. }\end{array}$ & $\begin{array}{l}\text { Unabdingbar } \\
\text { für eine Ge- } \\
\text { fährdungsab- } \\
\text { schätzung. }\end{array}$ & $\begin{array}{l}\text { Notwendiges } \\
\text { Kartenwerk. }\end{array}$ & $\begin{array}{l}\text { Notwendiges } \\
\text { Kartenwerk. }\end{array}$ \\
\hline Oral History & $\begin{array}{l}\text { Geeignet nur } \\
\text { bei einer } \\
\text { genügenden } \\
\text { Zahl von } \\
\text { Probanden. }\end{array}$ & $\begin{array}{l}\text { Bei intensiver } \\
\text { Untersuchung } \\
\text { sollte die Befra- } \\
\text { gung am Ende } \\
\text { des Aktenstudi- } \\
\text { ums durchge- } \\
\text { führt werden. }\end{array}$ & $\begin{array}{l}\text { Manchmal sehr } \\
\text { konkrete Hin- } \\
\text { weise auf Nut- } \\
\text { zungen, die in } \\
\text { keiner Akte } \\
\text { verzeichnet } \\
\text { sind. }\end{array}$ & $\begin{array}{l}\text { Schlechtes } \\
\text { Erinnerungs- } \\
\text { vermögen der } \\
\text { Befragten. Sehr } \\
\text { zeitintensiv in } \\
\text { der Befragung } \\
\text { und in der } \\
\text { Ausmerzung } \\
\text { widersprüchli- } \\
\text { cher Aussagen } \\
\text { schon bei der } \\
\text { Befragung } \\
\text { einer Person. }\end{array}$ \\
\hline
\end{tabular}


Tabelle 4: Rangfolge der Quellen für definierte Untersuchungsziele

\begin{tabular}{|c|c|c|c|}
\hline Quellengattung & $\begin{array}{l}\text { Erstverdachtsflä- } \\
\text { chenermittlung }\end{array}$ & $\begin{array}{l}\text { Vertiefte historische Stand- } \\
\text { ortuntersuchung }\end{array}$ & $\begin{array}{l}\text { Historische Erstgefähr- } \\
\text { dungsabschätzung }\end{array}$ \\
\hline $\begin{array}{l}\text { Altakten in } \\
\text { Archiven }\end{array}$ & & $\begin{array}{l}\text { Kommunalarchiv, } \\
\text { Landesarchiv, Bundes- } \\
\text { archive, Firmenarchive, } \\
\text { Kammerarchive. }\end{array}$ & $\begin{array}{l}\text { Wie vertiefte Stand- } \\
\text { ortuntersuchung. }\end{array}$ \\
\hline $\begin{array}{l}\text { Laufende Be- } \\
\text { hördenakten }\end{array}$ & $\begin{array}{l}\text { Umweltamt, } \\
\text { Gewerbeauf- } \\
\text { sichtsamt, } \\
\text { Gewerbeamt. }\end{array}$ & $\begin{array}{l}\text { Zusätzlich zur Erstver- } \\
\text { dachtsflächenermitt- } \\
\text { lung: } \\
\text { Bauaufsichtsamt, Ka- } \\
\text { taster- und Grund- } \\
\text { buchamt, Stadtentwäs- } \\
\text { serung, Müllabfuhr, } \\
\text { Bergamt, Wasserwirt- } \\
\text { schaftsamt, Gesund- } \\
\text { heitsamt, Handelsregis- } \\
\text { ter, Feuerwehr, Liegen- } \\
\text { schaftsamt, Wirt- } \\
\text { schaftsamt, Landes- } \\
\text { bauämter. }\end{array}$ & $\begin{array}{l}\text { Zusätzlich zur ver- } \\
\text { tieften historischen } \\
\text { Standortuntersu- } \\
\text { chung: } \\
\text { Geologisches Lan- } \\
\text { desamt, Meteorolo- } \\
\text { gisches Landesamt. }\end{array}$ \\
\hline $\begin{array}{l}\text { Druckwerke } \\
\text { und Literatur }\end{array}$ & $\begin{array}{l}\text { Einwohnerver- } \\
\text { zeichnisse, Bran- } \\
\text { chenverzeichnis- } \\
\text { se, Branchenbü- } \\
\text { cher. }\end{array}$ & $\begin{array}{l}\text { Zusätzlich zur Erstver- } \\
\text { dachtsflächenermitt- } \\
\text { lung: } \\
\text { Lokalhistorie, Firmen- } \\
\text { schriften, Verfahrens- } \\
\text { technik. }\end{array}$ & $\begin{array}{l}\text { Zusätzlich zur ver- } \\
\text { tieften historischen } \\
\text { Standortuntersu- } \\
\text { chung: } \\
\text { Patent- und Waren- } \\
\text { zeichenliteratur, } \\
\text { Wirtschaftsfachaus- } \\
\text { kunft, Bilanzen. }\end{array}$ \\
\hline Kartenmaterial & $\begin{array}{l}\text { Katasteramt, } \\
\text { Landesverwal- } \\
\text { tungsamt, Geo- } \\
\text { logisches Lan- } \\
\text { desamt, Archive: } \\
\text { TK } 25(1: 25000) \\
\text { DKG } 5(1: 5000) \\
\text { Luftbildkarte } \\
\text { 1:2500 }\end{array}$ & $\begin{array}{l}\text { Zusätzlich zur Erstver- } \\
\text { dachtsflächenermitt- } \\
\text { lung: Mutations- oder } \\
\text { Inselkarten, Flurkarte } \\
\text { 1:1000 oder Flurkarte } \\
\text { 1:500. }\end{array}$ & $\begin{array}{l}\text { Zusätzlich zur ver- } \\
\text { tieften historischen } \\
\text { Standortuntersu- } \\
\text { chung: } \\
\text { Hydrologisches } \\
\text { Landesamt, Meteo- } \\
\text { rologisches Lan- } \\
\text { desamt, Baugrund- } \\
\text { karten. }\end{array}$ \\
\hline
\end{tabular}




\begin{tabular}{|c|c|c|c|}
\hline Quellengattung & $\begin{array}{l}\text { Erstverdachtsflä- } \\
\text { chenermittlung }\end{array}$ & $\begin{array}{l}\text { Vertiefte historische Stand- } \\
\text { ortuntersuchung }\end{array}$ & $\begin{array}{l}\text { Historische Erstgefähr- } \\
\text { dungsabschätzung }\end{array}$ \\
\hline Fotos & & $\begin{array}{l}\text { Hauptstaatsarchive, } \\
\text { Kampfmittelräum- } \\
\text { dienst, USAF-Base } \\
\text { Montgomery, Univ. of } \\
\text { Keele, Kommunalar- } \\
\text { chiv, Landesarchiv, } \\
\text { Firmenarchiv, Private } \\
\text { Befliegungen, Luft- } \\
\text { meßblätter, Schrägauf- } \\
\text { nahmen, Postkarten, } \\
\text { Ansichtsfotos. }\end{array}$ & $\begin{array}{l}\text { Wie Standortunter- } \\
\text { suchung. }\end{array}$ \\
\hline Zeitzeugen & & $\begin{array}{l}\text { Betriebsangehörige, } \\
\text { Anwohner, Behör- } \\
\text { denmitarbeiter; Adres- } \\
\text { senbeschaffung über } \\
\text { Meldeamt oder den } \\
\text { Vergleich alter mit } \\
\text { neuen Straßenver- } \\
\text { zeichnissen. }\end{array}$ & $\begin{array}{l}\text { Wie Standortunter- } \\
\text { suchung. }\end{array}$ \\
\hline
\end{tabular}




\section{Die Umweltrelevanz der chemischen Reinigung ${ }^{10}$}

Chemische Reinigungen zählen nicht zu den üblichen Untersuchungsgegenständen der Umweltgeschichte. Wäschereien hingegen sind gelegentlich Gegenstand der Sozial- oder Gendergeschichte geworden. Es handelt sich weder um eine große Industriebranche, noch um ein scheinbar umweltrelevantes Thema. Am 9. September 2008 lud die Hamburger Umweltbehörde zu einem Workshop zum Thema Chemische Reinigung, bei dem sich, für die anwesenden Vertreter von Umweltbehörden und Umweltbüros nicht überraschend, herausstellte, dass diese Dienstleistung den urbanen Bereich vermutlich am Schlimmsten mit nachhaltig gefährdenden Stoffen verunreinigte. Es gab eine große Zahl von chemischen Reinigungen, die sich auf städtische Einkaufs- und Wohngebiete verteilte. Die Nutzung chlorierter Kohlenwasserstoffe (CKW) für die Reinigung wurde ohne Bedenken der Behörden genehmigt und die Entsorgung der verbrauchten Lösungen, Filterrückstände und des Kontaktwassers geschah über Jahrzehnte ohne Reglementierung, so dass die CKW zunächst die Dichtungen der Abwasserleitungen auflösten, dann in den Boden und später in das Grundwasser gelangten. Aufgrund des spezifischen Gewichts, das größer als das von Wasser ist, unterlagern diese Halogenverbindungen die einzelnen Grundwasserschichten und können dort mit dem Grundwasser zu den Trinkwasserfassungen wandern. Ein Abbau in Richtung Vinylchlorid findet ohne den Einfluss von Sauerstoff nicht statt, so dass die Schmutzfahnen der Reinigungsbetriebe alle größeren Ortschaften mit zentralen Funktionen unterlagern. Die folgende Übersicht über die Entwicklungsgeschichte dieser Branche ist ein Beispiel für die Aufgabe des Historikers, die Gefährdungslage einer umweltrelevanten Branche anhand des Stoffeinsatzes, der durch die Entwicklung der jeweiligen Stoffe und deren Kosten determiniert ist sowie anhand des Einsatzes von Maschinen zeitlich differenziert zu betrachten und Kategorien der Gefährdung abzuleiten.

Mit Hilfe der chemischen Reinigung werden Fette, Öle, Ruß, Schmierstoffen oder anderen Verschmutzungen aus Textilien und Leder entfernt. Die herkömmliche Wäscherei nutzt Wasser und Seife als Lösungsmittel, die chemische Reinigung nutzt ein leicht flüchtiges chemisches Lösungsmittel mit hohem Lösungsvermögen für Fette und Öle. Eine zeitgenössische Bezeichnung, „Trockenreinigung“, brachte zum Ausdruck, dass fast kein Wasser für die Reinigung benutzt wurde. Wasserlösliche Verunreinigungen wie Schweiß, Urin, Rotwein etc. wurden vor oder nach der Hauptreinigung durch eine Detachur beseitigt, während in der Neuzeit geringe Mengen wasserlöslicher waschaktiver Reinigungsmittel im Vollautomaten zugesetzt werden. Die chemische Reinigung als eigenständiger Dienstleistungsbetrieb

\footnotetext{
${ }^{10}$ Der Abschnitt über die chemischen Reinigungen ist die Kurzfassung eines vom Autor verfassten Branchenblattes „Chemische Reinigungen“ im Altlastenleitfaden Schleswig-Holstein, Band II, herausgegeben vom Landesamt für Natur und Umwelt des Landes Schleswig-Holstein, Kiel-Flintbek 2008 (im Druck).
} 
entstand im 19. Jahrhundert aus der Färberei. Für das Nachfärben gebrauchter Kleidung war es erforderlich, die gesäuberte Faser mit der Farbflotte in Berührung zu bringen. Das Bedürfnis nach anderen Waschverfahren für Kleidung entstand im 19. Jahrhundert, weil im Zuge der Industrialisierung immer mehr Fette und Schmieröle eingesetzt wurden und in der Luft ein zunehmender Anteil Ruß vorhanden war. Die üblichen Schmutzpartikel wie Staub etc. sammelten sich auf diesen wasserunlöslichen Flecken und konnten dann auch mit den üblichen Verfahren nicht entfernt werden. Der französische Färber Jolly Bellin wandte im Jahr 1826 Terpentinöl für die Beseitigung fetthaltiger und verharzter Flecken an und stellte dabei fest, dass dieses Mittel nicht allein für die Fleckenentfernung, sondern auch für die Wäsche des gesamten Kleidungsstückes geeignet war. Er konnte dann auf die bekannten Verfahren der Nasswäsche verzichten. Der Vorteil für die Oberbekleidung war offensichtlich: sie schrumpfte oder verfilzte sich nicht mehr. Zudem mussten Applikationen oder Mischgewebe, die zeitgenössisch gern als Zierde angebracht waren, nicht mehr abgetrennt und später wieder aufgenäht werden.

Die technische Ausstattung der Reinigung unterschied sich in keiner Weise von jener der Färberei oder den aufkommenden Großwäschereien in den Großstädten. Bereits Bellin hatte aus der Reinigung in seiner Schönfärberei einen eigenen Geschäftszweig gemacht, indem er diese Leistung auch unabhängig von der Färberei anbot. Als „Pariser Waschanstalt“ wurden ab 1850 in den europäischen Großstädten nach und nach Großbetriebe der Färberei und Reinigung gegründet, so z.B. 1853 in Hannover die „Färberei und Neueste Chemische Pariser Waschanstalt" von Friedrich A. Stichweh. 1854 wurde in Berlin von der Firma Spindler die erste Wäscherei und Färberei, die die trockene und chemische Reinigung anbot, gegründet.

Die Wandlung der Schönfärberei zur chemischen Reinigung mit Benzin war nur mit Ausnahmen möglich: der Umgang mit leichtflüchtigem Benzin war mit erheblichen Brandgefahren verbunden, so dass die Stadt Paris bereits vor dem Ende des 19. Jahrhunderts die Aufnahme dieses Gewerbes in der Stadt und deren Umfeld verbot. 1903 wurden vergleichbare Auflagen auch für das Deutsche Reich, in dem es zu diesem Zeitpunkt 1.540 Reinigungen gab, eingeführt. Wie die Firmen von Spindler oder Stichweh mussten die neuen Reinigungsbetriebe an die Peripherie ausweichen, wo die Gefährdung der Nachbarn durch Brände nicht so groß war, z.B. „Spindlerfeld“ in Berlin oder Winterhude/Barmbek in Hamburg. Um den Kontakt zu den Kunden zu halten, wurden deshalb in den Geschäftsvierteln der Städte Annahmestellen eingerichtet, wo die Kunden die Kleidung abgaben und wieder in Empfang nahmen. In den Betrieben wurden aus sicherheitstechnischen und arbeitsschutzrechtlichen Gründen nach und nach geschlossene Waschmaschinen, Schleudern und Trockner eingeführt, die sich deutlich von den Anfängen der Maschinisierung der Wäscherei unterschieden. Zur Vermeidung der Lösungsmittelverdunstung, die eine der Hauptursachen für vielfache Brände war, wurden die Maschinen mit Deckeln versehen, die an eine Absauganlage angeschlossen waren. Aus dieser Schutzmaßnahme entwickelte sich der Gedanke, das Benzin, den teu- 
ersten Verbrauchsstoff der Reinigung, zurückzugewinnen, indem die Maschinen an eine Redestillation angeschlossen wurden. Hieraus entstand allmählich ein fast geschlossenes System. Das Umladen von Maschine zu Maschine bei geöffneten Deckeln führte weiterhin zu Verdunstungen, so dass ab Mitte der 1920er Jahre die ersten Kombinationsmaschinen für Waschen, Schleudern und Trocknen aufkamen, in denen als Schutzgas Stickstoff eingesetzt wurde. Diese Maschinen benötigten allerdings eine große Stellfläche und hatten einen hohen Kraft- und Wärmebedarf sowie zahlreiche Nebenanlagen für die Kühlung und Redestillation, so dass nur Großbetriebe über derartige Anlagen verfügten. Eine weitere Ursache für die Brände in Benzinwäschereien bestand in der statischen Aufladung des Benzins in der Maschine, das besonders bei der Reinigung von wollenen Kleidungsstücken auftrat. Durch den Zusatz von „Benzinseife“, einem Magnesiumsalz, konnte seit dem Beginn des 20. Jahrhunderts die Gefahr gemindert werden. Mit Hilfe dieser Sicherheitseinrichtungen, die den Verlust des teuren Lösungsmittels deutlich verminderten und die Versicherungsprämien niedrig hielten, gelang es der Benzinwäscherei bis in die 1960er Jahre konkurrenzfähig zu anderen Reinigungsverfahren, die sich nach und nach etablierten, zu bleiben.

Bei den neuen Reinigungsmitteln handelte es sich in der Regel um chemische Lösungsmittel, die bereits aus der Detachur bekannt waren. Insbesondere wurden in der Fachliteratur seit ca. 1904 Tetrachlorkohlenstoff und seit ca. 1920 bzw. Ende der 1920er Jahre Tri- und Perchlorethen, Abfallprodukte der gewerblichen Acetylenherstellung, die nach dem Ersten Weltkrieg besonders durch die staatliche Förderung des Schweißens im Schiffbau entstand, zunehmend genannt. Diese Lösungsmittel waren der Chemie zwar bereits seit 1820 bzw. 1840 bekannt, wurden aber erst jetzt in großen Mengen von der Chemischen Industrie hergestellt, so dass der Preis langsam auf jenen von Benzol oder Benzin sank. Der große Vorteil dieser Chlorkohlenwasserstoffe (CKW) bestand darin, dass sie nicht brennbar waren und folglich auch in den offenen Altmaschinen, die bei vielen kleineren Betrieben immer noch in Gebrauch waren, genutzt werden konnten. 1949 wurden in den Vereinigten Staaten die ersten geschlossenen Reinigungsvollautomaten patentiert, 1951 wurde auch in Deutschland eine vergleichbare Maschine angeboten. Alle Waschvorgänge fanden innerhalb einer Maschine statt, so dass nur noch das Trocknen auf Gestellen außerhalb der Maschine erfolgte. Die Maschinen waren für die Anwendung der halogenierten Kohlenwasserstoffe und deren Redestillation in einem Wärmetauscher ausgelegt. Es handelte sich anfänglich noch um Geräte mit hohem Platz- und Energiebedarf, die bis zu $80 \mathrm{~kg}$ Wäsche und ca. 2001 Flotte enthielten. Ein Jahrzehnt später hatten sich die Dimensionen der Reinigungsautomaten verringert, weil sie nur noch für eine Beladung von bis zu $20 \mathrm{~kg}$ ausgelegt waren. In der Branche gab es vor der Einführung kleiner Automaten nur die Trennung von zentralem Reinigungsbetrieb an der Peripherie und Annahmestellen im Zentrum der Stadt. Aus den Annahmestellen konnten sich nun dezentrale Ladengeschäfte entwickeln, weil die Maschinen klein genug waren und die CKW als Lösungsmittel gemäß damaliger Arbeitsschutzauflagen auch in den Innenstädten 
einen sicheren Betrieb gestatteten. Für den Betrieb einer Schnellreinigung wurde daher seit 1954 keine abgeschlossene Lehre als Wäscher oder Reiniger verlangt, so dass auf der Basis eines bloßen Sachkundenachweises Reinigungsbetriebe in großer Zahl entstanden, die, vorwiegend von einem italienischen Maschinenbaubetrieb, mit den notwendigen Geräten auf Kredit ausgestattet wurden. Zusätzlich entstanden seit ca. 1960 zunehmend Waschsalons, in denen neben den Waschmaschinen, Schleudern und Trocknern auch Reinigungsautomaten im Selbstbedienungsbetrieb aufgestellt wurden. Der Bedarf an chemischen Reinigungen, der durch neue Kunstfasern gefördert wurde, und die Möglichkeit, diese Dienstleistung nunmehr auch selbständig in unmittelbarer Nachbarschaft der Kunden anzubieten, führte dazu, dass in den 1970er Jahren mehr als 30.000 Reinigungsbetriebe in der Bundesrepublik existierten. Von diesen waren deutlich mehr als die Hälfte als klein- oder mittelständisches Unternehmen geführt. In den Innenstädten, den Einkaufszentren, selbst in den Ladenzeilen der neuen Hochhaussiedlungen wurden chemische Reinigungen eine Selbstverständlichkeit. Seit der Verkündung des 2. BundesImmissionsschutzgesetzes 1990, das mit zahlreichen Umweltauflagen für die Reinigungen verbunden ist, nahm die Anzahl der Reinigungen bis auf gegenwärtig ca. 3.200 rapide $a b$.

Nach diesem ersten Überblick über die Geschichte der Branche ist es die Aufgabe des Historikers, die Arbeitsvorgänge von der Einführung bis zum aktuellem Verfahrensstand zu analysieren, ihnen bestimmte Stoffe und Gefährdungspotentiale zuzuordnen und damit auch eine differenzierte Bewertung der Gefährdung zu ermöglichen:

Die chemische Reinigung ist grundsätzlich mit folgenden vier Verfahrensschritten verbunden, die die Umwelt beeinträchtigen können:

- Die Reinigung, bei der gefährdende Lösungsmittel freigesetzt werden können;

- Die Trocknung, bei der Gase der Reinigungsmittel freigesetzt werden, die in der Umgebung kondensieren;

- Die Rückgewinnung des Lösungsmittels;

- Die Reinigung des Lösungsmittels durch Abscheidung von groben Verunreinigungen sowie die anschließende Reinigung durch Destillation.

Im Falle der chemischen Reinigung beginnt die Arbeit mit der Annahme und dem Sortieren sowie der Kennzeichnung. Diese Tätigkeiten sind ohne Relevanz, weil noch keine Stoffe eingesetzt werden. Während des Sortierens wird jedoch bereits eine Teilung gemäß der Pflegehinweise und eine Sichtung vorhandener Flecken durchgeführt. Sofern eine Vorbehandlung erforderlich war, wurde diese an einem Detachurtisch durchgeführt, bevor die Kleidung dann einer Vollreinigung in Benzin, Per oder einem Fluorchlorkohlenwasserstofflösungsmittel (FCKW) unterzogen wurde. Bis ca. 1950 musste die Kleidung von der Waschmaschine in eine Schleuder umgeladen werden. In den Automaten war die Schleuder integriert, so 
dass diese Handhabung, die mit Abtropfverlusten verbunden war, entfiel. Nach der Schleuder wurden die Kleidungsstücke bis zum Ende der 1980er Jahre zum Trocknen auf Gestellen getrocknet, so dass auch hierbei noch Abtropf-, überwiegend aber Verdunstungsverluste eintraten.

Nach der Ermittlung der wesentlichen Arbeitsschritte ist das Lösungsmittel und dessen Geschichte näher zu betrachten. Das Grundproblem jeder Reinigung besteht darin, ein lipophiles Lösungsmittel zu finden, das den verschmutzenden Stoff löst, ohne die Kleidung zu entfärben oder zu zerstören. Derartige Lösungsmittel standen erst mit der Entwicklung der Kohlechemie, der Petrochemie und der Chemischen Industrie in größeren Mengen zur Verfügung. In der Regel waren die Lösungsmittel bereits zuvor in Laboren entdeckt oder in kleinen Mengen produziert worden. Die Mengen jedoch, die von Reinigungen benötigt wurden, konnten Labore nicht produzieren oder sie waren so teuer, dass, angesichts der zunächst großen Verluste in den offenen Maschinen, sich ein Einsatz aus betriebswirtschaftlichen Gründen verbot. Ein weiterer Gesichtspunkt für die Wahl des Reinigungsmittels betraf die Anwendungssicherheit. Narkotisierende Mittel wie Äther, Chloroform, Benzol oder Aceton, die zudem noch brandgefährlich waren, wurden daher nur in kleinen Mengen für die Detachur eingesetzt. Benzine waren zwar ebenfalls feuergefährlich, konnten aber ab Ende des 19. Jahrhunderts zusammen mit den Benzinseifen unter Beachtung aller Sicherheitsvorschriften ohne größere Probleme genutzt werden. Nachdem ab Mitte der 1920er Jahre immer mehr geschlossene Maschinen auf den Markt kamen und nicht entzündliche Lösungsmittel zur Verfügung standen, begann die Automatisierung und Verkleinerung bei gleichzeitiger Rückgewinnung der Lösungsmittel, die letztlich zu den verhältnismäßig sicheren Vollautomaten der Neuzeit führte. Neue synthetische Textilfasern erforderten neue Lösungsmittel, die in den Fluorchlorkohlenwasserstoffen gefunden wurden, so dass eine breite Palette von Lösungsmitteln für unterschiedliche Textilfasern und Flecken zur Verfügung stand. Die folgende Übersicht bietet eine kurze Chronologie gebräuchlicher Trockenreinigungsmittel:

Terpentinöl, (1825 - 1860). Terpentinöl ist eine farblose, giftige Flüssigkeit, die nicht wasserlöslich ist. Es handelt sich um ein durch Destillation gereinigtes Kiefern- oder Kienöl. Der Nachteil des Öls besteht darin, dass dem unraffinierten Terpentin ein übler Geruch anhaftet. Mit gereinigtem Terpentinöl (Universalverdünner) wird auch heute noch gearbeitet.

Spiritus, Alkohol (seit 1850). Mit dem Anstieg der gewerblichen Brennerei als landwirtschaftlichem Nebengewerbe stand seit Mitte des 19. Jahrhunderts genügend Äthanol zur Verfügung, um in der Reinigung in Massen eingesetzt werden zu können. Aus steuerlichen Gründen wurde dem Alkohol ein Vergällungsmittel (Petroläther) zugesetzt.

Petroleum, $(1860-1900)$. Die zwischen $150^{\circ} \mathrm{C}$ und $220^{\circ} \mathrm{C}$ siedenden Fraktionen der Alkane kamen ab 1859 mit der Verbreitung der Petroleumlampe in zunächst sehr geringen Mengen über Apotheken auf den Markt. Mit zunehmendem Import von Rohöl und dem Aufbau der ersten Raffinerien in Bremen und Ham- 
burg ab 1865 wurde Petroleum (Lampenöl), aus dem dann später die Benzine destilliert wurden, so preiswert, dass es auch in der Reinigung eingesetzt wurde.

Leichtbenzin/Petroleumbenzin. $(1860$ - 1960). Die niedrig siedenden Mineralkohlenwasserstoffe der Alkane zwischen Pentan und Nonan kamen ab ca. 1860 in zunächst sehr geringen Mengen als Wundbenzin über die Apotheken auf den Markt und wurden wegen des hohen Preises und der Feuergefahr nur in der Detachur eingesetzt. Mit der Einführung der Vakuumdestillation in Europa ab 1904 stand Leichtbenzin in zunehmenden Mengen zur Verfügung und verbilligte sich ganz entscheidend, so dass die Benzinreinigung zum Standard wurde. Da dieses Reinigungsmittel sich sehr schnell entzündete, trug die Erfindung der Benzinseife entscheidend zur Ausbreitung bei.

Schwerbenzin (Handelsnamen: Kristallöl 30, Stoddard Solvent, White Spirit, Essovarsol, Testbenzin), (1860 - 1960). Es handelt sich um ein Gemisch von Alkanen zwischen Nonan und Dodecan, die oberhalb des Petroleums sieden. Die Schwerbenzine stehen den Paraffinen nahe und sind daher zwar brennbar, aber nicht so leicht entzündlich wie Benzin oder Leichtbenzin. Unter Berücksichtigung der Faktoren Wirkung, Kosten und Betriebssicherheit handelte es sich lange Zeit um ein konkurrenzloses Produkt. Schwerbenzin wird auch heute noch in vielen Ländern eingesetzt. Seit 1993 werden Paraffine oder Paraffinöle als sogenannte „neue Kohlenwasserstoffe“ (vgl. dort) auch in der Reinigung wieder verstärkt eingesetzt.

Benzol/Hüttenbenzin (1870ff.). Benzol $\left(\mathrm{C}_{6} \mathrm{H}_{6}\right)$ wurde als eines der ersten künstlich hergestellten organischen Lösungsmittel zunächst nur zögerlich in der Detachur eingesetzt, seitdem aber die Gasanstalten größere Mengen davon produzierten (ca. 1870), auch in der Trockenreinigung. Der Vorteil bestand darin, dass kein Geruch verblieb. Nachteilig war die große Feuergefährlichkeit und Giftigkeit sowie der sehr hohe Preis, der erst zum Ende des 19. Jahrhunderts sank. Der Einsatz von Benzol als Substitut zu Benzin ist aus Kostengründen unwahrscheinlich.

Tetrachlorkohlenstoff/ Tetrachlormethan (Handelsname z.B. Tetra oder Asordin) $(1904-1960)$. Seit 1904 ist das 1830 erfundene Tetra $\left(\mathrm{CCl}_{4}\right)$ in der Literatur als Alternative zu Benzol und den Benzinen genannt. Es hat den großen Vorteil gegenüber den bisherigen Lösungsmitteln, nicht brennbar zu sein und nahezu jeden Fleck entfernen zu können. Von Nachteil war allerdings der sehr hohe Preis, so dass ein verlustreicher Einsatz in den damaligen Maschinen nicht erfolgte.

Trichlorethen „Tri“ (Handelsnamen z.B.: Tri, Dynatri, Trilene, Westrosal, Drawinal), (1920 - 1930). Trichlorethen $\left(\mathrm{C}_{2} \mathrm{HCL}_{3}\right)$ verdankte seine Verwendung in der Reinigung einerseits seiner relativ hohen Wasserlöslichkeit, andererseits dem verhältnismäßig geringen Preis und der Tatsache, dass es wie Tetra nicht brennbar ist. Tri, wie auch das nachfolgend dargestellte Per, wurden von den Herstellern technischer Gase als Nebenprodukte der Acetylenherstellung auf den Markt gebracht. Zunächst als Reinigungsverstärker in der Nasswäsche eingesetzt, konnte es bald Benzol und Tetra in der Trockenreinigung verdrängen. Da es aber an der Luft unter Lichteinfluss schnell in Chlorwasserstoff, Kohlenmonoxid und Phosgen 
zerfällt, und zudem die Metallteile der Maschinen stark korrodiert, konnte es mit dem bald danach entwickelten Per nicht konkurrieren.

Perchlorethen/Tetrachlorethen „Per“. (Handelsname: Perawin, Dynaper, Dow-Per, Wacker-Per, Phillsolv, Percosolv) (1930 - aktuell) Perchlorethen (C2 CL4). war nicht teurer als Trichlorethen, zersetzt sich an der Luft aber nicht in dem hohen Maße und korrodiert auch die Maschinen nicht so intensiv. Zudem war es ebenfalls nicht brennbar, so dass Per bis heute in ca. $80 \%$ aller Reinigungen genutzt wird.

Monofluortrichlorethan (R 11) (Handelsnamen z.B.: Frigen 11, Freon 11, FriDohna F 11, Dional, Kaltron DC 11) (1960 - 1980). Mit der Einführung neuer Textilfasern (Nylon, Nyltest, Perlon, Dralon etc.) wurden in der Reinigung Lösungsmittel benötigt, die die Kunststofffaser nicht wieder auflösten. Die meisten Kunststofffasern werden bei der Herstellung in Lösungsmitteln verflüssigt, die nach der Passage der Spinndüsen schnell verdunsten, so dass Leichtbenzine, Aromaten (BTEX) und CKW für die Reinigung nicht mehr in Frage kamen. Als Alternative wurde die Gruppe der Fluorchlorkohlenwasserstoffe (FCKW) entdeckt, die aufgrund ihrer thermischen Stabilität bereits in den Kühlkreisläufen der Kühlschränke, Transformatoren und Gleichrichter eingesetzt wurden. Sie sind nicht brennbar, können Fette und Öle gut lösen und sind leicht zu redestillieren. R 11 $\left(\mathrm{CFCl}_{3}\right)$ hat, ähnlich wie ehedem Tri, allerdings den Nachteil, sehr flüchtig zu sein, so dass auch in geschlossenen Automaten der Verbrauch unwirtschaftlich war. Nachdem sich bis 1980 herausgestellt hatte, dass nicht alle modernen Stoffe, die mit dem Pflegesymbol F gekennzeichnet waren, dem R 11 widerstanden, wurde seine Nutzung in Automaten eingestellt.

Trifluortrichlorethan (R 113) (Handelsnamen z.B.: Frigen 113, Freon 113, FriDohna F 113, Kaltron DC, Valclene) $\left(1960\right.$ - 1990). R $113\left(\mathrm{C}_{2} \mathrm{~F}_{3} \mathrm{CL}_{3}\right)$ wurde zeitgleich mit R 11 eingeführt und besaß ähnliche Vorteile, verdunstete allerdings nicht in gleicher Menge und löste moderne Textilien weniger als R 11, so dass dieses Reinigungsmittel bis ca. 1990 neben Per und Schwerbenzin zum Standard der gebräuchlichen Lösungsmittel gehörte. Der Einsatz ist wegen erheblicher Gesundheitsgefährdungen verboten.

1,1,1-Trichlorethan (1985 - 1992). 1,1,1-Trichlorethan ist ein Reinigungsmittel, das kurz vor der Verabschiedung des Wasserhaushaltsgesetzes eingeführt wurde. Es handelt sich um ein Lösungsmittel, das bereits in der Metallentfettung mit Erfolg eingesetzt worden war und dann wegen seiner hohen chemischen und physikalischen Stabilität auch Eingang in die chemische Reinigung fand. Der Verdacht, dass dieses Mittel mutagen und krebserregend ist sowie die 1991 erfolgten Verbote und Nutzungseinschränkungen gegen die in der Reinigung eingesetzten CKW, zu denen auch dieser Stoff gehört, beendeten die Nutzung des 1,1,1-Trichlorethan ebenso wie die des Dichlorethan.

Kohlenwasserstofflösemittel (KWL) (Handelsnamen z.B.: Shellsol TK, Shellsolreiniger DSC, Rynex, Greenearth, n-Undecan, N 11, Actrel Dry, Clean 56). (1993 - aktuell). Die „,neuen Kohlenwasserstoffe“, die ohne Fluor- oder Chlorverbindungen auskommen, wurden nach Einführung der 2. BImSchV als Alternativen 
zu den umweltschädigenden CKW und FCKW propagiert. Es handelt sich in der Regel um Gemische von Alkanen, Cycloalkanen und Aromaten mit zehn bis zwölf C-Atomen: Paraffine von Decan bis Dodecan oder Cyclodecan bis Cyclododecan sowie um Methyl- und Dimethylnaphthaline. Ein Teil dieser Lösungsmittel wurde in der Geschichte der Reinigung bereits unter dem Namen „Schwerbenzin“ benutzt. Neben die Alkane traten nun aber auch die Cyclo- und Isoalkane, die ähnliche Reinigungseigenschaften besitzen. Insgesamt ist dieses Reinigungsmittel dem Dieselkraftstoff, der jahrzehntelang in der Entwachsung, Entfettung und Entölung von Fahrzeugen und Maschinen eingesetzt wurde, in der Zusammensetzung und Eigenschaften relativ ähnlich. Gegenwärtig werden ca. $20 \%$ der Reinigungen mit diesem Mittel durchgeführt.

\subsection{Halogenierte Lösungsmittel, der Abfall der ,großen Industrie“}

In der chemischen Reinigung traten erhebliche Verdunstungen des Lösungsmittels auf, denen sich die Verkleckerungen beim Umladen von einer Maschine zur nächsten hinzugesellten. Pro Waschgang war ein Verlust von bis zu 30 Prozent anzunehmen. Die Umstellung der Maschinen auf ein abgeschlossenes System begann in den 1920er Jahren und war bis in die 1930er Jahre im Wesentlichen abgeschlossen. Aus betriebswirtschaftlichen Gründen wurden geschlossene Maschinen an eine Redestillation angeschlossen, so dass sich die durchschnittlichen Lösungsmittelverluste pro Waschgang auf ca. 10 Prozent reduzieren ließen. Diese Verluste waren auf das Umladen von Maschine zu Maschine sowie die anschließende Lufttrocknung zurückzuführen. Mit der Einführung der geschlossenen Vollautomaten sank der Lösungsmittelverlust seit 1960 von ca. 10 auf gegenwärtig ca. 1 Prozent des eingesetzten Flottenvolumens. Die Reinigungsmaschinen werden seit 1990 (2. Verordnung zur Durchführung des BImSchV) in einem vollständig geschlossenen Kreislauf mit Filterkartuschen für die Gase, Aktivkohlefiltern für die gekühlte Abluft sowie gesondert zu entsorgenden Rückstandscontainern für Schmutzrückstände, verbrauchte Filter und Kontaktwasser betrieben. Die Maschinen können nicht geöffnet werden, bis der Vorgabewert in der Trockenluft unterschritten ist. Insgesamt ist eine kontinuierliche Verminderung der Lösungsmittelverluste durch eine stetige Verbesserung der Automaten zu beobachten. Für die chemische Reinigung gibt es keinen altlastirrelevanten Zeitraum, weil alle Lösungsmittel die Umwelt gefährdeten. Eine Differenzierung ist jedoch möglich, weil die eingesetzten Lösungsmittel ein unterschiedliches Abbauverhalten haben. Hierzu zählt z.B. Benzin, das in belüftetem Boden mikrobiologisch gut abgebaut wird. Da dieses Lösungsmittel insgesamt über einen Zeitraum von annähernd 100 Jahren die Reinigungsbetriebe an der Peripherie damaliger Städte dominierte, darf für diese Betriebe eine relativ geringe Gefährdung angenommen werden. Im Gegensatz zu anderen Branchen spielt bei den chemischen Reinigungen die geringere Betriebsgröße eine bedeutende Rolle für die Einschätzung des Gefährdungspotentials. Im Zeitraum zwischen 1954 und 1990 haben die Kleinbetriebe in innerstädtischen Lagen viel 
häufiger als die Großbetriebe mit CKW gereinigt, weil diese Lösungsmittel auch in solchen Publikumslagen genehmigungsfähig waren. Seit dem Beginn der 1990er Jahre, nachdem bestimmte Lösungsmittel verboten wurden und eine geregelte Abfallentsorgung für Filter- und Rückstandsmaterial vorgeschrieben war, hat sich die Umweltrelevanz dieser Branche nochmals verändert. Aufgrund technischer Sicherungen in den Maschinen sind Handhabungsverluste ausgeschlossen, so dass nur noch eine geringe Umweltgefährdung, verursacht z.B. durch unsachgemäßen Umgang und den Austausch der Filter und Destillationsrückstände, angenommen werden kann.

Für den Historiker ergeben sich, wie in dieser Kurzdarstellung angedeutet, mehrere Zugänge: technikhistorisch ist der Zugriff über die Entwicklung der Maschinentechnik vom offenen Färberbottich zur sondengesteuerten Technik, die die Handhabungsfehler ausschließt. Verfahrenstechnisch ist der Zugriff über die Lösungsmittel, die, entsprechend der Nebenproduktverwertung der ,großen Indust-

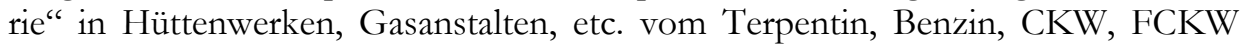
und Paraffine entsprechend der Kosten eingesetzt werden - damit ergibt sich auch ein Zugang aus der Wirtschaftsgeschichte. Sozialhistorisch ist die Wiederkehr der kleingewerblichen Wäscherei, die, wie besonders in Garnisonsstädten zu beobachten ist, von den Großwäschereien bereits abgelöst waren, zu beobachten. Auch die Rechtsentwicklung, die die Reinigungen zunächst an die Stadtränder verbannte, dann die chemische Reinigung ohne Auflagen für die Lösungsmittel und ohne nennenswerte Sachkenntnisse der Betreiber zuließ und letztendlich dafür sorgte, dass sowohl Maschinen wie auch Lösungsmittel harte Vorgaben einhalten müssen. Insgesamt ist also die chemische Reinigung, die die Menschen durch aufsteigende Gase unmittelbar in den Wohnungen und durch die Schmutzfahnen im Boden und im Trinkwasser bedroht, ein relevantes Thema der urbanen Umweltgeschichte. 


\section{3}

\section{Milzbrand, eine Zoonose als Altlast ${ }^{11}$}

Das Fehlen der sozialen Dimension in einem umwelthistorischen Aufsatz hatte Frank Uekötter vor Jahren in einer Rezension veranlasst, die Frage „Kann die Umweltgeschichte des Bodens mehr sein als eine schlichte Kontaminierungsgeschichte?" 12 zu stellen. Dass die Umweltgeschichte der Altlasten und mithin des Bodens von gesellschaftlicher Relevanz ist, ist nicht ernstlich zu bezweifeln. Ob es sich auch um ein mehrdimensionales historisches Thema handelt, ist nach der Lektüre der folgenden Ausführungen zum Milzbrand als Altlast zu fragen. Als Beispiel für die soziale Dimension einer derartigen Kontaminierungsgeschichte sei hier kurz eine Zusammenfassung zum Milzbrand in Neumünster, der einzigen Stadt, die durch Flyer seit 2003 die Einwohner vor dieser Gefährdung warnt, genannt:

Bereits im Oktober 1901 wurden dem preußischen Regierungspräsidenten Schleswig-Holsteins schriftlich die ersten Anliegerproteste gegen das Lederabwasser der holsteinischen Industriestadt Neumünster vorgelegt. Das Wasser der Stör sei oberhalb Kellinghusen ,in einer den Viehbestand außerordentlich schädigenden Weise" verunreinigt. ${ }^{13}$ Seither stand die Stör, ein Nebenfluß der Unterelbe, im Brennpunkt vieler bäuerlicher Anliegerproteste und einer großen Zahl von Untersuchungen, die durch die Aufsichtsbehörden veranlaßt wurden, bis im Jahre 1914 zunächst ein generelles Veränderungsverbot für die Lederfabriken erlassen wurde, das allerdings durch die Kriegsleder AG während des Ersten Weltkrieges unterlaufen wurde.

„Eine Besichtigung der Abwasserverhältnisse der Gerbereien der Stadt Neumünster im Herbst vorigen Jahres durch eine Kommission der beteiligten Ministerien hat ergeben, dass das Maß und die Art der Abwässer aus diesen Anlagen und ihre Beseitigung in polizeilichem Interesse eine grundsätzliche Neuregelung der gesamten Abwasserverhältnisse erfordert. Die Unzulänglichkeiten haben einen derartigen Grad erreicht, dass Veränderungen, welche wie die vorliegenden, einen Einfluss auf die Menge der Abwässer aus den Gerbereien in Neumünster auszuüben geeignet sind, eine Genehmigung des unterzeichnenden Ministers nicht eher

\footnotetext{
11 Das Kapitel über den Milzbrand geht zu Teilen auf folgendes unveröffentlichtes Gutachten des Autors zurück: Erfassung, Kategorisierung und Bewertung der Milzbrandgefährdungen an Fließgewässern in Schleswig-Holstein durch ledererzeugende Betriebe. Im Auftrag des Bearbeitungsgebietsverbandes 13, Oberlauf Stör, Bremen 2007 (128 Seiten). Da der Autor das Milzbrandthema zum Gegenstand einer eigenständigen Arbeit hat, handelt es sich zu weiteren Teilen um eine Zusammenfassung mit Werkstattcharakter, so dass nicht jede Aussage belegt ist, aber aus einem vorhandenen Fundus belegt werden könnte.

12 Frank Uekötter: Rezension von: Christoph Bernhardt (Hg.): Environmental Problems in European Cities in the 19th and 20th Century. Umweltprobleme in europäischen Städten des 19. und 20. Jahrhunderts: Waxmann 2001. In: Sehepunkte 3 (2003), Nr. 5 (15.05.2003), URL: <http://www.sehepunkte.de/2003/05/1494.html. (14.09.2008)

${ }^{13}$ Landesarchiv Schleswig-Holstein (LASH) Abt. 309, Nr. 10858 vom 11.10.1901.
} 
finden können, als bis die in Betracht kommenden Gerbereibesitzer ein Projekt der Abwässerbeseitigung zur Ausführung bringen, das den im öffentlichen Interesse zu stellenden Anforderungen genügt." 14

In einer Chronologie der Bauernproteste gegen die Lederabwässer fehlt seither kaum ein Jahr: nach jedem Frühjahrshochwasser der Stör waren hunderte Tiere am Milzbrand verendet. Obgleich nach dem Höhepunkt der Lederkonjunktur, dem Jahr 1924/25, einige große Lederfabriken in Neumünster schließen mussten, schwoll der Protest der Bauern derart an, dass sie die Landbevölkerung im August 1929 zu einem Totalboykott Neumünsters aufriefen. Dieses Ereignis fand seinen Niederschlag auch in dem 1931 in Berlin erschienenen Roman „Bauern - Bonzen - Bomben“ von Hans Fallada, der zu jener Zeit als Journalist in Neumünster tätig war. Eine Auswirkung dieses wirksamen Protestes und des daraus resultierenden Boykotts aller Waren und Dienstleistungen war, dass die Stadtverordnetenversammlung Neumünsters im Mai 1930 und erneut im März 1931 beschloß, alle Regressansprüche, die Bauern wegen milzbrandinfizierter Rinder anmeldeten, unmittelbar aus der Stadtkasse zu zahlen. Eine rechtliche Anerkennung als Verursacher solle jedoch ausgeschlossen bleiben. ${ }^{15}$ Auf dieser Basis wurden allein im Jahr 1931 die Ansprüche von 236 Bauern aus dem Kreis Steinburg und von 79 Bauern aus dem Kreis Rendsburg befriedigt. ${ }^{16}$

\subsection{Milzbrandsporen - ein Organismus ohne Lebensäußerung}

Zum Stichwort „Milzbrand“ führt eine der wichtigsten Enzyklopädien des 18. und 19. Jahrhunderts Folgendes aus: „Milzbrand, Milzseuche, Milzweh, Milzkrankheit, wildes Geblüt, heimliche Geblüt, Sommerseuche: eine Krankheit des Viehes, welche in einem rothlaufartigen Entzündungs-Fieber besteht, nicht ansteckt, eine bloße Witterungs- und Fütterungs-Krankheit ist, wo an gewissen Theilen des Körpers ausserlich sichtbare Beulen, Geschwülste und bisweilen bösartige Blutschwären zum Vorschein kommen, schnell tödtet. (...) Diese Krankheit, welche hier und da bey großen Viehherden manches Stück Vieh einzeln wegrafft, bisweilen herrschend ist, befällt alle Hausthiere ohne Unterschied. Man hat sie von jeher in verschiedenen Ländern wahrgenommen, von ihrer Natur und Ursachen sehr verschieden geurtheilet, und daher ihr auch verschiedene Nahmen beygeleget, weswegen man sie immer mit andern Krankheiten, ja selbst mit der wirklichen Viehpest verwechselt hat. (...) Gleichwie sie ihre Ursache allezeit ganz auf das Oertliche, Eigene und Besondere gewisser Umstände des Orts oder der Zeit bezieht: also schränket sie sich blos auf einzelne, gegen die Anzahl des Viehes im Orte oder in

\footnotetext{
14 Stadtarchiv Neumünster (StA-Nms), ungeordnete Bestände, Rekursbescheid Nr. III, Der Minister für Handel und Gewerbe, Berlin, den 30. April 1914.

15 Vgl.: StA-Nms. Magistratsarchiv Nr. 1680, Protokoll vom 7.5.1930 und vom 18.3.1931.

16 Vgl.: Klaus Schlottau (1994), Die Planung des Stör-Kanals von Itzehoe nach Neumünster als Binnenschifffahrtsweg und Abwasserkanal der Neumünsteraner Industrie (1891-1926). In: Mitteilungen des Canal-Vereins, Jg. 15, Rendsburg, S. 93-116, hier S. 112.
} 
der Gegend gerechnet, nur wenige Thiere, oft nur auf einen einzelnen Hof oder Ort, höchstens auf die Gegend ein; gemeiniglich, wo viele stockende und stillstehende Wasser sind.“"17

Milzbrand ist eine Erkrankung, die insbesondere Rinder, Schafe und Ziegen sowie deren wildlebenden Verwandten befällt ${ }^{18}$. Der Milzbranderreger ist ein sporenbildendes stäbchenförmiges Bakterium, das auf zweierlei Weisen zu Krankheiten führt: Es bildet einerseits im Körper ein Toxin, das den Zellstoffwechsel befällt und schützt sich andererseits im Körper gegen Abwehrzellen, indem es eine Kapsel bildet. Beide Eigenschaften beruhen auf extrachromosomalem genetischen Material, zwei Plasmiden, die beide vorhanden sein müssen, damit Infektionen hervorgerufen werden können. Unter ungünstigen Lebensumständen, z.B. beim Tod des befallenen Lebewesens, bilden die Bazillen Sporen, die keinen wahrnehmbaren Stoffwechsel besitzen. Sporen widerstehen sowohl großer Hitze als auch den meisten Chemikalien, so dass sie, trotz des Einsatzes vieler hochkonzentrierter Chemikalien auch die Gerbung und einer Lagerung in Böden, die voller Gerbereichemikalien sind, überstehen. Die Schwerpunkte des Milzbrandvorkommens befinden sich in Afrika, Asien sowie in Latein- und Südamerika. In Deutschland selbst ist der Erreger nicht heimisch, wurde aber durch Viehtrieb, Viehhandel und insbesondere durch den Hauthandel immer wieder eingeschleppt. Die Veterinärbehörden haben auf diese Erkrankung besonders geachtet und durch konsequentes Vorgehen gegen Verschleppung reagiert. Gelegentlich auftretende lokale Infektionen lassen sich in der Regel auf kontaminierten Erdboden oder auf importierte Futtermittel zurückführen. Tiere infizieren sich in der Regel über das Futter und verenden nach ca. zwei Tagen. Blutiger Ausfluß entleert sich aus allen Körperöffnungen und bildet unter Umwelteinfluß neue Sporen. Beim Menschen treten insbesondere drei Krankheitsbilder auf: der Hautmilzbrand, der Darmmilzband sowie der Lungenmilzbrand. Der Hautmilzbrand, der mit einem Anteil von 95 Prozent dominant ist, entsteht zumeist im Zuge von Transportarbeiten, bei denen die Sporen durch kleine Wunden in die Haut eindringen und dort Milzbrandkarbunkeln bilden, die in der Regel operativ entfernt werden können - die Behandlung mit Antibiotika, die allerdings früher nicht zur Verfügung standen, führt in der Regel zu einer völligen Ausheilung. Die Sterblichkeit des unbehandelten Hautmilzbrandes liegt bei 20 Prozent. Eingeatmete Sporen, die im Staub vorhanden sind, führen zu dem weit gefährlicheren Lungenmilzbrand. Sporen, die mit der Speise aufgenommen werden und die Magensäure unbeschadet passieren, verursachen den nicht minder gefährlichen Darmmilzbrand. Das Erscheinungsbild ähnelt einer Lungenentzündung und die Erkrankung ist selbst bei Einsatz von Antibiotika sel-

\footnotetext{
17 Johann Georg Krünitz (Hg.): Oekonomisch-technologische Encyklopädie, oder allgemeines System der Staats-, Stadt-, Haus- und Landwirtschaft und der Kunstgeschichte in alphabetischer Ordnung, 90. Theil (Meth bis Minderzwickel), Berlin, 1803, S. 709f.

18 Vgl. für das Folgende: Reinhard Böhm, Kap. 2.3 in: Erkundung ehemaliger Gerbereistandorte. Leitfaden. Umweltbundesamt. Projektträger Abfallwirtschaft und Altlastensanierung des BMBF, Berlin 1998, S. $51 \mathrm{ff}$
} 
ten zu heilen, so dass die Sterblichkeit sehr hoch ist. Eine Impfung gegen Milzband ist möglich, der Impfstoff ist jedoch nicht in Deutschland zugelassen. ${ }^{19}$

Die Geschichte der Entdeckung des Milzbranderregers sowie die Darstellung in Reinkultur durch Koch (1876) und die Entwicklung eines Impfserums durch Pasteur 1881 ist ausführlich dargestellt und diskutiert von Lottermoser. ${ }^{20} \mathrm{Da}$ es, seitdem Koch den Erreger identifizierte, eine Möglichkeit der Diagnostik gab, verlangten Viehversicherungen sowie die allmählich sich ausbreitenden staatlichen Viehseuchenentschädigungskassen in jedem Fall einen eindeutigen Nachweis, um dem Betrug vorzubeugen. Im Rahmen dieser Untersuchungen entstand 1911 die später häufig genutzte Ascoli-Reaktion. Sie beruht darauf, dass Eiweiße durch eine Fällungsreaktion bei Erhitzung, die sogenannte Präzipitation, nachweisbar sind. Milzbrandmaterial verliert diese Eigenschaft auch bei längerer Erhitzung nicht. Es handelt sich hierbei um eine schnelle aber ungenaue Labordiagnostik, bei der die Probe kurz in siedendes Wasser eingelegt wird und dann mit dem präzipitierenden Serum zusammen eine charakteristische Reaktion zeigt. Eine genauere aber zeitraubendere Methode bestand darin, Kulturen anzulegen und zu untersuchen. Derartige Kulturen bilden typische Formen und sind lange Jahre lagerungsfähig. So wurden 1952 in einem kriegszerstörten Labor 53 Milzbrandkulturen aufgefunden, die seit fast 50 Jahren gelagert worden waren. Von diesen Kulturen waren noch 32 virulent. In Milch sollen die Keime über 10 Jahre und in Wasser bis zu 18 Jahre lebensfähig sein. „Rein theoretisch werden, ausgehend von der Absterbekinetik der Sporen bei $3^{\circ} \mathrm{C}$, Halbwertzeiten von 620 Jahren im feuchten Zustand und 146 Jahre für getrocknete Präparate angegeben"..21 Nachgewiesen wurden bislang, je nach Umgebungsmilieu, Zeitspannen zwischen 47 und 71 Jahren. Die Lebensdauer der Sporen darf als extrem lang angesehen werden. In Württemberg wurde ein Milzbrandfall darauf zurückgeführt, dass bei Erdarbeiten auf einem Hof der Kadaver eines französischen Kavalleriepferdes aus den napoleonischen Kriegen nach fast 150 Jahren wieder freigelegt wurde. ${ }^{22}$ Die Virulenz der Milzbrandsporen war lange umstritten, bis 1927 bei Infektionsversuchen mit definierten Keimzahlen ermittelt wurde, dass bei einem Keim $28 \%$ der infizierten Mäuse verstarben, bei

19 Forschern des Max-Planck-Institutes für Infektionsbiologie gelang es 2005, das Protein alpha defensin aus den neutrophilen Granulozyten als verantwortliches Protein für die Vernichtung von Anthrax-Bazillen zu identifizieren. Vgl. Pressemitteilungen des MPI, PRI B 68/2005 (165) vom 11. November 2005.

20 Vgl.: Thomas Lottermoser: Milzbrand - ein Beitrag zur Geschichte der Krankheiten. Diss. Vet. Med. Berlin 1998, S. 51-68.

21 Vgl.: Frank Merkt, Milzbrand in der Bundesrepublik Deutschland, München 1990, S. 4; Ferner: Thomas Lottermoser: Milzbrand - ein Beitrag zur Geschichte der Krankheiten. Diss. Vet. Med. Berlin 1998, S. 74. Aus der Sowjetunion wurde ein Fund virulenter Sporen in einem ausgegrabenen 1300 Jahre alten russischen Dorf gemeldet. Es handelte sich jedoch um eine Propagandameldung, mit der ein Unfall in einer Biowaffenfabrik bei Sverdlovsk, in deren Verlauf 66 Personen an Milzbrand erkrankten, kaschiert werden sollte. (Vgl. ebd., zitiert nach I.S. Bezdeneznych und V.N. Nikiforov: Epidemiologische Analyse von Milzbrand-Erkrankungen in Sverdlovsk. In: Zeitschrift für Mikrobiologie, Nr. 5 (1980), S. 11 - 113.).

22 Albrecht Braun: Der Milzbrand in Württemberg. Eine statistisch-epidemologische Studie, Diss. Vet. Med. Gießen 1958, S. 37. 
einer Dosis von 2-10 Keimen starben bis zu $73 \%$ der Versuchstiere und ab 20 Keimen starben alle Labormäuse. Ein Milzbrandstamm wird gegenwärtig als vollvirulent definiert, wenn 10 bis 100 Sporen eine letale Dosis darstellen. Sterben die Versuchstiere erst bei einer Dosis von 1000 bis zu einer Million Keimen, gilt der Stamm als schwachvirulent. ${ }^{23}$

Milzbrand ist, statistisch betrachtet, in Nord- und Mitteleuropa ein Importseuche. In Südeuropa ist der Milzbrand allerdings, ebenso wie in Afrika, Asien und Südamerika, eine Bodenseuche. In Deutschland läßt sich seit dem Ende des 19. Jahrhunderts eine Verschiebung beobachten. Anfänglich sind die Milzbrandgebiete durch mangelnde Kadaverentsorgung gekennzeichnet und befinden sich in ländlich strukturierten Gebieten mit hohem Tierbestand oder vielen Tieren, die auf alten Handelswegen zum Markt getrieben wurden: Oberbayern, Erzgebirge, Schlesien, Brandenburg, Posen, Westpreußen und auch Schleswig-Holstein. Mit dem Beginn des Importes ausländischer Futtermittel verlagert sich der Schwerpunkt bis 1914 in Gebiete mit hoher Schweinemast sowie in Gebiete, in denen importierte Häute verarbeitet werden. Ab 1923 liegen die gefährdeten Gebiete ganz überwiegend entlang der Flußläufe, an denen importierte Häute und Felle bearbeitet wurden. Sobald diese Flußläufe reguliert wurden, gingen auch hier die Milzbrandinfektionen signifikant zurück. ${ }^{24}$

Boden ist das Reservoir für die Milzbranderreger. In der vegetativen Form können Sporen sich darin sogar vermehren, dafür sind eine Feuchtigkeit von $80 \%$ und eine Temperatur zwischen 30 und 39 Grad Celsius bei neutralem pH-Wert erforderlich, was in hiesigen Breiten aber gewöhnlich nicht geschieht. Bei tieferen Bodentemperaturen werden die Sporen konserviert, so dass sie bei geeigneten Lebensbedingungen wieder auskeimen können. Nach gegenwärtigem Kenntnisstand, der sich auf Untersuchungen in Neumünster bezieht, können die Keime auch nach 80 Jahren im Boden noch virulent sein. Betroffen von hohen Milzbrandsporenkonzentrationen sind daher alle alten Lederfabriken sowie deren Ablagerungen und im Bereich der Landwirtschaft schließlich alle ehemaligen Wasenplätze, Tierfriedhöfe und Tierkörperbeseitigungsanstalten. ${ }^{25}$

\subsection{Häute und Felle als Quellen für Milzbrandepidemien}

Durch den Import von Häuten und besonders Fellen, die mit Milzbrandsporen kontaminiert waren, gelangten die Erreger in den Produktionsprozess und mit dem Abwasser erfolgte bei Überschwemmungen eine Verschleppung in die nahe gelegenen Auenbereiche. Ein besonderes Risiko bestand für getrocknete Häute und Felle, die in den großen Fabriken Neumünsters jährlich zu Millionen verarbeitet wurden. Hierbei handelt es sich zumeist um südamerikanische Campo mataderos,

\footnotetext{
${ }^{23}$ Lottermoser: 1998, S. 78.

${ }^{24}$ Vgl.: ebd., S. 94.

${ }^{25}$ Vgl.: Merkt, 1990, S. $7 f$.
} 
um asiatische und afrikanische Kipse oder amerikanische Renderer-Hides, Einfuhren billigster Häute und Felle aus Steppengebieten, wo das Vieh ohne Veterinärkontrolle enthäutet wurde, bzw. um Häute aus der Abdeckerei. Im Zeitraum von 1885 bis 1910 hatte sich der Import von Kalbfellen von 8.236 t auf 32.102 t gesteigert. Der Import von Rindshäuten stieg von 45.973 auf 134.006 t. Der Roßhautimport wurde von 7.039 t auf 13.828 fast verdoppelt und nicht anders verhielt sich die importierte Menge an Schaf- und Ziegenfellen, die von $10.096 \mathrm{t}$ auf 18.795 anstieg. ${ }^{26}$ Alle genannten Häute und Felle, die den Rohstoff der Lederindustrie Norddeutschlands darstellten, waren in hohem Maße von Milzbrandbazillen verunreinigt.

Eine Konsequenz dieser Erkenntnis bestand darin, dass, in Ergänzung des Preußischen Viehseuchengesetzes, seit dem 1.12.1934 in Schleswig-Holstein eine Untersuchung aller Häute und Felle auf Milzbrandinfektionen mit Hilfe der AscoliReaktion vorgeschrieben wurde. ${ }^{27}$ Untersuchungen hatte ergeben, dass z.B. $4 \%$ aller Rindshäute aus China positiv getestet wurden. Schafffelle aus Jugoslawien waren mit 30,9\% Ascoli-positiv, gefolgt von der Türkei mit 15,8 \%, Brasilien mit $15,3 \%$ und Spanien mit 14,1\%. Ziegenfelle waren in einem noch höheren Maße betroffen: Felle aus der Türkei und Marokko waren je zu $44 \%$, aus Indien mit 37 $\%$ und aus Marokko mit $25 \%$ positiv getestet worden. ${ }^{28}$

\subsection{Milzbranduntersuchungen in Neumünster seit 1930}

Geht man von der Hypothese aus, dass der Rückgang der Milzbrandfälle entlang der mittleren Stör ab 1931 nicht durch die neue Kläranlage in Neumünster verursacht sein konnte, weil als Folge der Weltwirtschaftskrise kaum noch Rindshäute gegerbt wurden und Schafffellgerbereien verfahrensbedingt signifikant weniger Milzbrandprobleme hatten, dann ist zu untersuchen, ob, insbesondere für den Zeitraum nach dem 2. Weltkrieg, als die Lederindustrie für fast 20 Jahre wieder aufblühte, nicht das Pickelverfahren dafür sorgte, dass ein großer Teil der zweifellos auch weiterhin importierten Milzbrandsporen durch dieses Verfahren geschädigt oder abgetötet wurden, so dass sich ein Milzbrandverdacht überwiegend auf Boden oder Bodenschichten bezieht, die vor dem Pickelverfahren verunreinigt wurden. Der Fund virulenter Sporen in Bodenauffüllungen im Tal der Schwale, einen $\mathrm{km}$ unterhalb eines Industriegeländes, das zu Beginn des 20. Jahrhunderts als Rieselfeld mehrerer Lederfabriken diente - sowie die Funde virulenter Sporen in

\footnotetext{
${ }^{26}$ Vgl.: Fritz Adler: Die Entwicklung des deutschen Häutemarktes, Karlsruhe 1913, S. 135. Vgl. auch: Heinrich Siemons. Häute und Felle, 1962, Anlage 5a - 5c. Ferner: R. Trosdorf: Importhäute und Milzbrand - eine experimentell gestützte Studie. Diss. Vet. Med., Leipzig 1968. In Südamerika wurden durchaus auch sehr gute Häute angeboten, die aber in der Regel von britischen Fabriken aufgekauft wurden. Es handelte sich hierbei zumeist um sogenannte „Frigorifiques“, Rindshäute, die in den Großschlachtereien der Gefrierfleischfabriken und Fleischextraktfabriken (Maggi und LiebigGesellschaft) abgezogen worden waren.

${ }^{27}$ Vgl.: Willi Hausam: Die Bakteriologie in der Lederindustrie, Stuttgart 1946, S. 332 f.

${ }^{28}$ Vgl.: ebd., S. 103.
} 
den überdeckten Gerbgruben einer bereits 1927 stillgelegten Lederfabrik - würden zumindest mit dieser These korrelieren: bei allen anderen Untersuchungen betroffener Fließgewässer waren zwar alle Indikatorsubstanzen von Lederabfällen (Chrom, Arsen, Naphthalin) vorhanden, virulente Sporen aber konnten nicht nachgewiesen werden. ${ }^{29}$

Wissenschaftliche Untersuchungen zur Wirkung eines veränderten Gerbverfahrens wurden im Zusammenhang der Nutzung von Klärschlamm der Lederfabriken als Dünger zwischen 1930 und 1936 durchgeführt. ${ }^{30}$ Handelte es sich zunächst noch um eine Fragestellung, die der Gefahrenabwehr für die landwirtschaftlichen Unterlieger entlang der Stör dienen sollte, wandelte sich die Fragestellung ab 1933 dahingehend ab, ob und in welchem Maße der Dünger aus Klärwerken als ,Wertstoff im Rahmen der nationalsozialistischen Autarkiepolitik genutzt werden könne. Im Rahmen dieses Untersuchungsprogramms der Preußischen Landesanstalt für Wasser, Boden und Luft sollte daher nicht nur die Haltbarkeit von Milzbrandsporen, sondern auch anderer Krankheitskeime, insbesondere jener der Typhus- und Ruhrgruppe, die in kommunalen Klärwerken anfielen, im Klärschlamm und Boden getestet werden. Es war zwar bekannt, dass eine Desinfektion der Haut und damit eine Vernichtung der Sporen nicht möglich war. Am Ende der 1920er Jahre verbreitete sich jedoch das Pickelverfahren, mit dem die Häute vor der Gerbung und vor der Spaltmaschine angeschwellt wurden. Zufolge einer Untersuchung des staatlichen Veterinäramtes Potsdam in den Lederfabriken von H. G. Schmid und Wiese \& Söhne in Neumünster zwischen dem 23. und 28. August 1930 gelang es, alle 78 eindeutig milzbrandbefallenen Häute nach 68 Stunden mit einem verschärften Pickel im rotierenden Gerbfaß so zu behandeln, dass weder in der Haut, noch im Abwasser oder Bodensatz mikrobiologisch virulenter Milzbrand nachweisbar war. Die Abweichung gegenüber der gewöhnlichen Pickelflotte bestand darin, dass statt der üblichen $4501 \mathrm{HCl}(25 \%)$ und $453 \mathrm{~kg}$ Gewerbesalz pro $4,5 \mathrm{~m}^{3}$ nunmehr $5401 \mathrm{HCl}(25 \%)$ und $460 \mathrm{NaCl}$ pro 4,5 $\mathrm{m}^{3}$ Flotte gegeben wurde, durch diese Senkung des pH-Wertes drohte allerdings der Hautverlust. Nach der

\footnotetext{
${ }^{29}$ Im Rahmen des BMBF-Forschungsvorhabens: „Modellhafte Gefährdungsabschätzung und Sanierung ehemaliger Gerbereistandorte“, Projektlaufzeit 1992-1995, wurden von der Universität Hohenheim, Prof. Böhm, an 15 Beprobungsstellen in den Flußauen um Neumünster mikrobiologische Untersuchungen auf Milzbrandsporen im Boden durchgeführt. Virulente Sporen wurden mit Hilfe der Polymerasekettenreaktion (PCR) und Tierversuch in zwei Probenahmestellen ca. $1 \mathrm{~km}$ oberhalb der Einmündung der Schwale in die Stör nachgewiesen. Vgl.: BMBF-Forschungsvorhabens: Modellhafte Gefährdungsabschätzung und Sanierung ehemaliger Gerbereistandorte, Projektlaufzeit 19921995. H6: Standortbericht Auenprojekt, 05.06.1997, Bearbeiter Heinrich Kautzky, Ergebnisse der mikrobiologischen Untersuchungen S. 21f. (Dieser Teil des Forschungsberichtes wurde durch das Bundesumweltamt nicht veröffentlicht. Eine Kopie wurde dem Verfasser durch den Fachbereich IV, Natur und Umwelt, der Stadt Neumünster zur Verfügung gestellt.)

${ }^{30} \mathrm{Im}$ Anschluß an eine ausführliche Beschreibung der Rieselfelder und der Klärschlammkompostierung in Neumünster durch ORR Dr. Hailer 1933 begann zwischen diesem und seinem Vorgesetzten, dem Präsidenten des Staatlichen Untersuchungsamtes, Dr. Standfuß und dem Leiter de Preußischen Landesanstalt für Wasser-, Boden- und Lufthygiene, Prof. Dr. Bürger, eine entsprechende Diskussion, die 1936 in einem erfolglosen Projektantrag mündete. Vgl.: Bundesarchiv Koblenz, Abt. R 154, Nr. 755.
} 
Neutralisierung mit Soda wurden Proben für die mikrobiologische Untersuchung von der Haut, von der abgetropften Pickelflotte und von dem Bodensatz im Neutralisationsgefäß entnommen. Aus dem Neutralisationsgefäß wurden zur Kontrolle auf etwaige Keime nochmals nach 221, 290, 392 und 459 Stunden Bodensatzproben auf Milzbrandsporen untersucht. In keinem der Kultur- und Tierversuche mit den Proben von Haut, Pickelflotte oder Bodensatz konnten virulente Milzbrandsporen nachgewiesen werden. ${ }^{31}$ Das Pickelverfahren in der Rindsledergerbung hatte sich jedoch nicht wegen dieser keimtötenden Wirkung durchgesetzt, sondern wegen einer Veränderung des Ledermarktes: Leder wurde immer seltener nach Gewicht verkauft, so dass die erzeugte Lederfläche von entscheidender Bedeutung wurde. Da eine Rindshaut in zwei Flächen, einen Fleischspalt und einen Narbenspalt, aufgetrennt werden kann, wurde die bereits im 18. Jahrhundert erfundene Spaltmaschine weiterentwickelt und industriell eingesetzt. Voraussetzung dafür war das Pickelverfahren, bei dem sich die Zellen der Ober- und Unterhaut so prall füllen, dass die nasse schlüpfrige Haut in der Maschine dem Messer nicht ausweichen kann.

Obgleich Felle von Schafen und Ziegen beim Import meist hochgradig mit Milzbrandsporen verunreinigt sind, ist auffällig, dass in den Schafslederfabriken zwar viele Arbeitskräfte infiziert wurden, im Abstrom des Abwassers aber dennoch kaum Milzbrandinfektionen auftraten. Für diese Tatsache, die nicht nur für drei Betriebe Neumünsters, sondern auch für andere Fellgerbereien und seit dem Beginn der zentralen Wollwäscherei auch für die Wollspinnereien und Tuchmachereien zutrifft, bietet folgende These, die sich wiederum auf die Verfahrenstechnik bezieht, Argumente: Die Industrialisierung der Tuchmacherei basiert auf dem massenhaften Import von Wolle aus Übersee. Diese Wolle ist im gleichen Maße mit Sporen besetzt wie die oben genannten Schafshautimporte. Durch den zentralen Einkauf weniger großer Wollkämmereien in den Hafenstädten Hamburg und besonders Bremen und die dortige Wollwäscherei werden die Sporen aus der Wolle gewaschen. Sie müßten daher, wie auch die Sporen aus der in Neumünster als Nebenbetrieb der Gerbung betriebenen Hautwollwäscherei, im Abwasser auftreten. Ungewaschene Wolle besitzt einen hohen Fettanteil, an dem kleinste Partikel haften. Diese Eigenschaft wurde bereits in der griechischen Mythologie mit dem „goldenen Vlies“, in dem sich die Goldflitter kaukasischer Gebirgsbäche verfingen, beschrieben. Auch die Sporen haften an dem Wollfett, das wiederum ein begehrtes Nebenprodukt der Wollwäscherei war. Die Wollwäschereien unterschieden sich verfahrenstechnisch nur hinsichtlich der Größe von den oben beschriebenen chemischen Reinigungen, sie nutzten insbesondere Benzine sowie später nicht entzündende CKW, um das Fett aus der Wolle zu lösen. ${ }^{32}$ Mit Hilfe von Filtern wur-

\footnotetext{
${ }^{31}$ Vgl.: ebd., Bericht Dr. Standfuß vom Staatlichen Veterinär-Untersuchungs-Amt Potsdam vom 1. Dezember 1930 an den Minister für Landwirtschaft, Domänen und Forsten.

32 Vgl.: Klaus Schlottau (Histinvest): Branchenblatt Wollwäscherei. In: Altlastenleitfaden SchleswigHolstein, Band II, herausgegeben vom Landesamt Natur und Umwelt des Landes SchleswigHolstein, Kiel-Flintbek, 2003.
} 
den die Schwebstoffe aus den gelösten Fetten getrennt und durch Destillation wurde dann das Lösungsmittel zurückgewonnen. Die Filter und auch der Destillationsrückstände besitzen einen so hohen Brennwert, dass sie in den Kesselanlagen der Wollwäschereien verbrannt wurden. Das Verfahren der Lanolingewinnung im Nebenbetrieb der Wollwäscherei sorgte daher seit dem Ende des 19. Jahrhunderts kontinuierlich für eine Vernichtung von importierten Sporen.

Seit 1891 entstand jedoch eine weitere Quelle für den unbeabsichtigten Import von Milzbrandsporen. Nach dem Rücktritt des Reichskanzlers Otto von Bismarck wurde die von ihm eingeführte Schutzzollpolitik für landwirtschaftliche Güter durch den Nachfolger, Caprivi, aufgehoben. Als Folge entstand innerhalb weniger Jahre ein stark expandierender Handel mit Futtergetreide, insbesondere russischer Gerste aus dem Schwarzmeergebiet. Hinzu kamen sehr große Mengen an Ölsaaten für die aufstrebende Margarineindustrie, deren Abfälle zusammen mit dem Getreide und Schlachthofabfällen, Knochenmehl, getrocknetem Blut etc. als Mastfutter an die in Norddeutschland entstehenden Schweinemastbetriebe abgegeben wurden. Getreide und Ölfrüchte sind nicht der Lebensraum von Milzbrand. Die Sporen wurden aber in den Eisenbahnwaggons, besonders in den Laderäumen der Schiffe durch den Staub, der von vorheriger Ladung noch vorhanden war, verteilt. Als Folge dieser Entwicklung wurden vor dem Ersten Weltkrieg täglich Schlachtschweine in den Großschlachthöfen Hamburgs und Bremens aussortiert. Noch 1957 basierte eine Milzbrandepidemie in Schleswig-Holstein auf dem Import von Knochenmehl, das während des Transports mit Sporen verunreinigt wurde. ${ }^{33}$ Eine weitere Folge aber bestand darin, dass sich das jahreszeitliche Aufkommen der Milzbrandinfektionen von einer Frühsommererkrankung zu einer Stallfütterungskrankheit, die die Schweine ganzjährig und die Rinder im Winter betraf, wandelte.

Diese signifikante Verschiebung scheidet neuzeitliche von historischen Milzbrandepidemien: mit der Einführung von Kraftfutter zur Erhöhung der Milchoder Fleischleistung seit dem ersten Jahrzehnt des 20. Jahrhunderts trat eine neue importierte Gefahrenquelle auf: Knochen-, Fleisch-, Blut- oder Fischmehl, das aus den Kolonien oder aus unterentwickelten Ländern importiert wurde, um hier in Großmühlen mit Soja- oder Ölkuchen und Getreide und Mineralien vermischt und gemahlen zu werden. Der überwiegende Teil der in den 1930er Jahren untersuchten Knochenmehlproben war Ascoli-positiv, so dass davon ausgegangen werden kann, dass Kraftfutter oder Kraftfuttervorprodukte, die ohne vorherige Desinfektion eingeführt wurden, in den Stallungen, in denen sie verfüttert wurden, zu Milzbrand führten. Da die Einfuhr von Futtermittelzusätzen und der Einsatz von Kraftfutter fast zeitgleich zum Massenimport von Häuten für die Lederindustrie und von Wollen für die Tuchindustrie liegt, kann, angesichts der Tatsache, dass der neuzeitliche Milzbrand in Schleswig-Holstein sich empirisch als eine Stallinfektion

33 Vgl.: C. Piening: Milzbrandinfektion durch Futtermittel während der Stallperiode 1957/58 in Schleswig-Holstein. In: Berlin-Münchener tierärztliche Wochenschrift 1958, S. 474. 
erwies, und verfahrensbedingt die Leder- und Wollindustrie zunehmend für eine Beseitigung der Milzbrandsporen sorgten, die These aufgestellt werden, dass ein überwiegender Teil der Milzbrandfälle seit den 1920er Jahren auf Kraftfutterverunreinigungen zurückging.

Die Trennung von neuzeitlichem und historischem Milzbrand wirft die Frage nach der Geschichte des Milzbrandes nochmals auf. Einleitend wurde der Dissertation von Thomas Lottermoser, dem aktuellen Forschungsstand, zugestanden, die Geschichte dieser Krankheit dargestellt zu haben. ${ }^{34}$ Der Autor stellt in der Tat auch den Zusammenhang von Futtermitteln und Milzbrandhäufung dar, ohne daraus aber die Charakteristika der historischen wie auch der aktuellen Milzbranderkrankung abzuleiten: Es handelt sich, da die Krankheit in Deutschland nicht heimisch ist, aber die Sporen offenbar über viele Jahrzehnte oder Jahrhunderte virulent bleiben, um menschliche Tätigkeiten des Akkumulierens, des Wiederinverkehrbringens und des verfahrensbedingten Vernichtens von Sporen, die die Geschichte der Krankheit bestimmten. Hierin unterscheidet sich der Milzbrand auch von den Viehseuchen, deren Erreger außerhalb des Körpers nicht lebensfähig sind und daher auch nicht akkumuliert werden können.

Milzbrand ist daher schon in der Vergangenheit immer zu den spontan entstehenden, örtlich immer wieder auftretenden Seuchen, „Enzootien“, gezählt worden. In Unkenntnis des Erregers wurden daher bestimmte topographische Eigenschaften und daraus resultierende jahreszeitliche Seuchenausbrüche angenommen. ${ }^{35}$ Angesichts der Vielzahl von möglichen Seuchenerregern und einer gewaltigen Zahl von 200 Millionen allein in Kontinentaleuropa verendeter Tiere im 18. Jahrhundert ist es schwer, Milzbrand von anderen Seuchen zu trennen. Eine Retrodiagnostik ist angesichts der vielfältigen Beschreibungen der Symptome und regional abweichender Bezeichnungen ebenfalls nicht möglich. Verwechslungen z. B. mit der Lungenseuche, der Ruhrseuche und der Rinderpest machten den Zeitgenossen die Diagnose schwer. ${ }^{36}$

\footnotetext{
34 Vgl.: Lottermoser 1998.

35 Vgl. hierzu insbesondere: Hermann Wald: Das Vorkommen und die Entstehung des Milzbrandes. Gekrönte Preisschrift der Frage: „Tritt der Milzbrand in manchen Oertlichkeiten gar nicht, und in welchen regelmäßig oder häufig auf; und ist aus den physikalischen Bedingungen solcher Oertlichkeiten auf die Natur der Krankheit zu schließen?", Halle 1862. Wald bezieht sich besonders auf Brandenburg und das dortige Gewässernetz der Havel, das den Ausbruch der Krankheit miasmatisch hervorruft. Auch J. Feser, der den Milzbrandbazillus und die Sporen bereits kennengelernt hatte, argumentiert in seiner Veröffentlichung „Der Milzbrand auf den oberbayerischen Alpen“, München 1877, noch mit geologischen Beschreibungen und statistischen meteorologischen Daten. Vgl. insbesondere die Tabellen S. 216-222. Vgl. Auch: G. C. Haubner; Handbuch der Veterinär-Polizei, Dresden 1869, S. 280.

36 Beispielhaft für die Unsicherheit ist z.B. „Ueber den Milzbrand. Zur Belehrung und Warnung für die Viehbesitzer und Landleute. Publicirt auf Befehl des Senats, Gedruckt bey Joh. Aug. Meißner, Hamburg im September 1830.
} 


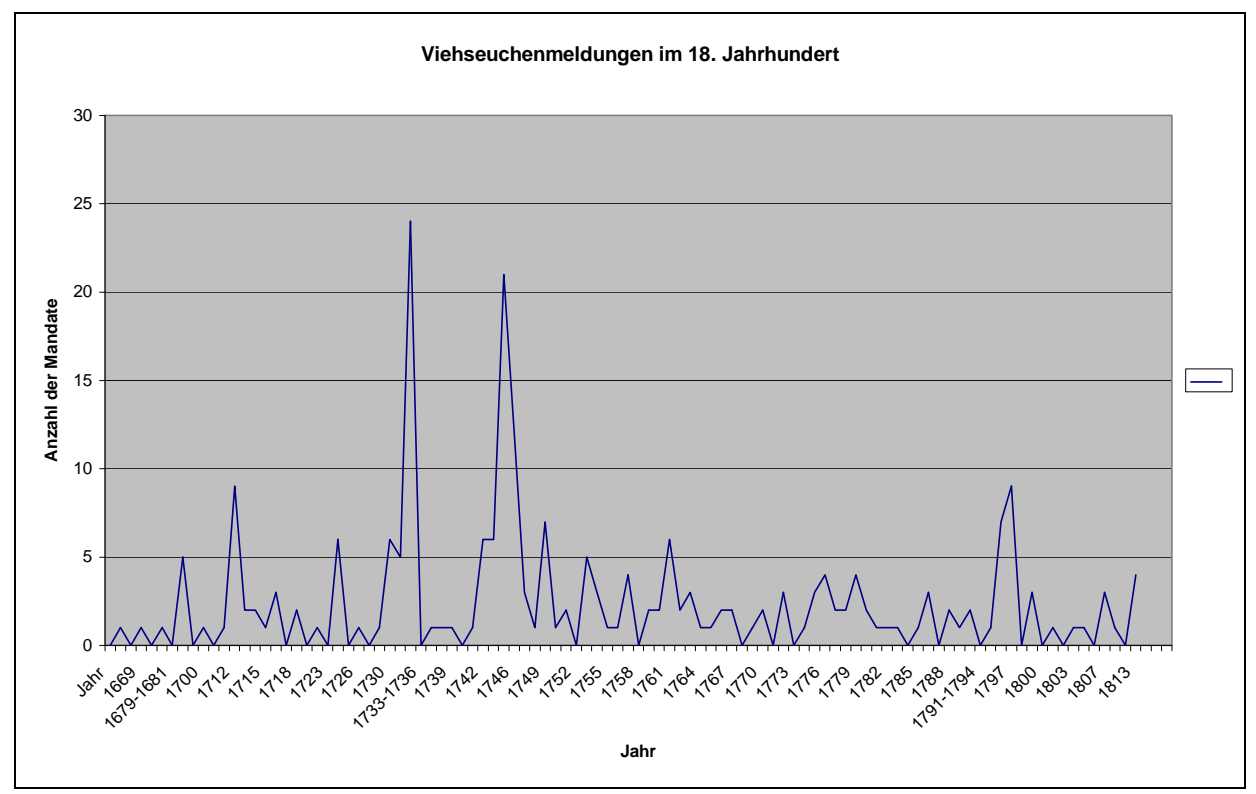

\section{Abbildung 1: Viehseuchenjahre des 18. Jahrhunderts. ${ }^{37}$}

Unterscheidungen zwischen den einzelnen Viehseuchen sind dennoch möglich, weil der Milzbrandbazillus ohne Ausnahme alle Warmblüter infiziert. Zeitgenössische Begriffe wie ,Rinderpest“ oder ,Hornviehseuche“ oder ,Pferdeseuche“, ,Drehkrankheit der Schafe' bringen zum Ausdruck, dass jeweils nur eine Tiergattung betroffen war. Es handelte sich mithin eindeutig nicht um Milzbrand, weil diese Krankheit Pferde, Rinder, Schafen, Ziegen, Rotwild, Schweine und Menschen ohne Unterschied betraf. Werden folglich in einer Quelle Todesfälle über die Gattungsgrenzen hinaus beschrieben, wird es sich mit hoher Wahrscheinlichkeit um Milzbrandinfektionen gehandelt haben. Unterscheidungen der genannten Art lassen sich z.B. über Zeitungsmeldungen der folgenden Art analysieren: "Wir haben hier seit kurzem eine Krankheit unter der Pferden und dem Hornvieh, die ich in ihrem Anfange ein hitziges, und in ihrem Fortgange ein faulendes Fieber nennen kann. Man fand beim todten Vieh hauptsächlich die Milz verbrannt (...)" 38

\footnotetext{
37 Die Darstellung basiert auf der Auswertung von 238 deutschsprachigen Seuchenmandaten und veröffentlichten Seuchenpredigten des 18. Jahrhunderts. Sie ist damit zwar noch unvollständig und auch quellenkritisch zu hinterfragen, gibt aber eine orientierende Übersicht, dass auch innerhalb dieses Seuchenjahrhunderts Jahrzehnte ohne Seuchenzüge vorkamen. Regional auffällig ist, bei einer Zuordnung der Mandate auf heutige Bundesländer, dass Bayern mit 50 Belegen weit vor Brandenburg (23), Niedersachsen (22), Sachsen (19), Mecklenburg-Vorpommern (19), Hessen (15), Nordrhein-Westfalen (15) und Sachsen-Anhalt (13) unter den Flächenländern führend ist. Dass aber der Stadtstadt Hamburg mit 10 Meldungen noch vor Schleswig-Holstein (8) liegt, ist wohl dem Handel geschuldet.

38 Hamburgischer Relations-Courier, Jahrgang 1768, Ausgabe 79. Brief aus Zürich. Für die Analyse wurden bislang, neben dem Relations Courier, die Hamburgischen Adreß-Comtoir-Nachrichten und der Hamburgische unpharteyische Correspondent, durchgesehen.
} 


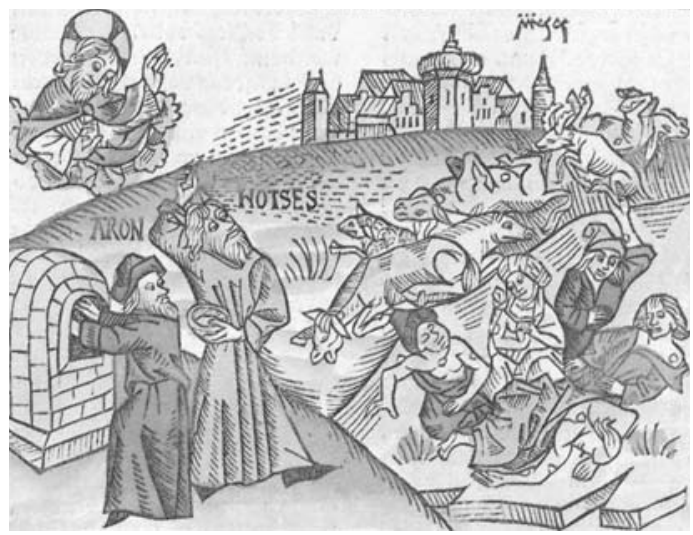

\section{Abbildung 2: Illustration der biblischen Beulenpest, Straßburg 1485. 39}

Auf der Abbildung 2 sind, neben den von der Pest gezeichneten Menschen, auch deren Haustiere sowie ein Hirsch abgebildet. Die Erfahrungen des Künstlers mit der zeitgenössischen Pest flossen in den Entwurf ein, so dass er das gemeinsame Sterben der Menschen, der Haustiere und des Wildes dokumentiert. Die zeitgenössischen Erfahrungen widersprechen allerdings den heutigen medizinischen Kenntnissen, die eine Infektion der Haustiere durch den Pesterreger ausschließt. In der Medizingeschichte wird seit Jahrzehnten bezweifelt, dass allein der Pesterreger für die in regelmäßigen Abständen von 10-15 Jahren auftretenden und sich mit einer Geschwindigkeit von ca. $8 \mathrm{~km}$ pro Tag ausbreitenden Epidemien des ausgehenden Mittelalters und der Frühen Neuzeit verantwortlich war:

„Der Milzbrand hat mit der Pest einige Symptome gemein; die Letalität bei Milzbrand ist hoch; er ist - anders als die Pest - eine hochkontagiöse Krankheit; ja selbst die klimatischen Bedingungen der 1340er Jahre würden eher zu Milzbrand passen als zur Pest. Milzbrandsporen halten sich am Boden und können durch Winde emporgetragen werden. Das könnte erklären, warum in den Alpen selbst in den oberen Höhenregionen ganze Bauernhöfe ausstarben. “40

Als Vektoren der Epidemien können die Ratten und deren Flöhe nur im Nahbereich, also in den Städten und Dörfern auftreten, weil sie nicht mehr als einen Kilometer pro Tag wandern, so dass der Mensch, seine Transportfahrzeuge und die wandernden Herden als Vektoren zu betrachten sind. In diesem Zusammenhang sind einerseits die großen Rindertrecks von den osteuropäischen extensiven Weidegebieten, die der Versorgung der westeuropäischen Bevölkerungszentren dienten und, andererseits, die wandernden Schafherden, die Transhumanz, zu nen-

\footnotetext{
39 Aus: Das große Sterben. Seuchen machen Geschichte, herausgegeben von Hans Wilderotter unter Mitarbeit von Michael Dorrmann, Ausstellungskatalog des Deutschen Hygiene-Museum Dresden, Berlin 1995, S. 97.

40 Manfred Vasold: Pest, Not und schwere Plagen. Seuchen und Epidemien vom Mittelalter bis heute, Augsburg 1999, S. 92.
} 
nen. Manfred Vasold nannte in der obigen Quelle insbesondere die Hochalpen, die unter starken Menschenverlusten litten und von Feser (1877) wissen wir, dass insbesondere die Almen stark vom Milzbrand betroffen waren. Die Verbreitung der Sporen durch den Wind würde eine Konzentration in windstillen Lagen bewirken. Die Wanderschäferei und die in den Alpen noch bis ins 18. Jahrhundert verbreitete „Küherei“, also die stetige Wanderung von Viehherden auf der Suche nach Mastweiden durch die Alpen (Jacobeit 1987), durchs französische Zentralmassiv,- oder auch über märkische Heiden - ist der wahrscheinlichere Grund für eine Verbreitung der Erreger, wie auch für die Konzentration auf den Almen, die im Mittelalter und in der Frühen Neuzeit noch keine Eigentümer hatten.

Schafe wurden selten als Mast- oder Milchschafe gezüchtet. Im Vordergrund stand stets die Nutzung von Schafswolle für die Tuchmacherei. Jedes ausgewachsene Schaf ergab pro Jahr fünf bis sechs kg Wolle. Vor der Schur mußten die Schafe gewaschen werden, um Erde, Kot, Urin und verfangene Pflanzenfasern zu entfernen. Dieses Ereignis fand regelmäßig im Frühjahr nach der „Kalten Sophie“ (15. Mai) und vor der sogenannten „Schafskälte“ (11. Juni), zwei meteorologischen Singularitäten, statt, um die nackten Schafe nicht ungeschützt den letzten Nachtfrösten auszusetzen. Bevor die industrielle Wäsche in den oben beschriebenen Wollwäschereien durchgeführt wurde, nutzten die Hirten und deren Helfer flache Gewässer mit geringer Strömung oder flache Seen, die sich bereits durch die ersten Schönwetterperioden des Frühjahrs aufgewärmt hatten. Stand ein Gewässer nicht zur Verfügung, wurden Gräben oder trogartige Holzkonstruktionen errichtet. Die Hirten trieben die Schafe einen Tag vor der Wäsche zum Einweichen in das Gewässer, um dann am nächsten Tag, im Wasser stehend, ein Schaf nach dem anderen erneut zu weichen, dann die Wolle mit den Händen kräftig auszudrücken und anschließend das Tier nochmals zu spülen. Nach der Wäsche, die viele Stunden oder gar Tage andauerte, benötigten die Tiere bis zu vier Tage, bevor die Wolle trocken genug zum Scheren war. Während dieser Zeit konnten die Wäscher die Unterkühlung und den Schnupfen, den sie sich trotz ständig kreisender Schnapsflasche meist zugezogen hatten, auskurieren. Im Frühsommer sind in der vorindustriellen Zeit regelmäßig Milzbrandepidemien auf den Weiden entlang der Flüsse ausgebrochen. Es ist daher zu vermuten, dass, obgleich keine Seifen eingesetzt wurden, bei der Schafswäsche aus den ausgewrungenen Erden und dem aufgelösten Kot so viele Sporen freigesetzt wurden, dass das Vieh sich an der Tränke oder auch an den Ufern unterhalb der Schafswäschen infizierte. Als Vektor der Ausbreitung fungiert in diesem Fall das Gewässer: bei den Seen setzten sich die Sporen unmittelbar am Ort des Geschehens und am Ufer ab; Fließgewässer transportieren die Sporen mit der Strömung in eine Stillwasserzone, wo die Sporen sedimentieren - solche kleinen Buchten mit Flachwasser wurden gerne als Tränken aufgesucht. Mit der "Schafskälte" beginnt daher auch das unerklärlich ortsfeste Milzbrandereignis jeden Jahres. Als Vektoren der Milzbrandverbreitung sind im bisherigen Gang der Argumentation folgende aufgetreten: 
Wandernde Schafherden sowie Kuhherden in prekären und eigentumsrechtlich noch nicht verbindlich vergebenen Gebieten (Alpenraum, Mittelgebirge, Heiden, Wüstungen);

Die Wollwäsche am lebenden Schaf sowie die Wäsche der Wolle in der Tuchmacherei und besonders in der frühen Tuchmanufaktur, wo in größerem Rahmen auch Waschhilfsstoffe zugesetzt wurden, damit sich das Wollfett zum Teil löste, was eine Voraussetzung für gleichmäßiges Spinnen ist - das Lösen des Fettes befreit einen großen Teil der Sporen;

Der Fernhandel mit Rindern von Ost nach West und Nord nach Süd; die "Ochsenwege“ kreuzten sehr häufig die festliegenden Routen der Wanderschafe und wurden zumeist auch als Heerstraßen und Handelsweg genutzt. Die Folge war ein stetiger Austausch von allen möglichen Seuchenerregern und eine Verbreitung in jede Richtung. Die Grenzquarantänestationen, Brandzeichen und die Begleitscheine, die z.B. Preußen und die Schweiz im 18. Jahrhundert einführten, halfen allerdings auch nicht besser als der über $2.000 \mathrm{~km}$ lange „Cordon sanitaire“ von der Adria bis in die Bukovina, den die Habsburger u.a. auch gegen die Einschleppung von Seuchen aufgebaut hatten.

Der Import spanischer Merinoschafe in großem Umfang seit dem 17. Jahrhundert zur Verbesserung der Wollqualität heimischer Rassen. Die iberischen Schafe waren noch im 20. Jahrhundert zu fast dreißig Prozent mit Sporen behaftet oder vom Milzbrand infiziert.

Der Handel mit Wolle, Häuten, Fellen und Haaren, also allen Stoffen, die in zeitgenössischen Seuchenmandaten entsprechend der miasmatischen Theorie der Kontagion als ,giftfangend“ bezeichnet wurden;

Die Verarbeitung der Häute und Felle in Gerbereien: die Gerberei beginnt immer mit einer intensiven Wäsche und dem Einweichen der Häute und Felle dabei sind den Gerbern nicht nur gelegentlich die „Felle weg geschwommen“, sondern regelmäßig auch alle Schmutzstoffe und Keime, die an der Haut und den Haaren hingen;

Als weitere Quellen verbleiben die Abfallprodukte der Tiere, also der Handel mit Knochen, Knochenschrot, Knochenmehl, Talg, Unschlitt, Blut und Därmen sowie deren Verarbeitung in verschiedenen Gewerben, z.B. Seifensieder, Kerzenzieher und die Mühlengewerbe einschließlich der frühen Kraftfutter- und Düngerherstellung, deren akkumulierende Funktion noch zu klären ist;

Als neuer Vektor tritt das Transportgewerbe mit Eisenbahnen und Frachtschiffen, deren Laderäume nach dem Transport von Häuten oder Wollballen nicht gereinigt wurden, bzw. die diese Fracht zusammen mit Getreide oder Ölsaaten im gleichen Frachtraum beförderte, hinzu.

Im Anschluß an die Darstellung der Ausbreitungsvektoren ist, bevor die Frage der Wiederinverkehrbringung erörtert werden kann, zunächst zu klären, wo und unter welchen Umständen sich Milzbrandkeime derart ansammeln können, dass ihre Konzentration mutmaßlich weit über der allgemeinen Streuung, aber auch über jener Konzentration, die ein einzelnes Tiergrab hat, liegt. Die Sporen wieder 
in den Umlauf zu bringen und ihnen damit das Auskeimen zu ermöglichen, setzt voraus, dass es Orte gibt, die in besonderem Maße Milzbrandsporen über Jahre, Jahrzehnte und Jahrhunderte akkumulieren - da die anderen Seuchenerreger spätestens nach einigen Monaten oder einem Frost nicht mehr virulent sind, ist die Verwendung des Begriffs „akkumulieren“ in diesem Fall auch für Keime ausnahmsweise gerechtfertigt. Solche Orte sind regelmäßig:

Abdeckereien, Wasenplätze, Schindanger, Tierfriedhöfe, Luderplätze und Wolfsgärten oder ähnliche Orte, die ausschließlich der Bestattung von Tierkadavern, deren Lagerung oder Verarbeitung dienen;

Langjährig genutzte Schafpferche, die im Winter als Standquartier von Wanderherden dienten;

Quarantänestationen entlang der Landesgrenzen sowie die Viehquarantänestationen der Hafenstädte;

Standorte der Ledererzeugung mit auffallend hoher Konzentration, z.B., unter anderen: Nördlingen, Reutlingen, Pößneck, Gera, Prag und Basel in der Frühen Neuzeit und Backnang, Offenbach, Straßburg, Neumünster, Elmshorn in der Industrialisierung. Jeder Fabrikstandort sowie deren Rieselfelder und Klärschlammaufhaldungen mit Vorflutbereichen ist in hohem Maße von Milzbrandsporen durchsetzt.

Standorte der Tuchindustrie und der Wollwäscherei. Signifikant zum Beispiel in der Umgebung Göppingens und Bradfords (England) am Ende des 19. Jahrhunderts, dort als „wool-sorters-disease“ bezeichnet sowie beim Ausbruch der Pest 1771/72 in Moskau, wo der Beginn in einer großen Tuchmanufaktur lag und binnen Tagen 800 der dort Arbeitenden starben;

Abfall- und Reststoffhandel, z.B. Lumpenhandel sowie die Deponien der nicht mehr wirtschaftlich nutzbaren Stoffe, Müllhalden oder, euphemistisch, „Reststoffdeponien". In den Entwicklungsländern gibt es daher einen berufs- und standesspezifischen Milzbrand, als „,rag-pickers-disease“ bezeichnet, der überwiegend bei den Personen vorkommt, die die Halden der Großstädte nach Verkäuflichem durchsuchen.

Von den Lumpensammlern gibt es einen schnellen Zugang zur Diskussion über die Wiederinverkehrbringung von Milzbrandsporen

Gefallene Tiere wurden in der Regel von amtlichen bestellten Personen, den Abdeckern oder Schindern beseitigt. Bis zur Mitte des 18. Jahrhunderts hatten sie die Aufgabe, die Kadaver zentral auf dem Schindanger oder Wasenplatz zu verarbeiten oder sie als Luder z.B. in einen der „Wolfsgärten“ zu schleppen, damit die nach dem 30-jährigen Krieg umherschweifenden Rudel in diesen Fallen bequem niedergeschossen werden konnten. Erst seit der Mitte des 18. Jahrhunderts hatte man den Milzbrand als besondere Krankheit erkannt und verbot jedweden Transport des Kadavers - er sollte an Ort und Stelle unabgedeckt mit zerschnittener Haut mindestens in einer Tiefe von ca. 1,5 m bestattet werden. Als weitere Schutzmaßnahme sollten Findlinge auf das Grab gerollt und das Areal mit Dorngesträuch 
bepflanzt werden. ${ }^{41}$ Ganz offensichtlich hatten die zuständigen Amtsträger - im Gegensatz zur akademischen Medizin - erkannt, dass von den Kadavern und deren abtropfenden Körperflüssigkeiten eine starke Gefährdung ausging. Die Verschleppung oder die Nutzung der Kadaver wurde mit Leib- und Lebensstrafen geahndet. Jahrhundertelang jedoch hatten Abdecker auch die Milzbrandkadaver auf ihren Betriebshof gebracht, die Kadaver aufgebrochen, die Häute verkauft, das Fett verkauft; Fleisch an die Jagdhunde der Herren verfüttert - manchmal auch selber gegessen - die Reste zu Unschlitt verkocht und die Knochen vergraben. Die Abdecker lebten vom Verkauf der Abfallprodukte und von den Gebühren, die die Bauern zu zahlen hatten: Viehseuchenjahre waren ökonomisch glänzende Jahre. Da die Betriebsflächen am Rande der Stadt oder des Amtssitzes beschränkt waren, hatten die Abdecker das Recht, in regelmäßigen Abständen, frühestens jedoch nach zehn Jahren, die Knochen wieder aus der Erde zu holen, um eine Teilfläche neu zu belegen.

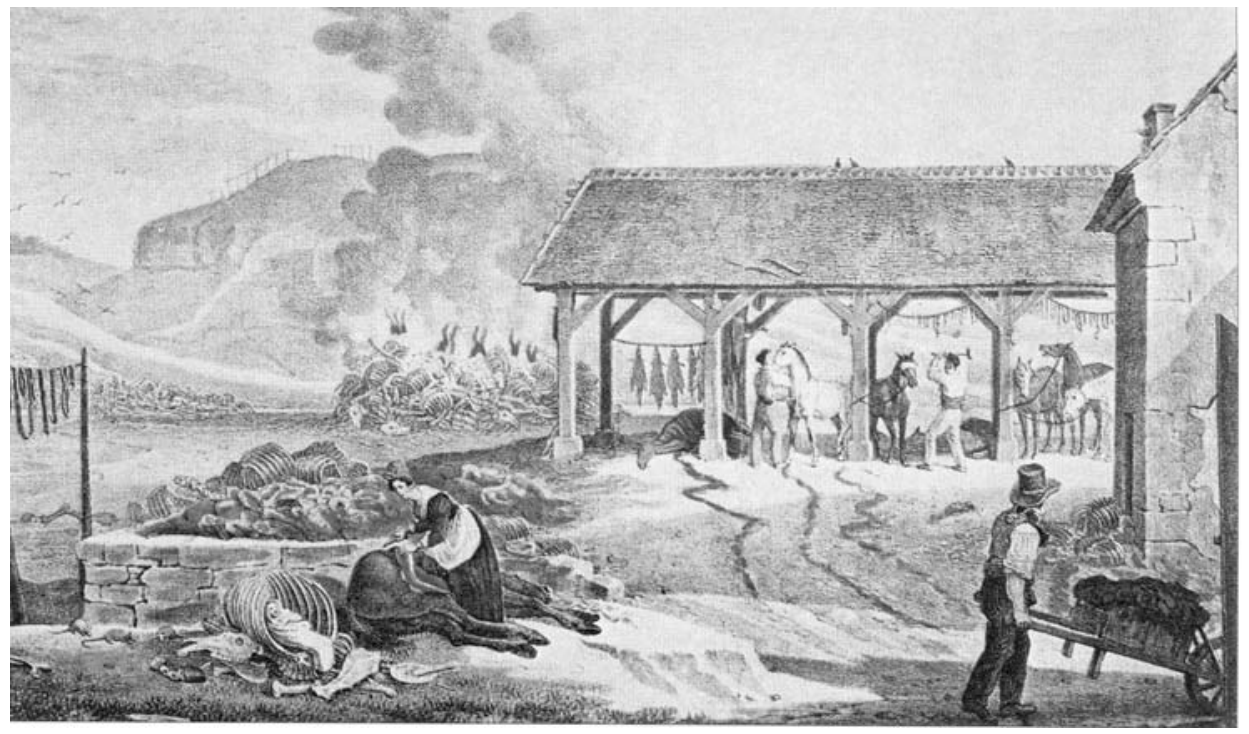

Abbildung 3: Ansicht der Abdeckerei auf dem Butte-Chamotte in Paris 1820. ${ }^{42}$

Bei Erdarbeiten treten auch heute noch die meisten Milzbrandinfektionen auf: sobald die Knochen wieder ans Tageslicht gezerrt wurden, begann eine neue Infektionswelle, die ihren Ausgang auf dem Wasenplatz hatte - ob auch die Pestzyklen

\footnotetext{
${ }^{41}$ In diesem Zusammenhang stellt sich die Frage, wie viele Milzbrandgrabstätten, die mit Findlingen eingefasst wurden, inzwischen als heimatkundlich bedeutende Hügelgräber unserer Ahnen geführt werden?

42 Aus: Angela von den Driesch und Joris Peters: Geschichte der Tiermedizin. 5000 Jahre Tierheilkunde. 2. Aktualisierte und erweiterte Aufl., Stuttgart/New York 2003, S. 152.
} 
mit diesem Flächenrecycling in einem Zusammenhang stehen, kann an dieser Stelle nicht beantwortet werden. Lange Jahrzehnte wußte niemand etwas mit den blanken Knochen anzufangen, so dass sie meist verbrannt wurden und gelegentlich als Knochenkohle, einem Vorläufer der Aktivkohle, sowohl in der Druckfarbenherstellung als auch für Raffinationszwecke z.B. im Zuckergewerbe, eingesetzt wurde. Frische und ausgegrabene Knochen wurden seit ca. 1770 in der Landwirtschaft als Dünger eingesetzt. Zu diesem Zweck wurden sie zerschlagen oder in Mühlen zerstampft und dann als Granulat in die Äcker eingepflügt. ${ }^{43}$ Der Nährwert der Knochen bemaß sich am Phosphorgehalt, der bei etwa zehn Prozent liegt. Neben dem Stallmist, dem Mergel sowie dem seit dem 17. Jahrhundert eingesetzten Woll- und Lumpenhackschnitzel, wurde das Knochenmehl zum wesentlichen Dünger der englischen Landwirtschaft des 18. Jahrhunderts. Auf dem Kontinent begann die Düngung mit Knochen erst zu Beginn des 19. Jahrhunderts, so dass ab 1820 eine große Zahl von Knochenmühlen entstand und jeder der oben genannten Vergrabungsplätze, aber auch große Schlachtfelder, z.B. Waterloo, wieder aufgegraben wurden. Die grobe Struktur des Knochendüngers ließ ihn als Depotdünger über einen Zeitraum von etwa fünf Jahren wirken. Für einen schnellen Ernteerfolg sorgte erst die Erfindung des Superphosphatdüngers in der Mitte des 19. Jahrhunderts, bei dem es sich um Knochenmehl handelt, das mit Schwefelsäure aufgeschlossen wurde, so dass die Mineralien im Boden unmittelbar aufgenommen und in einer Vegetationsperiode wirken konnten. Die Schwefelsäure wurde zu diesem Zeitpunkt in großen Mengen zu geringen Kosten von der aufstrebenden Montanindustrie angeboten. Aus der Montanindustrie entstand dem Superphosphat am Ende des 19. Jahrhunderts mit dem Thomasmehl, benannt nach dem Erfinder der metallurgischen Innovation des Windfrischens phospathaltiger Eisenerze im Thomas-Konverter, Sidney Thomas, ein Hauptkonkurrent, der die Landwirtschaft massenhaft mit billigem Phosphor-Kalk-Dünger aus der Schlacke der Hüttenindustrie versorgte.

Obgleich am Ende des 19. Jahrhunderts Knochenmehl nicht mehr zu konkurrenzfähigen Preisen verkauft werden konnte, stieg der Import von Knochenmehl in das Deutsche Reich kontinuierlich an. So wurden im Hamburger Hafen 1897 mehr als 30.000 t Knochenmehl allein aus Indien eingeführt. Warum? Bereits ab 1830 ist die Verwendung von Knochenschrot als Geflügelfutter nachweisbar. Für Geflügel ist der Milzbrandbazillus keine Gefahr, weil die höhere Körpertemperatur, ca. $41^{\circ} \mathrm{C}$, den Bazillus, der sich aus der Spore entwickelt, abtötet. Knochenschrot wurde in der Geflügelmast als Substitut für Getreide genutzt, nachdem die Nutzung des Knochenmehls als Dünger vom Thomasmehl abgelöst worden war, wurde eine neue Nutzung in der Kraftfutterindustrie gefunden. Die Kraftfutterindustrie siedelte sich vorwiegend in den drei größten deutschen Getreideimporthäfen des 19. Jahrhunderts, Hamburg, Bremen und Elmshorn, an, weil der Hauptbestandteil des Kraftfutters importierte Gerste war. Ein weiterer Bestandteil waren

${ }^{43}$ Vgl.: Krünitz, Bd. 41, S. 555f. Für den Hinweis auf Waterloo danke ich Prof. Dr. Ulrich Troitzsch. 
Ölkuchen, die direkt von den großen Ölmühlen und Magarinefabriken Norddeutschlands bezogen wurden. Salze kamen aus den nahegelegenen Revieren um Staßfurt und Süßungsmittel lieferten die Schnitzel- und Melassereste der Zuckerraffinerien. Phosphor wurde durch das Knochenmehl beigesteuert und Proteine lieferten die Abfälle der großen städtischen Schlachthöfe. Als Folge dieser Konzentration von Kraftfuttermühlen entstanden in Norddeutschland die Schweinemast im Großbetrieb sowie eine intensive Milchwirtschaft. Die Schweinemast hatte in den vorherigen Jahrhunderten immer weit hinter der Rinderhaltung zurückgestanden, weil Menschen und Schweine auf das gleiche Nahrungsangebot angewiesen sind - selbst die jahreszeitliche Eichelmast musste ausfallen, wenn in Hungersnöten das Brotgetreide mit Eichelmehl gestreckt wurde. Da die Menschen aber nicht bereit waren, Knochenmehl, Blutmehl, Fleischmehl oder Tierkörpermehl sowie gemahlene Federn, Eingeweide und Hühnerfüße als Nahrung zu akzeptieren, konnten diese Stoffe über den Umweg des Schweinemagens wieder in den Verkehr gebracht werden und dienten letztlich den Arbeiterfamilien des 19. und 20. Jahrhunderts als bezahlbare Fleischration. Da Schweine Allesfresser sind, gab es keine Probleme, die Ekelstoffe in das Futter zu mischen. Anders bei den Wiederkäuern, deren Fleisch- und Milchleistung mit dem Kraftfutter erhöht werden sollte: ihnen musste die Nahrungsergänzung mit Hilfe von Salzen und Zuckern erst schmackhaft gemacht werden. Die Folgen des Kraftfuttereinsatzes, in dem sich Sporen aus dem Import von Knochenmehl mit jenen aus dem Import von Getreide und Ölsaaten und jenen aus der Tierkörperverwertung akkumulieren, sind einleitend am Problem des Milzbrandes in der Störniederung geschildert worden: der Milzbrand wurde von einer Weideerkrankung zu einer Futtermittelinfektion. Gefallene Tiere wurden wieder in den Kreislauf eingespeist, so dass sich das Gefährdungspotential weiter erhöhte. Bei näherer Betrachtung lassen sich Parallelen zum BSE-Skandal und zu den diversen Ekelfleischskandalen der Gegenwart erkennen.

Ausgangsbasis der obigen Darstellungen zum Knochenmehl war die Abdeckerei, auf deren Areal regelmäßig Flächen wieder frei geräumt werden mussten. Jede Erdbewegung auf Plätzen der Milzbrandsporenakkumulation konnte neue Infektionswellen entstehen lassen. Zu diesen Erdbewegungen gehören regelmäßig auch die, die von den Salpeterern ausgehen. Salpeterhaltige Mineralien sind in Deutschland und auch in Europa eine große Seltenheit, so dass England, die Niederlande und auch Frankreich diesen Stoff aus den Kolonien Süd- und Südostasiens beschafften. Salpeter wird seit dem Ende des Mittelalters für die Herstellung von Schießpulver und Sprengstoff benötigt, ist aber, da Stickstoff ein inertes Gas ist, nur in geringsten Mengen und großer Verteilung vorhanden. Im Urin ist es im Harnstoff vorhanden und bei der Zersetzung organischen Materials bilden sich Nitride, Nitrite und Nitrate, die im Regelfall von der Bodenvegetation schnell wieder aufgenommen werden. Salpeterer waren Sammler von Salpeter und besaßen landesherrliche Privilegien, die ihnen gestatteten, nicht allein die Salpeterausblühungen von den Ziegel- und Lehmmauern der Ställe zu kratzen, sondern gleich 
den ganzen Stall und den Untergrund des Misthaufen aufzugraben und aus dem Boden eine Salpeterplantage zu machen. Das Niederreißen von Ställen konnte der Landbevölkerung nur in äußersten Mangellagen, also während eines Krieges, dessen Dauer die Kapazität der Pulvermagazine überstieg, zugemutet werden. Regelmäßige und einträgliche Salpeterplantagen konnten hingegen auf den Wasenplätzen der Abdecker oder in den Schafpferchen der Guts- und Domänenschäfereien eingerichtet werden. Von den Salpeterplantagen, die zum Teil seit dem 18. Jahrhundert ortsfest betrieben wurde, indem Urin und frische Stallerden sowie Fleischabfälle dorthin transportiert wurden, ging daher eine dauerhafte Gefährdung durch Verschleppung und Wiederinverkehrbringung von Sporen aus.

Gewerbe, die den Milzbrand wieder in den Umlauf bringen, nutzen in der Regel einen Teil des tierischen Abfalls als Rohstoff oder bringen mit Milzbrandsporen verschmutzte Stoffe wieder in den Verkehr. Es handelt sich hierbei, neben den oben dargestellten Gewerben, von denen eine große Gefährdung ausging, um Folgende:

Zunächst um das Gewerbe der Bürsten- und Pinselmacher, die ihre Produkte aus Borsten und Haaren von Tieren herstellen. Für Aufsehen sorgte besonders eine Masseninfektion während des Ersten Weltkrieges, als einer amerikanischen Einheit ein Kontingent milzbrandverseuchter Rasierpinsel zugeteilt wurde.

Das Gewerbe der Leimsiederei aus Knochen und Knorpeln sowie Lederabfällen und Falzspänen. Als neuzeitliche Fortsetzung dieses Gewerbes kann die Gelantineherstellung für z.B. Fruchtgummis betrachtet werden;

Das Gewerbe der Seifensiederei, die Fette, Talg und innere Organe nutzten;

Das Gewerbe der Lichterzieher, die sich den Rohstoff mit den Seifensiedern teilten;

Das Gewerbe der Lumpen- und Sekundärrohstoffsammler, die zumeist auch die dezentral anfallenden Kleintierfelle sammelten;

Die Papiermacherei, in der Lumpen in großer Menge verarbeitet wurden, so dass sich hier als Berufskrankheit die sogenannte ,Hadernkrankheit', die häufig mit Milzbrand gleichgesetzt wird, ausbreitete.

\subsection{Milzbrandinfektionen der Arbeitskräfte}

Der Milzbrand der Lederarbeiter wurde erst 1943 als Berufskrankheit anerkannt. Insgesamt gab es z.B. im Deutschen Reich im Zeitraum von 1910 bis 19252107 Fälle menschlicher Milzbrandinfektion (Vgl. Tabelle 5). Davon entfielen allein 50 Prozent auf Arbeitskräfte in der Landwirtschaft, gefolgt von nur 26 Prozent in der Lederindustrie, die nur geringfügig über dem Anteil der Textilindustrie lag. In den Transportgewerben gab es dann lediglich noch die verbleibenden 7 Prozent. ${ }^{44}$ In Bezug auf die absoluten Zahlen hatte die Landwirtschaft mit Abstand das größte

\footnotetext{
44 Vgl.: Graf, 1950, S. 63 sowie Friedrich-Karl Baumhöfener: Der Hautmilzbrand - Untersuchungen zur Infektkette, Diagnose und Therapie. Diss. med. Kiel 1965, S. 19.
} 
Risiko einer Infektion. Relativ hatte allerdings die Lederindustrie das größere Risiko, weil dort sehr viel weniger Arbeitskräfte beschäftigt waren. Bezogen auf die Stadt Neumünster, in der die verursachende Lederindustrie konzentriert war, gab es z.B. von 1907 bis 1921 insgesamt 70 Milzbranderkrankungen der Arbeitskräfte, von denen 7 letal waren. ${ }^{45}$ Eine Folge war, dass in den Fabriken für alle Arbeitskräfte in der Wasserwerkstatt eine Trennung von Arbeits- und Straßenkleidung vorgeschrieben war, die einer Trennung in Schwarz- und Weißbereich vergleichbar war. ${ }^{46}$

Tabelle 5: Menschlicher Milzbrand nach Gewerbezweigen $1912-1963 .{ }^{47}$

\begin{tabular}{|c|c|c|c|c|c|}
\hline Jahr & Leder-BG & $\begin{array}{l}\text { Fleischerei- } \\
\text { BG }\end{array}$ & $\begin{array}{l}\text { Land- } \\
\text { wirtschaft- } \\
\text { BG }\end{array}$ & Summe & Verstorbene \\
\hline 1912 & 78 & 72 & 64 & 260 & 32 \\
\hline 1913 & 65 & 53 & 40 & 198 & 28 \\
\hline 1914 & 56 & 41 & 54 & 194 & 38 \\
\hline 1915 & 15 & 17 & 18 & 64 & 13 \\
\hline 1916 & 7 & 10 & 12 & 35 & 5 \\
\hline 1917 & 4 & 15 & 8 & 32 & 9 \\
\hline 1918 & 13 & 5 & 2 & 25 & 6 \\
\hline 1919 & 6 & 6 & 2 & 18 & 2 \\
\hline 1920 & 10 & 7 & 17 & 35 & \\
\hline 1921 & 22 & 9 & 43 & 80 & \\
\hline 1922 & 21 & 9 & 56 & 118 & \\
\hline 1937 & & & & 78 & 7 \\
\hline 1938 & & & & 88 & 10 \\
\hline 1939 & & & & 46 & 8 \\
\hline 1940 & & & & 50 & 12 \\
\hline $\begin{array}{l}1941- \\
1947\end{array}$ & & & & 0 & 0 \\
\hline 1948 & 2 & 0 & 0 & 2 & 0 \\
\hline $\begin{array}{l}1949- \\
1952\end{array}$ & 10 & 4 & 0 & 14 & 0 \\
\hline 1953 & 5 & 1 & 1 & 7 & 0 \\
\hline
\end{tabular}

45 Vgl.: Gerbereiabwasseranlage Neumünster, Neumünster 1922, S. 7.

46 Vgl.: Klaus Schlottau: Die Geschichte der Lederindustrie in Neumünster. Ein Beitrag zur Industrialisierung Schleswig-Holsteins, Neumünster 1991, S. 101.

${ }^{47}$ Für die Zahlen 1912 bis 1919, vgl. K. Nieberle: Der Milzbrand der Tiere und gewerbliche Milzbranderkrankungen beim Menschen. In: Urania, Heft 5, Jahrgang 1926-27, S. 138-142, hier S. 140. Vgl.: Baumhöfer (1965), S. 10 für die Zahlen 1932 - 1963. Vgl. weiterhin: Paul Graf: Der Milzbrand vom Standpunkt des Arztes aus betrachtet. In: Lederindustrie-Berufsgenossenschaft : Maßnahmen zur Verhütung und Bekämpfung von Milzbrand, Mainz 1950, S. 63. 


\begin{tabular}{|l|l|l|l|l|l|}
\hline 1954 & 0 & 1 & 0 & 1 & 0 \\
\hline 1955 & 1 & 2 & 4 & 7 & 0 \\
\hline 1956 & 3 & 2 & 3 & 8 & 0 \\
\hline 1957 & 2 & 3 & 1 & 6 & 0 \\
\hline 1958 & 2 & 3 & 0 & 5 & 0 \\
\hline 1959 & 3 & 1 & 1 & 5 & 0 \\
\hline 1960 & 2 & 1 & 2 & 5 & 0 \\
\hline 1961 & 2 & 0 & 1 & 3 & 0 \\
\hline 1962 & 5 & 0 & 1 & 6 & 0 \\
\hline 1963 & 0 & 0 & 0 & 0 & 0 \\
\hline
\end{tabular}

„Durch die aufkommende Lederindustrie kam zwar kein einheimischer, aber ausländischer Milzbrand mit den Rohhäuten aus dem Osten nach Neumünster herein. Unter den großen Stapeln der rohen Häute steckte immer einmal die Haut eines von innerem Milzbrand befallenen Tieres, der man die Krankheit nicht ansehen konnte. Unsere Gerber bekamen aus der Gerbbrühe keinen inneren Milzbrand, sondern Karbunkeln auf den unbedeckten Stellen der Haut, vor allem an den Händen und Armen, aber auch im Gesicht und am Hals. Die Sporen überstanden den ganzen Gerbvorgang, wurden auf die Wiesen und Weiden der Schwale und Stör geschwemmt und steckten das holsteinische Vieh an. Das fing langsam an und brachte nach dem Ersten Weltkrieg einmal 1200 Stück Viehverlust.“48

48 Paul Graf: Aus dem Leben eines Arztes in Neumünster. Carl Barlach und seine Zeit 1846 - 1918, Sonderdruck, Neumünster 1950, S. 5. 


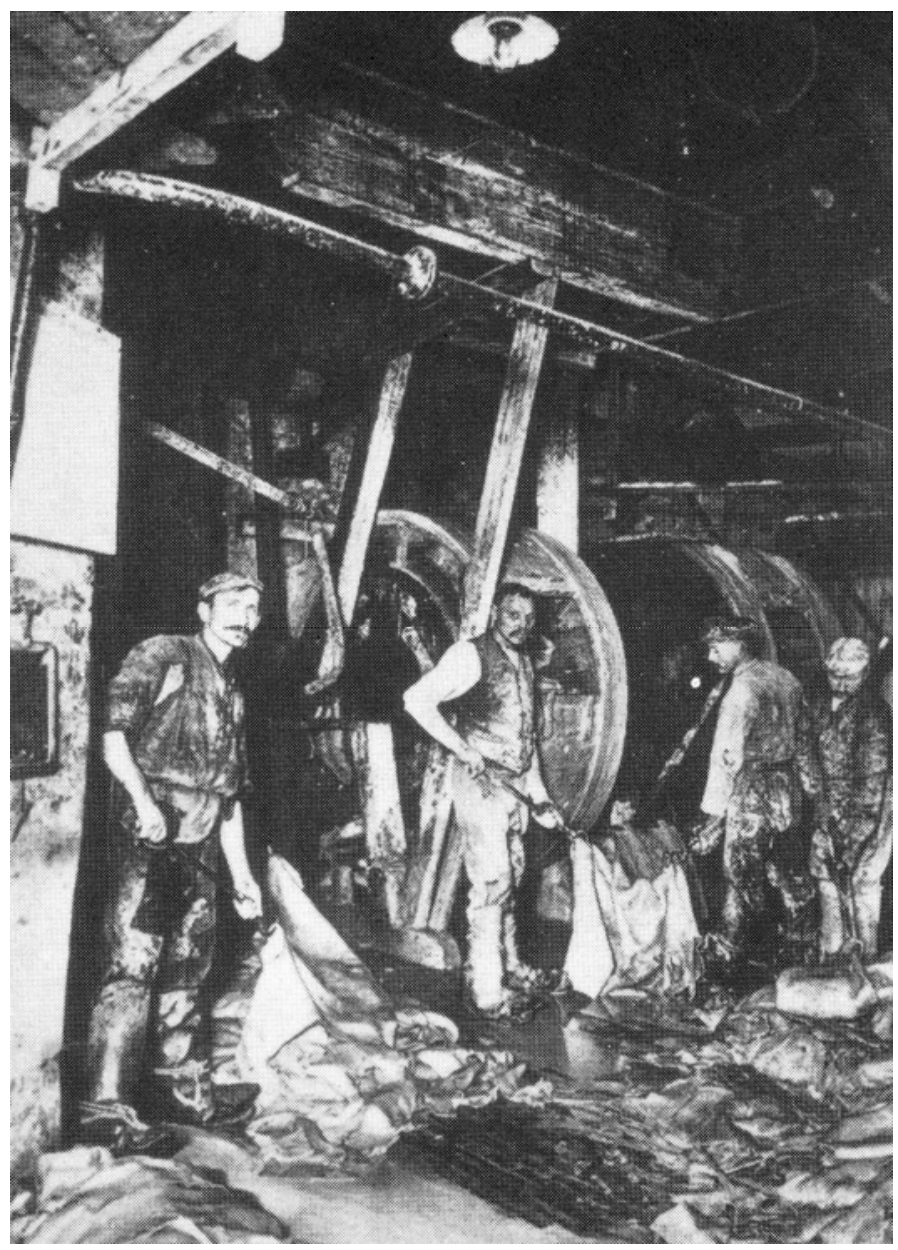

Abbildung 4: Milzbrandwarnhinweise auf einer Tafel am linken Bildrand in einer Lederfabrik Neumünsters im Jahre 1905. ${ }^{49}$

Die Milzbrandbehandlung durch Dr. Barlach in Neumünster erfolgte konservativ und war für die Zeitumstände vor der Einführung von Salvarsan sehr erfolgreich, weil er im Gegensatz zu den Behandlungen in Hamburg oder Dresden operative Eingriffe nach ersten Fehlschlägen mied: „unter den ersten zehn Fällen bis 1890 drei Tote, unter den nächsten 21 Fällen bis 1899 null Tote.(...) Bis zu seinem Tode (1918, d. V.) hatte er etwa 70 bis 80 Fälle behandelt mit zehn Prozent Sterblichkeit. (...) Gegen die Verseuchung der Wiesen unterhalb Neumünsters mit Sporen konn-

49 Vgl.: Schlottau, 1991, Titelblatt. 
te er nichts machen. Das teerfarbene Wasser glich um 1910 dem Styx der Unterwelt. Kein Frosch und kein Fisch mochte dort leben.“ 50

Nachdem seit 1903 im Deutschen Reich die Desinfektion von Wolle und Tierhaaren vor deren Verarbeitung in der Tuchindustrie Pflicht geworden war, traten in der Textilindustrie trotz der hohen Sporenzahl in importierten Wollen und Schafsfellen statistisch keine Milzbranderkrankungen mehr auf.

\subsection{Rechtsgeschichte des Milzbrandes.}

In den medizinischen Disziplinen setzt die Geschichte des Milzbrandes mit der Bibel und dem Gilgamesch-Epos ein. Auch die Viehseuche, die die Belagerung Trojas verlängerte, wird als Milzbrand interpretiert. Im europäischen Mittelalter werden ebenfalls einige beschriebene Viehseuchen mit dem Milzbrand in Verbindung gebracht. Selbst das Antoniusfeuer, das durch die Roggenmutterkornvergiftung entsteht und auch die schwarze Pest, die seit der Mitte des 14. Jahrhunderts durch Europa zog, wird von einigen Autoren als Milzbrand interpretiert. ${ }^{51}$ So gibt es auch viele Berichte über Seuchen, die die Viehbestände dezimierten, doch fehlen oft genaue Beschreibungen der Krankheitssymptome, so dass nicht mit Sicherheit gesagt werden kann, um welche Erkrankung es sich handelte. Die zeitgenössischen Ärzte bezeichneten jede epidemische Krankheit als pestis, sobald viele daran starben. Bis zum 15. Jahrhundert wüteten im gesamten Europa die verschiedensten Epidemien in relativ kurzen Abständen. Darunter nicht selten Rinderpest, Milzbrand, Maul- und Klauenseuche, Rotz, Tuberkulose, Räude und die Pockenseuche der Schafe.

In der Frühen Neuzeit werden weitere schwere Seuchen mit dem Milzbrand erklärt, so der Tod von 60.000 Menschen in Südeuropa im Jahr 1617 oder die Verheerungen, die die Seuche in Wallensteins Heer bei der Verfolgung des Mansfeldischen Heeres im Jahre 1623 anrichtete, als von 20.000 Mann nach einem langen Marsch über sechs Wochen und $800 \mathrm{~km}$ das Ziel, Nyitra, nur noch von 5.000 Mann, die über keine Pferde und Schlachtrinder mehr verfügten, erreicht wurde. ${ }^{52}$

Da die Erreger nicht bekannt waren und eine Therapie meist ohne Erfolg blieb, griffen die Staaten in Analogie zu den Pestmandaten zu vorsorgenden seuchenhygienischen Maßnahmen, um die Ausbreitung von Seuchen und die Übertragung auf Menschen zu verhindern. Eine erste Verordnung dieser Art erfolgte 1550 in Pommern, derzufolge alle Tiere einer Herde, in der eine Seuche ausgebrochen war, zu töten und zu beseitigen waren - eine Methode, heute „stamp out“ genannt,

\footnotetext{
50 Graf, 1950, S. 5.

${ }^{51}$ Vgl.: hierzu besonders: Lottermoser 1998, S. 21 ff. Der Autor bezieht sich in seinen Aussagen im Wesentlichen auf C. Fr. Heusinger: Die Milzbrandkrankheiten der Thiere und Menschen, Erlangen 1850. Die Aussage, dass die schwarze Pest als Milzbrand zu interpretieren sei, bezieht sich auf eine Ausbreitungsanalyse, derzufolge der Vektor Rattenfloh nicht für die Ausbreitungsgeschwindigkeit der Seuche ausreicht. Vgl. hierzu die bei Lottermoser genannten Autoren G. Twigg und Gl. Twigg: The black death: a biological reappraisal, London 1984.

52 Vgl.: Lottermoser, 1998, S. 34.
} 
die Albrecht von Haller nach intensiven Diskussionen am Ende 18. Jahrhundert auch in der Schweiz initiierte. Der Rinderhandel wurde durch Quarantänevorschriften z.B. in Sachsen in den Jahren 1712 und 1732 eingeschränkt. Das Öffnen und Enthäuten der Kadaver wurde verboten und erste Vorschriften zur Desinfektion der Werkzeuge, Kleidung und des Körpers derjenigen, die mit gefallenen Tieren Kontakt hatten, wurden erlassen. Die „Vieh-Seuchen-Ordnung“ des Fürstentums Neuburg aus dem Jahr 1753 forderte das Vergraben der Tiere, nachdem zuvor die Haut durch viele Schnitte unbrauchbar gemacht worden war. Die Kadaver mussten mit Kalk abgedeckt werden. Der Begräbnisplatz war einzuzäunen und durfte nicht genutzt werden. Eine zeitgleiche Seuchenordnung aus Kufstein forderte die Desinfektion des Stalles und der Werkzeuge sowie ein Vergraben des Kadavers in einer Tiefe von 5-6 Schuh (ein Schuh entspricht, je nach Region, einer Länge von 28 bis $37 \mathrm{~cm}$ ). Der Kadaver sollte dann mit Kalk abgedeckt und die Grube mit Erde wieder verfüllt werden. Um eine Folgeinfektion zu verhindern, sollte das Grab dann mit Findlingen abgedeckt und weiträumig mit Dornsträuchern bepflanzt werden, damit an dieser Stelle künftig kein Vieh weiden und kein Heu geerntet werden konnte. Auch in Preußen wurde der Rinderhandel, insbesondere die Einfuhr aus den östlichen Nachbarstaaten, durch eine Viehseuchenverordnung mit Quarantäne seit 1716 stark eingeschränkt. Durch eine Verordnung vom 29. April 1772 wurden ferner die Abdecker verpflichtet, jedes gemeldete Tier innerhalb eines Tages zu entsorgen - die Häute durften die Abdecker allerdings noch bis 1825 verwerten. In dem „Patent“ zur Abwendung der Viehseuchen und anderer ansteckender Krankheiten vom 2. April 1803 wurde die Meldepflicht für Viehseuchen eingeführt, das Verbot der Kadaveröffnung durch Privatpersonen festgelegt und der Futtermittelhandel in Seuchengebieten verboten. ${ }^{53}$ Eine wirkliche Verbesserung der Situation wird durch die Verordnungen, zu denen auch gleichartige in Bayern und anderen Staaten Europas zu zählen sind, nicht gebracht haben, weil mit der Meldung des Seuchenfalles keinerlei Entschädigung des Viehhalters oder Händlers verbunden war. Im Zweifelsfall wurden daher die Tiere geschlachtet, bevor sie zusammenbrachen, um das Fleisch und die Haut noch verkaufen zu können. Viehversicherungen, sogenannte „Kuhkassen“, gab es zu Beginn des 19. Jahrhunderts zwar bereits, aber die Prämien, besonders in den bekannten Milzbrandgebieten, waren so hoch, dass die Viehhalter freiwillig keine Versicherungen abschlossen.

Das norddeutsche Bundesgesetz vom 7. April 1869, Maßregeln gegen die Rinderpest betreffend, wurde 1875 auf das Reichsgebiet ausgedehnt. Im Falle der Viehseuchen schrieb das Bundes- (Reichs-) Gesetz vom 7. April 1869 die sofortige Tötung aller kranken und auch aller verdächtigen Tiere vor, um die Seuche schnell zum Erlöschen zu bringen. Gegenstand der Viehseuchengesetzgebung war und ist die Abwehr der Einschleppung aus Nachbarländern und die Unterdrückung der Seuchenherde im Inland. Die Abwehr erfordert permanente und vorübergehende

${ }^{53}$ Vgl.: ebd., S. $42 f$. 
Maßregeln an den Landesgrenzen, die in einer Überwachung des Grenzverkehrs mit Vieh und kontaminierten Dingen durch Grenztierärzte und in einem Verbot der Einfuhr von einzelnen oder mehreren Viehgattungen, von frischem Fleisch, Häuten, Heu, Stroh und anderen, der Anhaftung mit Ansteckungsstoffen verdächtigen Gegenständen, bestehen. Da die bei den Schlachttieren vorkommenden Viehseuchen zumeist aus östlichen Nachbarstaaten eingeschleppt wurden, war die Überwachung der Landesgrenzen zu Polen, Böhmen, Mähren und Österreich für Deutschland die wichtigste Abwehrmaßregel. Zum Schutz gegen die Viehseuchen verbot der Bundesrat des Kaiserreiches jede Einfuhr von Rindvieh über die östlichen Grenzen. Nur der Handel mit Arbeitsvieh war im Grenzverkehr zwischen Österreich und Sachsen, resp. Österreich und Bayern unter ausreichenden Kontrollmaßregeln gestattet. Die Maßregeln zur Unterdrückung der Viehseuchen im Inland erstreckten sich auf die Anzeigepflicht; die sachverständige Feststellung der Krankheit; die tierärztliche Überwachung der Viehmärkte; die Sperre des Stalles, des Gehöfts, des Ortes oder der Feldmark, selbst einer ganzen Provinz (wie bei der Rinderpest) gegen die Ausfuhr von Vieh; die Impfung (nur bei den Schafpocken); die Tötung von krankem und verdächtigem Vieh; die unschädliche Beseitigung der Kadaver; die Desinfektion und,- bezüglich der Rinderpest, der Lungenseuche und der Rotzkrankheit - auch die Teilentschädigung der auf polizeiliche Anordnung getöteten Tiere - nur verdächtige Tiere, welche auf polizeiliche Anordnung getötet und bei der Sektion für gesund befunden wurden, ersetzte der Staat gemäß revidierter Instruktion vom 9. Juni 1873 (Reichsgesetzblatt, S. 147 ff.) zum vollen Wert. Auch das Reichsgesetz vom 26. Februar 1876, betreffend die Beseitigung von Ansteckungsstoffen bei Viehbeförderungen auf Eisenbahnen, gehört hierher. Von den übrigen Viehseuchen handelt das Reichsgesetz vom 23. Juni 1880, betreffend die Abwehr und Unterdrückung von Viehseuchen, nebst Instruktion des Bundesrats vom 24. Februar 1881 (Zentralblatt für das Deutsche Reich, S. 37 ff.) sowie Nachtrag vom 2. Mai 1882. Dazu kamen die Ausführungsgesetze und Verordnungen der einzelnen Bundesstaaten des Deutschen Reiches. Die Gesetzgebung bot daher den Behörden ausreichende Mittel, um die Seuchen zum Erlöschen zu bringen. Die unschädliche Beseitigung der Kadaver stellte für die Veterinärbehörden das größte Problem dar. Hatte noch Robert Koch das Vergraben der Kadaver in sehr tiefen Erdgruben gefordert, wurden jetzt zunehmend Abdeckereien mit Verbrennungsöfen gefordert. Da aber in den Gesetzen für Milzbrand keinerlei Entschädigungen vorgesehen waren, meldeten die Viehbesitzer einen Milzbrandverdacht nur selten, weil damit erstens die Absperrung des Gehöftes und zweitens ein wirtschaftlicher Totalverlust verbunden war. Um diesen verbreiteten Reaktionen entgegenzuwirken, wurden 1880 in Baden, 1885 in Württemberg, 1886 in Sachsen und schließlich 1892 auch in Bayern und Preußen staatliche Entschädigungen eingeführt, so dass die Zahl der gemeldeten Milzbrandfälle wohl nicht zuletzt aus diesem Grunde plötzlich hochschnellte. ${ }^{54}$

${ }^{54}$ Vgl.: ebd., S. 85. 


\subsection{Ist der Milzbrand - eine Seuche - eine Altlast?}

Viehseuchen sind nach älterer Auffassung alle Krankheiten, die gleichzeitig eine größere Zahl von Tieren töten. In der neueren Zeit beschränkt man aber den Begriff auf die durch Ansteckung leicht übertragbaren Krankheiten der Haustiere und zählt zu diesen namentlich Rinderpest, Maul- und Klauenseuche, Lungenseuche des Rindes, Pockenseuche der Schafe, Beschälseuche, Bläschenausschlag, Räude der Pferde und Schafe, Milzbrand, Tollwut und Rotz. Die Zusammenfassung dieser Krankheiten als Viehseuchen knüpft zum Teil an die historische Tatsache an, dass zur Bekämpfung in allen europäischen Staaten Gesetze und Verordnungen erlassen worden sind. Die Rinderpest wurde im 18. Jahrhundert zu einer der größten Landplagen in Europa. Milzbrand, Rotz und Tollwut sind zugleich dadurch gefährlich, dass sie auf Menschen übertragen werden können und auch bei diesen zum Tod führen. Milzbrand wurde nach älterer Auffassung erst im 18. Jahrhundert über Italien und Frankreich nach Deutschland eingeschleppt, bereits zuvor aber entstanden die Milzbrandsynonyme - z.B. anthrax, pustula maligna, carbunculo sowie umgangssprachliche Varianten, z. B. Gelber Schelm etc.. 1712 wurde erstmals in Augsburg ein Zungenmilzbrand beschrieben. ${ }^{55}$ Bildliche und schriftliche Quellen, neuerdings aber auch mikrobiologische Untersuchungen alter Friedhöfe weisen darauf hin, dass Milzbrand regelmäßig auftrat.

Unter den Viehseuchen, die in den letzten Jahren verstärkt besonders von Historikern im Rahmen des Graduiertenkollegs interdisziplinäre Umweltgeschichte in Göttingen untersucht wurden, hat der Milzbrand vermutlich nur einen geringen Anteil. Seine Bedeutung begründet sich nicht allein darin, dass er auch Menschen betrifft, sondern besonders darin, dass er aufgrund seiner stofflichen Eigenschaften, die ihn von nahezu allen anderen Erregern abgrenzen, seine Virulenz über Jahrhunderte erhalten kann. Er kann durch menschliche Aktivitäten akkumuliert, importiert und weiterverbreitet werden, so dass die Orte, an denen er auftritt, nachhaltig kontaminiert werden. Die Bedeutung der Viehseuchen für die Geschichte ist mit Sicherheit noch nicht abschließend geklärt. Sie sind Gegenstand der Agrargeschichte, der Wirtschafts-, Sozial- und Umweltgeschichte. Mangel an Vieh ist nicht allein Mangel an Nahrungsmitteln, sondern auch Mangel an Zugvieh, Mangel an Stickstoff, also an Stallmist, mit dem die notwendigen Getreideäcker gedüngt werden mußten. Rinder sind in der Agrargeschichte bis zur Entwicklung der Kunstdünger ein notwendiges Übel, ohne die die Pflanzen nicht mit Stickstoff zu versorgen sind. Da das Kapital für die Industrialisierung Europas aus der Agrarwirtschaft stammt und über die Getreideproduktion erwirtschaftet wird, haben die Viehseuchen Kontinentaleuropas einen verheerenden Einfluss auf die Industrialisierung genommen. In diesem Zusammenhang ist besonders hervorzuheben, dass in Großbritannien im Jahr 1865, als durch den Import lettischer Zuchtkühe die Rinderpest im 19. Jahrhunderts die Insel erstmals erreichte und binnen kurzer

${ }^{55}$ Braun, 1958, S. 2. 
Zeit 650.000 Rinder starben, die britischen Veterinäre, die sich bis dahin als Pferdeärzte verstanden hatten, völlig überrascht waren und Delegationen nach Österreich-Ungarn entsandten, um sich fortzubilden. Bei einer Analyse des Periodikums „Hamburgischer unparteiischer Correspondent“, der auflagenstärksten, mehrmals wöchentlich erscheinenden Zeitung des 18. Jahrhunderts, stellte sich heraus, dass die letzte Seuchenmeldung für Hornvieh aus dem Jahr 1753 stammte, also mehr als 100 Jahre, während der gesamten Industrialisierungsphase, keine der klassischen Viehseuchen in Großbritannien auftrat. ${ }^{56}$ Die vergeblichen Versuche der Zeitgenossen, die „englische Landwirtschaft" auf den Kontinent zu transferieren oder vergleichbare Erfolge in der Viehzucht zu erreichen, mußten scheitern, weil es auf dem Kontinent gar nicht möglich war, eine ungebrochene Generationsfolge der Rinder für Versuche des Ein- oder Auskreuzens zu erhalten und so viele Tiere pro Hof zu ernähren. Viehseuchen könnten auf dem Kontinent als Nebeneffekt für Getreidemangel, - also auch für Hungerunruhen, höhere Sterberate, geringere Geburtenrate, niedrige Erträge der gewerblichen Wirtschaft etc. - Kapitalmangel und späte Industrialisierung verantwortlich sein. Erst die Ammoniakwasserabscheidung der Gasanstalten und Hüttenwerksretorten,- wiederum die Verwertung eines industriellen Nebenproduktes - konnte seit ca. 1870 den Mangel an Stickstoff aufheben, der durch Klee und Lupinen in der Brache nicht hatte beseitigt werden können.

Unter Berücksichtigung der Bedeutung der Seuchen für die gewerbliche Entwicklung in der Neuzeit und der besonderen Bedeutung des Milzbrandes für eine große Zahl von Industriezweigen - bis hin zur landwirtschaftlichen Fabrik des frühen zwanzigsten Jahrhunderts - kann dem Milzbrand eine historische Bedeutung nicht abgesprochen werden. Seine stofflichen Eigenschaften machen ihn zu einer Altlast, die auf bestimmten Industrieflächen gehäuft auftreten kann. Seine Qualität als Gegenstand der Geschichtswissenschaft bestimmt sich aus dem Diskurs über Viehseuchen, die seit dem 18. Jahrhundert andauert, aber auch aus den Massenprotesten gegen seine Auswirkungen, z.B. in holsteinischen Marschen.

\section{Anthropogene Stoffe der Nebenproduktanlagen als Gegenstand der Umwelt- und Altlastengeschichte}

Nebenproduktverwertungen wie z.B. der Ammoniakdünger, resultieren aus den Versuchen, einen Abfallstoff zu einem Gebrauchswert und einem Tauschwert zu machen. Aus der gleichen Quelle wie der Ammoniak stammen allerdings auch Benzol, Toluol, Xylol, Teer und Teeröle sowie frühe Chlorkohlenwasserstoffe, also

\footnotetext{
56 Der Hamburgische unparteiische Korrespondent wurde von seiner Erstausgabe 1736 bis 1850 systematisch auf Viehseuchenmeldungen, differenziert nach der Bezeichnung und, sofern vorhanden, nach den betroffenen Gattungen durchgesehen. Im Jahr 1753 wurde für die Grafschaften Norfolk und Suffolk eine Hornviehseuche gemeldet. Vgl. Ebd., Jg. 1753, Ausgabe 178. Alle weiteren Seuchenmeldungen aus Großbritannien betrafen ausschließlich Pferdeseuchen.
} 
nachhaltig verunreinigende Stoffe, die z. B. in der chemischen Reinigung Wasser, Boden und Luft dauerhaft unbrauchbar machen. Welche Gemeinsamkeiten haben die beiden gewählten Beispiele für die Umweltgeschichte, sofern sie nicht einfach Beispiele für Kontaminationen sind?

Es handelt sich in beiden Fällen um Stoffe, die der regionalen Umwelt unbekannt waren. Die chemische Reinigung wurde mit Stoffen durchgeführt, die in der Natur nicht vorkommen und erst durch die Verwertung von Abfällen eines Industriezweiges zu einem rentabel einsetzbaren Hilfsstoff wurden. Im Falle des Milzbrandes handelt es sich um eine Lebensform, die in Nordeuropa nicht lebensfähig ist. Eine Spore verhält sich jedoch aufgrund der stofflichen Qualität nicht wie ein Organismus, sondern wie ein Mineral und wird durch menschliches Handeln akkumuliert, weiter verbreitet und wieder in den Verkehr gebracht.

Beide dargestellten Problembereiche sind eng mit dem Bemühen der Menschen verbunden, aus Rest- und Abfallstoffen durch Stoffumwandlungen in Gewerben oder Industrieanlagen neue Wirtschaftsgüter herzustellen und damit Gewinn zu erwirtschaften - es handelt sich mithin häufig um Recyclingprobleme, die in der Geschichte der Umwelt Schaden zufügten. ${ }^{57}$

Das Gefahrenpotential beider Beispiele wird durch menschliches Handeln erzeugt, verstärkt, verbreitet oder, z. B. durch neue Technologien und rechtliche Grenzen, wieder abgebaut.

Ist folglich die Geschichte der Altlasten von Relevanz für die Umweltgeschichte und relevant für die Gesellschaft oder handelt es sich nur um eine Geschichte von Kontaminationen? Altlasten sind nicht schlichte Kontaminationen, davon gibt es genügend geogen entstandene: es handelt sich auch nicht um Stoffverlagerungen, die zu Altablagerungen werden, sondern um Verunreinigungen und Umweltveränderungen, die der bestehenden Natur durch menschliches Handeln hinzugefügt wurden und daher z.B. noch keine angepassten Verbraucher in Form von Mikroben, wie es beim Mineralöl der Fall ist, haben. Altlasten wie die Chlorkohlenwasserstoffe und die Milzbrandsporen haben eine vollständig von Menschen gemachte Geschichte, die ursächlich vom wirtschaftenden Menschen, der stets versuchte, auch die Neben- und Abfallprodukte durch Stoffumwandlung einer Nutzung zuzuführen, zu verantworten ist. Stoffumwandlung und Nebenproduktverwertung sind daher zentrale Begriffe einer Umweltgeschichte der Altlasten, die damit zu großen Teilen eine Geschichte der Verfahrenstechnik wird. Die Verfahrenstechnik wiederum ist abhängig davon, welche Sekundärrohstoffe sich wirtschaftlich umformen lassen - Gaswerke des 19. Jahrhunderts beispielsweise waren sehr stark von der Entwicklung der Teerchemie abhängig. Eine weitere Rahmenbedingung war von der Rechtsentwicklung und vom sogenannten ,Stand der Tech-

\footnotetext{
${ }^{57}$ Die Auswirkungen von Nebenproduktnutzungen und Recyclingvorgängen sind z.B. beschrieben in: Klaus Schlottau: (2003): Umweltprobleme beim Recycling der Metallabfälle spanabhebender Betriebe im 20. Jahrhundert. In: Roland Ladwig (Hg.): Recycling in Geschichte und Gegenwart. Die Technikgeschichte als Vorbild Moderner Technik. Georg-Agricola-Gesellschaft, Freiberg, S. 95-110.
} 
nik', womit neben der konkreten Maschinen- und Apparatetechnik auch der Stand der naturwissenschaftlich-technischen Entwicklung gemeint ist, abhängig.

Eine Geschichte der Altlasten unter Berücksichtigung der Geschichte der Verfahrenstechnik, der Wirtschaft, Technik, Naturwissenschaften und auch der Rechts- und Verwaltungsgeschichte sowie anderer betroffener Institutionen, z.B. der Verbände, ist daher in der Lage, Veränderungen der Gefährdungslagen für die Geschichte altlastrelevanter Branchen zu beschreiben und damit Vorgaben für eine künftige Sanierung verunreinigter Flächen zu schaffen. Bezogen auf die beiden genannten Beispiele bedeutet dies: von allen chemischen Reinigungen, die bis heute grundsätzlich als uneingeschränkt altlastenrelevant eingestuft sind, müssen etwas vereinfachend - nur noch jene untersucht werden, die als innerstädtische Kleinbetriebe zwischen etwa 1955 und 1990 existierten., weil nur in dieser Zeitspanne CKW für die Reinigung eingesetzt wurden. Das Beispiel des Milzbrandes wirft einen grundsätzlichen Altlastenverdacht z. B. auf Gerbereien und Lederfabriken. Folgt man jedoch der Geschichte der Verfahrenstechnik und der Geschichte der Wirtschaft, kommen nur noch wenige Lederfabriken in Frage, die sich z. B. durch - wiederum grob vereinfachend - importierte Häute letzter Wahl bei der Rohstoffversorgung und zugleich durch den Verkauf von Oberleder, besonders Lackleder, von anderen abgrenzen. Weitere Abgrenzungskriterien sind die Verkehrsinfrastruktur, überproportionale Wasserwerkstätten und gewaltig dimensionierte Kläranlagen oder Rieselfelder.

Die Relevanz der Altlastengeschichte im Rahmen der Umweltgeschichte ist darin begründet, dass neue Stoffe, von denen eine Gefährdung ausgeht, eine anthropogene Produktionsgeschichte haben, die von den Menschen mindestens zu Teilen auch als Auswirkung auf die Umwelt wahrgenommen wurden. Diese Geschichte kann mit historischen Mitteln und Methoden geschrieben und bewertet werden. Neben dieser Sinnstiftung für die Geschichtswissenschaft kommt der historischen Altlastenforschung auch eine Bedeutung als Handlungswissenschaft zu. Mit ihrer Hilfe lassen sich die vielen tausend Verdachtsflächen selektieren, prognostizieren, flächengetreu beproben und mithin schneller sanieren.

\section{Literatur}

Altlastenleitfaden Schleswig-Holstein. (2003): Bearbeitet von U. Ströh-Neben und V. Brill, herausgegeben vom Landesamt Natur und Umwelt des Landes Schleswig-Holstein, Kiel-Flintbek , Band 1: Erfassung; Band 2: Branchenblätter, bearbeitet von HistInvest-Dr. Schlottau, Bremen.

Anweisungen des Ministers der geistlichen, Unterrichts- u. Medizinalangelegenheiten zur Ausführung des Gesetzes, betreffend die Bekämpfung übertragbarer Krankheiten, (1906): Heft 8: Milzbrand. Amtliche Ausgabe, Berlin.

Baader, Ernst W. (1954): Gewerbekrankheiten, München und Berlin. 
Barkowski, Dietmar et al. (1993): Altlasten. Handbuch zur Ermittlung und Abwehr von Gefahren durch kontaminierte Standorte, 4. Auflage Karlsruhe.

Baumhöfener, Friedrich-Karl (1965): Der Hautmilzbrand - Untersuchungen zur Infektkette, Diagnose und Therapie. Diss. med. Kiel.

BAVG: Ausführungsvorschriften des Bundesrates zum Viehseuchengesetz vom 7.12.1911, zuletzt geändert durch die Viehverkehrsordnung vom 1.12.1982, Anlage A.

Bayerisches Landesamt für Wasserwirtschaft: Merkblatt Nr. 4.5/2-52, (2005): Hinweise zu Anhang 52 zur Abwasserverordnung (Chemischreinigung).

Bayerl, Günter (1980): Historische Wasserversorgung. Bemerkungen zum Verhältnis von Technik, Mensch und Gesellschaft. In: Ulrich Troitzsch und Gabriele Wohlauf (Hrsg.): Technik-Geschichte, Frankfurt am Main, S. 180212.

BGA. Bundesgesundheitsamt (Hg.) (1963): Verhütung und Bekämpfung des Milzbrandes beim Menschen - Ratschläge an Ärzte, Köln.

Böhm, Reinhard (1985): Milzbrand. In: Blobel-Schließer (Hg.): Handbuch der bakteriellen Infektionen bei Tieren, Bd. 5, Stuttgart-New York, S. 17-89.

Böhm, Reinhard (1988): Schnelle und automatisierbare Diagnosemethoden für Bacillus anthracis als Testkeim bei Untersuchungen zur Umwelthygiene, (Fortschritte der Veterinärmedizin. Beiheft zum Zentralblatt für Veterinärmedizin Heft 38), Berlin und Hamburg.

Bottler, M. (1908): Bleich- und Detachiermittel der Neuzeit, Wittenberg.

Braun, Albrecht (1958)t: Der Milzbrand in Württemberg. Eine statistischepidemiologische Studie, Diss. Vet. Med. Gießen.

Brühann, W. (1962): Zur Entwicklung der deutschen Tierseuchengesetzgebung. In: Berlin-Münchener tierärztliche Wochenschrift, Jg. 75, S. 309-313.

Bürger, B. und E. Nehring (1925): Die Abwässerbeseitigung der Stadt Neumünster in Holstein unter besonderer Berücksichtigung der dortigen Gerbereiabwässer, (Veröffentlichungen aus dem Gebiete der Medizinalverwaltung, XIX. Band, 10. Heft), Berlin.

Davies, L. (1988): Anthrax - A health hazard at the bottom of the garden. In: Community Health 3, S. $271-272$.

Elmers, S. (1982): Untersuchungen zur Überlebensdauer von Brucella abortus, Francisella tularensis und der Sporen von Bacillus anthracis sowie Bacillus globigii auf Oberflächen unter Freiluftbedingungen. Diss. Vet. Med. Giessen 
Engelken, K., Hünninger, D., Windelen, S. (Hg.): (2007) Beten, Impfen, Sammeln. Zur Viehseuchen- und Schädlingsbekämpfung in der Frühen Neuzeit, Göttingen.

Eye, J.D. und S.P .Graef (1967): Gerberei-Abwasser. In: Journal of American Leather Chemists Association, Jg. 62, S. 194-220.

Fahrenkrug, Peter (1978): Die Tierseuchensituation des schleswig-holsteinischen Landkreises Pinneberg in den Jahren 1954 bis 1977. Diss. Vet. Med.

Hannover.

Fallada, Hans (2006): Bauern, Bonzen und Bomben, 4. Auflage, (Lizenzauflage der Originalausgabe Berlin 1931) Hamburg.

Farag, Youssef A. (1965): Ein neues Verfahren zur Desinfektion milzbrandverdächtiger Borsten. Diss. Med. Hamburg.

Feikes, Lieselotte (1983): Ökologische Probleme der Lederindustrie, Frankfurt a. M..

Feser, Johann (1877): Der Milzbrand auf den oberbayerischen Alpen.

Beobachtungen darüber an Ort und Stelle mit experimentellen

Untersuchungen und geschichtlichen und statistischen Notizen, München.

Freudenberg, W. (1955): Die Häute- und Fellmärkte der Welt, Darmstadt.

Gesetz zum Schutz vor schädlichen Bodenveränderungen und zur Sanierung von Altlasten (Bundes-Bodenschutzgesetz - BBodSchG). Ausfertigungsdatum 17.03.1998 (BGB1. I S.3214) In:

http://bundesrecht.juris.de/bbodschg/BJNR050210998.html (Besucht am 18.07.2008).

Gesetz-Sammlung für die Königlichen Preußischen Staaten (1835).

Bekanntmachung des allerhöchst genehmigten und bestätigten Regulativs, die Sanitäts-Polizeilichen Vorschriften bei den am häufigsten vorkommenden ansteckenden Krankheiten enthaltend vom 28.10.1835, Berlin, S. 239 - 286.

Gillisen, G. (1961): Ein Verfahren zur Abtötung der Sporen von Bacillus anthracis in Abwässern von Lederfabriken, dargestellt im Großversuch. In: Archiv für Hygiene und Bakteriologie Jg. 145, S. 389-396.

Graf, Paul (1926): Zur Klinik und Behandlung des menschlichen Milzbrandes nach Erfahrungen des Städt. Krankenhauses Neumünster in den letzten 25 Jahren. In: Deutsche Zeitschrift für Chirurgie, Bd. 198, Heft 516.

Graf, Paul (1950): Der Milzbrand vom Standpunkt des Arztes aus betrachtet. In: Lederindustrie-Berufsgenossenschaft : Maßnahmen zur Verhütung und Bekämpfung von Milzbrand, Mainz, S. 55-71. 
Graf, Paul (1961): Aus dem Leben eines Arztes in Neumünster. Carl Barlach und seine Zeit 1846 - 1918, Sonderdruck, Neumünster.

Guillerme, A. (2001): Zur Geschichte industrieller Altlasten in Frankreich. In: Christoph Bernhardt (Hg.): Environmental Problems in European Cities in the 19th and 20th Century, Münster, (Cottbuser Studien zur Geschichte der Technik, Arbeit und Umwelt, Bd. 14) S. 41 - 52.

Gürich, Hans (1922): Die Milzbrandfälle des Allgemeinen Krankenhauses Eppendorf. Diss. Med. Hamburg .

Hanisch, Hans (1905): Deutschlands Lederproduktion und Lederhandel (Zeitschrift für die gesamte Staatswissenschaft, Heft 16) Tübingen.

Haubner, G. C. (1869): Handbuch der Veterinär-Polizei. Zum Gebrauche der Behörden, Verwaltungs- und Veterinär-Beamte, Aerzte und Thierärzte, Dresden.

Hausam, Willi (1944): Konservierung und Desinfektion der Haut. In: Grassmann, W. und J. Trupke (Hrsg.):Handbuch der Gerbereichemie und Lederfabrikation, Bd. 1, Teil 1, Wien, S. 769-895.

Hausam, Willi (1946): Die Bakteriologie in der Lederindustrie, Stuttgart.

Hausam, Willi (1950): Der Milzbrand gesehen vom Standpunkt des Bakteriologen und Gerbereifachmannes. In: Lederindustrie-Berufsgenossenschaft: Maßnahmen zur Verhütung und Bekämpfung von Milzbrand, Mainz, S. 31 54.

Heusinger, C. Fr. (1850): Die Milzbrandkrankheiten der Thiere und Menschen, Erlangen.

Hoßbach, H. (1962): Geschichte der Entwicklung der Tierseuchengesetzgebung auf dem Gebiete des Milzbrandes. Diss. Vet. Med., Leipzig.

Hoyer, Heinz (1951): Therapie des Milzbrandes in den letzten zehn Jahren, Diss. Med. Hamburg.

Hünemöder, K. F.: (2007), Zwischen , abergläubischem Abwehrzauber' und der ,Inokulation der Hornviehseuche'. Entwicklungslinien der Rinderpestbekämpfung im 18. Jahrhundert. In: Engelken, K., et al.. (Hg.): Beten, Impfen, Sammeln. Zur Viehseuchen- und Schädlingsbekämpfung in der Frühen Neuzeit, Göttingen, S. 21 - 56.

Hunziker, Samuel (1939): Über gewerblichen Milzbrand. (Diss. Vet. Med. Basel 1938), Amriswil.

Jacobeit, W. (1987): Schafhaltung und Schäfer in Zentraleuropa bis zum Beginn des 20. Jahrhunderts, 2. erw. Auflage, Berlin. 
Jedwillat, S. (1993): Eine historische Studie zur Entwicklung der Kenntnis und Theorie der Tierseuchen sowie ihrer Bekämpfung unter Berücksichtigung der Gesetzgebung im deutschsprachigen Raum vom Mittelalter bis zum Vorabend des Dreißigjährigen Krieges, Diss. Vet. Med., Berlin.

Jenko, L. und Kopf, E (1931).: Das Entfernen von Flecken aus Geweben (Detachieren), Leipzig und Wien.

Joclét, V. (1905): Die Kunst- und Feinwäscherei in ihrem ganzen Umfange. 4. gänzlich umgearbeitete Auflage, Wien und Leipzig

Jörissen, Franz (1909): Die deutsche Leder- und Lederwarenindustrie. Die Hilfsund Nebenindustriezweige derselben, sowie die einschlägigen Handelsgebiete in ihrer Entwicklung und heutigen Bedeutung, Berlin.

Kauker, Emil (1965): Globale Verbreitung des Milzbrandes um 1960

(Sitzungsberichte der Heidelberger Akademie der Wissenschaften, Mathematisch-Naturwissenschaftliche Klasse 1965/2), Heidelberg.

Kautsky, Heinrich (2001): Chemische und mikrobiologische Belastung von Gerbereistandorten. In: Altlasten Spektrum, Heft 5, S. 229-237.

Koller, Theodor (1921): Handbuch der rationellen Verwertung, Wiedergewinnung und Verarbeitung von Abfallstoffen jeder Art, Wien und Leipzig.

Kölsch, Friedrich (1918): Der Milzbrand. München.

Kosuch, N. (2004): Tierseuchen und ihre Bekämpfung an der Weser im Spiegel Nienburger Quellen (17- bis 19. Jahrhundert). Diss. med. vet. Hannover. Berlin.

Krünitz, Johann Georg (1803): Oekonomisch-technologische Encyklopädie, oder allgemeines System der Staats-, Stadt-, Haus- und Landwirtschaft und der Kunstgeschichte in alphabetischer Ordnung, 90. Theil (Meth bis Minderzwickel), Berlin.

Kurz, J. u.a. (1978): 125 Jahre Stichweh. Von Pompeji bis Hannover und 125 Jahre Stichweh. Herausgegeben von Färberei und Reinigung, Hannover.

Lappenberg, Johann Martin (Hg.) (1856): Sammlung der Verordnungen der Freien Hanse-Stadt Hamburg seit 1814. Verordnungen von 1855. Bekanntmachung betr. Die unter den Schweinen ausgebrochene Milzbrand-Krankheit, Hamburg 1856.

Laubender, B. (1815): Der Milzbrand der Hausthiere und seine Geschichte, München.

Lauffmann, R. (1917): Die Bedeutung des Milzbrandes in der Lederindustrie, Prag. 
Lindner, Friedrich (1987): Untersuchungen über die Möglichkeit der chemischen Desinfektion von Milzbrandsporen im Klärschlamm. Diss. Vet. Med., Gießen.

Löcher, W. und Kurz, J. (1970): Die Wäscherei. Theorie und Praxis moderner Betriebsführung, München.

Loescher, Hans-Ludwig (1936): Die norddeutsche Lederindustrie. Ihre Entwicklung und ihre Stellung in der gesamtdeutschen Lederindustrie. Diss. Hamburg .

Lottermoser, Thomas (1998): Milzbrand - ein Beitrag zur Geschichte der Krankheiten. Diss. Vet. Med. Berlin.

Lüthje, F. (1953): Nochmals: Ist mit einer Zunahme der Milzbranderkrankungen zu rechnen? Angriffe der Lederindustrie gegen den Verfasser. In: Tierärztliche Umschau, Jg. 8, S. 459-461.

Merkt, Frank (1990): Milzbrand in der Bundesrepublik Deutschland 1950 - 1988. Statistisch-epidemiologische Betrachtung. Diss. Vet. Med. München.

Niclauß, M. u. a (1989): Inventarisierung von Bodenkontaminationen auf Geländen mit ehemaliger Nutzung aus dem Dienstleistungsbereich. Umweltbundesamt Berlin, Forschungsbericht UBA-FB 89-053, Berlin.

Nieberle, K (1927):: Der Milzbrand der Tiere und gewerbliche

Milzbranderkrankungen beim Menschen. In: Urania, Heft 5, Jahrgang 1926-27, S. 138-142.

Nowosadtko, J. (2007): Milzbrand, Tollwut, Wölfe, Spatzen und Maikäfer. Die gesellschaftliche Verteilung von Zuständigkeiten bei der Bekämpfung der Viehseuchen und schädlichen Tiere in der Frühen Neuzeit. In: Engelken, K., et al.. (Hg.): Beten, Impfen, Sammeln. Zur Viehseuchen- und Schädlingsbekämpfung in der Frühen Neuzeit, Göttingen, S. 79 - 98.

Orland, B. (1991): Wäsche waschen. Technik und Sozialgeschichte der häuslichen Wäschepflege. Reinbek bei Hamburg.

Pedersen, K. P.: (2007). Als Gott sein strafendes Schwert über dem dänischen Sahnestück Fünen schwang. Über Verlauf und Bekämpfung der Viehseuche auf Fünen 1745-1770 unter besonderer Berücksichtigung des Bauernschreibebuchs von Peder Madsen auf Munkgaarde. In: Engelken, K., et al.. (Hg.): Beten, Impfen, Sammeln. Zur Viehseuchen- und Schädlingsbekämpfung in der Frühen Neuzeit, Göttingen, S. 57 - 78.

Phillips, C. R. und W. D. Phillips (1997): Spain's golden Fleece. Woll production und the wool trade from the Middle Ages to the Nineteenth Century, Baltimore/London. 
Piening, C. (1958): Milzbrandinfektion durch Futtermittel während der Stallperiode 1957/58 in Schleswig-Holstein. In: Berlin-Münchener tierärztliche Wochenschrift, S. 474.

Plehn, Bernhard (1903): Der staatliche Schutz gegen Viehseuchen. Ein Buch für die Praxis; mit Anhang: Die wichtigsten Viehseuchen, bearbeitet von Dr. Froehner, Kreistierarzt Fulda, Berlin

Poos, U. (1989): Die seuchenepidemiologische Bedeutung der äußeren Haut und ihre Behandlung im Tierseuchen- und Tierkörperbeseitigungsgesetz unter besonderer Berücksichtigung des Milzbrandes. Diss. Vet. Med. Hannover.

Radewald, F (1941): Der Milzbrand in Europa. Diss. Vet. Med. Hannover.

Rau, A. (1957): Untersuchungen über das Vorkommen von Bacillus anthracis in einem Wolle und Haare verarbeitenden Industriebetrieb, Diss. Vet. Med. Gießen.

Rebentisch (1926): Die Milzbrandstatistik der Lederindustrie-Berufsgenossenschaft 1906 - 1925. In: Zentralblatt für Gewerbehygiene und Unfallverhütung, N.F., Bd. 3, (1926), Nr. 6, S. 162-169.

Roggenhofer, G. (1927): Die Wäscherei in ihrem ganzen Umfange. Bearbeitet nach den neuesten Erfahrungen auf dem Gebiete der chemischen und Naßwäscherei unter Mitwirkung bewährter Fachmänner. 3. vermehrte und verbesserte Auflage, Wittenberg.

Rohde, R. G. (1950): Eine historische Studie über Milzbrand. Diss. Med. Frankfurt a. M..

Rohr, Chr. (2007): Zum Umgang mit Tierplagen im Alpenraum in der Frühen Neuzeit. In: Engelken, K., et al.. (Hg.): Beten, Impfen, Sammeln. Zur Viehseuchen- und Schädlingsbekämpfung in der Frühen Neuzeit, Göttingen, S. $99-134$.

Sändig, Johannes (1937): Die Lederindustrie in Deutschland. Eine wirtschaftsstatistische Betrachtung. In: Jahrbuch der Gerberei und Lederindustrie, Leipzig, S.117-149.

Schäfer, Michael (1974): Die schleswig-holsteinischen Bauernunruhen 1928 - 1932: eine Darstellung unter besonderer Berücksichtigung der Bestände der Kieler Bibliotheken, mscr. FH Hamburg.

Schlottau, Klaus (1991): Die Geschichte der Lederindustrie in Neumünster. Ein Beitrag zur Industrialisierung Schleswig-Holsteins, Neumünster.

Schlottau, Klaus (1993): Von der handwerklichen Lohgerberei zur Lederfabrik des 19. Jahrhunderts, Opladen (zugleich Diss. phil. Hamburg 1992.). 
Schlottau, Klaus (1994): Die Planung des Stör-Kanals von Itzehoe nach

Neumünster als Binnenschifffahrtsweg und Abwasserkanal der

Neumünsteraner Industrie (1891-1926). In: Mitteilungen des Canal-Vereins, Jg.

15, Rendsburg, S. 93-116.

Schlottau, Klaus (1998): Praktizierte Umweltgeschichte: Der Sachverständige für die „Durchführung und Beurteilung von standortbezogenen Erhebungen (historische Recherche)“ - Ein neues Berufsbild für Historiker. In: Günter Bayerl, Wolfhard Weber (Hrsg.): Sozialgeschichte der Technik. Ulrich Troitzsch zum 60. Geburtstag, Münster, New York, München, Berlin, S. 25 34.

Schlottau, Klaus (2000): Methodik und Probleme der Altlastenkartierung am Beispiel der Altstandorterfassung in Bremen In: Carl-Hans Hauptmeyer (Hrsg.): Mensch-Natur-Technik: Aspekte der Umweltgeschichte in Niedersachsen und angrenzenden Gebieten. Vorträge der Zusammenkünfte des Arbeitskreises Wirtschafts- und Sozialgeschichte der Historischen Kommission für Niedersachsen und Bremen am 8. November 1997 und am 7. März 1998. Bielefeld 2000, S. 159 - 179.

Schlottau, Klaus (2003): Eingriffe in den Bodenhaushalt. Industrielle Altlasten und ihre systematische Erkundung. In: Günter Bayerl, Torsten Meyer (Hrsg.): Die Veränderung der Kulturlandschaft. Nutzung-Sichtweisen-Planungen. (Band 2, Erfassung, Anlage 112 der Cottbuser Studien zur Geschichte von Technik, Arbeit und Umwelt), Münster, New York, München, Berlin 2003, S. 199-212.

Schlottau, Klaus (2003): Umweltprobleme beim Recycling der Metallabfälle spanabhebender Betriebe im 20. Jahrhundert. In: Roland Ladwig (Hg.): Recycling in Geschichte und Gegenwart. Die Technikgeschichte als Vorbild Moderner Technik. Georg-Agricola-Gesellschaft, Freiberg, S. 95-110.

Schmidt, Hans (1941): Milzbrand - Rotlauf - Brucellosis - Botulismus (Behringwerk Mitteilungen, Heft 20), Berlin.

Schneider, Ute (1988): Krätzekuren und Milzbrandbazillen: Das Krankenhaus 1847 bis 1924. In: Alfred Heggen und Klaus Tidow (Hrsg.): Industriekultur in Neumünster. Das "Manchester Holsteins" im 19. Jahrhundert, Neumünster, S. 157-163.

Scholz, Herbert (1956): Das Lederabwasser von Neumünster, Reutlingen.

Scholz, Herbert (1956): Ein wirkungsvolles chemisches Verfahren zur Reinigung von Lederabwasser. In: Die Reinigungsanlage für Lederabwasser in Neumünster/Holstein, herausgegeben von den Passavant-Werken, Michelsbacher Hütte, S. 1-5. 
Schütze, P. O. (1927): Schönfärberei und chemische Reinigung mit brenn- sowie nichtbrennbaren Lösungsmitteln. 2. Auflage, Wittenberg.

Seele, Hans (1950): Der Milzbrand gesehen vom Standpunkt der LederindustrieBerufsgenossenschaft. In: Lederindustrie-Berufsgenossenschaft : Maßnahmen zur Verhütung und Bekämpfung von Milzbrand, Mainz, S. 7 - 30.

Seidel, G. (1960): Historische Betrachtungen über den Milzbrand. In: Zeitschrift für die Geschichte der Naturwissenschaften, Technik und Mathematik, Bd. 1 (1960), S. 72-93.

Snoek, A (1929): Die Reinigung der Abwässer der Stadt Elmshorn unter besonderer Berücksichtigung der Lederfabrikabwässer. In: Collegium, 16. Hauptversammlung, November 1928, Hamburg, Bd. 1, S. 781.

Standfuss, R.: und G. Pohl (1930): Beitrag zur bakteriologischen Milzbrandfeststellung. In: Archiv der wissenschaftlichen und praktischen Tierheilkunde, 62 (1930/31), S. 178-197.

Stickelberger, Emanuel (1915): Versuch einer Geschichte der Gerberei, Berlin.

Ströh-Neben, Ulrike (2008): Sachgebiet 1 „Flächenhafte und standortbezogene Erfassung/historische Erkundung“. ITVA - Sitzung der Regionalgruppe Nord am 02.07.2008 in Hamburg. In:

http://www.hk24.de/produktmarken/recht_und_fair_play/schiedsgerichteme diationschlichtung/sachverstaendige/ordner (Besucht am 2.9.2008).

Thoms, R. (1964): Über die Geschichte des Haustier-Anthrax und die gegenwärtige Wandlung der Milzbrand-Problematik. Diss. Vet. Med. Leipzig.

Troitzsch, Ulrich: Umweltprobleme im Spätmittelalter und der Frühen Neuzeit aus technikgeschichtlicher Sicht. In: Bernd Herrmann (Hg.): Umwelt in der Geschichte. Beiträge zur Umweltgeschichte, Göttingen 1989, S. 89-110.

Trostorff, R. (1968): Importhäute und Milzbrand - eine experimentell gestützte Studie. Diss. Vet. Med. Leipzig.

Ueber den Milzbrand. (1830): Zur Belehrung und Warnung der Viehbesitzer und Landleute. Publicieret auf Befehl des Senats, Hamburg 1830.

Umweltbundesamt Berlin (Hg.) (1998): Erkundung ehemaliger Gerbereistandorte - Leitfaden, Berlin 1998.

Verordnung des Sächsischen Staatsministeriums für Umwelt und Landwirtschaft über Sachverständige nach \! ( BBodSchG) (SäschsSach VO). Vom 16. Dezember 2002. In: http:// www.umwelt.sachsen.de/lfug/abfallaltlasten_8428.html (Besucht am 18.07.2008).

Wald, Hermann (1862): Das Vorkommen und die Entstehung des Milzbrandes. Gekrönte Preisschrift der Frage „Tritt der Milzbrand in manchen 
Oertlichkeiten gar nicht, und in welchen regelmäßig oder häufig auf; und ist aus den physikalischen Bedingungen solcher Oertlichkeiten auf die Natur der Krankheit zu schließen?“, Halle.

Weber, Hans Hg. (1990): Altlasten. Erkennen - Bewerten - Sanieren, Berlin.

Weinheimer, C. (1895): Die Gesetze und amtlichen Belehrungen über die Krankheiten der Hausthiere einschließlich der Vorschriften über die Gewährleistung, 2. umgearbeitete Auflage, Schwäbisch-Hall 1895.

Weyl, Theodor (Hg. (1897): Handbuch der Hygiene, achter Band.

Gewerbehygiene, mit besonderer Rücksicht auf Fabrikgesetzgebung, Unfallschutz und Wohlfahrtseinrichtungen, Jena.

Wiedemann, Ulrike (1970): Staatlich angeordnete Maßnahmen zur Tierseuchenbekämpfung in Bayern im 18. Jahrhundert. Diss. Vet. Med. München.

Wiese, H. und J. Bölts (1966): Rinderhandel und Rinderhaltung im nordwesteuopäischen Küstengebiet vom 15. bis zum 19. Jahrhundert (Quellen und Forschungen zur Agrargeschichte Bd. XIV), Stuttgart.

Windel, Th. (1961): Unfallverhütungs- und sonstige Schutzmaßnahmen in der ledererzeugenden Industrie. In: Grassmann, W. und J. Trupke (Hrsg.):Handbuch der Gerbereichemie und Lederfabrikation, Bd. 3, Teil 1, 2. Aufl. Wien, S. 1529-1553.

Winkle, S. (1997): Geißeln der Menschheit: Kulturgeschichte der Seuchen, Düsseldorf/Zürich

Zündel (Hg.) (1911): Bericht über die XII. Plenarversammlung des Deutschen Veterinär-Rates zu Hamburg vom 5. Bis 8. April 1911. Darin: Entwurf einer Bundesratsinstruktion zum Viehseuchengesetz vom 26.6.1909 bezüglich der Duchführungsverordnung für Viehmärkte - Melkereien - Abdeckereien Verkehr mit Viehseuchenerregern und Impfstoffen - Viehkastrierer Milzbrand - Tollwut - Rotz - Maul- und Klauenseuchen - Lungenseuche Pockenseuche der Schafe - Beschälseuche und Bläschenausschlag - Räude der Einhufer und Schafe. Berlin 1911. 



\title{
Das Kyoto-Protokoll, oder: Was lässt sich aus der Geschichte umweltpolitischer Regulierung lernen?
}

\author{
Frank Uekötter
}

Das Jahr 2007 stand im Zeichen des Klimawandels. Die Berichte des Intergovernmental Panel on Climate Change (IPCC) wurden international zum Gegenstand intensiver Berichterstattung, die Klimaverhandlungen im indonesischen Bali wurden mit geradezu atemloser Spannung verfolgt, und gegen Ende des Jahres erhielt das IPCC zusammen mit dem amerikanischen Umweltpolitiker Al Gore den Friedensnobelpreis. Aber schon das folgende Jahr, in dem der hier dokumentierte Workshop in Göttingen stattfand, war von einer anderen, deutlich gedämpfteren Stimmung geprägt. Zwar war das Thema auch 2008 weiterhin in den Zeitungen, aber doch eher in den hinteren Seiten, während die Titelseiten anderen Problemen gewidmet wurden. War 2007 noch das Jahr, in dem die Dringlichkeit des Themas in grellen Farben ausgeleuchtet wurde, so war 2008 auch viel über die Kosten zu hören; im Zeichen eines rasanten Anstiegs der Energiepreise sowie der Erschütterung der Finanzmärkte war die Basis für eine energische Klimapolitik nun deutlich geschrumpft. Die zeitweise durchaus spürbare Erwartung, der endgültige Durchbruch der internationalen Klimapolitik stünde nun unmittelbar bevor, erwies sich als Illusion. Einiges spricht dafür, dass das Jahr 2007 der Klimadebatte lediglich eine neue Welle der öffentlichen Empörung bescherte. Seit dem öffentlichen Durchbruch des Themas in der zweiten Hälfte der 1980er Jahre sind immer wieder Konjunkturen der Klimadebatte zu registrieren, und 2007 war möglicherweise nur der jüngste Zyklus. 
Diese gegenwärtige Situation ist im hiesigen Zusammenhang nicht nur deshalb von Interesse, weil so schon die Ereignisse der jüngsten Vergangenheit zeigen, wie wichtig eine historische Perspektive für das Verständnis der Umweltdebatte ist. Es scheint auch, dass der skizzierte Diskussionsverlauf ein zentrales Problem der Umweltpolitik vor Augen führt, nämlich das Verhältnis von öffentlichem Protest und politischen Regelungen. Es bedarf keiner ausführlichen Begründung, dass der Aufschwung der Umweltpolitik in den vergangenen Jahrzehnten aufs Engste mit dem Entstehen einer breiten, populären Umweltbewegung verbunden ist. Ohne den Protest zahlreicher Personen von Brokdorf bis Buschhaus und die Bildung kräftiger politischer „pressure groups“ hätte sich die Umweltpolitik niemals zu einem eigenen Politikbereich mit Vertretung auf Ministeriumsebene gemausert soviel scheint man bei allem Streit der Historiker, wann dieser Übergang stattfand und wie dieser Zusammenhang überhaupt aussah, wohl sagen zu können. Aber zugleich ist doch deutlich, dass Umweltpolitik und Umweltbewegung ganz unterschiedlichen Verhaltenslogiken verpflichtet sind. Eine Umweltbewegung muss lebendig sein, attraktiv für breite Teile der Bevölkerung, sie muss mit Aktionen auf sich aufmerksam machen und Pressevertreter für sich gewinnen. Ganz anders die Imperative der Umweltpolitik: Sie muss Kompromisse zwischen unterschiedlichen Zielen und unterschiedlichen Fraktionen schmieden, sie muss Ziele in rechtlich verbindliche Vorgaben transformieren und deren Einhaltung sichern; während Bewegungen oft kurzfristig denken, nicht selten im Wochen- oder gar Tagesrhythmus, ist Umweltpolitik auf Jahre und Jahrzehnte orientiert. Oder noch kürzer: Umweltbewegung macht Spaß, Umweltpolitik oft nicht - so jedenfalls Raymond Tucker, eine Schlüsselfigur der amerikanischen Umweltpolitik im 20. Jahrhundert, der einmal bekannte, es gebe ,no romance“ in der Alltagsarbeit der Luftreinhaltung: „It is the unrelenting, day-by-day attention to detail that brings success. “1

Die Spannung zwischen Bewegungs- und Politikmodus bei Umweltproblemen ist kein Phänomen der jüngsten Vergangenheit. Einiges spricht sogar dafür, dass hier das zentrale Problem für den Umgang mit Umweltproblemen in offenen, demokratischen Gesellschaften liegt. Gewiss war es nicht das einzige Hindernis auf dem langen Weg zu einer Problemlösung. Natürlich mussten Umweltprobleme erst einmal als solche erkannt werden; aber das war in der Geschichte der Luftverschmutzung zumeist das geringste aller Probleme. Man nehme etwa die Kohlenrauchplage, also die bei der Verbrennung von Kohle entstehenden Rauch- und Rußemissionen, die über Jahrzehnte als wichtigste Luftverschmutzungsprobleme überhaupt galten und in der Genese der Umweltpolitik vielerorts eine Schlüsselrolle spielten: Die gräuliche Wolke über den Großstädten der Jahrhundertwende war für jeden Beobachter offenkundig, und die Folgen kannte jeder Stadtbewohner aus eigener Anschauung. Ein wichtiges Thema auf dem Weg zur Problemlösung war stets auch die Existenz von technischen Mitteln, die ein Problem zurückdrängten;

1 Raymond R. Tucker, Smoke Prevention in St. Louis, in: Industrial and Engineering Chemistry 33 (1941), S. 836-839; S. 839. 
aber auch hier fällt es schwer, einen echten „bottleneck“ zu erkennen. Es ist erstaunlich, wie früh viele technische Lösungsansätze existierten. Das Prinzip der katalytischen Abgasreinigung bei Automobilen war beispielsweise schon vor dem Ersten Weltkrieg bekannt. ${ }^{2}$ Kritisch wurde es erst dort, wo es darum ging, die technische Entwicklung bis zur Anwendungsreife zu forcieren und den Einbau der Technik durchzusetzen: Dazu brauchte es einen gewissen öffentlichen Druck und eine geschickte regulative Politik - aber vor allem bedurfte es einer Balance zwischen beidem. Und gerade diese Balance war in vielen Fällen das Schlüsselproblem.

Das zeigte sich zum Beispiel im späten 19. Jahrhundert, als die Großstädte der Welt aufgrund des steigenden Kohleverbrauchs immer mehr mit Rauch- und Rußbelästigungen zu kämpfen hatten. Gegen dieses Problem organisierten sich in amerikanischen Städten seit 1880 Bürger der urbanen Oberschicht. Sie gründeten in bürgerlichen Reformvereinen eigene Ausschüsse für Rauchbekämpfung und sogar spezielle Anti-Smoke Leagues, die keinem anderen Zweck dienten als dem Kampf gegen die Rauchplage. Zunächst standen diese Vereine und Kommissionen ganz im Zeichen einer radikalen Verfolgungsstrategie: Man wollte so viele Übeltäter bestrafen wie möglich. „We will not exclude anyone from prosecution in our hunt for the lawbreakers", lautete eine typische Devise. ${ }^{3}$ Aber im Laufe der Zeit wurde der Tenor deutlich konzilianter: Das Bestrafen übermäßigen Rauchausstoßes lief nämlich rasch auf einen Kampf gegen Windmühlenflügel hinaus. Manche Unternehmer verkündeten ganz offen, dass sie lieber eine monatliche Strafe von 50 Dollar bezahlten, als sich mit der komplizierten Materie der Rauchbekämpfung zu befassen. ${ }^{4}$ Andere vertrauten darauf, dass der Verfolgungseifer nach einiger Zeit schon wieder erlahmen würde, was sich in vielen Fällen auch als zutreffende Erwartung erwies: Nach wenigen Jahren des radikalen Kampfes gegen rauchende Unternehmen waren die meisten Bürger am Ende ihrer Energie. Ein Leitartikel der Zeitung St. Louis Republic sprach nach einigen wiederholten Kampagnen ziemlich unverhüllt vom Scheitern der Bewegung: „Does anybody doubt that the policy of threatening complaints, securing occasional convictions and recommending frequent remissions of fines on promises to do better, has been a failure?" 5 Daraus lernte die Bewegung nach einiger Zeit, mäßigte ihre Rhetorik und schuf Beratungsangebote als Ergänzung zur Strafe. Viele Stadtverwaltungen stellten „smoke inspectors" ein, zumeist geschulte Ingenieure, die den Unternehmern Ratschläge zur rauchfreien Verbrennung erteilten und beim Bau zweckmäßiger Feuerungsanlagen halfen; bestraft wurden nur noch jene Unternehmen, die trotz guter Ratschläge keine Besserung zeigten. Das kam bei den Unternehmern, bis dahin erbitterte

2 Frank Uekötter, Der Wert des Vorsorgeprinzips: Die gescheiterte Bekämpfung der Automobilabgase in Deutschland und den USA vor 1945, in: Technikgeschichte 69 (2002), S. 41-72; S. 47.

3 Baltimore Sun 18.1.1907 S. 12 Sp. 3.

4 Civic Improvement League of St. Louis, Second Annual Report of the Civic Improvement League, March 1904, St. Louis 1904, S. 27.

$5 \quad$ St. Louis Republic 9.2.1911 S. 6 Sp. 2. 
Gegner jeglicher Rauchbekämpfung, ausgesprochen gut an, und viele der Städte, die einen oder mehrere „smoke inspectors“ eingestellt hatten, vermeldeten nach einiger Zeit zwar nicht das Verschwinden der Rauchplage, aber doch einen spürbaren Rückgang. 6

Das beweist freilich nicht, dass Luftreinhaltung im lokalen Rahmen grundsätzlich erfolgreicher war als auf der nationalen oder gar supranationalen Ebene. Das genaue Gegenbild zur geschilderten Erfolgsgeschichte liefert der Konflikt um die Rauchschäden der Metallhütten, der in Deutschland seit Mitte des 19. Jahrhunderts ausdauernd und heftig geführt wurde. Vor allem Waldbesitzer protestierten heftig gegen die schwefelhaltigen Abgase solcher Fabriken, die ihren Besitz schädigten, sie organisierten sich wissenschaftliche Experten, um den Schaden zu belegen, wandten sich an die Industrieunternehmen und die Behörden, und erstaunlich oft bekamen sie nach mehr oder weniger langen Verhandlungen auch Recht. Aber das Ergebnis war vom Standpunkt des Emissionsschutzes zumeist enttäuschend: Es wurden keine Filter eingebaut, auch keine Produktion heruntergefahren oder Verfahren modifiziert, sondern lediglich zwischen Verschmutzern und Opfern eine Entschädigung ausgehandelt. Der Kreativität der Beteiligten waren dabei kaum Grenzen gesetzt. Eine Zinkhütte in Borbeck im nördlichen Ruhrgebiet leistete ihre Entschädigung in den 1880er Jahren zum Beispiel „,nicht in baar, sondern in Form von Düngestoffen (Kalk, Gyps, Mist u.s.w.)".7 Ein anderes Verschmutzungsopfer, der Fürst Metternich, vereinbarte mit einer Zink- und Rösthütte in BergischGladbach, „daß das bis auf eine gewisse Entfernung von der Hütte in den Forsten entstehende dürre Holz von der Hütte zum vollen Werthe gesunden Holzes entnommen werden muß." 8 Tatsächlich war es naheliegend, einen solchen Deal zu schließen: Die Opfer der Verschmutzung hielten sich zumindest finanziell mehr oder minder schadlos, und die Verschmutzer konnten sich eines unangenehmen Problems mit relativ geringem Aufwand entledigen. Zugleich sind solche Deals ein wichtiges Memento, bei Personen, die heftig über ein Verschmutzungsproblem klagen, genauer hinzuschauen. Die heute so beliebte Ansicht, jeder Gegner des Klimawandels sei ein Idealist (und jeder Zweifler ein Vertreter der Öllobby), lässt sich historisch rasch als naiv entlarven. Auch viele vermeintlich idealistische Klimaschützer verfolgen tatsächlich Sonderinteressen, nur ist die Bereitschaft, diese Interessen offenzulegen, bislang bemerkenswert unterentwickelt.

Die beiden geschilderten Episoden stammen aus einer Zeit, in der Luftreinhaltung eine geographisch eng begrenzte Angelegenheit war. Die im 19. Jahrhundert etablierten Umgangsweisen definierten Luftverschmutzung als ein durch und durch lokales Problem, für das man vor Ort Vereinbarungen treffen konnte, ohne

$6 \quad$ Vgl. Frank Uekötter, Von der Rauchplage zur ökologischen Revolution. Eine Geschichte der Luftverschmutzung in Deutschland und den USA 1880-1970, Essen 2003. In Kürze erscheint eine überarbeitete englische Fassung: Frank Uekoetter, The Age of Smoke. Environmental Policy in Germany and the United States, 1880-1970, Pittsburgh 2009.

7 Geheimes Staatsarchiv Preußischer Kulturbesitz Berlin Rep. 120 BB II a 2 Nr. 13 adh. Bl. 79.

8 GStA Rep. 120 BB II a 2 Nr. 13 adh. Bl. 11. 
sich um landesweite Gesetze und Verordnungen oder gar internationale Verträge sonderlich kümmern zu müssen. Diese Zeit endete in Deutschland schon kurz nach dem Zweiten Weltkrieg, als sich die Bundesregierung und vor allem die Landesregierung Nordrhein-Westfalen verstärkt um Luftverschmutzungsprobleme zu kümmern begann. Diese Aktivitäten sind bislang nicht in das kollektive Gedächtnis der Bundesrepublik eingegangen, das immer noch die hartnäckige Legende pflegt, die Bürger hätten sich vor Lachen auf die Schenkel geschlagen, als Willy Brandt den „blauen Himmel über der Ruhr“ forderte. Tatsächlich gab es aber schon in den 1950er Jahren einen enormen öffentlichen Unmut über den Zustand der Luft insbesondere im Ruhrgebiet. Das Westdeutsche Tageblatt wagte etwa schon im Oktober 1959, die Luftverschmutzung zum „Ruhr-Problem Nr. 1“ zu erklären.9 Zugleich gab es eine institutionelle Reaktion, Gesetze auf Bundes- und Landesebene wurden überarbeitet, zusätzliches Personal für den Immissionsschutz eingestellt und die VDI-Kommission Reinhaltung der Luft als Organ für die Entwicklung technischer Normen geschaffen. Und auch hier fiel es zunächst nicht leicht, Protestlogik und Bekämpfungslogik miteinander in Einklang zu bringen.

Es gab also, wenn man so will, nicht nur eine ökologische Revolution, sondern zwei Revolutionen: eine im Protestverhalten der breiten Bevölkerung und eine in den institutionellen Arrangements. Dabei war es auch keineswegs so, dass erstere der letzteren voranging, also der Protest der mündigen Bürger erst die träge Verwaltung auf Trab brachte. Lange Zeit wirkte es in Deutschland eher so, als ob die institutionelle Revolution der Revolution der Bewegungsmuster vorauseilte: Als 1964 die erste „Technische Anleitung Luft“ erschien und damit eine ungefähr zehn Jahre währende Zeit grundlegender Reformen abgeschlossen wurde, gab es deutschlandweit nur wenige Vereine oder Interessengemeinschaften, die gegen Luftverschmutzung protestierten. Auf der Ebene der maßgeblichen Gesetze hatte man jedenfalls seine Hausaufgaben gemacht, lange bevor Genscher als Bundesinnenminister die Umweltpolitik zu einem vielbeachteten Kernbereich seiner Amtsführung machte. Eine Besprechung von Ländervertretern erklärte 1968 klipp und klar: „Von der Rechtsgrundlage her besteht kein ,Notstand'“" 10 Das kann man auch im Rückblick durchaus so stehen lassen, denn als der Bundestag sechs Jahre später das Bundesimmissionsschutz verabschiedete (übrigens ohne eine einzige Gegenstimme ${ }^{11}$ ), war der Inhalt alles andere als revolutionär: Im Land NordrheinWestfalen, das damals die Avantgarde der Luftreinhaltung markierte, galt das Gros der vermeintlich neuen Bestimmungen bereits seit einem Dutzend Jahren. Selbst in den 1980er Jahren, bekanntlich das Jahrzehnt der Umwelt in der Geschichte der Bundesrepublik, blieb ein echter Politikwechsel, also eine Modifikation der politi-

$9 \quad$ Westdeutsches Tageblatt Nr. 249 (27.10.1959), S. 6 Sp. 1-3.

10 BayHStA MWi 28368, Ergebnisniederschrift über eine Länderbesprechung zum Entwurf eines Bundesimmissionsschutzgesetzes am 19.9.1968 im Arbeits- und Sozialministerium Düsseldorf, S. 2.

11 Verhandlungen des Deutschen Bundestages, 7. Wahlperiode (Stenographische Berichte Bd. 86), Bonn 1974, S. 4691. 
schen Steuerungsinstrumente, weitgehend aus. Einen schlagenden Beleg liefert die berühmte Großfeuerungsanlagen-Verordnung, die 1983 unter dem Eindruck der Waldsterbensdebatte erlassen wurde und die Entschwefelung aller deutschen Großkraftwerke innerhalb von fünf Jahren anordnete: Kaum ein Beobachter registrierte, dass damit eine Tradition administrativer Einzelanordnungen auf Regierungsebene fortgesetzt wurde, die bis weit ins 19. Jahrhundert zurückreicht. ${ }^{12}$

Das Schlüsselproblem dieses typisch deutschen Ansatzes der Emissionsbekämpfung war freilich stets der Vollzug. An guten Vorgaben von ministerieller Ebene hat es seit dem 19. Jahrhundert in Deutschland nur selten gemangelt - aber umso häufiger an dem Willen der unteren Behörden, diese Vorgaben auch systematisch umzusetzen. Bei der Großfeuerungsanlagen-Verordnung war dieses Problem relativ unbedeutend, da das Waldsterben so viel Aufsehen erregte, dass die Kraftwerksbetreiber sich tatsächlich streng an die ministerielle Verordnung hielten; aber das ist historisch beileibe nicht der Normalfall. Wer sich jahrelang durch Behördenakten wühlt, der lernt im Laufe der Zeit, dass die Welt des Vollzugs im Grunde genommen ein Paralleluniversum darstellte, in dem der Wunsch des Ministeriums nach strengem Vorgehen oft nicht mehr war als ein schwindendes Echo. Viele Behördenvertreter sahen wenig Grund für einen energischen Vollzug: Soll man sich wirklich mit mächtigen Industriellen anlegen, nur um einer ministeriellen Verordnung zu genügen? Und konnte das Ministerium überhaupt kontrollieren, wie die eigenen Verordnungen vollzogen wurden? Eine nennenswerte Kontrolle des Vollzugs gab es in der Geschichte der Luftreinhaltung nur in seltenen Ausnahmefällen, und so wurde die Umsetzung von Umweltnormen zu einer Schattenwelt, in der letztlich nichts unmöglich war. Erwähnt sei hier anstelle vieler Einzelbeispiele nur ein Fall, den das Gewerbeaufsichtsamt Dortmund 1959 schilderte: Hier ging es um eine Kohlenstaubfeuerung mit Schmelzkammerkessel, die zu diesem Zeitpunkt „bereits seit vier Jahren mit vorläufiger Genehmigung in Betrieb [war], ohne daß es bisher möglich war, sich mit dem Unternehmer über die endgültige Fassung der Bedingungen zu einigen. "13

Man kann diese Erfahrung als eine nachdrückliche Warnung vor einem „TopDown-Modus“ der Politikformierung interpretieren, also einer Politik, die ganz wunderbare Ziele formuliert und einfach davon ausgeht, dass diese Vorgaben schon irgendwie zu den Behörden vor Ort und den Unternehmen durchsickern würden. Ein solcher „Top-Down-Modus“ ist ein Kernelement des deutschen Regulierungsstils und wurde durch die Reformen der 1950er Jahre eher verstärkt als modifiziert; aber es ist sehr bezeichnend, dass die damit zusammenhängenden Probleme erst seit 1974 diskutiert wurden, als der Sachverständigenrat für Umweltfragen auf das Implementationsdefizit im Umweltrecht hinwies. ${ }^{14}$ Es drängt sich

12 Ausführlich dazu Kenneth Anders, Frank Uekötter, Viel Lärm ums stille Sterben: Die Debatte über das Waldsterben in Deutschland, in: Frank Uekötter, Jens Hohensee (Hg.), Wird Kassandra heiser? Die Geschichte falscher Ökoalarme, Stuttgart 2004, S. 112-138.

13 Hauptstaatsarchiv Düsseldorf NW 66 Nr. 436 Bl. 57.

14 Deutscher Bundestag, 7. Wahlperiode, Drucksache 2802, S. 177. 
geradezu der Eindruck auf, dass es in Sachen Vollzug eine Art Kartell des Schweigens gab: Die nachgeordneten Behörden vor Ort behielten die Umsetzungsprobleme für sich, und die Ministerialbeamten verzichteten ihrerseits darauf, allzu detailliert nachzufragen; und das scheint in der Klimadebatte der Gegenwart ganz ähnlich zu sein. Viel wird hier über die Folgen des Klimawandels gesprochen, viel auch über allgemeine Vorgaben, ein wenig redet man auch noch über die internationalen Klimaverhandlungen und das Kyoto-Protokoll. Aber danach wird es doch sehr ruhig, und diese Situation kommt einem als Historiker doch arg bekannt vor. Vollzug strenger Vorgaben war nie ein einfaches Unterfangen, immer wieder gab es vor Ort, wenn die abstrakten Vorgaben konkret wurden, Widerstände, Proteste, Verhandlungen und Kompromisse, manchmal tragfähige, oft faule. Die Hoffnung, das Kyoto-Protokoll würde sicherlich streng vollzogen werden, wenn nur endlich die USA unterschreiben würden, ist schlechterdings naiv.

Ein wichtiger Teil des Kyoto-Protokolls ist die Schaffung eines Marktes für Treibhausgasemissionen, um einen marktförmigen Weg in der Bekämpfung des Klimawandels zu ermöglichen; und auch hier lohnt ein Blick in die Vergangenheit. Die Geschichte solcher Überlegungen ist länger, als man zunächst vermuten würde: Sie beginnt mit dem Konzept für eine „Smog-Steuer“, die die Rand Corporation schon 1959 für Kalifornien formulierte. ${ }^{15}$ Wichtiger war langfristig die Coalition to Tax Pollution, die im Sommer 1971 als ein Dachverband amerikanischer Umweltverbände entstand und für eine Schwefelsteuer plädierte. ${ }^{16}$ Das war ein durchaus zukunftsweisender Vorschlag, denn tatsächlich kamen für Schwefelemissionen in den USA marktförmige Instrumente zur Anwendung - allerdings erst zwei Jahrzehnte später mit den Clean Air Act Amendments von 1990. Der darin vorgesehene Emissionshandel ist seither vielfach gelobt worden und gilt geradezu als Modell für den Marktmechanismus des Kyoto-Protokolls; aber auch hier sollte man genauer hinschauen. Der Markt für Schwefeldioxidzertifikate war in diesem Programm eng begrenzt und überschaubar, bei den Marktteilnehmern handelte es sich im wesentlichen um Kraftwerksbetreiber, und die Technik der Emissionsvermeidung, die berühmte Rauchgasentschwefelung, war längst verfügbar - das sieht beim Treibhauseffekt bekanntlich anders aus. Nicht zuletzt kam der Handelsmechanismus erst vor dem Hintergrund der zwei Jahrzehnte währenden Versuche zum Tragen, Schwefeldioxid auf dem klassischen Weg behördlicher Anweisungen, also der sogenannten „command-and-control“-Regulierung zu bekämpfen. Ein ähnliches Erlebnis fehlt beim Kyoto-Protokoll: Hier war der Marktmechanismus von Anfang an der Weg der Wahl. Interessanterweise schloss die erste umfassende Studie zu den Clean Air Act Amendments mit einer Mahnung, das Modell nur mit

15 Fort, D. M., Niskanen, W. A., Pascal, A. H., Sharpe, W. F., Proposal for a „Smog Tax“, Santa Monica 1959. Ausführlich zu dieser Geschichte Hugh S. Gorman, Barry D. Solomon, The Origins and Practice of Emissions Trading, in: Journal of Policy History 14 (2002), S. 293-320.

16 Department of Special Collections, Charles E. Young Research Library, University of California, Los Angeles, Collection 1199 Box 85 Folder 4, Coalition to Tax Pollution, The Sulfur Tax, 25.5.1972, Introduction. 
viel Vor- und Umsicht auf andere Problemfelder zu übertragen: „There is a potentially large distance between embracing emissions trading in principle and producing a detailed program that will perform well in practice." Ausdrücklich zielten die Autoren damit auf die Klimapolitik Marke Kyoto: „The gap between principle and practice appears quite large in the climate change context." ${ }^{\text {"17 }}$

Man erkennt, dass das Kyoto-Protokoll die Verhaltenslogiken der Umweltbewegung weitaus besser bedient als jene der Umweltpolitik. Die Klimapolitik Marke Kyoto ist global angelegt und spiegelt damit die Globalität der Problemlage, sie umfasst alle klimarelevanten Emissionen und beschränkt sich nicht auf jene, die relativ leicht $\mathrm{zu}$ regulieren sind, und sie verwendet mit dem Marktmechanismus einen Regulierungsmodus, der ganz unabhängig von seiner tatsächlichen Leistungsfähigkeit das Odium einer kühnen, innovatorischen Lösung verströmt. Die Kritik darf freilich an dieser Stelle nicht stehenbleiben, denn man kann die aktuelle Klimapolitik auch im breiten Referenzrahmen der internationalen Umweltpolitik betrachten. Eine internationale Vernetzung der Umweltdebatten ist schließlich schon vor dem Ersten Weltkrieg zu registrieren, aber sie führte erstaunlich selten zu greifbaren Resultaten. Auch im 21. Jahrhundert erscheint die Geschichte internationaler Abkommen in der Umweltpolitik nicht gerade ermutigend. Bis heute ist das Montreal-Protokoll von 1987 das wichtigste internationale Umweltabkommen, und dieses beschäftigte sich mit den Fluorchlorkohlenwasserstoffen, die als Verursacher des Ozonlochs identifiziert wurden. Die Verabschiedung und erfolgreiche Umsetzung des Montreal-Protokolls war gewiss keine geringe Leistung, aber doch etwas anderes als die Bekämpfung der Treibhausgase, die nahezu jeden Bereich unseres täglichen Lebens betreffen. ${ }^{18}$

Vermutlich ist es auch kein Zufall, dass internationale Abkommen im Bereich der Umweltpolitik so oft zahnlos sind. In der Geschichte der Umweltpolitik erkennt man, dass es lange Zeit eine gewisse Kongruenz zwischen politischem Aktionsradius und politischem Protest gab: Die Logiken von Protestbewegungen und Politiken waren nicht leicht vereinbar, aber immerhin umfassten sie grob den gleichen geographischen Rahmen. Die Rauchbekämpfer waren in amerikanischen Großstädten beheimatet - und so wurde Rauchbekämpfung ein Projekt der Kommunalverwaltungen. Das Ruhrgebiet lag im Herzen Nordrhein-Westfalens und so wurde Luftreinhaltung in den 1950er Jahren Ländersache. Beide Male das gleiche Phänomen: Ein lokaler bzw. regionaler Protest führte zu lokalen bzw. regionalen Lösungen. Ganz anders die derzeitige Situation beim Klimawandel: Hier fehlt eine einheitliche globale Problemdefinition, allen Bemühungen der vergangenen beiden Jahrzehnte zum Trotz. Es fällt nicht schwer zu erkennen, dass das Bewusstsein, das der Klimapolitik zugrunde liegt, eigentlich ein westeuropäischnordamerikanisches ist. Selbst in der EU gibt es enorme Divergenzen. Zum Teil

17 A. Danny Ellerman u.a., Markets for Clean Air. The U.S. Acid Rain Program, New York und Cambridge 2000, S. 321.

18 Vgl. Richard Elliot Benedick, Ozone Diplomacy. New Directions in Safeguarding the Planet, Cambridge, Mass. und London 1998. 
sind diese durch die unterschiedlichen Klimazonen Europas bedingt: Während in Spanien die durchaus reale Angst vor Desertifikation eine Rolle spielt, ist die Aussicht auf wärmere Zeiten für Finnland eigentlich nicht sehr furchterregend. Zum Teil handelt es sich aber auch um einen klassischen Ost-West-Gegensatz: Während Klimapolitik in Westeuropa als wichtige, ja unverzichtbare Selbstbeschränkung verkauft wird, wird sie in Ost- und Ostmitteleuropa als Neuauflage zentraler staatlicher Planung empfunden; man schaue nur auf das jüngst auch in deutscher Sprache erschienene Buch des tschechischen Präsidenten Václav Klaus. ${ }^{19}$ Und während die Gefahren des Klimawandels für die Dritte Welt immer wieder grell ausgemalt werden, ist das Interesse an den Folgen, die die Klimadebatte dort schon heute zeitigt, auffallend schwach. Zu den aufwühlendsten Studien im hiesigen Themenfeld gehört m.E. Anita Engels Dissertation über die Klimadebatte im Senegal. Dort kann man im Detail nachlesen, wie die Klimadebatte zu einer Verlagerung der Forschungsressourcen im Senegal führte: weg von den lokal brennenden Problemen wie der Küstenerosion, hin zum Klimaproblem, weil nach dem KyotoProtokoll auch der Senegal ein Klimakonzept entwickeln und den kritischen Gutachtern in der westlichen Welt präsentieren muss. Dabei liegt auf der Hand, dass Klimapolitik im Senegal purer Unsinn ist, denn das Land produziert etwa vier Millionstel der weltweiten Treibhausemissionen. ${ }^{20}$

Es dürfte deutlich geworden sein, dass die Skepsis des Verfassers gegenüber der gegenwärtigen Klimapolitik nichts mit einer Geringschätzung für das Problem zu tun hat. Trotzdem sei es zur Sicherheit noch einmal betont: Der Klimawandel ist eine der wichtigsten Herausforderungen der Menschheit im 21. Jahrhundert, und allein die Vorstellung, dass die Menschheit derzeit in der Atmosphäre ein globales Experiment mit ungewissem Ausgang durchführt, ist Grund genug zum entschlossenen Handeln. Nur scheint es gerade deshalb notwendig, vor dem Hintergrund der umweltpolitischen Erfahrungen gründlich über die gewählten Instrumente nachzudenken, und da ist das Ergebnis eigentlich ziemlich eindeutig: Wenn das Kyoto-Protokoll Erfolg haben sollte, wird es einen Großteil dessen widerlegen, was wir in vergangenen Jahrzehnten über Umweltpolitik gelernt haben. Im Grunde genommen ist die Frage nicht mehr $o b$, sondern woran das Klimaregime von Kyoto scheitern wird: am Widerstand einzelner Länder? An den kümmerlichen Zielvorgaben, die beim derzeitigen Stand ja nur das Wachstum des Problems ein wenig vermindern werden? Oder an einem großen Korruptions- und Manipulationsskandal? Gewiss lehnt man sich als Historiker mit solchen Prognosen weit aus dem Fenster, aber einiges spricht für das letztgenannte Szenario. Der Handels- und Implementationsmechanismus des Kyoto-Protokolls ist derart kompliziert, dass es ein Wunder wäre, wenn er nicht missbraucht würde. Längst gibt es Indizien für frisierte Daten und dubiose Klimaschutzprojekte - und dies zu einem Zeitpunkt,

19 Václav Klaus, Blauer Planet in grünen Fesseln. Was ist bedroht: Klima oder Freiheit? Wien 2007.

20 Anita Engels, Die geteilte Umwelt. Ungleichheit, Konflikt und ökologische Selbstgefährdung in der Weltgesellschaft, Weilerswist 2003. 
zu dem man mit $\mathrm{CO}_{2}$-Zertifikaten noch gar kein Geld verdienen kann. Es ist durchaus denkbar, dass eine Serie von Skandalen in den kommenden Jahren nicht nur den Kyoto-Prozess diskreditiert, sondern zugleich die gesamte Klimapolitik mit in den Abgrund reißt.

Wenn man sich skeptisch über das Kyoto-Protokoll äußert, dann kommt mit ziemlicher Wahrscheinlichkeit die Gegenfrage: Aber was ist die Alternative? Dabei ist es eigentlich nicht schwer, Alternativen zu finden. Kaum ein Umweltproblem erlaubt eine derartige Vielfalt von Lösungsansätzen: Man kann auf der nationalen Ebene ansetzen, auf der supranationalen und globalen, aber auch auf der regionalen und lokalen; man kann klassische Ordnungspolitik betreiben, aber auch marktförmige oder korporatistische Ansätze; man kann alle Verschmutzer in den Blick nehmen oder nur große industrielle Verschmutzer; ja man muss noch nicht einmal zwangsläufig auf politische Maßnahmen setzen, denn ein wesentlicher Grund der Verschmutzung ist die innige Verknüpfung des Energieverbrauchs mit unserem modernen Lebensstil, und Lebensstile sind ein Gegenstand, der sich staatlicher Regulierung gottlob entzieht. Natürlich sind einige dieser Wege besser als andere, manche werden von den einschlägigen Lobbies propagiert, denen die ganze Klimadebatte ungelegen kommt - aber könnte man über all das nicht auch eine konstruktive Debatte führen? Die Monomanie, mit der „Kyoto“ zur allein seligmachenden Lösung erklärt wird, ist jedenfalls rätselhaft.

Man kann sich leicht vorstellen, dass Umwelthistoriker einer kommenden Generation ein wenig ratlos sein werden, wenn sie in der Geschichte der Umweltpolitik auf die Klimapolitik zu sprechen kommen. Das Kyoto-Protokoll wirkt vor diesem Hintergrund der derzeit existierenden Traditionen wie ein im luftleeren Raum entstandener, letztlich ahistorischer Ansatz. Es knüpft nicht an historische Erfahrungen an, ja steht zum Teil in offenem Widerspruch zu diesen Erfahrungen, und es setzt sich erstaunlich leichtfüßig auch über die unterschiedlichen nationalen Regulierungsstile hinweg. Gewiss gibt es keinen Grund, landesspezifische Tradition für sakrosankt zu erklären: Das Modell der nationalen Regulierungsstile beschreibt einen Modus der Politikformierung, aber keineswegs einen „one best way“. Dennoch scheint es ratsam zu sein, die in den einzelnen Ländern gewonnenen Erfahrungen nicht einfach über Bord zu werfen, sondern vielmehr auf ihr Potential für eine effektive Klimapolitik hin abzuklopfen. Als ein wichtiger Vorzug der Klimapolitik im nationalstaatlichen Rahmen dürfte sich dabei ihre relative Überschaubarkeit erweisen: Ein internationales Klimaregime mit einer dreistelligen Zahl von Mitgliedsstaaten weist eine inhärente Unübersichtlichkeit auf, die in denkbar scharfem Kontrast zum demokratischen Gebot der Transparenz steht.

Es ist jedenfalls an der Zeit, einmal von Seiten der Umweltbewegung anzufangen, über Alternativen zu Kyoto nachzudenken und etwas zu entwickeln, was man als einen Plan B der Klimapolitik bezeichnen könnte. Das erscheint umso wichtiger, als von einer solchen Alternative vermutlich nicht nur die Klimadebatte profitieren würde, sondern auch das Kyoto-Protokoll selbst. Denn auch das kann man aus der Geschichte der Luftreinhaltung lernen: Oft bewegte sich politisch erst 
dann etwas, wenn es eine Alternative gab, die alle Parteien vermeiden wollten. Musterbeispiel Rauchdebatte: Wenn der amerikanische „smoke inspector“ so relativ erfolgreich war, dann auch deshalb, weil alle Beteiligten eine Rückkehr zum früheren, antagonistischeren Vollzugsmodus verhindern wollten. Auch in der frühen Nachkriegszeit fehlte es nicht an Drohkulissen: In einem Gespräch über Flugstaubprobleme bei nordrhein-westfälischen Braunkohlenkraftwerken warnten die Behördenvertreter 1954, es drohten ,„Notwehr'-Massnahmen des Regierungspräsidenten unter dem Druck der öffentlichen Meinung“'.21 Und in der Umweltpolitik der 1980er Jahre ist ohnehin unverkennbar, dass sie auch deshalb erfolgreich war, weil die wachsende Umweltbewegung nach weitaus radikaleren Maßnahmen dürstete. Auch die Klimapolitik braucht einen Plan B, wenn sie den zahlreichen Gegnern einer effektiven Klimapolitik entschlossen gegenübertreten möchte. Viel zu sehr hat sich die Umweltbewegung in den letzten Jahren darauf versteift, den Kyoto-Prozess nahezu um jeden Preis zu retten. Es ist an der Zeit, dass sie den Weg zu einer neuen, selbstbewussteren Haltung findet: Wir können auch anders.

21 Bundesarchiv Berlin R 154/86, Sitzung des Staubausschusses der Braunkohlekraftwerke im Kölner Bezirk vom 3.8.1954, S. 6. 



\title{
Naturkatastrophen: Was wurde aus ihnen gelernt?
}

\author{
Manfred Jakubowski-Tiessen
}

Wissenschaftliche Institutionen wie etwa das Fraunhofer Institut für intelligente Analyse- und Informationssysteme ${ }^{1}$, aber auch eine humanitäre Hilfsorganisation wie das Rote Kreuz gehen - wie selbstverständlich - davon aus, dass wir aus $\mathrm{Na}$ turkatastrophen etwas lernen können. ${ }^{2}$ In erster Linie wird angenommen, dass jeder Ausbruch einer Naturkatastrophe neue spezifische Erkenntnisse über Genese, Ablauf und Folgen von Naturkatastrophen bringen kann und dass diese neuen Erkenntnisse für die Katastrophenprognostik, Katastrophenprävention und Katastrophenbewältigung wertvoll sind, so dass Auswirkungen künftiger Naturgefahren begrenzt werden können.

Schaut man sich die Statistik über Naturkatastrophen der letzten 50 Jahre an, wie sie die beiden weltweit größten Rückversicherungsgesellschaften, die Münchner Rück (Abb. 1) und die Swiss Re (Abb. 2), aufgestellt haben, so wird allerdings erkennbar, dass die durch Naturkatastrophen verursachten Schäden in dieser Zeitspanne insgesamt keineswegs gesunken, sondern weltweit vielmehr enorm gestie-

\footnotetext{
1 „Aus Katastrophen lässt sich lernen. Mobile Response 2008“ hieß ein vom Fraunhofer Institut für intelligente Analyse- und Informationssysteme am 18. Juni 2008 veranstaltetes Internationales Symposium zu mehr Sicherheit und effizienterem Notfallmanagement durch IT.

2 Am 11. September 2004 veranstaltete das Deutsche Rot Kreuz in Dachau zum Thema „Aus Katastrophen lernen. Das Undenkbare planen“ zu einem interaktiven Training mit internationaler Beteiligung auf.. Beispielsweise rief auch der Club of Budapest unter dem Titel „Aus der Katastrophe lernen" zu einem öffentlichen Dialog über konkrete Schritte zu einer nachhaltigen Zivilisation auf.

Der Deutsche Geographentag befasste sich im September 2007 in Bayreuth ebenfalls mit diesem Thema. Siehe Nordbayrischer Kurier, Samstag/Sonntag 29./30. September 2007, S. 51.
} 
gen sind - vor allem in den letzten 30 Jahren. ${ }^{3}$ Diese Tatsache stimmt nachdenklich, und es drängt sich die Frage auf, ob und was nun wirklich aus Naturkatastrophen gelernt wurde. Wieso haben die Erkenntnisse, die wir aus den Analysen vergangener Katastrophen gezogen haben, nicht dazu geführt, die Auswirkungen von Naturkatastrophen zu begrenzen und die durch sie verursachten Schäden infolge von Präventivmaßnahmen weltweit drastisch zu minimieren?

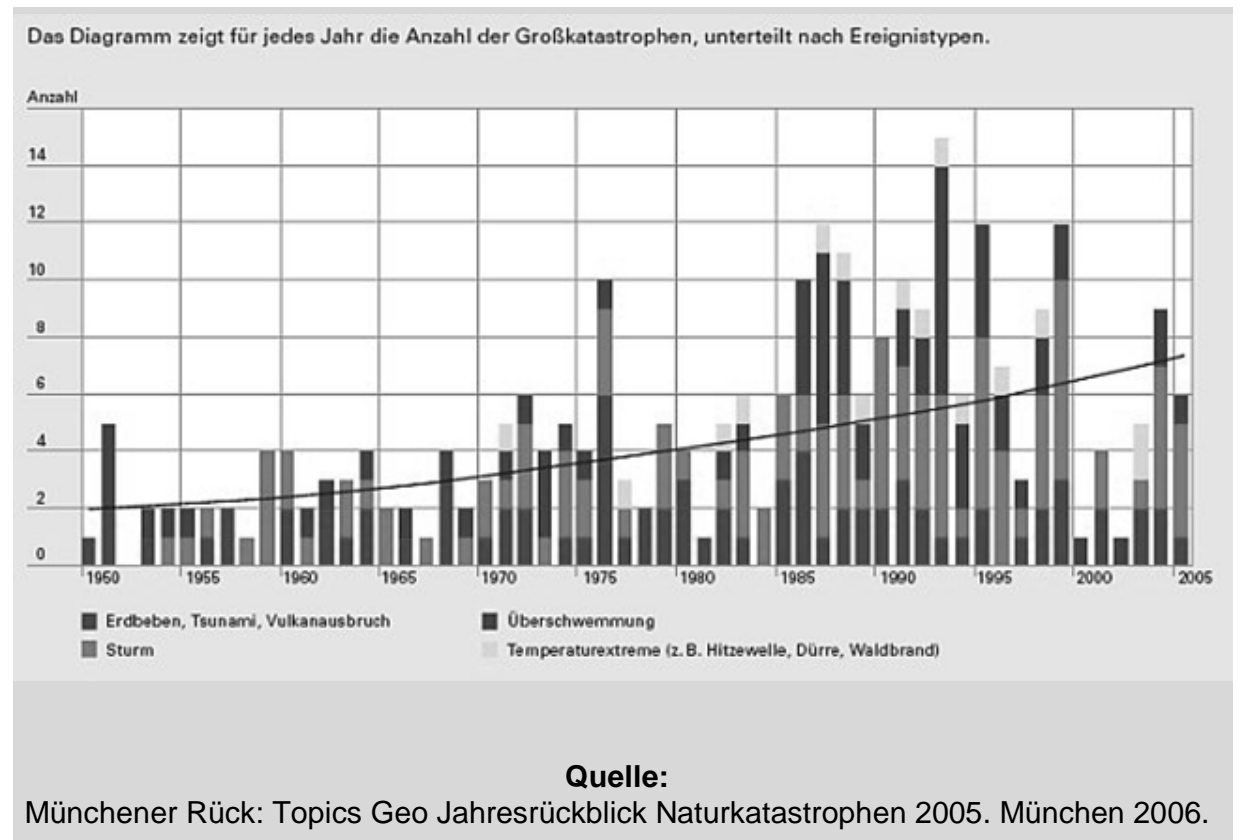

Abbildung 1: Statistik über Naturkatastrophen der letzten 50 Jahre der Rückversicherungsgesellschaft Münchner Rück.

${ }^{3}$ Renn / Klinke (2004). 


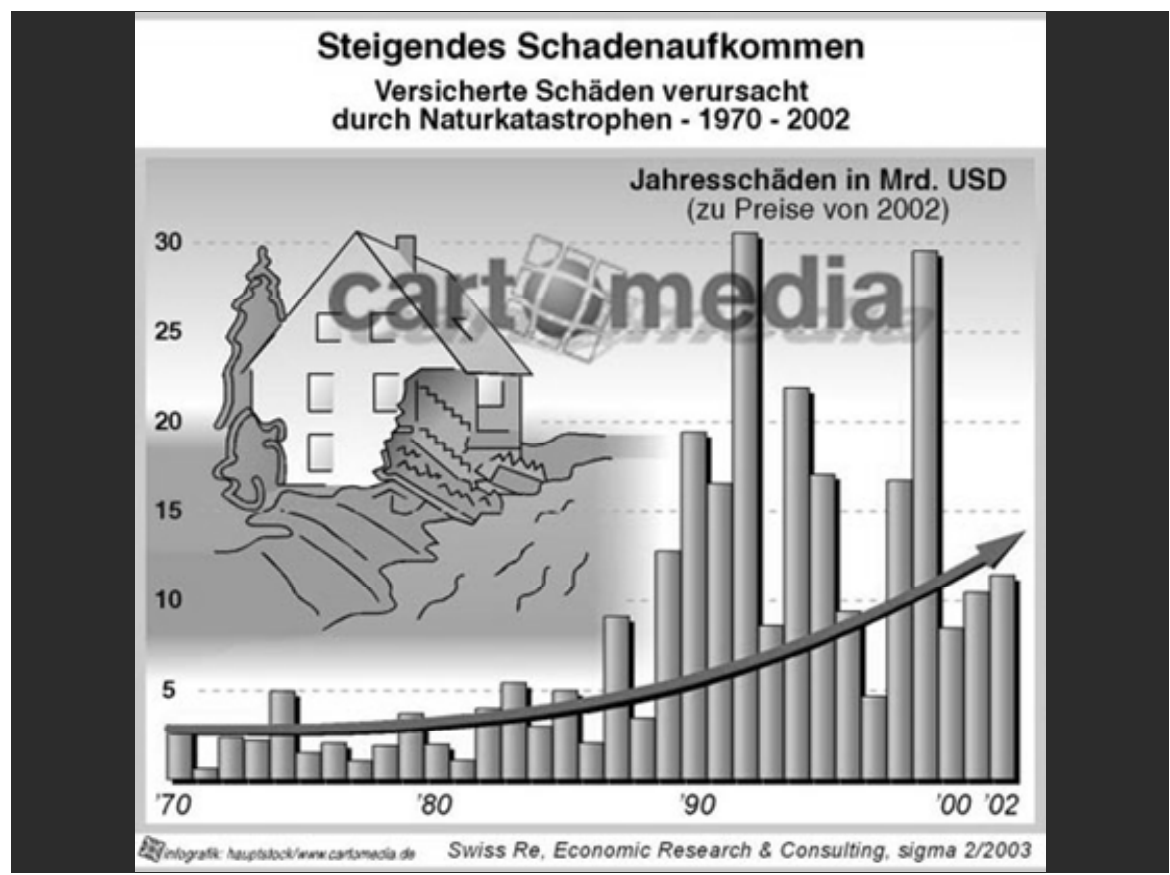

Abbildung 2: Statistik über Naturkatastrophen der letzten 50 Jahre der Rückversicherungsgesellschaft $S$ wiss $R e$.

Werfen wir zunächst einen Blick zurück in vergangene Jahrhunderte - in das 16., 17. und frühe 18. Jahrhundert, als Naturkatastrophen noch als Strafgerichte Gottes gedeutet wurden. ${ }^{4}$ Was hilft die Wiedererrichtung der Deiche, wenn Gott doch strafen will, fragten durch die verheerende Sturmflut des Jahres 1717 betroffene Einwohner an der Nordseeküste. Was könnten Sie gegen ein Strafgericht Gottes schon ausrichten? ${ }^{5}$ Dass es sinnvoll war, Deiche zu bauen, um vor der alltäglichen Flut geschützt zu sein, war allen Bewohnern der Nordseeküste einsichtig. Schließlich hatte erst der Bau von Deichen die Voraussetzung dafür geschaffen, in diesen neu gewonnenen Marschlanden an der Nordsee zu leben und zu arbeiten. Aber gegen die extraordinären, oftmals als „übernatürlich“ bezeichneten Sturmfluten eben jene als Strafgerichte Gottes gedeuteten Flutkatastrophen - konnte nach zeitgenössischer Auffassung kein noch so hoher Deich helfen. ${ }^{6}$ Aus Naturkatastrophen lernen - dass hieß in der damaligen Zeit zunächst einmal, den Blick auf das eigene Gottesverhältnis zu richten. Kirche und Obrigkeit, selbst durch diese religiösen Logiken ihrer Zeit geprägt, taten das ihre, um der durch die göttliche Strafrute

${ }^{4}$ Grundlegend für die kulturgeschichtliche Sicht von Katastrophen: Walter (2008); JakubowskiTiessen (2003a), S. 101-118.

Jakubowski-Tiessen (1992), S.142f.

${ }^{6}$ Ebd. S. 93. 
gepeinigten Bevölkerung wieder eine Perspektive für eine hoffnungsvolle Zukunft zu ermöglichen. ${ }^{7}$ Zunächst wurden vielfältige Hilfsmaßnahmen eingeleitet, sodann Buß- und Bettage angeordnet, auf denen zur Buße ermahnt wurde, und schließlich sozialdisziplinierende Gesetze und Verordnungen zur Vermeidung künftigen sündhaften Lebens erlassen; denn es galt, zunächst den Zorn Gottes zu besänftigen, indem die Ursachen der Katastrophe, nämlich das menschliche Fehlverhalten, korrigiert und abgestellt würde. ${ }^{8}$

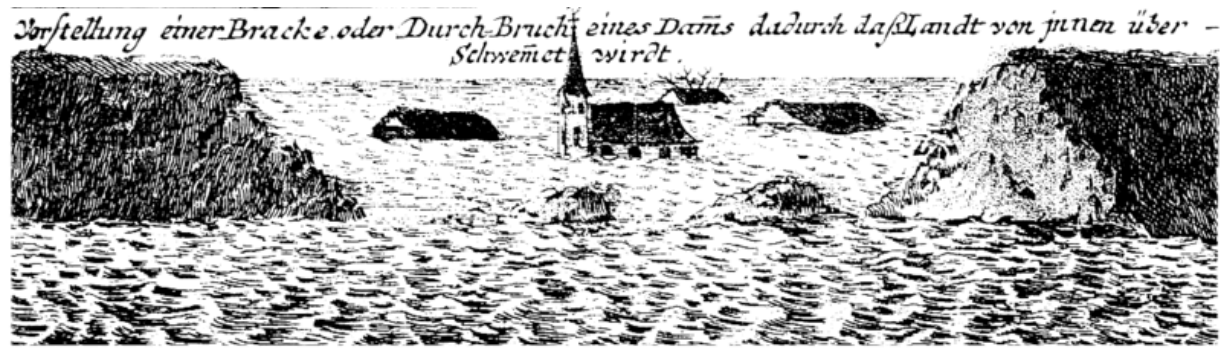

Abb. 3: Durchbruch eines Deiches

Gewiss, auch wurden große Bemühungen unternommen, die Durchbrüche in den Deichen wieder zu schließen; denn der Schutz vor dem täglich ein- und ausströmenden Meereswasser war Voraussetzung für ein Leben in den Marschregionen an der Küste. Auch wurden Kommissionen zur Aufnahme der Schäden eingesetzt ${ }^{9}$, aber noch stellte kein Gremium sich ernsthaft die Frage, wie die Auswirkungen künftiger Sturmflutkatastrophen mit besseren präventiven Maßnahmen zu begrenzen seien. ${ }^{10}$ Nur einzelne Personen aus den Kreisen der Deichbauspezialisten wollten in dem Desaster nicht allein die Hand Gottes wirken sehen, sondern sie fragten sich zugleich, ob nicht bessere Deiche doch auch einen besseren Schutz geboten hätten. ${ }^{11}$ Das waren aber nur einzelne Stimmen in der ersten Hälfte des 18. Jahrhunderts.

Als zu Beginn des 19. Jahrhunderts, im Jahr 1817, in einer Zeitschrift die Frage gestellt wurde, ob die Küstenbewohner heute wirklich sicher sein könnten, dass die Deiche einer Sturmflut wie im Jahre 1717 standhielten, wurde dieses eindeutig

\footnotetext{
7 Über die sich infolge der Sturmflut von 1717 ausbreitenden Hoffnungslosigkeit siehe ebd. S.141ff.

8 Jakubowski-Tiessen, (2003b), S. 194f; Jakubowski-Tiessen 2003a) S. 194.

9 Jakubowski-Tiessen (1992), S. 46ff.

10 Wenn auch gegen die als Strafgerichte Gottes gedeuteten Sturmflutkatastrophen in den Augen der meisten Zeitgenossen keine noch so großartigen technischen Präventivmaßnahmen erfolgreich sein konnten, so heißt das nicht, dass aus solchen Katastrophen und den mit Ihnen verbundenen Erfahrungen - neben den religiösen Konsequenzen - keine Lehren und neuen Einsichten gewonnen wurden, die auch zu Veränderungen in Verwaltung und Deichbauorganisation führen konnten. Diese Erkenntnisse resultierten vor allem aus der Katastrophenbewältigung, weil in den chaotischen Wochen nach derartigen Katastrophen oft Unzulänglichkeiten im administrativen Bereich sichtbar wurden, deren Behebung - auch unabhängig von der Katastrophenbewältigung - zu einer generellen Verbesserung von Verwaltungsabläufen führte. Vgl. dazu Poliwoda, (2007).
}

11 Ebd. S. 92 f. 
bejaht. Die Naturkräfte seien gewissen Gesetzen unterworfen, man hätte nun gelernt, deren Wirkung zu bekämpfen und unschädlich zu machen. ${ }^{12}$ Inzwischen hatte sich - allerdings noch keineswegs in allen Kreisen der Bevölkerung - ein Einstellungswandel vollzogen, nach dem Sturmfluten nicht mehr als Strafgerichte Gottes, sondern als Naturereignisse angesehen wurden, die sich allein aus den Gesetzen der Natur erklären ließen. Kennzeichnend für diesen Erkenntnisfortschritt ist ein Vortrag, den der von der Aufklärung geprägte dänische Regierungsrat und Philosoph Johann Nikolaus Tetens 1787 vor den Mitgliedern der SchleswigHolsteinischen Patriotischen Gesellschaft hielt. ${ }^{13}$ Der an der Nordseeküste aufgewachsene Tetens lehnte eine metaphysische Deutung von Sturmfluten generell ab. ${ }^{14}$ Im Rückblick auf vergangene Jahrhunderte stellte er allerdings fest: „In den noch ältern Zeiten sahe man die Fluthen als Strafen Gottes an, welche durch Deiche abhalten wollen, so viel sei, als dem Arm der Allmacht wehren wollen."15 Die Deutung einer Sturmflut als Gottesstrafe sei, so Tetens, ein „elende[s] Vorurtheil“", das den „Menschenverstand“ lange Zeit daran gehindert habe, „sich von dem Zwek, den man bei Aufführung der Deiche haben konte, und haben sollte, richtige Begriffe zu machen." 16

Das hier zum Ausdruck kommende, veränderte Naturverständnis und die neue wissenschaftliche Bemühung, auch hinter extremen Naturereignissen die naturgesetzlichen Abläufe zu ergründen, führte nicht allein zu anderen Erklärungsmustern, sondern wirkte sich auch auf die alltägliche Praxis im Umgang mit diesen Katastrophen aus. Denn erst die Rückführung extremer Naturereignisse auf rein natürliche Gesetzmäßigkeiten ermöglichte und legitimierte den Einsatz geeigneter Techniken der Prävention (vgl. Abb. 3). ${ }^{17}$

\footnotetext{
12 Jakubowski-Tiessen (199)7, S. 133.

13 Johann Nikolaus Tetens wurde 1736 in Tetenbüll in Eiderstedt geboren, studierte Philosophie, Mathematik und Physik in Rostock und Kopenhagen. 1763 wurde er o. Professor an der 1760 gegründeten und 1789 mit Rostock zusammengelegten Universität in Bützow und 1776 o. Professor der Philosophie und Mathematik an der Universität in Kiel. 1789 wurde er Assessor am Finanzkollegium und zweiter Finanzkassendirektor in Kopenhagen und später Etatsrat und Deputierter im dortigen Finanzkollegium. Er starb 1807 in Kopenhagen. Zu Tetens siehe Allgemeine Deutsche Biographie, Bd. 37, Leipzig 1894, S. 588-590 und Schleswig-Holsteinisches Biographisches Lexikon, Bd. 4, Neumünster 1976, S. 213-215. Dort auch weitere Literaturhinweise. Soeben erschienen ist ein Aufsatz über Tetens von Holm Tetens im Nordfriesischen Jahrbuch 44 (2009).

14 Zum folgenden siehe Allemeyer (2006), S. 365-371 und Allemeyer (2007), S. 45-49.

15 Johann Nikoalus Tetens, Über den eingedeichten Zustand der Marschländer, und die demselben anklebende Gefahr vor Überschwemmungen, eine Vorlesung, gehalten in der Versammlung der schleswig-holsteinischen patriotischen Gesellschaft den 3ten October 1787, in: SchleswigHolsteinische Provinzial-Berichte (1787), S. 641-665, hier S. 660. Die Vorstellung, dass Sturmfluten Strafgerichte Gottes seien, sei in den alten Zeiten ,allgemein“ gewesen, „und noch jetzo einen großen Theil von Menschen in dem Kopf". So ebenfalls in Tetens (1788), S. 6.

16 Tetens, Über den eingedeichten Zustand der Marschländer, S. 660.

17 Vgl. die Abb. 4, die zeigt, dass ein großer technischer Fortschritt in der Errichtung von Deichen erst im 18. Jahrhundert erreicht wurde..
} 


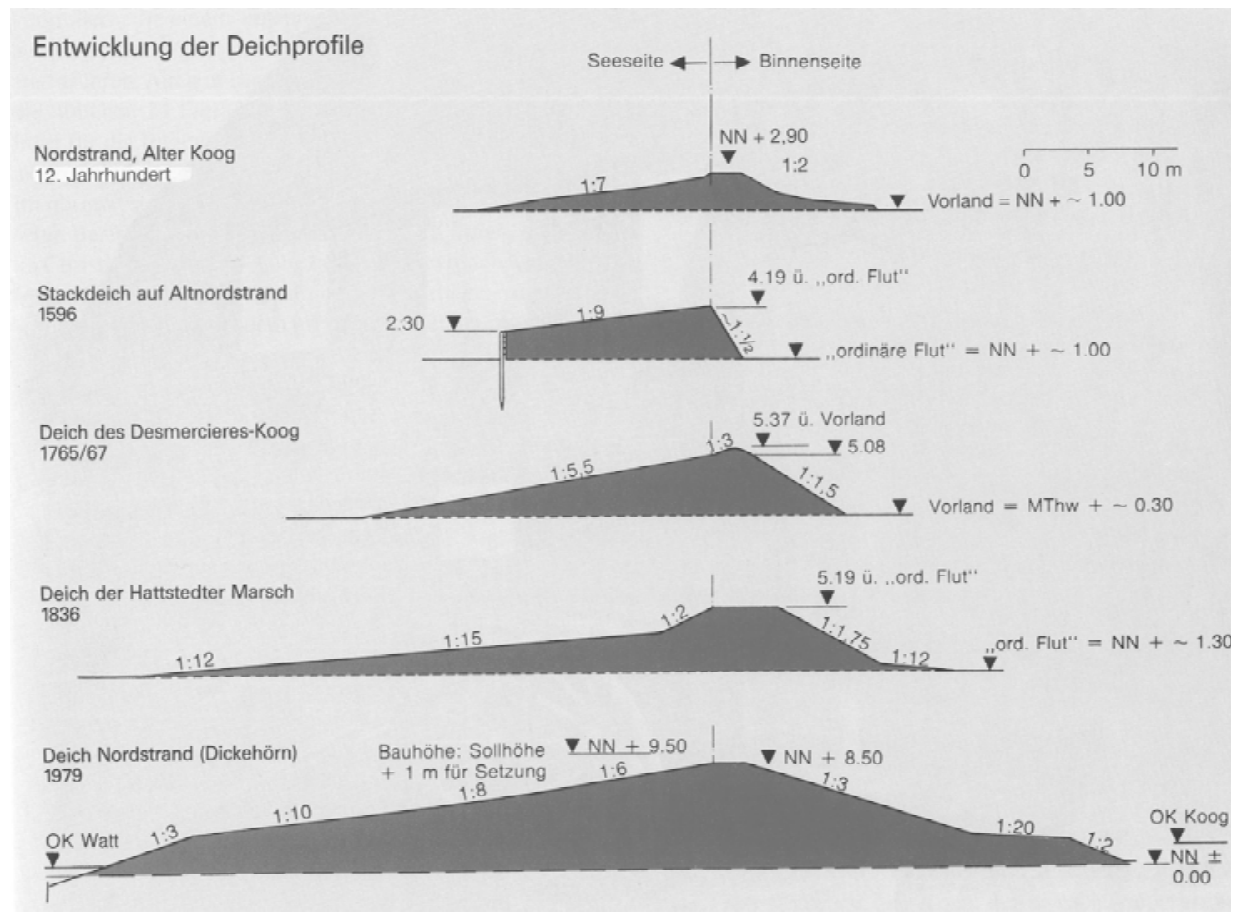

\section{Abbildung 4: Entwicklung der Deichprofile vom 12. Jahrhundert bis 1979}

Der jeversche Deichbaumeister Albert Brahms vertrat in seiner, bereits über zwanzig Jahre früher, im Jahr 1754 veröffentlichten Schrift „Anfangs-Gründe der Deich- und Wasser-Baukunst" 18 die Auffassung, dass im Grunde jede auf die Deiche einwirkende Kraft des Wassers messbar sei und somit auch jede noch so große Flut durch einen entsprechenden Bau der Deiche abgewehrt werden könnte:

„Es ist aus der Erfahrung mehr als zu bekannt, wie oftermalen bald hier, bald dort Einbrüche vom Wasser geschehen, wobey man jedoch keines weges gedenken muß, als ob selbige allemal ganz und gar unvermeidlich gewesen wären. Es ist im Gegentheil aus der Hydraulic zur Gnuege bekant, ... dass das Wasser doch nur eine abgemessene und determinirte Kraft habe. Also kommt es nur auf folgende Stuecke an: 1) Dass man untersuche, zu welcher Hoehe und Kraft das Wasser an diesem und jenem Orte zu einiger Zeit gestiegen, oder in denen allergroessesten und schweresten Fluthen, davon Nachricht vorhanden, angewachsen sey, damit man daraus urtheilen koenne, durch welche Verstaerkung der Deiche einem solchen Gewaesser haette Widerstand geschehen koennen und moegen. Sodann 2) $\mathrm{Ob}$ es vortheilhaft und profitabel, dass man die uebrige, und zur Verstaerkung 
annoch erfoderliche Kosten anwende, oder die Deiche nur so im Stande lasse, wie sie sind, und es darauf ankommen lasse."19

Wie dieses Zitat zeigt, spielten neben dem Glauben an die technische Machbarkeit auch wirtschaftliche Erwägungen für den Bau bzw. für die Verstärkung von Deichen eine Rolle. Und Brahms fügt seiner Argumentation noch ein weiteres, zu bedenkendes Kriterium hinzu: zunächst könne noch eine Risikoabwägung vorgenommen werden. ${ }^{20}$ Die Tatsache, dass im 18. Jahrhundert erstmals im Zusammenhang mit Naturkatastrophen von, Risiken' gesprochen wurde, zeigt eine neue Problemsicht. ${ }^{21}$ Die Kategorien ,Zufall' und ,Wahrscheinlichkeit' stehen nun in engem Verhältnis mit der Beherrschung von gesellschaftlich relevanten Zukunftsentscheidungen. ${ }^{22}$

Diesen Einstellungswandel formuliert Peter L. Bernstein in seinem Buch „Wider die Götter. Die Geschichte von Risiko und Risikomanagement von der Antike bis heute“ folgendermaßen: „Das Revolutionäre, das die Neuzeit von historischer Vergangenheit trennt, ist die Vorstellung der Risikosteuerung, der Gedanke, dass die Zukunft nicht bloß den Launen der Götter entspringt und dass Männer wie Frauen der Natur nicht passiv gegenüberstehen. Zuvor war die Zukunft nur ein Spiegelbild der Vergangenheit oder das Halbdunkel der Orakel und Weissager, die das Vorherwissen von Ereignissen monopolisiert hatten." 23

„Das Vertrauen in die Machbarkeit der Verhältnisse“ - wie Niklas Luhmann es nennt ${ }^{24}$ - war geschaffen, und damit die Vorstellung von der Möglichkeit einer weitgehenden Naturbeherrschung. Erst mit der Ablösung der alten Deutungs- und Erklärungsmuster für Naturkatastrophen wurde folglich der Weg geebnet, für eine Analyse der auf Gesetzmäßigkeiten beruhenden Ursache-Folgen-Beziehungen dieser Extremereignisse und damit die Idee geboren, unerwünschte Effekte durch geeignete Modifikationen der Ursachen oder durch die Begrenzung und Milderung der Konsequenzen zu vermindern. ${ }^{25}$

Erst diese mentalitätsgeschichtliche Wende schuf die Voraussetzung dafür, aus Naturkatastrophen zu lernen - zumindest in unserem modernen aufgeklärten Sinne. Doch die Lehren, die im festen Vertrauen auf die richtige Erkenntnis der Natur und ihrer Gestaltungsmöglichkeit gezogen wurden, entsprachen - wie könnte es

\footnotetext{
${ }^{19}$ Ebd. S. 33 f.

${ }^{20}$ Für den Deichbauexperten Albert Brahms ergibt sich bei einer solchen Risikoabwägung allerdings ein eindeutiges Ergebnis: „Vergleichet den Verlust und den Schaden mit denen anzuwendenden Kosten, um die Deich einer Provinz in einem haltbaren Stand zu setzen; so wird sichs finden, dass insgemein die anzuwendenden Kosten dem besorglichen Unheil und Schaden bey weitem nicht gleich kommen; es also besser sey, solche anzulegen, als Land und Leute in Gefahr zu exponiren." Ebd.

${ }^{21}$ Jakubowski-Tiessen (2006), S. 98.

22 Lübbe (1993), S. 27; Wiedemann (1993), S. 44; Bonß (1995); Plapp (2004), S. 9-14.

${ }^{23}$ Bernstein (1997), S. 9 (englisches Original: Against The Gods. The remarkable Story of Risk, New York u.a. 1996).

${ }^{24}$ Luhmann (1991), S. 21.

25 Plapp (2004), S. 13.
} 
auch anders sein - oftmals nicht dem heutigen Stand wissenschaftlicher Erkenntnis. Die Maßnahmen, die als Präventivmaßnahmen gegen Naturkatastrophen durchgeführt wurden, konnten nur auf der Basis der jeweiligen kulturellen Vorstellungen und des jeweiligen zeitgenössischen Wissensstandes geschehen. Im Rückblick waren sie oftmals unzulänglich und haben zuweilen nicht zu einer Vermeidung, sondern nur zu einer Verlagerung der Gefährdungen geführt. Eine solche Gefährdungsverlagerung versucht Verena Winiwarter am Beispiel der Ablösung der Miasmentheorie durch naturwissenschaftlich exaktere Konzepte der Bakteriologie im 19. Jahrhundert zu zeigen. Diese hätte dazu geführt, „dass vormals bekämpfte giftige Gewerbe und Industrieabwässer als weniger gefährlich empfunden wurden als die nun nach bakteriologischen Maßstäben bedenklicheren Fäkalien. “26 Und andererseits ist auch zu bedenken, dass sich manche - auch im Lichte heutiger Forschung - richtige Erkenntnis gegen die vorherrschende wissenschaftliche Richtung nicht durchzusetzen vermochte, was bekanntlich mitunter auch heute noch der Fall ist.

Die Geschichtsphilosophie des 18. Jahrhunderts sah den Gang der gegenwärtigen Geschichte, wie Odo Marquard formuliert, „als fortschrittliche Herbeiführung einer zukünftigen heilen Welt “27. Die Gelehrten der Aufklärungszeit wollten den Ursachen und Wirkungen der Naturkatastrophen auf die Spur kommen, und sie dadurch beherrschen - mit dem Ziel, eine sicherere Welt zu schaffen. Seit diesem Wahrnehmungswandel werden Naturkatastrophen als ein Problem betrachtet, das sich prinzipiell durch technische Maßnahmen lösen oder zumindest erheblich begrenzen lässt. Parallel zur fortschreitenden Technisierung der Welt hat sich diese Einstellung immer stärker verfestigt. In Mitteleuropa hat sich folglich ein Naturgefahrenmanagement entwickelt, das stark auf technische Regulation und Schutzmaßnahmen ausgerichtet ist, welche die "feindliche Natur" aus dem ,geordneten“" Lebensraum des Menschen heraushalten sollten. ${ }^{28}$

„Man hat mit einem Feinde zu thun, der keine Friedensschlüsse noch einen Waffenstillstand kent. [...] mit einem Feind [...], der offen handelt und sich immer gleich bleibet. Er sieht auf keine neuen Arten des Angrifs und auf keine Kriegslist, ist an allen Orten und zu allen Zeiten derselbige in seinem Verfahren." ${ }^{29}$ So noch einmal Tetens in seinem Vortrag von 1787, der hier das Meer als einen durchaus berechenbaren Feind ansieht, den es zu bekämpfen und zu besiegen gelte und der auch aufgrund der Erkenntnisfortschritte in der hydrologischen Wissenschaft zu besiegen sei. Diese auf die Natur angewandte Feind-Metapher setzt eine Unterscheidung von Gesellschaft und Natur voraus, die zu einem grundlegenden Merkmal neuzeitlicher Wissenschaftsgeschichte geworden ist. Mit der Ausbildung der Naturwissenschaften als eigenen Wissenschaftsbereich entsprach die Wissen-

\footnotetext{
26 Winiwarter / Knoll (2007), S. 189.

27 Marquard 2008), S. 209.

${ }^{28}$ Pfister. (2002); Engels (2003) S. 122-125.

${ }^{29}$ Tetens, (1787), S. 653 f.
} 
schaftsgeschichte dieser Dichotomie von Gesellschaft und Natur. Seit einiger Zeit ist jedoch festzustellen, dass diese arbeitsteilige Dichotomie zunehmend $\operatorname{erodiert}^{30}$, so dass sich die Trennlinie zwischen Natur und Gesellschaft immer mehr auflöst und verschiebt, wodurch auch traditionelle Modelle des Gefahrenmanagements zunehmend in die Kritik geraten.

In den letzten dreißig Jahren ist beispielsweise das bisher übliche Gefahrenmanagement im Hochwasserschutz an Flüssen immer wieder kritisiert und auch in Frage gestellt worden. ${ }^{31}$ Es zeigt sich, dass theoretisch entwickelte Modelle ihrerseits in der technischen Umsetzung wie auch durch die Aggregation kleinräumiger Nebenfolgen „Naturgefahren“ verstärken können, z.B. durch die Verringerung der natürlichen Retention von Hochwasser im Bereich der Flussauen. ${ }^{32}$ Ferner ist festzustellen, dass es insbesondere an Ufern, Küsten und in Gebirgen zum so genannten „Safe development paradox“33 kommt. Technische Schutzmaßnahmen erlauben eine verstärkte Siedlungsentwicklung in den nun vermeintlich sicheren Küstenund Uferregionen oder einst gefährlichen Gebirgslagen, wodurch die Vulnerabilität der Gesellschaft drastisch ansteigt und bei einem Versagen der Schutzeinrichtungen folglich sehr große Schäden entstehen. Der Hurrikan Katrina, der New Orleans im August 2005 traf, ist dafür ein bekanntes Beispiel unserer Tage. Auch hier zeigte sich, dass das hohe Schadenspotential das Paradox verschärft: Es scheint nun erst recht, als wären allein technische Schutzmaßnahmen geeignet, dem schon durch technische Eingriffe in die Natur verursachten Risiko zu begegnen.

Muss die Menschheit sich trotz technischen Fortschritts nicht gerade angesichts des Klimawandels und dessen Folgen das Scheitern an der Natur und das Scheitern des gesellschaftlichen Umgangs mit der Natur eingestehen? Ist der technisch-naturwissenschaftliche und ökonomische Blick auf die Natur ${ }^{34}$, die man seit der Aufklärungszeit erobert und sich zugleich kontemplativ-ästhetisch angeeignet hat, noch angemessen? ${ }^{35}$ Können die durch den Klimawandel hervorgerufenen Naturkatastrophen, die sowohl der Natur als zunehmend auch anthropogenen Einflüssen zuzuschreiben sind, noch mit den tradierten technisch-naturwissenschaftlichen Schutzstrategien in ihren Auswirkungen begrenzt werden? Sind $\mathrm{Na}$ turkatastrophen, die nach dem Katastrophensoziologen Lars Clausen immer Kulturkatastrophen sind ${ }^{36}$, dies nun im Hinblick auf den Klimawandel nicht in zweifacher Weise: hinsichtlich ihrer Genese und ihrer Auswirkungen? „Der enttäuschte

\footnotetext{
${ }^{30}$ Vgl. zur Dichotomie Natur - Gesellschaft aus der Perspektive der so genannten Akteur-NetzwerkTheorie den von Voss und Peucer herausgegebenen Sammelband (2006).

31 Vgl. Immendorf (1997). Siehe ferner Pörtge und Deutsch (2006) S. 244.

32 Dass die Verringerung der Retentionsflächen an den Fließgewässern nicht im Zusammenhang mit dem Klimawandel steht, sondern eine Folge von Flussbaumaßnahmen und Besiedlung ist, unterstreicht Bronstert in seinem Aufsatz „Klimaänderungen und Hochwasser - Zusammenhänge und Auswirkungen (1997), S. 179. Siehe auch Kruse (2008): S. 37-64

33 Burby (2006), S. 171-191.

32 Bayerl (1994) S. 29-56; Bayerl (2001)..

35 Vgl. Blackbourn (2007).

${ }^{36}$ Clausen und Dombrowsky (1983).
} 
Optimismus lebt fort. Freilich als versteckter, indirekter', mit negativem Vorzeichen versehener Optimismus. Weil er der Katastrophenurheber ist, liegt es nämlich beim Menschen, und bei ihm allein, Katastrophen zu verhindern. “"37

Die Stabilität oder Fragilität von Natur- und Weltdeutungen offenbart sich vor allem in der Krise; denn in einer Krise müssen sich bisherige Erfahrungen und Handlungsstrategien bewähren. Kulturelle Deutungsmuster können in Katastrophen- und Krisensituationen gefestigt, verworfen oder modifiziert werden. Befinden wir uns heute - ähnlich wie an der Wende vom 17. zum 18. Jahrhundert ${ }^{38}$ - in einer Zeit des Umbruchs, des beginnenden Wandels kultureller Wahrnehmungsmuster in Bezug auf die Natur. Werden die vorherrschenden technischnaturwissenschaftlichen Strategien noch ausreichen, den Klimawandel und die daraus entstehenden Naturkatastrophen einzudämmen? Werden wir nicht einen neuen Blick auf die Natur werfen müssen, wenn sie nicht ,,als geschundenes, wenn auch unberechenbares Opfer" 39 auf der Strecke bleiben soll? 40 Oder werden wir lernen müssen mit der Katastrophe zu leben?

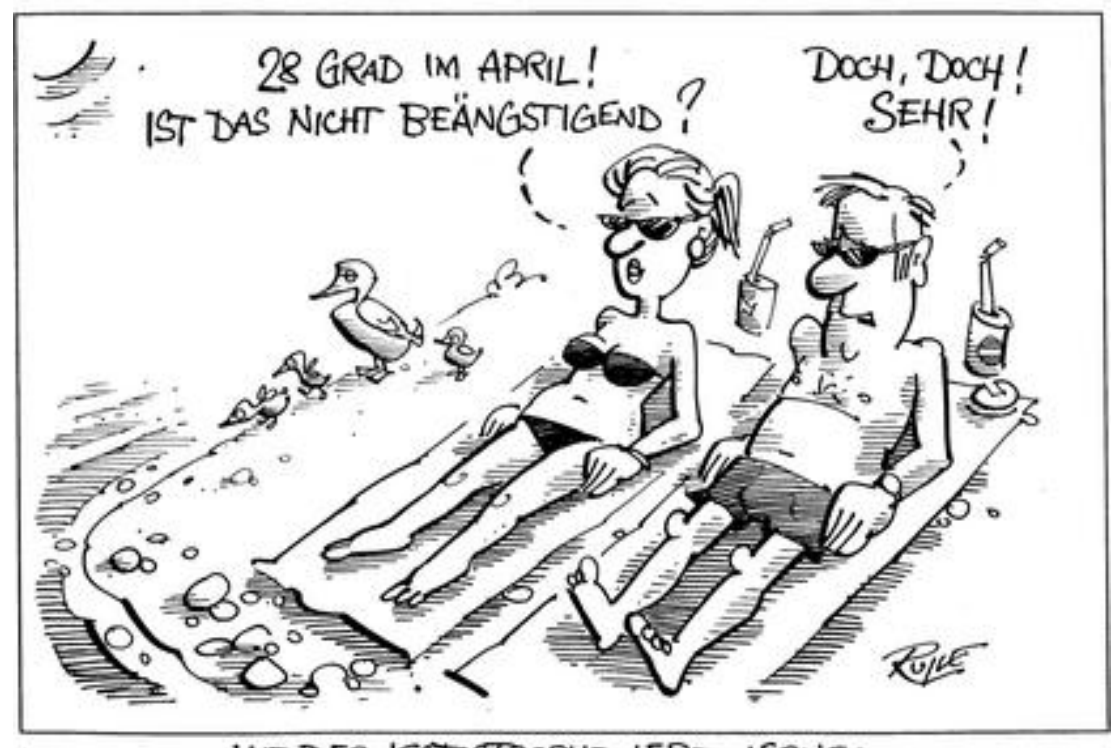

MIT DER KATASTROPHE LEBEN LERNEN ...

\footnotetext{
37 Engels (2003) S. 142.

38 Hazard (1939).

${ }^{39}$ Engels (2003) S. 141.

40 Böschen / Scheringer / Jaeger (2001) S. 207 betonen, ,,dass die naturwissenschaftliche Umweltforschung überwiegend immer noch dem Leitbild ,klassischer Naturforschung' folgt. „Die zentrale Aufgabe einer reflexiven Umweltforschung ist es daher, Werturteilsfragen und Sachfragen mit Hilfe natur-, sozial- und humanwissenschaftlichen Methoden systematisch in Beziehung zu bringen."
} 
Abschließend sei noch kurz auf einen anderen Aspekt der Fragestellung eingegangen: Was können Historiker und Umwelthistoriker bei der Beschäftigung mit Naturkatastrophen lernen? Anders als die naturwissenschaftlichen Katastrophenforscher, die vor allem an dem Naturereignis als solchem interessiert sind, werden Umwelthistoriker vor allem die Zeit nach der Katastrophe in den Blick nehmen und der Frage nachgehen, wie die von einer Naturkatastrophe betroffenen Länder, die staatlichen Organe ebenso wie die einzelnen Menschen, diese Naturkatastrophe mit ihren wirtschaftlichen und gesellschaftlichen Folgen bewältigten. ${ }^{41}$ Ferner wird zu fragen sein, welche Strategien der Gefahrenabwehr und des Naturgefahrenmanagements einzelne Gesellschaften entwickelten und ob und wie sich diese im Laufe der Zeit veränderten und ob dieser Wandel mit einer Veränderung des Mensch-Natur-Verhältnisses einherging.

Durch die Beschäftigung mit historischen Naturkatastrophen können die Historiker über die ereignisgeschichtlichen, ökonomischen und demographischen Aspekte hinaus wichtige Erkenntnisse über kulturelle Prozesse und mentale Veränderungen in früheren Gesellschaften gewinnen, denn die Betrachtung von „Katastrophen, die wie ein Blitz in die Dunkelheit einbrechen", können soziale und kulturelle Muster erhellen, die für die Geschichtsforschung ansonsten unsichtbar bleiben. ${ }^{42}$ In engem Zusammenhang mit der Frage nach den Bewältigungsstrategien steht die Frage, ob und wie eine Naturkatastrophe im kollektiven Gedächtnis nachgeborener Generationen gegenwärtig gehalten werden kann und konnte. Es scheint, dass die Erinnerung an Katastrophen nur dann Teil einer dauerhaft wirkenden kollektiven Gedächtniskultur werden kann, wenn mit der Erinnerungsleistung im Kontext ihrer jeweiligen Zeit ein aktives Deutungsangebot im Sinne einer Handlungslegitimation oder auch einer Selbstvergewisserung einhergeht, zum Beispiel als Perspektive für die Wieder - oder Neugewinnung einer kommunalen oder regionalen Identitätsbildung; vielleicht auch als dauerhafte Mahnung an die Nachgeborenen in gefährdeten Gebieten, sich der Naturgefahren stets bewusst zu sein und entsprechende Vorsorge zu leisten.

\section{Literatur}

Allemeyer, M L (2007): „In Deichsachen aber ist unter den Marschleuten immer Streit." Deichkonflikte in einer Küstengesellschaft der Frühen Neuzeit, in: Allemeyer, M. L., Jakubowski-Tiessen, M. und Rufino, S. R. (Hrsg.): Von der Gottesgabe zur Ressource, Konflikte um Wald, Wasser und Land in Spanien und Deutschland seit der Frühen Neuzeit, Essen, S. 27-55

\footnotetext{
${ }^{41}$ Vgl. Pfister (2002).

42 Ranft und Selzer (2004), S. 11.
} 
Allemeyer, M L (2006): „Kein Land ohne Deich...!“ Lebenswelten einer Küstengesellschaft in der Frühen Neuzeit (Veröffentlichungen des MaxPlanck-Instituts für Geschichte 222), Göttingen

Bayerl, G (1994): Prolegomenon der ,Großen Industrie’. Der technischökonomische Blick auf die Natur im 18. Jahrhundert, in: Abelshauser (Hrsg.): Umweltgeschichte. Umweltverträgliches Wirtschaften in historischer Perspektive, Göttingen (Geschichte und Gesellschaft 15), S. 29-56

Bayerl, G (2001): Die Natur als Warenhaus. Der technisch-ökonomische Blick auf die Natur in der Frühen Neuzeit, in: Hahn, S. / Reith, R. (Hrsg.), UmweltGeschichte. Arbeitsfelder, Forshcungsansätze, Perspektiven, Wien/München, S. 33-52

Bernstein, P L (1997): Wider die Götter. Die Geschichte von Risiko und Riskmanagement von der Antike bis heute, München (englisches Original: Against The Gods. The remarkable Story of Risk, New York u.a. 1996)

Blackbourn, D (2007): Die Eroberung der Natur. Eine Geschichte der deutschen Landschaft, München.. (Engl. Originalausgabe: The Concept of Nature. Water, Landscape and the Making of Modern Germany, London 2006)

Bonß, W (1995): Vom Risiko: Unsicherheit und Ungewissheit in der Moderne, Hamburg

Böschen, S / Scheringer, M / Jaeger, J (2001): Wozu Umweltforschung? - Über das Spannungsverhältnis zwischen Forschungstraditionen und umweltpolitischen Leitbildern, Teil II: Zum Leitbild „reflexive Umweltforschung“, in: GAIA 10, S. 203-212

Brahms, A (1754/57): Anfangs-Gründe der Deich- und Wasser-Baukunst (1754/57), 2 Bde., Leer 1989, ND der Ausgaben Aurich 1767 und 1773

Bronstert, A ( 1997): „Klimaänderungen und Hochwasser - Zusammenhänge und Auswirkungen“, in: Immendorf, Ralf (Hrsg.) Hochwasser, Natur im Überfluß? Heidelberg

Burby, R J (1998): Cooperating with Nature. Confronting Natural Hazards with Land-Use Planning for Sustainable Communities, Washington, D. C.

Burby, R J (2006): "Hurricane Katrina and the Paradoxes of Government Disaster Policy: Bringing about Wise Governmental Decisions for Hazardous Areas," Annals of the American Academy of Political and Social Science, S. 171-191

Clausen, L und Dombrowsky, W R (1983): Einführung in die Soziologie der Katastrophe, Bonn 
Engels, J I (2003): Vom Subjekt zum Objekt. Naturbild und Naturkatastrophen in der Geschichte der Bundesrepublik Deutschland, in: Groh, D, / Kempe, M. / Mauelshagen, F. (Hrsg.)

Hazard, P (1939): Die Krise des europäischen Geistes 1680-1715, Hamburg. (franzöische Erstausgabe: La crise de la conscience européenne 1680-1715, Paris 1935)

Immendorf, R (Hrsg.) (1997): Hochwasser. Natur im Überfluß? Heidelberg

Jakubowski-Tiessen, M (1992): Sturmflut 1717. Die Bewältigung einer

Naturkatastrophe in der Frühen Neuzeit, München

Jakubowski-Tiessen, M (1997): Mentalität und Landschaft. Über Ängste, Mythen und die Geister des Kapitalismus, in: Fischer, L. (Hrsg.), Kulturlandschaft Nordseemarschen, Bredstedt und Westerhever, S. 129-143

Jakubowski-Tiessen, M (2003a): Gotteszorn und Meereswüten. Deutungen von Sturmfluten vom 16. bis 19. Jahrhundert, in: Groh, D., Kempe, M. und Mauelshagen, F. (Hrsg.): Naturkatastrophen. Beiträge zu ihrer Deutung, Wahrnehmung und Darstellung in Text und Bild von der Antike bis ins 20 . Jahrhundert, Tübingen

Jakubowski-Tiessen, M (2003b): "Erschreckliche und unerhörte Wasserfluth" Wahrnehmung und Deutung der Flutkatastrophe von 1634, in: JakubowskiTiessen, Manfred und Lehmann, Hartmut (Hrsg.), Um Himmels Willen. Religion in Katastrophenzeiten, Göttingen, S. 179-200

Jakubowski-Tiessen, M (2006): Divine Judgement or Incalculable Risk? An Natural Disaster and its Consequences, in: Walter, F., Fantini, B. und Delvaux, P. (Hrsg) (2006): Les Cultures du Risque (XVI - XXIe siècle), Genf, S. 87-98

Kruse, S (2008): Structuring Multiple Perspectives in Environmental DecisionMaking: Flood Protection in the Middle Elbe River. In: Edmundson, R., Rau, H.(Hrsg.): Environmental Argument and Cultural Differences: Locations, Fractures and Deliberations. Oxford, S. 37-64

Lübbe, H (1993): Sicherheit. Risikowahrnehmung im Zivilisationsprozeß, in: Risiko ist ein Konstrukt: Wahrnehmungen zur Risikowahrnehmung, hrsg. von der Bayerischen Rückversicherung, München

Luhmann, N (1991): Soziologie des Risikos, Berlin

Marquard, O (2008): Die Krise des Optimismus, in: Lauer, G. und Unger, T. (Hrsg.), Das Erdbeben von Lissabon und der Katastrophendiskurs im 18. Jahrhundert, Göttingen, S. 205-215

Pfister, C (Hrsg) (2002): Am Tag danach: Zur Bewältigung von Naturkatastrophen in der Schweiz 1500-2000, Bern u.a. 
Pfister, C / Summermater, S (Hrsg.) (2004): Katastrophen und ihre Bewältigung. Perspektiven und Positionen, Bern u.a.

Plapp, T (2004): Wahrnehmung von Risiken aus Naturkatastrophen. Eine empirische Untersuchung in sechs gefährdeten Gebieten Süd- und Westdeutschlands, Karlsruhe, S. 9-14

Poliwoda, G N (2007): Aus Katastrophen lernen. Sachsen im Kampf gegen die Fluten der Elbe 1784 bis 1845, Köln u.a.

Pörtge, K-H / Deutsch, M (2006): „Wir sollten daraus lernen!“ Vorschläge und Maßnahmen zur Risikominderung angesichts schwerer Hochwasser (18911929), in: Forum für Hydrologie und Wasserbewirtschaftung, Bd. 1, Vorträge 1: Risikomanagement extremer hydrologischer Ereignisse. Beiträge zum Tag der Hydrologie 2006, 22./23. März 2006 an der Universität der Bundeswehr München, hrsg. von Disse, M. u.a.

Ranft, A und Selzer, S (2004): Städte aus Trümmern. Einleitende Überlegungen, in: Dies (Hrsg.): Städte aus Trümmern. Katastrophenbewältigung zwischen Antike und Moderne, Göttingen 2004, S. 11

Renn, O / Klinke, A (2004): Der gesellschaftliche Umgang mit Natrurisiken, in: Pfister, C / Summermatter, S. (Hrsg.), Katastrophen und ihre Bewältigung. Perspektiven und Positionen, Bern u.a.

Tetens, J N (1787): Über den eingedeichten Zustand der Marschländer, und die demselben anklebende Gefahr vor Überschwemmungen, eine Vorlesung, gehalten in der Versammlung der schleswig-holsteinischen patriotischen Gesellschaft den 3ten October 1787, in: Schleswig-Holsteinische ProvinzialBerichte, S. 641-665

Tetens, J N (1788): Reisen in die Marschländer an der Nordsee zur Beobachtung des Deichbaus in Briefen, Leipzig

Voss, M und Peucer, B (Hrsg.) (2006): „Verschwindet die Natur? Die AkteurNetzwerk-Theorie in der umweltsoziologischen Diskussion, Bielefeld

Walter, F (2008): Catastrophes. Une histoire culturelle XVIe - XXIe, Paris

Wiedemann, P M (2004): Tabu, Sünde, Risiko: Veränderungen der gesellschaftlichen Wahrnehmung von Gefährdungen, in: Risiko ist ein Konstrukt, Wahrnehmungen zur Risikowahrnehmung, hrsg. von der Bayerischen Rückversicherung, München

Winiwarter, V / Knoll, M (2007): Umweltgeschichte, Köln 


\title{
Invasive Arten - Freisetzungsexperimente in Vergangenheit und Gegenwart
}

\author{
Josef H. Reichbolf
}

\section{Einleitung: Unsere Natur im globalen Wandel}

Erstmals in ihrer Geschichte hat die Menschheit ein globales Netzwerk von Beziehungen und Austauschvorgängen aufgebaut, ,Globalisierung' genannt. Dass diese nicht allein Waren, Informationen und Menschen betrifft, sondern auch andere Lebewesen, liegt auf der Hand. Tiere, Pflanzen und Mikroben können innerhalb von Tagesfrist von Kontinent zu Kontinent oder von Meer zu Meer transportiert oder auch verschleppt werden. Wird sich daraus in absehbarer Zeit anstelle der bisher gebietstypischen Zusammensetzung ein globaler „Einheitsbrei“" von Arten entwickeln? Wird die Ausbreitung gebietsfremder die ortsheimischen Arten bedrohen oder gar vernichten? Solche Fragen bewegen nicht allein Naturschützer, sondern auch Epidemiologen und Ökonomen, weil die vordringenden, ,invasiven“ Arten neue Krankheiten mit sich tragen und ausbreiten oder ökonomische Schäden, insbesondere in der Landwirtschaft verursachen können. „Überfremdung“ wird befürchtet, weil fremde Arten auch dann nicht „,hierher passen“, so eine weit verbreitete Einstellung, wenn sie keine Schäden verursachen. Invasive Arten erregen öffentliche Aufmerksamkeit. Sie sind Medienthemen geworden. Wie gefährlich sind sie wirklich? 


\section{Problemstellung: Wie gefährlich sind „die Neuen“ wirklich und was macht sie invasiv?}

Die bloße Gegebenheit, dass Arten vordringen, sich ausbreiten oder sich (wieder) zurückziehen, gehört zur natürlichen Dynamik von Fauna und Flora. Einen festgelegten Zustand gab und gibt es zwar nicht, aber Veränderungen haben in aller Regel auch Folgen. Zu betrachten sind daher:

1. Geschwindigkeit der Veränderung

2. Einflussnahme fremder Arten auf heimische

3. Schäden, die sie verursachen (können)

Aus der von uns selbst erlebten Geschwindigkeit der globalen Veränderungen wird automatisch und unreflektiert geschlossen, dies müsse ganz allgemein, also auch für Pflanzen und Tiere, so gelten. Ist dies auch wirklich so?

Bei den meisten heimischen Arten verändern sich Vorkommen und Häufigkeit räumlich und zeitlich mehr oder weniger stark. Wirken die Neuen, die fremden Arten, nun in besonderem Maße beschleunigend, konkurrierend und bedrohend? Wie sieht es mit der Verdrängung heimischer durch fremde Arten aus? Warum kommt es gegebenenfalls zur Verdrängung? Fremde Arten können nicht allein deshalb, weil sie „fremd“ sind, den heimischen überlegen sein. Eher wäre das Umgekehrte anzunehmen, nämlich dass die gut etablierten den neuen Arten Widerstand entgegensetzen.

Welcher Art sind die Schäden? Sind ökonomische Verluste den „ökologischen" gleichzusetzen und warum treten sie überhaupt auf? Schäden verursachen viele heimische Pflanzen- (Unkräuter) und Tierarten (Schädlinge). In welchem Verhältnis stehen diese zu den Schäden, die neue, invasive Arten verursachen?

Schließlich ist zu klären, warum nur einige wenige Arten invasiv werden, die weitaus meisten aber unauffällig bleiben. Ein Blick in die Gärten zeigt die Verhältnisse schlaglichtartig auf: Gängigen Katalogen zufolge wachsen 4.000 oder mehr fremde Pflanzenarten in mitteleuropäischen Hausgärten, Parks und öffentlichen Anlagen. Wild wachsend („heimisch“) gibt es in Deutschland aber nur etwa 2.500. Zusammen mit den Nutzpflanzen, die nahezu ausnahmslos fremder Herkunft sind, leben in Deutschland natürlicherweise also nur etwa halb so viele Pflanzenarten wie vom Menschen angepflanzt vorkommen. Invasiv sind nur sehr wenige geworden, nämlich kaum mehr als ein Dutzend (Hartmann et al. 1995, Szyska 2004). Der Botaniker Ingo Kowarik formulierte dazu eine Häufigkeitsregel: Von 1.000 eingeführten Pflanzenarten siedeln sich etwa 100 spontan an, aber nur 10 können in naturnaher Vegetation auf Dauer existieren - und 20 in von Menschen stark veränderten Lebensräumen (Kegel 1999). Es geht also um ein bis zwei Prozent der Fremdlinge insgesamt. Ganz unabhängig von der Größe der Schäden oder der Probleme, die dabei verursacht werden, handelt es sich, auf das Ausgangsspektrum der fremden Arten bezogen, offenbar um Ausnahmefälle und nicht die 
Regel, dass fremde Arten invasiv werden. Zufall oder gute Gründe, das ist die Frage. Doch da auch „heimische“ Arten sehr invasiv werden können, zum Beispiel wenn Birken beginnen, (geschützte) Hochmoore zu überwachsen, Brennnesseln an Orten große Bestände bilden, an denen sie nicht erwünscht sind, Brombeergeranke wuchert oder (heimische!) Algen sich zur so genannten, den Sauerstoffhaushalt stark beeinträchtigenden Wasserblüte schier explosionsartig vermehren, kann es nicht am „Fremdsein“ der Arten liegen, wenn sie sich stark vermehren und ausbreiten. Wann ist überhaupt eine Art „heimisch“, wann „fremd“? Diese scheinbar überflüssige Frage trifft nämlich einen ganz zentralen Aspekt der Problematik. Was heimisch oder fremd ist, lässt sich „,von Natur aus“ gar nicht festlegen, weil die Vorkommen so gut wie aller frei lebenden Arten „Geschichte“ haben, Naturgeschichte nämlich.

\section{Heimisch und fremd: Die Zeitfrage}

Vor 10.000 Jahren sahen die Landschaften Mitteleuropas ganz anders aus als heute. Am Ende der letzten Eiszeit, des Würm- bzw. Weichsel-Glazials, gab es nur vereinzelt Bäume oder lockere Baumbestände. Das Land zwischen den abschmelzenden Alpengletschern und dem sich zurückziehenden nordwesteuropäischen Eisschild war überwiegend noch mit eiszeitlicher Tundravegetation, der so genannten Mammutsteppe, bedeckt. Langsam, mit zunehmender Wärme sich beschleunigend, setzte die Wiederausbreitung der Wälder und ihrer Begleitflora aus den südwestund südosteuropäischen Refugialgebieten ein. Die nacheiszeitliche Vegetationsentwicklung ist recht gut nachvollziehbar, weil die im Torf von Mooren gespeicherten Pollen die großräumige Zusammensetzung der Flora und ihre Veränderung über die Jahrtausende dokumentieren (Schroeder 1998). Für die letzten 8.000 Jahre der Ausbreitung von Wäldern bedeutet dies, dass - aneinandergereiht - gerade erst einmal rund ein Dutzend Baumgenerationen vergangen sind. Die Eiszeiten, von denen es vier Hauptglaziale und mehrere kleinere, kürzere Kaltzeiten in den letzten zwei Jahrmillionen gegeben hatte, vernichteten insbesondere in den mittleren und nördlichen Breiten Europas zahlreiche Arten, so dass Europa erheblich artenärmer wurde als die entsprechende geographische Region in Ostasien, wo die Wirkung der Eiszeiten und ihrer Wechsel mit Warmzeiten aus geographischen Gründen nicht annähernd so massiv ausgeprägt war wie an der Westseite Eurasiens.

Nach dem Zurückweichen des Eises mit Beginn der gegenwärtigen Warmzeit, des Holozäns, herrschte also ein stark verarmter Zustand von Flora und Fauna, ein Artendefizit.

Dass Europa nördlich der Alpen dennoch wieder vergleichsweise artenreich wurde, lag am einsetzenden Wirken des Menschen. Mit der Ausbreitung der Landwirtschaft wurden Verhältnisse geschaffen, die es sehr vielen Arten aus dem mediterranen und dem pontischen Raum ermöglichten, nach Mittel- und Nord- 
westeuropa einzuwandern. Dazu gehören die meisten Tier- und Pflanzenarten des Offenlandes, also der Fluren. Deutschland wäre ohne diese in historischer Zeit zugewanderten Arten kaum halb so artenreich wie gegenwärtig.

Der Prozess der Zu- und auch der Abwanderung dauert an. Zu keinem Zeitpunkt der Vergangenheit lässt sich ein Zustand von Fauna und Flora festlegen, welcher ökologisch oder biogeographisch „der Richtige“ wäre. Folglich ist es unmöglich, fremd und heimisch zeitlich objektiv voneinander zu trennen. Beliebige Festlegungen, die praktische Gründe haben, werden vorgenommen. So gelten Arten nach dem Deutschen Naturschutzgesetz als heimisch, wenn sie sich längere Zeit schon im Gebiet erfolgreich und selbständig fortgepflanzt haben. Andere Einteilungen gehen vom Jahr 1900 aus. Was vorher da war, gilt als heimisch, was danach kam als fremd bzw. neu. Genauso gut ließe sich allerdings das Jahr 2000 als ein noch „runderer“ Zeitpunkt dafür verwenden. Aus biogeographischen Erwägungen heraus wird 1492, das Entdeckungsjahr Amerikas, wie von Ragnar Kinzelbach (2001) vorgeschlagen, vorgezogen, weil danach ein besonders intensiver transkontinentaler Austausch von Arten und die „Europäisierung“ ganzer Kontinente (Crosby 1986) einsetzte.

Der Prozess der Ansiedlung fremder Arten verläuft kontinuierlich über die Zeiten, mal schneller, dann auch wieder langsamer. Entsprechendes gilt für die räumliche Verbreitung. Es ist völlig normal und natürlich, dass sich die Areale von Tieren und Pflanzen verschieben, wenn sich die Umweltbedingungen ändern. Die Kultivierung von Naturland, die seit Jahrtausenden läuft, verstärkte diese natürliche, hauptsächlich von Wetter und Klima verursachte Dynamik. Die Vorgänge sind nicht „neu“. Vielmehr gibt es sie seit jeher. Die letzten 500 Jahre wurde jedoch ganz besonders stark ,globalisiert“. Tiere und Pflanzen, die ohne Zutun des Menschen auf natürlichem Weg Europa nicht hätten erreichen können, wie etwa die Kartoffel und der Mais aus Amerika oder die Eukalyptusbäume aus Australien, wurden hier heimisch gemacht, wie Kaninchen in Australien und Feldhasen in der Pampa Argentiniens. Im 19. Jahrhundert gab es in Europa „Akklimatisierungsgesellschaften“, die es sich zur Aufgabe gemacht hatten, die Tauglichkeit fremder Arten für die Einbürgerung zu testen. Australische Kängurus kamen auf diese Weise (erfolglos) ins Rheinland (Niethammer 1963) und afrikanische Elenantilopen nach Russland. Umgekehrt verfrachteten die Europäer ihre Nutzpflanzen und Nutztiere, wie Rinder, Schafe, Ziegen, Hunde, Katzen und diverse Vögel, in alle Welt, so dass ganze Kontinente, wie Australien, davon weithin „europäisiert“ worden sind. Im Vergleich dazu nimmt sich die gegenwärtige Globalisierung mit ihren Begleiterscheinungen wie ein fast bedeutungsloses Nachspiel zu den großen Veränderungen der letzten Jahrhunderte aus. Heimisch und fremd haben vor diesem Hintergrund weitgehend ihren Sinn verloren. „Neu“ trifft besser, wenn es sich tatsächlich um Arten handelt, die erst in allerletzter Zeit in für sie fremde Gebiete gekommen sind. Sie werden Neozoen ( $=$ neu angesiedelte Tiere) und Neophyten (= neu angesiedelte Pflanzen) genannt. Anfangs sind die Neuen fast immer selten und unauffällig. Erst wenn sie häufig genug geworden sind, wird ihre Anwesenheit 
bemerkt. Fehleinschätzungen kommen auf diese Weise zustande. So werden die gegenwärtig typisch invasiven Problemarten, wie der Riesenbärenklau Heracleum mantegazzianum, das Drüsige oder Indische Springkraut Impatiens glandulifera und die Kastanienminiermotte Cameraria obridella ziemlich gleichermaßen als „neu“ erachtet. Doch Riesenbärenklau und Springkraut gibt es bereits seit dem späten 19. Jahrhundert in Mitteleuropa. Beide würden somit nach dem Bundesnaturschutzgesetz klar zu den heimischen Arten gehören. Legt man jedoch Kinzelbach's Jahr 1492 zugrunde, sind sie Neulinge (Neobiota), wie sehr viele weitere Tier- und Pflanzenarten unserer Fluren auch. Erst seit den 1970er Jahren wurden Riesenbärenklau und Drüsiges Springkraut „invasiv“. Die sich seit Anfang der 1990er Jahre ausbreitende Miniermotte an Rosskastanien ist zwar tatsächlich ein vorher nicht da gewesener Neuling, aber sie befällt die gebietsfremde, nämlich vom südöstlichen Balkan stammende Rosskastanie. Diese gibt es allerdings seit mehreren Jahrhunderten in Mitteleuropa. Als Baum schafft sie es aber kaum, sich frei in Wäldern zu etablieren. Die Miniermotte holte ihren Wirtsbaum somit lediglich mit ziemlicher Verspätung ein. An ,heimischen“ Baumarten kommt sie nicht vor. Es verhält sich mit ihr ganz ähnlich wie mit Kartoffel und Kartoffelkäfer. Der wesentliche Unterschied besteht in der Art des Schadens. Während starker Befall der Kartoffeln mit Leptinotarsa decemlineata erhebliche wirtschaftliche Verluste verursachen kann, beschränkt sich die Wirkung der Miniermotte auf eine verfrühte Verbräunung der Kastanienblätter im Hoch- oder Spätsommer, was als Beeinträchtigung der Biergartenatmosphäre angesehen wird. Finanziell bezifferbare Schäden sind in den beiden Jahrzehnten des bisherigen Hierseins der Miniermotte dadurch nicht aufgetreten.

\section{$4 \quad$ Ursachen der Invasivität}

Die oben getroffene Feststellung, dass nur sehr wenige Arten invasiv geworden sind, wirft die Frage nach den eigentlichen Ursachen der Invasivität auf. Die weitaus meisten Neozoen und Neophyten, mehr als $95 \%$, etablieren sich, ohne heimische Arten zu verdrängen und ohne wirtschaftliche Schäden anzurichten oder Krankheiten zu verbreiten. Sie werden Teil der Dynamik von Fauna und Flora. Sichtbare Veränderungen können nostalgische Reaktionen hervorrufen, weil es nicht mehr so wie vorher (früher) ist. Die Zeit geht darüber hinweg. Das frühere Vordringen von blauer Kornblume Centaurea cyaneus und Rotem Mohn Papaver rhoeas, von Feldlerchen Alauda arvensis und Feldhasen Lepus europaeus in ein Land, das ohne die landschaftsgestaltenden Eingriffe des Menschen weitestgehend von Wald bedeckt wäre, lässt sich rückblickend schwerlich als unakzeptabel oder gar als ökologische (Invasions)Katastrophe werten. Wir würden auch die einstens mediterranen Wohlriechenden Veilchen Viola odorata oder die Vielzahl der inzwischen „besonders geschützten“ Orchideenarten aus dem Süden nicht missen wollen und manch anderes mehr in unserer Natur, weil wir längst daran gewöhnt sind. Die 
Arten, die wir „kennen“, werden freimütig als hierher gehörig eingestuft. Das uns noch (weitgehend) Unbekannte, das Neue, wirk hingegen fremd (und wird meistens unbewusst auch ablehnt). Manch eine schon lange vorhandene, aber unauffällig gebliebene Art kann plötzlich wieder zum kritisch betrachteten Fremdling werden, wenn sie aus irgendwelchen Gründen beginnt, sich in Massen zu vermehren und dabei auffällig wird. Gerade solche Fälle bieten die besten Möglichkeiten, zu erforschen, woran es liegt, dass manche Arten invasiv werden.

Invasiv werden Arten, für die lebenswichtige Ressourcen in großem Umfang zur Verfügung stehen oder durch menschliche Aktivitäten verfügbar gemacht werden. Das zeigen solch schnellwüchsige und nährstoffbedürftige Pflanzen, wie Riesenbärenklau und Drüsiges Springkraut oder auch die lokaler invasiv gewordenen ostasiatischen Riesenknöteriche der Gattung Reynoutria. In einer Vegetationsperiode wachsen sie zwei oder mehr Meter hoch, bilden zahlreiche Blütenstände mit vielen Samen oder armstarke, fast stammartige Stängel. Um so schnell so viel Biomasse produzieren zu können, brauchen solche Pflanzen große Mengen insbesondere an Stickstoffverbindungen und Phosphaten. Unsere übermäßig gedüngte Landschaft bietet diese Nährsalze. Seit den 1970er Jahren ist die Nährstoffbilanz zwischen Eintrag (durch Düngung) und Entzug (durch die Ernte) in Mitteleuropa flächendeckend „positiv“ geworden. Das deutsche Bundesamt für Naturschutz bezifferte bereits Anfang der 1990er Jahre den durchschnittlichen StickstoffÜberschuss auf 104 Kilogramm pro Hektar und Jahr. Große Flächen in Nordwestund Süddeutschland liegen mit über $200 \mathrm{~kg} / \mathrm{ha} / \mathrm{Jahr}$ jedoch weit darüber. Solcherart überreich gedüngte Böden bereiten buchstäblich den Nährboden für wucherndes Wachstum und invasive Arten, die von den Nährstoff-Überschüssen profitieren. Abb. 1 zeigt die zeitliche Entwicklung in der Stickstoffbilanz. Von der anfänglich (durchschnittlichen) Unterversorgung schlug sie innerhalb eines Jahrzehnts zur Überdüngung (Eutrophierung) um. Das ist der Hintergrund dafür, dass Pflanzen, die schon seit dem 19. Jahrhundert im Land waren, wie Riesenbärenklau (ursprünglich zur Verbesserung der „Bienenweide“ im Spätsommer eingeführt!) und Drüsiges Springkraut, eine Gartenzierpflanze, erst seit den 1970er Jahren „invasiv“ geworden sind und nicht schon viel früher. 


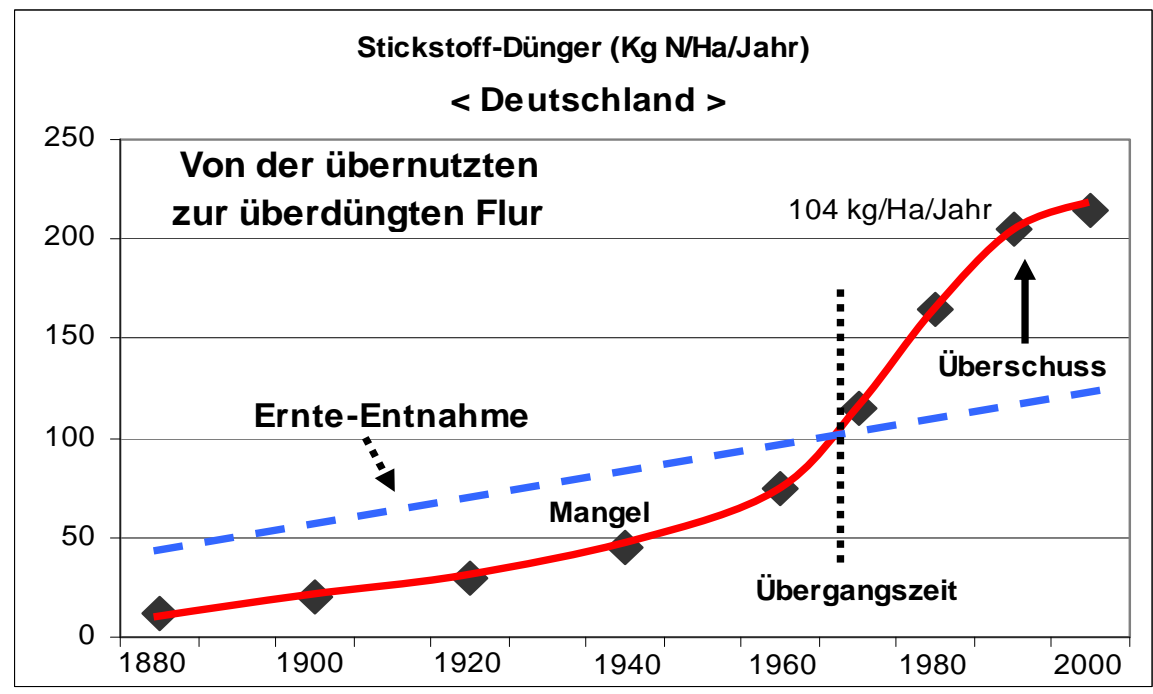

Abbildung 1: Trend von Nährstoffeinträgen durch Düngung und Entnahme über die Ernte in Deutschland von 1900 bis 2000. Die Stickstoffbilanz schlug in den 1970er Jahren zur Überdüngung um; vor allem mit der Einführung der Güllewirtschaft (aus Reichholf 2004).

Zwei Jahrzehnte vorher hatte sich eine ganz ähnliche Entwicklung in vielen Gewässern Deutschlands vollzogen. In den 1950er und 1960er Jahren wucherte in vielen Gewässern die so genannte Wasserpest Elodea canadensis und es kam zur Massenentwicklung von Blaugrünalgen (Cyanobakterien). Ursache war die Überfrachtung der Gewässer mit Phosphaten aus den Waschmitteln. Mit Einbau der Phosphatfällung in die Kläranlagen und Verminderung des Phosphatzusatzes in den Waschmitteln gingen Wasserpest und „Wasserblüten“ (AlgenMassenvermehrungen) rasch fast bis zur Bedeutungslosigkeit zurück. Die invasive Wasserpest, die aus Nordamerika stammte, ist inzwischen weithin selten geworden und kaum noch zu finden (Reichholf 2005).

Entsprechendes gilt für invasive Tierarten. Der aus Nordamerika stammende Kartoffelkäfer konnte, nachdem er nach Europa eingeschleppt worden war, deshalb invasiv werden, weil es großflächigen Anbau von (ursprünglich südamerikanischen) Kartoffeln in Monokulturen gab. Gegenwärtig ist mit der Ausbreitung des Maisanbaus (Herkunft Mittelamerika) der Maiszünsler Ostrinia nubilalis, eine Kleinschmetterlingsart, dabei, problematisch zu werden. 


\title{
Aktuelle Biodiversität: Bilanz zwischen Ansiedlungs- und Aussterberate
}

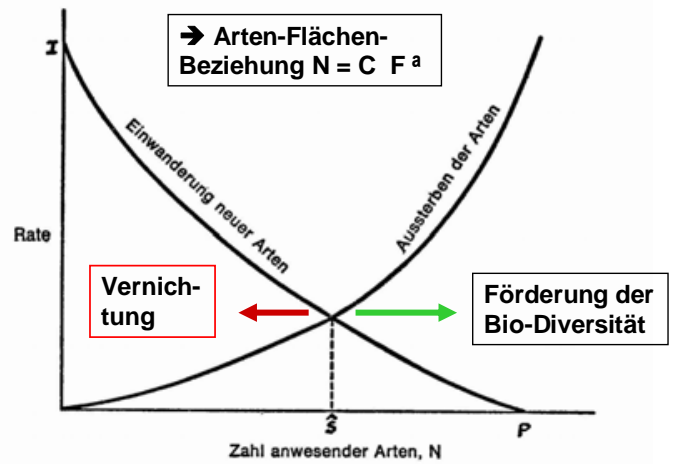

\begin{abstract}
Abbildung 2: Modell der Artenzahl einer Fläche als Funktion von Zuwanderungs- und Aussterberate (nach MacArthur \& Wilson 1967). Je kleiner und entfernter eine isolierte Fläche von einer Artenquelle, desto niedriger bleibt der Artenbestand und umgekehrt. Auch auf zusammenhängenden Kontinentalflächen läuft an Ort und Stelle diese Dynamik von Neuankömmlingen und Aussterben weiter, gleicht sich aber über größere Flächen aus.
\end{abstract}

Wo hingegen die Vegetation weitgehend natürlich und artenreich entwickelt ist, spielen invasive Arten nur eine geringe oder gar keine Rolle. Im Allgemeinen entsprechen die Möglichkeiten der Etablierung gebietsfremder Arten dem biogeographischen Grundmodell von MacArthur \& Wilson (1967), demzufolge sich immer weniger Arten neu etablieren können, je artenreicher die örtliche Flora und Fauna geworden ist (Abb. 2). Dieses „ökologische Modell“ lässt sich grundsätzlich auf kontinentale und insulare Verhältnisse gleichermaßen anwenden. Kleine, abgelegene ozeanische Inseln sind aber erheblich artenärmer als gleich große Flächen auf Festländern im selben Klimabereich, weil die Trefferwahrscheinlichkeit neu ankommender Arten mit der der Entfernung von einer „Artenquelle“ abnimmt. Daher sind häufig eigenständige evolutionäre Entwicklungen zustande gekommen. Vom Menschen dorthin verfrachtete Arten können sehr invasiv werden und die Besonderheiten verdrängen. Es mangelt den Inselarten an Konkurrenzfähigkeit. Die Invasivität verläuft daher auf Inseln, zumal wenn diese klein und entlegen sind, ganz anders als auf Kontinenten (MacArthur \& Wilson 1967, MacArthur 1972, Pielou 1979). Die grundlegenden Unterschiede bleiben jedoch zumeist in der allgemeinen Diskussion um gebietsfremde und invasive Arten, insbesondere im Naturschutz in Deutschland, unberücksichtigt. Was auf fernen Inseln geschah, wurde ohne entsprechende Einschränkungen der Übertragbarkeit auf unsere europäi- 
schen Verhältnisse bezogen (Kegel 1999). Entsprechendes gilt für die „europäisierten" Kontinente, auf denen, wie in Australien und Amerika, riesige Flächen ursprünglicher Natur für die europäischen Formen von Landwirtschaft, Düngung mit eingeschlossen, umgestaltet worden sind. Tier- und Pflanzenarten aus Europa konnten folglich leicht in diese „Neo-Europas“, wie sie Crosby (1986) sehr treffend nannte, eindringen und invasiv werden. Das bekannteste Beispiel hierfür sind die europäischen Kaninchen Oryctolagus cuniculus in Australien.

Prinzipiell vergleichbar verhält es sich bei der Ausbreitung von Krankheiten und Parasiten. Gerade die jüngsten Epidemien von Vogelgrippe zeigen dies in aller Deutlichkeit: Nicht die Wildvögel waren und sind das Problem, sondern die Massenhaltung von Hühnern, Gänsen und Enten auf engstem Raum. Maul- und Klauenseuche, Rinderpest und andere Krankheiten wurden und werden immer wieder epi- oder pandemisch. Auch potenziell für Menschen gefährliche Parasiten von Wildtieren, wie Kleiner Fuchsbandwurm, sind die Folgen künstlich überhöhter (Wild)Tierbestände. Malaria gab es in Mitteleuropa bis ins ausgehende 19. und beginnende 20. Jahrhundert, also unter ungünstigeren klimatischen Verhältnissen als gegenwärtig. Ihre Ausbreitung in außertropische Regionen wird ungleich stärker von Tourismus und Migrationen von Menschen als von Wetter und Klima begünstigt.

Zur Invasivität kann es also kommen, wenn umfangreiche, vorher wenig oder ungenutzte Ressourcen für die passenden Arten von Pflanzen, Tieren oder Krankheitserregern verfügbar werden. Sie ist kein Attribut der Arten an sich, sondern fast immer anthropogen. Nur sehr selten ereignen sich erdgeschichtlich umfangreiche Invasionen von Faunen und Floren in andere Kontinente. Es geschah dies als lange isolierte Kontinente oder Meere durch tektonische Vorgänge Kontakt zu einander bekommen hatten, wie zum Beispiel beim interamerikanischen Faunenund Florenaustausch als sich am Ende des Tertiärs, vor rund drei Millionen Jahren, durch plattentektonische Verschiebungen die mittelamerikanische Landbrücke zwischen Nord- und Südamerika gebildet hatte (Simpson 1980). Im Vergleich dazu ist die über den Suezkanal erzeugte Verbindung von Rotem Meer mit dem Mittelmeer ein lokaler Effekt geblieben und viel weniger bedeutsam für Fauna und Flora des Mittelmeeres geworden als die anthropogene Überdüngung und Vergiftung dieses Meeres.

\section{5 Überfremdung}

Wirtschaftliche Gewinne oder Verluste sind nicht die einzigen bedeutsamen Aspekte, wenn es um invasive Arten geht. Häufig wird seitens des Naturschutzes die von den fremden Arten verursachte „Überfremdung“ hervorgehoben. Sie würden das typische, natürlich „gewachsene“ Bild der heimischen Natur beeinträchtigen. Dieser nostalgischen Haltung stehen zwei klare Befunde entgegen. 
Erstens gibt es naturnahe Landschaften in Europa ohnehin nur in winzigen Restflächen. Mitteleuropa ist nahezu ausschließlich Kulturlandschaft. Über $99 \%$ der Fläche unterliegen Nutzungen. Weder die Fluren, noch die Wälder und Gewässer enthalten annähernd natürliche Zusammensetzungen von Pflanzen- und Tierarten. Massenkulturen wie Getreideanbau, Kartoffeln, Mais und Fichtenforste anstelle standortgemäßer Mischwälder bestimmen zusammen mit Städten, Dörfern und Verkehrsanlagen das Landschaftsbild. Wir leben von dieser Kulturlandschaft und nicht von der Natur Mitteleuropas. Nicht einmal die wenigen Prozent Naturschutzgebiete repräsentieren ursprüngliche Natur, denn die meisten geschützten Flächen sind aus früheren Nutzungen hervorgegangen. Die Rückentwicklung zum (mitteleuropäischen) Urwald würde, geschähe sie großflächig, das heutige Artenspektrum drastisch reduzieren. Denn mehr als die Hälfte aller Arten, die gegenwärtig in Mitteleuropa leben, siedelten sich in historischen Zeiten hier an. Die Kultivierung eröffnete ihnen großflächig diesen neuen Lebensraum.

Zweitens sind die gegenwärtig (noch) fremden Arten in Bezug auf das ,heimische“ Artenspektrum nicht einmal in den Großstädten prägend. Die Prozentanteile der Fremdarten liegen sehr niedrig (Abb. 3). Gäste (Durchzügler) und Irrgäste werden alljährlich etwa in der mitteleuropäischen Vogelwelt in großer Zahl festgestellt. Zu spontanen Ansiedlungen kommt es höchst selten, bei den Irrgästen so gut wie nie. Die Vögel verhalten sich in dieser Hinsicht ganz ähnlich wie die Gartenpflanzen zur wild wachsenden Flora. Die eigentliche Artendynamik spielt sich an den Arealrändern ab. Östliche oder nördliche, südwestliche oder südliche Arten breiten sich aus oder weichen zurück, je nachdem, wie sich die Witterung von Jahr zu Jahr entwickelt. Mitteleuropa liegt im Überschneidungsbereich dreier Klimazonen, nämlich der Atlantischen im Westen, der Pontisch-Mediterranen im Süden und Südosten sowie der Borealen im Osten und Nordosten. Klimatische Grenzräume zeichnen sich von Natur aus durch eine höhere Rate der Veränderung aus als zentral-kontinentale mit beständigerem Klima. Ein bestimmter Status für die Artenzusammensetzung ließe sich im Hinblick auf diese mehr oder weniger intensive, natürliche Dynamik nicht festlegen. Deshalb bleibt die Einstufung, wie eingangs schon betont, in „heimisch“ und „fremd“ reichlich willkürlich. 


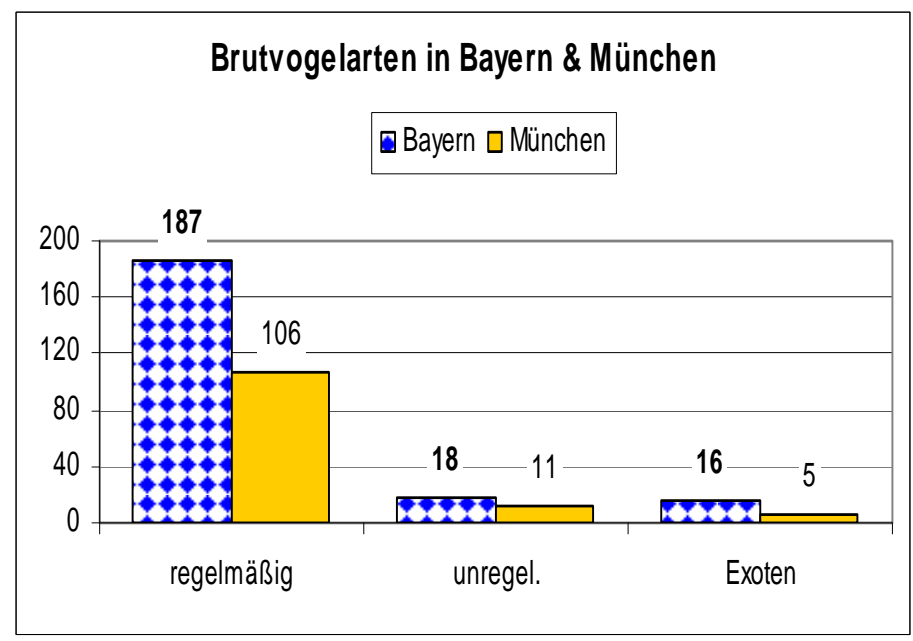

Abbildung 3: Anteil fremder Vogelarten an der Vogelwelt Bayerns und der Stadt München (Stand 2005). Die wenigen Fremdarten fallen mit 5\% nicht ins Gewicht zur Kennzeichnung der Vogelwelt.

\section{Gefährdung der Biodiversität}

Invasive Arten und Klimawandel gelten gegenwärtig als Hauptbedrohung für die Biodiversität, die Lebensvielfalt der Erde. Während ozeanische Inseln, insbesondere solche mit eigenständigen Evolutionen in tropisch-subtropischer Lage, tatsächlich durch solche Arten gefährdet sind, die von den Menschen eingeführt oder eingeschleppt worden waren (Ziegen, Ratten, Mäuse, Katzen sowie diverse europäische Pflanzen vor allem), trifft diese Einschätzung für die Mehrzahl der Inseln nahe an Kontinenten und für die Kontinente selbst sowie für die Meere nicht zu (Reichholf 2007). Weitaus stärkere Gefährdungen der Artenvielfalt gehen von der Landwirtschaft und ihren Folgen aus. Für Viehweiden und neue Anbauflächen von Futtermitteln und Getreide ist im 20. Jahrhundert allein die Hälfte des Ende des 19. Jahrhunderts noch vorhandenen Waldbestandes der Erde gerodet worden. Das Lebendgewicht der Rinder übertrifft gegenwärtig das aller Menschen um das gut Dreifache. Alle Nutztiere der Menschen zusammen verzehren rund das Zehnfache dessen, was die Menschheit direkt an Nahrung verbraucht. Die europäische Landwirtschaft mit ihren Importen von Futtermitteln für die Massenhaltung von Vieh, Geflügel und pflanzlicher Biomasse für die Biospriterzeugung nimmt mit weitem Abstand vor allen anderen die erste Stelle unter den globalen Gefährdungsfaktoren für die Biodiversität ein. Unser Stallvieh „,frisst“ seit Jahrzehnten höchst artenreiche Tropenwälder. Die Erzeugung von Biosprit verstärkt diesen Vernichtungseffekt für die tropische Biodiversität. Diese ist ungleich reichhaltiger als die Artenvielfalt der gemäßigten Breiten (Abb. 4). Die Umwandlung tropischer Wälder in 
Nutzland für landwirtschaftliche Export-Produkte gefährdet daher die Biodiversität weitaus stärker als das aus den bloßen Flächenveränderungen hervorgeht. Dem prognostizierten Klimawandel und den wenigen wirklich invasiven Arten wird kaum noch Biodiversität zum Opfer fallen, weil diese längst ausgerottet ist, bis sich das Klima stark genug geändert hat, wenn die Rate der Umwandlung tropischer Naturflächen im gegenwärtigen Tempo weiter läuft (Abb. 5). Hauptnutzer der Tropen sind Europa, insbesondere auch Deutschland, und China, aber nicht die arme örtliche Bevölkerung, die neues Land zum Überleben braucht (Reichholf 2004).
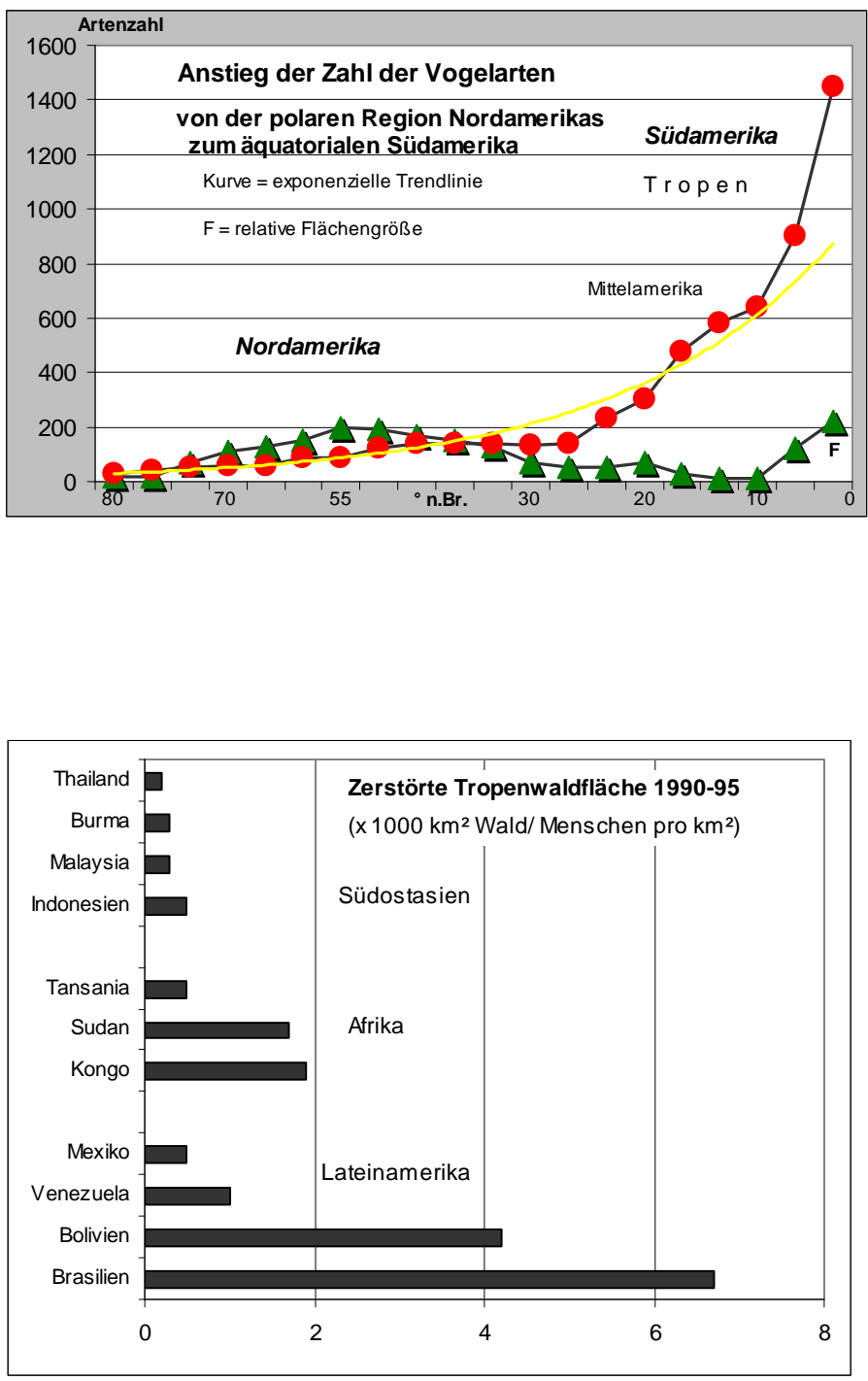

\begin{abstract}
Abbildung 4: Anstieg des Artenreichtums am Beispiel der Vogelwelt vom nördlichsten Nordamerika über Mittelamerika zum Äquator (Amazonien). Die Dreiecke geben die relative Flächengröße in den zugehörigen Breiten wieder. Das vergleichsweise winzige Costa Rica hat mehr Vogelarten als die riesigen Landflächen Nordamerikas.
\end{abstract}
Abbildung 5: Vernich- tung von Tropenwald in den verschiedenen Tropenregionen, be- zogen auf die Bevöl- kerungsdichte (Men- schen $/ \mathrm{km}^{2}$ ). Die weitaus größten Flä- chen wurden in Süd- amerika für Rinder- weiden und Sojaan- bau abgeholzt. 


\section{$\begin{array}{ll}7 & \text { Fazit }\end{array}$}

Pflanzen und Tiere sind nicht von Natur aus ,invasiv“. Einige wenige sind dies geworden, weil die Lebensbedingungen für sie so sehr verbessert wurden. Sie sind keine „bösen Arten“, sondern Ausdruck für das Wirken des Menschen, das sie als Bio-Indikatoren mit ihrem Vordringen und ihrer Häufigkeit anzeigen. Schäden verursachen sie, wenn sie Nutzungsansprüche von Menschen beeinträchtigen oder gefährden. Heimische Arten tun das prinzipiell nicht anders als neue Arten. Die früheren Ackerunkräuter, die Jahrhunderte lang intensiv bekämpft worden sind, drohen von den modernen Pflanzenschutzmitteln ausgerottet zu werden. Mit vielen Millionen Euro finanziert nun die EU die Erhaltung dieser in Ackerwildkräuter umbenannten Arten.

Klimatische Fluktuationen, wie das ,Mittelalterliche Klimaoptimum' und die ,Kleine Eiszeit' verstärkten die Dynamik von Flora und Fauna, die von den sich ändernden Formen der Landbewirtschaftung, die Entwicklung von Städten und Verkehrssystemen ausgelöst worden waren. Ein „richtiger Zustand“ der Natur lässt sich für keinen Zeitpunkt festlegen; nicht einmal, wenn man 10.000 Jahre bis zum Ende der letzten Eiszeit zurückgeht. Die Natur ist dynamisch. Dynamik ist die Vorbedingung für Evolution und Geschichtlichkeit. Ebenso wenig wie es in der menschlichen Geschichte eine Zeit oder einen Zustand gibt, auf den alle anderen bezogen werden könnten, lässt sich ein solcher für die Natur festlegen. Dass menschliche Einflüsse Geschwindigkeit und Ausmaße der natürlichen Veränderungen enorm verstärken können, liegt auf der Hand. Sicher gehört unsere Zeit zu einer Phase besonders intensiven Wandels. Die Herausforderung besteht daher darin, im Rahmen der weiteren Entwicklung so viel wie möglich von der Lebensvielfalt der Erde zu erhalten. Naturschutz ist, so verstanden, kein Gegensatz zu Wandel und Dynamik, sondern Bewahrung des Bewährten aus der Vergangenheit für die Zukunft. In dieser Hinsicht sind die invasiven Arten besonders lehrreich.

\section{Literatur}

Bayerische Akademie der Wissenschaften (2001) Gebietsfremde Arten, die Ökologie und der Naturschutz. Rundgespräch 22 der Kommission für Ökologie. F. Pfeil, München

Crosby AW (1986) Ecological Imperialism. The Biological Expansions of Europe, 900 - 1900. Cambridge University Press, Cambridge

Hartmann H, Schuldes H, Kübler R \& Konold W (1994) Neophyten. Ecomed, Landsberg

Kegel B (1999) Die Ameise als Tramp. Von biologischen Invasionen. Ammann, Zürich 
Kinzelbach R (2001) Das Jahr 1492: Zeitenwende für Flora und Fauna? In:

Bayerische Akademie der Wissenschaften. Rundgespräch 22: 15 - 27

MacArthur RH (1972) Geographical Ecology. Harper \& Row, San Francisco

MacArthur RH \& Wilson, EO (1967) The Theory of Island Biogeography.

Princeton Univ. Press, Princeton, New Jersey

Niethammer G (1963)Die Einbürgerung von Säugetieren und Vögeln in Europa. Parey, Hamburg

Pielou EC ( 1979) Biogeography. John Wiley \& Sons, New York

Reichholf JH (2004) Der Tanz um das goldene Kalb. Der Öko-Kolonialismus Europas. Wagenbach, Berlin

Reichholf JH (2005) Die Zukunft der Arten. C.H. Beck, München

Reichholf JH (2007) Ende der Artenvielfalt? S. Fischer, Frankfurt

Schroeder, F-G (1998) Lehrbuch der Pflanzengeographie. UTB Quelle \& Meyer, Heidelberg

Simpson GG (1980) Splendid Isolation. Yale Univ. Press, New Haven

Szyska B (2004) Neophyten. BfN Skripten 108. Bundesamt für Naturschutz, Bonn 


\title{
Was macht eigentlich das Waldsterben?
}

\author{
Roland Schäfer, Birgit Metzgger
}

\section{Einleitung}

„Erst stirbt der Wald, dann stirbt der Mensch“: In diesem populären Spruch drückte sich die Zukunftsangst vieler Menschen im Westdeutschland der 1980er Jahre aus. Befürchtet wurde nicht nur ein Absterben des Waldes auf großen Flächen und damit eine „Versteppung“ Deutschlands, sondern eine umfassende Umweltkrise, die auch die menschliche Existenz bedrohe. Von einem „ökologisches Hiroshima“, ja einem „ökologischen Holocaust" war die Rede (Der Spiegel 14.2.1983, S. 72, S. 74). Führende Wissenschaftler, Politiker und ein großer Teil der Bevölkerung der Bundesrepublik in den 1980er Jahren teilten diese Angst. Sie waren überzeugt, dass die durch die Industriegesellschaft verursachte Luftverschmutzung, der „Saure Regen“, jahrzehntelang auf den Wald eingewirkt und ihn so stark geschädigt habe, dass er in absehbarer Zeit sterbe - sofern man nicht schnell etwas ändere. „In absehbarer Zeit“ hieß je nach Prognose in 5 bis 20 Jahren.

Das Katastrophenszenario eines großflächigen Waldsterbens, das Wissenschaftler 1979 erstmals prognostizierten (Ulrich et al. 1979), traf auf offene Ohren: Ab Mitte 1981 entwickelte sich eine breite und aufgeregte öffentliche Debatte. In den folgenden Monaten und Jahren war das Thema Waldsterben ständig in Presse und Rundfunk präsent, es wurde zum Gegenstand von massenhaft publizierten populären Aufklärungsbüchern (siehe z.B. Abbildung 1), von Wahlkämpfen und Aufsehen erregenden Protestaktionen. Das „Waldsterben“ avancierte zum „Umweltproblem Nr.1“ - so das Ergebnis einer Umfrage der deutschen Presseagentur im Sommer 1982 (zitiert im General-Anzeiger, 27.8.1982). Bundes- und Landesre- 
gierungen reagierten verhältnismäßig schnell auf den öffentlichen Druck und veranlassten weitreichende Maßnahmen, die regelmäßige Waldzustandsberichte, Verordnungen zur Luftreinhaltung und eine außergewöhnliche Forschungsförderung für die Forstwissenschaft umfassten (BMI 1985).

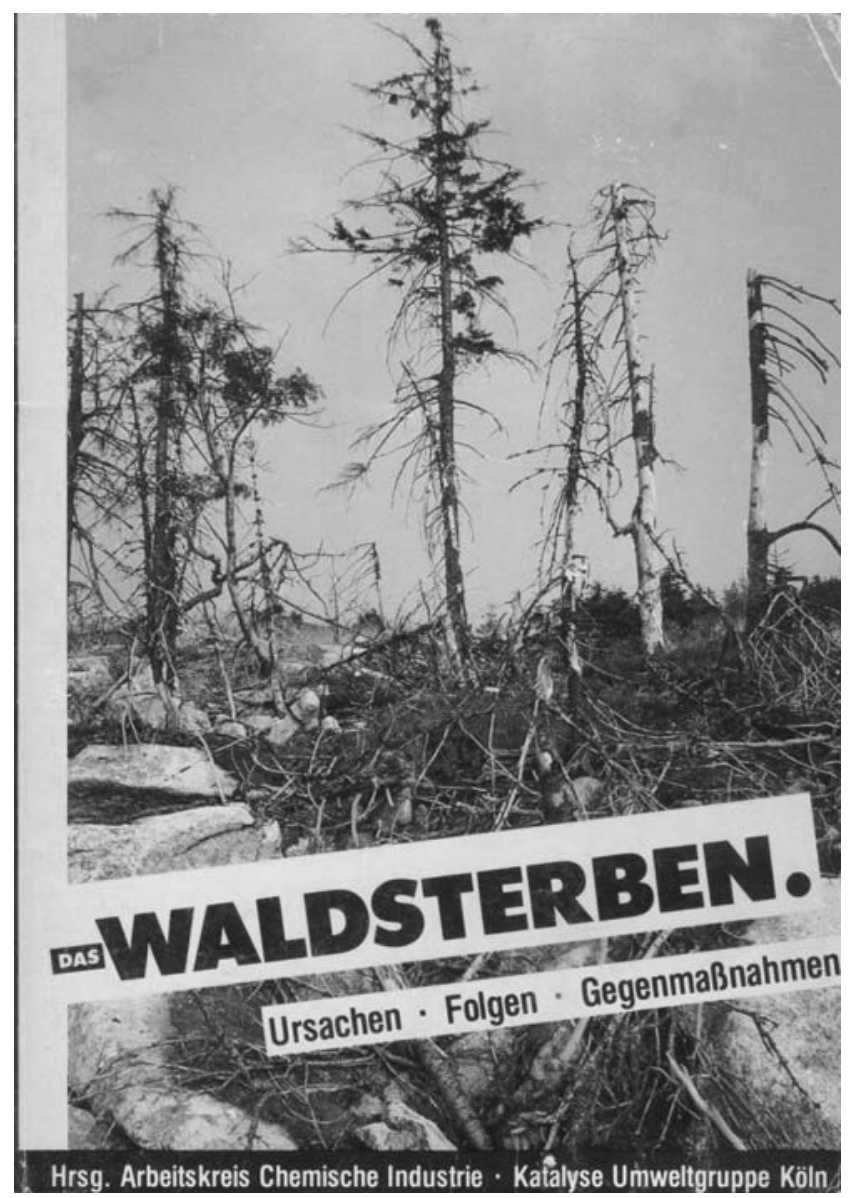

Abbildung 1: Sterbender Wald als Titelbild eines populären Aufklärungsbuches aus dem Jahr 1983

Heute lässt sich mit einem Blick aus dem Fenster feststellen, dass die prophezeite Katastrophe nicht eingetreten ist: Weiterhin wächst Wald in Deutschland. Vom Waldsterben als aktuellem Umweltproblem ist kaum noch die Rede, der Umweltdiskurs wird heute von der „Klimakatastrophe“ dominiert. War also die gesamte Debatte um das Waldsterben nur ein emotional aufgeladenes Medienereignis, eine Hysterie mit vergleichsweise kleinem Faktenkern, wie einige Kritiker behaupten (vgl. z.B. Holzberger 1995, S. 234-236; Müller-Ullrich 1996)? Oder ist das Waldsterben vielmehr als Beispiel erfolgreicher Umweltpolitik zu verstehen? Und wel- 
che Schlüsse lassen sich aus dem „Waldsterben“ für die Klimadebatte ziehen? Das sind Fragen, die heute immer wieder gestellt und ganz unterschiedlich beantwortet werden. Wir wollen uns diesen Fragen in etwas allgemeinerer Form annähern: Wie ist die Debatte der 1980er Jahre aus heutiger Perspektive zu verstehen und historisch einzuordnen? Was ist aus dem Thema geworden, das einst die gesamte westdeutsche Gesellschaft bewegte und in eine Katastrophenstimmung versetzte? Welche umwelthistorischen Lehren können wir aus dem Beispiel Waldsterben ziehen?

Dieser Aufsatz schlägt einen Bogen von den 1980er Jahren bis heute, um einen Überblick über die Entwicklung des Themas Waldsterben zu geben. Anhand der Kategorien Experten, Politik und Öffentlichkeit schildern wir zunächst die Charakteristika und den Verlauf der historischen Waldsterbensdebatte (Abschnitt 2). Daran anschließend gehen wir auf den aktuellen Stand der Debatte ein (Abschnitt 3). Aufbauend auf diesem Überblick versuchen wir auf einer übergeordneten Ebene, am Beispiel des Waldsterbens Probleme und Potenziale der Umweltgeschichte aufzuzeigen, und zwar für die historische Forschung einerseits und für das Verständnis aktueller Umweltdebatten andererseits (Abschnitt 4).

\section{Charakteristika der Waldsterbensdebatte in den 1980er Jahren}

\subsection{Ein modernes Umweltproblem und die Rolle der Wissenschaftler}

Es waren Forstwissenschaftler und Forstpraktiker, die Ende der 1970er Jahre auffällige Schäden an den Bäumen entdeckten und Politik und Öffentlichkeit darauf aufmerksam machten. Wissenschaftler waren auch die ersten, die vor großflächigen Waldschäden und vor einer ökologischen Katastrophe warnten; so zum Beispiel Karl Friedrich Wentzel in einer Anhörung des Innenausschusses des Bundestages 1978 (UBA 1978), Peter Schütt im Spiegel (8.9.1980), oder Bernhard Ulrich in der Süddeutschen Zeitung (15.11.1980). Auch in den folgenden Jahren behielten die Stellungnahmen von Wissenschaftlern und Forstpraktikern eine große Relevanz in der öffentlichen und der politischen Debatte. Die Fachleute waren in den Medien präsent und wurden zu politischen Anhörungen und öffentlichen Vorträgen eingeladen.

Die Tatsache, dass die Experten für die Kenntnisnahme des Problems in der Politik und einer breiteren Öffentlichkeit bedeutend waren, erscheint auf den ersten Blick absurd: War doch das Waldsterben ein Phänomen, an dem die zumeist eher abstrakte Umweltgefährdung und die weitgehend unsichtbare Luftverschmutzung konkret und für jeden anschaulich wurde (z.B. Beck 1986, S. 73). Zwar waren die damals festgestellten Waldschäden grundsätzlich für jeden sichtbar. Allerdings musste erst einmal die Aufmerksamkeit der Laien auf das Problem und seine Symptome gelenkt werden. Fachleute mussten also das Phänomen „Waldsterben durch 
Luftverschmutzung“ erst einmal definieren und erklären, um es für die öffentliche und politische Diskussion zugänglich zu machen (Beck 1986, z.B. S.68; allgemein dazu Bocking 2004). Zudem handelte es sich bei dem Thema „Waldschäden durch Luftverschmutzung“" um ein kompliziertes und unübersichtliches Umweltproblem, das für die Experten viele ungeklärte Fragen aufwarf. Unter anderem stellte die schnelle geographische und biologische Ausbreitung der beobachteten Schäden die Fachleute vor Rätsel. Nicht umsonst nannte man das Problem auch ,neuartige Waldschäden“ (SRU 1983: S. 100-102). Zwar waren sich am Anfang die meisten Wissenschaftler weitgehend einig, dass Luftschadstoffe, vor allem Schwefeldioxid und Stickoxide, eine wesentliche Ursache für die Schäden waren. Den Anteil der möglichen Schadstoffe und die genauen Ursachen-Wirkungszusammenhänge kannte man jedoch nicht (siehe z.B. in einem Aufruf deutscher Forstwissenschaftler an Regierung und Bürger der BRD (Anonymus 1983a; BMI 1985). Umgekehrt konnte man auch nicht ausschließen, dass noch andere, bisher unbekannte Faktoren eine Rolle spielten. Um diese Fragen zu klären, was ja Voraussetzung war, um wirksame Gegenmaßnahmen ergreifen zu können, wurde eine große Anzahl von Forschungsprojekten initiiert und mit Geld ausgestattet. Laut Umweltbundesamt wurden zwischen 1982 und 1992 in der Bundesrepublik mehr als 850 Forschungsvorhaben mit insgesamt 465 Mio. DM gefördert (UBA u. BMU 1997).

Aus den regen Forschungsaktivitäten resultierte eine Vielzahl unterschiedlicher Ursachen-Hypothesen: 167 zählten schwedische Wissenschaftler 1985 (Süddeutsche Zeitung, 5.2.85). Die Thesen reichten von den schon lange bekannten Rauchschäden über natürliche Einflüsse wie Klimaextreme oder Schädlinge bis hin zum schädigenden Einfluss von Radioaktivität aus Kernkraftwerken (einen Überblick gibt Nöthig 1986, S. 148-190). Einige Hypothesen wurden schnell wieder verworfen, wie etwa die Radioaktivitätsthese. Allerdings konnte trotz der enormen Forschungsförderung und der Vielzahl der Forschungsprojekte nie abschließend geklärt werden, welchen Anteil die in Frage kommenden Ursachen tatsächlich an den festgestellten Schäden hatten. Stattdessen entwickelten sich mit der Zeit zunehmend kontroverse Standpunkte. Mitte der 1980er Jahre wurden auch Stimmen lauter, die sich skeptisch über das prophezeite Waldsterben äußerten und die Bedeutung der Luftschadstoffe als Ursache relativierten (z.B. Rehfuess in BILD, 25.1.1985; Kandler im Mannheimer Morgen, 26.1.1985).

Neben der Vielzahl ungeklärter Fragen war das engagierte Selbstverständnis einiger Wissenschaftler bedeutsam für ihre Stellung in der Debatte: Beispielsweise empfand es Bernhard Ulrich, ein Göttinger Ökosystemwissenschaftler, der als Experte großen Einfluss auf die öffentliche und politische Debatte nahm, als Verpflichtung, die Gesellschaft frühzeitig vor Risiken zu warnen (Ulrich 1987, S. 975; ähnlich Peter Schütt in Neuweiler 1983). Seine Aussagen betrafen nicht nur sein wissenschaftliches Arbeitsgebiet, sondern beinhalteten auch zivilisationskritische und apokalyptische Warnungen: Der Mensch müsse sein Wirtschaften nach den thermodynamisch begründeten Regeln der Ökosysteme ausrichten, also beispielsweise Energie sparen, sonst drohe das Aussterben der Menschheit (z.B. Ulrich 
1982). Auch der bereits erwähnte Aufruf von 132 westdeutschen Forstwissenschaftlern an die Regierung der Bundesrepublik ist ein Ausdruck dieses politischen Selbstverständnisses (Anonymus 1983a).

\subsection{Das Waldsterben in der Bundespolitik}

Das Waldsterben wurde schnell zu einem politischen Thema. Von der Wissenschaft gelangte es 1980/81 über die Medien und die Umweltverbände einerseits und durch die Ministerialbürokratie andererseits in die Landes- und Bundespolitik. Dabei wandelte es sich von einem wissenschaftlichen zu einem umweltpolitischen Problem, bei dem die Frage danach, was zu tun sei, im Vordergrund stand.

Eine erste Reaktion von Seiten der politisch Verantwortlichen bestand darin, sich einen Überblick über die Situation und einen Einblick in die Problematik zu verschaffen. Dazu lud man Experten zu Stellungnahmen in verschiedene Gremien ein, zuerst im Sommer 1981 ins Bundeslandwirtschaftsministerium (vgl. Der Spiegel, 13.7.1981). Da die Lage entsprechend den Aussagen vieler Experten als sehr ernst eingestuft wurde, brachte die Bundesregierung verhältnismäßig schnell jedoch unter ständigen Druck der Öffentlichkeit - eine Reihe von Maßnahmen auf den Weg. Erste Schritte wurden noch unter der sozial-liberalen Koalition Helmut Schmidts vorbereitet und zum Teil schon eingeleitet. Dazu gehörten erste Bestandsaufnahmen der Schäden, die Einberufung einer Expertenkommission, erste Forschungsprogramme sowie die Vorbereitung von Verordnungen zur Verminderung der Luftverschmutzung. Daran knüpfte die Regierung Kohl wenig später an und beschloss weiterreichende Maßnahmen. Diese umfassten die jährliche und systematisierte Bestandsaufnahme in den Waldschadensberichten, die Forschungskoordinierung und -förderung, nationale und internationale Initiativen zur Luftreinhaltung (Großfeuerungsanlagenverordnung 1983, TA Luft 1982/85, Katalysator für Kraftfahrzeuge, bleifreies Benzin 1985/86) sowie waldbauliche Maßnahmen zur unmittelbaren Schadensminderung. Zusammengefasst wurden diese Maßnahmen im „Aktionsprogramm Rettet den Wald“, das 1983 verabschiedet und bis 1989 mehrfach aktualisiert und verlängert wurde (BMI 1985; BMU 1989).

Vor dem Hintergrund der fortdauernden wissenschaftlichen Unsicherheit und der zunehmenden Kontroverse über die Ursachen des Waldsterbens ist besonders bemerkenswert, dass schnell konkrete politische Maßnahmen ergriffen wurden. Dabei beruhten die politischen Entscheidungen ausschließlich auf der Luftverschmutzungs-These. So betrafen die wichtigsten Beschlüsse den Ausstoß von Schwefeldioxid bei Kraftwerken, der bis Ende des Jahrzehnts um über 70\% gegenüber dem Stand von 1980 gesenkt wurde, sowie den Ausstoß von Stickstoffoxiden, der auch wesentlich reduziert wurde - beim Hauptverursacher Straßenverkehr durch die Einführung des Katalysators. Lediglich bei der Forschungsförderung berücksichtigte man andere Ursachentheorien (vgl. z.B. Gregor 1986), die aber nie Eingang in konkrete politische Maßnahmen fanden. 
Diese schnelle Festlegung auf die Luftverschmutzung im politischen Bereich hatte verschiedene Gründe: Zum einen konnte man auf eine lange Erfahrung mit Schäden an Vegetation und menschlicher Gesundheit durch Luftverschmutzung zurückgreifen, forstwissenschaftliche Forschungen zu diesem Problemkomplex gab es seit über 100 Jahren (vgl. z.B. Wislicenus 1985). Zudem waren der „Saure Regen“ und seine Auswirkungen auf die Umwelt seit den 1970er Jahren auf internationaler Ebene ein Thema (Wetstone u. Rosencranz 1985). Etwa zeitgleich wurden auch Smog in den Städten und die gesundheitlichen Folgen der Luftverschmutzung zunehmend als Problem wahrgenommen (vgl. z.B. Prittwitz 1990, S. 13-15). Die Bundesregierung befasste sich ebenfalls seit den 1970er Jahren mit dem Problem der Luftverschmutzung und der Frage, wie diese durch gesetzliche Maßnahmen eingeschränkt werden könne. Gesetzesnovellen im Bereich der Luftreinhaltung wurden in den 1970er Jahren diskutiert, konnten allerdings bis Anfang der 1980er Jahre aufgrund verschiedener politischer und wirtschaftspolitischer Widerstände nur begrenzt durchgesetzt werden (vgl. dazu Müller 1986, S. 206315). Diese Faktoren trugen dazu bei, dass die These „Waldschäden durch Luftverschmutzung" zu Beginn der 1980er Jahre große Plausibilität aufwies und trotz aller Unsicherheiten schnell eine breite Akzeptanz fand. Die Einigkeit über die Diagnose und über die Notwendigkeit, umweltschützende Maßnahmen zu ergreifen, bestand zu Beginn der 1980er Jahre Parteien übergreifend. Das Thema wurde von den GRÜNEN bis hin zur CSU aufgegriffen.

Dennoch gab es kontroverse Diskussionen, die vor allem die Maßnahmen betrafen. Umstritten waren die Struktur der Energieversorgung, die genauen Grenzwerte bei den Verordnungen zur Luftreinhaltung oder die Integration ökonomischer Anreize in den Umweltschutz: Sollte man die Kohlevorrangpolitik fortführen oder die Atomenergie zur Verminderung der Schwefeldioxid-Emissionen ausbauen? Sollten ökonomische Anreize in den Umweltschutz integriert werden und wenn ja, auf welche Weise? Sollte man geschädigte Waldgebiete kalken oder nicht?

Hervorzuheben ist, dass die Positionen quer zu den Parteigrenzen verliefen. Zum Beispiel war die Bundesregierung aus CDU/CSU und FDP gegen ein Tempolimit, jedoch sprachen sich das unionsregierte Baden-Württemberg und UnionsVerbände auf der kommunalen Ebene dafür aus (Mackenthun 1984). In der SPD forcierte das Thema Waldsterben die programmatische Kontroverse über das Verhältnis von Ökonomie und Ökologie (z.B. VEBA 1984).

Trotz kontroverser Punkte überwiegt insgesamt das Bild einer übergreifenden Einigkeit. Kaum jemand bestritt in den Jahren 1982 bis 1985 ernsthaft, dass das Waldsterben überhaupt ein Problem war (z.B. Gesamtverband des Deutschen Steinkohlenbergbaus 1983), ja sogar die wissenschaftlich zunehmend umstrittene These, dass Luftverschmutzung die Hauptursache sei, wurde öffentlich kaum kontrovers diskutiert. Das „Aktionsprogramm Rettet den Wald“ wurde von allen als das Mindeste, was zu tun sei, akzeptiert. Am deutlichsten wurde dies vielleicht beim Regierungswechsel 1982/83: Die neu gewählte christlich-liberale Koalition knüpfte nahtlos an die von der sozialliberalen Koalition 1981 und 1982 vorbereite- 
ten Maßnahmen an, ergänzte und beschloss diese. Allerdings war die Bundesregierung unter Helmut Kohl nicht bereit, über die im „Aktionsprogramm Rettet den Wald“ genannten Maßnahmen hinaus zugehen. Ein Tempolimit, eine Abgabe auf Schwefelemissionen oder strengere Grenzwerte lehnte sie strikt ab. Dies wurde in der umweltinteressierten Öffentlichkeit sehr kritisch wahrgenommen und bildete wiederholt Anlass für Protest.

\subsection{Das Waldsterben in der Öffentlichkeit}

Die große öffentliche Aufmerksamkeit, die das Thema Waldsterben in den 1980er Jahren erfuhr, und die damit verbundene Aufregung sind die Charakteristika, die aus heutiger Sicht besonders auffallen. Nach ersten Thematisierungen in Medien, Parlamenten, Ministerialverwaltungen sowie bei Umwelt- und Forstverbänden im Laufe der Jahre 1980 und 81 wurde das Thema mit einer dreiteiligen Titelserie im Spiegel Ende 1981 endgültig im Fokus der Öffentlichkeit etabliert (Der Spiegel, 16./23./30.11.1981).

Aufregung und Krisenstimmung der öffentlichen Debatte drückten sich in Katastrophenszenarien und emotionalen Schlagworten aus. Diese entsprangen zum Teil rhetorischen Übertreibungen von Journalisten und Umweltaktivisten, waren aber auch Ausdruck einer als krisenhaft wahrgenommenen Situation. Für viele umweltbewusste Menschen war das Waldsterben das erste unbestreitbare Zeichen einer herannahenden ökologischen Katastrophe (SRU 1983). Denn gemäß den Prognosen einiger Wissenschaftler rechnete man tatsächlich mit einem großflächigen Absterben des Waldes in naher Zukunft. Bezeichnende Begriffe und verbreitete Schlagworte, mit denen die befürchtete Katastrophe dargestellt wurde, waren zum Beispiel: „Säuresteppe“, „Erst stirbt der Wald, dann der Mensch“, „ökologisches Hiroshima“ oder „ökologischer Holocaust“ (z.B. Der Spiegel, 14.2.1983). Vergleiche der Situation mit einem Krieg, gar einem Dritten Weltkrieg, waren keine Seltenheit. So heißt es beispielsweise in einem Liedtext des Sängers Peter Horton: „Vielleicht heißt der Dritte Weltkrieg schlicht: ,Saurer Regen.“" (Horton 1985). Mit diesen Vergleichen erhielt das Thema in einem spezifisch deutschen Bezugsrahmen eine existenzielle und moralische Bedeutung, mit der Gegenmaßnahmen als absolut unumgänglich dargestellt wurden. Gleichzeitig stellte die Wahl von Begriffen aus dem Bedeutungsfeld „Krieg““ (vgl. auch Holzberger 1995, S. 234-236) einen direkten Bezug zur einflussreichen Friedensbewegung her und war Ausdruck der zu Beginn der 1980er Jahre verbreiteten Krisenstimmung (Engels 2006, S. 295, 396 f.; Wirsching 2006, S. 429-433). Eine emotionale und nationale Aufladung erfuhr das Thema auch durch den Rückgriff auf verbreitete Begriffe und Bilder, die einen Bezug herstellten zur Romantik und zum „deutschen Wald“ (Metzger et al. 2009). Die schnelle und große Resonanz auf das Thema wurde vermutlich begünstigt durch die eindringlichen Katastrophenszenarien, durch bekannte Deutungs- 
muster und Anknüpfungen an andere aktuelle Themen, wie die Friedens- und die Anti-Atombewegung.

Neben diesen diskursiven Anknüpfungspunkten wurde das Problem konkret und für jeden sichtbar: Dazu trugen etwa Fotos von toten Bäumen bei (vgl. Abbildung 1), wobei Bilder aus dem Erzgebirge, wo es tatsächlich auf großen Flächen abgestorbenen Wald gab, eine besondere Bedeutung hatten (z.B. Allgemeine Zeitung, 6.9.1984; Redaktion Kölner VolksBlatt und Katalyse Umweltgruppe Köln e.V. 1982, S. 7-8). Auch die fast flächendeckend angebotenen Waldschadensexkursionen, Waldsterbe-Lehrpfade und die populäre Aufklärungsliteratur machten das Problem für viele Menschen fassbar. Die abstrakten Themen „Luftverschmutzung“ und „Umweltverschmutzung“" wurden auf diese Weise im Waldsterben gegenständlich und anschaulich.

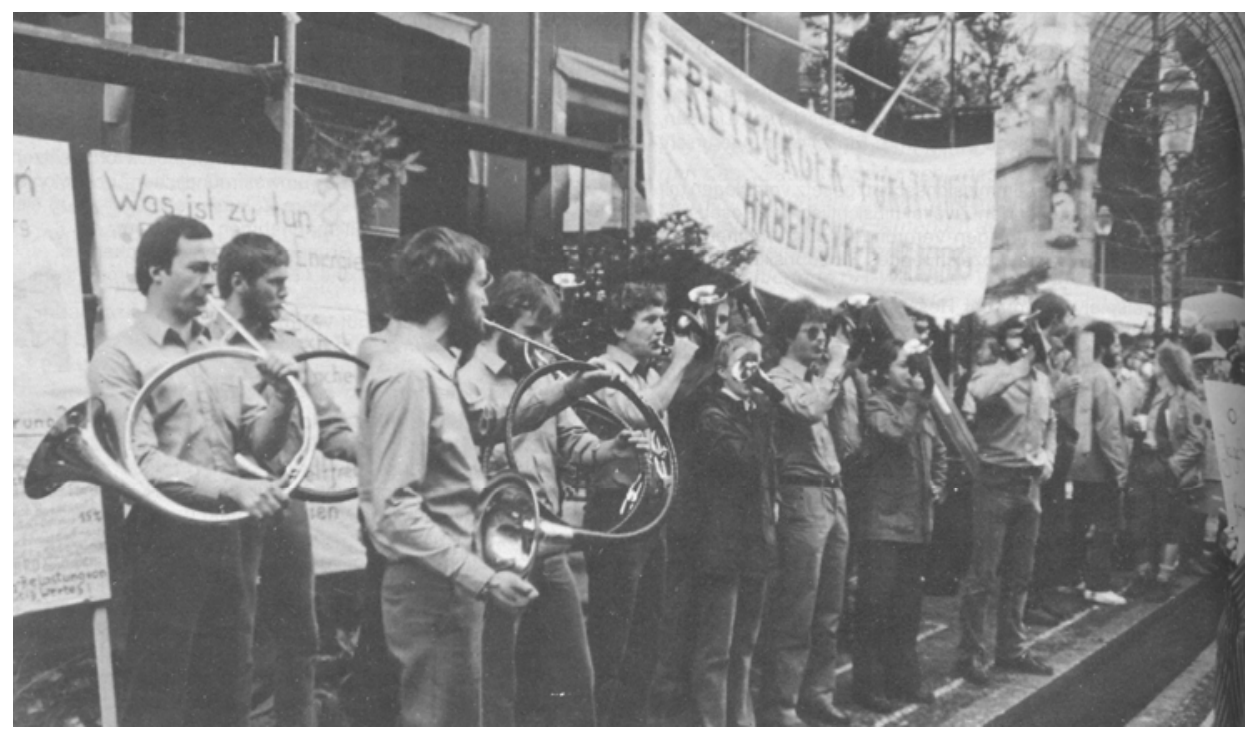

Abbildung 2: Freiburger Forststudenten demonstrieren am 7. Mai 1983 in traditioneller Kluft (aus Anonymus 1983b)

Wie sehr das Waldsterben quer durch die Gesellschaft als drängendes Thema wahrgenommen wurde, zeigt sich an der großen Anzahl von Veröffentlichungen zum Thema oder auch in Meinungsumfragen. 1984 und 1985 nannten viele Bundesbürger das Waldsterben als das wichtigste politische Thema nach der Arbeitslosigkeit; und zwar unabhängig von Alter, Geschlecht, sozialer Lage und parteipolitischen Präferenzen (Dierkes u. Fietkau 1988, S. 76; Ipos 1984, S. 5-15; Ipos 1985). Es bildeten sich breite Bündnisse, beispielsweise die Stiftung Wald in Not, die Freudenstädter Aktionsgemeinschaft gegen das Waldsterben, oder die Deutsche Aktionsgemeinschaft Kampf gegen das Waldsterben. Alle drei Organisationen bündelten ein breites Spektrum von am Umweltschutz interessierten Personen und Verbänden. In den beiden 
Aktionsgemeinschaften reichte das Bündnis von Adeligen bis zu Autonomen, wie es die tageszeitung (4.10.1983) ausdrückte. Konkret waren damit Umweltverbände, Forstverbände, Gewerkschaften und Waldbesitzer gemeint. Zur Erhaltung des Waldes war man bereit, für eine begrenzte Zeit über politisch und ideologisch völlig unterschiedliche Vorstellungen hinweg gemeinsam zu handeln. Zu den gemeinsamen Aktionen zählten öffentliche Demonstrationen, Resolutionen, populärwissenschaftliche Veröffentlichungen (vgl. Abbildung 1), Vortragsveranstaltungen und Exkursionen in betroffene Waldgebiete. Die Milieu-übergreifende Einigkeit bestand hinsichtlich der Diagnose „Waldsterben durch Sauren Regen“ und der Motivation, den Wald zu erhalten. Einig war man sich auch, dass dieses Ziel nur durch durchgreifende Maßnahmen im Umweltschutz, vor allem im Bereich der Luftreinhaltung, erreicht werden könnte. Die Maßnahmen der Bundesregierung, so die verbreitete Annahme, seien zwar „ein erster Schritt in die richtige Richtung“, aber viel zu lasch und zu langsam greifend, um den Wald noch zu retten. Aus diesem Grund war man zum Protest bereit: Traditionell konservative Forstleute gingen neben links-alternativen Umweltschützern auf die Straße (vgl. Abbildung 2). Auch den Zeitgenossen fiel auf, dass hier etwas Außergewöhnliches geschah (z.B. die tageszeitung, 12.11.1984; Süddeutsche Zeitung, 12.11.1984). Einige Aktivisten sahen hier das Potenzial für eine breite Volksbewegung für den Umweltschutz mit revolutionärem Charakter (vgl. z.B. Niemann o.J. ca. 1983).

Dazu kam es allerdings nicht; das breite Engagement ermattete ab 1985 zusehends. Das mag zum einen daran liegen, dass tatsächlich politische Maßnahmen ergriffen worden waren, die allmählich Wirkung entfalteten und so dem Protest einen Teil seines Gegenstandes nahmen. Zum anderen waren die inhaltlichen und formalen Unterschiede zwischen den Gruppierungen zu groß, um auf Dauer überbrückt zu werden. Immer wieder kam es zu Konflikten, die etwa die Aktionsformen betrafen: Beispielsweise waren die spektakulären Aktionen der kleinen Gruppe Robin-Wood konservativeren Gruppierungen ein Dorn im Auge (vgl. Schwarzwälder Bote, 20.10.1986). Denn mit dem Stil wurden auch Inhalte transportiert, etwa grundsätzlicher Protest gegen die Politik der Bundesregierung.

Abschließend ist festzuhalten, dass das Problem Waldsterben / Luftverschmutzung in der Öffentlichkeit und der Politik Westdeutschlands als sehr drängend wahrgenommen wurde. Das Thema bot für Akteure aus den unterschiedlichsten Milieus und politischen Spektren Anknüpfungspunkte und war Anlass zu konkreten politischen Maßnahmen.

\section{Das Waldsterben von den 1990ern bis heute}

Nach der hitzigen Debatte in den frühen 1980er Jahren ebbte die Aufmerksamkeit für das Waldsterben ab Mitte des Jahrzehnts deutlich ab. Im Laufe der 1990er Jahre kamen die groß angelegten und großzügig geförderten Forschungsprojekte zu einem meist wenig beachteten Abschluss. In Politik und Medien beschränkte 
sich die Thematisierung des Waldsterbens zusehends auf die jährlich erscheinenden Waldzustandberichte. Die eigens anlässlich des Waldsterbens 1983 gegründete Stiftung „Wald in Not" wird aufgelöst (Anonymus 2008). Was macht also das Waldsterben heute? Zunächst ist festzustellen: Den deutschen Wald gibt es noch, er hat, statt abzusterben, an Fläche sowie an Holzvorrat je Hektar zugenommen (BMELV 2002).

\subsection{Das Waldsterben in der Forstwissenschaft}

In der Forstwissenschaft ist heute von einem Waldsterben nicht mehr die Rede. Nachdem dieser Begriff anfangs durchaus als wissenschaftlicher Fachbegriff verwendet wurde (siehe z.B. Wentzel 1982; Schütt u. Summerer 1983; Ulrich u. Matzner 1983), wurde er ab 1983 zusehends durch die Neuschöpfung ,neuartige Waldschäden“ ersetzt (erstmals in SRU 1983), doch auch dieser Begriff wird immer seltener verwendet. In einem Handbuch, das 2007 einen umfassenden und abschließenden Stand des Wissens zu den Waldschäden darzustellen versucht, hat sich der Gegenstand zu einer „Schädigung von Waldökosystemen“ gewandelt (Elling et al. 2007). Bereits im Laufe der 1990er Jahre hatte sich in der Wissenschaft zunehmend die Ansicht durchgesetzt, dass von einem stattfindenden oder unmittelbar bevorstehenden großflächigen „Waldsterben“ nicht die Rede sein könne (siehe z.B. Rehfuess 1990; Elling 1992; Ulrich 1995).

Keinerlei Einigkeit herrscht allerdings über die heutige sowie die nachträgliche Bewertung der damaligen Waldschäden. Viele Wissenschaftler vertreten die Meinung, dass die Wälder in den 1980er Jahren stark unter Luftschadstoffen litten und auch heute noch leiden (Wentzel 2001; Hildebrand 2003; Elling et al. 2007). Die ergriffenen Maßnahmen zur Luftreinhaltung hätten „viele sterbende Fichtenwälder gerade noch gerettet" (Wentzel 2008, S. 31). So vertreten auch Ulrich und einige seiner Schüler die Ansicht, dass die Waldsterbe-Prognosen im Prinzip korrekt waren und ihr Eintreten nur durch die aufgrund dieser Prognosen erfolgte drastische Reduktion der Luftschadstoff-Emissionen verhindert werden konnte (Ulrich 1997a, 1997b; Ulrich in die tageszeitung, 27.09.2008; Beese u. Schulte-Bisping 2006). Auf der anderen Seite sahen bereits in den 1990er Jahren zahlreiche Wissenschaftler keinen oder lediglich geringen Einfluss von anthropogenen Luftverunreinigungen auf den Zustand der Wälder (siehe z.B. Rehfuess 1990; Kandler 1994; Zöttl 1995; Hüttl 1998). Das gehäufte, synchrone Auftreten verschiedener Waldschäden in den 1980er Jahren wird von dieser Seite oft mit Witterungsextremen wie Trockenjahren und Frostereignissen erklärt. Einzelne Forscher äußern wiederum die Befürchtung, dass uns die wirklich großen Schäden erst noch bevorstehen, sei es aufgrund der weiterhin überhöhten Stickstoff-Immissionen (Hildebrand 2003) oder aufgrund des Klimawandels (Spiecker in Die Welt, 08.12.2004).

Angesichts dieser divergierenden Beurteilungen erstaunt es, dass eine ernsthafte Debatte über das Phänomen Waldsterben nicht mehr stattfindet; die verschiedenen Meinungen stehen unvermittelt nebeneinander. Als Beispiel seien Äußerun- 
gen Freiburger Forstwissenschaftler in Massenmedien im September 2008 angeführt, deren sich widersprechende Bewertungen keinerlei Bezug aufeinander erkennen lassen: Spiecker und Hildebrand äußerten sich pessimistisch über die Zukunft der Wälder in Deutschland (in einer Sendung des SWR2, 08.09.2008), Aldinger und Bauhuss nur vier Tage später optimistisch (Der Tagesspiegel, 12.09.2008). Auch eine innerwissenschaftliche Aufarbeitung der Waldsterbensdebatte fand nur in Ansätzen statt (Oesten 2004, S. 150). Von außerhalb werden hingegen massive Vorwürfe gegen die (Forst-)Wissenschaftler und ihr Verhalten in der Waldsterbensdebatte erhoben: Die „Willfährigkeit“ und mangelnde Selbstkritik der Wissenschaftler wird angeprangert (Lehmann 1999, S. 265), es sei den beteiligten Forschern um Gelder und Karrieren gegangen (Horeis 2005), sie hätten die Medien und die Politik vor sich hergetrieben (Keil u. Kellerhoff 2006, S. 231). Keil (2004) schreibt in der ZEIT: „Der Schaden für die Wissenschaftler ist enorm. Nun glaubt ihnen keiner mehr.“ Die Wissenschaft habe in der Debatte um das ,angebliche Waldsterben" ihre Reputation und ihre Glaubwürdigkeit verloren (Storch in Berner Zeitung, 16.02.2008; Lehmann 1999, S. 265).

\subsection{Politik und Waldzustandsinventur}

Auch in der Politik ebbte die Aktivität nach den einschneidenden Maßnahmen der 1980er Jahre ab. Die verschärften Regelungen zur Luftreinhaltung führten zu deutlichen Senkungen der relevanten Emissionen. Insbesondere die außerordentliche Reduktion der Schwefeldioxid-Emissionen gilt als großer Erfolg der Umweltpolitik (Newig 2004, S. 832), während die Stickstoff-Emissionen weniger deutlich abgesenkt werden konnten (Vestreng u. Støren 2000, S. 42-43).

Die Vorstellung des Waldzustandberichtes gab allerdings alljährlich noch Anlass für parlamentarische Debatten sowie auch für Waldsterbens-Berichterstattung in den Massenmedien. Die Waldschadensinventuren und -berichte waren eine der dauerhaftesten und symbolträchtigsten Institutionalisierungen der Waldsterbensdebatte; mehrfach wurde der jährliche Bericht und die darauf konzentrierte, kurzfristige Thematisierung des Waldzustandes kritisch als „Ritual“ bezeichnet (z.B. Hartenstein in Deutscher Bundestag 1994, S. 17714; Horeis 2005, S. 16; Kommission für Ökologie der Bayerischen Akademie der Wissenschaften 2005).

Die Inventur des Waldzustandes wird seit 1984 nach einem einheitlichen Stichprobenverfahren durchgeführt, das einen Vergleich der Inventurergebnisse bis heute zulässt. ${ }^{1}$ Dabei wird einmal jährlich im Sommer der Kronenzustand der Bäume an den Stichprobenpunkten von geschulten Inventurteams visuell einge-

\footnotetext{
${ }^{1}$ Am Anfang der Waldschadenserhebungen stand 1982 eine bundesweite Befragung in den Forstämtern mit dem Ziel, den Umfang der neuartigen Schäden abzuschätzen. 1983 wurde die Befragung in leicht abgewandelter Form wiederholt, wobei in einigen Bundesländern bereits Stichprobenverfahren zum Einsatz kamen.
} 
schätzt. Ausschlaggebend ist der „Nadel-/Blattverlust“ bzw. der Grad der „Kronenverlichtung" der Inventurbäume im Vergleich zu einem optimal belaubten/benadelten Baum. Vergilbungen der Blätter/Nadeln und einige weitere Faktoren können ebenfalls in die Bewertung einfließen (BMVEL 2006). Seit 1989 werden die Inventurergebnisse nicht mehr unter der Bezeichnung „Waldschadenserhebung“ veröffentlicht, sondern unter dem Titel „Waldzustandsbericht“ bzw. „Bericht über den Zustand des Waldes“. Diese Verschiebung der Begrifflichkeiten hin zu vermeintlich neutralen und nüchternen Ausdrücken, die sich auch schon im Wandel vom „Waldsterben“ zu den „neuartigen Waldschäden“ zeigte, wurde von einigen Autoren als Verharmlosung kritisiert (Luhmann 1992; Kenneweg 1997; zur Geschichte der Waldzustandsinventur siehe auch Anders u. Uekötter 2004, S. 127134).

Der Bericht zum Jahr 2006 war der letzte seiner Art. Infolge einer Neuordnung des Berichtswesens des zuständigen Ministeriums für Ernährung, Landwirtschaft und Verbraucherschutz wird von nun an einmal pro Legislaturperiode ein umfassender „Waldbericht“ erscheinen. Die Ergebnisse der weiterhin jährlich stattfindenden Inventuren werden in stark verkürzter Form im Internet bekannt gegeben; die Inventurergebnisse von 2007 erschienen somit unter dem Titel „Ergebnisse der Waldzustandserhebung 2007“ (BMELV 2008). Diese Änderung hat im Vorfeld zu Protesten geführt (etwa von Robin Wood und der Stiftung „Wald in Not") und wurde auch im Bundestag lebhaft diskutiert. Die GRÜNEN-Abgeordnete Cornelia Behm äußerte die Befürchtung, dass ohne jährlichen Bericht die Aufmerksamkeit für die Waldschäden in der Öffentlichkeit nachlassen werde (Deutscher Bundestag 2007, S. 12938). Als die Ergebnisse der Waldzustandserhebung 2007 im Januar 2008 online erschienen, ohne in einen Bericht eingebettet zu sein und vor allem ohne Einberufung einer Pressekonferenz, fiel das Medien-Echo im Vergleich zu den Vorjahren tatsächlich gering aus. Die Zeitungsartikel bezogen sich zudem meist auf eine Pressemitteilung des BUND (BUND 2008), in der von einem weiterhin besorgniserregenden Waldzustand die Rede ist; diese Pressemitteilung erscheint als der eigentliche Anlass für die Berichterstattung (z.B. Der Tagesspiegel, 31.01.2008; die tageszeitung, 31.01.2008). 


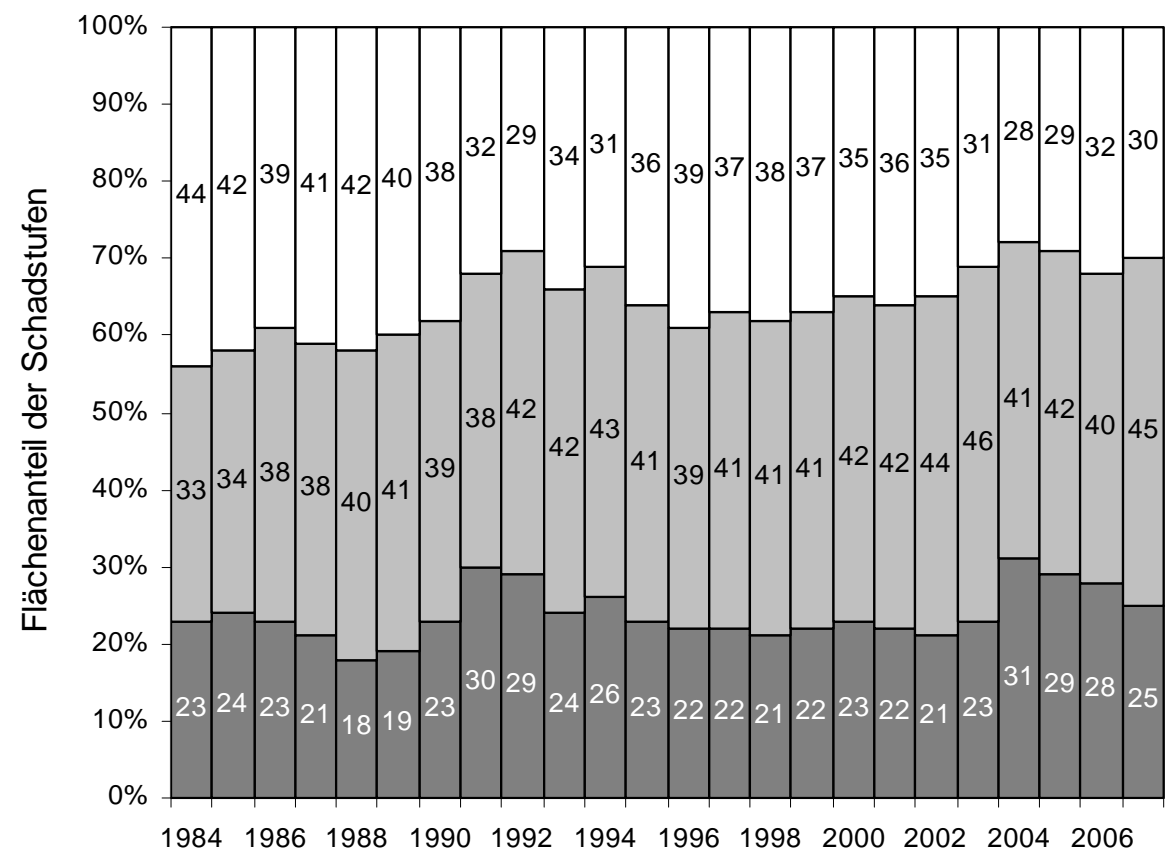

$\square$ ohne Verlichtung (Schadstufe 0: 0-10\% Nadel-/Blattverlust)

$\square$ schwache Kronenverlichtung (Schadstufe 1 bzw. Warnstufe: 11-25\% Nadel-/Blattverlust)

$\square$ deutliche Kronenverlichtung (Schadstufen 2-4: 26-100\% Nadel-/Blattverlust)

\section{Abbildung 3: Die Ergebnisse der Waldzustandsinventuren 1984 bis 2007 in der BRD für alle Baumarten (aus BMELV 2008)}

Wie steht es also mit dem deutschen Wald laut Waldzustandsinventur? In Abbildung 3 sind die Ergebnisse der Inventur von 1984 bis $2007 \mathrm{zu}$ sehen. Demnach zeigten 2007 lediglich 30\% der Bäume keinerlei Schäden, während 25\% aller Bäume deutlich geschädigt waren. Über den gesamten Zeitraum ergibt sich eine deutliche Seitwärtsbewegung der Schadstufen mit einem schwachen Trend hin zu größerer Kronentransparenz (Abnahme der Bäume ohne Verlichtung, Zunahme der schwachen Kronenverlichtung).

Die Aussagekraft dieser Daten ist allerdings umstritten. Der Kronenzustand der Bäume unterliegt starken natürlichen Schwankungen, und Kronenverlichtungen stellen ein sehr unspezifisches Symptom dar (Ellenberg 1996, S. 96-102). Selbst mit einer um 30 bis 60\% Prozent reduzierten Blatt- oder Nadelmasse können Bäume noch normales Wachstum aufweisen (Literatur siehe bei Hüttl 1998, S. 136). Die Festlegung der „Schadstufen“ erfolgte unter Gesichtspunkten der Praktikabilität und nicht aufgrund etwaiger faktisch gegebener Krankheitsschwellen (BMELV 1997, S. 132). Mehrfach wurde von wissenschaftlicher Seite harsche 
Kritik an der Inventur geäußert und ihre Abschaffung oder doch starke Modifikation gefordert (z.B. Hüttl 1998; SRU 2000; Kommission für Ökologie der Bayerischen Akademie der Wissenschaften 2005; hingegen nach kritischer Diskussion befürwortend: Elling et al. 2007, S. 44-54). Für den Geobotaniker Ellenberg hat diese mangelhafte Inventurmethode wesentlich zur Konstruktion eines allgemeinen Waldsterbens beigetragen: „Die beängstigende Ausdehnung neuartiger Waldschäden in Deutschland und in Europa war und ist mithin zu einem beträchtlichen Teil ein Konstrukt [ ... ], das durch Anwendung einheitlicher Schätzungshilfen auf standörtlich unterschiedliche Waldflächen und in witterungsmäßig ungleichen Jahren zustande kam.“ (Ellenberg 1996, S. 99, Hervorhebung im O.).

\subsection{Das Waldsterben in der Öffentlichkeit}

Am Gesamtzustand der Bäume hat sich also laut Waldzustandsinventur in den letzten Jahren wenig geändert. Die öffentliche Aufmerksamkeit für das Waldsterben hat sich indessen stark gewandelt: In Abbildung 4 ist der Thematisierungsverlauf des Waldsterbens in führenden deutschen Printmedien dargestellt. ${ }^{2}$

Nach einem schnellen, steilen Anstieg, der 1983 kulminierte, ging die Berichterstattung fast ebenso rasch wieder zurück und hat inzwischen ein recht niedriges Niveau erreicht (man beachte den Kontrast des Aufmerksamkeitsverlaufes zur „realen“ Entwicklung des Waldzustandes, wie er in Abbildung 3 dargestellt ist). Damit unterliegt das Thema Waldsterben wie andere Themen auch dem Konjunkturverlauf der medialen Aufmerksamkeit (dazu allgemein: Kolb 2005; zu vergleichbaren Thematisierungsverläufen des Waldsterbens in den Printmedien kommen Otto (2001, S. 145) für die BRD 1980-1990 sowie Zierhofer (1998, S. 200) für die Schweiz).

\footnotetext{
${ }^{2}$ Der Graph ist das Ergebnis einer Volltext-Suche nach dem Stichwort „Waldsterben“ im Pressearchiv von Gruner+Jahr; erfasst wurden: Spiegel, Stern, ZEIT, Welt, Welt am Sonntag, Bild, Bild am Sonntag, Frankfurter Allgemeine Zeitung, Frankfurter Allgemeine Sonntagszeitung, Frankfurter Rundschau, Süddeutsche Zeitung, tageszeitung, Focus. Die Anzahl der Waldsterben-Artikel pro Jahr wurde durch die Gesamtzahl an Artikeln der genannten Printmedien desselben Jahres geteilt und mit 100.000 multipliziert, um eine Vergleichbarkeit der Ergebnisse über den gesamten Zeitraum zu erreichen. Somit wird hier der relative Anteil der Waldsterbensberichterstattung an der Gesamtberichterstattung wiedergeben. Wir danken Ulrich Schnabel und Christoph Drösser von der ZEIT für die Weitergabe Ihrer Ergebnisse.
} 


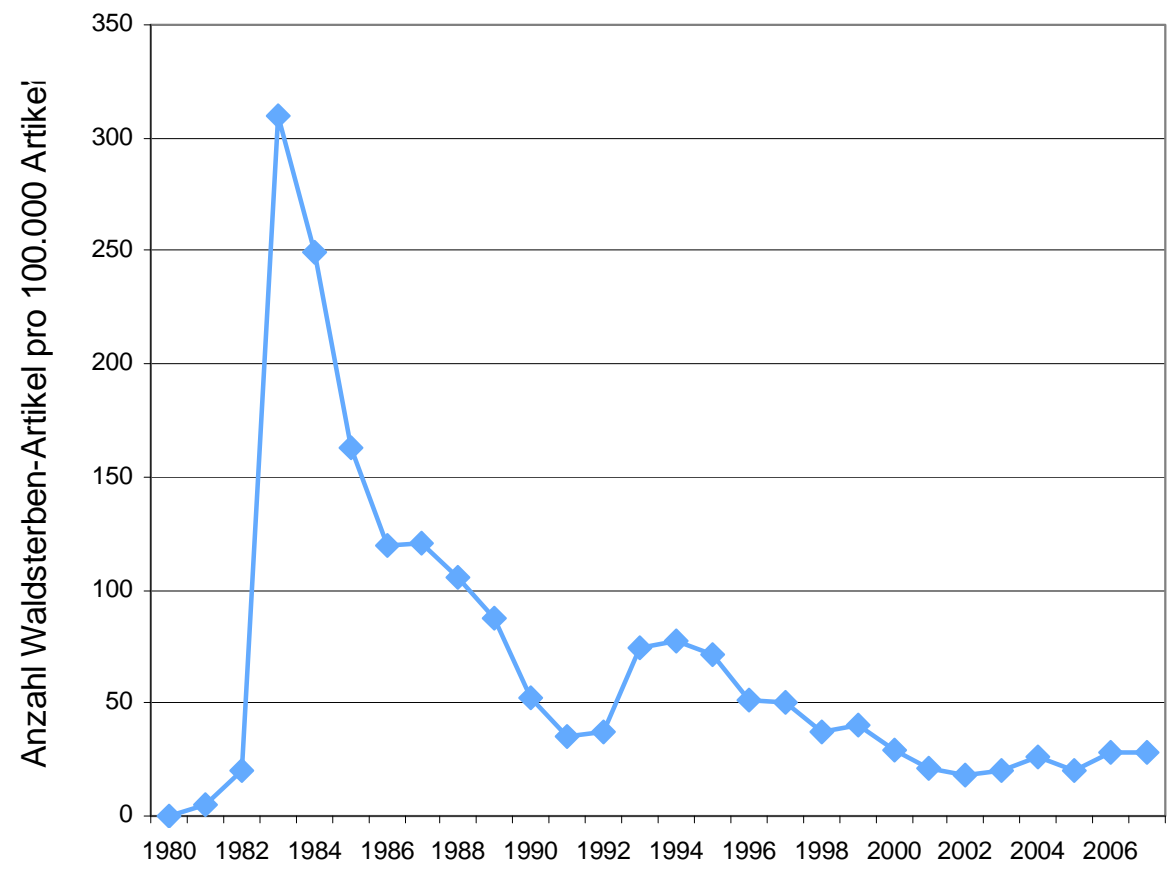

\section{Abbildung 4: Verlauf der Medienberichterstattung zum Waldsterben (nach Schna- bel 2008)}

Im allgemeinen Sprachgebrauch ist der Begriff „Waldsterben“ fest verankert. Auffällig ist das breite Spektrum an Bewertungen zu dem Phänomen „Waldsterben“: Autoren, von denen viele den liberalen „Ökoskeptikern“ zuzurechnen sind, kommen zu dem Schluss, dass es ein Waldsterben nie gegeben habe, dass die Sorgen und Ängste übertrieben waren und dass Medien, Umweltverbände und Wissenschaftler Panikmache betrieben hätten (siehe z.B. Holzberger 1995; Müller-Ullrich 1996; Maxeiner u. Miersch 1998; Keil u. Kellerhoff 2006). In der vielzitierten Dissertation des Journalisten Holzberger (1995) und auch beim Publizisten MüllerUlrich wird das Waldsterben auf ein reines Medienphänomen reduziert: „Die volle Wahrheit über das Waldsterben, die da lautet: Es war nur ein Rauschen im Zeitungsblätterwald, ist kaum je gedruckt worden." (Müller-Ullrich 1996, S. 26). Auf der anderen Seite sehen „Umweltschützer“ (siehe z.B. BUND 2008; Robin Wood 2008) und Interessensverbände (AGDW 2008) den Wald weiterhin als stark gefährdet an.

In der öffentlichen Umweltdebatte hat der Klimawandel dem Waldsterben als wichtigstes Thema längst den Rang abgelaufen (siehe dazu auch Schnabel 2008, S. 32). Das Waldsterben lebt allerdings als Kampfbegriff und argumentative Figur innerhalb der Klimadebatte fort, und wiederum lassen sich eine „ökoskeptische“ 
und eine „umweltschützerische“ Bewertung unterscheiden: Die „,Öko)-Skeptiker“ sehen im Waldsterben ein typisches Beispiel für eine irrationale, hysterische Umweltdebatte und möchten Klimawandel-Befürchtungen mit dem WaldsterbensVergleich diskreditieren (siehe z.B. Bartsch et al. 2007; Horx 2007; Bandulet 2008). Auch in der Politik wird das Waldsterben auf diese Art argumentativ eingesetzt (z.B. Wolfgang Clement in Westfälische Rundschau, 04.09.2008; Michael Glos in Waldportal 2007). Hingegen nennen „Klimaschützer“ das Waldsterben als positives Beispiel für eine erfolgreiche öffentliche Thematisierung eines Umweltproblems: Einschneidende politische Maßnahmen hätten eine drohende Umweltkatastrophe abgewendet, und dies spreche für entschlossenes politisches Handeln angesichts des Klimawandels. Dabei befinden sich die „Klimaschützer“ bemerkenswerterweise zumeist in einer defensiven Position: Sie führen das Waldsterben nicht von sich aus als positives Beispiel an, sondern in Reaktion auf die „ökoskeptische“ Deutung (siehe z.B. Kromp-Kolb in Wiener Zeitung, 16.04.2008; Rauchhaupt 2007). Auch diese Argumentation findet sich in der Politik wieder (Troge in Süddeutsche Zeitung, 06./07.10.2007; Gabriel in ovb-online 2008). Aktuell umstritten ist das Waldsterben somit vor allem als historisches Objekt, das in der Klimadebatte konkurrierenden Deutungen unterliegt.

\section{Fazit: Können wir etwas aus dem Waldsterben lernen?}

Ein Blick auf die heutige Umweltdebatte zeigt, dass das Waldsterben zwar nach wie vor als Umweltproblem wahrgenommen wird, jedoch keine drängende Aktualität mehr besitzt. Gleichzeitig erlebt das Waldsterben in der Klimadiskussion eine Renaissance als beliebig einsetzbare Argumentationsfigur: Es dient wahlweise als Beispiel für eine fehlgeleitete oder eine erfolgreiche Umweltdebatte. Hierfür bietet sich das Waldsterben auch deshalb an, da es gewisse Charakteristika aufweist, die als typisch für moderne Umweltprobleme angesehen werden können. Dazu gehören etwa die Komplexität des Problems, die damit verbundene wissenschaftliche Unsicherheit und die zentrale Bedeutung von Experten. Gleichzeitig stößt heute die enorme gesellschaftliche und politische Aufmerksamkeit auf Interesse, die das Problem Waldsterben in den 1980er Jahren erfuhr und die zu konkreten umweltpolitischen Veränderungen führte.

Welche Erkenntnisse und Perspektiven aber bietet eine Beschäftigung mit diesem Beispiel für das Verständnis aktueller Umweltprobleme einerseits und für die historische Forschung andererseits? Für die aktuelle Umweltdebatte ist zunächst von Interesse, inwieweit das Waldsterben als ein prototypisches modernes Umweltproblem einerseits und als spezifisches, historisch bedingtes Umweltproblem andererseits anzusehen ist. Auf einer wissenschaftstheoretischen Ebene geraten die Unterschiede zwischen realistischen und konstruktivistischen umwelthistorischen Ansätzen ins Blickfeld. Im Mittelpunkt steht dabei das Verhältnis von Natur und 
Gesellschaft bzw. die gesellschaftliche Problematisierung von Umweltveränderungen.

Einen Ansatz zur Erklärung bietet die Problemdruckthese, also die Vorstellung, dass Umweltpolitik als Reaktion auf die steigende Umweltbelastung erfolge und durch naturwissenschaftliche oder technische Analyse verstanden werden könne. Diese These ist trotz wissenschaftlicher Kritik (vgl. Prittwitz 1990a, S. 103107) weiterhin verbreitet. Auf diese Weise erklärt beispielsweise der Historiker Andreas Wirsching das Waldsterben in seiner Geschichte der Bundesrepublik der 1980er Jahre. Den gesamtgesellschaftlichen Konsens über dieses zentrale umweltpolitische Thema jener Zeit führt Wirsching auf die objektive Dringlichkeit des Problems zurück: „,...] die objektivierbaren Schäden [seien] viel zu offenkundig und gravierend [gewesen], als dass sie nicht die Sorge der Bevölkerung sowie der politischen Akteure erregen mussten." (Wirsching 2006, S. 365). Mit Verweis auf die Waldschadensberichte nimmt Wirsching die Waldschäden als objektiven Tatbestand an, der zwangsläufig zu politischen Reaktionen führte.

Im Fall des Waldsterbens zeigen sich aber deutlich die Grenzen des Erklärungspotenzials der Problemdruckthese. Allein wenn man den Verlauf der öffentlichen Thematisierung des Waldsterbens (Abb. 4) mit den Ergebnissen der Waldzustandsinventur der letzten 25 Jahre (Abb. 3) vergleicht, wird deutlich, dass kein direkter Zusammenhang zwischen dem beobachtbaren Waldzustand und dessen Thematisierung besteht. Die Problemdruck-These, die solch einen unmittelbaren Zusammenhang von Umweltzustand und Umweltproblematisierungen unterstellt, kann daher nicht zum Verständnis der Waldsterbensdebatte beitragen. Dazu müssen vielmehr andere, soziale und historische Faktoren berücksichtigt werden.

Auf einige dieser Faktoren sind wir in den vorhergehenden Abschnitten eingegangen: Zunächst spielten Wissenschaftler mit ihren Forschungsergebnissen über den Wald einerseits und mit einem bestimmten Selbstverständnis andererseits eine führende Rolle. Einige von ihnen sahen sich in einer Rolle als engagierte Wissenschaftler und traten mit politischen, teils gesellschaftskritischen Warnungen an die Öffentlichkeit. Viele von ihnen verfolgten das Ziel, den politischen Umweltschutz voranzubringen. Auch viele andere gesellschaftliche Akteure teilten die Überzeugung, dass auf dem Gebiet des Umweltschutzes, besonders bei der Luftreinhaltung, dringender Handlungsbedarf bestehe. Voraussetzung dafür war, dass sich bis Ende der 1970er Jahre in Teilen der Ministerialverwaltung und mit der verhältnismäßig starken Umweltbewegung ein neuartiges Umweltbewusstsein etabliert hatte, ohne dass nach zeitgenössischer Wahrnehmung angemessene Maßnahmen zur Luftreinhaltung erfolgt wären. Um 1980 standen somit genügend Kapazitäten bereit, um das Thema schnell aufzugreifen (zur Kapazitätenthese siehe Prittwitz 1990, S. 107-112). Einiges spricht also dafür, dass es im Kern der Debatte nicht allein um den Wald ging, sondern um einen umfassenden Ansatz zum Umweltschutz allgemein. Solch ein Ansatz wurde in der zu Beginn der 1980er Jahren ver- 
breiteten Krisenstimmung (Wirsching 2006, S. 429-435) für dringend erachtet. Damit ließe sich auch erklären, warum die Ursachendebatte in der Öffentlichkeit wenig Raum einnahm, die Maßnahmendebatte dafür umso mehr: Für viele Akteure ging es von vornherein um die Frage, wie der Umweltschutz besser in die Gesellschaft integriert werden könne. Auf welche Weise das geschehen sollte, war freilich umstritten.

Die Problematisierung der ,,neuartigen Waldschäden“ kann also als ein komplexer sozialer Prozess verstanden werden, bei dem der Umweltschutz zum Gegenstand des Zusammenwirkens unterschiedlichster Akteure aus Wissenschaft, Politik und einer breiten Öffentlichkeit wurde. Welche Bedeutung aber hatte der reale Wald für die Debatte? Mit Sicherheit ist zu sagen, dass die gesellschaftliche Debatte nicht im luftleeren Raum schwebte, sondern sich immer wieder auf die „realen“ Wälder und die physische Umwelt bezog. Wissenschaftler untersuchten „kranke“ Bäume, Journalisten gingen in den Wald, um „sterbende“ Wälder zu fotografieren und zu filmen, Politiker und Umweltbewegte unternahmen Exkursionen in Schadensgebiete, Inventuren dokumentierten den Schadensverlauf, die Umweltbewegung veranstaltete Waldschadensführungen.

Dabei eignete sich besonders der Wald als Kristallisationspunkt für eine grundsätzliche Umweltdebatte, wurden hier doch die Gefahren der zumeist unsichtbaren Luftverschmutzung für alle sichtbar. Da der Wald für viele Bundesbürger als Verkörperung der Natur schlechthin galt, wurde seine Bedrohung als besonders katastrophal angesehen. Das Waldsterben wurde zum Sinnbild der von vielen befürchteten ökologischen Krise. Daneben bot der Wald vielfältige Anknüpfungspunkte für ganz unterschiedliche Akteure: Waldbesitzer und Forstleute fühlten sich ebenso betroffen wie Natur- und Umweltschützer. So war es möglich, dass sich unter dem Thema Waldsterben die heterogene Umweltbewegung mit ihren unterschiedlichen politischen Strömungen, Konservative wie Progressive und Alternative, bündelte. Zudem konnte an bekannte Deutungs- und Interpretationsmuster vom „romantischen Wald“, vom „deutschen Wald“ und „sterbenden Wald“ angeknüpft werden - auch das trug sehr wahrscheinlich zur Breitenwirkung bei.

Der Wald war als konkreter Bezugspunkt der Debatte unbestreitbar von Bedeutung. Aber war er auch wirklich geschädigt? Und wenn ja, wodurch? Viele Studien, die sich aus kulturwissenschaftlicher Sicht rückblickend mit dem Waldsterben beschäftigen, versuchen, auch diese Frage zu beantworten. Ein rein konstruktivistischer Ansatz befriedigt angesichts des Phänomens Waldsterben offenbar nicht. So führt etwa der Umwelthistoriker Engels (2008) die Waldschäden auf Witterungsextreme zurück, der „Ökoskeptiker“ Reichholf (2002, S. 75) verweist auf die erfolgreiche Filterung basisch wirkender Stäube sowie gestiegene StickstoffImmissionen. Der Journalist Holzberger (1995, S. 284) führt das Wenige, was am Waldsterben „,real“, also nicht auf die Medienhysterie reduzierbar sei, auf Monokulturen zurück. 
Bereits die Zeitgenossen waren mit der Komplexität des Problems, der Unbeweisbarkeit von Kausalketten und mit wissenschaftlicher Unsicherheit konfrontiert; die nachträgliche Bewertung ist noch weitaus schwieriger. Zur Beurteilung der historischen Situation sind wir zu einem großen Teil auf die dokumentierten Wahrnehmungen der Zeitgenossen angewiesen. Mit Sicherheit gab es Anfang der 1980er Jahre einen Wandel der Deutungsmuster und eine Neuausrichtung der Aufmerksamkeit. Phänomene, die vorher als mehr oder weniger normale Schäden aufgrund von Frost, Schädlingen oder Sommertrockenheit gedeutet wurden, erschienen nun als Symptome der „Walderkrankung“ aufgrund von Immissionseinflüssen. Großflächige Erhebungen des Benadelungsgrades von Baumkronen hatte es vor den Waldschadensinventuren nie gegeben. Wie bereits erwähnt, waren auch viele Forstexperten grundsätzlich der Meinung, dass die Schadstoff-Emissionen zu hoch seien und dass die Industriegesellschaft sich der Natur gegenüber rücksichtslos verhalte. Ein nachträgliches Urteil über den naturwissenschaftlichen Realitätsgehalt des Waldsterbens wird auch dadurch erschwert, dass zu keinem Zeitpunkt eine einheitliche Definition des Begriffes „Waldsterben“ vorlag.

Bei allen Einschränkungen der Erkenntnismöglichkeiten über die damaligen Entwicklungen in den Wäldern scheint doch einiges darauf hinzuweisen, dass die Wälder in Deutschland Anfang der 1980er Jahre tatsächlich „schlechter“ oder zumindest anders aussahen als vor- und nachher. Dies ergibt sich aus der Zusammenschau von Zeitzeugengesprächen mit der zeitgenössischen forstwissenschaftlichen Fachliteratur oder auch aus aktueller dendrochronologischer Forschung (Elling et al. 2007, S. 54-60, 181-197). Selbst zeitgenössische Kritiker der Debatte, etwa aus der Industrie, stritten damals Veränderungen am Waldzustand nicht ab. Fragen nach den Ursachen sowie der Entwicklung und Ausbreitung der Schäden sind allerdings noch schwieriger zu beantworten als die nach den Veränderungen selbst.

Die wissenschaftliche Unsicherheit bei gleichzeitiger Abhängigkeit von Expertenwissen, die Unmöglichkeit, Kausalzusammenhänge zu beweisen, der aus diesem Grund gebotene Rückgriff auf das Vorsorgeprinzip und die Komplexität, die das Waldsterben auszeichnen, machen es zu einem Prototypen für moderne Umweltprobleme, zu denen auch das Ozonloch oder der Klimawandel zu rechnen sind. Insofern kann eine Analyse des Waldsterbens zum Verständnis aktueller Umweltprobleme beitragen. Im Hinblick auf die Umweltgeschichte verdeutlicht das Beispiel Waldsterben, dass man sowohl die sozialen Geschehnisse als auch die physische Umwelt bei der Untersuchung von Umweltdebatten berücksichtigen muss. Ein rein konstruktivistischer Ansatz scheint uns ebenso wie ein rein realistischer Ansatz nicht weiterzuführen.

Gleichzeitig wird auch deutlich, dass die Umweltgeschichte nicht nur in Bezug auf die Umweltthematik Erkenntnisse ermöglicht, sondern auch allgemeiner einen Zugang zur Geschichte bietet. Das Beispiel der Waldsterbensdebatte ermöglicht einen vertieften Blick auf die Gesellschaft und die Politik der Bundesrepublik der 
1980er Jahre. Hier zeigt sich auch, dass das Waldsterben nicht nur typische Charakteristika eines modernen Umweltproblems aufwies, sondern auch Elemente, die aus einer spezifischen historischen Situation resultierten. Besonders weil es sich für die Zeitgenossen um eine Krisensituation handelte, zeigen sich elementare Strukturen der damaligen Gesellschaft in der Waldsterbensdebatte. Das Waldsterben ist nicht nur ein Stück Umweltgeschichte, sondern auch ein Stück Zeitgeschichte.

\section{Literatur}

AGDW (Arbeitsgemeinschaft deutscher Waldbesitzerverbände) (2008)

Waldzustand weiter besorgniserregend. Pressemitteilung, 30.1.2008

Allgemeine Zeitung (6.9.1984) Energiesparen und Tempolimit retten den Wald

Anders K, Uekötter F (2004) Viel Lärm ums stille Sterben - Die Debatte über das

Waldsterben in Deutschland. In: Uekötter F, Hohensee J (Hrsg.) Wird

Kassandra heiser? - Die Geschichte falscher Ökoalarme. Steiner, Stuttgart. S. $112-138$

Anonymus (1983a) Gefährdung der Wälder durch Luftverunreinigungen. Aufruf an die Regierung der Bundesrepublik Deutschland und die Bürger der Bundesrepublik Deutschland [ohne Ort]

Anonymus (1983b) Freiburger Forststudenten informieren auf dem Münsterplatz. Allgemeine Forstzeitschrift, H. 21, S. 528-529

Anonymus (2008) Stiftung Wald in Not soll aufgelöst werden. AFZ-DerWald, H. 17, S. 945

Bandulet B (2008) Ökologismus: Die Klimamacher. Ein Bericht über den Sozialismus des 21. Jahrhunderts. ef-magazin.de.

http://ef-magazin.de/2008/09/02/623-oekologismus-die-klimamacher zuletzt besucht am 09.12.2008

Bartsch C, Ederer G, Horx M, Lotter W, Maxeiner D, Reichholf JH, Weimer W (2007) Wir müssen Urängste relativieren. Frankfurter Allgemeine Zeitung, 05.09.2007, S. 35

Beck U (1986) Risikogesellschaft : auf dem Weg in eine andere Moderne. Suhrkamp, Frankfurt Main

Beese F, Schulte-Bisping H (2006) Ökosysteme reagieren, wenn die Politik agiert: Interaktionen eines dynamischen Verknüpfungsgebildes. Vortrag auf: Symposium: „Zukünftige Entwicklung der Waldzustandserhebung: Geben die Waldzustandsberichte ein realistisches Bild der Situation unserer Wälder wieder". Stiftung Wald in Not, Gotha, 20.11.2006 
Berner Zeitung (16.02.2008) Die verkappte Moral des Klimas. S. 38

BILD (25.1.1985) Wald: Die Nadeln kommen wieder

BMELV (Bundesministerium für Ernährung, Landwirtschaft und Verbraucherschutz) (1997) Waldzustandsbericht 1997. (Bundestagsdrucksache 13/9442)

BMELV (2002) Die zweite Bundeswaldinventur - BWI2. Das Wichtigste in Kürze. http://www.bundeswaldinventur.de/enid/5982fce304a769dcb28361e6f22f1b6 $0,0 / 31 . h t m l$ zuletzt besucht am 09.12.2008

BMELV (2006) Bericht über den Zustand des Waldes 2006 - Ergebnisse des forstlichen Umweltmonitorings. BMELV, Berlin

BMEVL (2008) Ergebnisse der Waldzustandserhebung 2007 http://www.bmelv.de/cln_045/nn_753668/DE/06Forstwirtschaft/Waldberichte/_waldberichte_node.html_nnn=true zuletzt besucht am 10.12.2008

BMI (Bundesministerium des Inneren) (1985) Aktionsprogramm Rettet den Wald. Bonn. (Umweltbrief, Nr. 32)

BMU (Bundesministerium für Umwelt, Naturschutz und Reaktorsicherheit) (1989) Aktionsprogramm „Rettet den Wald“ (3. Fortschreibung). Bonn

Bocking S (2004) Nature's Experts. Science, Politics, and the Environment. Rutgers University Press, New Brunswick, New Jersey, London

BUND (Bund für Umwelt und Naturschutz Deutschland) (2008) Über zwei Drittel des Waldes krank. BUND fordert waldverträgliche Agrar-, Verkehrsund Energiepolitik. Pressemitteilung, 30.1.2008

Der Spiegel (8.9.1980) Alle kaputt. H. 37, S. 81-82

Der Spiegel (13.7.1981) Etwas stirbt immer. H. 29, S. 72

Der Spiegel (16./23./30.11.1981) Säure-Regen: „Da liegt was in der Luft“. H. 47, S. 96-106; H. 48, S. 188-200; H. 49, S. 174-190

Der Spiegel (14.2.1983) „Wir stehen vor einem ökologischen Hiroshima“. H. 7, S. $72-92$

Der Tagesspiegel (12.9.2008) Hartes Holz

Der Tagesspiegel (31.1.2008) Waldsterben - die meisten deutschen Eichen sind krank

Deutscher Bundestag (1994) Plenarprotokoll 12/205 
Deutscher Bundestag (2007) Plenarprotokoll 16/123

Dierkes M, Fietkau H-J (1988) Umweltbewusstsein - Umweltverhalten.

Kohlhammer, Karlsruhe

die tageszeitung (4.10.1984) Adelige und Autonome

die tageszeitung (12.11.1984) Sauer macht lustig - der Wald lacht sich kaputt

die tageszeitung (31.01.2008) Buchen gesundgebetet. S. 8

die tageszeitung (27.09.2008) „Da hält man besser das Maul“. S. 10-11

Die Welt (8.12.2004) Deutsche Wälder in Not. S. 15

Ellenberg H (1996) Vegetation Mitteleuropas mit den Alpen in ökologischer, dynamischer und historischer Sicht. Ulmer, Stuttgart

Elling W (1992) Waldschäden und Waldschadensforschung: Eine kritische Zwischenbilanz. Naturwissenschaftl. Rundschau, H. 5, S. 184-189

Elling W, Heber U, Polle A, Beese F (2007) Schädigung von Waldökosystemen:

Auswirkungen anthropogener Umweltveränderungen und Schutzmaßnahmen. Elsevier, Spektrum Akademischer Verl., Heidelberg, München

Engels JI (2006) Naturpolitik in der Bundesrepublik. Ideenwelt und politische Verhaltensstile in Naturschutz und Umweltbewegung 1950-1980. Schöningh, Paderborn

Engels JI (2008) „Inkorporierung“ und „Normalisierung“ einer Protestbewegung am Beispiel der westdeutschen Umweltproteste in den 1980er Jahren.

Zeitschrift für soziale Bewegung, H. 1 [in Druck]

General-Anzeiger (27.8.1982) Waldsterben: Umweltproblem Nummer eins

Gesamtverband des Deutschen Steinkohlenbergbaus (1983) Saurer Regen und Forstschäden: Eine Dokumentation. Essen

Gregor H-D (1986) Wissenschaftliches Symposium "Neue Ursachenhypothesen". Berlin

Hildebrand EE (2003) Neuartige Waldschäden - Realität oder Sturm im Wasserglas? AFZ-DerWald, H. 25, S. 1311-1313

Holzberger R (1995) Das sogenannte Waldsterben. Zur Karriere eines Klischees:

Das Thema Wald im journalistischen Diskurs. Eppe, Bergatreute

Horeis H (2005) Begrabt das Waldsterben! Novo, H. 11/12, S. 16-18

Horton P (1985) Saurer Regen. In: ZDF (Hrsg.) Mein Freund der Wald, Mainz (Schallplatte) 
Horx M (2007) Epidemien der Angst: Warum ich nicht an die Klimakatastrophe glaube. Die Welt, 14.03.2007, S. 3

Hüttl R (1998) Neuartige Waldschäden. In: Berlin-Brandenburgische Akademie der Wissenschaften (Hrsg.) Berichte und Abhandlungen, Band 5. Akademie Verlag, Berlin. S. 131-215

Ipos (Institut für praxisorientierte Sozialforschung) (1984) Meinungen zum Umweltschutz. Mannheim

Ipos (1985) Meinungen zum Umweltschutz. Mannheim

Kandler O (1994) Vierzehn Jahre Waldschadensdiskussion - Szenarien und Fakten. Naturwissenschaftl. Rundschau, H. 11, S. 419-430

Keil G (2004) Chronik einer Panik. Die ZEIT, 09.12.2004

Keil L-B, Kellerhoff SF (2006) Gerüchte machen Geschichte: Folgenreiche Falschmeldungen im 20. Jahrhundert. Links, Berlin

Kenneweg H (1997) Ist "Das Waldsterben" tot? Unser Wald, H. 1, S. 12-13

Kolb S (2005) Mediale Thematisierung in Zyklen. Theoretischer Entwurf und empirische Anwendung. Halem, Köln

Kommission für Ökologie der Bayerischen Akademie der Wissenschaften (2005) Stellungnahme der Kommission für Ökologie der Bayerischen Akademie der Wissenschaften zu den Waldzustandsberichten des Bundesministeriums für Verbraucherschutz, Ernährung und Landwirtschaft.

Lehmann A (1999) Von Menschen und Bäumen: Die Deutschen und ihr Wald. Rowohlt, Reinbek

Luhmann H-J (1992) Warum hat nicht der Sachverständigenrat für Umweltfragen, sondern der Spiegel das Waldsterben entdeckt? Jahrbuch Ökologie 1992. Beck, München. S. 292-307

Mackenthun G (1984) dpa-Zusammenstellung: Umweltschutz: Problem und Aufgabe der Gegenwart. Die Diskussion um Tempo 100 (2 Teile), 30.10 .84

Mannheimer Morgen (26.1.1985) Wissenschaftler: Kein Anlass zur Panik

Maxeiner D, Miersch M (1998) Lexikon der Ökoirrtümer: Überraschende Fakten zu Energie, Gentechnik, Gesundheit, Klima, Ozon, Wald und vielen anderen Umweltthemen. Eichborn, Frankfurt a.M.

Metzger B, Bemmann M, Schäfer R (2009) „Erst stirbt der Wald, dann stirbt der Mensch“: Was hatte das Waldsterben mit dem deutschen Waldmythos zu tun? [erscheint 2009 im Katalog zur Ausstellung „Mythos Wald“ des Landschaftsverbandes Westfalen-Lippe] 
Müller-Ullrich B (1996) Medienmärchen: Gesinnungstäter im Journalismus. Blessing, München

Müller E (1986) Innenwelt der Umweltpolitik: Sozial-liberale Umweltpolitik (Ohn)macht durch Organisation? Westdeutscher Verlag, Opladen

Newig J (2004) Symbolische Gesetzgebung: Umweltpolitik unter gesellschaftlichen Macht- und Informationssymmetrien. Zeitschrift für Politikwissenschaft, H. 3, S. $813-851$

Niemann HG (o.J. ca. 1983) Rumpelstilzchen - ein Aktionsplan. In:

Koordinationsbüro Waldsterben Hagen (Hrsg.) Den Wald retten. Arbeitsmappe, Hagen.

Nöthig Z (1986) Das Waldsterben : Literaturauswertung zum Stand der Kenntnisse und zu den Erklärungshypothesen. Diplomarbeit Institut für Kraftfahrwesen, Rheinisch-Westfälische Technische Hochschule Aachen.

Oesten G (2004) War da was? Über die Auswirkung der „,Waldsterbens“-Debatte auf die Forstwissenschaften im Allgemeinen und die Forstökonomik im Besonderen. In: Löwenstein W, Olschewski R et al. (Hrsg.) Perspektiven forstökonomischer Forschung. Sauerländer, Frankfurt a.M. S. 139-152. (Schriften zur Forstökonomie, Nr. 25)

ovb-online (2008) Umweltschutz durch Hightech.

http://www.ovb-online.de/news/landkreis_rosenheim/LK-Rosenheim-laquoDieUmweltschutz-durch-Hightech;art4107,1094730

zuletzt besucht am 10.12.2008

Otto K (2001) Thematisierungsstrategie in den Massenmedien und ihre Auswirkung auf die Bevölkerung und die politischen Entscheidungsträger. Thematisierung und Deutung des Problems "Waldsterben" in den achtziger Jahren. Dissertation, Universität Dortmund

Prittwitz V von (1990) Das Katastrophenparadox. Elemente einer Theorie der Umweltpolitik. Leske+Budrich, Opladen

Rauchhaupt U von (2007) Ist es nicht schon zu spät? Frankfurter Allgemeine Sonntagszeitung, 03.12.2007

Redaktion Kölner VolksBlatt und Katalyse Umweltgruppe Köln e.V. (1982) Saurer Regen - Ursachen, Folgen, Gegenmaßnahmen. Verlag Kölner VolksBlatt, Köln

Rehfuess KE (1990) Übersicht über den Stand der Waldschadensforschung unter besonderer Berücksichtigung bodenkundlicher Forschungsansätze. In: Weiß JP (Hrsg.) Die Entwicklung der Waldschäden. Wilhelm-Münker-Stiftung, Siegen. S. 36-60 (Beiträge zur Lebensqualität, Walderhaltung und Umweltschutz, Volksgesundheit, Wandern und Heimatschutz, Nr. 30) 
Robin Wood (2008) ROBIN WOOD zum Thema Waldsterben. http://www.robinwood.de/german/wald/waldsterben/index.htm zuletzt besucht am 10.12.2008

Schnabel U (2008) Die Konjunktur der Ängste. Die ZEIT, Nr. 26, 19.6.2008, S. 31-32

Neuweiler G (1983) Waldsterben - darf die Wissenschaft schweigen? Um-bits, H. 5, S. 1-4

Schütt P, Summerer H (1983) Waldsterben-Symptome an Buche.

Forstwissenschaftliches Centralblatt, S. 201-206

Schwarzwälder Bote (20.10.1986) Transparente sorgen für Ärger

SRU (Der Rat von Sachverständigen für Umweltfragen) (1983) Waldschäden und Luftverunreinigungen. Kohlhammer, Stuttgart/Mainz

SRU (Der Rat von Sachverständigen für Umweltfragen) (2000) Umweltgutachten 2000: Schritte ins nächste Jahrtausend. Bundestags-Drucksache 14/3363

Süddeutsche Zeitung (15.11.1980) Der Nadelwald steht unter Streß

Süddeutsche Zeitung (12.11.1984) Der große Protest gegen das Waldsterben

Süddeutsche Zeitung (5.2.1985) Zweifel an Ursachen des Waldsterbens

Süddeutsche Zeitung (6./7.10.2007) „Stromsparen wird in Mode kommen“. S. 24

SWR2 (8.9.2008) SWR2 Impuls: Weltuntergänge - und was aus ihnen wurde (1):

Das Waldsterben. (Radiosendung)

UBA (Umweltbundesamt) (1978) Medizinische, biologische und ökologische Grundlagen zur Bewertung schädlicher Luftverunreinigungen:

Sachverständigenanhörung, 20. bis 24.2.1978, Berlin

UBA u. BMU (Umweltbundesamt und Bundesministerium für Umwelt, Naturschutz und Reaktorsicherheit) (1997) Auswertung der

Waldschadensforschungsergebnisse (1982-1992) zur Aufklärung komplexer Ursache-Wirkungsbeziehungen mit Hilfe systemanalytischer Methoden. Erich Schmidt Verlag, Berlin

Ulrich B (1982) Die Versauerung - Giftstoffe reichern sich an. Bild der Wissenschaft, H. 12, S. 108-119

Ulrich B (1987) Zum Stand der Waldschadensdiskussion: Stellungnahme zu Presseverlautbarungen beim Internationalen Botaniker-Kongreß. Allgemeine Forstzeitschrift, H. 38, S. 973-975 
Ulrich B (1995) The history and possible causes of forest decline in central Europe, with particular attention to the German situation. Environmental Reviews, H. 3/4, S. 262-276

Ulrich B (1997a) Ökosystem Wald - Funktion und Nutzen. In: Haury H-J, Aßmann G, et al. (Hrsg.) Patient Wald - sterbenskrank oder kerngesund? GSF

- Forschungszentrum für Umwelt und Gesundheit, Neuherberg, S. 1-11 (Journalistenseminar der Information Umwelt, Nr. 20)

Ulrich B (1997b) Waldschäden - Problematik und neue Erkenntnisse. Unser Wald, H. 1, S. 14-16

Ulrich B, Matzner E (1983) Ökosystemare Wirkungsketten beim Wald- und Baumsterben. Forst- und Holzwirt, H. 18, S. 468-474

Ulrich B, Mayer R, Khanna PK (1979) Deposition von Luftverunreinigungen und ihre Auswirkungen in Waldökosystemen im Solling. Sauerländer's, Frankfurt a.M.

VEBA (1984) Brief des Betriebsrats der VEBA Kraftwerke Ruhr AG an die SPD BT-Fraktion am 27.7.84. Archiviert in: Archiv der Sozialen Demokratie der Friedrich-Ebert-Stiftung, Bestand SPD-Fraktion, Ordner 2/BTFJ 0000042

Vestreng V, Støren E (2000) Analysis of UNECE/EMEP Emission Data: MSC-W Status Report 2000. EMEP/MSC-W Note 1/00

Waldportal (2007) Glos gegen „Katastrophenszenarien“ bei Klimawandel http://www.waldportal.org/allgemein/news.allgemein2007/news.allg.2007042 4/print.html zuletzt besucht am 10.12.2008

Wentzel K-F (1982) Ursachen des Waldsterbens in Mitteleuropa. Allgemeine Forstzeitschrift, H. 45, S. 1365-1368

Wentzel K-F (2001) Was bleibt vom Waldsterben? Bilanz und Denkanstöße zur

Neubewertung der derzeitigen Reaktion der Wälder auf Luftschadstoffe.

HochschulVerlag, Hamburg

Wentzel K-F (2008) Forstpolitischer Rückblick auf das Waldsterben-Debakel 1980/90. proWALD, H. 5, S. 30-32

Westfälische Rundschau (04.09.2008) Clement greift SPD-Führung an

Wetstone GS, Rosencranz A (1985) Weltbedrohung Saurer Regen.

Abwehrversuche in Europa und Nordamerika; eine Studie des Environmental Law Institute f.d. German Marshall Fund of the United States. Dreisam Verlag, Freiburg

Wiener Zeitung (16.04.2008) „Rasch und entschieden handeln“ 
Wirsching A (2006) Abschied vom Provisorium: 1982 - 1990. Deutsche VerlagsAnstalt, München

Wislicenus H (1985) Waldsterben im 19. Jahrhundert. VDI-Verlag, Düsseldorf

Zierhofer W (1998) Umweltforschung und Öffentlichkeit: Das Waldsterben und die kommunikativen Leistungen von Wissenschaft und Massenmedien. Westdeutscher Verlag, Opladen/Wiesbaden

Zöttl HW (1995) Waldschäden und Ursachen. Forst und Holz, H. 2, S. 54-57 



\title{
Was macht eigentlich das Geschwindigkeitslimit?
}

\author{
Kurt Möser
}

Die Debatte um Begrenzung und Entgrenzung der Geschwindigkeit von Motorfahrzeugen ist mindestens so alt wie diese Fahrzeuge selbst. Sie bildet eine Parallelgeschichte zur Geschichte der Motorfahrzeuge und ist ein integraler Teil der Geschichte der Mobilität. Geschwindigkeitslimits für Fahrzeuge sind aber älter als das Automobil, älter auch als die Reaktionen auf die Sattelzeit der Individualmobilität um 1880. Nach der „Kalmierung“ der Reiter und einer Entschleunigung der Kutschen (Köppen) war es dann insbesondere das „Herunterbremsen“ der mechanischen Mobilitätsmaschine Fahrrad auf das Geschwindigkeitsregime des restlichen Verkehrs, das den argumentativen Trend und die Konfrontationsstrukturen der späteren Geschwindigkeitsdebatten bestimmte. Denn das Fahrrad, als biogen betriebene Mobilitätsmaschine, hatte ein beträchtlich höheres Geschwindigkeitspotential als die ebenfalls biogenen Kraftmaschinen des Straßenverkehrssystems. Konsequenterweise war man bestrebt, diese zum Maßstab zu machen und die systemstörende Geschwindigkeit einzuhegen.

Die Erfahrungen im Umgang mit der früher eingeführten Mobilitätsmaschine und deren Disziplinierung wurden nun für das Automobil aktiviert. So durften die neuen Mobilitätsmaschinen, betrieben mit ,elementarer Kraft“, wie es in den badischen Verkehrsvorschriften hieß, nicht die Geschwindigkeit eines „mäßig trabenden Pferdes“ überschreiten (Möser 2002). Der erste Maßstab der Begrenzung war kein abstraktes Geschwindigkeitsmaß, sondern ein an den biogenen „Motoren“ orientiertes. Abstrakte, quantifizierende Messungen folgten in einem weiteren Schritt. Davor allerdings entstand die Notwendigkeit eines „Verstehens“ oder „Empfindens“ der gefahrenen Geschwindigkeiten. 
Im kollektiven Umgang mit den Geschwindigkeiten der Motorisierung ging es einmal um Anerziehung und Übung des „Geschwindigkeitssinns“; und schließlich um objektive Messung. Das waren zwei zunächst getrennte Zielrichtungen: einmal sollte die neue „Geschwindigkeitselite“ ein Geschwindigkeitsgefühl entwickeln. Genau das mussten aber auch die nichtmotorisierten Beobachter von außen: Die Disziplinierer und Überwacher, also konkret, die „Organe“ der Polizeibehörden, mussten Geschwindigkeit zuverlässig schätzen lernen, obwohl sie keine unmittelbaren teilnehmenden Erfahrungen besaßen (Dorfpolizisten fuhren bis in die 1950er Jahre in Deutschland allenfalls Rad). Frühe Geschwindigkeitsübertretungen wurden in der Regel durch Zeugenaussagen von Polizeibeamten bestätigt, die Fälle von nicht weiter quantifiziertem „Rasen“ anzeigten. Geahndet wurden sie von meistens ebenso unmotorisierten Justizbeamten. Dabei gab es in der Regel einen konfliktbelasteten Widerspruch der zwei Geschwindigkeitsperzeptionen, derjenigen des Fahrers und die der Instanzen des Überwachens und Strafens.

Die Notwendigkeit, Geschwindigkeit objektiv messbar zu machen, war evident. Zunehmend wurden Geschwindigkeitsmesser eingebaut, und Fahrer lernten sie zu benutzen. Tachometer wurden dann mit „reiferer“ Motorisierung zu Pflichtinstrumenten in Motorfahrzeugen. Diese Geräte sind die materiellen Substrate der Objektivierung des „Geschwindigkeitssinns“, der sich mit den Messdaten abzugleichen und damit zu präzisieren hatte. Ihr Gegenstück bekam diese Formierung des „Geschwindigkeitssinnes“ auf der Seite der Kontrolle: Die subjektiven Geschwindigkeitseindrücke der Polizisten wurden ersetzt durch Objektivierung und Messung. Das konnte zunächst recht einfach erfolgen, durch die gestoppte oder nur geschätzte Zeit für das Durchfahren einer fixen Strecke. Die Technisierung der Geschwindigkeitsüberwachung wurde im Verlauf der Mobilitätsgeschichte immer weiter verfeinert: durch impulsgebende Messkabel, Lichtschranken, Radiomessung (Radar), Dopplerradar, Infrarotgeräte und Laser.

Mit der Messbarmachung und Objektivierung von Straßengeschwindigkeit einher ging eine zunehmende Transparenz der lokalen Geschwindigkeitsregeln. Während es anfangs nur erforderlich war, dass eine Veröffentlichung der Limits in dem jeweiligen Gesetzesblatt erfolgte, oder durch Aushänge, gab es teilweise auch Tafeln an den Verwaltungsgrenzen. Einheitliche Regelungen waren durch die Reste der Kleinstaaterei im Deutschen Reich behindert. Eine konkrete Beschilderung von Geschwindigkeitslimits auf der Straße - und zwar nicht eine solche zur Temporeduktion von Gefahrenstellen, sondern von generellen Limits - setzte sich erst in der Zwischenkriegszeit durch. Davor waren regionale Unterschiede üblich. Fahrer, die bei Fernfahrten den Geltungsbereich ganz unterschiedlicher „Fahrordnungen“ durchmaßen, warfen den Kontrollbehörden vor, „Autofallen“ aufzustellen und „Willkür“ auszuüben - ein Vorwurf, der sich durch die Geschichte der staatlichen Geschwindigkeitsüberwachung des Straßenverkehrs zieht (Merki 2002). Die Antwort der klaren Limits und der Objektivierbarkeit der Messung wurde nicht wirklich als Problemlösung empfunden. Ebenso wenig begrüßt wurde eine Vereinheitlichung der vielen unterschiedlichen Geschwindigkeitsregelungen: 1910 
wird die höchstzulässige Geschwindigkeit innerorts erstmals reichseinheitlich geregelt. In geschlossenen Ortsteilen beträgt sie $15 \mathrm{~km} / \mathrm{h}$ für PKW (Seherr-Thoss 1986, 76).

Die Spannung zwischen dem älteren Muster der Orientierung am biogenen Verkehr, der staatlichen Regulierung und des „freiheits“-suchenden Geschwindigkeitspotentials der Motorisierung eskalierte schon früh. Carl Benz erzählt in seiner Autobiographie, wie sein „Fahrmeister“ T(h)um zwei hohe badische Regierungsbeamte vom Bahnhof abholte. Er hielt sich, von Benz strikt angewiesen, an die in Mannheim geltenden Geschwindigkeitsregeln von $6 \mathrm{~km} / \mathrm{h}$ inner- und $12 \mathrm{~km} / \mathrm{h}$ außerorts. Die Beamten fragten Tum, ob er nicht schneller fahren könne. ,'Können tu ich's schon', sagte der Mann am Steuer, ,aber ich darf nicht, es ist polizeilich verboten.' ,Ei was, fahren Sie einmal zu, sonst fährt uns ja jede Milchkutsch' vor'.“ (Benz 1925,88).

Carl Benz' Geschichte beschreibt eine Standardsituation des Spiels zwischen Geschwindigkeitspotential und regulierender Limitierung dieses Potentials, zwischen individuellen Mobilitätsmöglichkeiten und staatlich-autoritären Regulativen, zwischen Freiheitsanspruch des mobilen Individuums und behördlicher Fürsorge für das Allgemeine, in diesem Fall für das Gesamt-Verkehrssystem. Die Ironie besteht darin, dass die Behördenvertreter in dem Moment, indem sie selber zu motorisierten Verkehrsteilnehmern werden, ihre Rollen wechseln und das Interesse an der Aufhebung von Limits vertreten. Benz kommentiert, dass damit die „Freizügigkeit des Motorwagens“ nicht mehr an die ,engherzigen Geschwindigkeitsvorschriften einer veralteten Kutscherepoche" gebunden sei (Benz 1925, 88), wobei er auch den modernisierenden Effekt der neuen technogenen Geschwindigkeit ins Spiel brachte.

Das Argument für die Limitierung von Geschwindigkeit war aber von Anfang an ein Sicherheitsargument: Es sollten die übrigen Nutzer der Straßen und Verkehrsflächen vor den Geschwindigkeits-Eindringlingen geschützt werden, zum anderen aber auch die „Geschwindigkeitspioniere“ untereinander. Schon „rasende“ Kutschen, oder Radfahrer, die sich und untereinander Rennen lieferten, gefährdeten nicht nur für die übrigen Verkehrsteilnehmer, sondern auch sich selber. Immer ging es darum, dass sich alle Systemteilnehmer aus Sicherheitsgründen in das Mobilitätssystem einfügen mussten. Die Spannung zwischen dem Potential individueller, sich als un- oder antisystemisch verstehender Mobilität und den inhärenten, funktional motivierten Zwängen des real existierenden Mobilitätssystems war damit gegeben.

Die Besitzer von Automobilen empfanden die Versuche der forcierten Systemeinpassung nicht nur als Beschränkung der freien Nutzung ihrer Mobilitätsmaschinen, sondern auch als Parteinahme des Staates für die NichtAutomobilisten. Und da Motorfahrzeuge zunächst Fahrzeuge der oberen Klassen waren, trug der Streit um die Geschwindigkeit und die staatlichen Anstrengungen zur Disziplinierung von Anfang an auch einen Klassenauseinandersetzungscharakter: Die Automobilisten, die sich selber als Angehörige der staatstragenden Schicht 
sahen, empfanden die Parteinahme der Autoritäten für die unmotorisierten HaveNots des Verkehrs als parteiisch, unfair, und als durchaus äquivalent zu den Sozialistengesetzen (Möser 2004).

Insbesondere der Streit um die Haftpflichtversicherung und anderer staatlicher Regelungsmaßnahmen, die um das Jahr 1906 zu einer umfassenden Verregelung und Verrechtlichung der motorisierten Komponente des Straßenverkehrs führte, wurde von des Automobilisten so interpretiert. Vom großen Rest der Verkehrsteilnehmer aus gesehen, schienen jedoch gerade die gefährlichen, rücksichtslosen, unfallverursachenden und arroganten „Autler“ einen Klassenkampf von oben auf der Straße zu führen (Möser 2002). Im zeitgenössischen Kontext wurde die Auseinandersetzung um den auf die Geschwindigkeit fokussierten Automobilismus empfunden und dargestellt als Kampf zwischen modernen und alten Mobilitätsformen. Im Kern ging es um einen durchaus auch politisch verorteten Hierarchieanspruch der technosozial fortschrittlicheren Mobilitätsmaschine, um einen Machtkampf zwischen Mobilitätshierarchien.

So lange es Geschwindigkeitsbegrenzungen und deren Durchsetzung gab, wurden sie offenbar symbolisch aufgeladen, und zum Aktionsfeld sozialer und politischer Gegensätze. Sie konnten sowohl die gegensätzlichen Haltungen gesellschaftlich Agierender zur Motorisierung betreffen wie auch zur technischindustriellen Moderne insgesamt. Und sie konnten zum Indikator werden nicht nur für den Kontrast des erstrebenswerten Neuen zum vormodernen Alten, wie dies Benz formulierte, sondern gerade auch für die Stellung zum Verhältnis von Staat und Individuum. Disziplinierungsanstrengungen mit dem Anspruch des Gemeinwohls und des Schutzes der Individuen vor dem Missbrauch ihrer Freiheiten stand damit einem Anspruch auf Selbstverantwortung und Freiheit des Individuums entgegen (Möser 2004). Verkürzt kann man sagen, dass der alte Gegensatz zwischen autoritärem und liberalem Staat auf dem symbolisch-kulturellen Spielfeld der Freiheit auf den Straßen sich austrug, wobei die älteren politischen Grenzen zwischen „progressiv" und „konservativ“" sich durchaus verschoben.

Während dieses Konfrontationsmuster in den ersten Jahrzehnten der Motorisierung in Deutschland sich wenig wandelte, erfolgte eine Zäsur in der staatlichen Haltung zur Motorisierung und also auch im automobilen Geschwindigkeitsregime im Jahr 1933. Die bis dahin starke Regulierungsbetonung wurde umgekehrt, und die Geschwindigkeitslimits wurden abgeschafft. Die Freigabe der Geschwindigkeit nach 1933 gehört in den großen Komplex eines umfassenden systemischen Zugriffs der Nationalsozialisten auf den Straßenverkehr, der kaum eine Systemkomponente unberührt ließ. Abbau von Geschwindigkeitsbeschränkungen war eine der Ebenen der Motorisierungsförderung der Nationalsozialisten, neben der Einführung des „Wagens für das Volk“, den Nur-Autostraßen-Bau, die Steuerbefreiung für Neufahrzeuge, die Erleichterung des Führerscheinerwerbs, das Primat von Motorfahrzeugen in einer neuen Straßenverkehrsordnung, oder das breite Ausbildungsprogramm durch NS-Organisationen (Kopper 1995). Die konkrete wie auch symbolisch-politische Bevorzugung und Förderung des Automobils sollte 
paradoxerweise die Regulation des Individualverkehrsmittels durch den Staat reduzieren: Der NS-Staat reorganisierte das Verkehrssystem zugunsten der motorisierten Individualität. Eine der Konsequenzen bestand darin, dass die Abschaffung der Geschwindigkeitsbeschränkungen auf die individuelle Verantwortung des motorisierten Verkehrsteilnehmers setzen musste.

Auch wenn flankierend zur Geschwindigkeitsfreigabe Appelle an das Verantwortungsgefühl für die „Volksgemeinschaft“ erfolgten, ist es doch bemerkenswert, und interpretationsbedürftig, dass die NSDAP beim Auto auf zwang-lose Maßnahmen baute, auf individuelles Handeln. Das scheint kontraintuitiv: Ausgerechnet auf dem Feld der Motorisierung verzichtete sie auf staatliche Reglementierung und Autoritätsausübung. Hierarchie, Autorität und Kontrolle wurden, im Gegensatz zum gesamten gesellschaftspolitischen Trend des Nationalsozialismus, im Straßenverkehr gerade abgelehnt. Gerade auch Hitler war der Exponent einer Ablehnung der Geschwindigkeitskontrollen im Straßenverkehr. Er schloss sich damit dem schon vorher sozial wirksamen Trend einer kleinbürgerlichen, vielleicht auch rechts-anarchischen, jedenfalls aber motorisierungsenthusiastischen Opposition gegen staatliche Eingriffe im Straßenverkehr an.

Die schließliche Wiedereinführung von Limits 1938 erfolgte nicht aus politischen Gründen, etwa durch einen Wandel der Haltung zum Straßenverkehr. Ursache war vielmehr die NS-Kriegsvorbereitungspolitik. Schon zuvor war eine treibstoff-fressende Individualmotorisierung in (gar nicht so latentem) Widerspruch zum Streben nach energetischer Autarkie und zur Präferenz der militärischen Technik über zivilem Konsum gestanden. Mit der forcierten Aufrüstung und entsprechender Ressourcenknappheit, und auch mit dem sich klar abzeichnenden Fehlschlag einer Energiepolitik, die auf heimische Rohstoffe und neue technische Verfahren zur Treibstoffherstellung gesetzt hatte, waren Restriktionen des Privatverkehrs offenbar unumgänglich geworden. Zudem stiegen - und dies war die zweite Ursache für die Wiedereinführung von Limits - die Unfallzahlen außerordentlich an. Programme und Appelle an Fahrer, Jugendverkehrserziehungsmaßnahmen und striktere Disziplinierung waren ebenso erfolglos wie disziplinarische Initiativen insbesondere des NSKK. Mit der (mindestens für Hitler selber) contre ceur- Wiedereinführung von Tempolimits hatte sich 1938 die autoritätsferne Politik gegenüber dem Automobilisten umgekehrt. Nach Kriegsbeginn setzte sich eine somit schon vorbereitete restriktivere Individualmotorisierungspolitik fort und führte zu weiteren strikten Limitierungen des Autoverkehrs.

Während der scheinbaren Normalisierung des Straßenverkehrs nach der Gründung der Bundesrepublik setzte man zunächst wiederum auf eine Freigabe der Geschwindigkeit. Die verkehrspolitische Haltung zur Höchstgeschwindigkeit kann durchaus als Apercu zur generellen Debatte um Kontinuitäten vor und nach 1945 dienen. In der frühen Bundesrepublik wurde nämlich mit dem Argument der individuellen Verantwortung des einzelnen Fahrers, die den Grundsätzen eines freiheitlichen und demokratischen Staates genau entsprechen sollte, auf ein Limit verzichtet. Von der früheren, von vielen Fahrern noch erlebten Geschichte der 
Freigabe von 1933 war allerdings überhaupt nicht die Rede. Im Gegenteil: In der frühen Bundesrepublik war die Geschwindigkeitsdebatte in die Abgrenzung von den beiden totalitären Regimen eingebunden: der Distanz zum Nationalsozialismus und zu den sozialistischen Staaten. Beide wurden portraitiert als Regimes, die ihre undemokratische, autoritäre Kontroll-Lust gerade im Straßenverkehr ausagierten. Das Image eines befreiten Straßenverkehrs wurde so schon früh mit der neuen demokratischen Identität der Bundesrepublik verknüpft. Der ADAC verlieh konsequenterweise dem späteren Tempolimitgesetz das „Odium eines ,Nazigesetzes““ (Klenke 1993, 97). Dass dies ein sehr schiefes und ahistorisches Bild war, spielte für die Genese der Images keine Rolle.

Die Opposition gegen Geschwindigkeitsbegrenzungen in der Frühphase der Bundesrepublik richtete sich zudem noch -- wenn auch weniger explizit -- gegen eine harsche Regelungsinstanz, nämlich gegen die alliierten Besatzer. Insbesondere die Amerikaner führten massiv Kontrollen in ihrer Besatzungszone durch, und dies neben und mitunter gegen die westdeutschen Behörden (Klenke 1993, 97). Die Besatzer waren aber nun aber kaum des Totalitarismus zu verdächtigen. Aber auch hier hatte eine Betonung der „freien Fahrt“ identitätsschaffende Funktionen: Eine Abkehr vom strikten US-Geschwindigkeitsregime auf deutschem Boden schien in symbolischem Konnex mit dem Ende der Besatzungszeit.

Ungeregelter Straßenverkehr - allerdings nur für ,freie Bürger“ im PKW, nicht für abhängig Beschäftigte, etwa für LKW- und Busfahrer - wurde schließlich repräsentativ für den Widerstand gegen politische Entmündigung und als Feld für die politisch angestrebte Erziehung des Bundesbürgers zum eigenverantworteten Handeln (Klenke 1993, 102; Südbeck 1994). Spätestens nach den Geschwindigkeitsdebatten der 1970er Jahre, wohl aber schon in den Debatten der frühen 1950er etablierte sich eine enge Verbindung von „staatsbürgerlichen Persönlichkeitsideale(n) der Selbstverantwortung und Freiheit“" (Klenke 1993, 94) mit der individuellen Mobilität, und mit dem Artefakt Automobil als Bedeutungsträger gerade auch für die junge westdeutsche Demokratie. Auch die Korrelation von individuell verfügbarer Geschwindigkeit und Moderne, die schon Benz betont hatte, wurde immer wieder aufgerufen. Der wirtschaftlichen und sozialen Rolle, die dem Automobil zuwuchs oder zugeschrieben wurde, entsprach also die politischedukative. Geschwindigkeitsbegrenzungen hatten hier nichts zu suchen.

Mit der Aufladung der fahrerischen Freiheit bildete sich auch ein sozial wirksames symbolisches Feld aus, das besonders im Tagesjournalismus und in der Rhetorik der Parteienauseinandersetzung heftig ausgebeutet wurde. „Dieses Volk muss bei der nächsten Wahl klar entscheiden, wohin es fahren will: Mit dem Auto in die Wohlstandsgesellschaft oder mit dem Bus in den Wohlfahrtsstaat", so der BildChefredakteur Peter Boenisch 1974 (Andersen 1997, 174). Das latent Anarchische solcher Positionen war nicht auf Deutschland beschränkt; Joe Moran spricht von einem „resilient Tory anarchism of the road“ (Moran 2008), der bis heute die politische Auseinandersetzung um die Verkehrspolitik in Großbritannien bestimme. 
Ein schiefes Bild ergäbe sich aber, wenn man für die Bundesrepublik einen generellen Gegensatz von Regelungslust von oben und deren Ablehnung von unten konstruieren würde. Denn auch ganz oben war laissez-faire in Mode. Bezeichnenderweise erklärte in einer frühen Debatte zum Fall des Tempolimits der Bundesverkehrsminister Seebohm eine regelnde Gesetzesinitiative für völlig überflüssig (Klenke 1993, 92). In dieser Debatte gab auch der BDI-Ausschuss seine Meinung ab, mit einer Begründungsstruktur, die, nach Klenke, als „argumentative Waffe“ Geschichte machte, weil sie einen Begründungstrend setzte, der die Debatten bis heute bestimme. Danach lag die Ursache der außerordentlichen Gefährdung im Straßenverkehr nicht im Fehlverhalten der Fahrer, sondern in wenig, geschwindigkeitsuntauglichen' Straßen und in gravierenden Defiziten der Verkehrinfrastruktur und der staatlich bestimmten Verkehrsorganisation. Zudem sollten nach dieser Argumentation Pauschalvorschriften, wozu besonders die undifferenzierten Tempolimits gehörten, die Eigenverantwortlichkeit hindern und das Streben nach situationsgerechter und ,angepasster“ Fahrweise substituieren, wodurch die Sicherheit eher reduziert würde.

Ein biologistisches Argument flankierte dieses nicht undialektische Argument: dass nämlich ein gleichmäßig fließender, eher langsamer Straßenverkehr zu gefährlicher Ermüdung und Unaufmerksamkeit führen sollte, oder aber auch zu gefährlichen, aber dann doch durchaus verständnisvoll bewerteten Ausbruchsversuchen gegen dergleichen unverständliche Geschwindigkeitsgleichmacherei. Dass Aufregung als aufmerksamkeitsgenerierend gilt, und Adrenalinausstoß als sicherheitsfördernd, ist auch heute durchwegs noch zu hören.

Die symbolische Rolle der Geschwindigkeitsbegrenzung als demokratischer Freiheits-Prüfstein spielte auch in der bundesrepublikanischen ParteienAuseinandersetzung eine Rolle. Sich als liberal verstehende Parteien favorisierten eine Geschwindigkeitsfreigabe, mit dem Argument der Selbstverantwortung und Bürgerfreiheit, während Regelungsanstrengungen als Ausfluss sozialistischautoritärer-staatsdominierender Haltungen dargestellt werden konnten. Konkret erwies sich die Verkehrspolitik der liberalen Partei, der FDP, als durchwegs kontroll- und damit limitskeptisch und traf damit die generelle Stimmung des fahrenden Wahlvolks weit eher als die Sozialdemokratie, deren Staatsorientierung gerade auch an ihrer Neigung zu Geschwindigkeitslimits festgemacht und damit politisch kritisiert werden konnte.

Die schließliche Einführung von Geschwindigkeitslimits in der Bundesrepublik war zunächst motiviert durch die steigenden Unfallzahlen mit ihren „Opfern“ von Toten und Verletzten, die sogar in einer Gesellschaft, die eine bemerkenswerte und bislang von Mobilitätshistorikern nicht befriedigend erklärte Toleranz gegen den Straßentod besaß, nicht hinnehmbare Dimensionen annahmen. Die Kulmination der „Verluste“ war im Jahr 1970, in dem es 21.000 Tote auf westdeutschen Straßen gab. Die Entwicklung stagnierte in den Jahren danach auf diesem hohen Niveau, und traf schließlich mit einer Ressourcenkrise zusammen. Beide Argumente für 
strikte Begrenzungen, Sicherheits- wie Spritsparaspekte, kamen nach 1970 zusammen und führten zu einer erneuten Restriktionsphase.

Denn mit der ersten Öl(preis)krise trat ein weiteres Argument, das des Ressourcenverbrauchs, in den Vordergrund (Hohensee 1994). Es hatte aber schon 1938 im NS eine Rolle gespielt hatte. Langsameres Fahren wurde nun als effizientes Mittel zum Spritsparen gesehen, das ohne technische Eingriffe und ohne staatliche Investitionen (einmal abgesehen von Kontrollmaßnahmen) kurzfristig wirksam werden konnte. $\mathrm{Zu}$ dem Bündel recht radikaler treibstoffsparender Maßnahmen, mit denen der Ölkrise begegnet wurde, gehörten neben den Fahrverbotssonntagen zwischen 25. November und 16. Dezember 1973 strikte Geschwindigkeitslimits von $80 \mathrm{~km} / \mathrm{h}$ auf Landstraßen und $100 \mathrm{~km} / \mathrm{h}$ auf Autobahnen.

Die Aufhebung dieser Geschwindigkeitsbegrenzung zum 14. März 1974, nach dem scheinbaren und vorläufigen Ende der Ressourcenkrise, war das Ergebnis einer der signifikantesten Debatten um Tempolimits. Von den Kritikern der Maßnahmen wurde nicht nur die systemische Wirksamkeit bezweifelt, also der Spareffekt relativiert, sondern auch politische Argumente wurden wiederum mobilisiert. Der notorische Slogan „Freie Fahrt für freie Bürger“ entstand in dieser Phase der Debatte. Geprägt wurde er vom ADAC, dessen Kampagne, in Verbindung mit Vertretern der Autoindustrie und mit zahlreichen limitskeptischen Artikulationen „von unten“, schließlich zur Aufhebung bzw. Modifikation der harsch scheinenden Limits führte. Eine Geschwindigkeitsgrenze von $120 \mathrm{~km} / \mathrm{h}$ wurde schließlich geblockt. Dies geschah in einem komplexen, für die Verkehrspolitik der Bundesrepublik sehr typischen Prozess der Hearings, der Abstimmungen, der Aktionen und Äußerungen von Interessengruppen im Interesse einer vorgeblichen „Kompromissfindung“. Typisch dafür war ein „Hearing“ am 26. Februar 1974: Gegen $120 \mathrm{~km} / \mathrm{h}$ waren die CDU/CSU-Fraktion, VDA, ADAC, DTC, ZAV, DVR. Die eingebrachte Gesetzesvorlage für $120 \mathrm{~km} / \mathrm{h}$ wurde schließlich am 16.3.1974 vom Bundesrat zu Fall gebracht (Seherr-Thoss 1986, 628).

Am Ende stand ein Verzicht auf ein generelles Limit - und dies bezeichnenderweise gegen den politischen Ressortleiter, Bundesverkehrsminister Lauritzen, der für eine Beibehaltung der krisengenerierten Regulierung war. Ein weiteres als Kompromiss verstandenes Ergebnis war, neben der Deklaration der noch bestehenden Limitierung als befristeter „Großversuch“, die Einführung einer „Richtgeschwindigkeit“ von $130 \mathrm{~km} / \mathrm{h}$. Die „Richtgeschwindigkeit“ war ein bis heute geltendes, jetzt kaum mehr wahrgenommenes merkwürdiges Konstrukt zwischen funktionalen Einsichten und politischer Durchsetzbarkeit, zwischen Akzeptanz einer Grenze problematischer Geschwindigkeitseffekte und der Balance zwischen Regulierung und Freiwilligkeit der Nutzer, bei dessen Entwurf klar regulierungsskeptische Positionen dominierten.

In dieser wichtigen Phase der Debatten stabilisierten sich auch die Positionen und Argumente der Limitgegner, die bis heute topisch vorgebracht werden: einmal ein industriepolitisches Argument, nämlich der positive Effekt unlimitiert hoher 
Geschwindigkeiten auf die deutsche Automobilindustrie, und hier besonders auf die Verkäufe im oberen „Klassen“-Segment. Die Möglichkeit schnellen Fahrens für alle erscheint so als indirekte symbolische Subventionierung deutscher Autobauer. Zweitens taucht das Argument immer wieder auf, dass die nicht-regulierten Strecken sowieso unbedeutend seien, drittens dass die absolute Geschwindigkeit sowieso nichts über die Gefährdung aussage, und die Autobahnen mit ihrem Geschwindigkeitsregime die inhärent sichersten Straßengattung sei. Viertens sei neben dem Sicherheits- auch der Ressourcenspareffekt von Limits marginal. Fünftens soll der technische Fortschritt pauschale Limits überflüssig zu machen; den Sicherheits- und Ressourcenschonungs-Effekten von Maßnahmen wie sparsamen Motortechnologien oder fahrzeugbezogener Sicherheitstechnik wird eine viel größere Wirksamkeit zugeschrieben als allen Geschwindigkeitsreduzierungen. Das, was Limits leisten können, wird automobiltechnischen Innovationen aufgegeben kurz: wo Regulierung ist, soll Technik werden.

Die deutsche Politik im vereinigten Europa ist in einem double bind: Einerseits müsste, gemäß der Forderungen nach einem vereinigten Europa, auch Deutschland seinen Sonderweg im Straßenverkehr beenden. Andererseits will man unpopuläre Geschwindigkeitslimits, deren Diffamierung sich über Jahrzehnte stabilisierte, auch um den Preis von anti-europäischer Verkehrspolitik vermeiden. In der Alleinstellung Deutschlands im durchwegs geschwindigkeitsgeregelten Verkehr des vereinigten Europa muss nun aber - ein sechstes Argument - nicht zwingend Homogenisierungsbedarf gesehen werden. Vielmehr kann die deutsche Geschwindigkeitsfreigabe als anti-bürokratisches Pionierfeld innerhalb eines übermäßig geregelten Europa gesehen werden. Im Hinblick auf Tempolimits bekommt so Deutschland die Funktion eines Praeceptor Europae.

Diese von Industrie und Lobbyverbänden vorgegebenen und in öffentlichen Debatten stabilisierten Argumente erfahren nach wie vor eine breite, wenngleich leicht schwindende Unterstützung „,von unten“, von den fahrenden und beifahrenden Bürgern. Argumente von Fachleuten der Verkehrs-, vor allem aber der Umweltbehörden der Länder und es Bundes werden kaum ernsthaft diskutiert. Ein relativ neues Argument, das eng mit den Zumutungen des modernen Massenverkehrs gekoppelt ist, bezieht sich auf eine, weiche' Wirkung eines Limits: dass nämlich die Geschwindigkeitsdifferenzen und damit vor allem die Ursachen für Aggressivität und kompetitives Verhalten der Verkehrsteilnehmer reduziert werden könnten, und dass damit eine indirekte Erhöhung von Sicherheit erzielt würde.

Nachdem alle Argumente längst ausgetauscht wurden, und das in Repetition, scheint es inzwischen kaum mehr um die vernünftige Begründung und den Austausch von Positionen zu gehen. Die Haltung zum Limit in der Auseinandersetzung um die Funktion des Privatautomobils ist zur Gretchenfrage geworden, zum handlichen Prüfstein für die Positionierung als Autogegner oder Autobefürworter - und dies wiederum stellvertretend für die Haltung zur Industriegesellschaft generell. Bezeichnenderweise wurden um 1970 Kapitalismus- mit Automobilkritik verbunden und damit Debatten um industrielle Verschwörungen und Indoktrinierung 
automobiler Konsumenten in Gang gebracht (Krämer-Badoni u.a. 1971). Die Akzeptanz oder Ablehnung der Forderung nach einem generellen Geschwindigkeitslimit ist damit ein Indikator für die jeweiligen ideologischen Zuordnungen und Identifikationen „für" und „gegen“ das Automobil, und weiterhin „für“ oder „gegen“ die real existierende westliche Wirtschaftsordnung. Die Stellung zum Geschwindigkeitslimit ist ein Mittel zur Identifikation von Freund und Feind geworden, im Sinn von Carl Schmitt.

\section{Literatur}

Andersen, A (1997): ,und läuft und läuft...“. In: Andersen, Arne, Der Traum vom guten Leben. Alltags- und Konsumgeschichte vom Wirtschaftswunder bis heute. Frankfurt / New York, S. 154-175

Benz, C (1936): Lebensfahrt eines deutschen Erfinders. 22.-26.Tsd (Erstaufl. 1925)

Hohensee, J (1994): Und sonntags wieder laufen... Die erste Ölkrise 1973/74 und ihre Perzeption in der Bundesrepublik Deutschland. Salewski, Michael / Stölken-Fitschen, Ilona (Hg.), Moderne Zeiten. Technik und Zeitgeist im 19. und 20. Jahrhundert. Stuttgart, S. 175-196

Klenke, D (1993): Bundesdeutsche Verkehrspolitik und Motorisierung Konfliktträchtige Weichenstellungen in den Jahren des Wiederaufstieges. Stuttgart

Klenke, D (1995): "Freier Stau für freie Bürger". Die Geschichte der bundesdeutschen Verkehrspolitik 1949 - 1994. Darmstadt

Klodt, M von (2002): Alarmzustand im Gehirn. Das freiwillige Tempolimit der Hersteller auf $250 \mathrm{~km} / \mathrm{h}$ wankt. Wächst das Risiko auf der Überholspur? In: Focus 36/2002, S. 112f

Köppen, T (2005): Tempolimit und Fahrverbot. In: Achse, Rad und Wagen 13, S. $82-93$

Kopper, C (1995): Modernität oder Scheinmodernität nationalsozialistischer Herrschaft. Das Beispiel der Verkehrspolitik. Jansen, Christian / Niethammer, Lutz / Weisbrod, Bernd, Von der Aufgabe der Freiheit. Festschrift für Hans Mommsen. Berlin 1995, S. 399-411

Krämer-Badoni, T, Grymer, H. \& Rodenstein, M (1971): Zur sozioökonomischen Bedeutung des Automobils. Frankfurt /M. (= edition suhrkamp 540)

Merki, C M (2002): Der holprige Siegeszug des Automobils. Zur Motorisierung des Straßenverkehrs in Frankreich, Deutschland und der Schweiz. Wien / Köln / Weimar 
Möser, K (2002): Geschichte des Automobils. Frankfurt/M. / New York

Möser, K (2004): Der Staat und seine Regelungslust. Landesmuseum für Technik und Arbeit in Mannheim (Hg.), Lust am Auto. Ausstellungskatalog Mannheim

Moran, J (2008): The Myth of Top Gear. The Guardian, 10.6.2008, S. 24

Radkau, J (1991): Auto-Lust: Zur Geschichte der Geschwindigkeit. In: Königs, T. / Schäffer, R., (Hg.), Fortschritt vom Auto? München, S. 113-130

Seherr-Thoss, H C, Graf v. (1979): Die deutsche Automobilindustrie. Eine Dokumentation von 1886 bis 1979. Stuttgart, 2., korr. u. erw. Aufl.

Südbeck, T (1994): Motorisierung, Verkehrsentwicklung und Verkehrspolitik in der Bundesrepublik Deutschland der 1950er Jahre. Stuttgart

Thorbrietz, P (1995): Die Auto-Wende. Tempo-Limit, Autobahngebühren, CityMaut, Öko-Benzin: Mit neuen Techniken aus der Krise. In: Die Woche 6.1.1995, S. 1 



\title{
Wie vermittelt man Umweltgeschichte in der Schule?
}

\author{
Bodo von Borries
}

\section{Methode}

Das gestellte Thema kann man entweder normativ angehen (Was sollte sein? Was ist wünschbar und möglich?) oder deskriptiv (Was ist der Fall? Was findet tatsächlich statt?). Zur normativen und pragmatischen Seite wurden bereits mehrmals Beiträge geliefert (v. Borries 1988/89/2004, 1990/2006, 1990, 1992/2006,1995/2006, 1996, 2006), die - trotz des großen Zeitabstandes - im Kern noch immer zutreffen dürften. Deshalb soll hier - auch wenn das ein wenig größenwahnsinnig wirkt versucht werden, die wirklichen Verhältnisse und Bedingungen in der gegenwärtigen Schule abzubilden bzw. wenigstens anzudeuten.

Schulpraxis ist höchst komplex, widersprüchlich und vielfältig. Relativ bequem ist ein Versuch, sie in erster Näherung mit Richtlinien/Lehrplänen und mit Schulbüchern/Lernmaterialien abzubilden. Dazu gibt es in einem Kurzvortrag eigentlich keine Alternative. Aber auch den Weg über die amtlichen Vorgaben darf man sich nicht zu einfach vorstellen. Sechzehn Bundesländer haben auf der Sekundarstufe I im Mittel drei Schulformen (und im Durchschnitt vier Jahre Geschichtsunterricht). Für die Sekundarstufe II (ab 16 Jahren) ist die Sache keineswegs einfacher; jenseits des Gymnasiums gibt es allerdings nur teilweise überhaupt noch eine obligatorische historische Unterweisung. Das bedeutet etwa 80 bis 100 amtliche Dokumente, die hier nicht im Detail analysiert werden können und selbst mit Hilfe des Bil- 
dungsservers - und der Suchfunktion für Begriffe wie „Umwelt“, „Natur“, „Ökologie“, „Energie“, „Wasser" - nicht trivial aufzuarbeiten sind.

Bei den Schulbüchern ist die Vielfalt des Materials kaum geringer. Es gibt - allein für die Sekundarstufe I - gewiss etwa 50 grundsätzlich unabhängige Lehrwerke mit je drei bis vier Bänden und oft zahlreichen partiell abweichenden Länder- oder Regionalausgaben. Anders als bei den Richtlinien (mit ihrer Digitalisierung im Bildungsserver) hat man auch keine Chance, diesen Materialwust mit der Suchfunktion auf Zentralbegriffe wie „Umwelt“, „Natur“/,natürlich“, „Öko-/Ökologie/ökologisch“, „Energie“, „Boden“, „Rohstoff“ usw. durchzugehen. Für die Oberstufe (Sekundarstufe II) ist das Angebot - auch einschließlich der Themenhefte - etwas enger; hier gibt es aber seit einigen Jahren drei thematische QuasiSchulbücher zur Umweltgeschichte, zu denen eine vergleichende Rezension vorgelegt wurde (v. Borries 2004).

In beiden Fällen kommen die überaus wichtigen Überschneidungen mit dem Unterricht in Nachbarfächern, vor allem Geografie/Erdkunde (z.B. „Desertifikation" in der Sahara, „Bewässerungsrisiken“), Politik (z.B. Maßnahmen gegen den „Klimawandel"), Religion (z.B. „Bewahrung der Schöpfung") und Deutsch (z.B. Raabes „Pfisters Mühle") bei der Analyse nicht zureichend in den Blick. „Umwelt" ist per se ein interdisziplinäres Thema, schon gar zwischen den drei sozialwissenschaftlichen Fächern. Ebenso wie bei Themen wie „Migration“ und „Nord-SüdKonflikt" kommt es ja primär nicht darauf an, in welchem Fach „Desertifikation“, „Brandrodungsfeldbau“, „Wölbäcker“ oder „Monokulturen und Latifundien“ behandelt werden, sondern dass das geschieht (und zwar auch mit einem historischen Blick). Bei der Zusammenarbeit mit Physik, Chemie und Biologie müsste es normativ gesprochen - ähnlich aussehen, aber davon verstehe ich weniger.

Leider steht es um den Beweischarakter von Richtlinien und Schulbüchern als Vertretern („Indikatoren“) der Unterrichtspraxis keineswegs besonders günstig. Beide Textsorten sind vielmehr hoch normativ aufgeladen und Dokumente amtlicher Illusionen. Von den Schulbüchern ist durch die PISA-Studien (2000, 2003, 2006) und durch experimentelle geschichtsdidaktische Untersuchungen (z.B. v. Borries u.a. 1992, 1995, 2005, 2006, Beilner 2002, Langer-Plän 2003) zureichend gesichert, dass sie von einem großen Teil - oft der Mehrheit - ihrer Adressaten nicht einmal in einem basalen Sinne verstanden werden. Schließlich sind reichlich ein Fünftel der Jugendlichen in Deutschland funktionale Analphabeten bzw. Minimalleser; bei den Jungen ist die Quote viel höher. Das hat für die Kennzeichnung von Unterrichtspraxis eine kaum zu überschätzende Bedeutung.

Richtlinien werden seit vielen Jahren im „Pädagogischen Indikativ“ formuliert, nicht im Imperativ (Aufträge oder „haben zu“) oder Optativ (Konjunktiv oder „sollen“). Dadurch verwandeln sich - bis zur unfreiwilligen Komik - höchst pathetisch-schwülstige und unrealistisch-übertreibende Absichtserklärungen in „Beschreibungen von - angeblich tatsächlichem - Schülerverhalten“. Erfahrene Pädagogen und Testkonstrukteure wissen, wie wenig solche indikativischen Forderungen - in der Kenntnis - wie in der Kompetenzdimension - mit der sozialen Wirk- 
lichkeit in Klassenzimmern zu tun haben. Das ist auch durch eine Reihe von Befragungen und Schulleistungsvergleichen belegt (vgl. v. Borries u.a. 1995, 2005, Torney-Purta e.a. 2001). Noch jede empirische Studie zu jedem Thema - übrigens auch jedem Fach - hat im Vergleich zu den Richtlinien bescheidene bis kümmerliche tatsächliche Leistungen gefunden. Demgegenüber wirken Richtlinien/Lehrpläne wie Gemälde des „,sozialistischen Realismus“ mit „Helden der Arbeit" und „Muster-Traktoristinnen“.

\section{Richtlinien als Indikator}

Es mag verständlich sein, mit dem eigenen Bundesland zu beginnen und dabei verschiedene Schulformen und -stufen einzubeziehen: Hamburg hat vor wenigen Jahren Richtlinien für das achtstufige Gymnasium in Kraft gesetzt (2004), arbeitet aber mit Hochdruck schon wieder an einem neuen, noch stärker kompetenzorientierten Modell (auf diesen zugänglichen, aber noch nicht in Kraft gesetzten Entwurf wird hier nicht eingegangen). In den Richtlinien bildet „Wirtschaft und Umwelt“ einen von zehn Schwerpunkten, die als „,kategoriale Aspekte“ gewissermaßen bei der Strukturierung der Inhalte und didaktischen Thematisierung ständig zu berücksichtigen, wenigstens zu prüfen sind (S. 8).

Dabei steht der Zugang „Wirtschaft und Umwelt“ also neben anderen wichtigen, aber auf einigermaßen verschiedenen Ebenen liegenden Gesichtspunkten wie „Revolution und Evolution“, „Welt- und Menschenbild“, „Kultur““ oder „Rolle der Frau und Geschlechterbeziehungen“. Es erfolgen - anders als bei manchen anderen Schwerpunkten - sogar noch Konkretisierungen wie „die Auseinandersetzungen der Menschen mit ihren natürlichen Lebensbedingungen“ und „das Spannungsverhältnis von Beherrschung, Benutzung und Bewahrung der Natur" (S. 9). So überzeugend die ständige Rückkoppelung von Wirtschaft und Umwelt ist, erscheint doch schon damit das Risiko, meist, nämlich in den anderen neun „kategorialen Aspekten“, die Umweltgeschichte unter den Tisch fallen zu lassen.

Das ist - fast naturgemäß - beim Thema „Von Sammlern und Jägern zu Ackerbauern und Viehzüchtern“ nicht so (S. 13), wo von einem „bewussten Eingriff in die Natur“ die Rede ist, allerdings die Stichworte „Kulturlandschaft" oder „Bodenerschöpfung“ nicht auftauchen. Auch bei „Ägypten - Hochkultur am Nil“ (S. 14) sind „Einflüsse von Klima und Landschaft“ und „Umwelt: Der Nil - Freund oder Feind der Ägypter" noch präsent. Danach aber treten Umweltfragen völlig zurück. Im Mittelalter wird das Beispiel Lüneburg (Salzsiederei, Entwaldung) immerhin als methodische Möglichkeit erwähnt (S. 22). Bei „Industrialisierung und ,soziale(r) Frage"“ schaffen die Richtlinien es, die Umweltaspekte vollständig auszulassen (S. 30). Für die weiteren Themen bis zur Gegenwart finden sich „Ökologie“, „Natur“" „Umwelt" usw. überhaupt nicht mehr.

Für die Nachkriegszeit allerdings wird bei den „Verweise(n) auf andere Fächer und Aufgabengebiete“ unter „Umwelterziehung“ das Thema „Entsorgung, Um- 
gang mit Energie" erwähnt (S. 39), ohne dass es dabei ernsthaft um Historisches ginge. Und unter „Globale Entwicklungen bis zum Beginn des 21. Jahrhunderts“ (S. 40) wird - neben fünf anderen Aspekten - auch an „ökologische“ gedacht; eine Konkretisierung fehlt, außer in dem erneuten Verweis auf „Umwelterziehung“ mit „Entwicklung der Menschheit bei gerechter Verteilung der Ressourcen - Verminderung und Vermeidung von Bevölkerungswachstum“. Wie gesagt, das geschieht in Richtlinien, die auf der programmatischen und theoretischen Ebene „Wirtschaft und Umwelt" als einen von zehn Schwerpunkten ausweisen.

Im „Bildungsplan Integrierte Gesamtschule“ (2003), wo das Fach „Gesellschaft" heißt, stellen sich die historischen Bezüge ebenfalls als minimal heraus. Wenn überhaupt „Umwelt“ ins Spiel kommt, geschieht das so gut wie ausschließlich in geografischen Zusammenhängen, z.B. 5./6. Klasse „Naturraum prägt Tätigkeiten - Tätigkeiten verändern Natur“" und „Umwelt und Raumordnung in den Medien“ (S. 11). Die „Zeitbezüge“ (,Tätigkeiten und Berufe in früheren Zeiten“, "Öffentlichkeit und Privatleben in früheren Zeiten") und die "Geschichte" („Hochkulturen im Altertum“, „Leben im Mittelalter“) bei diesen Einheiten haben bezeichnenderweise nicht (oder kaum) mit Umwelt zu schaffen. In der 7./8. Klasse (z.B. „Lebensraum Erde“) (S. 20) und in der 9./10. Klasse (z.B. „Umwelt und nachhaltige Entwicklung“) (S. 28) sieht es ähnlich aus: Bei „Umweltgeschichte“ ergibt sich also eine Fehlanzeige.

Im „Rahmenplan Geschichte/Politik“ des „Bildungsplan(es) Hauptschule und Realschule Sekundarstufe I“ (Hamburg, 2003) wird verlangt: „Der Unterricht regt Schülerinnen und Schüler zur aufmerksamen Beobachtung ihrer Umwelt an." (S. 9) Das ist buchstäblich alles; aber darüber sollte man nicht lachen. Das ,historische Lesen“ einer Landschaft, einer Agglomeration, eines Gebäudes ist in der Tat eine wichtige und schwierige Kompetenz, und zudem umweltgeschichtlich relevant. Nur beigebracht wird das nach diesen Richtlinien nicht; jedenfalls fehlt jede Konkretion.

Dieses Urteil ändert sich nicht, wenn man den „Rahmenplan Aufgabengebiete“ wirklich genau ansieht, in dem die „Besonderen Bildungs- und Erziehungsaufgaben in der Hauptschule und Realschule" unter neun Gesichtspunkten - darunter „Umwelterziehung“ (S. 37-40) - im Detail aufgelistet sind. Was da gefordert wird, ist in der Tat eine umfassende, anspruchsvolle gegenwarts- und zukunftsbezogene Umwelterziehung mit Listen von Einsichten und Leistungen auf vier vollen Seiten. Fraglich ist dagegen die Historizität. Dass menschliches Leben sich „,in einem über Jahrtausende vom Menschen gestalteten Naturraum“ vollzieht, wie der Vorspann sagt (S. 37), ist gewiss zutreffend, bleibt aber ohne jede Folge für das Curriculum.

Im Text selbst ist nur einmal von „Energieversorgung früher und heute, bei uns und in anderen Kulturen“ (S. 40) die Rede, wozu auch als Leistung das „vergleichen“ (S. 40) gefordert wird. Unterscheidung und Vergleich von „früher und heute" ohne jede weitere Spezifizierung aber sind eine typische Grundschulformel und können nur bedingt als ,historisches Denken“ angesehen werden. Es bleibt mithin dabei, dass „Umweltgeschichte“ in der Haupt- und Realschule - wie in der 
Gesamtschule - nicht thematisiert werden; eher wird um Umweltgeschichte bewusst ein großer Umweg gemacht.

Für die gymnasiale Oberstufe („Bildungsplan Geschichte“, 2004) wird einmal das Wort „Umweltgeschichte“ erwähnt (S. 19). Es handelt sich um die Einheit „Modernisierung in Wirtschaft und Gesellschaft", wo aus Max Webers Theorie zum Kapitalismus als „schicksalsvollste(r) Macht unseres modernen Lebens“ die sozialwissenschaftliche Wendung von Geschichtsforschung und -unterricht abgeleitet wird, wozu dann auch - neben den drei anderen Richtungen „Alltags-“, „Kultur-“ und „Geschlechtergeschichte“ - die „Umweltgeschichte“ gehört. Freilich wird gerade sie auf der gleichen Seite unter den ,verbindlichen Unterrichtsinhalten" schon wieder vergessen. Auf den folgenden drei Seiten erscheinen dann als Stichworte allenfalls „Ökologiebewegung“ (S. 20) und „Wandervogel“ (S. 21). In Wahrheit geht es nämlich bei diesen „Bewegungen“ sehr wohl um „Sozialgeschichte $^{\text {“ }}$ und „Mentalitätsgeschichte“. Der Begriff „Umweltgeschichte“ aber ist schlicht! - erschlichen.

Wenigstens ein zweites Beispiel, d.h. ein anderes Bundesland, soll noch erwähnt werden, und zwar diesmal in einem Längsschnitt, aber nur für eine einzige Schulform. Die Bestimmungen für die Realschule in Baden-Württemberg („Bildungsplan für die Realschule“, 1994), immerhin dem drittgrößten Bundesland, sind besonders typisch. Das Wort „Umwelt“ ist in ihnen überaus häufig (hunderte von Erwähnungen). Es meint an vielen Stellen ebenso ,technische (menschengemachte) Umwelt“ wie „natürliche Umwelt“. So gelten „Reizüberflutung“ und „Lärm“ durchaus als Umweltprobleme. In praktisch allen Fächern, so Mathematik, Chemie, Biologie, Kunst, Gemeinschaftskunde, Englisch, werden „Umwelt“ und „Umweltschutz" thematisiert.

Vorweg wird unter „Erziehungs- und Bildungsauftrag“ aber ausdrücklich darauf hingewiesen (S. 10), dass bei der „Umwelterziehung“ auf „ein ausgewogenes Verhältnis von Ökologie und Ökonomie zu achten ist" (was immer das heißen und wie immer das möglich sein soll). Bestimmend ist allerdings, dass all diese Umweltbezüge strikt bloß gegenwarts- und zukunftsbezogen sind und mit Geschichte nichts zu tun haben. Daran, dass auch die jeweiligen fachlichen Erwähnungen von „Umwelt“ ihre historischen Aspekte haben müssten (z.B. Chemie und Altlasten, Biologie und ausgestorbene Tierarten), wird kein Gedanke verschwendet. Es gibt sogar ein Wahlpflichtfach „Mensch und Umwelt“, das allerdings nur die Hauswirtschaftslehre umfasst und besonders unhistorisch angelegt ist.

Nur im Fach Geschichte sieht es anders aus. Unter „Die Industrialisierung verändert Leben und Arbeitswelt“ (S. 227) erscheint als ein Punkt „Auswirkungen auf Natur und Gesellschaft“, der wiederum in „Ausbeutung der Ressourcen, zunehmende Umweltzerstörung“ und „Gesellschaftliche Umschichtungen, Gliederung der Gesellschaft" aufgegliedert wird. Das ist im Bereich Geschichte schon alles: Nicht einmal die Entstehung der deutschen Kulturlandschaft durch den Landesausbau im Mittelalter (Rodungsperiode) kommt vor. 
Absolut zentrale Themen wie „Wohlstand auf Kosten der Umwelt“ und „Armut zerstört die Umwelt" (beides ohne Fragezeichen!) finden sich im Fach Erdkunde (S. 375). Hier wird auch der „Veränderung des Weltklimas“ Rechnung getragen; historische Bezüge fehlen. Die gesamte Einheit „Geschichte und Zukunft der Ökosphäre: Globale Umweltfragen“ ist unter „Biologie“ eingeordnet (S. 392) und allenfalls „naturhistorisch“ ausgerichtet; „,humanhistorische“ Fragen spielen keine Rolle, wohl aber die Zukunftsrisiken.

Wie soll man als Umwelthistoriker darauf reagieren? Soll man sich freuen, dass die Umweltfrage so wichtig genommen wird (genau genommen: wurde, denn durch die „Standards“ des Landes [s.u.] haben sich diese Bildungspläne inzwischen erledigt)? Soll man beleidigt sein, dass um das eigene Fach Geschichte - fast absichtlich - ein großer Bogen geschlagen wird? Soll man gute Miene machen, weil „Umweltgeschichte“ ja kein Selbstzweck sein kann/darf und fast alle Umweltfragen einen hochgradig interdisziplinären Charakter tragen? Oder soll man klagen, weil ohne historische Dimension eben auch „ökosystemare“ Ansätze, die durchaus eingeführt werden, unverständlich und irrelevant bleiben? Soll man sich beschweren, weil die „historisch-anthropologische“ Frage so gar nicht in den Blick kommt?

Baden-Württemberg ist inzwischen (2004) zu „Bildungsstandards für Geschichte" übergegangen. Freilich befinden sich der alte Bildungsplan weiterhin auf dem deutschen Bildungsserver unter „Lehrplan-Datenbank“ der KMK (hier ID: LP 11038, letzter Zugriff 23.08.2008), nicht mehr aber auf dem „Landesbildungsserver Baden-Württemberg“. Da andererseits die Schulen Mustercurricula entwickeln sollen, die ebenfalls auf dem Bildungsserver erscheinen, stellt sich - trotz zentraler Prüfungen - die Frage der Gültigkeit und möglicher Konflikte seltsam verwirrend, wenn nicht unlogisch dar (das gilt auch für andere Bundesländer, die ihre älteren Bildungspläne bei den neuen Bildungsstandards nicht wirklich außer Kraft gesetzt haben). Jedenfalls ist der relativ hohe Stellenwert ökologischer Fragen von 1994, der - mit einigen Jahren Abstand - natürlich das starke Umweltkrisenbewusstsein der späten achtziger Jahre gespiegelt hat, schlicht wieder auf dem Müllhaufen der Geschichte gelandet.

In den „Bildungsstandards für Geschichte Realschule Klassen 6, 8, 10“ (2004) kommt Umwelthistorisches nur zweimal ganz am Rande ins Spiel: Dabei geht es zum einen um den Übergang von „Zweckgemeinschaften [zum] Überleben in der Natur" zu „ersten Formen des Gemeinschaftswesens“ und in diesem Zusammenhang auch um die Entwicklung vom ,Jäger und Sammler zum Viehzüchter und Ackerbauern“ (S. 106). Es sei offen gelassen, ob Ethnologen „Sammlerinnen und Jägern“ oder „Wildbeutern“, wie sie meist sagen, keine Kultur, kein Gemeinschaftswesen, zusprechen. Jedenfalls werden Umwelt und Umweltveränderung hier nicht explizit ins Spiel gebracht. Das zweite Themenbeispiel ist natürlich die Industrialisierung mit ihren „Auswirkungen industrieller Entwicklungen auf die Umwelt" und lösungsorientierten Diskussionen „des verantwortungsvollen Umgangs mit den natürlichen Ressourcen“ (S. 112). Das ist schon alles: schmal und zudem mehr als vage. Wiederum gilt, dass im Paralleldokument „Bildungsstandards für 
den Fächerverbund Erdkunde - Wirtschaftskunde - Gemeinschaftskunde Realschule Klassen 6, 8, 10“ (2004) „Umwelt“, „Klima“, „Ökologie“ usw. deutlich häufiger ins Spiel kommen, allerdings gänzlich unhistorisch.

Es wäre leicht möglich, weitere der rund achtzig bis hundert Richtlinien- bzw. Lehrplan-Dokumente durchzugehen. Das erscheint aber als wenig fruchtbar; denn das Bild gleicht sich in der Tiefenstruktur, obwohl auf der Oberfläche große Differenzen auftreten können. Lohnend ist also nur noch eine Diskussion der Tiefenebene, die aber ans Ende dieses Textes gehört.

\section{Schulbücher als Indikator}

Natürlich ist es nötig, einen zweiten Indikator der Schulpraxis zu untersuchen und dafür die Schulbücher zu wählen. Diese haben heute meist eine Struktur aus geschlossenen Doppelseiten und verfügen neben Auftaktseiten und Autorentexten (mit Grafiken, Tabellen, Karten und Rekonstruktionszeichnungen oder -modellen) sowie Text- und Bildquellen auch über Arbeitsaufgaben und Methodenseiten (z.B. „Werkzeugkasten“, „Gewusst wie“). Die folgenden Beispiele stammen alle aus überdurchschnittlichen Schulbüchern und thematisieren Umweltgeschichte in vergleichsweise herausragender Qualität. Bewusst sind verschiedene Schulformen berücksichtigt, aber auch Längsschnitte und Querschnitte, bloße Zusammenfassungen und breite Entfaltungen, vorwiegende Textdarstellungen und vorwiegende Bildquelleneditionen, eher aktuell-politische und eher historisch-analytische $\mathrm{Zu}$ gänge. Es zeigt sich, dass mehr möglich ist, als man nach den Richtlinien erwarten sollte. Aber das heißt noch lange nicht, dass solche Schulbuchteile sowohl oft benutzt als auch zureichend verstanden werden (s.o.).

In „Forum Geschichte“ (II, 2001, 14-49) gibt es ein Kapitel „Bauern und Adlige im Mittelalter" mit einer randständigen Erwähnung von Umweltgeschichte. Immerhin werden zu „Von der Feldgras- zur Dreifelderwirtschaft“, „Wachsende Bevölkerung - knappe Böden“ und „Naturverbrauch durch Rodung und Siedlung“" jeweils ganze Abschnitte im Autorentext gebracht (S. 18-20), denen noch sechs Zeilen einer Textquelle (Notiz des Abtes von Prüm 1222 über die Landschaftsänderungen seit 893) und drei Bildquellen (Buchmalereien mit Hakenpflug und Räder-Streichbrettpflug sowie das berühmte Eichelmastbild des Duc de Berry), eine Textdarstellung (Historikerzitat „Umwelt und Ernährung im Mittelalter"), eine Bilddarstellung, eine Karte und eine Art Tabelle beigegeben sind. Darauf folgt die Anleitung zum Besuch eines Freilichtmuseums (S. 20). Die Wirtschaftsgeschichte wird hier also in elementarer Weise zugleich als Umweltgeschichte deutlich gemacht. Man kann sich höchstens fragen, ob die wenigen vorausgehenden Seiten zum Bauernleben mit konkreteren Texten und Bildern (17f.) genügen, damit so junge Kinder so abstrakte Zusammenhänge zuverlässig erfassen können.

Völlig anders sieht es in „Geschichte konkret“ (II, 1997) aus. Hier gibt es zwei eigene Kapitel „Umwelt hat Geschichte“ (120-135) und „Die Geschichte der Ener- 
gie“ (218-227), die man im ersten Falle als systematischen Querschnitt - angesichts der Erweiterung um die Neue Welt - (,Tiere, Pflanzen und Krankheiten breiten sich aus“ [Nutzpflanzen, Haustiere, Baumrinde gegen Malaria, Pocken], „Die Menschen verändern die Umwelt" [,grüne Revolution', Umweltzerstörung]) und im zweiten - bei der Industrialisierung - als typisierenden Längsschnitt („Energie aus der Natur“ [Muskelkraft, Holz], „Die Suche nach neuen Energiequellen“ [Kohle]) ansprechen kann. Wie man sieht, sind das absolut zentrale, exemplarische Punkte in weiser Beschränkung und Vereinfachung. Das wäre, wenn es auf andere Lernwerke übertragen würde, wirklich ein Durchbruch, wenn auch die Bäume nicht in den Himmel wachsen können. Herausgeber des Arbeitsbuches ist, nicht zufällig, einer der prominentesten Geschichtsdidaktiker, Hans-Jürgen Pandel.

Aber diese Gestaltung ist durchaus nicht typisch; in „Geschichte (und) Geschehen“ (I, 2003) bekommt das Kapitel „Die Frühzeit des Menschen“ (24-47) einen Schluss mit einet auch umweltgeschichtlichen Zusammenfassung: „Mensch Natur - Technik“ (45f). Bezeichnenderweise geschieht dies bei einem der ,groBen“ ökologischen Themen, der „,neolithischen Revolution“. Zutreffend wird festgestellt: „Das Verhältnis der Menschen zur Natur und Umwelt wandelte sich. Bisher hatten die Menschen nur genutzt, was ihnen die Natur von sich aus bot. Nun begannen die Menschen selbst in die Natur einzugreifen und sie zu verändern. Aus der natürlichen Umwelt begann eine vom Menschen gestaltete Kulturlandschaft zu entstehen (...)“ (45) Weiter werden eine Reihe von Details angeführt, wobei die Frage der Wirtschaft im Zentrum bleibt. Der Gedanke wird dann - am Beispiel der Getreideernte und mit vier Abbildungen - als Ausblick über die Metallzeit bis zur Industrialisierung und zur Gegenwart weitergeführt (45f.). Dabei gewinnt erneut ein anderer Geschichtssektor die Vorhand, diesmal die Technikgeschichte.

„mitmischen in Geschichte und Politik“ (III, 2001) verfährt noch weit gegenwartsbezogener und ist handlungsorientierter gestaltet. Das Kapitel „Der letzte Baum“ (258-287) beginnt zwar nach der aktuellen Auftaktdoppelseite mit einem Unterkapitel „Umwelt hat Geschichte“ (260-261) und lässt dann unter „Mit der Natur im Einklang leben“ (262-263) ein Unterkapitel über den gewissermaßen zeitlosen Früh-Typ der Wildbeuter (hier als „Naturvölker“ bezeichnet) folgen. Die weiteren Abschnitte (,Sechs Milliarden und kein Ende“, „Men made desert“, Aralsee, „Treibhaus Erde“, Braunkohlenabbau Garzweiler, „Sonne - Wasser - Wind“ als Energie, Lokale Agenda 21, Stromsparen, Radfahren, Flusssterben, Fragebogen zum Umweltbewusstsein, 264-285) beziehen sich durchweg auf die allerletzten Jahre. Das soll keine Kritik sein, zumal an der Hauptschule in NRW Geschichte und Politik zusammen unterrichtet werden. Der im engeren Sinne umwelthistorische Anteil bleibt in diesem herausragenden Buch (hauptschulgerecht viele Bilder und kurze Texte) jedoch einigermaßen bescheiden.

Die wenigen Beispiele zeigen, dass durchaus mehr möglich ist und vermutlich auch von manchen Kolleg(inn)en realisiert wird, als die Richtlinien vorschreiben (spannender ist, was sie überhaupt erlauben). Im Bereich der Praxiszeitschriften gibt es durchaus zahlreiche Unterrichtsmodelle, manchmal ganze Themenhefte 
(„Umwelt und Geschichte“ 1986, „Umweltgeschichte“ 1988, „Umweltgeschichte“ 1994, „Umweltkonflikte...“ 1995, „Mensch und Umwelt“ 1997), wenn auch kaum aus der letzten Jahren. Die Hochkonjunktur historisch-ökologischen Denkens scheint vorbei zu sein (vgl. eben deshalb v. Borries 2006). Von drei Oberstufenbüchern bzw. Themenheften (Bachsleitner u.a. 2001, Schmal 2001, Radkau 2002) war bereits die Rede. Mit anderen Worten: Wer als Lehrer(in) Umweltgeschichte unterrichten will, findet nicht nur jede Menge Projektchancen, sondern auch genügend vorstrukturierte und realistische Einheiten. Lebhaft angespornt wird er/sie aber durch Staat, Buchmarkt und Fachdidaktik nicht (mehr).

\section{Diskussion}

Das Ergebnis ist enttäuschend. Zunächst einmal muss man sich jedoch den extremen Abstand zwischen dem, was Geschichtswissenschaftler, schon gar spezialisierte Umwelthistoriker, für gut und nützlich und nötig halten, und der Praxis an Schulen immer wieder brutal klar machen. Das sind - auch in anderen Geschichtssektoren - zwei Welten; die Kümmerlichkeit des Alltags und die enge Begrenztheit des Möglichen (Lernzeit, Stundentafeln, Vergessenskurve, Motivationsmangel, Leseunfähigkeit, Konzentrationsschwäche usw.) gilt es sich immer wieder vor Augen zu führen. Dem Hochschulmenschen ohne Schulkontakte ist das vermutlich kaum eindrücklich genug deutlich zu machen. Alle unsere Erwartungen und Hoffnungen pflegen weit über das Mögliche - jedenfalls außerhalb des Gymnasiums Mögliche - hinauszuschießen.

Dennoch gilt: Der große Aufbruch der mittleren und späten achtziger Jahre er war nicht nur in der Spezialforschung, sondern auch in der Geschichtsdidaktik und manchmal sogar in Schulbüchern und Richtlinien und erst recht in den Antworten (nicht der Praxis!) befragter Lehrender und Lernender (vgl. v. Borries u.a. 1995) zu verspüren - ist längst verpufft. Sich darüber hinwegzutäuschen, wäre eine Illusion. Für den großen Rückschlag gibt es gute und schlechte Gründe.

\subsection{Falsch verstandene Methoden- und Kompetenzorientierung}

Seit einiger Zeit ist in der Bildungspolitik nur noch von Standards, Kompetenzen und Outcome-Steuerung die Rede. Das kann hier nicht ausführlich erklärt und kritisiert werden. Unverkennbar ist, dass dadurch zwingend eine gewisse Vereinheitlichung ausgelöst wird; zentralisierte Prüfungen sind nur eine Form der Umsetzung. Das wäre dann vielleicht nicht besonders tragisch, wenn der Kerngedanke des Kompetenzansatzes ernst genommen würde. Als „Kompetenzen“ sind domänenspezifische, auf neue Situationen übertragbare Fähigkeiten und Bereitschaften zur Problemfindung und Problemlösung definiert. Die bestehen aber nicht in Katalogen von Informationen und Kenntnisbeständen, sondern in Fragestellungen, 
Methodenanwendung, Orientierungsvermögen und Kategoriensystemen (vgl. Körber/Schreiber/Schöner 2007).

Natürlich geht es nicht ohne beispielhaftes kontextbezogenes „Wissen“ (für die jeweilige Arbeitsaufgabe), aber zu den wichtigsten Fähigkeiten gehört es, sich neues Wissen in neuen Situationen schnell, organisiert und effektiv anzueignen. „Stoffe" und „Themen“ stehen also nicht im Vordergrund, sondern die Einübung von Zugangsweisen, Methoden und Konzepten (natürlich anhand thematischer Beispiele, die aber relativ frei wählbar sind). Ob man - in unserem Falle - das Ziel „reflektiertes und (selbst-)reflexives Geschichtsbewusstsein“, „historisches Denken“ oder „narrative Kompetenz" nennt, ist relativ gleichgültig. Dass es jedoch nicht ,sicherer Überblick über die Nationalgeschichte“ oder „Kenntnis der Grunddaten der Weltgeschichte" heißen darf, ist offenkundig.

In der Praxis gibt es nun zwei Anwendungen dieses Konzepts, das eigentlich von der typischen Kanonisierung und Stofforientierung im Fach wegführen müsste. Das eine ist die offenkundige Umkehrung bei der Verwirklichung: „Wo Kompetenz draufsteht, muss nicht Kompetenz drin sein." Der Etikettenschwindel bei der Standard-Festlegung ist recht häufig. „Content-Standards“ sind - nicht nur in Deutschland - weit üblicher als „Performance-Standards“ und „Opportunity-toLearn-Standards“. Dass aber bei einer neuen „Stofforientierung" des Geschichtslernens, die über „objektive“ Vergleichsarbeiten und zentrale Prüfungsverfahren strikt durchgesetzt wird, gerade die Umweltgeschichte kaum Chancen hat, liegt auf der Hand. Die Reduktion auf das (so genannte) gesicherte Grundwissen bedeutete schon immer - das hat gerade der Konservative Erich Weniger $(1969,25)$ betont das Geschäft der Reaktion.

\subsection{Richtig verstandene Methoden- und Kompetenzorientierung}

Wenn man aber intellektuell redlich mit dem Kompetenz-Konzept umgeht, ist die Umweltgeschichte auch noch nicht automatisch auf der Siegerstraße. Jetzt muss ja der maßgebliche Aufbau des Curriculums in einer Lernprogression der fachspezifischen Denkfiguren (nicht bloß Arbeitstechniken und Medienumgangsweisen!) bestehen. Und nebenbei sind sekundär jeweils solche Themen zu wählen, die sich an lebensweltliche Erfahrungen und Orientierungsbedürfnisse der Lernenden gut anpassen (und sei es als „Alteritätserfahrung“/,Verfremdung“"), die also „historische Sinnbildung" überhaupt zu einem biografisch relevanten Prozess für die Lernenden machen können.

Dieser Auswahlprozess ist allerdings von den Konventionen in der Gesellschaft keineswegs unabhängig. Selbst wenn die einzelnen Lehrer und Klassen ihre Auswahl autonom betreiben würden (und die Lehrer über Umweltgeschichte bestens informiert wären), blieben historisch-ökologische Fragen vorläufig eher ein gesamtgesellschaftlich - und meist auch subkulturell - nicht anerkanntes und nicht gestütztes Gegenprogramm. Das kann man (explizit argumentierend) vereinzelt machen, aber nicht ständig. Denn die Schüler müssen ja lernen, sich kommunikativ 
und motivational (wenn auch kritisch!) in die Geschichtskultur ihrer Gesellschaft (und deren Subkulturen) hineinzuarbeiten. Historie ist eine Konstruktion, aber eben nicht nur eine individuell-beliebige, sondern eine im sozialen Prozess bestimmte (schon gar bei Kindern und Jugendlichen). Wer sich ganz ernsthaft um stufenweise Förderung von historischer Kompetenz kümmert, wird leider die „Umweltgeschichte“ - oder andere unkonventionelle Themen - nicht im Schwerpunkt seiner Didaktik haben können, sondern nur als programmatischen Kontrapunkt, als expliziten Kritik- und Oppositionsversuch.

Offen gesagt: die „Umweltgeschichte“ scheint in der Schule zunächst weitgehend als „historische Geografie“ der „Humangeografie“ und damit dem Fach „Erdkunde“ zugeteilt worden zu sein, wofür ja - pragmatisch wie normativ durchaus gute Gründe geltend gemacht werden können. In der zweiten Runde ist dann wohl - unter dem Druck von Kürzungen in den Stundentafeln, als Kapitulation vor der Stofffülle und auch wegen der begrenzten eigenen Kompetenz von Richtlinienmacher(inne)n und Lehrer(inne)n - innerhalb der Humangeografie die „historische Geografie“ und erst recht die „Umweltgeschichte“ wieder fast total vergessen worden. Das ist eine Hypothese; wirklich zuverlässig prüfen könnte man sie nur durch eine Detailanalyse von geographischen Curricula, Standards, Schulbüchern und Unterrichtsstunden.

\subsection{Fixierung auf Wiedervereinigung und deren Krisenerscheinungen}

Das große politische Erdbeben von 1989/91 mit dem Ende des sozialistischen Lagers und der deutschen (Wieder-)Vereinigung hat zweifellos die Aufmerksamkeit wie die Interessenlage nachhaltig verändert. Ähnliches gilt für den zweiten Schock des internationalen Terrorismus (11. September 2001) und die späte Einsicht in den Charakter Deutschlands als einer „Einwanderungsgesellschaft“. Die erste Wandlung spiegelt sich bereits deutlich im Geschichtsunterricht (zumal ja der Charakter der Vereinigung als „Erfolgsgeschichte“ inzwischen lebhaft bezweifelt wird), die zweite steht als nächste Transformationswelle an (nicht zuletzt wegen der beklagenswerten Bildungserfolge der Kinder mit Migrationshintergrund). Dass „Umweltgeschichte“ gegen beide jetzt gesponserten Großthemen eine nennenswerte Chance auf Aufwertung hätte, kann man wohl nicht hoffen, vielleicht nicht einmal hoffen wollen.

Vor allem scheint die neue „Umwelt-und-Klimaschutz-Debatte“ gerade kein Wasser auf die Mühlen der Umweltgeschichte zu leiten, vielleicht wegen der alten Einsicht von Radkau (1986) und anderen (z.B. Sieferle 1986), dass die Probleme heutiger Umweltschädigungen (weltweit, vernetzt, unsichtbar, irreversibel) so neuartig sind, dass historische Vergleiche nur bedingt und indirekt zur Problemlösung taugen. Das ist richtig, stellt sich aber in anderen Sektoralgeschichten (Krieg, Friedenssicherungssysteme, Technologie, Wirtschaft, Menschenrechtsfrage) keineswegs anders dar. Ganz allgemein und themenunabhängig gilt: Von Geschichtsunterricht darf man eben keinesfalls nur „traditionale“ und „exemplarische“ Sinnbil- 
dung, d.h. unmittelbare Anwendbarkeit, erwarten. Die hilft oft wenig weiter oder führt gar in die Irre. Stattdessen kommt es auf „genetische“ und „kritische“ Sinnbildung an (vgl. Rüsen 1994), die ziemlich genau dem entspricht, was der „Club of Rome“ (vgl. Botkin u.a. 1979) vor Jahrzehnten - leider mit wenig Beachtung - als „partizipatorisches und antizipatorisches Lernen“ bezeichnet hat.

\subsection{Anhaltender Wachstumsfetischismus}

Vor Jahrzehnten gab es die noch zögerliche und zaghafte Einsicht; dass es ohne Verzicht in den Industrieländern nicht gehen wird („Brundtlandt-Bericht“, vgl. Hauff 1987, „Unsere gemeinsame Zukunft“ 1990), dass das Raumschiff Erde begrenzt ist und dass ein exponentielles Wachstum auf Dauer auch logisch unmöglich ist („Schachbrett-Aufgabe"). Diese Einsicht ist mittlerweile wieder verloren gegangen, weil nicht mehr die Frage der Versorgung von Bevölkerungen, sondern des Funktionierens von liberalen Marktwirtschaften („Kapitalismus") interessiert. Und dieses Funktionieren scheint an Wachstum gebunden, weil sonst private Gewinne („Profite“) in einem Nullsummenspiel nur noch aufgrund von anderer Leute Verlusten realisierbar sind (was zur gefährlichen - kriegstreibenden - Politik des „beggar your neighbour“ führt). Die Dreiheit von Wachstum, TechnologieInnovationen und Massenkonsum bildet eben ein vorzügliches Schmiermittel der Wirtschaft, ja sogar der Demokratie.

Es ist also kein Wunder, dass vereinzelte Mahner und Warner (vgl. Biedenkopf 2004, Zitat v. Borries 2006, 391) schlicht sofort überhört, nicht einmal wahrgenommen werden. Stattdessen benehmen sich praktisch alle Spitzenpolitiker nach dem Motto des Pompeius, der - bei der Ausfahrt zum Seeräuberkrieg - den Warnenden entgegen geschleudert hat: „Navigare necesse est, vivere non necesse est“. Genau so tönt es heute beim „Tanz ums goldene Kalb“: „,crescere necesse est, vivere non necesse est". Die Politik der Sackgasse - um nicht zu sagen des Selbstmords - ist amtliches Programm. Muss ich noch sagen, dass angesichts der Entwicklung - und des Standes - der Weltbevölkerung ein Prozess von „Wachstum als Transformation zur Nachhaltigkeit" natürlich unverzichtbar ist: „Überleben ist nötig; Wachsen ist nur noch als Konversion zu relativ stabilen Dauerlösungen (ich wage nicht zu sagen: Gleichgewichten) erlaubt." Aber auf die Steuerung kommt es an - und die wird nicht einmal versucht.

Offenbar hat man die Auffassung, angesichts der Logik des Kapitalismus einerseits und der Grenzen des Staates (sowie der Demokratie) andererseits könne man sowieso nicht viel ändern oder gestalten. $\mathrm{Da}$ wäre es doch gänzlich unangemessen, auch noch Umweltgeschichte zu betreiben und sich dadurch die Laune verderben zu lassen. Wenn gar nichts geht, verdrängt man lieber auch die Vorgeschichte der Gefahr. Die mentale Haltung des „Nach uns die Sintflut!“ wird nicht immer voll bewusst - fortgesetzt. Auch orientalische Gottkönige wollten lieber feiern und richteten schlimmstenfalls die Boten hin, die ihnen militärische Niederlagen meldeten... 


\subsection{Umwelt als bloßer „Luxus“}

In Schülergesprächen - im Rahmen von Unterrichtsversuchen wurden eine Reihe davon geführt - werden die neuen gesellschaftlichen Selbstverständlichkeiten ziemlich rasch klar. Es gibt ein beachtliches Feeling über die Unmöglichkeit, den gegenwärtigen ökonomisch-ökologischen Weg - von Wachstum, Vergeudung und Verschmutzung - langfristig fortzusetzen. Gleichzeitig gibt es einen massiven Widerstand gegen irgendeine Umstellung des eigenen Lebensstils oder irgendeine Konsequenz aus der eigenen Einsicht.

Das schlägt massiv auf den „Umwelt“-Begriff selbst durch. Als Umwelt wird nicht das eigene Habitat der (deutschen, europäischen, weltweiten) Gesellschaft aufgefasst, was eindeutig längst „,natürliche“ wie „kulturelle“ wie „technische“ Teile enthält und alles andere als bloß eine außer-menschliche Natur umfasst. Unter „Umwelt“ werden vielmehr die menschenfernen kleinen Reservate (z.B. Naturschutzgebiete, Naturparks) verstanden. Umwelt wird - auch ganz explizit - als „Luxus“ dargestellt, den wir normalen Leute uns - nach Schülermeinung - aber leider gar nicht mehr (oder nur in äußerst kleinem Ausmaß) leisten können. Das ist höchstens etwas für Millionäre...

Wer - eine einigermaßen intakte und überlebensfähige - „Umwelt“ aber nicht als Voraussetzung und Teil allen menschlichen Lebens erfasst („conditio sine qua non"), sondern als seltenes Sonntagsausflugsziel, der geht an entscheidenden Einsichten vorbei und macht aus einer Überlebensfrage mit Zwangscharakter (für alle) eine Konsumfrage mit Wahlfreiheit (für Reiche). Wer so denkt (und das tut die große Mehrheit selbst im Gymnasium), hätte Umweltgeschichte - nicht nur als Thema, sondern auch als Kategoriengerüst - für sein historisches Denken, seine Orientierungskompetenz, dringend nötig. Es hat noch nie eine Gesellschaft gegeben, die man ohne ihren dauerhaften Austausch mit ihrem Habitat und ihren Umgang mit dessen - selbst erzeugten - Wandlungen hätte begreifen können.

\subsection{Universitätsstudium und Massenmedienpraxis als „geheimer Lehrplan“}

Lehrende unterrichten gewöhnlich noch stärker nach dem Schulbuch als nach den Richtlinien; und sie fühlen sich offenbar vor allem bei den Themen wohl, die sie einmal im Hochschulstudium kennen gelernt haben. Jedenfalls gilt das für die Gymnasiallehrer (in der Hauptschule wird ohnehin sehr viel fachfremder Unterricht gegeben). Das macht die massive Nationalstaatslastigkeit und Politiklastigkeit der Universitätsausbildung in der Historie zu einem großen Problem. Was heute an Hochschulen gelehrt wird, dürfte sich - angesichts geringer Lehrerfortbildung - an den Höheren Schulen für einige Jahrzehnte halten (,geheimer Lehrplan“). Umweltgeschichte kommt dabei so wenig vor, dass Studierende vielfach nicht einmal eine Vorstellung davon haben, was das sein könnte. Ich habe das mehrfach durch kleine schriftliche Befragungen in Seminaren erhoben und glaube nicht, dass es an anderen Hochschulen - oder im Geographiestudium - wesentlich anders aussieht. 
Natürlich muss versucht werden, die Kultusministerien und die Schulbuchverlage weiterhin - auch mit Lobbyarbeit und mit überzeugenden Argumenten - auf die Umweltgeschichte zu bringen. Aber die Wirksamkeit wird ausgesprochen gering bleiben (bestenfalls „Pyrrhussiege“), wenn sich in den Hochschulen selbst nichts ändert. Deshalb sind Durchbrüche in der Geschichtswissenschaft (und ihrer „Vermachtungs- und Vermarktungsstruktur") entscheidend. Solange die Umweltgeschichte nur eine schmale zusätzliche Sektoralgeschichte am äußersten Rand darstellt, solange man sich nicht blamiert, wenn man die Geschichte eines Landes oder einer Epoche ohne die Analyse des Habitats und seiner Veränderungen (übrigens auch der Population und ihres Wandels) darstellt, solange wird es keine substanzielle Besserung geben.

Nur wenn die „Lufthoheit über den intellektuellen Stammtischen“ von Fernsehsendern (besonders ARD, ZDF) und Historikerverband (sowie Historischen Fachbereichen und Instituten) auch der „Umweltgeschichte“ zufällt, wird sich etwas ändern. Es genügt nicht, dass - wie in mehreren anderen historischen Feldern mit innovativen Minderheiten und begeisternden Büchern - Umwelthistorie eine blühende und gedeihende Sonder-Kultur wird oder bleibt. Angesichts des massiv strukturkonservativen Verhaltens der genannten großen „Partner“ mit betonhaft etablierten Epochalgliederungen (,Alte Geschichte“, „Mittelalter“, „Frühe Neuzeit", „Neuzeit") - und ihrer konstitutiven Übermacht über die kleine Gruppe von innovativen Protestlern - stehen die Chancen dazu gegenwärtig bemerkenswert schlecht.

\section{Erwähnte Literatur}

Artelt, Cordula u.a.: Lesekompetenz: Testkonstruktion und Ergebnisse, in: PISA 2000. Basiskompetenzen von Schülerinnen und Schülern im internationalen Vergleich; Opladen (Leske \& Budrich) 2001, S. 69-137.

Bachsleitner, Karl, Conrad, Franziska und Engelke, Rolf: Umweltgeschichte. Fragestellungen und Quellen zum Geschichtsunterricht, Sek II; Wiesbaden (Hessisches Landesinstitut für Pädagogik - HeLP) 2001.

Bachsleitner, Karl u.a.: "In der Nähe solcher Fabriken erstirbt fast jede Vegetation" Die chemische Industrie um die Jahrhundertwende - Auseinandersetzungen um Folgen und Risiken, in: Geschichte lernen 7. Jg., 1994, Heft 41, S. 47-52.

Beilner, Helmut: Empirische Zugänge zur Arbeit mit Textquellen in der Sekundarstufe I, in: Schönemann, Bernd und Voit, Hartmut (Hrsg.): Von der Einschulung bis zum Abitur. Prinzipien und Praxis des historischen Lernens in den Schulstufen; Idstein (Schulz-Kirchner) 2002, S. 84-96.

Biedenkopf, Kurt: Wachstum schafft noch keine Arbeit, in: Die Zeit, 25.11.2004, S. 8. 
Bildungsplan für die Realschule, Geschichte; Stuttgart (Ministerium für Kultus und Sport Baden-Württemberg) 1994.

Bildungsstandards für Geschichte Realschule Klassen 6, 8, 10; Stuttgart

(Ministerium für Kultus und Sport Baden-Württemberg) 2004.

Bildungsstandards für den Fächerverbund Erdkunde - Wirtschaftskunde -

Gemeinschaftskunde Realschule Klassen 6, 8, 10; Stuttgart (Ministerium für Kultus und Sport Baden-Württemberg) 2004.

Borries, Bodo v.: Kolonialgeschichte und Weltwirtschaftssystem. Europa und Übersee zwischen Entdeckungs- und Industriezeitalter 1492-1830; Düsseldorf (Schwann) 1986, bes. 122ff., 132ff., 382ff. (= Geschichtsdidaktik. Studien, Materialien 36), 2. Aufl. Münster/Hamburg (Lit) 1992, bes. S. 122ff., 132ff., 382ff. (= Global 2).

Borries, Bodo v.: Umweltgeschichte - Vergessene Einsichten und neuartige Herausforderungen, in: Geschichte lernen, Heft 4, 1988, 8-13. Erweiterter Wiederabdruck in: Calließ, Jörg u.a. (Hrsg.): Mensch und Umwelt in der Geschichte; Pfaffenweiler (Centaurus) 1989, S. 353-375. Wiederabdruck in: Borries, Bodo v.: Lebendiges Geschichtslernen. Bausteine zu Theorie und Pragmatik, Empirie und Normfrage; Schwalbach/Ts. (Wochenschau) 2004, S. 58-78 (= Forum Historisches Lernen).

Borries, Bodo v.: Umweltgeschichte im Schullandheim, in: Klenk, Gerald (Hrsg.): Umwelterziehung im Schullandheim; Burgthann-Mimberg (Bayr. Akad. für Schullandheimpädagogik) 1990, 155-193 und Bilderheft 1-24. Veränderter Wiederabdruck als: Regionale und lokale Umweltgeschichte. Spurensuche in Gelände, Bildervorrat und Archiv, in: Borries, Bodo v.: Durchbrüche von Wirtschaft und Wissenschaft - Krisen von Umwelt und Innenwelt? Versäumte Lektionen zur Ökologie- und Mentalitätsgeschichte; Herbolzheim (Centaurus) 2006, S. 306-341 (= Reihe Geschichtswissenschaft 51).

Borries, Bodo v.: Lernpotentiale der Umweltgeschichte. Kategoriale Einsichten, regionale Beispiele und praktische Erfahrungen, in: Internationale Schulbuchforschung 12. Jg. 1990, S. 9-33.

Borries, Bodo v.: Umwelt und Natur-Mensch-Verhältnis im Geschichtsunterricht? Perspektiven historisch-ökologischen Lernens am Beispiel "Altchinesische Hochkultur", in: Niemetz, Gerold (Hrsg.): Vernachlässigte Fragen der Geschichtsdidaktik; Hannover (Metzler) 1992, S. 7-46. Veränderter Wiederabdruck als: Umweltdebatte und Natur-Mensch-Verhältnis in der altchinesischen Hochkultur. Habitat-verwüstende Praxis und rational-reflexiver Diskurs, in: Borries, Bodo v.: Durchbrüche von Wirtschaft und Wissenschaft Krisen von Umwelt und Innenwelt? Versäumte Lektionen zur Ökologie- und 
Mentalitätsgeschichte; Herbolzheim (Centaurus) 2006, S. 46-79 (= Reihe Geschichtswissenschaft 51).

Borries, Bodo v. und Tabaczek, Martin: Umwelt- und Technikgeschichte, in: Emer, Wolfgang und Horst, Uwe (Hrsg.): Praxis eines demokratischen Geschichtsunterrichts. Perspektiven - Lernorte - Methoden; Bielefeld (Oberstufen-Kolleg des Landes NW) 1995, S. 83-117 (= Ambos 40). Stark veränderter Wiederabdruck als: Industrialisierung in Deutschland (1870-1914). Technikfortschritt, Naturbelastung und Mentalitätswandel, in: Borries, Bodo v.: Durchbrüche von Wirtschaft und Wissenschaft - Krisen von Umwelt und Innenwelt? Versäumte Lektionen zur Ökologie- und Mentalitätsgeschichte; Herbolzheim (Centaurus) 2006, S. 342-390 (= Reihe Geschichtswissenschaft 51).

Borries, Bodo v.: Didaktische Möglichkeiten und Grenzen der Umweltgeschichte, in: Bayerl, Günter u.a. (Hrsg.): Umweltgeschichte. Methoden - Themen Potentiale; Münster/New York (Waxmann) 1996, S. 309-324.

Borries, Bodo v.: Umweltgeschichte für den Unterricht (Rezension), in: Geschichte lernen 17. Jg., 2004, Heft 97, S. 6-7.

Borries, Bodo v.: Das Verhältnis von Gesellschaft und Natur: Umweltgeschichte im Unterricht, in: Meyer, Torsten und Popplow, Marcus (Hrsg.): Technik, Arbeit und Umwelt in der Geschichte. Günter Bayerl zum 60. Geburtstag; Münster u.a. (Waxmann) 2006, S. 501-513.

Borries, Bodo v.: Durchbrüche von Wirtschaft und Wissenschaft - Krisen von Umwelt und Innenwelt? Versäumte Lektionen zur Ökologie- und Mentalitätsgeschichte; Herbolzheim (Centaurus) 2006 (= Reihe Geschichtswissenschaft 51).

Botkin, James W. u.a.: Das menschliche Dilemma. Zukunft und Lernen; Wien (Fritz Molden) 1979.

Forum Geschichte Bd. 2. Das Mittelalter und der Beginn der Neuzeit, hrsg. von Christoph Kunz u.a.; Berlin (Cornelsen) 2001.

Geschichte konkret Bd. 2. Ein Lern- und Arbeitsbuch, hrsg. von Hans-Jürgen Pandel; Hannover (Schroedel) 1997.

Geschichte (und) Geschehen Bd. 1, Sekundarstufe I, von Ursula Fries u.a.; Leipzig u.a. (Ernst Klett) 2003.

Hauff, Volker (Hrsg.): Unsere gemeinsame Zukunft. Der Brundtlandbericht; Greven (Eggenkamp) 1987.

Körber, Andreas, Schreiber, Waltraud und Schöner, Alexander (Hrsg.):

Kompetenzen historischen Denkens. Ein Strukturmodell als Beitrag zur 
Kompetenzorientierung in der Geschichtsdidaktik; Neuried (ars una) 2007 (= Kompetenzen: Grundlagen - Entwicklung - Förderung, Bd. 2).

Langer-Plän, Martina: Problem Quellenarbeit, in: Gesch. in Wiss. und Unterr. 54. Jg., 2003, S. 319-336.

Mensch und Umwelt (= Praxis Geschichte Heft 4/1997).

mitmischen in Geschichte und Politik Bd. 3 Nordrhein-Westfalen (Hauptschule), von Siegfried Cornell u.a.; Leipzig u.a. (Ernst Klett) 2001.

PISA-Konsortium Deutschland (Hrsg.): PISA 2003 - Der zweite Vergleich der Länder in Deutschland. Was wissen und können Jugendliche?; Münster (Waxmann) 2005.

Prenzel, Manfred u.a. (Hrsg.): PISA 2006: Die Ergebnisse der dritten internationalen Vergleichsstudie; Münster u.a. (Waxmann) 2007.

Radkau, Joachim: Vorsorge und Entsorgung. Geschichte und historischer Augenblick in der Mensch-Umwelt-Beziehung; in: Geschichtsdidaktik 11. Jg. 1986, S. 209-222.

Radkau, Joachim: Mensch und Natur in der Geschichte. Leipzig (Klett) 2002 (= Historisch-politische Weltkunde. Kursmaterialien Geschichte Sekundarstufe II/Kollegstufe).

Rahmenplan Geschichte, Bildungsplan achtstufiges Gymnasium Sekundarstufe I; Freie und Hansestadt Hamburg (Behörde für Bildung und Sport) 2004.

Rahmenplan Geschichte, Bildungsplan gymnasiale Oberstufe; Freie und Hansestadt Hamburg (Behörde für Bildung und Sport) 2004.

Rahmenplan Geschichte/Politik, Bildungsplan Haupt- und Realschule Sekundarstufe I; Freie und Hansestadt Hamburg (Behörde für Bildung und Sport) 2003.

Rahmenplan Gesellschaft, Bildungsplan integrierte Gesamtschule Sekundarstufe I; Freie und Hansestadt Hamburg (Behörde für Bildung und Sport) 2003.

Rüsen, Jörn: Historisches Lernen. Grundlagen und Paradigmen; Köln (Böhlau) 1994.

Schmal, Stephan: Umweltgeschichte. Von der Antike bis zur Gegenwart; Bamberg (C. C. Buchners Verlag) 2001 (= Buchners Edition Geschichte).

Sieferle, Rolf Peter (Hrsg.): Menschen gegen neue Technik; Stuttgart (dva) 1986 (= Bild der Wissenschaft 9/ 1986 - Sonderdruck).

Torney-Purta, Judith, Lehmann, Rainer, Oswald, Hans and Schulz, Wolfram: Citizenship and Education in Twenty-eight Countries. Civic Knowledge and Engagement at Age Fourteen; Amsterdam (IEA) 2001. 
Umwelt und Geschichte (= Geschichtsdidaktik 11. Jg. 1986, Heft 3).

Umweltgeschichte (= Geschichte lernen Heft 4, 1. Jg. 1988).

Umweltgeschichte (= Unterricht Biologie Heft 195, 18. Jg. 1994).

Umweltkonflikte in der Geschichte; Villingen-Schwenningen (Neckar-Verlag) 1995 (= Politik und Unterricht IV/1995).

Unsere gemeinsame Zukunft. Bericht der Weltkommission für Umwelt und Entwicklung; Berlin (Staatsverlag der DDR) 2. Aufl. 1990.

Weniger, Erich: Neue Wege im Geschichtsunterricht; Frankfurt/M. (SchulteBulmke) 4. Aufl. 1969. 


\title{
„Warum wir Umweltgeschichte studieren und erforschen"
}

\author{
Markus Schwarzer, Ole Sparenberg
}

Dieser Beitrag basiert auf den Erträgen mehrerer Diskussionen unter den Kollegiaten ${ }^{1}$ der zweiten Kohorte des Graduiertenkollegs „Interdisziplinäre Umweltgeschichte". Keiner der Kollegiaten hat einen Abschluss im Studienfach Umweltgeschichte erworben, weil es bislang noch nicht als grundständiger Studiengang etabliert ist. Aber alle haben während des Studiums, meist auf ganz unterschiedliche Weise, Kontakt zu umweltgeschichtlichen Fragestellungen bekommen. Diese Vorkenntnisse sind einerseits geprägt durch die jeweiligen Herkunftsdisziplinen Geschichte, Politikwissenschaft, Geographie und Archäologie, Geologie und Biologie, Forstwissenschaft und schließlich Landschaftsarchitektur und -planung. Andererseits ist das umweltgeschichtliche Vorwissen maßgeblich beeinflusst durch individuelle Interessen, die Wahl von Schwerpunkten im Studium und nicht zuletzt auch durch das Interesse der jeweiligen Dozenten an umweltgeschichtlichen Perspektiven.

In der Anfangsphase der internen Diskussionen im Graduiertenkolleg lag daher auch ein Schwerpunkt auf der Verständigung über unterschiedliche Sichtweisen auf umweltgeschichtliche Fragen. Dabei zeigte sich, dass es für das Interesse der Kollegiaten an der Umweltgeschichte durchaus gemeinsame Motive gibt. Diese können in der Differenz zu Aspekten des allgemeinen Umweltbewusstseins folgendermaßen gekennzeichnet werden: Zum einen bezieht sich die Thematisierung

\footnotetext{
${ }^{1}$ Auch in diesem Beitrag ist mit der männlichen stets auch die weibliche Form gemeint.
} 
von „Umwelt“ in populären Medien bisweilen ausschließlich auf aktuelle Problemlagen wie die Knappheit naturaler Ressourcen oder den Klimawandel. Mit einer zum Teil diffusen oder ins Katastrophische gesteigerten Sorge um die Lebensgrundlagen dieser und künftiger Generationen werden dabei Umweltthemen häufig vermischt, auch um die vermeintliche Sensationslust der Leserschaft zu bedienen. Zum anderen scheint in der Öffentlichkeit die Auffassung weit verbreitet zu sein, für die Erforschung der Umwelt seien ausschließlich Naturwissenschaften zuständig. Es wird in der Regel gar nicht nach den historischen Bedingungen von Umweltthemen gefragt. Vielmehr bemüht man entweder eine pauschale Untergangsrhetorik, ohne differenzierte Fortschrittskritik zu üben oder es werden politische Ansätze vorgestellt, die Probleme technisch zu lösen.

Im Gegensatz dazu besteht innerhalb des Kollegs Einigkeit darüber, dass solche populären Auffassungen unzureichend sind. Denn die Erscheinungsformen der Umwelt sind bekanntermaßen nicht ausschließlich naturgegeben, sondern in hohem Maße durch menschliches Verhalten und Handeln geprägt. Daher können Umweltthemen nur aus einer Perspektive angemessen verstanden werden, welche menschliches Verhalten und Handeln im Verlauf der Geschichte als grundlegende Gesichtspunkte in die Forschung einbezieht. Denn Umweltprobleme sind - unabhängig von deren Aktualität - grundsätzlich historisch bedingt. Diese Erkenntnisse, die das populäre Umweltbewusstsein übersteigen, können trotz der unterschiedlichen disziplinären Herkunft als ein gemeinsamer motivierender Ausgangspunkt im Graduiertenkolleg angesehen werden. Dies beinhaltet auch, dass Umweltgeschichte hier weder als Verfallsgeschichte eines vermeintlich natürlichen Idealzustandes konzeptualisiert wird, noch einem technokratischen Fortschrittsoptimismus das Wort geredet werden soll. ${ }^{2}$ Es gilt vielmehr die unterschiedlichen Strömungen der jeweiligen zeitgenössischen Wahrnehmung und Nutzung von Umwelt zu beleuchten und die dabei verwendeten Begriffe, Normen und Wertungen offenzulegen.

\section{Interdisziplinarität im Kolleg und das Verständnis von Umweltgeschichte}

Ein weiterer Ausgangspunkt, der auch Antworten auf die Frage liefert, warum die Kollegiaten Umweltgeschichte studieren, ist die Interdisziplinarität des Graduiertenkollegs. Diese dürfte dem außerordentlichen Facettenreichtum der historischen Umweltthemen ebenso wie bestimmten Strömungen des Zeitgeistes geschuldet sein. Es soll hier nicht die Rede sein von den Schwierigkeiten der Interdisziplinarität, etwa dem Problem, dass die wissenschaftliche Reputation in der Regel diszipli-

\footnotetext{
2 Eine Art von Niedergangsgeschichte war in frühen umwelthistorischen Debatten, die eng mit der Umweltbewegung verbunden waren, durchaus nicht unüblich. Solche Positionen können nach Uekötter in der Umweltgeschichte als überwunden gelten. Frank Uekötter, Umweltgeschichte im 19. und 20. Jahrhundert (Enzyklopädie Deutscher Geschichte; 81), München 2007.
} 
när strukturiert ist, oder die Frage erörtert werden, ob Umweltgeschichte als Disziplin zugleich interdisziplinär wie im Graduiertenkolleg sein kann. ${ }^{3}$ Vielmehr gilt es in diesem Zusammenhang zu betonen, dass trotz solcher Schwierigkeiten die Interdisziplinarität von den Kollegiaten zumeist als spannende Herausforderung angesehen wird. Insbesondere die problembezogene Auseinandersetzung mit theoretischen und methodischen Ansätzen aus dem wissenschaftlichen Umfeld kann bei der Konzeption der Dissertationen im Kolleg als typisch angesehen werden. So werden beispielsweise akteurszentrierte Ansätze aus den Sozialwissenschaften aufgegriffen, kulturwissenschaftliche Konzepte zur Analyse von Naturwahrnehmung herangezogen oder geographische Informationssysteme zum Vergleich historischer Karten. Gerade durch die Diskussion solchermaßen konzipierter Forschungsprojekte im Doktorandenkolloquium kann die interdisziplinäre Ausrichtung, ohne überzogene Erwartungen zu hegen, als Bereicherung und Motivation angesehen werden. Eine Grundlage für diese interdisziplinäre Kommunikation ist vor allem durch die Lektüre umweltgeschichtlicher Basistexte in einem Seminar gelegt worden. Die Auseinandersetzung mit den kontroversen Positionen innerhalb der Umweltgeschichte, wie sie exemplarisch in der Holznotdebatte vorliegen und auch denjenigen Standpunkten, über die ein Konsens besteht, erweiterte den umwelthistorischen Horizont der Kollegiaten. Vor diesem Hintergrund lässt sich die interdisziplinäre Verständigung innerhalb des Graduiertenkollegs durchaus schlagwortartig mit dem gesellschaftlich angestrebten Dialog zwischen den Geistes- und den Naturwissenschaften bezeichnen.

Während der Diskussion der Doktoranden haben sich zwei grundlegende Aspekte des Verständnisses von Umweltgeschichte herauskristallisiert. Beide begründen letztlich auch die Motivation und das Interesse der Kollegiaten an dieser Forschung:

Erstens ist zu betonen, dass umweltgeschichtliche Themen wie die Nutzung natürlicher Ressourcen oder die Eindämmung von Naturgefahren auf kulturell tradierten und sozial eingespielten Sinngebungen und Bedeutungen basieren. Es ist daher ein zentrales Moment umwelthistorischen Forschens, die zeitgenössischen Begriffe und Bilder ebenso wie die Wahrnehmungen und Idealvorstellungen der unterschiedlichen Facetten von „Natur“, „Umwelt“ oder „Landschaft“ zu beleuchten. Wie sahen die Menschen jener Zeit die umgebende Natur; welche Ideen leiteten deren Nutzung und Gestaltung, Verehrung oder Ausbeutung? Eine Auseinandersetzung mit diesen Fragen führt zu der grundlegenden Erkenntnis, dass die Wahrnehmung und Nutzung der Umwelt ebenso wie ihre begrifflichen und bildlichen Repräsentationen einem kulturellen Wandel unterliegen. Daher kommt der

\footnotetext{
${ }^{3}$ Es ist auffallend, dass in den beiden bislang einzigen deutschsprachigen Einführungen zur Umweltgeschichte diese als „historische Subdisziplin“ (Uekötter 2007) bzw. trotz ihres interdisziplinären Charakters „als historisches Fachgebiet“ (Winiwarter und Knoll 2007) behandelt wird. Wegen der geringen Institutionalisierung schlägt Bernd Herrmann vor, weniger von „Disziplin“ als vielmehr von einem „Wissenszusammenhang“ zu sprechen (siehe den Beitrag von Herrmann in diesem Band).
} 
Historisierung dieser grundlegenden Aspekte ein ganz entscheidender Stellenwert innerhalb der Umweltgeschichte zu.

Der zweite Grundgedanke liegt in der mit dem Umweltbegriff verbundenen Fokussierung auf die materiellen Grundlagen menschlicher Gesellschaften. Wenngleich Rohstoffvorkommen oder natürliche Extremereignisse nicht als determinierend für historische Prozesse angesehen werden, so ist die politische, wirtschaftliche und soziale Entwicklung ohne Betrachtung der materiellen Basis nicht angemessen zu verstehen und in gewisser Weise unvollständig. Weil Umweltbedingungen dem menschlichen Handeln Möglichkeiten bieten, aber auch Grenzen setzen, kann Umweltgeschichte einen Beitrag zu einem erweiterten Geschichtsverständnis liefern. In diesem Zusammenhang kann die Interpretation von nicht-schriftlichen Quellen, die mit naturwissenschaftlichen Methoden erschlossen wurden, eine besondere Rolle spielen. ${ }^{4}$

Auf welchem der beiden Aspekte der Schwerpunkt liegt, variiert mit der Art der Fragestellung. Von grundsätzlicher Bedeutung ist jedoch, dass beide Facetten bei der Konzeption umweltgeschichtlicher Arbeiten bedacht werden. Martin Knoll und Verena Winiwarter folgern in ihrer Einführung in die Umweltgeschichte diesbezüglich: „Die Beziehungen zwischen Erfahrungen, Wahrnehmungen, den vielfältigen Formen von Repräsentationen und der materiellen, biophysischen Welt, die als ,Natur' bezeichnet und so behandelt wird, sind dialektisch: Theorien über die Natur sind Repräsentationen, die sich auf die Wahrnehmung von Naturdingen entscheidend auswirken, doch ist die Theorieentwicklung nur auf der Basis von Wahrnehmungen denkbar. ${ }^{{ }^{5}}$ Vor diesem Hintergrund ist Umweltgeschichte - unabhängig davon, ob sie im weiteren Sinn als interdisziplinärer Wissenszusammenhang konzipiert ist oder als historische Disziplin - als eine Wissenschaft zu begreifen, die kritisches Wissen generiert und Orientierung in Bezug auf bestimmte Umweltfragen bietet. Diese Antworten auf die Ausgangsfrage beschreiben zugleich grundlegende Aspekte der gesellschaftlichen Relevanz von Umweltgeschichte. Sie führen jedoch auch zu den Fragen, in welchem Verhältnis das umweltgeschichtliche Wissen zu demjenigen der Herkunfts- oder Nachbardisziplinen steht und in welche Anwendungskontexte das Orientierungswissen der Umweltgeschichte einfließen könnte.

\section{Der Beitrag der Umweltgeschichte zu den „Nachbardisziplinen“}

Wie eingangs erwähnt, stammen die Stipendiaten des Kollegs aus einer großen Bandbreite unterschiedlicher Disziplinen. Ein weiteres Argument für die Umweltgeschichte besteht darin, dass sie zu den einzelnen Herkunftsdisziplinen einen Bei-

\footnotetext{
${ }^{4}$ Vgl. dazu auch die Ausführungen in der Einleitung dieses Bandes von Kreye und Schwarzer.

${ }^{5}$ Verena Winiwarter/ Martin Knoll, Umweltgeschichte. Eine Einführung, Stuttgart 2007, S. 299.
} 
trag leisten kann. ${ }^{6}$ Die Umweltgeschichte wurzelt nicht nur in vielen verschiedenen etablierten universitären Fächern, sie kann ihrerseits diesen Disziplinen helfen, bessere, also aussagekräftigere und vollständigere Resultate hervorzubringen.

Was die naturwissenschaftlichen „Ursprungsdisziplinen“ von der Umweltgeschichte übernehmen können, ist eine bestimmte Art der Historisierung ihrer Untersuchungsgegenstände. Für die Biologie, die Forstwissenschaften und die Geowissenschaften, die zwar auch eine eigene Fachgeschichte betreiben, liegt der Nutzen einer umwelthistorischen Betrachtung darin, dass der Umweltzustand sich oftmals besser aus einer Perspektive verstehen lässt, in der gesellschaftliche Veränderungen stärker in den Blick genommen werden als in den jeweiligen Fachgeschichten. Das gilt für Magerrasen, Waldformen oder Flusstäler nicht weniger als für Bergbaulandschaften. Gemeint ist nicht die letztlich triviale Erkenntnis, dass heutige Zustände in einer zeitlichen Abfolge entstanden sind, sondern dass ihre Ausformung wesentlich auf menschliches Handeln bzw. auf nichtintendierte Folgen dieses Handelns zurückgeht. Der anthropogene Einfluss springt bei einem Bergbaugebiet meist ins Auge, bei anderen Landschaften oder Biotopen wird er gerne übersehen. Um diesen Einfluss des Menschen verstehen zu können ist aber schon der interdisziplinäre Brückenschlag nötig, also die Verwendung historischer Quellen und die Beachtung der kulturellen Dimension von menschlichen Verhaltensweisen. Menschliche Einflussnahme folgt häufig eben nicht nur ökonomischen oder physiologischen Notwendigkeiten, sondern kulturell tradierten Vorstellungen. Der Forstwissenschaft dient die Geschichte auch zur Selbstvergewisserung ihrer Profession und Tradition. ${ }^{7}$ Das ist im Einzelnen kritisch zu hinterfragen, wirkt aber mit Sicherheit zumindest unterschwellig als kulturelles Leitbild wieder auf den Ist-Zustand des Waldes zurück.

Ein weiterer Gewinn für die Biologie sowie Forst- und Geowissenschaften liegt in der Erschließung neuer Daten durch die Umweltgeschichte. Schriftliche, historische Quellen bedürfen sicherlich einer ganz anderen Untersuchungsweise nämlich einer geschichtswissenschaftlichen Analyse und Deutung - als naturwissenschaftlich gewonnene Daten. So würde beispielsweise die Rekonstruktion früherer Landschaftszustände anhand naturwissenschaftlicher Daten ohne die Interpretation schriftlicher Quellen ein nur unzureichendes Bild historischer Landnutzungsformen ergeben.

Die Landschaftsarchitektur und -planung ist ebenso wie Teile der Forstwissenschaft anwendungsorientiert. Planerische Konzepte zur Gestaltung der Landschaft folgen bestimmten Leitbildern. Beispielsweise soll die Wiederherstellung eines vermeintlich „,natürlichen“ Zustandes gemäß populärer Forderungen von Naturschützern und Landschaftsplanern durch das Zulassen der „natürlichen Dynamik“

\footnotetext{
${ }^{6}$ Während auch von Winiwarter und Knoll dieser Aspekt betont wird, fällt auf, dass er in der engen Fassung von Umweltgeschichte als „,historische Subdisziplin“ bei Uekötter ausgeblendet wird.

7 Vgl. zu der älteren Forstgeschichte und ihrer Lesart der Holznot-Debatte des 18. und 19. Jahrhunderts: Frank Uekötter, Umweltgeschichte im 19. und 20. Jahrhundert (Enzyklopädie Deutscher Geschichte; 81), München 2007, S. 51-56.
} 
entstehen wie es das mittlerweile auch im Naturschutzgesetz verankerte Konzept des Prozessschutzes vorsieht. Diese Sichtweise lässt sich als naturalistisch und ahistorisch bezeichnen. Denn die kulturelle Dynamik von Traditionen und Praktiken einer Gegend, die zu dem aktuellen Zustand geführt haben und diesen prägen, werden ausgeblendet zugunsten jener Auffassung, dynamisch sei nur „die Natur“" und menschliches Handeln ein „Eingriff“" oder eine „Störung“. Dies lässt sich exemplarisch an den Konflikten zwischen der lokalen Bevölkerung und der Verwaltung der Nationalparke Schleswig-Holsteinisches Wattenmeer bzw. Bayerischer Wald erkennen. ${ }^{8}$ Die anwendungsorientierten Professionen zur Planung und Gestaltung der Landschaft können grundsätzlich von einer umwelthistorischen Perspektive profitieren: Denn Leitbilder, die zum Teil aus der Gesellschaft an sie herangetragen werden, gilt es kritisch zu reflektieren, damit diese angemessen und nachvollziehbar begründet in demokratische Entscheidungsprozesse einfließen können.

Im Fall der Geschichtswissenschaft ist das Defizit, dem die Umweltgeschichte abhelfen kann, offensichtlich nicht das Fehlen einer historischen Perspektive. Der Beitrag der Umweltgeschichte zur (allgemeinen) Geschichtswissenschaft liegt vielmehr darin, das Augenmerk auf die materiellen Grundlagen menschlicher Gesellschaften zu richten. ${ }^{9}$ Gemeint sind nicht ein historischer Materialismus im marxistischen Sinne und auch kein ausschließlicher Umweltdeterminismus. Aber was die Umweltgeschichte ins Licht rücken kann, ist die Bedeutung der konkreten belebten und unbelebten Natur, also von Wasser, Luft, Boden, mineralischen Ressourcen, Pflanzen und Tieren. Die Geschichte von Politik, Wirtschaft und gesellschaftlichen Beziehungen war zu allen Zeiten zwar nicht determiniert, aber tief geprägt von der sie umgebenen materiellen Welt, von Stoff- und Energieflüssen. Es ist an sich trivial, wird aber in der Geschichtswissenschaft oft nicht thematisiert, dass Menschen unumgehbar darauf angewiesen sind, zu essen, zu trinken, zu wohnen, sich zu kleiden und sich Werkzeuge zu verschaffen. Für all dies sind sie auf die sie umgebende materielle Natur angewiesen. Der somit notwendige Zugriff auf die Umwelt wird in der Industriegesellschaft nicht weniger sondern intensiver.

Der grundsätzliche Beitrag der Umweltgeschichte für die Geschichtswissenschaft besteht darin, dies bewusst zu machen und das Geflecht der unterschiedlichen Akteure, deren leitende Konzepte und ihr Handeln zu analysieren. Zudem

\footnotetext{
8 Vgl. zu dieser Problematik: Manfred Jakubowski-Tiessen, „Ökodiktatur? Nein danke!“ Der Streit um den „Nationalpark Schleswig-Holsteinisches Wattenmeer“, in: Marie Luisa Allemeyer / Manfred Jakubowski-Tiessen / Salvador Rus-Rufino (Hrsg.), Von der Gottesgabe zur Ressource. Konflikte um Wald, Wasser und Land in Spanier und Deutschland seit der Frühen Neuzeit. Essen 2007, S. 85 96. Für den Bayerischen Wald siehe: Ellen Brouns, Ist Wildnis planbar? Werte- und Interessenskonflikte in der raumbezogenen Umweltplanung anhand von ausgewählten Wildnisprojekten. München 2003.

${ }^{9}$ Ellen Stroud, Does Nature Always Matter? Following Dirt Through History, in: History and Theory, Theme Issue 42 (December 2003), S. 75-81. Eine Synthese aus Geschichte und Natur wurde in der Historiographie - oft allerdings in der Gestalt eines geographischen und klimatischen Determinismus - bereits seit der Antike wiederholt angestrebt, vgl.: Joachim Radkau, Natur und Macht. Eine Weltgeschichte der Umweltgeschichte, München 2002, S. 11.
} 
kann die Umweltgeschichte sowohl einerseits neue Blickwinkel auf bekannte Quellen eröffnen als auch andererseits neue, verstärkt auch nichtschriftliche, naturwissenschaftlich gewonnene Quellen in ihre Interpretation einbeziehen. Die Umweltgeschichte hilft somit der Geschichtswissenschaft, ein umfassenderes Bild der Vergangenheit zu entwerfen. Ähnliches gilt für die Ur- und Frühgeschichte als archäologische Disziplin, die allerdings stets schon von ihrer Quellenbasis her materiellen Aspekten gegenüber aufgeschlossener war als die klassische Geisteswissenschaft Geschichte. Jedoch gilt auch hier, dass ohne die Einbeziehung von Umweltaspekten ein prägnantes Bild einer menschlichen Gemeinschaft nicht zu gewinnen ist.

Diese Beispiele, inwiefern umwelthistorische Perspektiven die Herkunfts- und Nachbardisziplinen der Kollegiaten bereichern können, zeigen: Umweltgeschichte hat innerhalb des universitären Wissenschaftsbetriebes ihre Berechtigung, indem sie gemäß ihrem interdisziplinären Charakter bestimmten natur- und geisteswissenschaftlichen Disziplinen helfen kann, jeweils einige spezifische blinde Flecken zu überwinden.

\section{Die Relevanz von Umweltgeschichte außerhalb des Wissenschaftsbetriebes}

Eine sich herausbildende Disziplin wie die Umweltgeschichte sollte sich ihrer gesellschaftlichen Relevanz auch außerhalb des rein wissenschaftlichen Feldes bewusst sein. Weil längst nicht für jeden Kollegiaten einst einmal ein Lehrstuhl bereitsteht, sollte es daher (im Bereich der Lehre) darum gehen, Voraussetzungen zu schaffen, damit Absolventen auch in nicht-wissenschaftlichen Berufsfeldern Fuß fassen können. Obwohl die Umweltgeschichte kein direkt anwendbares Wissen produziert, sollten doch Brücken gebaut werden zwischen Umwelthistorikern und den dezidiert anwendungsorientierten Umweltwissenschaften bzw. umweltbezogenen Praxisfeldern. Heute wird zwar kaum jemand mehr behaupten wollen, man könne aus der Geschichte lernen, in der Weise, dass die Geschichte ein auf die Gegenwart unmittelbar übertragbares Erfahrungswissen bereithalte, ${ }^{10}$ und auch die Umweltgeschichte kann nicht versprechen, dass dem, der sie studiert hat, alle Handlungschancen und -folgen in der Beziehung zur Umwelt offenbar werden. Es lassen sich aber dennoch gute Gründe dafür anführen, dass Umwelthistoriker auch in anwendungsorientierten Feldern einen wertvollen Beitrag leisten können.

Ein weites Feld, das hier in Frage kommt, ist die Wissensvermittlung. Im Journalismus sind Umweltthemen seit einigen Jahrzehnten immer wieder aktuell. Daher ist auch hier Bedarf für umwelthistorisches Hintergrundwissen, nicht zuletzt als kritisches Korrektiv in öffentlichen Debatten. Für die Vermittlung von Geschichte in Schulen und Museen oder auch im Journalismus gilt Ähnliches wie für

\footnotetext{
${ }^{10}$ Benjamin Herzog, Historia magistra vitae, in: Stefan Jordan (Hrsg.), Lexikon Geschichtswissenschaft. Hundert Grundbegriffe, Stuttgart 2002, S. 145-147.
} 
die Geschichtswissenschaft: ${ }^{11}$ Die Einbeziehung von materiellen Umweltbeziehungen ermöglicht es, ein vollständigeres Bild von der Vergangenheit zu zeichnen. Außerdem nimmt die Beschäftigung mit der Umwelt heutzutage einen hohen Stellenwert in der Öffentlichkeit ein. Der Blick auf umwelthistorische Fragestellungen kann also ein neuer Weg sein, um Menschen für Geschichte zu interessieren. Bei der Vermittlung von naturwissenschaftlichen Zugängen zu Umweltthemen schadet es dagegen gewiss nicht, stets die Historisierung kulturell geprägter Vorstellungen, Begriffe und Bildern von Natur im Auge zu behalten.

Eine weitere Nische für Umwelthistoriker kann man in den Feldern Politikberatung, Verwaltung, NGOs oder auch privaten Planungsbüros ausmachen, die sich im weiteren Sinne mit Umweltfragen beschäftigen. ${ }^{12}$ Hier kann die Umweltgeschichte wiederum Hintergrundwissen bereitstellen über die Geschichtlichkeit von bestimmten Zuständen, die anderenfalls fälschlicherweise als „natürlich“ angenommen werden. Dies ermöglicht eine kritische Reflexion über Konzepte und Leitbilder. Ein historischer Blick auf Umweltplanung und Landschaftsgestaltung im weitesten Sinne wird auch ins Gedächtnis rufen, dass solche Prozesse meist mit Konflikten einhergehen: Stets ist der Nutzen mit Kosten verbunden; selten sind Nutzen und Kosten gleichmäßig auf die Menschen verteilt worden. Nicht zuletzt kann der Praktiker profitieren von umweltgeschichtlichen Einsichten in die Auswirkungen und Grenzen menschlicher Planung und Gestaltung. Ein weiteres, direkt anwendungsbezogenes Tätigkeitsfeld für Umwelthistoriker bildet die Rolle als Sachverständiger für die Untersuchung und Bewertung von Altstandorten, deren Böden möglicherweise Schadstoffe oder auch Krankheitserreger enthalten. ${ }^{13}$ Hier sind die Begutachtung von altlastverdächtigen Grundstücken und die Anlage von Altlastenkatastern, die belastete Standorte verzeichnen, unmittelbar auf umwelthistorische Recherchen angewiesen.

Gründe, Umweltgeschichte zu studieren und zu erforschen, gibt es also viele. Die Kollegiaten sind überzeugt, dass die Umweltgeschichte zu jedem der genannten Felder etwas beizusteuern hat und dass dieser Beitrag auch benötigt wird. Offen bleibt noch die Frage, ob dieser Beitrag auch angenommen und nachgefragt wird: Ist es dem jungen Fach Umweltgeschichte bisher gelungen, andere akademische Disziplinen und außeruniversitäre Berufsfelder von ihrer Berechtigung und ihrem Wert zu überzeugen? Es scheint, dass es eine klare gesellschaftliche Relevanz von Umweltgeschichte gibt, diese sollte jedoch über die fachlichen und wissenschaftlichen Grenzen hinaus stärker kommuniziert und damit auch popularisiert werden.

\footnotetext{
11 Vgl. zum Stellenwert der Umweltgeschichte im Geschichtsunterricht den Beitrag von Borries' in diesem Band.

12 Zur Umweltpolitik auf der nationalen und internationalen Ebene vgl. die Beiträge von Müller und Uekötter in diesem Band.

13 Vgl. den Beitrag von Schlottau in diesem Band.
} 


\section{Literatur:}

Brouns, Ellen: Ist Wildnis planbar? Werte- und Interessenskonflikte in der raumbezogenen Umweltplanung anhand von ausgewählten Wildnisprojekten. München: Ökom-Verlag, 2003.

Herzog, Benjamin: Historia magistra vitae, in: Stefan Jordan (Hrsg.), Lexikon Geschichtswissenschaft. Hundert Grundbegriffe, Stuttgart: Reclam, 2002, S. 145-147.

Jakubowski-Tiessen, Manfred: „Ökodiktatur? Nein danke!“ Der Streit um den „Nationalpark Schleswig-Holsteinisches Wattenmeer“. In: Marie Luisa Allemeyer / Manfred Jakubowski-Tiessen / Salvador Rus-Rufino (Hrsg.), Von der Gottesgabe zur Ressource. Konflikte um Wald, Wasser und Land in Spanier und Deutschland seit der Frühen Neuzeit. Essen: Klartext-Verlag, 2007, S. 85-96.

Radkau, Joachim: Natur und Macht. Eine Weltgeschichte der Umweltgeschichte, München: C.H. Beck, 2002.

Stroud, Ellen: Does Nature Always Matter? Following Dirt Through History, in: History and Theory, Theme Issue 42 (December 2003), S. 75-81.

Uekötter, Frank: Umweltgeschichte im 19. und 20. Jahrhundert (Enzyklopädie Deutscher Geschichte; 81), München: Oldenbourg, 2007.

Winiwarter, Verena / Knoll, Martin: Umweltgeschichte. Eine Einführung, Stuttgart: UTB, 2007. 



\section{Die Autoren}

Bodo von Borries, Prof. Dr., geboren 1943, Studium der Geschichte und Germanistik, 1968 Promotion in Verfassungs-, Sozial- und Wirtschaftsgeschichte und erstes Staatsexamen, hauptberufliche hochschul- und bildungspolitische Arbeit, 1972 zweite Staatsprüfung, danach Schul- und Hochschultätigkeit. Von 1976 bis 2008 Professor an der Universität Hamburg für Erziehungswissenschaft unter besonderer Berücksichtigung der Didaktik der Geschichte, mit den üblichen Neben-Aktivitäten als Herausgeber, Juror, Beirat, Fellow, Fortbildner, Vortragsreisender, Gutachter... Seit 1988 drittmittelfinanzierte empirische Untersuchungen zum Geschichtsbewusstsein von Jugendlichen in der ,alten“ Bundesrepublik, im ostdeutsch-westdeutschen (1990, 1992) und im osteuropäisch-westeuropäischen Kulturvergleich in 27 Ländern (1995), Publikationen auch in über einem Dutzend Fremdsprachen.

Bernd Herrmann, Prof. Dr., geboren 1946, ist seit 1978 Professor für Anthropologie der Biologischen Fakultät und kooptiertes Mitglied des Seminars für Mittlere und Neuere Geschichte der Universität Göttingen, mit Arbeitsschwerpunkten zur Biologie historischer menschlicher Bevölkerungen, zur Biologischen Spurenkunde und der Umweltgeschichte. Er ist Sprecher des Göttinger Arbeitskreises Umweltgeschichte und des Graduiertenkollegs 1024 „Interdisziplinäre Umweltgeschichte“. Herrmann war Fellow des Berliner Wissenschaftskollegs und ist Mitglied der Nationalen Akademie der Wissenschaften LEOPOLDINA. Er ist einschlägig Verfasser von Aufsätzen vor allem zu biologisch ausgerichteten Themen der Umweltgeschichte, zuletzt zur Schädlingsthematik.

Lars Kreye, geboren 1972 in Lemgo, studierte Geschichte und Sozialwissenschaften für das Lehramt Sek. II an der Universität Bielefeld und der Johns Hopkins University in Baltimore (USA). Er promoviert seit Juli 2007 an der Universität 
Göttingen am DFG-Graduiertenkolleg 1024 „Interdisziplinäre Umweltgeschichte“. Der Arbeitstitel seines Dissertationsprojekts lautet: „Der Deutsche Wald in Übersee: transnationale koloniale Forstwirtschaft in Tansania, 1891-1916.

Christof Mauch, Prof. Dr., geboren 1960, studierte Geschichte, Literaturwissenschaft, Philosophie, Ev. Theologie, Religious Studies, Drama und Sprachen an den Universitäten Tübingen, am King's College und Leo Baeck College London sowie an der Universidad de Salamanca. Promotion 1990 an der Universität Tübingen. Habilitation 1998 an der Universität Köln. Er unterrichtete an den Universitäten Tübingen, Bonn und Köln und war Gastprofessor an der American University in Washington. Von 1999 bis 2007 war er Direktor des Deutschen Historischen Instituts in Washington. Er veröffentlichte zahlreiche Arbeiten zur amerikanischen und deutschen Umwelt- und Politikgeschichte. Seit 2007 lehrt er als Professor für Amerikanische Geschichte und transatlantische Beziehungen am Amerika-Institut der Ludwig-Maximilians-Universität München.

Birgit Metzger, Dipl. Kulturwissenschaftlerin, geboren 1978 in Marburg, studierte Historisch orientierte Kulturwissenschaften und Interkulturelle Kommunikation an der Universität des Saarlandes in Saarbrücken und der Université Laval in Kanada. Sie schloss ihr Studium ab mit einer Diplomarbeit zum Thema „Kreative Milieus in der Stadt - Geschichte und Gegenwart. Chinatown an der Saar?". Seit 2006 ist sie Doktorandin am Freiburger DFG-Projekt „Und ewig sterben die Wälder. Das deutsche ,Waldsterben' im Spannungsfeld von Wissenschaft und Politik. Zur Funktionsweise von Politikberatung und modernem Umweltschutz." Sie arbeitet an einer Dissertation zum Thema „Das Waldsterben als Politikum in der Bundesrepublik der 1980er Jahre.“

Kurt Möser, Dr. phil. habil, geboren 1955 in Memmingen / Allgäu, studierte Literaturwissenschaft und Sozial- und Technikgeschichte an der Universität Konstanz. Promotion 1982. Er unterrichtete an den Universitäten Erlangen, Mannheim und St. Gallen und war DAAD-Lektor an den Universitäten Oxford und New Delhi. 1987 wurde er Konservator am Landesmuseum für Technik und Arbeit in Mannheim und betreut die Sammlungen und Ausstellungen zur Mobilitätsgeschichte. Er veröffentlichte zahlreiche Arbeiten zur Mobilität und zu Kultur und Technik. Von 2006 bis 2008 war er abgeordnet an das Institut für Geschichte der Universität (TH) Karlsruhe. Er habilitierte sich 2007 mit einer Arbeit zu „Fahren und Fliegen in Frieden und Krieg - Kulturen individueller Mobilitätsmaschinen 1880 bis 1930“.

Edda Müller, Dipl. Pol., Dr. res publ., Studium an der ENA, Honorarprofessorin für Politikwissenschaft an der Hochschule für Verwaltungswissenschaft Speyer. Bis Juli 2007 Vorstand des Verbraucherzentrale Bundesverbands e.V. Zuvor Vizedirektorin der Europäischen Umweltagentur, Kopenhagen, Leiterin der Abteilung Klimapolitik des Wuppertal Instituts, Ministerin für Natur und Umwelt des Landes 
Schleswig-Holstein, Ministerialdirigentin im Bundesministerium für Umwelt, Naturschutz und Reaktorsicherheit, zuständig u.a. für Klimapolitik. Weitere berufliche Stationen: Umweltbundesamt, Planungsabteilung des Bundeskanzleramts, Verfassungsabteilung des Bundesministeriums des Innern. Edda Müller hat sich in zahlreichen Veröffentlichungen mit Fragen der Umwelt-, Klima- und Verbraucherpolitik sowie dem politischen Interessensausgleich in modernen Demokratien beschäftigt.

Josef H. Reichholf, Prof. Dr., geboren 1945 in Aigen am Inn, Niederbayern, studierte Biologie, Chemie, Geographie und Tropenmedizin an der Universität München. Er promovierte 1969 über die Biologie wasserlebender Schmetterlinge. Neben der Leitung Hauptabteilung Wirbeltiere und der Sektion Ornithologie der Zoologischen Staatssammlung in München lehrte er an beiden Münchner Universitäten in Evolutionsbiologie und Tiergeographie (LMU, bis 2005), Ökologie und Naturschutz (Technische Universität München bis 2008). Forschungsreisen führten ihn nach Südamerika, Afrika, Südasien und Australien sowie zu Inseln im Indischen und Pazifischen Ozean. Er ist Autor von mehr als 30 Büchern und erhielt zahlreiche wissenschaftliche Auszeichnungen.

Roland Schäfer, Dipl.-Forstwirt, geboren 1978 in Pinneberg, studierte Forstwissenschaften an der Albert-Ludwigs-Universität Freiburg und an der ETSIAM in Córdoba/Spanien. Sein Studium beschloss er mit einer Diplomarbeit zum Thema „Landschaftsdarstellung und -symbolik im Kinofilm ,Der Herr der Ringe - Die Gefährten"،. Nach dem Studium folgte eine Ausbildung zum Fach-/ Wissenschaftsredakteur am mibeg-Institut Medien, Köln. Anschließend arbeitete er als Lektor für Lehrfilme bei Hagemann Bildungsmedien in Düsseldorf. Seit 2006 ist er Doktorand in einem DFG-Projekt zur Geschichte des Waldsterbens an der Universität Freiburg und behandelt dort insbesondere die Rolle der Wissenschaft in der Waldsterbensdebatte.

Klaus Schlottau, Dr., geboren 1954 in Mölln/Hzgt. Lauenburg, studierte nach einer Ausbildung zum Chemielaboranten Volkswirtschaftslehre, Philosophie, Politologie sowie Sozial- und Wirtschaftsgeschichte in Hamburg. Nach dem Magisterexamen wurde er mit einer technikhistorischen Arbeit zum Dr. phil. promoviert. Von 1987 bis 1992 leitete er das Industriemuseum und das Stadtarchiv Neumünster und eröffnete dann das umwelthistorische Büro Histinvest. Seit 1995 wissenschaftlicher Mitarbeiter der Arbeitsstelle „Sozialgeschichte der Technik und Umwelt" am Institut für Sozial- und Wirtschaftsgeschichte der Universität Hamburg, seit Oktober 2004 am Historischen Seminar, Arbeitsbereich Deutsche Geschichte, Schwerpunkt Sozial- und Wirtschaftsgeschichte.

Markus Schwarzer, Dipl. Ing., geboren 1974, ist seit Juli 2007 Doktorand im Graduiertenkolleg „Interdisziplinäre Umweltgeschichte“ in Göttingen. Er untersucht den planerisch-gestalterischen Diskurs zur Bergbaufolgelandschaft in Ost- 
deutschland nach 1990. Von 2001-2007 studierte er Landschaftsarchitektur und Umweltplanung an der Technischen Universität München. Von 1997-2001 absolvierte er eine Ausbildung im Garten- und Landschaftsbau und war berufstätig in Heidelberg und Erlangen.

Ole Sparenberg, M. A., geboren 1976, arbeitet als Stipendiat des Graduiertenkollegs 1024 „Interdisziplinäre Umweltgeschichte“ an einem Projekt zu Fischerei und Walfang im Rahmen der nationalsozialistischen Autarkiepolitik in den 1930er Jahren. Er studierte Mittlere und Neuere Geschichte, Politikwissenschaft sowie Öffentliches Recht an der Georg-August-Universität in Göttingen und der Universidad Complutense, Madrid.

Manfred Jakubowski-Tiessen, Prof. Dr., ist Lehrstuhlinhaber am Seminar für Mittlere und Neuere Geschichte an der Universität Göttingen und stellvertretender Sprecher des Graduiertenkollegs 1024 „Interdisziplinäre Umweltgeschichte“. Zuvor war er Professor am Max-Planck-Institut für Geschichte in Göttingen. Veröffentlichungen zur Sozial-, Mentalitäten-, Umwelt- und Kirchengeschichte der Frühen Neuzeit. Buchveröffentlichungen u.a.: Sturmflut 1717. Die Bewältigung einer Naturkatastrophe in der Frühen Neuzeit, 1992; (Hg.), Krisen des 17. Jahrhunderts. Interdisziplinäre Perspektiven, 1999; (Hg. zus. mit K.-J. Lorenzen-Schmidt), Dünger und Dynamit. Beiträge zur Umweltgeschichte Schleswig-Holsteins und Dänemarks, 1999; (Hg. zus. mit H. Lehmann), Um Himmels Willen: Religion in Katastrophenzeiten, 2003; (Hg), Religion zwischen Kunst und Politik: Aspekte der Säkularisierung im 19. Jahrhundert, 2004.

Frank Uekötter, Dr. phil., geboren 1970 in Münster, studierte Geschichte, Sozialwissenschaften und Politikwissenschaft an den Universitäten Freiburg und Bielefeld sowie der Johns Hopkins University in Baltimore (USA). Er promovierte 2001 an der Universität Bielefeld mit einer Arbeit zur Geschichte der Luftreinhaltung und organisierte 2002 den Fachkongress "Naturschutz und Nationalsozialismus“ im Auftrag des Bundesumweltministeriums und forscht seither zur Wissensgeschichte der Landwirtschaft. Zu seinen Veröffentlichungen zählt der Band „Umweltgeschichte im 19. und 20. Jahrhundert“ in der Reihe „Enzyklopädie Deutscher Geschichte" des Oldenbourg-Verlags. 
limawandel, Artensterben, Viehseuchen und Naturkatastrophen bestimmen vielerorts die Debatten um die globale Zukunft. Hierbei erscheint es oft, als seien diese Phänomene völlig neuartige Entwicklungen, die erst jetzt unsere Aufmerksamkeit geweckt haben. Dabei sind die meisten gegenwärtigen Probleme durchaus nicht neu. Frühere Generationen haben sich sehr wohl Gedanken um ihre Umwelt gemacht und viele der gegenwärtigen Diskussionen sind historisch bedingt. Daher erfordert die Bewältigung unserer Probleme nicht nur zukunftsorientiertes Denken, sondern auch ein historisches Verständnis der politischen, ökonomischen, sozialen und ökologischen Hintergründe unserer gegenwärtigen Situation.

Unter den Themenkomplexen „Spektrum der Umweltgeschichte“, „Aus der Geschichte lernen“, „Fast vergessene Debatten der Umweltgeschichte“ und "Umweltgeschichte im Bildungssystem“ erörtern die Autoren die Bedeutung der Umweltgeschichte für die Lösung zukünftiger Probleme.

Der vorliegende Band ist das Ergebnis eines Workshops, den das DFG-Graduiertenkolleg "Interdisziplinäre Umweltgeschichte. Naturale Umwelt und gesellschaftliches Handeln in Mitteleuropa" am 16. und 17. Juli 2008 in Göttingen unter dem Titel „Umweltgeschichte und Umweltzukunft - Zur gesellschaftlichen Relevanz einer jungen Disziplin" veranstaltet hat. 